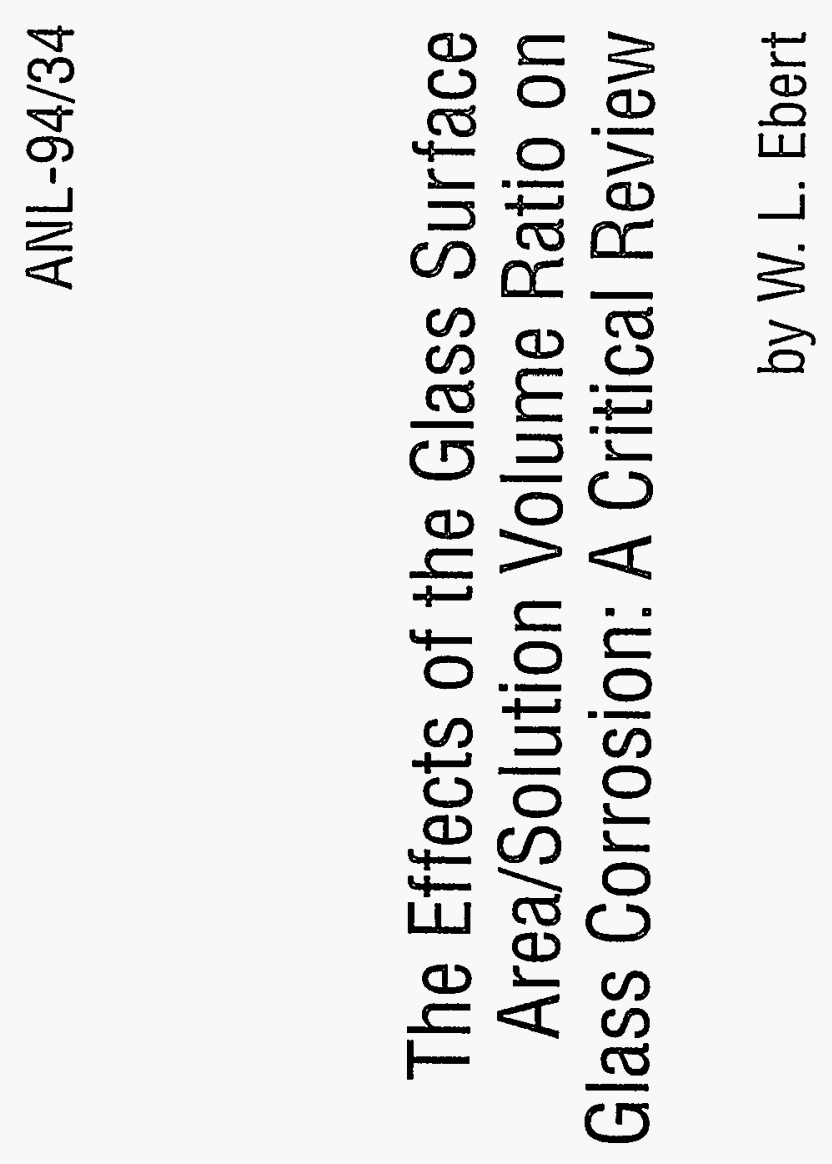

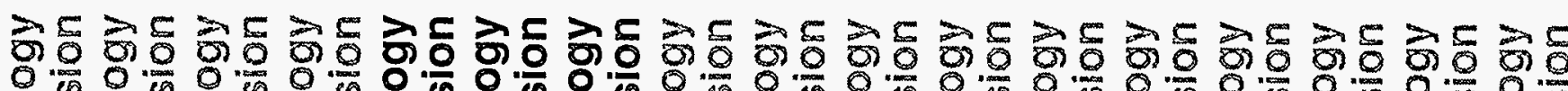

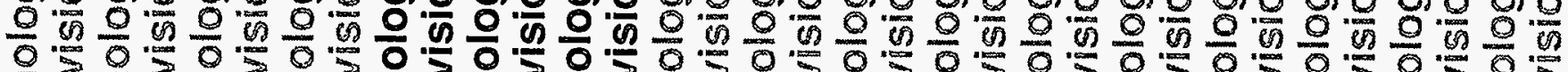

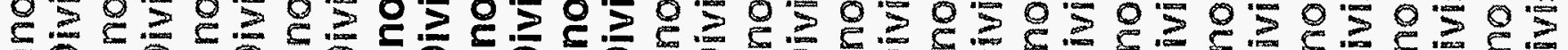

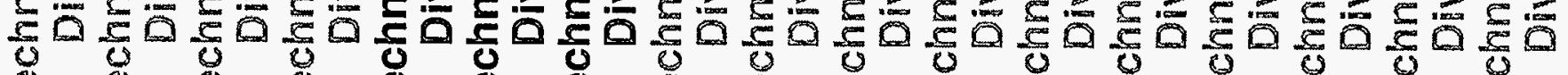

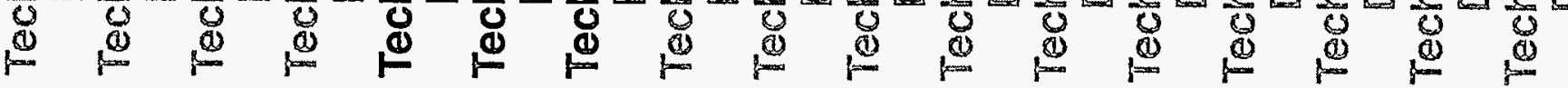

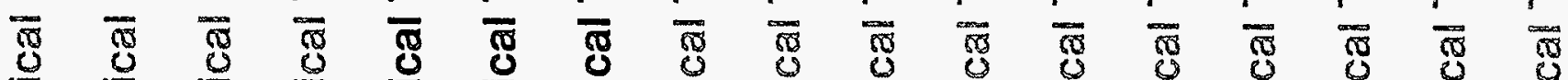

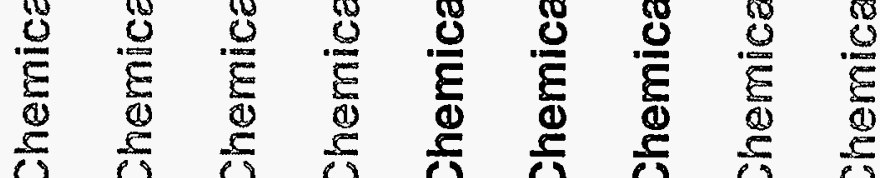

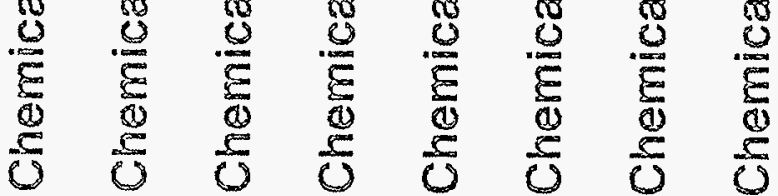

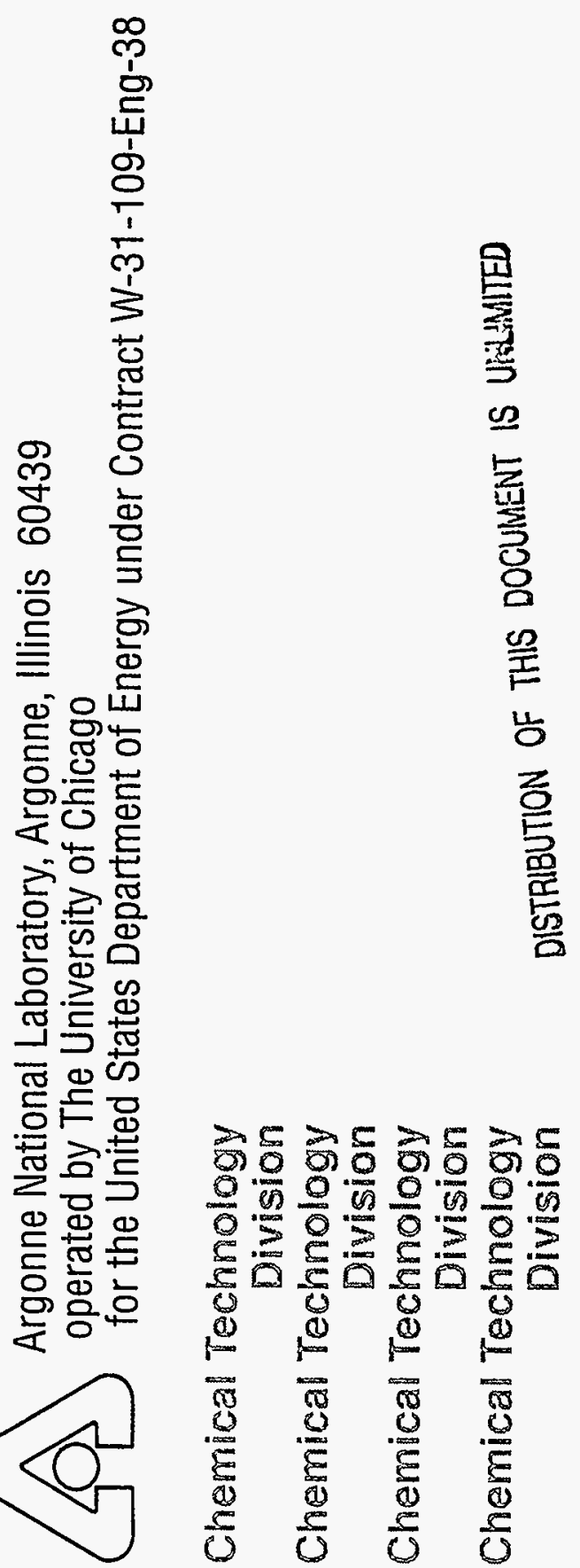


Argonne National Laboratory, with facilities in the states of Illinois and Idaho, is owned by the United States government, and operated by The University of Chicago under the provisions of a contract with the Department of Energy.

\section{DISCLAIMER}

This report was prepared as an account of work sponsored by an agency of the United States Government. Neither the United States Government nor any agency thereof, nor any of their employees, makes any warranty, express or implied, or assumes any legal liability or responsibility for the accuracy, completeness, or usefulness of any information, apparatus, product, or process disclosed, or represents that its use would not infringe privately owned rights. Reference herein to any specific commercial product, process, or service by trade name, trademark, manufacturer, or otherwise, does not necessarily constitute or imply its endorsement, recommendation, or favoring by the United States Government or any agency thereof. The views and opinions of authors expressed herein do not necessarily state or reflect those of the United States Government or any agency thereof.

Reproduced from the best available copy.

Available to DOE and DOE contractors from the Office of Scientific and Technical Information P.O. Box 62

Oak Ridge, TN 37831

Prices available from (615) 576-8401

Available to the public from the National Technical Information Service

U.S. Department of Commerce 5285 Port Royal Road Springfield, VA 22161 


\section{DISCLAIMER}

Portions of this document may be illegible in electronic image products. Images are produced from the best available original document. 
Distribution Category:

High-Level Radioactive Waste Disposal in Tuff

(UC-814)

ANL-94/34

\section{ARGONNE NATIONAL LABORATORY}

9700 South Cass Avenue

Argonne, Illinois 60439

\section{THE EFFECTS OF THE GLASS SURFACE AREA/SOLUTION VOLUME RATIO ON GLASS CORROSION: A CRITICAL REVIEW}

by

William L. Ebert

Chemical Technology Division

March 1995

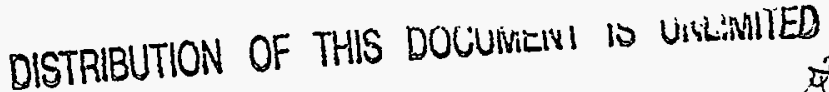




\section{TABLE OF CONTENTS}

Page

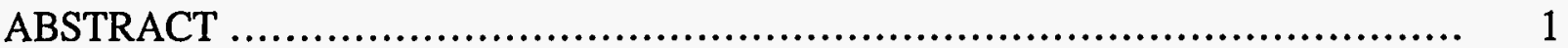

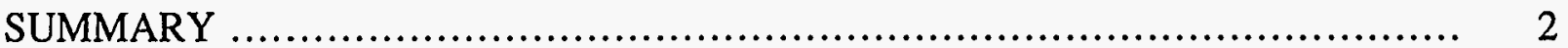

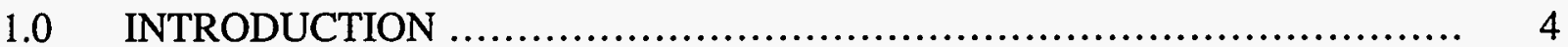

2.0 DESCRIPTION OF GLASS STRUCTURE ................................. 7

$2.1 \quad$ Chemical Description of Glass Corrosion ............................... 9

2.1.1 Alkali Release ..................................................... 9

2.1.2 Water Diffusion ............................................... 12

2.1.3 Dealkalization ................................................ 12

2.1.4 Release of Network-Forming Elements ......................... 16

2.1.5 Glass Corrosion .............................................. 17

2.2 Experimental Measures of the Extent of Glass Corrosion ................ 17

2.3 Measurement of the Corrosion Rate ...................................... 21

2.4 A Phenomenological Model of Corrosion Behavior ...................... 22

$2.5 \quad$ Standard Test Methods .................................................... 25

2.5.1 Static Tests ...................................................... 27

2.5.1.1 MCC-1 Test ......................................... 27

2.5.1.2 Product Consistency Test ............................ 32

2.5.1.3 MCC-3 Solubility Test .............................. 34

2.5.1.4 Argonne National Laboratory Vapor

Hydration Tests ...................................... 35

2.5.1.5 Buffered Solution/pH-Stat Tests ................... 36

2.5.2 Dynamic Tests ............................................ 37

2.5.2.1 Soxhlet Tests ...................................... 37

2.5.2.2 Single-Pass Flow-Through Tests .......................... 37

2.5.2.3 Periodic Replenishment Tests ......................... 38

2.6 Measurement of Surface Area ............................................ 40

$2.7 \quad$ Effect of Surface Finish ..................................................... 46

$2.8 \quad$ Measurement of Leachant Volume ................................................ 47

$2.9 \quad$ Changes in $\mathrm{SA} / \mathrm{V}$ as the Glass Corrodes ............................. 48

3.0 OBSERVED EFFECTS OF THE SOLUTION CHEMISTRY

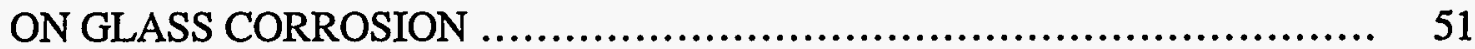

3.1 Tests Conducted to Distinguish between Mass Transport and Surface Reaction Effects .......................................... 52

3.2 Observed Effects of Silicic Acid Concentration on Glass Corrosion ............................................................... 60

3.3 Observed Effects of the Leachate $\mathrm{pH}$ on Glass Corrosion ................. 63

3.4 Observed Effects of Other Species on Glass Corrosion .................. 72

3.5 Glass Corrosion under Silicon Saturated Conditions:

The Residual Rate ...................................................... 73

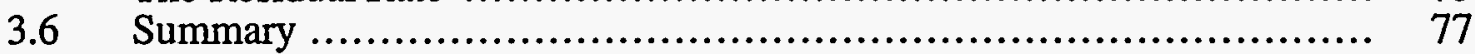




\section{TABLE OF CONTENTS (contd.)}

Page

4.0 OBSERVED EFFECTS OF THE GLASS SURFACE AREATO-SOLUTION VOLUME RATIO IN STATIC TESTS $\ldots \ldots \ldots \ldots \ldots \ldots \ldots \ldots \ldots . . . \ldots$

4.1 Effects of SA/V on the Solution Chemistry ........................... 78

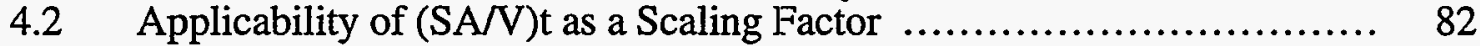

4.3 Modeling the Effects of the Solution $\mathrm{pH}$.............................. 92

4.4 Effects of SA/V on Alteration Layers ................................... 95

4.5 Tests Conducted to Attain Highly Concentrated Solutions ............... 97

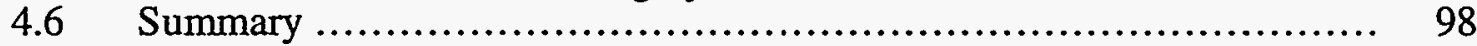

5.0 OBSERVED EFFECTS OF THE FLOW RATES IN DYNAMIC

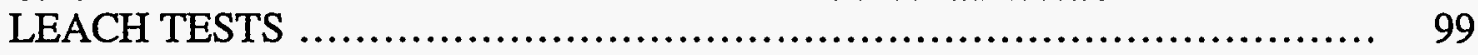

6.0 DETERMINATION OF MODEL PARAMETERS FROM LABORATORY TESTS

7.0 EFFECTS OF SA/V ON RADIONUCLIDE DISPOSITION $\ldots \ldots \ldots \ldots \ldots \ldots \ldots \ldots \ldots$

7.1 Summary of the Chemistries of Tc, $\mathrm{U}, \mathrm{Np}, \mathrm{Pu}$, and $\mathrm{Am} \ldots \ldots \ldots \ldots \ldots \ldots . . . . .117$

7.2 Experimental Measurement of Radionuclide Release .................... 119

7.2.1 Tests Conducted under Dilute Solution Conditions ............... 119

7.2.2 Tests under Concentrated Solution Conditions .................. 127

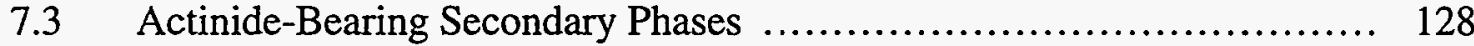

7.4 Effects of Other Materials on Actinide Disposition ...................... 129

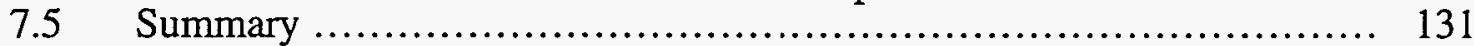

8.0 SUMMARY OF CRITICAL REVIEW OF EFFECTS OF SA/V ON GLASS CORROSION AND DISCUSSION OF REMAINING

UNCERTAINTIES

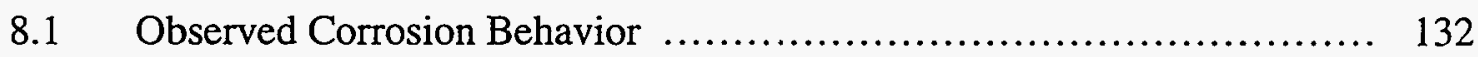

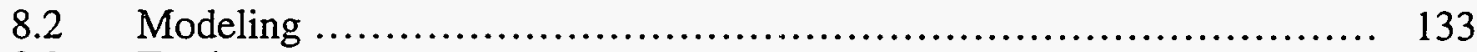

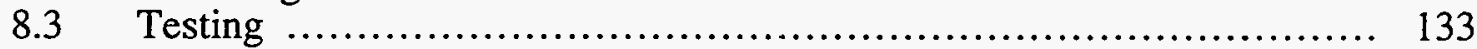

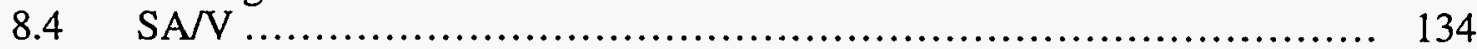

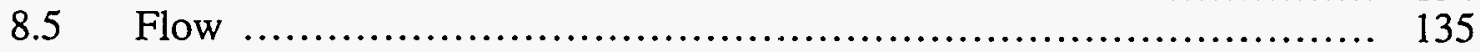

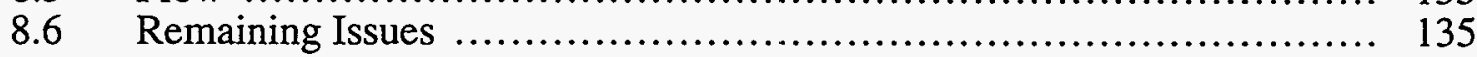

9.0 ANL TASK "RELATIONSHIP BETWEEN HIGH SA/V TESTS

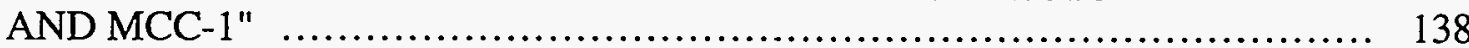

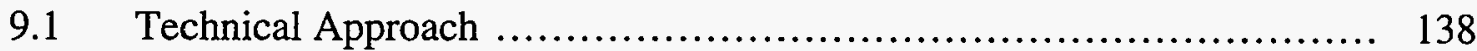

9.2 Observed Effects of the SA/V on the Leachate Chemistry $\ldots \ldots \ldots \ldots \ldots \ldots . . . . .141$

9.2.1 Leachate $\mathrm{pH}$................................................... 141

9.2.2 Glass Matrix Components .................................... 143

9.2.3 Observed Effects of SA/V on the Behavior

of Radionuclides ............................................. 153

9.3 Effects of the SA/V on the Alteration of the Glass Surface ................... 161

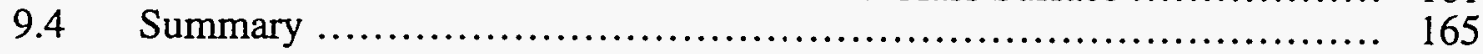




\section{TABLE OF CONTENTS (contd.)}

Page

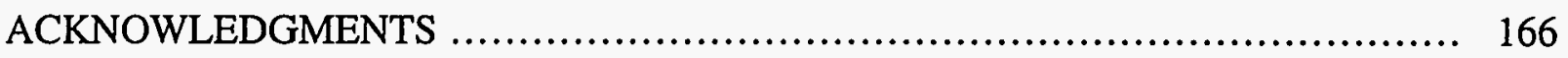

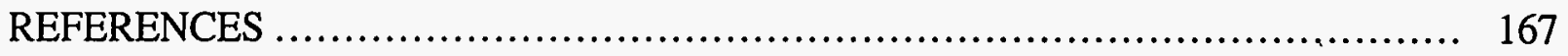

APPENDIX A. Definitions ................................................... 187

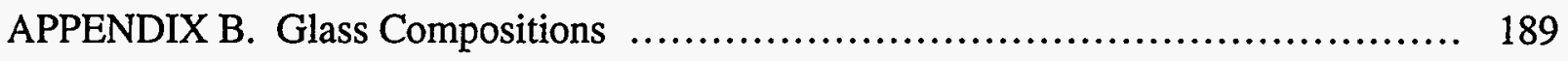

APPENDIX C. Derivation of Rate Equation .................................... 191

APPENDIX D. Data Generated in the Study of the Relationship between High SA/V Tests and MCC-1 Tests .................................. 196 


\section{LIST OF FIGURES}

No.

Title

Page

1. Schematic Plot of the Fraction of $\equiv \mathrm{SiO}$ - Sites Associated with

Protons or Other Cations as a Function of $\mathrm{pH}$ in $1 \times 10^{-3} \underline{\mathrm{M}}$

Cation Solutions

2. Schematic Plot of the Fraction of $\equiv \mathrm{AlO}^{-}, \equiv \mathrm{BO}^{-}$, and $\equiv \mathrm{SiO}^{-}$

Sites Associated with Protons or Sodium as a Function of $\mathrm{pH}$

in $1 \times 10^{-3} \underline{\mathrm{M}}$ Cation Solutions

3. Measured Concentrations of Sodium, Hydrogen, and Excess

Hydrogen at the Surface after Corrosion in Silicate Solution at $100^{\circ} \mathrm{C}$

4. Product Consistency Tests Showing Nonstoichiometric Release

of Glass Components

5. Schematic Plot Showing Instantaneous and Integrated Rates

for a Specific Reaction Time "A" on a Typical Release Curve

6. Schematic Plot Showing Corrosion Behavior as the

Corrosion Progresses

7. Measured Boron Concentrations in 28-Day Replacement Tests

8. Photomicrographs of $-100+200$ Mesh Size Fraction of Crushed Glass

9. Test Results for Different Size Fractions of Crushed Glass

10. Photomicrograph of Corroded SRL EA Glass Grain

11. Variations of MCC-1 Test with Glass A

12. Variations of MCC-1 Test with Glass B

13. Variations of MCC-1 Test with Glass C

14. Variations of MCC-1 Test with MCC 76-68 and

C31-3 Glass in Deionized Water

15. Variations of MCC-1 Test with MCC 76-68 and

C31-3 Glass in $\mathrm{MgCl}_{2}$ Solution

16. Measured Extent of Corrosion vs. Time for Tests with

Variously Treated Samples

17. Boron Release from PNL 76-68 Glass vs. Silicic Acid

Concentration Synthetic Solutions and Groundwaters 


\section{LIST OF FIGURES (contd.)}

No. $\quad$ Title $\quad \underline{\text { Page }}$

18. Glass Corrosion Rate vs. the Ion Activity Product of Silicic Acid .............. 61

19. Release of $\mathrm{B}, \mathrm{Na}, \mathrm{C}, \mathrm{Al}$, and $\mathrm{Si}$ from CSG Glass as a

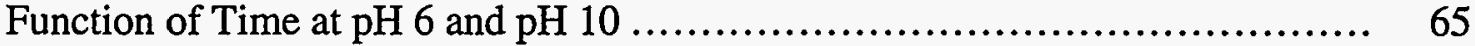

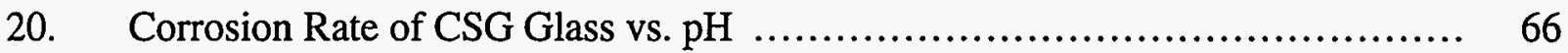

21. Corrosion Ratio of R7T7 Glass vs. pH ....................................... 66

22. Alkali Release Rates from Simple Glasses vs. $\mathrm{pH}$............................ 67

23. Silicon Release from Fused Silica vs. $\mathrm{pH}$ and Silicon Release

vs. $\mathrm{pH}$ from $33 \mathrm{Li}_{2} \mathrm{O} \cdot 67 \mathrm{SiO}_{2}$ and $15 \mathrm{~K}_{2} \mathrm{O} \cdot 85 \mathrm{SiO}_{2}$ Glasses ................... 69

24. Release of Sodium, Calcium, and Si from

$20 \mathrm{Na}_{2} \mathrm{O} \cdot 20 \mathrm{CaO} \cdot 60 \mathrm{SiO}_{2}$ vs. $\mathrm{pH}$

25. Release of Aluminum and Sodium vs. $\mathrm{pH}$ from Glass 1

and from Glass 2

26. Effects of $\mathrm{Si}, \mathrm{Al}, \mathrm{Mg}$, and $\mathrm{Ca}$ on CSG Dissolution

27. Normalized Mass Loss of Boron and Silicon from

C-31-3EC Glass in $\mathrm{NaCl}$ Solutions at $200^{\circ} \mathrm{C}$

28. Normalized Sodium Mass Loss and $\mathrm{pH}$ vs. Time at

Different SA/V Ratios

29. Measured $\mathrm{pH}$ and $\mathrm{Si}$ in Tests with PNL 76-68 Glass

Conducted in Deionized Water at Different SA/V Ratios

30. Concentration vs. (SA/V)t for Release of Boron and

Silicon from PNL 76-68 Glass at $90^{\circ} \mathrm{C}$

31. Measured Boron Concentrations in Tests with SRL 165

Glass vs. (SA/V)t

32. Boron Release vs. (SA/V)t in Tests of SRP Black Frit

Glass at Various SA/V Ratios

33. Measured Silicic Acid Concentration vs. (SA/V)t for Tests

with SRL 165 Glass at SA/V Ratios of 7.9 and $85 \mathrm{~m}^{-1}$

34. Measured Silica Concentration vs. Log Time for Tests

with $\mathrm{Li}_{2} \mathrm{O} \cdot 2 \mathrm{SiO}_{2}$ Glass

35. Corrosion of HW-39 Glass at Various SA/V Ratios 


\section{LIST OF FIGURES (contd.)}

No.

Title

Page

36. Measured Concentrations of Boron and Silica in Tests

with R7T7 Glass

37. Measured Boron Concentration vs. (SA/V)t and Measured $\mathrm{pH}$

vs. Time for Tests with SRL 202 Glass at SA/V Ratios of

10,2000 , and $20,000 \mathrm{~m}^{-1}$

38. Normalized Mass Loss vs. Reaction Time for Tests with

SRL 200R and SRL 200S Glasses at $20,000 \mathrm{~m}^{-1}$

39. Mass Loss vs. Corrosion Time for SRL 131 Glass at

$90^{\circ} \mathrm{C}$ at Various Flow Rates

40. Results Obtained by Adiga et al. Plotted as Glass Corrosion

Rate vs. Flow

41. Measured Boron Concentration in Partial Replenishment

Tests Conducted at Constant $\mathrm{T}_{\mathrm{r}}=112$ Days

42. Boron Release from SRL 131 Glass as a Function of Flow

Rate as Simulated by Periodic Replenishment of the Solution

43. Corrosion Rate vs. Ion Activity Product of Silicon

44. Normalized Boron Mass Loss from MW Glass vs. Reaction Time

45. Log of Normalized Boron Mass Loss vs. $\log (\mathrm{SA} / \mathrm{V}) \mathrm{t}$ for

Tests with WV205A.01, ECM85-13, WV205, and SRL 165 Glasses

46. Release of Boron from SRL 202A Glass as $\log [B]$ vs.

$(\mathrm{SA} / \mathrm{V}) \mathrm{t}$ for Tests at $\mathrm{SA} / \mathrm{V}$

47. Measured Normalized Mass Losses in Tests with

Actinide-Doped R7T7 Glasses in Tests at $90^{\circ} \mathrm{C}$ and $50 \mathrm{~m}^{-1}$

48. Measured Normalized Mass Losses in Tests with

Actinide-Doped R7T7 Glasses in Tests at $50^{\circ} \mathrm{C}$ and $50 \mathrm{~m}^{-1}$

49. Calculated Retention Factors vs. Reaction Time for Tests at $90^{\circ} \mathrm{C}$ and $50^{\circ} \mathrm{C}$ with R7T7 Glass Doped with $\mathrm{U}, \mathrm{Np}$,

$\mathrm{Pu}-238, \mathrm{Pu}-239$, and $\mathrm{Am}-241$

50. Normalized Mass Loss of Plutonium-238 from SRL 165

Glass in Tests at $100 \mathrm{~m}^{-1}$ Based on ${ }^{238} \mathrm{Pu}$ in Solution and

${ }^{238} \mathrm{Pu}$ in Solution Plus on Vessel Walls 


\section{LIST OF FIGURES (contd.)}

No.

Title

$\underline{\text { Page }}$

51. Calculated Retention Factors and Measured $\mathrm{pH}$ Values for

Tests with Actinide-Doped SRL 165 Glass at Tests at $30 \mathrm{~m}^{-1}$

52. Measured $\mathrm{pH}$ Values in ANL Tests with SRL 202A Glass, SRL 202U Glass, and SRL $131 \mathrm{~A}$ Glass in Tests at $90^{\circ} \mathrm{C}$ at $10,340,2000$, and $20,000 \mathrm{~m}^{-1}$ in EJ-13 Water and at 340, 2000 , and $20,000 \mathrm{~m}^{-1}$ in Deionized Water

53. Measured Cation Concentrations in ANL Tests with SRL 202A Glass Conducted at $90^{\circ} \mathrm{C}$ at 10,2000 , 20,000 , and $20,000 \mathrm{~m}^{-1}$

54. Measured Cation Concentrations in ANL Tests with SRL 131A

Glass Conducted at $90^{\circ} \mathrm{C}$ at 10,2000 , and $20,000 \mathrm{~m}^{-1}$

55. Measured Cation Concentrations in ANL Tests with SRL 202U

Glass Conducted at $90^{\circ} \mathrm{C}$ at 340 and $20,000 \mathrm{~m}^{-1}$ in EJ-13

and at 340,2000 , and $20,000 \mathrm{~m}^{-1}$ in Deionized Water

56. Normalized Boron Mass Loss from Glasses vs. Reaction

Time in Tests Conducted at ANL

57. Logarithm of Measured Boron Concentration vs. Logarithm

of (SA/V)t for Tests with SRL 131A, SRL 202A, and

SRL 202U Glasses Conducted at SA/V of 10, 340, 2000,

and $20,000 \mathrm{~m}^{-1}$ in EJ-13 Water, and 340, 2000, and

$20,000 \mathrm{~m}^{-1}$ in Deionized Water

150

58. Normalized Mass Loss vs. Reaction Time for Tests with

SRL 202A Glass at $2000 \mathrm{~m}^{-1}$, Tests with SRL 202A Glass

at $20,000 \mathrm{~m}^{-1}$, Tests with SRL $131 \mathrm{~A}$ Glass at $2000 \mathrm{~m}^{-1}$,

and Tests with SRL $131 \mathrm{~A}$ Glass at $20,000 \mathrm{~m}^{-1}$

155

59. Photomicrographs of Alteration Layers Formed on

SRL 202U Glass

162 


\section{LIST OF TABLES}

No.

Title

Page

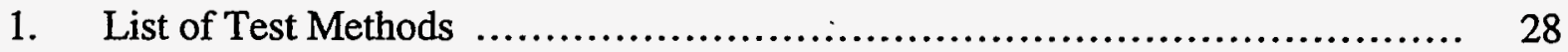

2. Analysis of Leachants by Participants in the MCC Round

Robin of the MCC-1 Test .................................................. 31

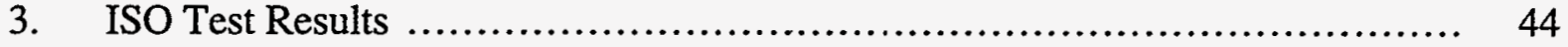

4. Compositions of Three Glasses Used by Chick and Pederson f................. 52

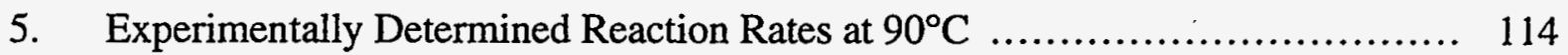

6. Leach Rates from UK 208 Glass in Distilled Water at $60^{\circ} \mathrm{C} \ldots \ldots \ldots \ldots \ldots \ldots \ldots . . \ldots 121$

7. Compositions of Tuff Groundwater from Well J-13 and

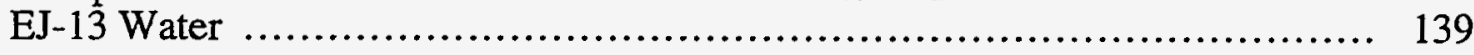

8. Summary of Test Matrix for ANL Task ................................... 140

9. Results of Filtering Leachate Solutions from Tests with SRL 202U Glass through $6 \mathrm{~nm}$ Filters

10. Anion Concentrations in Tests with SRL 131 and

SRL 202 Glasses

11. Normalized Mass Losses for Radionuclides .................................. 154

12. Mass of Actinides in Various Fractions …................................ 157

13. Highest Actinide Concentrations Measured in Leach Tests ................... 158

14. Radionuclide Retention Factors for Tests with SRL 202A

and SRL 131A Glasses

15. Measured Composition of Alteration Layers Formed on

SRL 202 Glass Reacted in DIW at $20,000 \mathrm{~m}^{-1}$ for 364 Days ................... 164

16. Measured d-Spacings from Selected Area Electron

Diffraction Analysis of SRL 202 Glass Reacted at

$20,000 \mathrm{~m}^{-1}$ for 364 Days 


\title{
THE EFFECTS OF THE GLASS SURFACE AREA/SOLUTION VOLUME RATIO ON GLASS CORROSION: A CRITICAL REVIEW
}

\author{
William L. Ebert
}

\begin{abstract}
This report reviews and summarizes the present state of knowledge regarding the effects of the glass surface area/solution volume (SA/V) ratio on the corrosion behavior of borosilicate waste glasses. The SA/V ratio affects the rate of glass corrosion through the extent of dilution of corrosion products released from the glass into the leachate solution: glass corrosion products are diluted more in tests conducted at low SA/V ratios than they are in tests conducted at high SA/V ratios. Differences in the solution chemistries generated in tests conducted at different $\mathrm{SA} / \mathrm{V}$ ratios then affect the observed glass corrosion behavior. Corrosion of borosilicate waste glasses results in the dissolution of soluble components and the accumulation of sparingly soluble components in a layer on the surface of the corroding glass. Laboratory tests performed to distinguish the effects of the solution chemistry and the surface layer on the glass corrosion rate have shown the rate to be controlled primarily by the solution chemistry. Therefore, any testing parameter that affects the solution chemistry will also affect the glass corrosion rate. The results of static leach tests conducted to assess the effects of the SA/V are discussed with regard to the effects of SA/V on the solution chemistry. The effects of the SA/V ratio observed in static tests can be understood based on the effects of the solution chemistry on glass corrosion behavior. Test results show several remaining issues with regard to the long-term glass corrosion behavior: Can the SA/V ratio be used as an accelerating parameter to characterize the advanced stages of glass corrosion relevant to long disposal times? Is the alteration of the glass surface the same in tests conducted at different SA/V, and in tests conducted with monolithic and crushed glass samples? What are the effects of the SA/V and the extent of glass corrosion on the disposition of released radionuclides? These issues will bear on the prediction of the long-term performance of waste glasses during storage. The results of an experimental program conducted at ANL to address these and other remaining issues regarding the effects of $\mathrm{SA} / \mathrm{V}$ on glass corrosion are described.
\end{abstract}




\section{SUMMARY}

This report reviews and summarizes the present state of knowledge regarding the effects of the glass surface area/solution volume ratio (SA/V) on the corrosion behavior of borosilicate waste glasses. The effects of the SA/V occur through dilution of corrosion products and through the effects of the corrosion products on the glass corrosion mechanism and rate. Therefore, the effects of the SA/V on glass corrosion are discussed in terms of the various effects of the solution chemistry. This is accomplished by first presenting a brief summary of glass structure, the processes leading to the alteration and dissolution of the glass, and laboratory tests that have been applied to characterize waste glass corrosion. This background information is required to characterize and quantify the corrosion rate and mechanism, and the influence of the SA/V on the corrosion behavior. Laboratory tests performed to elucidate the corrosion process are then reviewed to assess how various test results are related to the corrosion process and the importance of the solution chemistry on glass corrosion. The results of static leach tests conducted to assess the effects of the SA $N$ are then discussed based on what is known about the effects of the solution chemistry on glass corrosion. Of course, the understanding of glass corrosion behavior, the effects of the solution chemistry, the effects of the SA/V, and the interpretation of test results has not been achieved independently. The understanding of the effects of test conditions and various aspects of the solution chemistry has advanced simultaneously through the accumulation of a wide and varied data base. Remaining issues identified through the literature review include uncertainties regarding the use of tests at high $\mathrm{SA} / \mathrm{V}$ to attain advanced stages of glass corrosion relevant to long disposal times, the relationship between tests conducted at different SA/V and between tests with monolithic and crushed glass samples, and the effects of the SA/V and the extent of glass corrosion on the disposition of released radionuclides. Finally, the results of an experimental program conducted at ANL to address remaining issues regarding the effects of SA/V on glass corrosion are summarized. Many of the published results are utilized in the literature review sections where they provide insight into the corrosion behavior and the effects of the SA/V.

Work done to distinguish the effects of the fluid chemistry and glass alteration layers on glass dissolution has shown that the effects of the fluid chemistry dominate the glass corrosion rate. The silicic acid concentration and the $\mathrm{pH}$ affect the rate most strongly, with the reaction to release silicic acid usually being modeled as the key corrosion step. The rate of silicic acid release from the glass depends primarily on the temperature, the glass composition, the concentration of silicic acid in solution, and the solution $\mathrm{pH}$. For a given glass composition at a given temperature, any test conditions that affect the silicic acid concentration or the solution $\mathrm{pH}$ will affect the glass corrosion rate. The silicic acid concentration and the solution $\mathrm{pH}$ are affected by several factors: the release of other glass components as the glass corrodes; the volume of solution available to dilute released glass components; the refreshment of the leachate solution by solution flow, if any; and the formation of secondary phases. The relative importance of these will be determined by the glass and initial fluid compositions, and by the test method and test conditions used in a particular experiment. In general, the glass and solution compositions are determined by the waste form and the disposal site, while the test conditions may be selected to simulate particular environmental conditions, highlight a particular reaction step, or characterize the general corrosion behavior.

Glass corrosion is well-described by a rate equation that combines a rate coefficient that depends on the glass composition, a $\mathrm{pH}$ dependence, and a reaction affinity term expressed as the difference between the actual silicic acid concentration and a "saturation" value. Use of this equation requires the experimental determination of (1) the rate coefficient and its temperature dependence, (2) the $\mathrm{pH}$-dependence of the rate coefficient, and (3) the saturation value to characterize the corrosion behavior of each particular glass. In addition, secondary phases that precipitate from highly concentrated solutions and control the silicic acid concentration after 
long corrosion progress must be identified. The corrosion of borosilicate waste glasses can be described as occurring in three stages, which are related to the solution chemistry. The first stage occurs when the solution concentration of silicic acid is very low and the glass corrodes at a relatively high rate that depends on the glass composition and the solution $\mathrm{pH}$. This is sometimes referred to as the "forward" or "intrinsic" rate, since it depends on the glass composition and is not affected by the affinity term. The second stage occurs as the silicic acid concentration increases to a maximum level and the glass corrosion rate decreases to a minimum value. The decrease in the corrosion rate is due to a decrease in the reaction affinity. The maximum concentration is usually referred to as the "saturation concentration" for the particular glass, as if the solution was saturated with respect to the glass phase. Of course, the solution cannot equilibrate with the thermodynamically metastable glass phase. However, the corrosion behavior of high-level waste glasses behaves as if the solution approaches saturation. The third stage occurs when secondary phases precipitate from solution to establish a silicic acid solubility limit that may be lower than the "saturation" concentration established by the glass during the second stage of corrosion. This affects the rate through the affinity term. We emphasize that secondary phases do establish thermodynamic solubility limits for glass components in contrast to the glass itself, which establishes "apparent" solubility limits.

Both the glass and leachant compositions and the testing method will determine which stage or stages of glass corrosion are observed in laboratory tests, and laboratory tests can be used to highlight particular aspects of the corrosion process. Tests with large solution volumes relative to the exposed glass surface area, such as MCC-1 and flow-through tests, will maintain dilute conditions and will be unaffected or weakly affected by silicic acid in solution. These tests are used to determine the intrinsic corrosion rate and the $\mathrm{pH}$-dependence of the corrosion rate. Tests that allow silicic acid to accumulate in the solution, such as the MCC-3 test and the Product Consistency Test (PCT), show the effects of the affinity term on the corrosion rate and the approach to saturation. Under some test conditions, secondary phases will precipitate from the saturated solutions and the corrosion process may enter the third corrosion stage, although reaction times of several months or years may be required before precipitation occurs.

The corrosion behavior of high-level waste glasses in these three stages must be known to project their long-term performance. The SA/V ratio used in laboratory tests provides a means of driving the corrosion progress to these different stages within reasonable testing times to characterize the corrosion behavior of a waste glass. Characterization of the disposal site and anticipated storage conditions can be used to determine which corrosion stages will be important during the service life of the storage site. Test results describing the corrosion behavior can then be coupled with anticipated storage conditions in existing performance models to project glass durability over long-time periods. 


\subsection{INTRODUCTION}

This report reviews and summarizes the present state of knowledge regarding the effects of the glass surface area/solution volume ratio (SA/V) on the corrosion behavior of borosilicate waste glasses. The effects of the SA $\mathrm{V}$ occur through dilution of corrosion products and through the effects of the corrosion products on the glass corrosion mechanism and rate. Therefore, the effects of the SA/V on glass corrosion are discussed in terms of the various effects of the solution chemistry. This is accomplished by first presenting a brief summary of glass structure, the processes leading to the alteration and dissolution of the glass structure, and laboratory tests that have been applied to characterize waste glass corrosion. This background information is required to characterize and quantify the corrosion rate and mechanism, and the influence of the $\mathrm{SA} / \mathrm{V}$ on the corrosion behavior. Laboratory tests performed to elucidate the corrosion process are then reviewed to assess how various test results are related to the corrosion process and the importance of the solution chemistry on glass corrosion.

The results of static leach tests conducted to assess the effects of the SA/V are then discussed based on what is known about the effects of the solution chemistry on glass corrosion. Of course, the understanding of glass corrosion behavior, the effects of the solution chemistry, the effects of the $S A / V$, and the interpretation of test results has not been achieved independently. The understanding of the effects of test conditions and various aspects of the solution chemistry has advanced simultaneously through the accumulation of a wide and varied data base. Remaining issues identified through the literature review include uncertainties regarding the use of tests at high $S A / V$ to attain advanced stages of glass corrosion relevant to long disposal times, the relationship between tests conducted at different $S A / V$ and between tests with monolithic and crushed glass samples, and the effects of the SA/V and the extent of glass corrosion on the disposition of released radionuclides. Finally, the results of an experimental program conducted at ANL to address remaining issues regarding the effects of SA $V$ on glass corrosion are summarized. Many of the published results are utilized in the literature review sections where they provide insight into the corrosion behavior and the effects of the SA/V.

Stabilization of high-level waste destined for disposal in a geologic repository will be accomplished by fixing the waste in a borosilicate glass matrix, pouring the glass into a stainless steel canister, placing the canister in a metal container, and placing the assembled waste package in a mined repository. The repository will be engineered so that radioactive waste components will be isolated from the biosphere for the tens of thousands of years that some will remain toxic. The radioactivity of high-level waste will be dominated initially by fission products that disintegrate primarily through beta decay (mainly ${ }^{90} \mathrm{Sr}\left[\mathrm{t}_{1 / 2}=28 \mathrm{yr}\right]$ and ${ }^{137} \mathrm{Cs}\left[\mathrm{t}_{1 / 2}=30 \mathrm{yr}\right]$, which will account for about $95 \%$ of the radioactivity for the initial 100 years [1]). While the presence of these radionuclides is an issue during waste vitrification, they are relatively shortlived and will be of minor consequence after about 500 years. Radionuclides having longer halflives disintegrate primarily through alpha decay (mainly ${ }^{239} \mathrm{Pu}\left[\mathrm{t}_{1 / 2}=24,400 \mathrm{yr}\right],{ }^{240} \mathrm{Pu}$ $\left[\mathrm{t}_{1 / 2}=6580 \mathrm{yr}\right]$, and $\left.{ }^{241} \mathrm{Am}\left[\mathrm{t}_{1 / 2}=458 \mathrm{yr}\right]\right)$. These radionuclides will become the dominant source of radioactivity after about 500 years. Thus, while fission products present significant handling and processing difficulties, high-level radioactive waste forms must be designed to stabilize the long-lived transuranic elements for a period of on the order of 10,000 years or longer.

Radionuclides will be isolated from the accessible environment by multiple barriers. For radionuclides to be mobilized from emplaced waste forms, liquid water must contact and corrode first the container, then the canister, and then the waste glass. Radionuclides could then be released from the glass matrix as it corrodes and could enter the fluid either as dissolved or as 
suspended material. The radionuclides could then be transported away from the repository by groundwater flow. Subsequent interactions with the surrounding geology could result in the radionuclides being removed from the groundwater by filtration or sorption onto mineral surfaces. Assessment of the suitability of a disposal system for the storage of radioactive waste requires that the performance of each barrier be evaluated. This report summarizes the current knowledge of the chemical durability of high-level waste glasses.

Corrosion and the subsequent dissolution of a glass waste form occur when interaction with a corrosive fluid causes chemical bonds to break and reform, generating an energetically more stable system. While glasses are thermodynamically unstable with respect to an assemblage of mineral phases compatible with their compositions, transformation in the solid state is effectively prohibited by very slow reaction kinetics. Contact between glass and a corrosive fluid provides an energetically favorable pathway for the glass to convert to other, more stable phases. Glass components may become solvated and enter solution, or may form new bonds and restructure to generate new solid phases at the glass surface. Secondary phases may also precipitate from the fluid as it becomes saturated. One goal of waste stabilization through vitrification is to produce glasses which corrode at sufficiently low rates that waste components remain immobile for long periods.

To evaluate the durability of a candidate waste glass, its long-term durability must be projected under anticipated environmental conditions in a repository. Such projections are made by using computer simulations that are based on models derived from laboratory experiments. Because of this experimental basis, the effects of various reaction parameters on glass corrosion are accounted for separately. For accurate modeling, it is important to understand the effects on the corrosion mechanism of the SA/V ratios and flow rates used in laboratory tests.

The effects of the SA/V used in static leach tests and the flow rate used in dynamic tests on the observed glass corrosion behavior can be understood (and related to glass corrosion behavior in the repository) in terms of the effects of the solution chemistry and, to a lesser extent, the alteration layers formed on the corroded glass. Laboratory tests conducted under controlled conditions may generate solutions which are very similar to fluids that may contact the glass during some period in the repository. It is important that the corrosion of waste glasses in the laboratory tests be understood in a way that can be used to simulate the corrosion of the same glasses in the repository. Therefore, both the corrosion mechanism of the glass and the relation between test results and the corrosion process must be understood.

The first part of this report provides background information, including descriptions of the glass structure and important corrosion processes, a simple model of the glass reaction to support experimental measures of the extent of glass corrosion in laboratory tests, a brief description of commonly used laboratory tests, and a discussion of how various SA/V ratios are attained in the laboratory. The observed effects of sample preparation techniques and various aspects of test protocols are also discussed.

The second part of this report consists of a review of key studies regarding the glass corrosion mechanism and the effects of testing conditions, including the solution chemistry, $\mathrm{SA} / \mathrm{V}$, and flow. It is important to recognize that the SA/V is a testing parameter that, by itself, does not directly influence the corrosion behavior. That is, in contrast to the solution chemistry and the test temperature, the glass is "unaware" of the SA/V being used, except for its effects on the solution chemistry as the glass corrodes. Because the SA/V used in a static leach test affects the dilution of glass components, the need to understand how the SA/V affects glass corrosion can be represented in terms of the need to understand the effects of the solution chemistry on glass corrosion. Therefore, a large part of this review is concerned with assessing important effects of the solution chemistry, including the effects of the solution $\mathrm{pH}$ and of various dissolved glass components, on the glass corrosion behavior to better understand the effects of 
the SA/V. Experimental studies related to the effects of the SA/V can then be interpreted in terms of specific effects of the solution chemistry on the glass corrosion behavior. When reviewing the literature, the following are considered: the soundness of approach, quality of results, validity of conclusions, and consistency of results with other work. This evaluation is accomplished by presenting the data in the context of a description of the reaction path for the dissolution of high-level waste glass, and by assessing how specific test results contribute to this description. While the effects of the SA/V are of primary interest, only a limited number of tests have been performed so as to isolate the effects of the SA $/ V$. Therefore, other effects arising from differences in the glass and leachant compositions, the glass geometry and/or preparation technique, the reaction temperature, and other test variables are considered when appropriate. In general, the papers reviewed will be discussed chronologically according to publication date, except when more recent work provides a basis for interpreting of earlier work. Although experiments performed with simple glasses having only a few components are utilized to demonstrate basic phenomena, the emphasis of the review is on multicomponent glasses relevant to high-level borosilicate glasses.

Four appendices are included: a list of definitions and acronyms is given in Appendix A, the compositions of glasses discussed in the text are compiled in Appendix B, and a derivation of the rate equation used in most computer simulations is given in Appendix C. Appendix D contains data spreadsheets for the ANL Task "Relationship between tests at high SA/V and MCC-1" performed during fiscal years 1991 through 1994. 


\subsection{DESCRIPTION OF GLASS STRUCTURE}

A basic description of glass structure provides a convenient starting point for discussing the breakdown of a glass as it corrodes. Glasses used in high-level waste management are generated when a viscous melt cools sufficiently fast that elemental constituents become fixed in a noncrystalline arrangement. Although the bonding and coordination requirements of glass components are met in the glass phase, strain and distortion of chemical bonds cause the free energy of the glass phase to be higher than that of a corresponding crystalline state. This excess free energy will likely affect the energetics of the corrosion process.

Glasses have only short-range order, which corresponds to the hybridization of their component elements. Long-range periodic order is absent because bond lengths and bond angles may vary. High-level waste glasses are almost exclusively based on silicate glasses, and this review is focused on this family of glasses. The silicate network can be described as a threedimensional polymeric matrix comprised of $\left[\mathrm{Si}^{-\mathrm{O}_{4 / 2}}\right]$ units in which each silicon atom is bonded to four oxygen atoms in a tetrahedron (the tetrahedral coordination around the silicon atom is a result of its $\mathrm{sp}^{3}$ hybridization) and each oxygen is part of an adjacent silica unit. Oxygen atoms bonded to two silicon atoms are often referred to as "bridging oxygen," since they bridge two silica units. The silicon-oxygen bonds have about $60 \%$ covalent and $40 \%$ ionic character. While the bond lengths and bond angles are constrained in individual tetrahedra, rotation about the bonds linking tetrahedral units contributes to the lack of long-range order.

Silicate glasses have been selected as host matrices for stabilization of radioactive wastes in part because most components of waste streams can be incorporated into the silica structure. The extent to which other elements can be incorporated depends on their size, coordination, and valence, as discussed in the following paragraphs.

Cations with a +4 charge and radii similar to silicon and can fit into four-coordinate sites are highly soluble in silicate glasses. Trivalent cations such as aluminum and iron(III) typically have coordination numbers of 6 and have low solubilities in silicate glasses (typically on the order of a few weight percent). However, the solubilities of these trivalent cations can increase significantly when they are associated with alkali metals and form paired cations such as $\left[\mathrm{Na}-\mathrm{Al}-\mathrm{O}_{4 / 2}\right]$ units, which can substitute into four-coordinated sites (typically to levels in excess of 10 weight percent). The formation of such units perpetuates the silicate polymeric network. Boron can participate in such units (to form reedmergnerite groups [Na-B-O $\left.{ }_{4 / 2}\right]$, or it can hybridize as sp $\mathrm{s}^{2}$ and form a glass phase composed of trigonal planar $\left[\mathrm{BO}_{3 / 2}\right]$ units. The coordination of these trigonal units is incompatible with the tetrahedral silicate network and results in phase separation. Boron may exist in both four-fold sites in the silicate phase and in three-fold sites in the borate phase. It is likely that an equilibrium exists between three- and four-coordinated boron in most alkali borosilicate glasses, and that the amounts of three- and four-coordinated boron depend on the alkali concentration. If sufficient alkali is present, boron will exist primarily in four-coordinated sites and yield a more durable glass. It is important to note that, because of the greater difference in charge potential, alkali ions will form stronger bonds with aluminate groups than with borate groups, so that reedmergnerite groups will form only after all aluminate groups (and most other trivalent cation groups) have been "charge balanced." In addition, cations with low charge density are more effective in "charge balancing" trivalent groups than those with high charge density. Thus, sodium is more effective than calcium. 
Phosphorous exists in glass mainly in the +5 oxidation state and is $\mathrm{sp}^{3}$ hybridized. Although phosphorous coordinates with four oxygen atoms, its +5 oxidation state requires one double bond, so that only three oxygen atoms are available for polymerization. The nonequivalence of the oxygen results in severe distortion of the phosphorous tetrahedra, which are not incorporated into the silicate network. Instead, a separate phosphorous glass phase may form. Phosphorous also readily forms apatites with alkaline earths, titanium, and zirconium. These apatites may occur as primary inclusions as the glass solidifies.

The behavior of monovalent and divalent cations in silicate glasses is strongly influenced by their ionic size, their coordination, and their ionic charge. Their bonds with oxygen are highly ionic. Oxygen bonded to monovalent cations cannot propagate the polymeric network, and those oxygen atoms are often referred to as "nonbridging oxygen." While the formation of nonbridging oxygen bonds decreases the rigidity of the network and the durability of the glass, the addition of alkali metals and alkaline earth elements to waste glasses is important because the presence of nonbridging oxygen lowers the melting point and allows waste forms to be processed under conditions that can be attained in commercial glass melters.

Cations of higher valance typically have large coordination shells and form strong bonds with oxygen. They will preferentially coordinate with oxygen atoms to fill their coordination shells at the expense of the silicate network. These groups are difficult to link into the silicate network and have very low solubilities in silica glasses. For example, most actinide elements are six-fold coordinated and are incorporated into alkali silicate glasses at levels that are typically less than $1 \mathrm{~mol} \%$. An important exception is uranium(VI), which forms uranyl units that are better accommodated in the network. Uranium has a solubility on the order of $10-15 \%$ in alkali silicate glasses, while other actinides have solubilities of 2-4 wt\% [2]. The solubilities of highly coordinated components are increased by the presence of alkali metals, which generate nonbridging oxygen and provide charge balance.

Anions are generally large and highly polarizable, and do not fit into the silica structure. Most anions are volatilized at typical melting temperatures of glass waste forms $\left(1050-1150^{\circ} \mathrm{C}\right)$ and their incorporation into silica glasses is usually not considered when discussing waste glass compositions. Only fluoride is incorporated into silica glasses to an appreciable extent (5-10 wt\%) because of its ability to replace hydroxide (i.e., as $\equiv \mathrm{Si}-\mathrm{F})$. The other halides are volatilized, although these and other anions may occur in waste glasses as trapped gases or insoluble phases. Sulfur forms sulfates on the top of glass melt in joule-heated melters under oxidizing conditions, but it is only soluble in silica to about $1 \mathrm{wt} \%$. Carbon and nitrogen are oxidized and volatilized, though some may remained trapped in the melt because of incomplete oxidation. Technetium $[3,4]$ and other waste components, such as mercury, will volatilize during vitrification.

While anions generally do not contribute to the formation of the glass network, their presence in included phases may affect the corrosion behavior and performance of the waste form as these phases dissolve. For example, sulfate, phosphate, chloride, fluoride, nitrate, and nitrite all complex released radionuclides, and so the presence of these anions in the glass will affect the disposition of waste components as the glass corrodes.

Various components in the glass structure are commonly categorized in the literature as filling "network forming" and "network modifying" roles, depending on whether the component continues or discontinues the polymeric network structure, or, in other words, whether the component supports bridging or nonbridging oxygen. For several components, their role in the glass structure depends on the presence or absence of other components; i.e., boron requires charge compensation by an alkali metal. Such elements are sometimes called "intermediates," since they may fill network forming or network modifying roles depending on the glass 
composition. While this terminology is not rigorous, it is used throughout the glass literature and will be used in this report when relating literature results and where it improves clarity. In general, silicon and trivalent elements that are coupled with alkali are referred to as networkforming elements, while monovalent and divalent elements are referred to as network-modifying elements.

\subsection{Chemical Description of Glass Corrosion}

Glass corrosion can be described as occurring through a series of chemical reactions to break one or more oxygen bonds such that component species become solvated and released into solution or the bonding scheme is altered in the corroded glass. The release of different species to solution will depend on the number and strength of cation-oxygen bonds that must be broken to generate a detached unit that can be solvated and enter solution. While the strain energy of a bond in a glass will affect some reactions, it can be assumed that the strain is removed as neighboring bonds are broken. When contacted by an aqueous solution, bonds are broken through hydrolysis reactions, which can be written as

$$
\mathrm{R}-\mathrm{O}-\mathrm{R}^{\prime}+\mathrm{H}_{2} \mathrm{O}=\mathrm{R}-\mathrm{O}-\mathrm{H}+\mathrm{H}-\mathrm{O}-\mathrm{R}^{\prime}
$$

where $R$ and $R^{\prime}$ are cations that may or may not be linked to the glass network. Depending on the size and chemistry of the $R$ and $R^{\prime}$ groups, the products of the reaction in Eq. 1 may remain part of the glass network or may be released into the leachate as either dissolved or colloidal material. Glass corrosion is conveniently discussed in terms of the identities of the $R$ and $R^{\prime}$ groups based on their role in the glass structure. In the following discussion, the $\mathrm{R}$ group is considered to include cations capable of forming the polymeric network (i.e., network formers), primarily silicon, aluminum, and boron. Because of the high concentration of silica in most waste glasses, $\mathrm{R}$ is represented as $\equiv \mathrm{Si}$ - in the following chemical reactions unless other elements are of specific interest. This depiction identifies the bond involved in the chemical reaction. The $\mathrm{R}^{\prime}$ group may be either network formers, in which case Eq. 1 describes network corrosion, or elements that do not contribute to polymerization, such as monovalent and divalent cations.

\subsubsection{Alkali Release}

The reaction to release alkali metal ions from a nonbridging oxygen can be

written as

$$
\equiv \mathrm{SiO}-\mathrm{M}+\mathrm{H}_{2} \mathrm{O}=\equiv \mathrm{SiO}-\mathrm{H}+\mathrm{M}^{+}+\mathrm{OH}^{-}
$$

where $\equiv \mathrm{SiO}-\mathrm{H}$ represents a silanol group and $\mathrm{M}^{+}$is an alkali metal ion. The net effect of the hydrolysis reaction is the replacement of an alkali atom in the glass by a proton from solution. Alkali release is often written as an ion exchange, since the reaction can also be written as

$$
\equiv \mathrm{SiO}-\mathrm{M}+\mathrm{H}^{+}=\mathrm{SiO}-\mathrm{H}+\mathrm{M}^{+}
$$

or

$$
\Rightarrow \mathrm{SiO}-\mathrm{M}+\mathrm{H}_{3} \mathrm{O}^{+}=\equiv \mathrm{SiO}-\mathrm{H}+\mathrm{M}^{+}+\mathrm{H}_{2} \mathrm{O}
$$


While Eq. $2 \mathrm{~b}$ gives the basic reaction, an unhydrolyzed proton is not likely to be stable either in solution or in the glass [5]. Equations $2 a, 2 b$, and $2 c$ are related through the ionization reaction of water. Both the activity of the alkali ions in solution and the solution $\mathrm{pH}$ will affect the dealkalization of the glass through the equilibria described in Eqs. 2a-c. An expression for the equilibrium constant for Eq. $2 \mathrm{~b}$ can be written as

$$
\log \mathrm{K}_{\text {eq }}=\log \left(\frac{\equiv \mathrm{SiO}-\mathrm{H}}{\equiv \mathrm{SiO}-\mathrm{M}}\right)+\log \left(\mathrm{M}^{+}\right)+\mathrm{pH}
$$

Similar equilibrium constants can be written for Eqs. $2 \mathrm{a}$ and $2 \mathrm{c}$, and the equilibrium constants for the three equations differ only by including $\mathrm{K}_{\mathrm{w}}$. Equilibrium constants have been measured for exchange of alkali metals and alkaline earths with silica gels by Dugger et al. [6]. They found the values of $\log \mathrm{K}_{\mathrm{eq}}$ (for the reaction as written in Eq. $2 \mathrm{~b}$ increased as $\mathrm{K}<\mathrm{Na}<\mathrm{Li}<<\mathrm{Mg}, \mathrm{Ca}$, and Sr. This ordering is consistent with the general order for ion-exchange of alkali metals and alkali earths, i.e., that the larger, more polarizable ion will be replaced in an ion-exchange solid by the smaller, less polarizable ion. Dugger et al. also concluded that the acidity of the silanol group in silica gel is similar to that of water. The equilibrium in Eq. $2 \mathrm{~b}$ will be affected by the solution $\mathrm{pH}$ : high $\mathrm{pH}$ values will shift the equilibrium to the right and favor the formation of $(\equiv \mathrm{SiO}-\mathrm{M})$. Conversely, $(\equiv \mathrm{SiO}-\mathrm{Na})$ groups will be protonated more easily than $(\equiv \mathrm{SiO}-\mathrm{Li})$ groups as the proton concentration increases. This relationship is shown schematically in Fig. 1, where the results of Dugger et al. are utilized to describe the pH effect on the replacement of a proton on silanol with sodium, lithium, or calcium in a $1 \times 10^{-3} \mathrm{M}$ solution of the exchanging ion [7]. For sodium, the silanol will exist entirely in the protonated form (as $=\mathrm{SiO}-\mathrm{H}$ ) at $\mathrm{pH}$ values below about 8 , and entirely in the sodium form (as $=\mathrm{SiO}-\mathrm{Na}$ ) at $\mathrm{pH}$ values above about 12.5 . Both forms will occur at $\mathrm{pH}$ values between about 8 and 12.5. Of course, the $\mathrm{pH}$ values bounding the regions of coexistence of $\equiv \mathrm{SiO}-\mathrm{H}$ and $\equiv \mathrm{SiO}-\mathrm{Na}$ will decrease as the solution concentration of sodium increases.

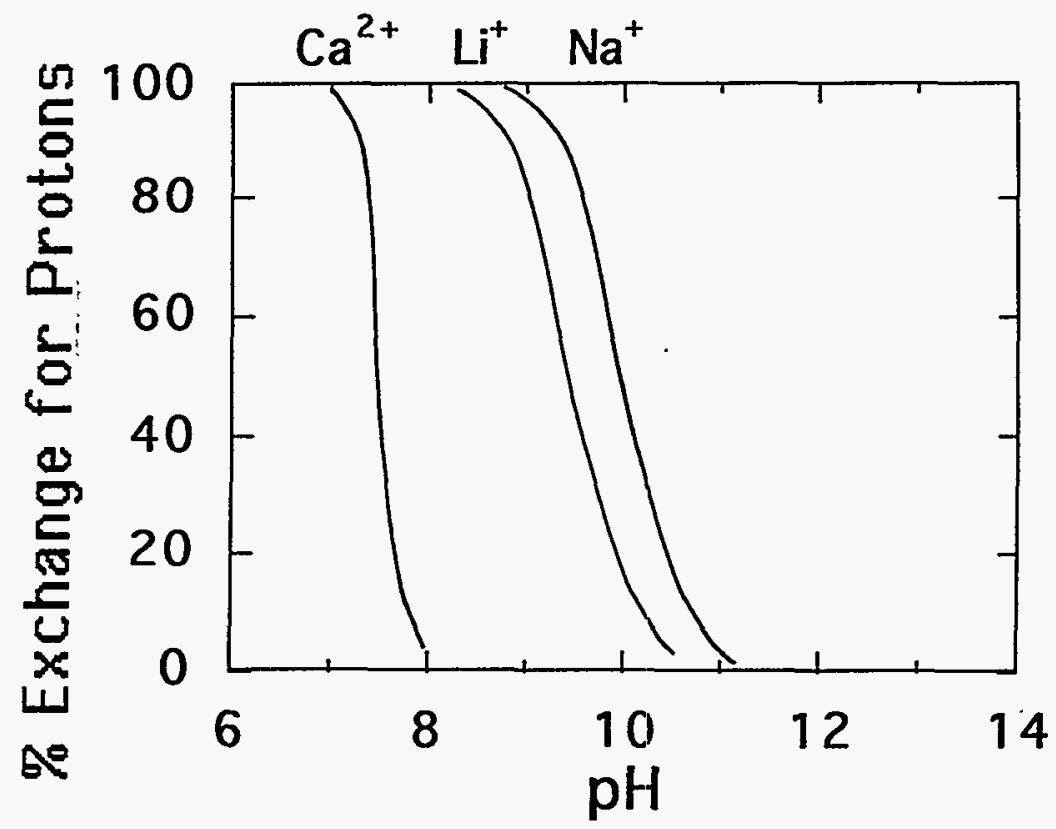

Fig. 1. Schematic Plot of the Fraction of $\equiv$ SiO- Sites Associated with Protons or Other Cations as a Function of $\mathrm{pH}$ in $1 \times 10^{-3} \underline{\mathrm{M}}$ Cation Solutions (after [7]). 
Similar ion exchange reactions can be written for alkali bonded to oxygen atoms that are coordinated with aluminum, boron, or other elements. The alkali metal-oxygen bond energies will differ slightly from the energies for oxygen bonded to other $\mathrm{R}$ groups, such as $\equiv \mathrm{AlO}-\mathrm{Na}$, and so the values of $\mathrm{K}_{\mathrm{eq}}$ will differ, although the relative order is expected to remain the same. By analogy to the behavior of aluminum described by Barrer and Klinowski [8], Bunker et al. reasoned that ion exchange at tetrahedral boron sites differs from exchange at silica sites, and that alkali will be released from nonbridging oxygen in the order $\left(\equiv \mathrm{AlO}^{4-}\right)<\left(\equiv \mathrm{BO}^{4-}\right)<$ ( $\equiv \mathrm{SiO}^{4-}$ ) [9]. That is, an alkali metal ion will be released from a nonbridging oxygen bonded to silicon more easily than from a nonbridging oxygen bonded to boron or aluminum. This prediction is shown schematically in Fig. 2, where the $\mathrm{pH}$-dependence of the ion exchange is shown for a $1 \times 10^{-3} \mathrm{M}$ solution of sodium. At $\mathrm{pH}$ values higher than the curves, protons on silanol groups will be replaced by sodium. At $\mathrm{pH}$ values lower than the curves, protons will remain with silanol groups. This is consistent with the experimental observation that sodium atoms bonded to silicate groups are released more easily than sodium atoms bonded to aluminate groups in aluminosilicate glasses [10].

Ion-exchange reactions may occur between the glass and the solution as a result of competition between alkali metals and alkaline earths. Ions bonded to the glass will be replaced by smaller ions having higher charge densities. Thus, alkaline earths and other divalent cations will replace alkali metals in the glass. The greater stability of these bonds may protect the glass against further corrosion, perhaps by inhibiting water penetration. For example, Buckwalter et al. found that the presence of lead, and to a lesser extent the presence of aluminum, in the leachate solution quenched the corrosion of PNL 76-68 glass [11]. Lehman and Kuchinski determined that both metallic lead and lead(II) oxide greatly reduced the extent of glass corrosion, relative to that occurring in the absence of any lead, while lead(I) oxide enhanced the extent of glass corrosion [12]. The incorporation of magnesium from solution has

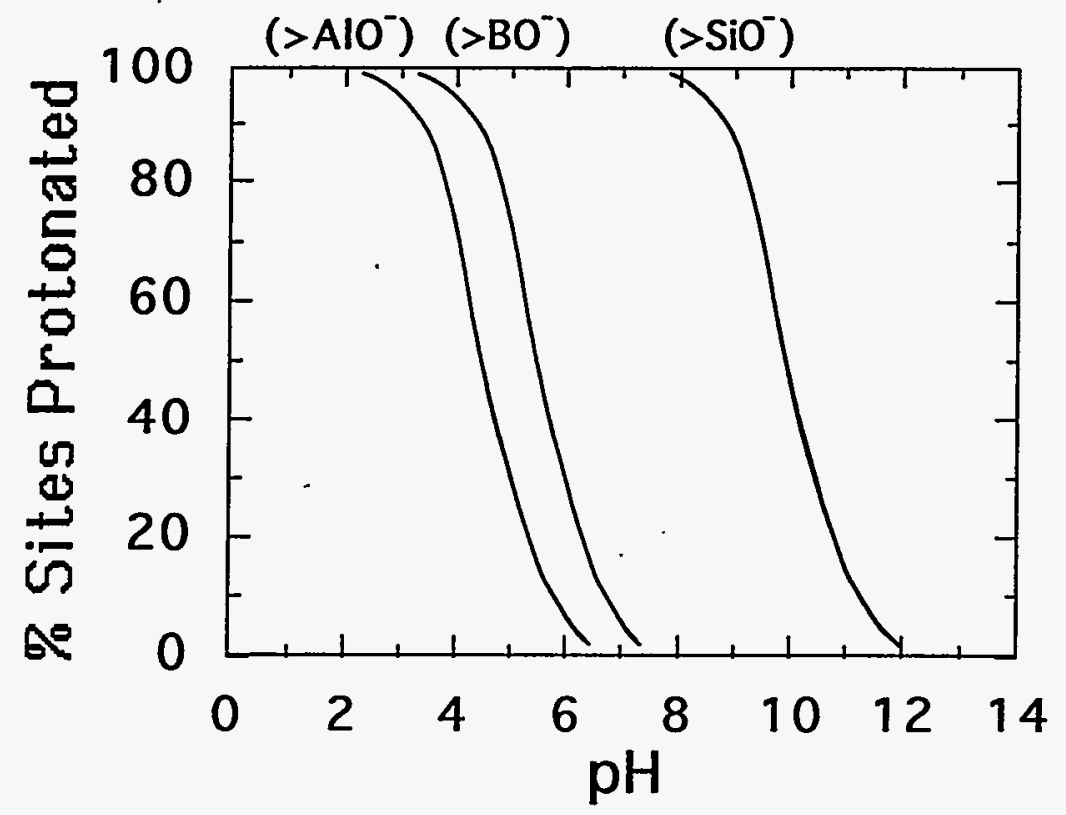

Fig. 2. . Schematic Plot of the Fraction of $\equiv A 1 O^{-}, \equiv \mathrm{BO}$, and $\equiv \mathrm{SiO}^{-}$Sites Associated with Protons or Sodium as a Function of $\mathrm{pH}$ in $1 \times 10^{-3} \underline{\mathrm{M}}$ Cation Solutions (after [7]). 
also been found to reduce glass corrosion $[13,14]$. The mechanism by which these cations affect the corrosion cannot be determined conclusively based on the available experimetal evidence. The effects may occur in the solution by the formation of secondary phases or at the glass surface.

\subsubsection{Water Diffusion}

While alkali metals at the glass surface may be immediately released through hydrolysis reactions upon contact with an aqueous fluid, continued alkali release requires a proton source, such as water, to diffuse into the glass to reach other alkali/nonbridging oxygen bonds. That dealkalization and water diffusion are interrelated is evidenced by the complimentary and S-shaped appearance of hydrogen and alkali profiles commonly obtained at the surfaces of corroded glasses. Experimental results have been interpreted based on the diffusion of molecular water and of hydronium into glasses. However, the small opening size in the silica polymeric structure is known to prevent diffusion of molecular water in many zeolites and fused silica [9]. In these materials, diffusion is instead explained as occurring through hydration/dehydration reactions between water and $\equiv \mathrm{Si}-\mathrm{O}-\mathrm{Si} \equiv$ bonds [15-19]. Nevertheless, defects in the silicate structure may provide diffusion channels large enough to permit molecular water diffusion. For example, water diffusion is known to occur without affecting the silicate network of obsidian [20-23]. In most instances, tests of alkali silicate glasses in silicate solutions also show dealkalization to occur without affecting the silicate network [24]. The difference between water diffusion in silica glass or zeolites and water diffusion in alkali silicate is likely the presence of percolation channels in the latter formed by neighboring nonbridging oxygen atoms $[25,26]$. These channels may be formed by neighboring silanol groups in obsidian and by alkali and alkaline earths in waste glasses. It is likely that the presence of nonbridging oxygen and "the nature of cations bonded to non-bridging oxygen atoms control the hydration of silicapoor glasses" [27] and the rate of water diffusion into high-level waste glasses.

\subsubsection{Dealkalization}

Early work with simple alkali silicate glasses showed the release of alkali metal ions to be proportional to the square root of the reaction time initially, then to be proportional to time after longer reaction times. The root time dependence was interpreted to evidence a diffusion controlled rate and the linear time dependence a surface dissolution controlled rate. The diffusion coefficient for alkali release can be obtained from short-term leaching data as

$$
\mathrm{D}_{\exp }=\frac{\left(\frac{\pi}{4 \mathrm{C}_{\mathrm{i}}^{2}}\right)\left(\mathrm{M}_{\mathrm{i}}\right)^{2}}{\left(\mathrm{SA} \bullet \mathrm{t}^{1 / 2}\right)^{2}}
$$

where $D_{\exp }$ is the effective diffusion coefficient in the experiment (in $\mathrm{cm}^{2} / \mathrm{s}$ ), $\mathrm{C}_{\mathrm{i}}{ }^{2}$ is the concentration of alkali metal ion $i$ in the glass (in moles $/ \mathrm{cm}^{3}$ ), $\mathrm{M}_{i}$ is the moles of alkali released into solution, $\mathrm{SA}$ is the surface area of the glass (in $\mathrm{crn}^{2}$ ), and $\mathrm{t}$ is the reaction time (in seconds). 
Alkali release from simple silicate glasses has been described in terms of the "interdiffusion" of hydronium and alkali metal ions, where the rate of dealkalization is related to the diffusivities of the exchanged species [28-33]. The interionic diffusion coefficient is given by

$$
D=\frac{D_{M} D_{H}}{N_{M} D_{M}+N_{H} D_{H}}
$$

where $D$ is the interionic diffusion coefficient, $D_{M}$ and $D_{M}$ are the diffusion coefficients of the alkali metal and hydronium ions in the glass, and $N_{M}$ and $N_{H}$ are the fractions of alkali metal and hydronium ions bonded to silanol groups in the glass [33]. This model is based on an alkalifor-hydronium exchange mechanism, as given in Eq. $2 \mathrm{c}$, and is supported by the similarity between the hydrogen penetration depth and the alkali depletion depth and by the observation that the measured proton to alkali exchange ratio is often near three, as predicted by this model [34]. However, exchange ratios are affected by the glass composition, corrosion, and analytical conditions, and ratios as low as 1.5 have been measured $[35,36]$. This discrepancy between the expected and measured exchange ratios may be due in part to dehydration of the sample in the vacuum chamber, which would lead to lower measured amounts of hydrogen in the altered glass layers and thus lower exchange ratios [37]. The effects of dehydration should be greatest at the outer surface of the layer and become less significant deeper into the layer. Cooling the sample during analysis has been shown to reduce the loss of water during analysis in vacuum [33].

The measured proton/alkali ratio can be affected by other reactions that may obscure the stoichimometry of the exchange reaction. For example, hydrolysis of a silanol/alkali metal bond by molecular water according to Eq. 2 a followed by sorption of a second water molecule would be expected to give a ratio of $3: 1$. Silanol is known to strongly sorb water which may remain fixed in silicate glasses even under vacuum conditions [38-41].

Leach tests of several sodium-potassium-silicate glasses in deionized water at room temperature showed the interdiffusion rate to be much more weakly dependent on the glass composition than would be calculated using the interdiffusion model [18]. Monolithic samples were leached in deionized water under conditions that were used to monitor the initial stages of dealkalization (i.e., where the kinetics were linear with the square root of time). While the SA/V used in the tests was not reported, it is assumed that the $S A / V$ was sufficiently low to maintain dilute conditions. Results were reported by Bunker et al. for tests conducted at temperatures of 22,30 , and $60^{\circ} \mathrm{C}$. The leachates were titrated with $\mathrm{HCl}$ or $\mathrm{KOH}$ solutions to maintain a constant solution $\mathrm{pH}$ as the glasses dissolved using the $\mathrm{pH}$-stat technique. The net amount of acid added to the leachate was used to measure the extent of corrosion (dealkalization) and determine the effective diffusion coefficient. The interdiffusion model, which utilizes the bulk diffusivities of sodium and potassium, predicted the observed diffusion coefficient would have a minimum near the $0.5 \mathrm{Na}_{2} \mathrm{O} \cdot 0.5 \mathrm{~K}_{2} \mathrm{O} \cdot 3 \mathrm{SiO}_{2}$ composition, whereas the data showed the coefficient to increase smoothly between $\mathrm{Na}_{2} \mathrm{O} \cdot 3 \mathrm{SiO}_{2}$ and $\mathrm{K}_{2} \mathrm{O} \cdot 3 \mathrm{SiO}_{2}$. In addition, the magnitude of the predicted coefficient was between 2 and 7 orders of magnitude smaller than the measured values. These results demonstrate that the diffusion of sodium and potassium in the glass does not control the kinetics of dealkalization. Instead, diffusion through the hydrated glass surface controls the dealkalization rate.

A different model assumes that it is molecular water, rather than hydronium, that diffuses into glass $[5,42,43]$. In this model, ion exchange reactions occur rapidly compared to the diffusivity of molecular water, which controls the rate of dealkalization [10]. This model is supported by the observation of molecular water in leached glasses by infrared spectroscopy [44-46], near infrared spectroscopy and differential thermogravimetry [39]. Of course, molecular water may also be produced by condensation of silanol within the glass (see below). 
The mechanism of molecular water diffusion was proposed by Smets and Lommen to be operative in the $\mathrm{pH}$ range of 4 to 7 . This mechanism is likely to be favored at the higher $\mathrm{pH}$ values commonly attained during corrosion of high-level waste glasses, where the concentration of hydronium is very low.

The dealkalization mechanism has been studied by corroding simple glasses and simulated waste glasses in isotopically labeled solutions. Pederson et al. reported that $18 \mathrm{O}$ penetrated a soda lime silicate glass to the same depth as sodium was depleted, which they interpreted to indicate interaction between molecular water and the silica $[47,48]$. Their tests also showed that more oxygen was fixed to the glass than deuterium. This result indicates that the diffusing species, whether molecular or ionic water, was not incorporated into the glass through a simple insertion mechanism. The isotope effect observed by Pederson et al. when a sodium silicate glass was corroded in $\mathrm{D}_{2} \mathrm{O}$ and in $\mathrm{H}_{2} \mathrm{O}$ showed that a hydrogen (or deuterium)oxygen bond was broken during the dealkalization process [48]. The authors ascribe the isotope effect to hydrolysis of the silicate network, rather than mass transport, for this glass.

In similar tests of more durable soda aluminosilicate glasses using isotopically labeled water, Pederson et al. did not observe a kinetic effect [49]. Their results implied that very little structural rearrangement occurred following ion exchange, and the authors suggest that the observed corrosion rate is controlled by mass transport instead of chemical reaction. These results are probably more representative of high-level waste glasses than are the results for soda silicate glasses discussed earlier.

The dealkalization of a soda-lime silicate glass was investigated in tests conducted at 100 and $200^{\circ} \mathrm{C}$ in deionized water, in a silicate solution (152 ppm $\mathrm{SiO}_{2}$ and $37 \mathrm{ppm}$ $\mathrm{NaCl}$ ), and in a $5 \mathrm{M}$ sodium chloride solution to discriminate between models based on molecular water and on hydronium diffusion [50]. Both the hydrogen and sodium profiles in the alteration layers were measured by using resonance nuclear reaction spectroscopy. Results for a sample reacted in silicate solution at $100^{\circ} \mathrm{C}$ is shown in Fig. 3. Shown in the plot are the measured concentrations of hydrogen and sodium and the "exceess" hydrogen not required in the ion exchange reaction. Note that the decrease in the measured hydrogen concentration at the outer surface is probably due to dehydration in the vacuum. The hydrogen penetration and sodium depletion depths were the same after corrosion at $100^{\circ} \mathrm{C}$ in deionized water or in the silicate solution. This suggests the mechanism is controlled by an exchange reaction between sodium and a proton in both leachants. The "excess" hydrogen is due to the presence of molecular water, which accompanies the proton as it penetrates the glass. The depth of hydrogen penetration and sodium depletion was slightly greater in tests conducted in the silicate solution than those in the deionized water, which is consistent with less dissolution of the hydrated glass in the silicate solution. The hydrogen penetration and sodium depletion depths were much less after corrosion in the sodium chloride solution. Only the outer 20-60 nm of the glass was chemically altered. This dealkalization could have occur by exposure to air prior to initiating the test, as has been shown by Hench [51]. These results suggest that in the absence of dealkalization, penetration of hydrogen (either as molecular water or as an ionic species) did not occur. In other words, stopping the ion exchange stopped the penetration of water into the glass.

Tests in deionized water and in the silicate solution conducted at $200^{\circ} \mathrm{C}$ showed similar behavior to that observed at $100^{\circ} \mathrm{C}$ except the alteration depths were greater. Tests in the $\mathrm{NaCl}$ solution at $200^{\circ} \mathrm{C}$ showed sodium to be depleted to a depth similar to that measured in tests conducted in deionized water. However, hydrogen had penetrated deeper into the glass than sodium was depleted. Thus, penetration of water occurred in the absence of dealkalization at $200^{\circ} \mathrm{C}$. 


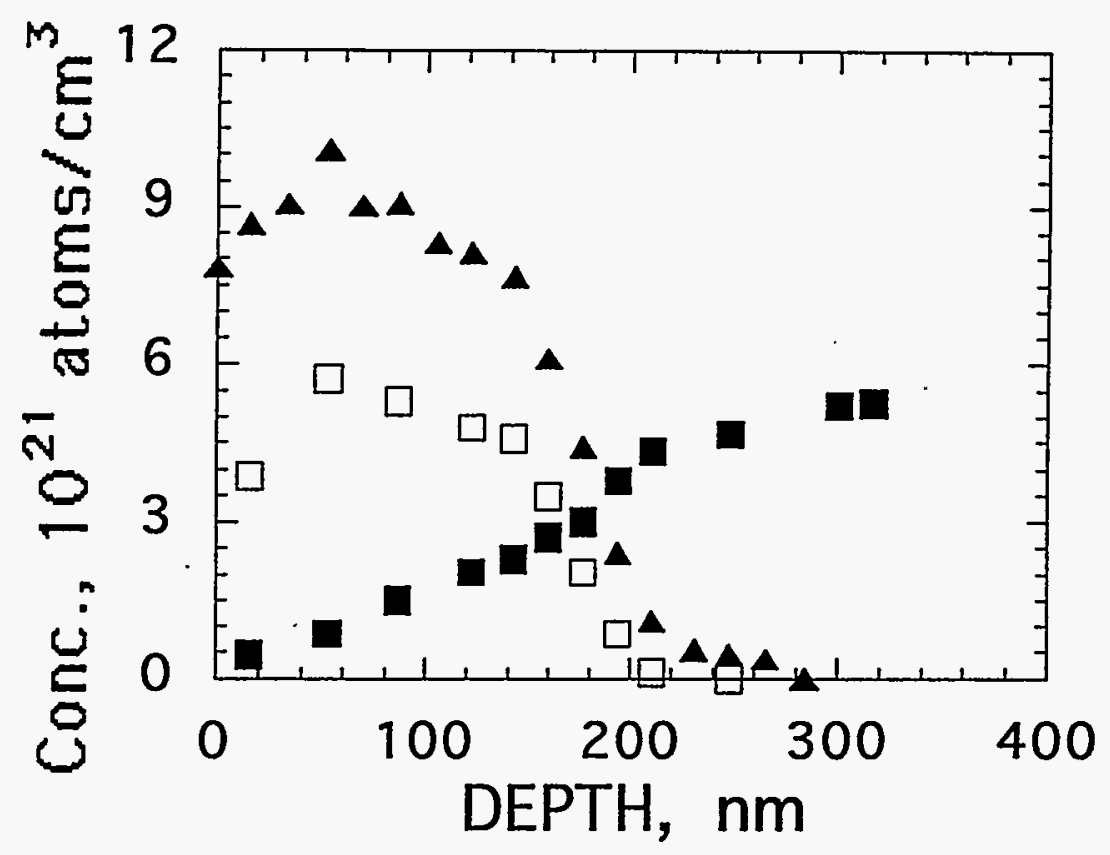

Fig. 3. Measured Concentrations of ( $\mathbf{A})$ Sodium, ( $(\mathbf{\square})$ Hydrogen, and ( $\square$ ) Excess Hydrogen at the Surface after Corrosion in Silicate Solution at $100^{\circ} \mathrm{C}$ (after [50]).

Dran et al. also found that the $\mathrm{H} / \mathrm{Na}$ ratios increased from about 1.8 to about 2.5 in at $100^{\circ} \mathrm{C}$, and from about 1.8 to about 2.5 in tests $200^{\circ} \mathrm{C}$, in both tests in deionized water and in silicate solution, as the layer/glass interface was approached. The similarities in the ratios with depth suggest that the layers are not diffusion barriers. The slight increase in the ratio of $\mathrm{H} / \mathrm{Na}$ as the pristine glass was approached was interpreted to indicate a decrease in the extent of alteration of the alkali-depleted layer, such as hydrolysis of the silicate network, and to represent the ion exchange reaction in the absence of competing reactions. The authors interpreted the results to indicate that ion exchange and water permeation occur simultaneously, and that the test conditions determine which is the controlling process [50]. At $100^{\circ} \mathrm{C}$, ion exchange controls the dealkalization rate and controls the water penetration rate. High concentrations of alkali in the leachate slow the dealkalization and thereby hinder water penetration. At $200^{\circ} \mathrm{C}$, some water penetration can occur independent of dealkalization.

The corrosion of a nuclear waste glass showed similar behavior [52]. Reaction in deionized water at $120^{\circ} \mathrm{C}$ resulted in anticorrelated hydrogen and sodium profiles, while reaction in a $5 \underline{\mathrm{M} \mathrm{NaCl}}$ solution showed very little dealkalization. At $200^{\circ} \mathrm{C}$, hydrogen penetrated into the glass to a greater depth than sodium was depleted in tests conducted in either deionized water or the $\mathrm{NaCl}$ solution. Dran et al. determined that the $\mathrm{H} / \mathrm{Na}$ ratio never reached a value of three, and concluded that hydronium was not involved in the hydration reaction [52]. Other analyses showed the altered layer to restructure and insoluble components to accumulate in the layer [24]. The restructuring of the layer was found to generate molecular water due to the condensation of silanol groups (see Eq. 5a below) [47,53]. 
Studies with simple glasses have shown the models developed using test results for some glasses cannot be applied to some other glasses. The extension of models developed based on simple glasses may be even less applicable to complex high-level waste glasses. While the specific models may not be directly applicable, knowledge gained regarding the processes that occur in simple glasses provide valuable insight into the study of high-level waste glasses.

These different results also underscore the importance of accounting for how the test conditions influence the observed extent of corrosion and the effects of possible analytical artifacts. For example, the work of Bunker et al. shows the dealkalization reactions to be highlighted by maintaining dilute conditions and preventing the $\mathrm{pH}$ from increasing. The work of Dran et al. shows the dealkalization reactions to become quenched in the presence of high sodium solution concentrations. Different experimental approaches consistently show a net exchange of $\mathrm{H}^{+}$for $\mathrm{M}^{+}$during dealkalization, although uncertainties remain regarding the identity of the speciation of the inwardly diffusing proton source $\left(\mathrm{H}^{+}\right.$or $\left.\mathrm{H}_{3} \mathrm{O}^{+}\right)$. Distinction of the proton source is of mostly academic interest, and is mostly irrelevant to characterizing the behavior of high-level waste glasses, where the release of waste components is of prime importance. The identity of the hydrogen species involved in a particular reaction may not be experimentally accessible due to the extremely high mobility of a proton in water. If, rather than an isolated water molecule, a linear array of molecules penetrates the glass through a percolation channel, then it may be impossible to distinguish whether the end of the array is $\mathrm{H}_{2} \mathrm{O}$ or $\mathrm{H}_{3} \mathrm{O}^{+}$.

\subsubsection{Release of Network-Forming Elements}

The degradation and eventual dissolution of the glass occurs by hydrolysis of the bonds forming its polymeric network. Hydrolysis of $\equiv \mathrm{Si}-\mathrm{O}-\mathrm{Si} \equiv$ linkages may occur through nucleophilic attack on a silicon atom by either molecular water or hydroxide. Silicate glasses are typically quite resistant to acid attack. Hydrolysis is modeled to occur through formation of a five-coordinate activated complex, which may degrade by breaking the network bond $[54,55]$. The net reactions can be written as

or

$$
\equiv \mathrm{SiO}-\mathrm{Si} \equiv+\mathrm{H}_{2} \mathrm{O}=\equiv \mathrm{SiOH}+\mathrm{HO}-\mathrm{Si} \equiv
$$

$$
\equiv \mathrm{SiO}-\mathrm{Si} \equiv+\mathrm{OH}^{-}=\equiv \mathrm{SiO}^{-}+\mathrm{HO}-\mathrm{Si} \equiv
$$

Hydrolysis of terminal $-\mathrm{Si}(\mathrm{OH})_{3}$ groups leads to dissolution of the glass as

$$
=\mathrm{SiO}-\mathrm{Si}(\mathrm{OH})_{3}+\mathrm{H}_{2} \mathrm{O}=\equiv \mathrm{SiOH}+\mathrm{H}_{4} \mathrm{SiO}_{4(\text { aq })}
$$

or

$$
\equiv \mathrm{SiO}-\mathrm{Si}(\mathrm{OH})_{3}+\mathrm{OH}^{-}=\equiv \mathrm{SiO}^{-}+\mathrm{H}_{4} \mathrm{SiO}_{4(\mathrm{aq})}
$$

The forward reaction in Eqs. $5 \mathrm{a}$ and $5 \mathrm{~b}$ is referred to as a network hydrolysis reaction and the forward reaction in Eqs. $6 \mathrm{a}$ and $6 \mathrm{~b}$ is referred to as a network dissolution reaction. The reverse reactions are referred to as condensation reactions The $\equiv \mathrm{SiO}^{-}$groups generated in Eqs. $5 \mathrm{~b}$ and $6 \mathrm{~b}$ may become protonated by interacting with another water molecule. Note also that silicic acid is a weaker acid than silanol (at $90^{\circ} \mathrm{C}, \mathrm{pK}_{\mathrm{a}}($ silanol $)=6.8$ [56], and $\mathrm{pK}_{\mathrm{a} 1}$ (silicic acid) =9.7 [57]). Because hydroxide is a stronger nucleophile than molecular water and because experimental observations indicate that the pH affects the rate of silicon release (see Sec. 3.3), it is likely that hydroxide is the dominant nucleophile in neutral and alkaline solutions, and that Eqs. $5 \mathrm{~b}$ and $6 \mathrm{~b}$ more accurately represent the hydrolysis reactions. 
Similar reactions can be written for the hydrolysis of other terminal metal-oxygen bonds, such as for boron at terminal sites

$$
\Rightarrow \mathrm{SiO}-\mathrm{B}(\mathrm{OH})_{2}+\mathrm{OH}^{-}=\mathrm{SiO}^{-}+\mathrm{B}(\mathrm{OH})_{3}
$$

The release of boron may consume a second hydroxide as

$$
\mathrm{B}(\mathrm{OH})_{3}+\mathrm{OH}^{-}=\mathrm{B}(\mathrm{OH})_{4}^{-}
$$

The $\mathrm{pK}_{\mathrm{a}}$ for this reaction is 9.23 at $20^{\circ} \mathrm{C}$ [58] and 8.81 at $90^{\circ} \mathrm{C}$ (calculated from the results of [59]).

\subsubsection{Glass Corrosion}

It is clear from considering the dissolution of individual glass components that all processes are interrelated through their occurrence in a common fluid; that is, changes in the solution chemistry due to the release of one component will affect the release of other components. Hydroxide produced during alkali release through Eq. 2a, for example, will catalyze the hydrolysis of nearby $\equiv \mathrm{SiO}-\mathrm{B}=$ bonds. The replacement of alkali in the glass with protons and the hydrolysis of network bonds will increase water diffusion into the glass, and larger amounts of water in the glass will promote the ion exchange reactions. Water subsequently diffuses into the glass and exchanges with alkali metal ions that are encountered. The release of alkali metal ions via ion exchange generates hydroxide, which then catalyzes hydrolysis reactions.

The relative rates of individual reaction steps will also affect the overall glass corrosion rate because these rates control the number of reaction sites exposed. Initially, the number of reaction sites is proportional to the surface area. As water penetrates into the glass and a dealkalized surface layer forms, the number of structural $\equiv \mathrm{Si}-\mathrm{O}-\mathrm{Si} \equiv$ bonds available for reaction increases significantly and becomes proportional to the volume of the layer. Glass components having limited solubilities may participate in reactions to form alteration phases at the glass surface. The corroded surface layers of many waste glasses have been observed to be transformed into clays having compositions similar to the glasses on which they form [60-65].

\subsection{Experimental Measures of the Extent of Glass Corrosion}

As described in Section 2.1, contact of glass by an aqueous fluid results in the diffusion of water into the glass, partial dissolution of the glass, and physical and chemical alteration of the glass surface in contact with the fluid. Because glass corrosion involves several processes which lead to the alteration of the network, it is difficult to precisely define or operationally measure the extent of glass corrosion. Different measures of corrosion can be defined in terms of each reaction process, and different measures may be appropriate during different stages of corrosion and in different tests.

Although the extent of reaction may be determined by the depth of water penetration into the glass, the penetration depth of water is difficult to measure and is not a useful monitor of the glass corrosion. Indeed, diffusion of water into the glass does not constitute glass alteration or corrosion. The extent to which leachable components are released into solution provides an upper limit on the glass corrosion rate and an appropriate measure for modeling the release of leachable radioelements such as ${ }^{137} \mathrm{Cs}$ and ${ }^{99} \mathrm{Tc}$. The release of other radioelements requires the dissolution of the glass matrix, and may be better modeled using the dissolution rates of matrixforming elements such as silicon and aluminum. 
The most common measure of the extent of corrosion utilizes the measured amounts of glass components that are released into solution. The mass of a component $i$ in solution is usually normalized to the surface area and weight fraction of that element in the glass to permit the results of tests with different glass performed at different $S A / V$ ratios to be compared directly. The normalized elemental mass loss of $i, N L(i)$, is given by the function

$$
N L(i)=\frac{m_{i}-m_{i}^{\circ}}{S A \bullet f_{i}}
$$

where $m_{i}$ is the mass of element $i$ measured in the leachate, $m_{i}{ }^{\circ}$ is the mass of element $i$ in the initial leachant solution, $S A$ is the surface area of the glass, and $f_{i}$ is the weight fraction of element $i$ in the glass, i.e., mass /mass $_{\text {glass. }}$. Alternatively, the masses in Eq. 9 can be replaced by the concentrations $\left(c_{i}\right.$ and $\left.c_{i}{ }^{\circ}\right)$ if the $S A$ term is replaced by $(S A / V)$ to give

$$
N L(i)=\frac{c_{i}-c_{i}^{\circ}}{(S A / V) \cdot f_{i}}
$$

If $c_{i}$ and $c_{i}{ }^{\circ}$ are expressed in $g / \mathrm{m}^{3}$ and SA/V in $\mathrm{m}^{-1}$, then NL(i) will have units of $\mathrm{g} / \mathrm{m}^{2}$. It is important to note that, because of the $f_{i}$ term, the mass reflected in the units of NL(i) is the mass of glass, not mass of species $i$. The value of NL(i) represents the amount of glass that has reacted, as calculated using the solution concentration of species $i$, not the amount of $i$ that is released.

If all glass components are released stoichiometrically, then the values of NL(i) will be the same when calculated using the solution concentrations of different species . Otherwise, the value of NL(i) will be different for each species used in the calculation. High-level radioactive waste glasses generally contain many chemical components that have different solubilities and may be released from the glass at different rates. They are usually found to corrode nonstoichiometrically under most test conditions. For example, Fig. 4 shows the normalized mass losses calculated from the solution concentrations of several major glass components for a glass relevant to the Defense Waste Processing Facility (DWPF) [66,67]. The glass was reacted in a tuff groundwater solution at $90^{\circ} \mathrm{C}$ following the Product Consistency Test (PCT) protocol (see Sec. 2.5.1.2). Alkali metals and boron are released to a greater extent, and uranium to a lesser extent, than silicon. Clearly, the measured extent of glass corrosion is highly dependent on the element selected to calculate NL(i); for example, the integrated rate (see Sec. 2.3) based on lithium release is about 3.5 times that calculated based on the release of silicon. For this reason, the element used to calculate the normalized mass loss should be identified, and the corrosion of different glasses should be compared based on the release of the same element. Notice in Fig. 4 that the differences in the NL(i) calculated using different elements become nearly constant for tests conducted beyond 181 days; the rates become much more similar after that that time. This change may reflect a change in the corrosion mechanism occurs after about 181 days that results in nearly stoichiometric release. That is, these results imply that the reaction conditions, not the glass composition, determines whether corrosion occurs stoichiometrically or nonstoichiometrically. An increase in the leachate $\mathrm{pH}$ as the glass corrodes is responsible, in part, for the change from nonstoichiometric release to nearly stoichiometric release seen in the tests.

Early work with borosilicate glasses used silicon as a measure of the extent of glass reaction because it is the most abundant cation in the glass, is the predominant network-forming species, and is present in most glasses tested. However, the solution concentration of silicon may be a poor indicator of the extent of glass reaction because several glass components may be 


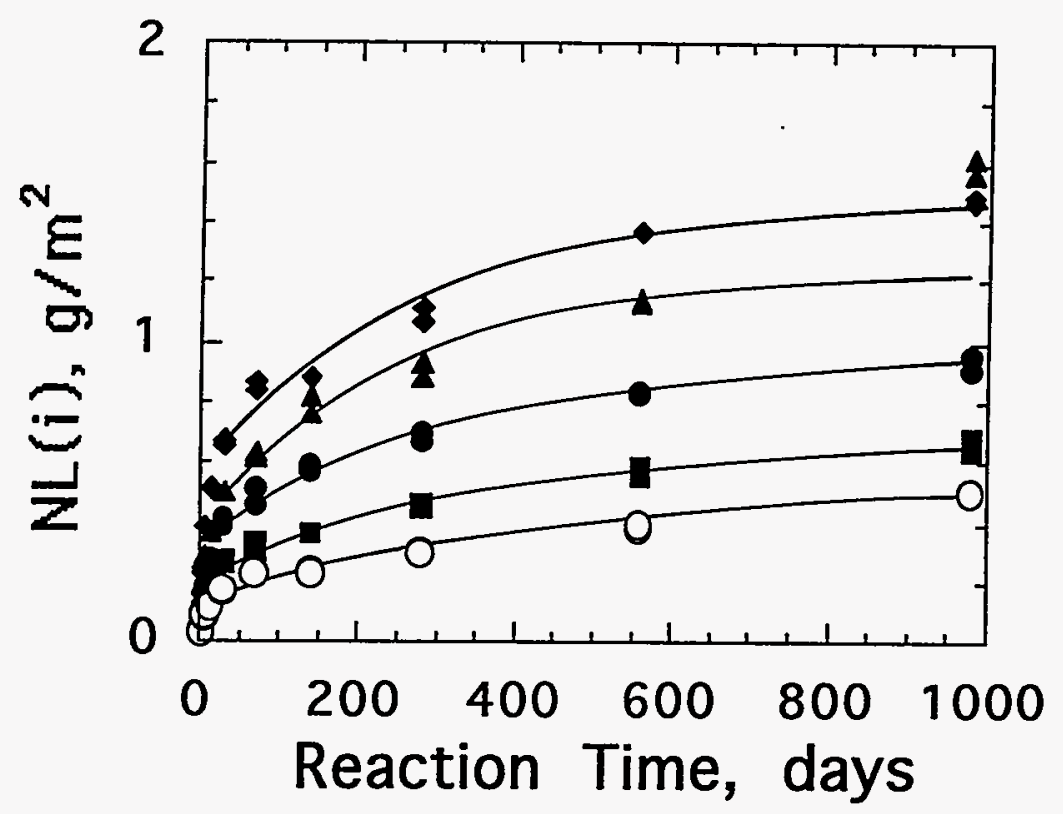

Fig. 4. Product Consistency Tests Showing Nonstoichiometric Release of Glass Components. (O) $\mathrm{Si},(\boldsymbol{\square}) \mathrm{K},(\bullet) \mathrm{B},(\mathbf{\Delta}) \mathrm{Na}$, and $(\bullet) \mathrm{Li}$ (after [67]).

released faster than silicon. Also, many secondary phases that have been seen to form during glass dissolution contain silicon. The solution concentration of silicon will not be indicative of the amount of silicon lost from the glass if some of the silicon released from the glass is contained in secondary phases. It should be noted that early work was conducted using tests at lower SA/V ratios in which the nonstoichiometry of glass corrosion was not as obvious as that seen in Fig. 4.

Water diffusion into the glass and the release of alkali metal ions and boron from the glass generally occur to a greater extent than hydrolysis reactions and the release of silicon and most other glass components. The solution concentrations of alkali metals and boron are often used as a measure of the extent of glass reaction in part because they represent the upper limit of elemental release rates (as seen in Fig. 4). A benefit of using boron as a measure of the glass reaction progress instead of an alkali metal is that boron is contained in very few secondary phases [68]. Alkali metals are contained in zeolite and clay phases that commonly form during glass corrosion. Boron release has been shown to be sensitive to reaction conditions such as temperature [69], $\mathrm{pH}[70,71]$, and silicic acid concentration [72,73], and it usually provides the best single measure of the extent of glass corrosion. The boron release has also been shown to be more sensitive to changes in the corrosion rate than other glass components [65]. In addition, boron is rarely present in appreciable concentrations in groundwaters or in other components that may be present in the repository, such as minerals in the host rock or engineering components, and so the glass corrosion can be measured in laboratory tests in the presence of other components without the need for background corrections.

One shortcoming of using boron to measure the extent of corrosion is that boron is not present in naturally occurring glasses or most historic and artifact glasses. These glasses have been used to derive correlations between glass corrosion rates and glass compositions that can be used during production of high-level waste glasses [74]: These glasses cannot be included in correlations based on boron release. 
The analysis of the corrosion rate using solution concentrations can be complicated by the formation of colloids and suspended solids, including suspended glass particles in tests performed using crushed samples. While leachates are readily filtered, filtration may introduce artifacts as a result of sorption of material to the filtering material, dissolution of the filtering material, etc. Whether a leachate needs to be filtered or not should be determined by the test being conducted (i.e., if the sample is a monolith or crushed) and the information desired. If the purpose of the test is to determine how much material has been lost from the glass, then both dissolved and colloidal material should be included in the analysis. However, if crushed glass is used, coarse filtration may be required to remove suspended glass particles. If the purpose of the test is to provide a measure of the dissolved concentrations for use as simulation parameters for computer modeling, then filtration may be required. The need to filter solutions is sometimes incorporated into standard test protocols and sometimes left to the discretion of the experimenter. In either case, it is important to consider possible effects of filtration on the measured solution concentrations.

The normalized mass loss and normalized release rate (which is simply the normalized mass loss divided by the test time) provide upper bounds on the extent of corrosion (assuming that the element that is released to the greatest extent is used to calculate it) in terms of the mass of glass that dissolves per square meter of exposed glass surface and per unit time. Direct application of the normalized release to assessing the performance of a waste form requires knowledge of the surface area, from which can be calculated the fractional release rate.

The mass loss of a sample after corrosion can be measured directly and used as a measure of the extent of corrosion. The measured mass loss has been used extensively in tests utilizing monolithic samples. The measured mass loss is related to the normalized mass loss calculated based on solution analyses: the value of $\mathrm{NL}$ is an estimate of the total mass dissolved assuming the glass dissolves congruently, and will vary depending on which element is used in the calculation. Nonstoichiometric dissolution may result in NL being greater than the measured mass loss if the solution concentration of a leached component (alkali metal or boron) is used. Direct measurement of the mass loss is complicated by the formation of alteration layers or precipitation of secondary phases on the glass surface. Alteration layers and secondary phases are usually physically removed before measuring the mass loss.

Another measure of the extent of glass reaction is the thickness or mass of the outer glass layer that becomes depleted of alkali metals and boron as the reaction progresses. The thickness of the layer can be measured in cross section by using microscopic techniques, or the layer can be removed from monolithic samples and weighed directly. Neither the layer thickness nor the mass give an absolute measure of the extent of glass reaction; rather, they give the difference between the dissolution rates of the alkali metals and boron from the interior of the glass and the dissolution rate of silicon from the glass surface. The layer thickness will also be affected by drying the sample for analysis, sample preparation, and the environment in which the sample is analyzed. It is best to couple the layer thickness and solution data to attain a material balance for the reaction process $[75,76]$. Analysis of the reaction layer provides a valuable -and sometimes the only- measure of the extent of glass reaction in systems where solution analyses cannot be performed, such as in field tests [77-80] or in tests with water vapor [81]. In this case, the enrichment of insoluble components in the layer may provide a measure of the extent of etching of the reacted surface. That is, the amount of insoluble material left as residue on the reacting surface as the rest of the glass dissolves can be compared to the bulk concentrations to calculate the volume of glass that must have dissolved to generate the measured amount of residue. The total volume corroded (including the amount dissolved, if known, and the amount remaining in 
the alteration layer) is related to the normalized release through the density of the glass. For example, the extent of corrosion leading to a layer with a uniform thickness can be estimated by multiplying the thickness of the layer (in $\mu \mathrm{m}$ ) by the density of the glass (in $\mathrm{g} / \mathrm{cm}^{3}$ ) to obtain the normalized mass loss (in $\mathrm{g} / \mathrm{m}^{2}$ ).

The use of solids analyses to monitor the extent of glass corrosion should be related to a viable corrosion model and should assure material balance. For example, the precipitation of secondary phases on the surface should be excluded from the layer thickness measurements, since most of the material in these phases was made available after chemical alteration of the glass surface, and its inclusion would, in effect, double count the release of those components. In some cases, an abundant secondary phase may settle evenly across the glass surface so that the thickness of the layer formed by the secondary phase can be used as a measure of the reaction progress in lieu of the alteration layer formed on the glass. Otherwise, the general abundance of secondary phases can provide a qualitative measure of the extent of glass reaction.

\subsection{Measurement of the Corrosion Rate}

The corrosion rate is determined based on the measured extent of corrosion as a function of the corrosion time. Corrosion rates can be calculated based on any of the measures of the extent of corrosion discussed above. The corrosion rate can be expressed in terms of the overall extent of corrosion measured over the total time tested, which is referred to as the integrated rate, or as the rate at a specific time, which is referred to as the instantaneous rate. The difference between the two rates is illustrated using the measured lithium normalized mass losses from data in Fig. 4, where the normalized lithium mass loss is used to measure the extent of corrosion. These data are shown in Fig. 5 . The integrated rate is determined by simply dividing the extent of reaction at the point of interest be the reaction time, and gives the average rate from the time the test was initiated through the time of interest. The integrated rate after a reaction time of

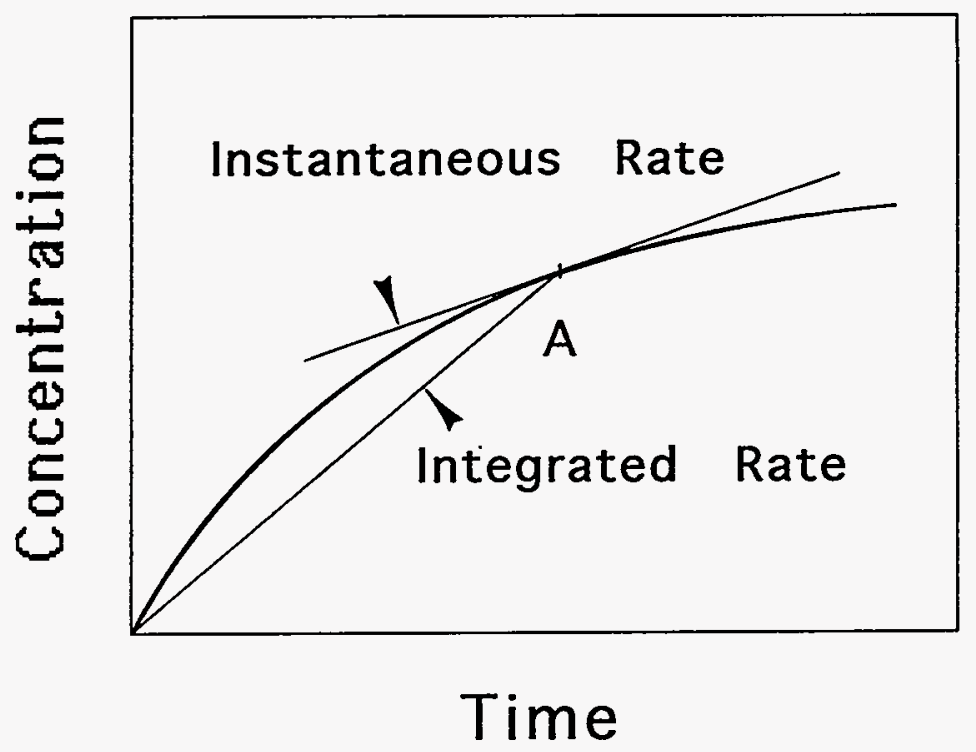

Fig. 5. Schematic Plot Showing Instantaneous and Integrated Rates for a Specific Reaction Time "A" on a Typical Release Curve. 
560 days is calculated to be $\left(1.37 \mathrm{~g} / \mathrm{m}^{2}\right) /(560$ days $)=2.5 \times 10^{-3} \mathrm{~g} / \mathrm{m}^{2} / \mathrm{d}$. The instantaneous rate is given by the tangent to the corrosion curve at the point of interest, and is usually approximated using data points collected after two different reaction times. From the lithium data in Fig. 5, the normalized lithium mass loss after 280 days is $1.10 \mathrm{~g} / \mathrm{m}^{2}$ and after 980 days is $1.47 \mathrm{~g} / \mathrm{m}^{2}$ so that the instantaneous rate from the data between 280 and 560 days is $(1.37-1.10) /(560-280)=9.6 \mathrm{x}$ $10^{-4} \mathrm{~g} / \mathrm{m}^{2} / \mathrm{d}$, and between 560 and 980 days is $(1.47-1.37) /(980-560)=2.4 \times 10^{-4} \mathrm{~g} / \mathrm{m}^{2} / \mathrm{d}$. The average instantaneous rate calculated from these test data is $6 \times 10^{-4} \mathrm{~g} / \mathrm{m}^{2} / \mathrm{d}$, which is less than one-fourth the integrated rate. The values of the integrated and instantaneous rates are similar in the early stage of the corrosion when the glass corrodes rapidly, but become quite different beyond the steep portion of the curve at short reaction times. This is because all integrated rates account for the initial rapid corrosion while the instantaneous rate does not. Which rate is being discussed should be specified when presenting rate data.

Only the integrated rate can be determined for a test in which only one time period is used, as in product consistency tests. While the integrated rate can be used to compare the relative durabilities of different glass compositions under specific conditions, it is not particularly useful for characterizing the glass corrosion behavior or projecting long-term behavior. The integrated rate is strongly influenced by the initial corrosion rate and is only weakly sensitive to changes in the corrosion rate at long time periods. Also, rates that become constant at long time periods do not give constant integrated rates. Instantaneous rates are sensitive to changes in the corrosion rate and are better suited to characterizing the long-term corrosion behavior.

As described earlier, several different reactions and processes are involved during glass corrosion, and which controls the observed corrosion rate may change as the corrosion progresses and may differ under different test conditions. Utilizing a single measure of the extent of corrosion throughout the corrosion process may be misleading, since that measure may or may not track the rate-determining process. For example, dealkalization dominates the initial stage of glass corrosion while the release of silicic acid does not occur until the $\mathrm{pH}$ increases above about 9 . Tracking the silicon concentration does not characterize the initial stages of corrosion. The intermediate stages of corrosion can be followed using the silicon concentration in solution, since the glass dissolves nearly stoichiometrically. Secondary phases formed after significant corrosion contain silicon, so that the silicon concentration no longer provides a good measure of the extent of corrosion. Likewise, measurement of the thickness of alteration layers depends on the relation between dealkalization and water penetration, which establish the layer/glass interface, and hydrolysis and dissolution, which establish the layer/solution interface. Early in the corrosion, dealkalization occurs rapidly and dissolution occurs slowly, while in the intermediate stages they occur at similar rates and during later stages precipitated material may settle on the glass surface. Therefore, it is important to relate what is being used to measure the extent of corrosion to the corrosion mechanism, and it is beneficial to utilize more than one measure to assure that changes in the rate controlling step will be accounted for.

\subsection{A Phenomenological Model of Corrosion Behavior}

As will be described in this report, an extensive and diverse range of laboratory tests have led to a generally accepted description of high-level waste glass corrosion. The following description of corrosion in a static system is referred to as phenomenological because it does not rely on the identification of specific chemical reactions or processes, rather it summarizes various experimental observations. It is presented to support the discussion of particular aspects of corrosion in later sections of the report. Figure 6 schematically illustrates the corrosion path followed as a waste glass corrodes under static conditions, where the extent of corrosion is indicated by the solution concentration of a highly soluble glass component. (Test conditions where the leachate solution is diluted due to flow or periodic replenishment will prevent the 


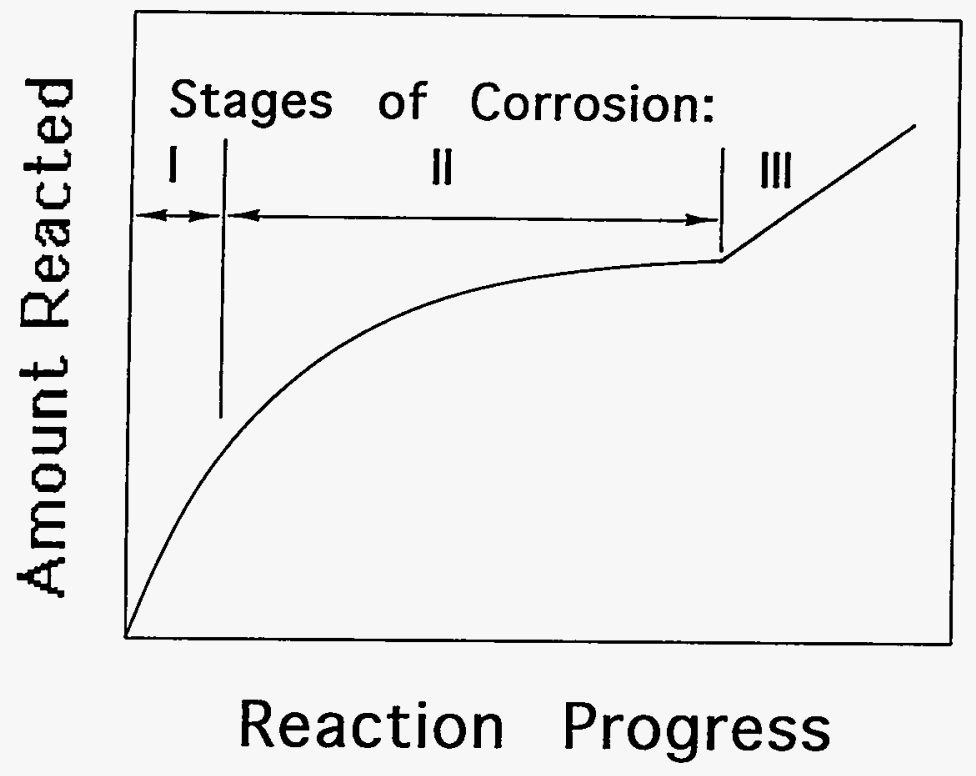

Fig. 6. Schematic Plot Showing Corrosion Behavior as the Corrosion Progresses. Stage I occurs when the solution concentrations of glass components are too low to significantly affect the corrosion rate. Stage II occurs when glass components approach an apparent saturation concentration and slow the corrosion to a negligible rate. Stage III occurs when secondary phases maintain glass corrosion products at concentrations below the apparent saturation values.

environmental conditions from evolving. The change in the solution chemistry allowed by the test conditions will depend on the specific flow or replenishment rate. The effects of specific test conditions on the corrosion behavior are addressed below where pertinent.) The reaction path described by the curve is consistent with existing quantitative models discussed in Section 3 . The reaction progress variable shown as the $\mathrm{x}$-axis in the figure includes the effects of the reaction time and various test conditions, including the SA $/ \mathrm{V}$. The reaction progress variable is sometimes represented as the product of time and the glass surface area/solution volume ratio when plotting experimental data. However, it will be shown that the different $\mathrm{pH}$ values attained in tests at different $\mathrm{SA} / \mathrm{V}$ complicate direct comparison of tests at different $\mathrm{SA} / \mathrm{V}$ (see Section 4.2) [81].

The curve drawn in the figure describes the evolution of a particular corrosion system where the solution chemistry freely evolves as the glass corrodes over time. Hence, several important aspects of the system change as the reaction progresses, including the solution $\mathrm{pH}$, the concentrations of glass components in the leachate, and the development of an alteration layer on the glass surface due to nonstoichiometric glass corrosion and secondary phase formation. The $y$-axis represents the measured amount of glass corroded, and different measures of the extent of corrosion will yield slightly different curves. The curve in Fig. 6 is meant to represent the solution concentration of a highly soluble glass component (such as boron). The corrosion rate is given by the derivative of the curve. Different curves will exist for different glass compositions, leachant compositions, and temperatures. The following discussion is with regard to a particular system of glass, leachant, and temperature.

The corrosion is described as occurring in three stages which are represented by changes in the solution concentration of boron in Fig. 6 . (Boron is commonly used as a measure of the extent of glass corrosion and, for convenience of discussion, will be used for that purpose here.) 
During the initial stage of corrosion, referred to here as Stage I, corrosion occurs at a constant rate that depends on the glass composition and the solution $\mathrm{pH}$ that is often referred to as the forward rate. The boron concentration increases at a constant rate during this stage of the corrosion, and is not measurably affected by the presence of glass corrosion products in solution. The rate measured in the absence of effects of dissolved glass components (the forward rate) provides a valuable characteristic of the glass for modeling its long-term corrosion behavior. The forward rate is affected by the glass composition, the test temperature, and the solution $\mathrm{pH}$. Different glass components may also be released at different forward rates. That is, the glass may dissolve nonstoichiometrically during Stage I. Dynamic tests conducted under high flow rates or frequent leachate replenishment can be used to maintain the corrosion in Stage I.

As the glass continues to corrode, the corrosion rate will eventually decrease as corrosion products accumulate in the solution. This is referred to as Stage II. The transition from Stage I to Stage II occurs gradually as the corrosion rate decreases as the solution becomes more concentrated in corrosion products. The distinction between Stages I and II is somewhat arbitrary, and depends, in part, on the ability to measure small changes in the corrosion rate. The rate will also decrease as alteration layers form on the glass surface and slow the release of glass components through mass transfer effects. The amounts of glass components released to solution will also decrease as the surface area of the dissolving glass decreases, although the dissolution rate itself will not necessarily decrease. Corrosion may continue in Stage II for very long time periods. Eventually, the rate will decrease to near zero as the chemical potential difference between components in the glass and in the solution approach minimum values. The rate measured experimentally is referred to as the "saturation rate," since the solution appears to become saturated with respect to the glass. Corrosion is Stage II is observed in most static leach tests where dissolved glass components are allowed to accumulate in the leachate solution.

Secondary phases will eventually precipitate from the concentrated solution and will alter the solution chemistry. The change in the solution chemistry will then affect the corrosion rate. The formation of secondary phases that affect the corrosion rate marks the beginning of Stage III. The extent to which secondary phases affect the corrosion rate depends on the assemblage of phases formed and how they alter the solution chemistry. In fact, some secondary phases form without measurably affecting the corrosion rate. Clays commonly form early during the corrosion of borosilicate waste glasses, but their formation does not measurably affect the corrosion rate. This is probably because their effect on the solution chemistry is negligible. The increased corrosion rate shown in Fig. 6 is similar to that observed for high-level waste glasses under some test conditions. Corrosion in Stage III has been observed in static leach tests conducted at very high SA/V and elevated temperatures where highly concentrated leachate solutions are generated.

The corrosion rate observed in Stage III depends on the relative rates of secondary phase precipitation and glass dissolution, that is, whether or not the secondary phases equilibrate with the solution. If secondary phases precipitate fast enough to maintain equilibrium with the solution, then the observed glass dissolution rate will be higher than that prior to secondary phase formation and will be constant (as shown in the figure). If secondary phases precipitate slowly, such that the solution remains supersaturated, then the observed rate will slow over time. The ability of the secondary phases to remove glass components from the solution will also depend on the relative surface areas of the glass and the secondary phases. Continued corrosion in Stage III involves the maturation of the assemblage of secondary phases as the initially formed phases are replaced by more stable phases in a paragenetic sequence. As more stable phases form, the solution chemistry and glass corrosion rate will be affected. Corrosion of waste glasses for extremely long time periods will be controlled by changes in the solution chemistry that occur during the evolution of the assemblage of secondary phases. 
Glass corrosion in Stages I and II is commonly observed in most laboratory tests where the corrosion products are allowed to accumulate in the leachate solution. Progression into Stage III is not commonly seen in laboratory tests simply because the glass corrosion does not reach Stage III under the test conditions used within the reaction times tested. Several examples exist where the last data point collected is observed to be higher than expected based on the trend shown by tests conducted for shorter time periods; these tests have likely progressed to Stage III. By performing tests under conditions that lead to highly concentrated solutions within a relatively short time, the corrosion progress of most glasses can be driven to Stage III.

The corrosion behavior of a glass in each Stage cannot be predicted from its measured behavior in any other Stage because what controls the corrosion rate is different in each Stage. In Stage I, the corrosion is controlled by the glass composition and leachant $\mathrm{pH}$. In Stage II, it is controlled by the solution chemistry (primarily silicic acid concentration and $\mathrm{pH}$ ) which is determined by the glass composition. In Stage III, the corrosion rate is controlled by the solution chemistry that equilibrates with the assemblage of secondary phases that is formed. While the compositions of the secondary phases is determined in part by the glass composition (the solution from which they precipitate is composed of dissolved glass), the identity of secondary phases that form initially and the effects of their formation on the glass corrosion rate must be determined experimentally.

The prediction of glass corrosion behavior over long time periods requires the corrosion progress curve of the waste glass be known and related to storage conditions. This requires characterization of the glass corrosion behavior using several test methods to determine (1) the forward reaction rate, (2) the solution saturation conditions and the saturation rate, and (3) the identity of the assemblage of secondary phases formed and determination of how they affect the corrosion rate. Once the corrosion curve of the glass is determined, how far along the curve corrosion will progress under particular disposal scenarios can be estimated based on projected groundwater flow rates, amounts of groundwater expected to contact the waste packages, chemical effects due to interaction of the groundwater with the surrounding geology, radiation effects, etc. This report now focuses on the test methods used to describe the glass corrosion behavior and experiments conducted to establish the curve and analytical model of the corrosion rate.

\subsection{Standard Test Methods}

Laboratory tests are performed on glass waste forms to provide information on their chemical durabilities. Test results can be used to guide formulation of waste form compositions and to monitor their production, or to determine waste form corrosion mechanisms and predict their long-term durabilities under potential disposal environments. The information needed will determine the test methodology that should be used and the required detail of analysis of the reacted sample. Tests designed to monitor the processing of waste forms are discussed in detail because of the extensive database that is available for these tests and because of the characterization of the glass corrosion process that these tests provide. Other tests have been designed to highlight a particular corrosion step or to provide values for use in reaction models.

The American Society for Testing and Materials (ASTM) has issued a Standard Practice for relating laboratory tests to the prediction of glass corrosion (ASTM C1174-91: "Prediction of the Long-Term Behavior of Waste Package Materials Including Waste Forms Used in the Geologic Disposal of High-Level Nuclear Waste"). In this Practice, several categories of tests are identified, including attribute tests, characterization tests, accelerated tests, service condition tests, analog tests, and confirmation tests. The purpose of these tests is to provide parametric values to be used in support of a mechanistic model of the corrosion process and to verify the relevance of the model. The Practice does not identify specific test procedures to be used as attribute tests, characterization tests, accelerated tests, etc., rather it "covers the general approach 
for proceeding from the statement of a problem in prediction of long-term behavior of materials, through the development and validation of appropriate models, to formulation and confirmation of actual predictions" [82].

The corrosion of a glass observed in a laboratory test can be utilized simply as a measure of the response of the glass to those particular test conditions, or the results can be further interpreted as a measure of a characteristic property of the glass, usually with respect to a specific corrosion model. Different test methods have been developed to compare the responses of different glasses under standard test conditions without regard to the corrosion mechanism. These tests have been created to assist in the development of waste forms and the monitoring of product consistency. Other tests have been developed to characterize the corrosion behavior of a glass under conditions believed to highlight a certain aspect of the corrosion process. In some cases, the same test method can be used to compare the response of a glass to that of a reference glass and to ascertain a corrosion characteristic of the glass. Of course, the distinction between a response test and a characterization test is primarily in how the results are utilized. However, as will be discussed in detail in this report, the corrosion mechanism should be considered when interpreting the results of response tests because corrosion of different glasses under the same test conditions may result in different characteristic corrosion processes dominating the observed responses. That is, the test may measure different corrosion characteristics for different glasses and, therefore, not measure the same aspect of corrosion for different waste forms. This may or may not affect the utility of the test for determining product consistency.

Service condition tests are designed to simulate environmental conditions anticipated to exist during waste disposal, such as temperature, amount and chemistry of groundwater, surrounding geology, etc. In general, the test conditions used to characterize the glass corrosion behavior are very different from those anticipated to exist during actual storage of the glass. This is because the characterization tests are typically designed to isolate one aspect of the corrosion behavior, while the corrosion of the glass in service condition tests is affected by several interactions simultaneously. Additionally, in an actual disposal site, most conditions are expected to vary over time, while constant environmental conditions are usually maintained in characterization tests. For example, the temperature in a repository is expected to decrease over time while most tests are conducted at a constant temperature. Water can enter the repository only very slowly, first as water vapor, then as small volumes of liquid water. Laboratory tests are generally conducted with much larger volumes of water than are likely to contact the waste in a repository, and generally do not replicate the slow increase in the amount of water present. Thus, the results of characterization tests can only be related to repository performance after careful consideration of the anticipated environmental conditions and an understanding of the glass corrosion mechanism and how it is affected by parameters such as the temperature, water volume and fluid chemistry, and how these conditions change over time. Although specific service conditions and the evolution of the environment in a waste repository are not discussed in this review, the relevance of specific test methods designed to characterize the corrosion behavior of waste glasses to glass performance under likely storage conditions is discussed.

Laboratory tests of chemical durability are performed by contacting the glass sample with a solution and measuring changes in either the glass or the solution. Standard testing procedures have been developed to directly compare results obtained at different laboratories for different glass compositions. These tests can be categorized as dynamic tests, in which the solution is continuously or periodically refreshed, or as static tests, in which the leachate is not refreshed during the test. (Note that in the present context, the specification of static and dynamic refers only to the replacement of leachate solution as the test is conducted.) Tests may be performed on either monolithic samples or crushed samples to provide various glass surface areas that may be contacted by different solution volumes. A summary of test methods used to measure the 
chemical durability of high-level waste glasses has been compiled by the International Atomic Energy Agency (IAEA) and is included with other test methods in Table 1 [83]. Some commonly used tests relevant to further discussions in this report are discussed in more detail below.

\subsubsection{Static Tests}

\subsubsection{MCC-1 Test}

The MCC-1 static leach test (sometimes referred to as MCC-1P) was developed by the Materials Characterization Center to provide a comparison of the durabilities of candidate waste forms developed for the stabilization of high-level nuclear wastes [84]. The method calls for placing a monolithic specimen of known geometric surface area into a volume of leachant such that the SA/V is $10 \mathrm{~m}^{-1}$. The test may be performed in deionized water, a reference silicate solution, or a reference brine. The reference silicate and brine compositions as analyzed in Round Robin tests conducted for the MCC-1 are given in Table 2 . The silicate solution is dominated by sodium and silicon, while the brine is dominated by sodium and potassium. Tests are to be conducted in perfluoralkoxy (PFA) Teflon containers, unless the absorbed dose to the container due to the radiation field will exceed $10^{4} \mathrm{rad}$ (100 Gy) over the course of a test, in which case a vessel constructed from an inert material (such as stainless steel or fused silica) is to be used. Teflon vessels cannot be used in high radiation fields because of the possibility of fluoride being leached from the vessel [85]. The reference temperature is $90^{\circ} \mathrm{C}$ and the reference test time is 28 days, although temperatures of 40 or $70^{\circ} \mathrm{C}$ and other testing times can be used. (The MCC-2 test procedure is a variation of the MCC-1 procedure for reaction temperatures greater than $90^{\circ} \mathrm{C}$ [86].) The measured solution concentrations of component species are used to monitor the extent of glass corrosion.

Monolithic specimens can be prepared for testing by fracturing, cutting, or grinding the sample material. The surface finish resulting from the surface preparation is known to have a measurable effect on the measured corrosion rate, which increases with surface roughness (see Section 2.7). The increase is due to both the increase in the actual surface area with the surface roughness (the geometric surface area generally does not account for the surface finish) and the increased surface strain. Test results for short-term tests of a few days are strongly influenced by the initial surface condition and the presence of fines. The MCC-1 test provides a solution-dominated system in that the leachate remains dilute as the glass dissolves. Because there is little interaction between released glass components and the glass, the corrosion is strongly influenced by the glass composition. However, tests conducted for long periods may be affected by the leachate solution chemistry. Samples corroded in MCC-1 tests show details of the chemical and physical alteration of the glass surface that cannot be seen in other tests. Detailed analyses of monolithic samples corroded during this test have provided valuable insight into the mechanism controlling the initial stage of corrosion [87-89].

The precision of the MCC-1 test method was evaluated by Kingston et al. by testing glasses with tightly controlled key procedural steps and experimental conditions [90]. Aware of the effects of surface finish on the test result, they ground all test specimens to a 600-grit final finish to improve the uniformity of the samples. They determined that "all the imprecision contributed by the test parameters and the glass samples combined was found to be insignificant compared to the analytical technique used for leachate analysis under these conditions" [90]. Analytical error is typically near 10-15\% of the amount present for multicomponent analyses required during the performance of these tests. The precision determined from the MCC Round Robin test program was 6-11\% within a laboratory and $25-40 \%$ between laboratories [91]. Much of the difference between laboratories was probably due to sample preparation. 
Table 1. List of Test Methods (adapted from [83])

\begin{tabular}{|c|c|c|c|c|c|}
\hline Test Name & Temp. $\left({ }^{\circ} \mathrm{C}\right)$ & Leachant & Flow Rate & Sample Description & Ref. \\
\hline Soxhlet & $50-100$ & DIW & $1.5 \mathrm{~mL} / \mathrm{min}$ & Plate, $S=3 \mathrm{~cm}^{2}$ & 1 \\
\hline Modified Soxhlet & $35-100$ & DIW & Variable & Plate or crushed & 2 \\
\hline Hot-Cell Soxhlet & 100 & DIW & $80 \mathrm{~mL} / \mathrm{h}$ & Beads, plates, chips & 3 \\
\hline MCC-5 Soxhlet & 100 & DIW & $1.5 \mathrm{~mL} / \mathrm{h}$ & Plate, $S=4 \mathrm{~cm}^{2}$ & 4 \\
\hline Soxhlet (PNC) & 70,100 & DIW & $60-225 \mathrm{~mL} / \mathrm{h}$ & Bar, $\mathrm{S}=\mathrm{cm}^{2}$ & 5 \\
\hline $\begin{array}{l}\text { HIPSOL } \\
\text { (HT Soxhlet) }\end{array}$ & $100-300$ & DIW & $100-900 \mathrm{~mL} / \mathrm{h}$ & Powder or block & 6 \\
\hline IAEA & 25 & DIW & Periodic replacement & Cylinder & 7 \\
\hline ISO Buffer & $23-100$ & $\begin{array}{l}\text { DIW, buffers, or } \\
\text { sea water }\end{array}$ & Periodic replacement & Monoliths & 7 \\
\hline ANS 16.1 & 25 & $\begin{array}{l}\text { DIW or synthetic } \\
\text { seawater }\end{array}$ & Periodic replacement & $\begin{array}{l}\text { Cylinders, } \\
\mathrm{S} / \mathrm{V}=10 \mathrm{~m}^{-1}\end{array}$ & 8 \\
\hline DLT & 25 & $\begin{array}{l}\text { DIW or synthetic } \\
\text { seawater }\end{array}$ & Periodic replacement & Cylinders, variable & 9 \\
\hline Powder (P1) & $95-200$ & DIW & Periodic replacement & $100-200 \mu \mathrm{m}$ & 10 \\
\hline Powder (P2) & $40-100$ & DIW & Daily replacement & $100-150$ mesh & 11 \\
\hline MCC-4 & $40,70,90$ & $\begin{array}{l}\text { DIW or } \\
\text { reference } \\
\text { groundwater }\end{array}$ & $0.1-0.001 \mathrm{~mL} / \mathrm{min}$ & Plate & 4 \\
\hline Low Flow & $25-90$ & DIW & $1 \mathrm{~mL} / \mathrm{wk}$ & Plate & 12 \\
\hline Dynamic & $35-90$ & DIW & $3-1200 \mathrm{~mL} / \mathrm{h}$ & Crushed or monolith & 13 \\
\hline Grain Titration & 100 & DIW & Static & Crushed & 14 \\
\hline $\begin{array}{l}\text { Time-Dependent } \\
\text { Method }\end{array}$ & $20-60$ & Buffer & Static & Disc & 12 \\
\hline MCC-1 & $40,70,90$ & $\begin{array}{l}\text { DIW or } \\
\text { reference } \\
\text { groundwater }\end{array}$ & Static & $\begin{array}{l}\text { Monolith, } \\
\mathrm{S} / \mathrm{V}=10 \mathrm{~m}^{-1}\end{array}$ & 4 \\
\hline MCC-2 & $110,150,190$ & $\begin{array}{l}\text { DIW or } \\
\text { reference } \\
\text { groundwater }\end{array}$ & Static & $\begin{array}{l}\text { Monolith, } \\
\mathrm{S} / \mathrm{V}=10 \mathrm{~m}^{-1}\end{array}$ & 4 \\
\hline MCC-3 & $\begin{array}{l}40,90,110 \\
150,190\end{array}$ & $\begin{array}{l}\text { DIW or } \\
\text { reference } \\
\text { groundwater }\end{array}$ & Static (agitated) & $\begin{array}{l}\text { Crushed, } 149-175 \mu \mathrm{m} \text {, } \\
\text { or }<45 \mu \mathrm{m}\end{array}$ & 4 \\
\hline HTLC (CEC) & $\begin{array}{l}90,110,150, \\
190\end{array}$ & DIW & Static & $\mathrm{S}=4 \mathrm{~cm}^{2}$ & 15 \\
\hline Autoclave (HMI) & $150-200$ & DIW or brine & Static & Beads, chips & 16 \\
\hline Autoclave (KfK) & $\begin{array}{l}100,150,200, \\
250\end{array}$ & DIW or brine & Static & $\begin{array}{l}\text { Cylinders, } \\
\mathrm{S}=20 \text { or } 5 \mathrm{~cm}^{2}\end{array}$ & 17 \\
\hline $\begin{array}{l}\text { Repository } \\
\text { Simulation }\end{array}$ & $25-90$ & Granitic water & & Plate, $S=3 \mathrm{~cm}^{2}$ & 12 \\
\hline $\begin{array}{l}\text { Waste/Water/ } \\
\text { Rock Leach }\end{array}$ & 98 & $\begin{array}{l}\text { DIW or granitic } \\
\text { water }\end{array}$ & & $\begin{array}{l}\text { Cube, } S=6 \mathrm{~cm}^{2} \\
20 \text { g granite powder }\end{array}$ & 5 \\
\hline MCC-14 & $25-290$ & $\begin{array}{l}\text { Repository } \\
\text { groundwater }\end{array}$ & $\begin{array}{l}\text { Static or periodic } \\
\text { sampling }\end{array}$ & Monoliths or powders & 4 \\
\hline PCT A & 90 & DIW & Static & Crushed, $74-149 \mu \mathrm{m}$ & 18 \\
\hline PCT B & Variable & Variable & Static & Crushed & 18 \\
\hline $\mathrm{ALT}$ & Variable & DIW & Periodic replacement & Cylinder & 19 \\
\hline TCLP & 25 & Acetate solution & Static (agitated) & Crushed $(<0.375$ in. $)$ & 20 \\
\hline
\end{tabular}


Table 1 - contd.

\begin{tabular}{llllll}
\hline Test Name & Temp. $\left({ }^{\circ} \mathrm{C}\right)$ & Leachant & Flow Rate & Sample Description & Ref. \\
\hline Cal. WET & 25 & $\begin{array}{l}\text { DIW or citrate } \\
\text { solution }\end{array}$ & Static (agitated) & Crushed & 21 \\
\hline MEP & 25 & $\begin{array}{l}\text { Acetate solution } \\
\text { followed by } \\
\text { synthetic acid } \\
\text { rain }\end{array}$ & & & 20 \\
\hline MWEP & 25 & $\begin{array}{l}\text { DIW or synthetic } \\
\text { site water }\end{array}$ & Static & Crushed or monolith & 22 \\
\hline ELT & 25 & DIW & Static & Crushed & 9 \\
\hline ANC & 25 & $\begin{array}{l}\text { Nitric acid } \\
\text { solution }\end{array}$ & Static & Crushed & 9 \\
\hline SCE & 25 & Acid solution & Static & Crushed & 9 \\
\hline Unsaturated Test & $25-90$ & $\begin{array}{l}\text { Repository } \\
\text { groundwater }\end{array}$ & $\begin{array}{l}75 \mu \mathrm{L} / 3.5 \text { days } \\
\text { (variable) }\end{array}$ & Monolith & 23 \\
\hline Vapor Hydration & $70-250$ & DIW & Static & Monolith & 24 \\
\hline
\end{tabular}

[1] H. Nakamura and S. Toshiro, Progress Report on Safety Research of High-Level Waste Management for the Period April 1982 to March 1983, Japan Atomic Energy Research Institute Report JAERI-M-83-076 (1983).

[2] M. Hussain and L. Kahl, "Incorporation of Precipitation from Treatment of Medium-Level Liquid Radioactive Waste in Glass Matrix or Ceramics Together with High-Level Waste," Ceram. in Nucl. Waste Manag. Proc. Int. Symp (1979).

[3] Commission of the European Communities, Testing and Evaluation of Solidified High-Level Waste Forms, Commission of European Communities (CEC) Joint Annual Progress Report EUR 10038 (1983).

[4] Nuclear Waste Materials Handbook (Test Methods), Technical Information Center, DOE Report DOE/TIC-11400 (1981).

[5] H. Igarashi et al., "Leaching Test of Simulated HLW Glass in the Presence of Rock," presented at the Annual Meeting of the Atomic Energy Society of Japan (1983).

[6] M. Senoo et al., High-Pressure Soxhlet-Type Leachability Testing Device and Leaching Test of Simulated High-Level Waste Glass at High Temperature, Japan Atomic Energy Research Institute Report JAERI-M8571 (1979).

[7] Draft International Standard Report ISO/DIS-6961 (1979).

[8] American Nuclear Society, La Grange Park, Illinois.

[9] Dynamic Leach Test (DLT), Equilibrium Leach Test (ELT), Acid Neutralization Capacity (ANC), and Sequential Chemical Extraction (SCE), "Investigation of Test Methods for Solidified Waste Evaluation-A Cooperative Program, Appendix B: Test Methods for Solidified Waste Evaluation," Environment Canada and Alberta Environmental Center (1991).

[10] V. M. Oversby and A. E. Ringwood, "Leaching Studies on SYNROC at $95^{\circ} \mathrm{C}$ and $200^{\circ} \mathrm{C}$," Radioact. Waste Manag. 23, 223 (1982).

[11] K. D. Reeve et al., "The Development of Testing of SYNROC for High-Level Radioactive Waste Fixation," Waste Manag. Proc. Symp. 1, 249-266 (1981).

[12] P. Van Iseghem et al., Chemical Stability of Simulated HLW Forms in Contact with Clay Media, Commission of European Communities Report EUR-8424 (1983).

[13] G. A. Vaswani et al., Development of Improved Leaching Techniques for Vitrified Radioactive Waste Products, Bhabha Atomic Research Centre Report BARC-1032 (1978).

[14] Deutsches Institut fur Normung, DIN Leach Test, Report 12111 (1976).

[15] European Community Static High Temperature Test Summary, Commission of European Communities Report EUR 9772 (1985).

[16] F. K. Altenhein et al., Scientific Basic for Nuclear Waste Manag. Proc. Int. Symp., 363-370 (1981).

[17] L. Kahl, M. C. Ruiz-Lopez, J. Saidl, T. Dippl, Preparation and Characterization of an Improved Borosilicate Glass for Solidification of High-Level Radioactive Fission Product Solutions, Part 2: Characterization of the Borosilicate Glass Product GP 98/12, Kernforschungszentrum Karlsruhe Report KFK-3251e (1982). 
Table 1 - contd.

[18] C. M. Jantzen, N. E. Bibler, D. C. Beam, W. G. Ramsey, and B. J. Waters, Nuclear Waste Glass Product Consistency Test (PCT) Version 5.0(U), Westinghouse Savannah River Co. Report WSRC-TC-90-539, Rev. 2 (1992).

[19] M. Fuhrmann, J. H. Heiser, R. F. Pietrzak, E. M. Franz, and P. Colombo, "Method for Accelerated Leaching of Solidified Waste," Brookhaven National Laboratory Report BNL-52268, November (1990).

[20] U.S. Environmental Protection Agency, "Toxicity Characteristic Leach Procedure," Federal Register, March 29, 1990, pp 11798-11877, and June 29, 1990, pp 26986-26998.

[21] California Waste Extraction Test, "Criteria for Identification of Hazardous and Extremely Hazardous Wastes," California Code, Title 22, Article 11.

[22] Monofill Waste Extraction Procedure, "A Procedure for Estimating Monofilled Solid Waste Leachate Composition," Technical Resource Document SW 924, First Edition, U.S. Environmental Protection AGency, Cincinnati, OH (1968).

[23] A. B. Woodland, J. K. Bates, and T. J. Gerding, Parametric Effects on Glass Reaction in the Unsaturated Test Method, Argonne National Laboratory Report ANL-91-36 (1991).

[24] J. K. Bates, L. J. Jardine, and M. J. Steindler, The Hydration Process of Nuclear Waste Glass: An Interim Report, Argonne National Laboratory Report ANL-82-11 (1982). 
Table 2. Analysis of Leachants by Participants in the MCC Round Robin of the MCC-1 Test ${ }^{\mathrm{a}}$

\begin{tabular}{ccc}
\hline Element & $\begin{array}{c}\text { Silicate Solution } \\
\text { Average, ppm }\end{array}$ & $\begin{array}{c}\text { Brine Solution } \\
\text { Average, ppm }\end{array}$ \\
\hline $\mathrm{B}$ & 0.161 & 1.412 \\
$\mathrm{Ca}$ & 0.072 & 1.014 \\
$\mathrm{Cs}$ & 0.110 & 0.520 \\
$\mathrm{Fe}$ & 0.006 & 0.867 \\
$\mathrm{~K}$ & 0.782 & 20650 \\
$\mathrm{Na}$ & 49 & 37214 \\
$\mathrm{Sr}$ & 0.004 & 0.094 \\
$\mathrm{Si}$ & 22.76 & 3.596 \\
$\mathrm{pH}$ & & 6.48 \\
$\mathrm{pH}$ & \\
aNumber of significant figures reported by Johnston and \\
Daniel [91].
\end{tabular}

While the MCC-1 method was originally designed to compare the relative chemical durabilities of candidate waste forms, the test can be used to characterize several aspects of the corrosion process in conjunction with a corrosion mechanism. Tests conducted for short periods (on the order of 28 days or less, depending on the reactivity of the waste form) provide a simple and useful measure of the glass corrosion rate under dilute conditions. Variations of the MCC-1 method conducted in buffer solutions or in solutions spiked with various glass components can be used to determine the effects of the leachate chemistry on the glass corrosion rate [92]. Longer term tests can be run to monitor the alteration of the glass surface during corrosion to investigate the corrosion mechanism [87,93-97].

Application of the MCC-1 test to various materials has several advantages. First, heterogenous waste forms can be analyzed before and after testing to monitor the corrosion behavior of the different phases that are present. Second, sample characterization prior to testing can be performed to assure that representative samples of heterogenous materials are be tested. Third, samples can be prepared with geometric shapes amenable to post-test physical and chemical analytical methods, such as fracture tests and chemical analysis of the reacted surface. One disadvantage of the method is that it may be difficult to prepare monolithic samples of glasses that have not been annealed for testing. The removal of strains in the glasses during annealing may affect the performance of the glass in the test. Preparation of samples of radioactive glasses may also be difficult if remote handling is required.

The MCC-1 test conditions are not representative of disposal conditions that are currently under consideration. The very low SA/V used in the MCC-1 test represents the highly unlikely event of the head space of a waste canister being instantaneously filled with groundwater. More likely, water vapor will first fill the canister and condense on the glass. The amount of water contacting the glass would slowly increase, possibly filling the head space if no drainage from the canister occurred. The glass would continually corrode as more water entered the canister, such that the highly dilute conditions measured in the MCC-1 test may never occur under disposal conditions.

The MCC- 1 test has recently been standardized by the ASTM through committee C-26 as Standard C1220-92, "Test Method for Static Leaching of Monolithic Waste Forms for Disposal of Radioactive Waste" [98]. Despite possible difficulties in application with 
radioactive samples and its being irrelevant to service conditions, the $\mathrm{MCC}-1$ test provides a simple method of comparing the relative durabilities of different homogeneous waste glass compositions under conditions that are sensitive to the glass composition. The results of MCC-1 tests conducted for short time periods (typically 7 days or less) provide a useful estimate of the forward corrosion rates of candidate waste glasses. The MCC-1 tests are easier to perform than other tests designed to measure the forward corrosion rate, such as Soxhlet and single-pass flowthrough tests, which are described below.

\subsubsection{Product Consistency Test}

The Product Consistency Test (PCT) [74] was developed for use at the DWPF to monitor the chemical durabilities of the glasses to be produced and assure that all waste forms exceed the benchmark measure of durability $[99,100]$. Variations of the PCT are used to monitor the product consistency (PCT method A) and to characterize the corrosion behavior (PCT method B). In the PCT method A, a mass of crushed glass in the size fraction between 100 mesh and 200 mesh sieves is combined with a mass of deionized water ten times that of the glass. The test time is seven days and the test temperature is $90^{\circ} \mathrm{C}$. At the end of the test, the leachate solution is filtered using a relative coarse filter (a $0.45-\mu \mathrm{m}$ filter is called for in the procedure) to remove suspended glass. This solution is analyzed to measure the boron, sodium, and silicon concentrations. The PCT method A was developed to remotely monitor the consistency of glass produced by the DWPF. The PCT method A specifies that stainless steel reaction vessels be used, since the glasses to be tested will be highly radioactive and other materials, such as Teflon, may be affected by the radiation fields [85]. The PCT method A is a "response test," in which the response of a sample glass is compared to the benchmark response of a reference glass, which for the DWPF has been identified as the "SRL EA" glass [100].

The PCT method B allows variation in the size fraction of the glass used, the glass mass-to-leachant mass ratio, the leachant composition, the test temperature, and the test duration. The PCT method B was designed to investigate the effects of various parameters on the response of a specimen so that the results could be used in the further development of waste forms. In effect, the PCT method B encompasses all variations of crushed-sample, static leach tests. The method does not specify the use of a particular reaction vessel; studies have shown the effect of the vessel used in the test to generally be small $[67,101,102]$.

There is some concern regarding the effect of using crushed glass in tests, since waste forms placed in storage will not be crushed and since uncertainties exist regarding the effects of the particle size on the test results. Particle size effects are very difficult to separate from other effects in laboratory tests, in part because the surface area of crushed glasses is hard to measure (see Section 2.6) and the surface area may decrease significantly over the course of the test (see Section 2.9). The surface area is an important test parameter that is approximately proportional to the number of reaction sites available for each corrosion process (water diffusion through channels, ion exchange, and hydrolysis). The surface area is usually combined with the solution volume in a single test parameter, namely SA/V. The SA/V of a test determines the rate of change of solution concentrations as a waste form dissolves, which affects the corrosion rate. The PCT method A avoids addressing surface area effects by specifying that glass of a particular size fraction be used in the test. An acceptably reproducible surface area can then be attained by accurately weighing the glass. However, different glass compositions may fracture differently, and so the total surface areas in tests with different glasses may not be the same. This is probably an unavoidable limitation of using crushed glass.

Also, glasses of different density will result in different numbers of grains being used in the test. That is, fewer grains are present in $1 \mathrm{~g}$ of a heavy glass than in $1 \mathrm{~g}$ of a lighter glass. If the grains of heavy and light glass are the same size, as assumed for the 
same sieve fraction, then less surface area will be present in tests with the heavy glass than with the light glass. The solution concentrations of components released from the heavy glass will be lowered due to the smaller surface area. The solution concentrations could be normalized by multiplying by the densities of the glasses, although this is not done in practice. The correction factors would be of the order of the differences in densities. That is, a glass that has a density $10 \%$ higher than another glass is expected to have about $10 \%$ less surface area per gram and so the solution concentration is expected to be about $10 \%$ lower due to the lower surface area, irrespective of the durability of the glass.

While the surface area is not specified by the PCT procedure, it must be known to compute the normalized mass loss, which is need to compare PCT results for various size fractions to other test results. (Use of the PCT method A as a response test does not require computation of the normalized mass loss, since all results are compared to other PCT method A results.) The surface area is usually calculated by assuming that the crushed particles have a convenient geometric shape, such as a sphere or a cube, that can be related to the sieve size. Such an approximation suggests the SA/V used in the PCT method A test, for glasses having a density of about $2.7 \mathrm{~g} / \mathrm{cm}^{3}$, is about $2000 \mathrm{~m}^{-1}$ (see also Section 2.6 ).

Despite the uncertainty in the absolute surface area, the SA/V used in the PCT method provides a convenient means of accelerating the approach of the solution chemistry to saturation. The reaction system is dominated by the surface area of glass that is available for reaction (in contrast to the MCC-1 method in which the system is dominated by solution). Because the corrosion of a very small amount of glass produces large changes in the solution chemistry under high SA/V conditions, the effects of the solution chemistry and the glass corrosion products on the glass corrosion process are highlighted. Tests that are run for only a few days may attain solution compositions that MCC-1 tests would require several years to attain. Alteration of the glass surface progresses rapidly in response to the solution chemistry, and alterations observed after a year or more in MCC-1 testing can be observed within a few days in PCT testing [81]. Similarities in the alteration of the glass surface demonstrate that the corrosion mechanism is the same under MCC-1 and PCT conditions. Thus, PCT tests can easily be used to accelerate the corrosion process, and PCT tests run for periods between a few days and several months can be used to map the evolution of the solution chemistry as it approaches saturated conditions. These tests can be used to provide parameters to mechanistic models used to predict the long-term behavior of waste glasses under a wide range of corrosion conditions. The composition of the saturated solution is needed for computer modeling of the corrosion process (see Section 6). In addition, secondary phases can be generated in PCT tests conducted for periods on the order of several months or more, depending on the glass durability. These tests can be used to determine the corrosion rate attained upon the formation of secondary phases, which represents the long-term corrosion rate. Analysis of the corroded solids in tests which have progressed into the long-term corrosion stage provides insight into the corrosion mechanism, allows corrosion products to be identified, and confirms that the corrosion mechanism has not changed [65].

Saturated solution conditions, secondary phase formation, and the longterm corrosion rate are attained faster in tests at higher SA/V. Higher SA/V conditions can be attained by simply performing the PCT test using $-100+200$ mesh glass at a greater glass massto-solution volume ratio or by using a smaller particle size. Again, the absolute surface area provided by a particular grain size can only be approximated, although the relative surface area is expected to scale with the mass of glass used in the test. Therefore, tests performed with different masses of the same grain size are expected to scale according to the masses used. Since the rate decrease of surface area as the glass corrodes will be higher for smaller particles, tests with different grain sizes may not scale in a predictable fashion. The loss of surface area will affect the actual SA/V of the test and complicate comparison of the results to tests at other SA/V 
(see also Section 2.9). The effect will depend on the relative durability of the glass being tested: durable glasses will show less effect than non-durable glasses. If different grain sizes are used to attain a particular SA/V, some tests should be conducted using glass in the 100 to 200 mesh size fraction to relate the performance of the glass being tested to other glasses and to assess the effects of the grain size.

Complications of using crushed glass include the possibilities that nonrepresentative sampling may occur due to preferential sieving, that the physical shapes of the glass particles affect the measured corrosion rate (i.e., glasses which fracture to produce particles with high energy surfaces and sharp edges will react more than glasses which fracture to form low energy surfaces without sharp edges, regardless of the differences in the intrinsic reactivities of the glasses).

Other complications may arise when applying the test method to some materials. Non-representative samples may be taken when crushing heterogenous waste forms due to the generation of different particle size distributions during fracturing and sieving. Interpretation to the test results may also be difficult if the relative surface areas of various phases exposed to the leachate are not known. Highly soluble phases may be partially or totally dissolved during the washing step to remove fines and so not be detected during the test.

The PCT has been standardized by the ASTM as Standard C1285-94, "Standard Test Methods for Determining Chemical Durability of Nuclear Waste Glasses: The Product Consistency Test (PCT)" [103].

\subsubsection{MCC-3 Solubility Test}

The PCT test described above is similar to the MCC-3 test, which was designed to measure the maximum solubility of a waste form in the solution of interest [86]. The MCC -3 test is a crushed glass test that originally specified that glass passing through a 325 mesh (about $40 \mu \mathrm{m}$ ) screen is to be reacted with a mass of solution ten times the mass of the glass. Tests were to be conducted until the solution composition did not vary with the reaction time (i.e., until the glass "saturated" the solution). However, application of the test method showed that (1) constant solution compositions were not achieved within a few weeks, and (2) the solution composition was dependent on the size fraction of the glass used. The first observation is a direct result of the glass reactivity, while the second is due to the different SA/V that result from the different total surface areas of different sieve fractions. As discussed in this report, tests conducted at higher SA/V are expected to generate more concentrated solutions. This was observed in MCC-3 tests conducted with PNL 76-68 glass [86]. The MCC-3 procedure has been modified such that (1) a single size fraction is specified, and (2) the test vessel is continuously agitated during the test. The PCT test has effectively replaced the MCC-3 test in most laboratories, although all of the complications associated with the use of crushed glass found during development of the MCC-3 tests are common to the PCT procedure.

The development of the MCC-3 test was based on the premise that different glasses having different compositions would generate saturated solutions having different concentrations. Crushed glass was used to accelerate the saturation of the solutions. However, the observed effects of the particle size and the SA/V on the solution composition show that unique solution compositions are not obtained, and that the solution compositions depend on the test conditions. The difference in the solution concentrations are due in large part to the fact that the glasses corrode nonstoichiometrically and that the $\mathrm{pH}$ achieved in the test depends on the SA/V that is used. 


\subsubsection{Argonne National Laboratory Vapor Hydration Tests}

The vapor hydration test developed at Argonne is a static test in which a monolithic sample is exposed to water vapor in a sealed vessel [104-108]. At relative humidities above about $80 \%$, a thin film of water condenses on the sample in which the glass corrodes [41]. Tests are commonly performed in $100 \%$ relative humidity at temperatures above $100^{\circ} \mathrm{C}$, although tests have been conducted at lower humidities and temperatures. These tests combine the effects of high SA/V and high temperature to accelerate the corrosion process. At the completion of a test, the sample is removed from the vessel, and the reacted surface is analyzed. Corrosion of the sample results in the formation of discrete secondary mineral precipitates and alteration of the glass surface. The solution evaporates from the sample when the test is terminated and is not available for analysis. Material is left as a residue on the corroded sample as the solution evaporates. While most secondary phases form during the test, some secondary phases may form as the fluid evaporates from the sample. These can usually be distinguished by consideration of the solubilities and morphologies of the phases. (Evaporite phases are expected to contain highly soluble components, such as boron, and are usually small, poorly crystalline or amorphous, and widely distributed.) This material can be redissolved by recondensing vapor on the sample at room temperature then removing the solution using a micropipette [81], or by submerging the entire sample in deionized water, as in an MCC-1 test [109]. Both methods have shown high concentrations of soluble glass components to exist on the sample surface, though the latter method is complicated by the disintegration of the layer itself.

The extent of corrosion is determined by measuring the volume of glass that has been altered or the volume of alteration phases generated. Alteration products generally include a clay layer that uniformly covers the sample and precipitated phases that nucleate at various sites on top of or within the clay layer. The extent of corrosion is conveniently measured using the thickness of the alteration layer, which can be assumed to be proportional to the volume of glass corroded. Secondary phases precipitated on the surface are generally excluded from the measurement of the layer, since they are typically localized. Most high-level waste glasses tested to date have formed relatively uniform clay layers at the glass surface that were used to quantify the extent of corrosion [81,104-108]. The relative amounts of secondary phases formed have also been used to compare the extents of reaction [109]. Secondary phases can be analyzed in situ or removed from the sample for analysis and identification.

These tests provide a convenient means of generating secondary phases for analysis within a short period. A knowledge of the assemblage of phases formed is needed to simulate the corrosion process using computer models (see Section 6). In addition, these tests provide a qualitative measure of the effect of secondary phase formation on the long-term corrosion rate; that is, if the formation of secondary phases increases the corrosion rate. This information is important to final waste form development because the long-term corrosion rate depends on the glass composition and the assemblage of phases formed. From these test results, it can be determined whether a candidate final waste form will show an unacceptable increase in the corrosion rate upon secondary phase formation. Such compositions can therefore be avoided. While similar information can be obtained from PCT B tests run for very long time periods, vapor hydration provides results within a few days or weeks.

Vapor hydration tests are performed in a batch mode, in which a separate test is run for each period. Tests can be run for several different durations to determine when the secondary phases form and how they affect the corrosion rate. Vapor hydration tests can be performed at any temperature, though higher temperatures significantly accelerate the individual reaction rates (i.e., diffusion, ion exchange, and hydrolysis reactions). The rate measured at high temperatures can be extrapolated to lower temperatures if the activation energy is known, although the rate-determining step may be different at lower temperatures. Tests at lower temperature may also be required to confirm that the same secondary phases form, but the 
known stability ranges of specific phases can be utilized instead. This information is incorporated into geochemical equilibration computer codes such as EQ3NR, which is being utilized to simulate long-term glass corrosion behavior [110]. However, since the EQ3NR and other equilibration codes require complete specification of the solution chemistry, application of vapor hydration data is currently restricted to identifying the phases that form experimentally for comparison to those that are predicted to form by the model [111], due to the lack of solution data. Nevertheless, identification of the assemblage of secondary phases is crucial to long-term modeling, since these phases control the glass corrosion rate. Phases identified to form in vapor hydration tests are generally different than those predicted to form by computer simulation $[111,112]$. The difference is likely due to the fact that the phases found to form experimentally are generally those favored kinetically while the therrnodynamically most stable phases are predicted to form in the computer models. The phases formed initially will likely be replaced by more stable phases through diagenetic reactions over long time periods. In fact, paragenetic sequences have been observed in vapor hydration tests conducted over extended time periods $[113,114]$.

Corrosion in a vapor environment represents a high SA/V system dominated by the solution chemistry, since very little glass must dissolve to saturate the thin film of sorbed water. While the geometric surface area of the specimen is easily measured, the volume of water in contact with the glass specimen will vary as the corrosion progresses. Initially, only about 50 to 100 monolayers condense on the glass [41] to form a film estimated to be about $0.01 \mu \mathrm{m}$ thick (assuming each monolayer is about $0.15 \mathrm{~nm}$ thick [115]). This is equivalent to an $\mathrm{SA} / \mathrm{V}$ of $1 \times 10^{8} \mathrm{~m}^{-1}$. Additional water condenses as the glass corrodes during the test, until a maximum of about $0.05 \mathrm{~mL}$ condenses on a sample having a surface area of about $1.5 \mathrm{~cm}^{2}$. (Enough water is added to the test vessel to allow $0.05 \mathrm{~mL}$ to condense.) If the water is evenly distributed, then the minimum SA/V is about $3000 \mathrm{~m}^{-1}$, similar to that of the PCT test. However, a significant amount of corrosion will have occurred before the SA/V approaches $3000 \mathrm{~m}^{-1}$ in the vapor hydration test, whereas the SA $/ \mathrm{V}$ is about $2000 \mathrm{~m}^{-1}$ at all times in the PCT test, so the two tests cannot be directly compared. Glass corrosion will be more progressed in vapor hydration tests than in PCT tests because of the initially higher SA/V in the vapor environment. Nevertheless, the glass corrosion path is expected to be the same in long-term PCT tests and in short-term vapor hydration tests. Indeed, hydrothermal leach tests similar to the PCT that were conducted at high temperatures have resulted in the formation of secondary phases within a year $[60,116]$.

\subsubsection{Buffered Solution/pH-Stat Tests}

In $\mathrm{pH}$-stat tests, a constant solution $\mathrm{pH}$ is maintained by the periodic addition of strong acid or base solutions. Tests in buffer solutions and $\mathrm{pH}$-stat tests are performed to suppress changes in the solution $\mathrm{pH}$ as the waste form corrodes. These tests are equivalent to those performed in buffer solutions, except buffers contain counter-ions than could affect the corrosion of the waste form. Strong acid and base solutions are used in pH-stat tests to assure that the counter-ions will not interfere with the waste form.

Corrosion of borosilicate waste glasses generally results in an increase of the solution $\mathrm{pH}$ as a result of dealkalization. The increased $\mathrm{pH}$ then affects the glass corrosion rate. External control of the solution $\mathrm{pH}$ suppresses the natural $\mathrm{pH}$ change during corrosion of borosilicate glasses, although glass corrosion products are free to build up in the solution. Control of the fluid $\mathrm{pH}$ by materials other than the glass may be important in disposal conditions. For example, large amount of backfill material may maintain lower fluid $\mathrm{pH}$ values than those attained during laboratory tests conducted in the absence of these materials. 
Neither $\mathrm{pH}$-stat nor $\mathrm{pH}$ buffering constitute test methods per se, rather they are modifications of other test methods. The need to periodically add acid or base in the $\mathrm{pH}$-stat technique may restrict application to some test methods, and the technique is not widely used in testing high-level waste glasses. The use of buffers to control the solution $\mathrm{pH}$ during both static and dynamic laboratory tests is common (see Section 3.3).

\subsubsection{Dynamic Tests}

\subsubsection{Soxhlet Tests}

Soxhlet tests provide a simple and convenient method for assessing glass corrosion rates under highly dilute conditions. Several variations of the soxhlet test have been utilized. Soxhlet tests are performed by reacting a sample with condensed fluid in a refluxing apparatus. Water is boiled from a reservoir, recondensed in a reflux tube, then allowed to drip into a small cup that contains the sample. The solution in the sample cup is refreshed as condensate (leachant) drips into the sample cup and leachate solution is displaced from the sample cup and drips into the reservoir. Complete replacement typically occurs every few minutes; the precise replenishment time depends on the refluxing conditions. The refluxing action provides an effective flow rate that can be varied by controlling the boiling of the liquid and the condensation of the vapor. Tests are usually run to attain a flow rate sufficiently high that the solution in the sample cup remains highly dilute and does not affect the corrosion rate. The corrosion rate can be measured by periodically removing a sample of the solution in the reservoir for analysis. Different reaction temperatures can be tested by varying the pressure in the apparatus to maintain boiling over a wide temperature range [69]. Because the leachant solution is recondensed vapor, only deionized water or an azeotrope solution can be used as a leachant. Soxhlet tests can be used to provide a measure of the corrosion rate in highly dilute solutions and over a wide range of temperatures. Either crushed or monolithic specimens can be used: however, test conditions must be such that crushed glass is not flushed out of the sample cup. Most tests are conducted using monolithic samples. The MCC-5 Soxhlet test specifies the use of a monolithic sample and an all-Teflon apparatus [86]. Teflon is specified to prevent contamination of the solution by Pyrex glassware.

Soxhlet tests are commonly utilized to measure the initial corrosion rate of glasses as a function of the temperature [69,117]. The glass continues to react at the forward rate because the leached glass components accumulate in the reservoir as leachate solution drips from the reaction cell, while the freshly distilled leachant accumulates in the reaction cell. The fluid contacting the sample remains highly dilute, thus maintaining the forward corrosion rate, while the fluid in the reservoir becomes concentrated, which facilitates analysis. Soxhlet tests have also been performed for long corrosion times on a single sample by periodically replacing the solution in the reservoir with fresh deionized water [118]. Soxhlet tests have also been used to characterize the alteration of the surface after extensive corrosion [119]. Note that the alteration phases formed during the Soxhlet tests are due to differences in the solubilities of different glass components and are not necessarily identical to secondary phases formed from the

saturated leachate in static tests. The leachate solution in contact with the sample in Soxhlet tests remains highly dilute.

\subsubsection{Single-Pass Flow-Through Tests}

In single-pass flow-through tests, leachant solution is passed over a monolith surface or through crushed glass at a constant rate. The sample is contained in a reaction cell that can be modeled as a well-stirred reactor with leachant solution entering the cell and leachate solution exiting the cell at the same rate. Solution is pumped through the cell by a peristaltic pump; the solution leaving the cell is periodically collected and analyzed. The singlepass flow-through apparatus designed at Lawrence Livermore National Laboratory has been 
described by Weed et al. [120] and by Coles [121,122]. The corrosion rates of glass waste forms are known to increase with the leachant flow rate up to a maximum corrosion rate, which is the forward reaction rate (see Section 5). Low flow rates can be used to simulate conditions in particular disposal sites. Alternatively, solution can pumped through the cell at a rate high enough that the glass reacts at its characteristic forward rate. The forward rate has been reached if further increases in the flow rate do not increase in the corrosion rate. The forward corrosion rate is an important characteristic of waste forms that is needed for computer simulations of corrosion behavior (see Section 6).

The forward rate represents the maximum corrosion rate of the waste form at a particular solution $\mathrm{pH}$ and temperature. The corrosion rate is known to decrease as silicic acid and other glass components become concentrated in the solution. The leachant solution used in a single-pass flow-through test can be varied to measure the effects of the solution composition on the forward reaction rate. Such tests have been applied to determine the effects of $\mathrm{pH}$, and of silicon, calcium, aluminum, and other glass components on the glass corrosion rate $[70,71]$. In addition, the reaction cell can be placed in an oven to determine the effects of temperature on the corrosion rate. Such tests are valuable in assessing the corrosion mechanism of waste forms, and they provide values for parameters needed to simulate their long-term corrosion behavior (see Section 6).

Flow tests are usually conducted for several days at a constant flow rate, with samples being collected periodically until the analyses show that a constant (sometimes referred to as "steady-state") rate has been reached. Determination of the corrosion rate requires the glass surface area be known at all times. Tests are complicated by the need to maintain negligible concentrations of corrosion products at the glass surface but to generate leachates that can be analyzed to monitor the reaction rate. The test data must also be interpreted to account for the effects of the initial surface finish of monolithic samples, the presence of fines, the reduction in surface area as the glass corrodes, and the possible formation of surface layers and precipitates. Fines present on the freshly prepared sample provide a very high initial surface area that usually cannot be quantified, and so data from the initial reaction is usually ignored. Tests must also be conducted so that the loss of surface area during the test is negligible. Therefore, crushed glass samples should be sufficiently large that the surface area does not decrease significantly over the test duration. Monolithic samples provide a readily measured but small surface area. Crushed glass samples provide a higher surface area, but the surface area is difficult to quantify. Crushed glass samples are also more likely to contain fines. Also, the surface area of crushed glass samples decreases faster than that of monolithic samples. The loss of surface area will lead to a decrease in the measured corrosion rate over time. The loss of surface area can be determined by directly measuring the reacted glass or estimated based on the solution analyses and the test duration. Therefore, intermediate reaction times most faithfully represent the corrosion rate, while results from short times are complicated by fines and the results from long times are complicated by the decrease in surface area. In practice, rates are computed from data showing "steady state" behavior, i.e., for which the solution concentrations remained nearly constant with time $[70,71]$.

The large volumes of leachant required and the large volumes of leachate solution generated may preclude use of this test in cases where a limited volume of leachant is available, such as when groundwaters are used, or where disposal of the leachate solution is of concern, such as when radioactive glasses are used.

\subsubsection{Periodic Replenishment Tests}

Several test methods have been developed in which leachate solution is periodically removed from an ongoing static test and replaced with an equal volume of fresh leachant solution. Such replacement tests can be used to simulate very low flow rates that cannot 
be attained by using mechanical pumps [123,124]. Either monolithic or crushed samples can be used, and different leachant compositions can be used. Specific test methods have different replacement schedules and call for different fractions of the total solution volume to be replaced. For example, the test designated by the International Organization for Standardization (ISO) calls for the entire solution volume to be replaced daily for the first week, every week for eight weeks, monthly for six months, and twice yearly thereafter [125]. The American Nuclear Society test ANS 16.1 and variations of the ANS 16.1, such as the Dynamic Leach Test (DLT) and the Accelerated Leach Test (ALT), also require the total solution volume of leachate to be exchanged periodically. The variations differ in the replacement schedules. For example, the ALT calls for replacement of the entire solution after 2, 5, and 17 hours and then daily for 10 days. The total accumulated contact time in the ALT is 11 days [126]. Other replacement tests replace a only a small fraction of the leachate that is removed for analysis [127].

The effect of the solution replacements on the corrosion of the waste form depends on the amount of leachate replaced and on the frequency of the replacement. If the solution is refreshed often and completely, as in the initial stages of the ISO and ALT methods, reaction products will be prevented from accumulating in the solution and the solution will remain dilute. The solution chemistry will have a minor effect on the corrosion of the waste form under these conditions. These tests will be similar to static tests with very low SA $N$. If only a small amount of leachate is replaced, reaction products will accumulate and the solution will approach saturation. Under these conditions, the corrosion rate will slow between refreshments but then attain at a slightly higher rate after refreshment until the solution returns to near saturation.

Differences in the presentation of test results in terms of an incremental leach rate and the cumulative leach rate, as in the ISO method have been discussed by Ogard and Bryant [128], who caution against possible misinterpretation of test results. Plotting the rate against the reaction time increment does not account for when a particular time increment occurred. In effect, the sample is assumed to be unaffected by previous leaching increments. This assumption is not valid for most waste glasses, which dissolve nonstoichiometrically under most conditions. Formation of an altered surface layer is known to affect glass corrosion, though only slightly for most waste glasses (see Section 3.1). The leach rates measured between samplings will be affected by the duration of the leaching increment because of the feedback effects of corrosion products, which tend to decrease the corrosion rate. Plotting the extent of reaction against the reaction time increments will group rates obtained at common durations and more clearly show the effects of changes in the solution composition. In contrast, plotting against the cumulative time will more clearly show the effects of the generation of a surface layer.

The perturbing behavior of leachate replacement is shown in Fig. 7 , where the boron concentrations measured over seven exchange intervals with $\Delta \mathrm{V} / \mathrm{V}=0.25$ and $\Delta t=28$ days $\left(T_{r}=112\right.$ days $)$ are plotted against the cumulative exposure time [127]. The boron concentration is seen to rise over each interval and then decrease by about $25 \%$ upon replenishment. The observation that the maximum and minimum concentrations reach nearly constant values after three exchanges (four exchanges corresponds to complete volume replacement under these conditions) indicates that the corrosion layer, which is assumed to continually increase with time, has a negligible effect on the corrosion rate. The perturbations seen in the boron concentration due to leachate replacement will similarly affect the silicic acid concentration and the $\mathrm{pH}$. Indeed, it is probably the periodic reduction in the silicic acid and the $\mathrm{pH}$ that affect the glass corrosion rate and lead to the cycling in the boron concentration (see Section 3). 


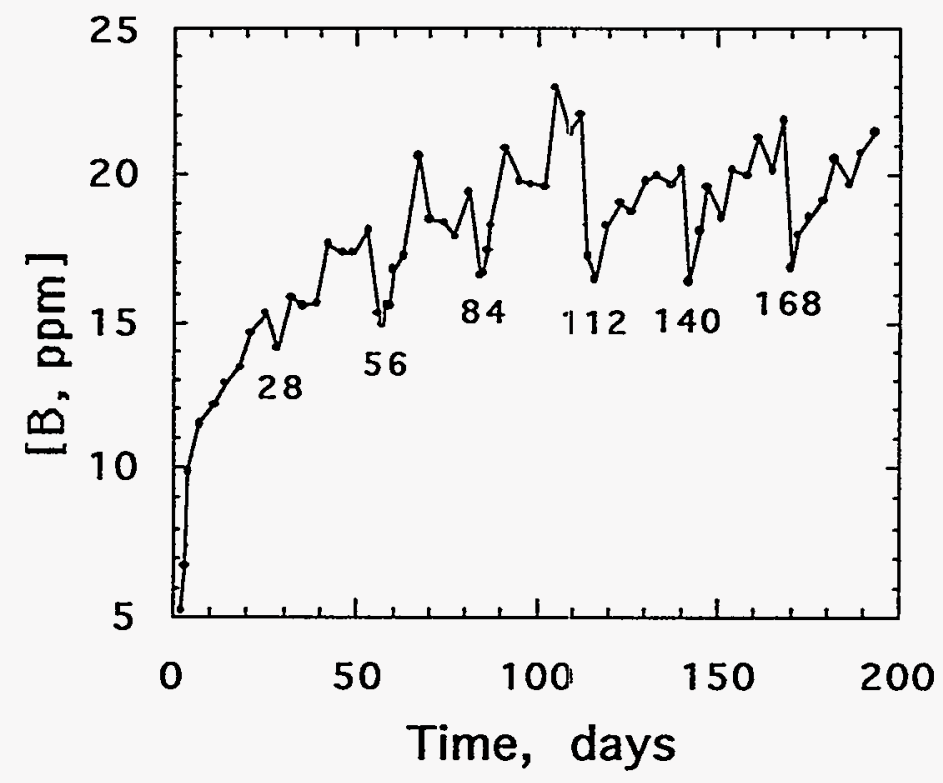

Fig. 7. Measured Boron Concentrations in 28-Day Replacement Tests (after [127]).

Refreshment of the corroding fluid may significantly affect the glass corrosion mechanism by preventing the solution from becoming saturated for long periods, and long-term tests conducted in static solutions and those conducted with partial replenishment may show very different glass performance. Leachant replacement results in a cycling of the corrosion process as the control over the corrosion rate alternates between the glass and the solution chemistry under highly dilute and more concentrated solutions, respectively. These perturbations may have a significant effect on the observed corrosion behavior (see Sec. 5.0). Even small perturbations of an otherwise static test may prevent precipitation of secondary phases and progression of the corrosion into the long-term stage. Replacement tests cannot be conveniently used for characterizing the long-term corrosion process because of uncertainties about the effects of the solution perturbations. They are better used as response tests to index durabilities of candidate waste forms relative to a benchmark of performance. Even for comparative purposes, it is important that the same replacement schedules be followed.

An effect that has been observed in partial replenishment tests that has not been observed in continuous flow tests is transient leach rate excursions $[129,130]$. These excursions are characterized by a suddent temporary increase in the leach rate. Barkatt et al. have speculated that these excursions are due to cracking of the surface layer and suddent exposure of fresh glass, but have not provided convincing evidence. They also point out that the excursions are transient and do not appear to significantly affect the corrosion rate of typical high-level waste glasses (see Sec. 3.1).

\subsection{Measurement of Surface Area}

Most test procedures require a measurement of the glass surface area used in the test. The glass surface area that is available for reaction must be known to determine the reaction rate from the measured solution compositions in all tests methods. Monolithic samples may be prepared with regular shapes that can be measured with calipers. Finely crushed glasses have high surface areas, which can be measured by means of gas adsorption. In this technique, the amount of an inert gas that condenses on a sample of powdered glass is measured at a temperature near the boiling point of the gas. The amount of gas condensed on the sample is 
measured by the pressure or volume change in the system upon exposure to the sample, or by direct weighing of the sample before and after sorption. When one monolayer of gas is sorbed onto the glass, the surface area of the glass covered by gas can be calculated from the known size of the gas molecule or atom and the amount sorbed. Inert gases such as nitrogen and krypton are usually used for surface area measurements. What is measured in these analyses is the surface area accessible to nitrogen or krypton, which may be different than that accessible to water molecules. The surface areas measured using different gases may also be different. For example, the surface areas measured using adsorption of $\mathrm{N}_{2}$ was about twice that measured using adsorption of $\mathrm{Kr}$ [131]. The area measured by the adsorption of $\mathrm{Kr}$ was about 15 times the area estimated using the sieve size. These gases are larger than water and so may not contact glass in small pores or channels that are accessible to water. Of course, geometric measurement of monoliths will not include pores or cracks in the glass, either. The surface area that is available during the reaction may be greater than that measured geometrically or by gas adsorption.

The surface area accessible to water may be measured by the sorption of water vapor directly. The interaction of water vapor with the glass surface may play an important role in the reactivity of waste glasses in a hydrologically unsaturated repository, where the glass will likely be contacted by water vapor before liquid water reaches it. Water vapor is known to chemisorb strongly onto surface silanol groups even at very low water vapor pressures. Sorption onto other sites is generally weak. Water sorbs initially onto the silanol sites and then forms small drops of several molecules that are anchored to the glass surface by the silanol group. As more water condenses, the drops coalesce to minimize the water surface area and form a thin film covering the surface. The surface density of silanol groups depends on the glass compositions and has an upper value of about 4.6 silanol groups $/ \mathrm{nm}^{2}$ in silica gel [40]. As the silica content of the glass decreases, the surface density of silanol groups will also decrease. Sorption studies on a typical waste glass composition showed that increased amounts of water may condense as a result of reaction between the glass and the sorbed water [41]. Exposed alkali metal ions can ion exchange with condensed water if enough water is present to hydrate the released ion. The increased hygroscopicity of the condensate will result in more water condensing on the glass.

Alternatively, the grain sizes of crushed glass can be approximated on the basis of the specific sieve fraction used in the test by assuming the grains have a simple geometry, such as cubic [132], spherical [133], or tabular [134]. The specific shape does not significantly affect the computed surface area. In this approach, the average dimension of the particle is taken as the average mesh opening of the sieve fraction used, and the distribution of the particles is assumed to be Gaussian and centered on the average mesh size. The surface area per unit mass is then computed on the basis of the glass density.

For example, consider crushed glass in the sieve fraction of $-100+200$ mesh where the particles are approximated to be spheres. The opening of the 100 mesh sieve is $0.149 \mathrm{~mm}$ and the opening of the 200 mesh sieve is $0.74 \mathrm{~mm}$. The arithmetic average opening is $0.112 \mathrm{~mm}$. The volume and surface area of a sphere can be readily calculated as volume $=\pi \mathrm{d}^{3} / 6$ and surface area $=\pi \mathrm{d}^{2}$, where $\mathrm{d}$ is the diameter. A sphere having a diameter of $0.112 \mathrm{~mm}$ has a volume of $7.4 \times 10^{-7} \mathrm{~cm}^{3}$ and a surface area of $3.9 \times 10^{-4} \mathrm{~cm}^{2}$. If the density of the glass is assumed to be $2.7 \mathrm{~g} / \mathrm{cm}^{3}$, then there are $5 \times 10^{5}$ glass particles per gram and $2 \times 10^{-2} \mathrm{~m}^{2}$ of surface area per gram of glass. Larger size fractions will have smaller surface areas per gram and smaller size fractions will have larger surface areas per gram. Glasses with densities greater than 2.7 will have less surface area per gram and glasses with densities less than 2.7 will have more surface area per gram. 
A photomicrograph of glass in the $-100+200$ mesh sieve size fraction is shown in Figs. 8 a and $8 \mathrm{~b}$ for two different glasses. It is not obvious that the particles of either glass could be well represented by cubes, spheres, or tablets. The crushed glasses shown in Figs. 8a and $8 \mathrm{~b}$ also have different particle habits. The particles of crushed WV 6 glass are relatively uniform in size and shape. The particles are generally free from sharp edges, having been rounded when crushed. Three distinct morphologies of SRL EA glass particles can be seen in the photomicrograph: chunk-like particles with a somewhat sperical shape, rod-like particles, and plate-like particles. The particles of SRL EA glass have sharper fracture points and edges than the particles of WV 6 glass. The WV 6 particles are all of a similar size, whereas the SRL EA particles have a wider size distribution, and are generally bigger than the WV 6 particles. Clearly the crushed WV 6 and SRL EA glasses have different surface areas per unit mass. In addition to differences in the total surface area, the particles with sharper edges and points are likely to be more reactive than the particles with more rounded surfaces simply due to the higher surface energy of the sharper particles. The fracture behavior depends largely on the cooling of the glass from the melt: rapidly quenched glasses are expected to fracture to form particles with sharper edges than annealed glasses.

The exposed surface areas of various size fractions have been compared experimentally in terms of the extent of corrosion under test conditions that are sensitive to the surface area. Fig. 9 shows the sodium concentrations in solutions attained in tests using different size fractions of crushed glass. In these tests, glass mass/solution mass ratios were selected to yield a common $\mathrm{SA} / \mathrm{V}$, namely $77 \mathrm{~m}^{-1}$, based on a geometric approximation of the particle surface area [135]. The results show that the sodium release is the same for all size fractions, within experimental error, and that the glass corrosion rate is not affected by the particle size over this time period. Longer reaction times are expected to show particle size effects because smaller particles will lose surface area faster. These results imply that the geometric approximation is sufficient, compared to the testing errors.

Several studies have compared the surface area estimated by sieve size to that measured by adsorption based on the experimentally measured extent of reaction. For example, Oversby compared the extent of corrosion of various sieve fractions of crushed SYNROC in modified ISO tests at $90^{\circ} \mathrm{C}$ [131]. The surface areas of the different size fractions were measured by $\mathrm{Kr}$ adsorption and the geometric surface area was calculated by assuming the grains to be cubes having an edge length equal to the average sieve opening. Tests were conducted for one, two, and three days, where the entire leachate volume was replaced and analyzed daily. Tests were conducted using the sieve fractions listed in Table 3. The surface area measured by adsorption, the surface area calculated by assuming the grains to be cubes, and the approximate SA/V (based on the geometric surface area) are given for each size fraction.

Oversby reasoned that all size fractions should corrode at a similar rate under these conditions. The leach rates of aluminum, calcium, sodium and silicon calculated using the geometric surface areas are consistently higher for tests conducted at lower SA/V ratios. The sodium release rates are included in Table 3 . The differences are consistent with the effects of the $\mathrm{SA} / \mathrm{V}$ ratio observed in other tests. Using the surface areas measured using gas adsorption, SA (ads), which were higher than the geometric surface areas, SA (geom.), amplifies the differences in the different size fractions. Oversby concluded that the smaller differences calculated using the geometric surface areas were more consistent with her expectation that all size fractions would corrode at a similar rate. She estimated that the "real" surface area of the crushed glass was about 1.5 times that calculated geometrically due to the grain shapes. 
(a)

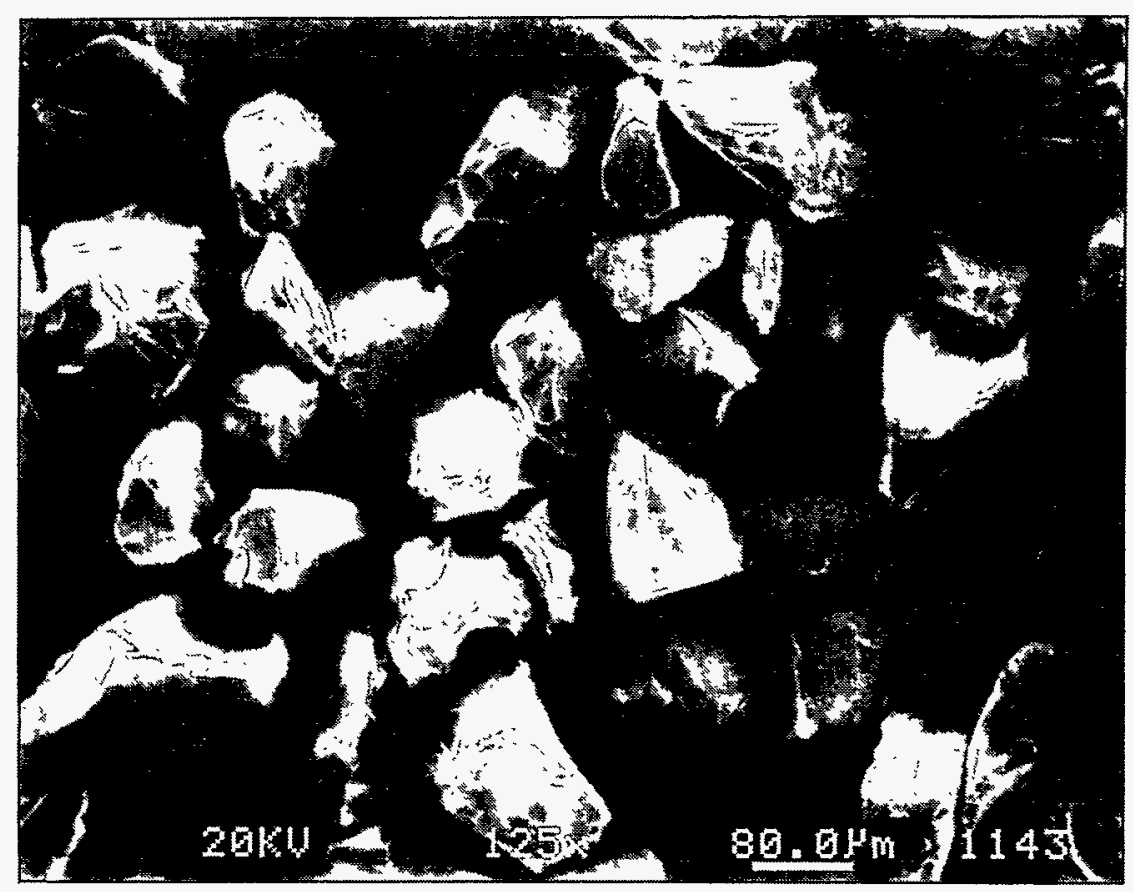

(b)

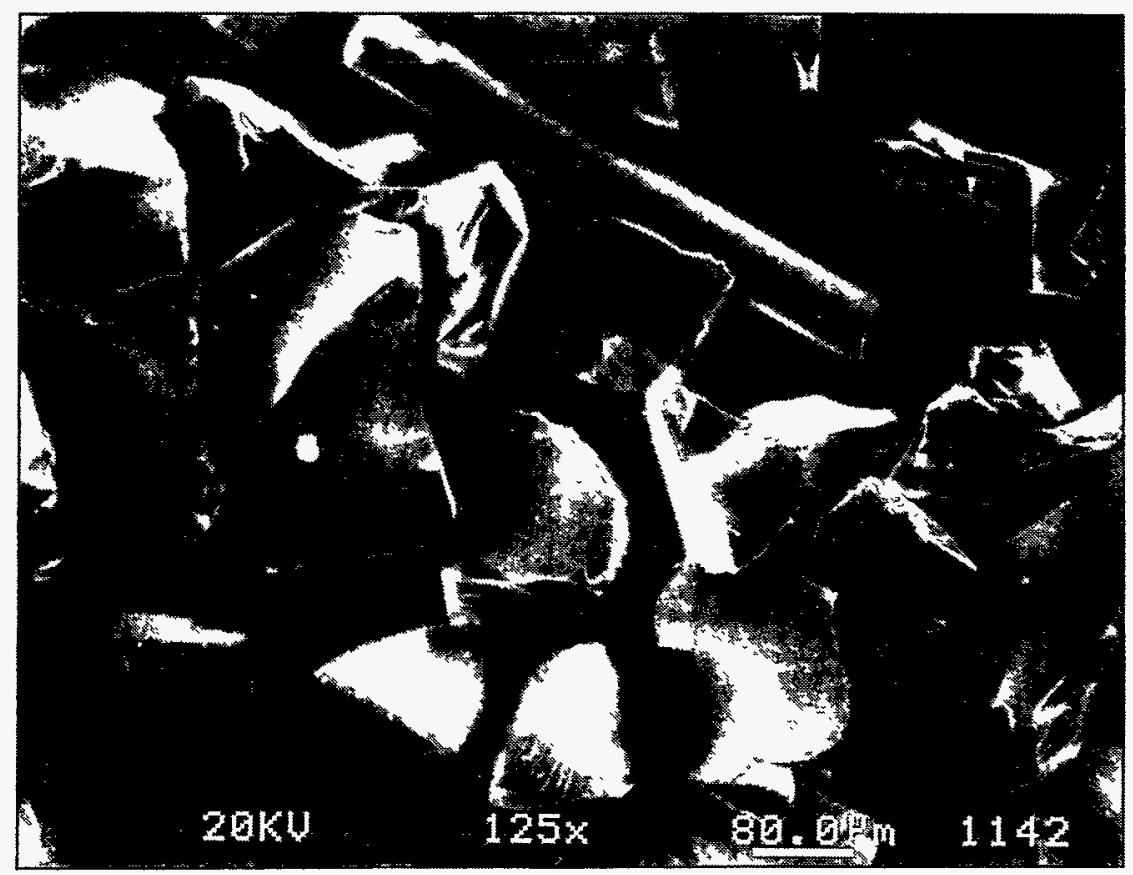

Fig. 8. Photomicrographs of $-100+200$ Mesh Size Fraction of Crushed Glass 


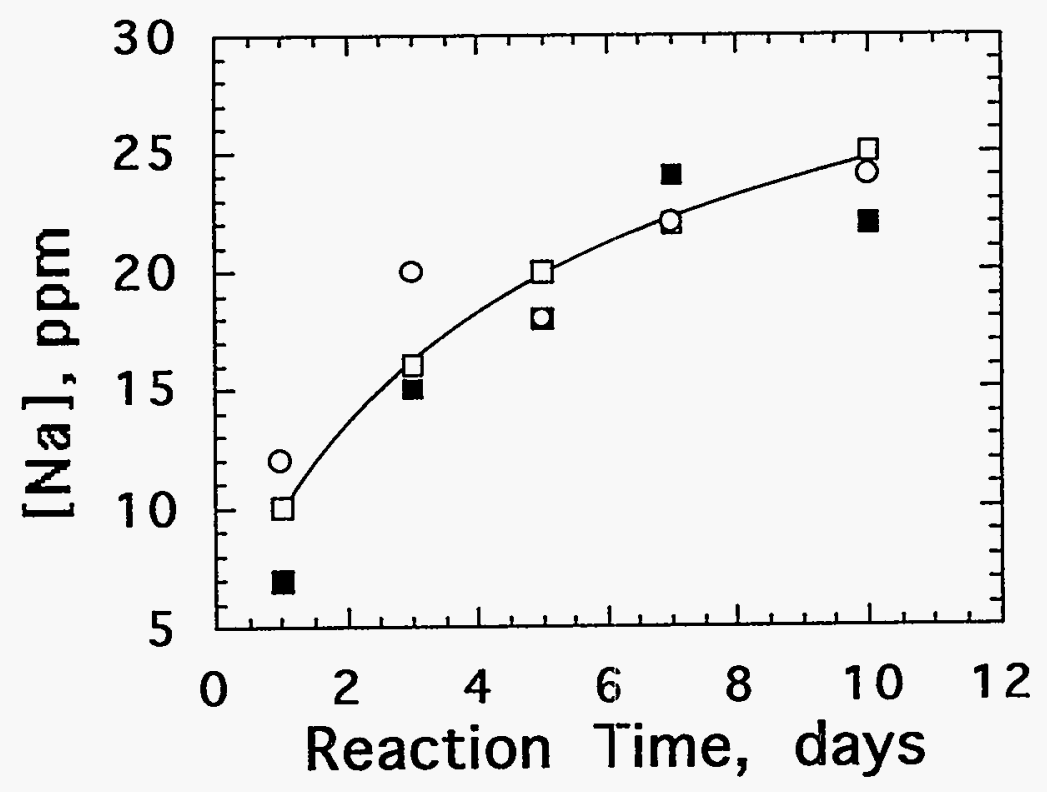

Fig. 9. Test Results for Different Size Fractions of Crushed Glass: (a) $-4+10$ Mesh, (E) $-45+60$ Mesh, and (O) $-325+400$ Mesh (after [134])

Table 3. ISO Test Results [131]

\begin{tabular}{lcccc}
\hline $\begin{array}{l}\text { Size Fraction, } \\
\text { mm }\end{array}$ & $\begin{array}{c}\text { SA (ads.), } \\
\mathrm{cm}^{2}\end{array}$ & $\begin{array}{c}\text { SA (geom.), } \\
\mathrm{cm}^{2}\end{array}$ & $\begin{array}{c}\text { SA (ads.)/V, } \\
\mathrm{m}^{-1}\end{array}$ & NL(Na)c \\
\hline $177-246$ & 187.7 & 3.16 & 20 & 5.6 \\
$246-417$ & 27.0 & 3.93 & 24 & 5.0 \\
$417-840$ & 39.3 & 4.94 & 31 & 3.7 \\
$840-1650$ & 69.2 & 6.95 & 44 & 2.1 \\
\hline
\end{tabular}

aSurface area measured by gas adsorption.

bSurface area estimated based on sieve size.

In $\mathrm{g} / \mathrm{m}^{2} \mathrm{~d}$. 
Oversby also compared the results of a 3 day MCC-1 test using a monolithic sample having a known geometric surface area to the results of a one day ISO test [131]. The leach rate measured in the MCC-1 test was in good agreement with the results of the ISO test where the "real" surface area was used (that is, 1.5 times the surface area calculate by assuming cubic geometry).

Tests were conducted by Barkatt et al. to compare the reactivities of monolithic samples to that of crushed samples [136]. Tests were conducted by placing a monolithic sample of measured geometric surface area and a known mass of crushed glass samples of the size fraction $-42+60$ mesh in a Teflon reaction vessel with deionized water and placing the sealed vessel in a $70^{\circ} \mathrm{C}$ oven. Enough water was added in an effort to maintain low SA/V conditions throughout the test periods. The water was replaced at frequent intervals and analyzed. Tests were conducted using three different glasses, PNL 76-68, and unidentified CUA and SRL glasses. By comparing the silica concentrations measured after several reaction intervals in tests with monolithic with that measured in tests with crushed glass, Barkatt et al. concluded that the effective surface area of the crushed glass in the size range $-42+60$ was $97 \pm 11 \mathrm{~cm}^{2} / \mathrm{g}$. This is in good agreement with the value calculated assuming spherical particles, which is about $117 \mathrm{~cm}^{2} / \mathrm{g}$ (average mesh opening is $190 \mu \mathrm{m}$ ). The range of silica concentrations attained in the tests ranged between 1.65 and $11.1 \mathrm{ppm}$ in tests with the PNL 76-68 glass, between 0.09 and $0.59 \mathrm{ppm}$ in tests with the CUA glass, and between 1.92 and $42.3 \mathrm{ppm}$ in tests with the SRL glass. The higher concentrations attained in tests with the SRL glass may have reduced the corrosion of both monolithic and crushed glasses slightly. However, similar concentrations were attained in tests with monolithic and crushed glasses, so the comparison of surface areas is expected to be unaffected.

Using the measured extent of reaction to estimate the surface area of crushed glass is based on the premise that tests at similar products of the SA/V and the reaction time generate equivalent solution compositions. This approach is best applied by using tests that are dominated by the initial stages of corrosion and maintain dilute solution conditions, under which the build-up of glass corrosion products has a negligible effect on the corrosion rate.

Because of the uncertainty in the measuring the precise surface area of crushed glass, some test protocols (for example, the PCT) specify only the size fraction and mass of crushed glass to be used, rather than the surface area or the SA/V. Crushed glass is sieved to isolate a specific size fraction, which is carefully washed to remove fines from the particles. (The PCT procedure includes the option of not washing the crushed sample to remove fines if the presence of highly soluble included phases is anticipated or determined. Of course, the presence of fines will then likely result in higher solution concentrations of all elements present in the fines. The trade-off of losing soluble material when washing fines from the sample or including the effects of fines in the test result is a consequence of using crushed glass. It may be possible to wash the crushed glass with a nonaqueous solvent in which the phases have low solubility.) It is assumed that the total surface area per mass of crushed glass is reproducible for a given size fraction. The fracture behavior probably depends on the glass composition and the method used to crush the glass. Tests can be performed at different $\mathrm{SA} / \mathrm{V}$ either by using crushed glass of different size fractions or by using different masses of crushed glass of the same size fraction. While the SA/V attained is only approximate for either method, using the same size fraction allows direct comparison of tests at different relative $\mathrm{SA} / \mathrm{V}$, since the area per gram, while it may not be known very accurately, can be expected to be constant. That is, the error introduced by using sieve size to approximate the surface area per gram of glass will cancel in test results at different

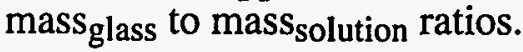




\subsection{Effect of Surface Finish}

The actual surface area of a monolithic glass sample that is available for reaction in a laboratory test will be affected by how the sample is prepared. Different surface finishes may be achieved by cutting with various wafering blades, grinding with abrasives, fracturing, flame polishing, etc. The microscopic surface areas generated during sample preparation may differ significantly among samples that have the same geometric surface areas but were prepared differently. For example, it has been reported that abrasion with 100-grit increased the surface area of PNL 76-68 glass available for reaction by about 33\% and abrasion with 600-grit increased the surface area by about $17 \%$ over the geometrically measured surface area [137].

The release of glass components during corrosion will increase with the surface roughness because of both the greater area and the higher strain. Because the strain induced by the abrasion penetrates into the glass to a finite depth, corrosion becomes equivalent after the corrosion front penetrates to that depth. The effects of surface finish are expected to be most important during the initial stages of glass corrosion and to diminish as the corrosion process proceeds. Tests used to measure the effects of surface finish and surface strain on glass corrosion must be sensitive to the initial stages of glass corrosion and minimize the effects of the solution chemistry on the corrosion. Such tests must maintain dilute solution conditions and be dominated by the reaction of the topmost millimeter of glass, and typically they must be shortterm tests (on the order of a few days).

Differences in reactivities have been measured for samples with different surface finishes. In Soxhlet tests with R7T7 glass, the normalized boron mass loss was higher by a factor of about two for a saw-cut sample compared to the loss for a sample polished with 1 micron diamond powder [138]. In MCC-1 leach tests with PNL 76-68 glass, the boron loss was higher by factor of about 3 for glass ground to 100-grit finish compared to loss for a sample polished with 1 micron diamond powder [137]. The differences between samples polished with 1 micron diamond powder and samples ground to a 600-grit finish were small (less than about $20 \%$ ) for both glasses. The soxhlet and MCC-1 test methods highlight the initial stages of glass corrosion during which the effects of materials released to solution on the continued glass corrosion are small, and emphasize the effects of the glass surface area. These test results indicate that the higher surface areas of the more coarsely ground surfaces (i.e., the saw-cut sample and the sample with a 100-grit finish) generate higher solution concentrations than the samples with the finer polish (1 micron finish).

Tests were performed by Bates et al. to assess the importance of surface strain to glass reactivity in a water vapor environment at elevated temperatures [139]. Glass samples were either polished, fractured thermally, or fractured by percussion. Some glasses were thermally annealed after fracturing to partially relieve the stresses induced during fracturing. It was found that the annealed samples and those polished to 600-grit were the least reactive and that the fractured and 320-grit samples reacted similarly. Thus, both stresses and increased surface area promoted corrosion under these conditions.

Fractures may occur at the sample surface during mechanical preparation of monolithic samples (core drilling, wafering, etc.). These fractures may be removed by subsequent annealing or grinding of the surfaces. The effect of such surface fractures on test results depends on the crack width and whether water can enter the crack. An investigation of the effect of various crack widths was carried out by Perez and Westsik following a MCC-1 type test procedure [140]. Various crack sizes were simulated by placing different sized platinum wires between monolithic samples. They found that leaching from cracks was less than that from free surfaces, and that leaching was nil from tight cracks. However, analysis of corroded monoliths in cross-section have shown dealkalization to occur to similar depths around surface cracks and at free surfaces [73]. This suggests that solution analyses are not sufficiently sensitive to distinguish the effects 
of leaching from cracks. Surface analysis also shows that little or no etching occurs in the crack, while significant etching may occur on free surfaces. Thus, material remains contained within the crack as the glass corrodes. Insight into the effects of cracks is provided by an understanding of the effects of the solution chemistry on glass corrosion. Of course, the effects of the cracks will disappear as the corrosion front penetrates into the glass deeper than the cracks.

\subsection{Measurement of Leachant Volume}

Measurement of the leachant volume is trivial in static leach tests because the amount of leachant is added to the reaction vessel either by volume or by weight. However, several test procedures will be discussed for which the leachant volume or effective leachant volume is not directly measurable. These will include partial-replenishment tests, in which the leachate is periodically refreshed; continuous flow tests, in which the leachant is continuously flowing past the glass; and static tests in water vapor, in which the leachant is condensed on the sample from the vapor phase. The dynamic tests require the definition of a contact or residence time in which a measurable volume of solution tends to react with the glass in a quasi-static system.

Alternatively, the time required to completely replace the leachate solution in a confined volume can be determined.

In a vapor system, water may condense on the sample to form a film many monolayers thick. (A monolayer is a film one molecule thick covering the surface.) The amount of condensed water depends on the glass surface composition, the water vapor pressure, and the temperature. If the the sorbed water is corrosive towards the surface, for example, if dealkalization occurs, a hydrophilic solution may be generated that will increase the amount of water that is condensed. While the amount of water condensed at ambient temperatures can readily be measured [41], condensation at higher temperatures is not easily quantified. Instead, the maximum volume of water that can be supported on the sample without dripping off can be approximated and used as the leachant volume.

Initially, only about 50 to 100 monolayers condense on the glass [41] to form a film estimated to be about $0.01 \mu \mathrm{m}$ thick (assuming each monolayer is about $0.15 \mathrm{~nm}$ thick [115], i.e., equals the diameter of a water molecule). The formation of this layer occurs rapidly [41]. For a sample having a surface area of about $2 \mathrm{~cm}^{2}$, about $2 \times 10^{-6} \mathrm{~mL}$ of water would be required to form a layer $0.01 \mu \mathrm{m}$ thick. The corresponding SA/V can be easily calculated by considering the $0.01 \mu \mathrm{m}$ thick layer to form on a plane surface, such that the volume of water per square meter is about $1 \times 10^{-8} \mathrm{~m}^{3}$ and the $\mathrm{SA} / \mathrm{V}$ is about $1 \times 10^{8} \mathrm{~m}^{-1}$. Additional water condenses as the glass corrodes and releases alkali metals and other glass components into the film during the test. The amount of water on the glass increases steadily until a maximum of about $0.05 \mathrm{~mL}$ condenses on the sample. Only enough water to allow $0.05 \mathrm{~mL}$ to condense is added to the test vessel. Condensation of more than $0.05 \mathrm{~mL}$ is likely to result in water dripping from the sample. For a sample having a surface area of about $1.5 \mathrm{~cm}^{2}$, and assuming that the $0.05 \mathrm{~mL}$ of water is evenly distributed, the minimum SA/V achieved during the test is about $3000 \mathrm{~m}^{-1}$.

The actual SA/V of the system will decrease from the values calculated from the initial surface area of the glass and the amount of water added because some of the water will diffuse into the glass as corrosion occurs. This water is no longer available to dilute corrosion products. Alteration layers on the surface of corroded high-level waste glasses are generally found to include about 4 to $7 \mathrm{wt} \%$ water [46]. Assuming the layer to contain $7 \mathrm{wt} \%$ water, the density of water in the layer of a glass having an initial density of $2.7 \mathrm{~g} / \mathrm{cm}^{3}$ would be about $0.2 \mathrm{~g} / \mathrm{cm}^{3}$. Formation of a $10 \mu \mathrm{m}$ thick layer would consume about $2 \mathrm{~mL}$ of water per square meter $\left(2 \times 10^{-5} \mathrm{~mL} / \mathrm{cm}^{3}\right)$. For a sample having a surface area of about $2 \mathrm{~cm}^{2}$, about $4 \times 10^{-5} \mathrm{~mL}$ of water would be consumed by formation of a $10 \mu \mathrm{m}$ thick layer. Note that this is about 20 times 
the water needed to form the initial $10 \mu \mathrm{m}$ thick film on the glass surface, but is negligible compared to the formation of a $0.05 \mathrm{~mL}$ drop of water as corrosion proceeds. Presumably, water entering the glass is replaced by freshly condensed glass as alkali metal ions are released into the surface film.

Water is also consumed as crushed glass corrodes. If the alteration layer contains about 4 to $7 \mathrm{wt} \%$ water, then the maximum amount of water consumed can be calculated from the known volume of glass in the test. For a PCT A test, 1 gram of glass is added to $10 \mathrm{~g}$ of water. About $0.07 \mathrm{~g}$ of water is required to hydrate all the glass to $7 \mathrm{wt} \%$ water. This is negligible compared to the $10 \mathrm{~g}$ added to the test.

\subsection{Changes in SA/V as the Glass Corrodes}

Changes in either the surface area of the glass available for reaction or the volume of solution available to dilute the corrosion products as the reaction progresses will change the $\mathrm{SA} / \mathrm{V}$ of the system. Changes in the solution volume can usually be assumed to be negligible, as discussed in the preceding section. The surface area may either decrease or increase as the glass corrodes. Consider the simple case of spherical particles. The initial radius is given by $\mathrm{r}_{\mathrm{i}}$ and the radius after corrosion is given by rf. The initial surface area is simply $4 \pi r_{i}{ }^{2}$ and the final surface area is simply $4 \pi \mathrm{rf}^{2}$, so that the net loss in surface area is $\Delta \mathrm{A}=4 \pi\left(\mathrm{ri}^{2}-\mathrm{rf}^{2}\right)$. The volume of material dissolved is given by $\Delta \mathrm{v}=(4 / 3) \pi\left(\mathrm{ri}^{3}-\mathrm{rf}^{3}\right)$, and the mass dissolved is given by $\Delta \mathrm{v} \rho$, where $v$ is the volume of the particle and $\rho$ is the density of the glass. Since the differential change in the volume decreases as the square of the change in radius $\left(\mathrm{dV} / \mathrm{dr}=4 \pi \mathrm{r}^{2}\right)$ and the differential change in the surface area decreases proportional to the change in radius $(\mathrm{dSA} / \mathrm{dr}=8 \pi \mathrm{r})$, the volume (and mass) of the particle will decrease faster than the surface area (until the radius is less than 2). The SA/V of the system will change directly proportional to the change in the surface area as the glass corrodes, assurning that the solution volume remains constant. Therefore, the initial SA/V can be defined as $4 \pi \mathrm{Nr}_{1}^{2} / \mathrm{V}$, where $\mathrm{N}$ is the number of particles and $V$ is the solution volume, while the final SA/V is $4 \pi N r f^{2} / V$. The initial SA/V is greater than the final SA/V.

The decrease in surface area is illustrated by the photomicrograph of a crosssectioned corroded particle of SRL EA glass in Fig. 10. The glass was reacted in tuff groundwater at $90^{\circ} \mathrm{C}$ at an SA/V ratio of $20,000 \mathrm{~m}^{-1}$ for 98 days. The inner core of material is pristine SRL EA glass and the outer crust has been depleted of soluble glass components. The crust was formed as soluble glass components dissolved and sparingly soluble components formed the outer layer. As the particle continues to corrode, soluble elements are released from the surface of the inner core of glass, which has a much smaller surface area than the original particle.

The model of the glass as a dissolving sphere and the analytical relation between the radius, surface area, and volume has the important consequence that if the glass dissolves at a constant rate (i.e., the mass loss is proportional to $t^{1}$ ), then the surface area (and the radius of the particle) will decrease proportional to the square of time $\left(t^{2}\right)$. Conversely, if the radius decreases linearly over time, the mass loss will decrease proportional to the square root of time $\left(\mathrm{t}^{1 / 2}\right)$. Hence, the observed time dependence of the corrosion rate provides evidence of the ratecontrolling phenomenon: if the corrosion rate is controlled by alteration of the glass, through processes such as diffusion through alteration layers, then the radius of the particles is expected to decrease linearly with the reaction time and the mass loss is expected to decrease with the 


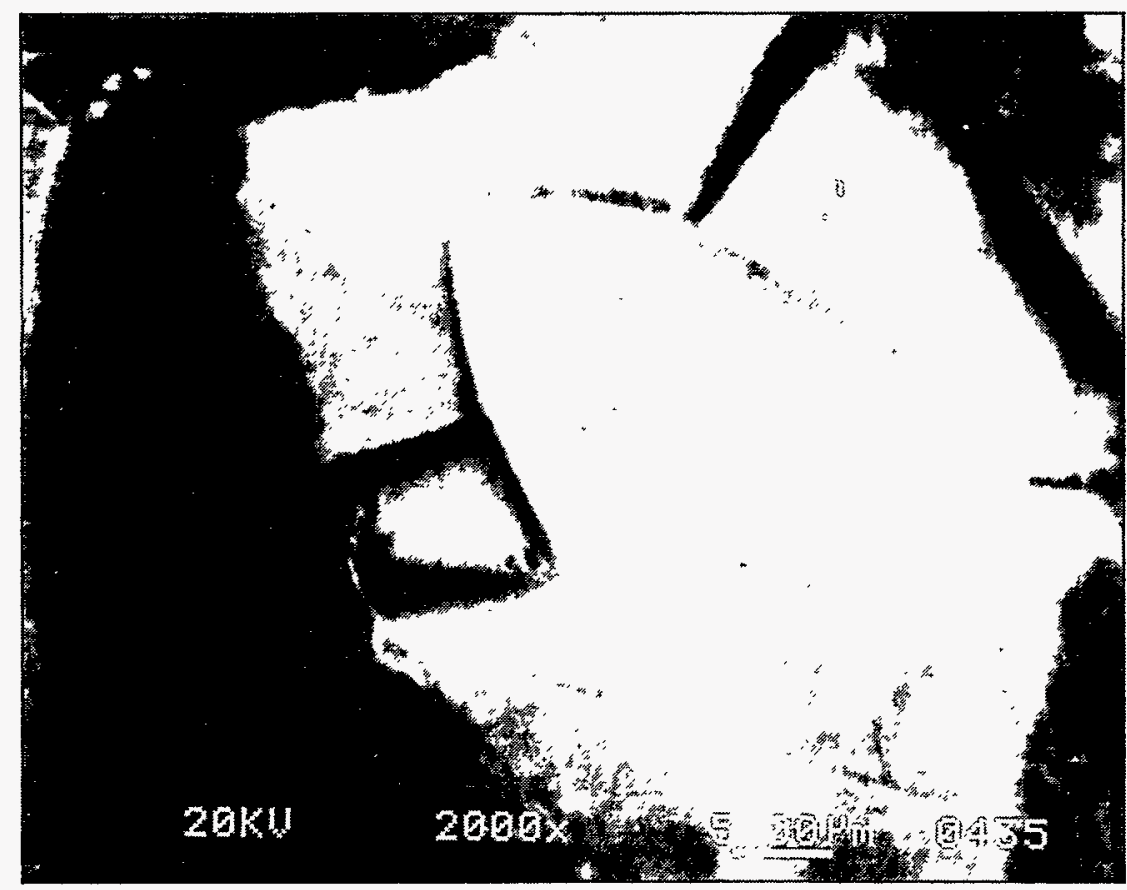

Fig. 10. Photomicrograph of Corroded SRL EA Glass Grain

square root of the reaction time. If the corrosion rate is controlled by the solution, then the mass loss is expected to increase linearly with the reaction time, and the radius of the particle (and the surface area) will decrease as the square of the reaction time.

For example, the results of tests conducted at ANL show the boron concentration to increase linearly with respect to time in tests conducted with SRL 202 glass at an initial SA/V of $20,000 \mathrm{~m}-1$ for 364 days or longer (see Section 4.2). This indicates that the volume of glass corroded was constant with respect to time and that the radii of the glass particles decreased at an ever increasing rate (proportional to $\mathrm{t}^{2}$ ). Since any mass transport effects would result in a root time dependence, the corrosion layers are seen not to affect the long-term corrosion rate. Instead, the long-term corrosion rate is controlled by the solution chemistry.

Nonuniform dissolution can lead to the formation of pits and pores and a net increase in the surface area. Nonstoichiometric corrosion can also lead to an increase in the effective surface area for some glass components. For example, boron may be depleted from the glass to a depth corresponding to the surface for $\mathrm{r} f$, while much of the silicate structure remains in the boron-depleted region. Thus silicon-oxygen bonds are exposed to water throughout the altered volume, while boron-oxygen bonds are exposed only on the surface at $r_{f}$. While the number of reaction sites for boron release continually decreases as the glass corrodes, the number of reaction sites for silicon release continually increases.

Knowledge of the maximum possible values of the normalized mass loss function is important when conducting long-term tests to (1) recognize effects of shrinking particles, and (2) avoid having glasses corrode too much (or completely). Glasses that completely corrode during the test cannot be used to determine the corrosion rate, since it cannot be determined when the corrosion was complete. The maximum possible normalized mass loss values can be calculated by assuming the grains to be spheres of uniform diameter. Consider the complete 
reaction of 1 gram of $-100+200$ mesh glass containing 3 elemental wt\% boron to completely dissolves in $10 \mathrm{~mL}$ of deionized water. The surface area of the size fraction is $2 \times 10^{-2} \mathrm{~m}^{2} / \mathrm{g}$. The normalized mass loss based on the release of $0.03 \mathrm{~g}$ boron can be calculated as

$$
\mathrm{NL}(\mathrm{B})=\frac{\left(\frac{0.03 \mathrm{~g} \mathrm{~B}}{10 \mathrm{~mL}}\right)}{\left(\frac{1 \mathrm{~g} \text { glass }}{10 \mathrm{~mL}}\right)\left(2 \times 10^{-2} \mathrm{~m}^{2} / \mathrm{g}\right)\left(\frac{0.03 \mathrm{~g} \mathrm{~B}}{\text { g glass }}\right)}
$$

which equals $1 /\left(2 \times 10^{-2} \mathrm{~m}^{2} / \mathrm{g}\right)=50 \mathrm{~g} / \mathrm{m}^{2}$. The maximum $\mathrm{NL}(\mathrm{B})$ depends on the size fraction of the glass but is independent of the $\mathrm{SA} / \mathrm{V}$. Smaller size fractions have larger maximum NL values. A similar calculation can be performed for monolithic samples. For a right cylinder, the surface area per gram is $1 \times 10^{-3} \mathrm{~m}^{2} / \mathrm{g}$ for a glass density of $2.7 \mathrm{~g} / \mathrm{cm}^{3}$ and the maximum NL value is $1000 \mathrm{~g} / \mathrm{m}^{2}$.

The loss of surface area during the test is usually not accounted for when computing corrosion rates from test data, though it is clearly important when evaluating the corrosion rates of poorly durable glasses or of durable glasses over very long time periods. The solution results are normalized to the surface area to obtain a measure of the rate that is independent of the glass surface area, such that rates measured in tests with different initial surface areas can be compared. Because the surface area of the starting material is used, the normalized mass function only describes the integrated extent of dissolution, i.e., the average rate between the start of the test and the point at which the solution concentration is analyzed (see Sec. 2.3). The normalized mass losses computed after two different reaction times may not provide a valid approximation of the instantaneous corrosion rate since the surface area of the glass will be less than that at the start of the test. The difference may or may not be significant, depending on the reaction times and the corrosion rate of the glass. For example, the perimeter of the remaining glass core in Fig. 10 is less than half that of the outer crust. The corresponding surface area of the core is certainly less than half that of the original surface. Expressing the continued corrosion rate in terms of the normalized mass loss, which is calculated using the original surface area, clearly underestimates the corrosion rate. An appropriate means of estimating the effects of decreasing surface area on the long-term corrosion rate of crushed glass samples needs to be developed. 


\subsection{OBSERVED EFFECTS OF THE SOLUTION CHEMISTRY ON GLASS CORROSION}

The composition and chemistry of the fluid that contacts a high-level waste glass will affect the glass corrosion rate and the release of glass components into solution. The chemistry of the groundwater that may eventually enter the repository will be determined initially by its interactions with the atmosphere and various phases in the host rock, then with engineering components such as metal containers and backfill materials, and by radiolysis of the gas phase and the groundwater itself. The amounts of dissolved air, iron, and other redox-sensitive species are likely to establish the redox potential of the fluid which initially contacts the waste glass [141]. The chemistry of the solution in contact with the glass will change considerably as the glass corrodes and glass components are released into solution. These changes will affect both the corrosion rate of the glass and the disposition of various glass components as the glass reacts. The relative importance of interactions with the surrounding environment and the corroding waste glass to the fluid chemistry will depend strongly on the volume of groundwater that contacts the waste form; the chemistry of small volumes of groundwater will be more affected by interactions with the waste form than that of larger volumes. Since a key design goal of engineered high-level waste repositories will likely be to minimize the amount of groundwater that may contact waste, it may be assumed that the fluid chemistry will be dominated by glass corrosion products. This may not be the case if the entering fluid is a brine, however. In order to assess the long-term corrosion behavior of waste glasses, it is necessary to understand how their long-term corrosion is affected by the chemistries of both the fluid initially contacting the waste and that generated after interaction.

Static leach tests commonly show the amounts of glass components released into solution to decrease in a parabolic fashion with the reaction time (i.e., approximately in proportion to the square root of time). As described in Section 2.4, the interaction of a high-level waste glass with an aqueous solution results in the dissolution of the soluble glass components and the formation of alteration layers containing less soluble glass components at the glass surface. Both the solution composition and the alteration layer may affect the continued rate of glass corrosion. The decrease observed in the corrosion rate over time may be due either to mass transport through the layers that develop as the glass corrodes or to the concentrations of glass components in the solution, which slow the individual reactions releasing those components.

It should be emphasized that concentration gradients are likely to exist in the solution, especially when layers form. A nearly static fluid having a chemistry that may be very different from the bulk solution will exist within the layer and at the surface of the glass [142]. While the composition of the bulk solution is analyzed in laboratory tests, it is the chemistry of the static fluid at the glass surface that will affect the corrosion rate. While the chemistry of the static fluid may be difficult to measure, possible differences should be borne in mind when modeling glass corrosion behavior.

Static leach tests have also shown high-level waste glasses to dissolve nonstoichiometrically. This may be due to the incorporation of some elements into secondary phases [143], or simply to different release kinetics. Several models have been proposed based on these observations, including models based on mass transport dominance $[18,75,119,144-148]$ and surface reaction dominance [149-154]. Both mass transport and surface reaction effects probably affect the corrosion of most glasses, although one or the other effect may be dominant for specific glass compositions or reaction conditions. 


\subsection{Tests Conducted to Distinguish between Mass Transport and Surface Reaction Effects}

Consideration of solution results alone is not sufficient to distinguish whether mass transport or chemical reaction effects dominate measured corrosion behavior, since static leach test data can be fitted equally well by both diffusion and surface reaction models [143]. Instead, other tests have been performed to make this distinction. These tests typically involve comparison of the extent of reaction of glass samples in two sets of conditions: (1) with and without the physical removal of alteration layers and (2) with and without removal of corrosion products from the solution through leachant replacement. If the surface reactions control the glass corrosion rate, then changes in the solution composition as the glass corrodes are expected to dominate the corrosion rate, and the presence or absence of an alteration layer should not affect the measured extent of reaction. If mass transport controls the glass corrosion rate, then the presence or absence of an alteration layer is expected to dominate the corrosion rate, and changes in the solution composition will have little effect.

Chick and Pederson conducted tesits based on the MCC-1 protocol and three variations of it test to assess the effects of the solution composition and mass transfer through the alteration layer on the corrosion rate [155]. The compositions of the three glasses used are given in Table 4. In the first series of tests, glass A was reacted in deionized water under MCC-1 conditions for several reaction times between 7 and 56 days. The measured boron release in these tests is shown as filled circles in Fig. 11. The normalized boron mass loss increased rapidly over the first few days and then leveled off at longer reaction times. In the first variation of the MCC-1 test, samples of glass A were reacted for 56 days and then removed from the leachate, carefully rinsed, and placed in fresh deionized water and reacted for additional times up to 56 days. This variation tests the corrosion of a sample having an alteration layer in a leachate solution without any reaction products, where only the alteration layer is expected to affect the reaction. The upper dashed line in Fig. 11 shows the trend observed through the initial 56 days of reaction translated to days 56 through 112. Data from tests continued after the layers were

Table 4. Compositions (wt\%) of Three Glasses Used by Chick and Pederson [155]

\begin{tabular}{lccc}
\hline & \multicolumn{3}{c}{ Simulated Waste Glass } \\
\cline { 2 - 4 } Oxide & $\mathrm{A}$ & $\mathrm{B}$ & $\mathrm{C}$ \\
\hline $\mathrm{Al}_{2} \mathrm{O}_{3}$ & 6.1 & 6.9 & 5.9 \\
$\mathrm{~B}_{2} \mathrm{O}_{3}$ & 4.2 & - & 9.1 \\
$\mathrm{CaO}$ & 2.1 & 7.1 & - \\
$\mathrm{CeO}$ & - & - & 1.2 \\
$\mathrm{Fe}_{2} \mathrm{O}_{3}$ & 11.5 & 12.8 & 0.5 \\
$\mathrm{Gd}_{2} \mathrm{O}_{3}$ & - & - & 9.1 \\
$\mathrm{Li}_{2} \mathrm{O}$ & 6.7 & - & 4.6 \\
$\mathrm{MoO}_{3}$ & - & - & 1.7 \\
$\mathrm{Na}_{2} \mathrm{O}$ & 8.3 & 9.0 & 9.7 \\
$\mathrm{Nd}_{2} \mathrm{O}_{3}$ & - & 0.1 & 2.6 \\
$\mathrm{NiO}$ & 1.1 & 0.7 & 0.3 \\
$\mathrm{P}_{2} \mathrm{O}_{5}$ & 0.8 & - & 2.2 \\
$\mathrm{SiO}_{2}$ & 57.5 & 59.0 & 48.1 \\
$\mathrm{UO}_{2}$ & - & 1.8 & - \\
$\mathrm{Others}$ & 1.7 & 2.6 & 5.0 \\
\hline
\end{tabular}




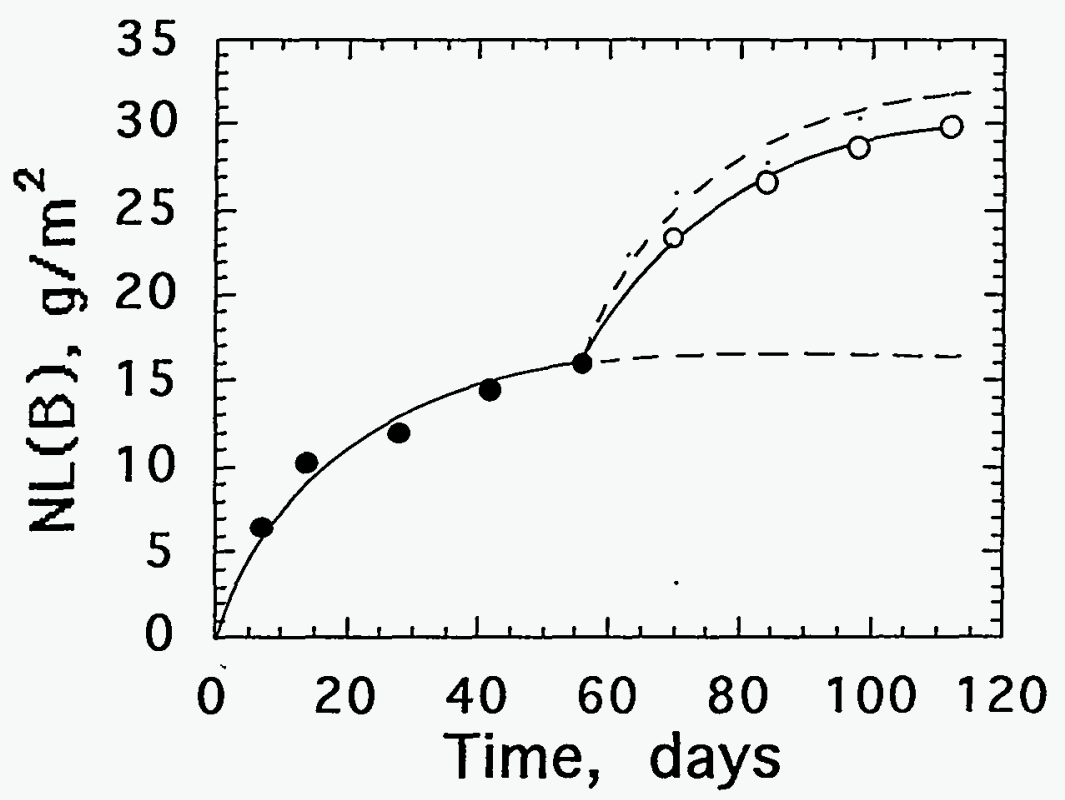

Fig. 11. Variations of MCC-1 Test with Glass A: (O) MCC-1 Tests, (๑) MCC-1 Tests in which Leachate is Replaced by Fresh Leachant after 56 Days (after [155]).

removed were expected to follow this curve if the corrosion was affected only by the solution chemistry. The lower dashed curve shows the extrapolation of the trend observed during the initial 56 days through 112 days. Data from tests continued after the layers were removed were expected to follow this curve if the corrosion was affected only by the mass transport through the layer. The normalized boron mass loss from glass $\mathrm{A}$ in tests continued in fresh leachant after 56 days shows a rapid increase after the leachate was replaced with fresh leachant, followed by a leveling off at longer times. This behavior is similar to that shown during the initial 56 days of reaction and indicates that the layer formed during the first 56 days of reaction had a minor effect on the corrosion rate during the second 56 days of reaction.

A different glass, glass B, was also reacted under MCC-1 conditions for times up to 56 days. The normalized silicon mass loss values measured in the MCC-1 tests are shown in Fig. 12 (open circles). The silicon release from glass $B$ follows the same trend as the boron release from glass A, namely an initial rapid rise followed by a leveling off. In a second variation of the MCC-1 test, samples of glass B were reacted for half the prescribed test duration and then removed from the leachate and replaced with fresh (unreacted) samples which were reacted for the remaining time. This test measures the reactivity of a glass sample having no alteration layer in a leachate solution that contains dissolved glass constituents from the corrosion of the first sample. In this variation, the corrosion products remaining in the leachate are expected to affect the corrosion rate. The normalized silicon mass losses for these tests (filled circles in Fig. 12) continue the trend established by the initial glass samples during the first 56 days of reaction. This result indicates that the solution components from the corrosion of the first glass specimen dominate the corrosion rate of the second glass specimen. The observation that the normalized silicon release from the variant tests (in which the samples were replaced after half the reaction time, filled circles in Fig. 12) is slightly higher than that from the standard tests (open circles in Fig. 12) indicates that the layer does have a minor effect on the glass corrosion rate. 


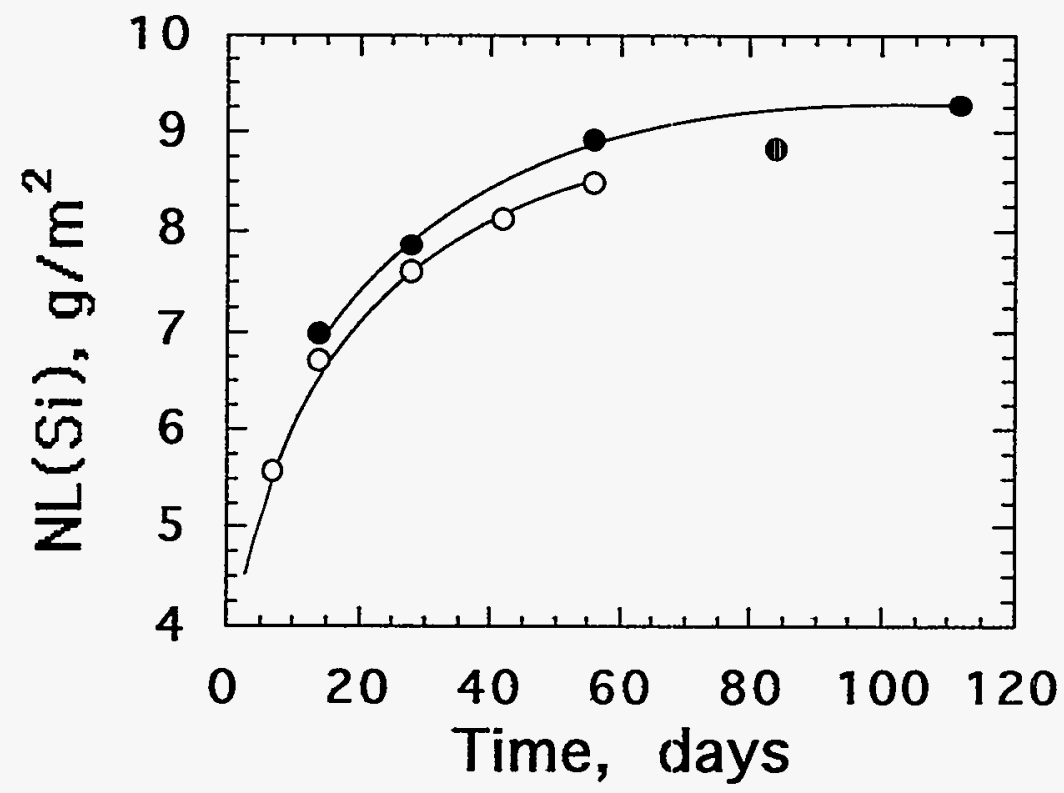

Fig. 12. Variations of MCC-1 Test with Glass B: (O) MCC-1 Tests, (๑) MCC-1 Tests in which Glass Samples are Replaced by New Samples after Half the Scheduled Reaction Time (after [155]).

In a third variation of the $\mathrm{MCC}-1$ test, samples of a third glass, glass $\mathrm{C}$, were removed from the leachate solution, rinsed, and placed in fresh leachant every 7, 14, or 28 days. For comparison, glass $\mathrm{C}$ was also reacted for several different time periods under standard MCC-1 conditions; these data are plotted as circles in Fig. 13. (Note that this test variation is a periodic replacement test. See Sections 2.5.2.3 and 5.) Under these conditions, the alteration layer is expected to continue to grow on the monolithic glass sample, while the glass reaction products are periodically removed from the solution. It can be seen in Fig. 13 that the normalized sodium mass loss increases nearly linearly, with the rate of increase highest in the tests with the greatest solution replacement rate. Tests in which the leachate was replaced every 7 days maintain the most dilute solutions, but also generate the thickest alteration layers. Thus, for this glass, the solution effects are more important than the mass transfer effects. The results from these variations of the MCC-1 test all show that the solution chemistry has a much greater effect on the glass corrosion rate than does the generation of an alteration layer. This implies that mass transport of water and reaction products (i.e., boron from glass A, silicon from glass B, and sodium from glass $C$ ) through the alteration layer is faster than the reactions leading to their release from the glass. We note also that boron, sodium, and silicon fill different roles in the glass structure and are released via different reaction mechanisms (see the Introduction to Sections 2 and 2.1) Nevertheless, the release of all three is controlled primarily by the solution chemistry.

Grambow and Strachan used a different modification of the MCC-1 method to study the effects of the alteration layer on the corrosion of two glass compositions in deionized water and in a $\mathrm{MgCl}_{2}$ solution [152]. Monolithic glass samples were reacted following the MCC-1 procedure for 57 days. At that point, samples in some tests were removed from the leachate, and the layers that had formed were physically removed from the samples. The remaining glass was then replaced in the same leachate and allowed to react for up to an additional 91 days (for a total 


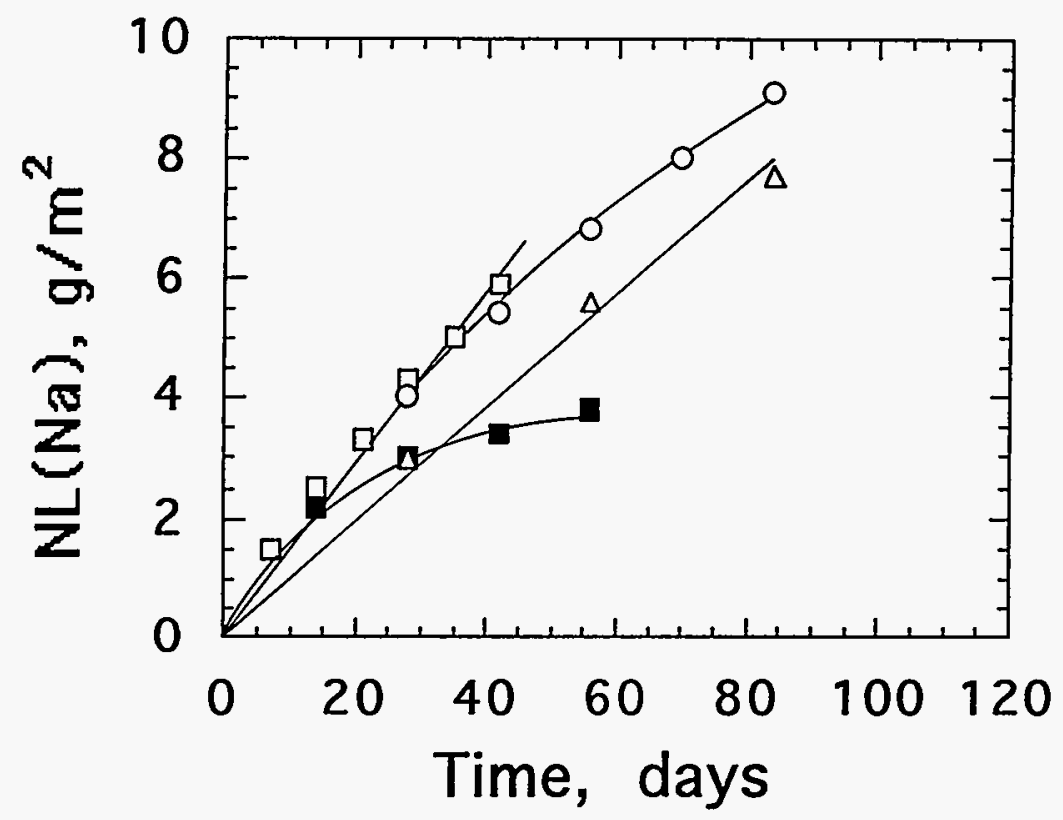

Fig. 13. Variations of MCC-1 Test with Glass C: (匹) MCC-1 Tests, MCC-1 Tests in which Leachate is Replaced by Fresh Leachant Every $(\Delta) 7$ Days, (O) 14 Days, and (ㅁ) 28 Days (after [155]).

reaction time of 148 days). Other tests were conducted without disruption, for comparison. The normalized boron and silicon mass losses for the glass MCC 76-68 (also known as PNL 76-68) and C31-3 glass in deionized water are plotted in Fig. 14. The boron and silicon releases are essentially the same whether the layer was removed or not, which shows that the layer had little effect on the corrosion rate.

In other tests in which MCC 76-68 and C31-3 glasses were reacted in a $0.001 \mathrm{M} \mathrm{MgCl} 2$ solution, removal of the layer resulted in an increase in the normalized boron and silicon mass losses, as shown in Fig. 15. However, while the layer formed on the MCC 76-68 glass in the $\mathrm{MgCl}_{2}$ solution may have been protective, Grambow and Strachan emphasized that the normalized release of boron (and to a lesser extent of silicon) from C31-3 glass was greater in the $\mathrm{MgCl}_{2}$ solution than in deionized water, both with and without the layer present. This observation indicates that the increase is due to the solution chemistry and not the layer. Grambow and Strachan suggested that the affinity for glass corrosion was increased in the presence of magnesium because of the formation of a magnesium silicate, such as sepiolite, which reduced the silicic acid concentration in solution and accelerated the corrosion. Sepiolite is formed by the following reaction:

$$
2 \mathrm{Mg}^{2+}+3 \mathrm{H}_{4} \mathrm{SiO}_{4}=\mathrm{Mg}_{2} \mathrm{Si}_{3} \mathrm{O}_{6}(\mathrm{OH})_{4}+2 \mathrm{H}_{2} \mathrm{O}+4 \mathrm{H}^{+}
$$

While magnesium was abundant in the leachant solution, silicic acid had to be supplied by corrosion of the glass. The reaction to form sepiolite (or perhaps another magnesium-bearing phase, such as magnesium-montmorillonite) approached equilibrium as silicic acid was released by corrosion of the glass during the initial 57 days. Removal of the layer after 57 days caused an increase in the forward reaction to generate more sepiolite. Sufficient magnesium was present in the solution, but additional silicic acid was generated by an increase in the glass corrosion rate. 
(a)

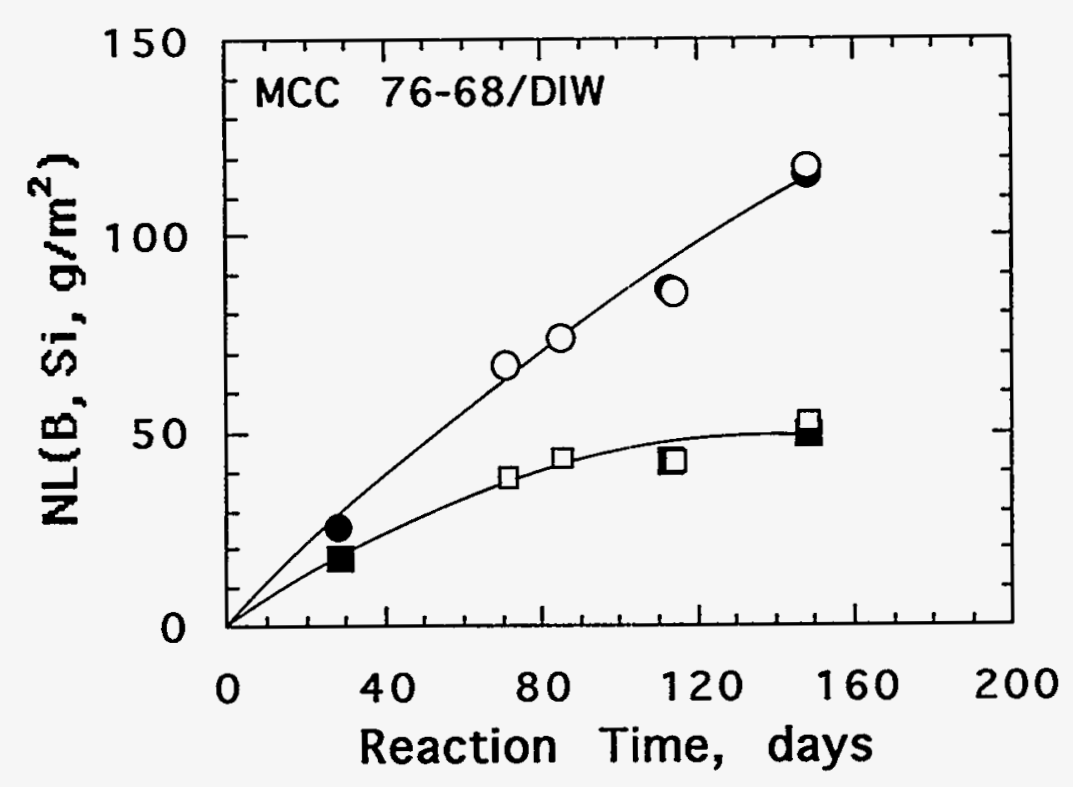

(b)

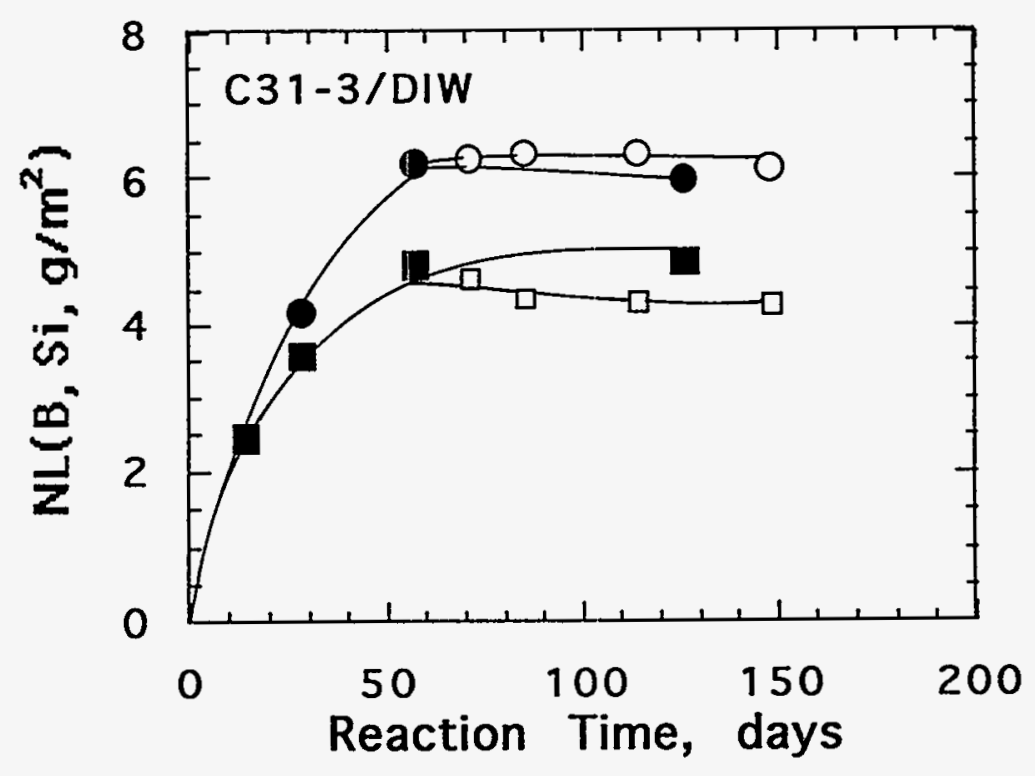

Fig. 14. Variations of MCC-1 Test with (a) MCC 76-68 and (b) C31-3 Glass in Deionized Water: (O) B in Tests in which Layer is Removed after 57 Days, $(\bullet)$ B in Tests in which Layer is Not Removed, ( $\square$ ) Si in Tests in which Layer is Removed after 57 Days, and (ם)Si in Tests in which Layer is Not Removed (after [152]). 
(a)

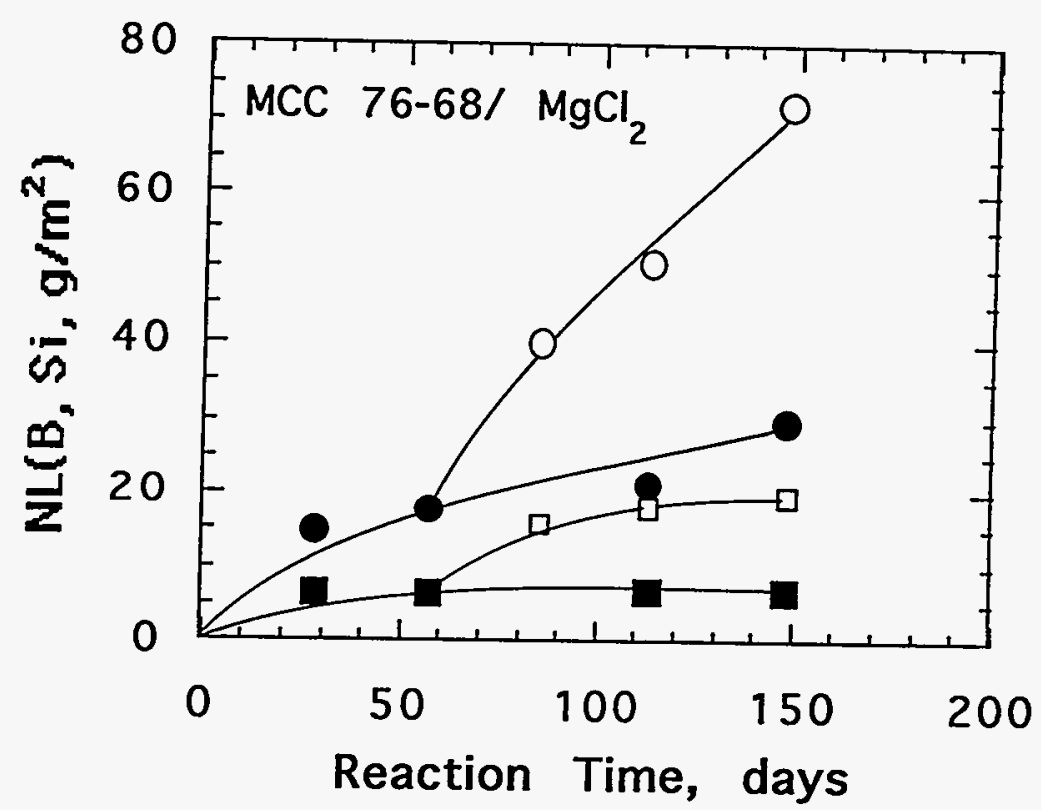

(b)

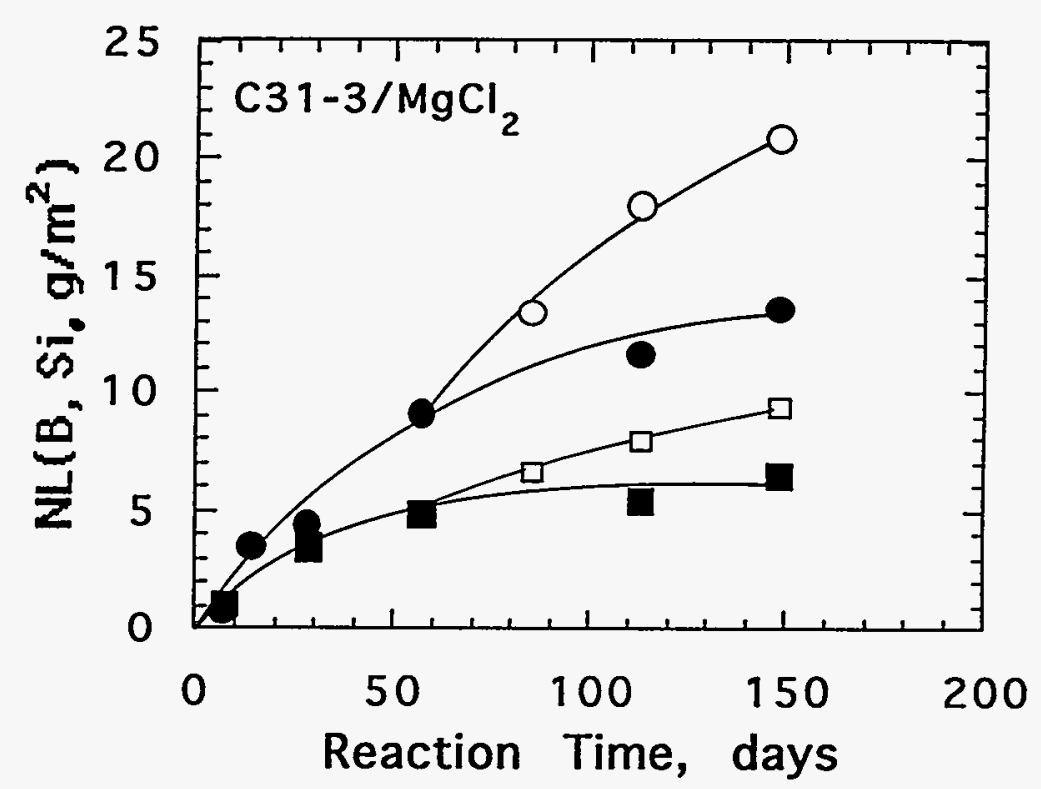

Fig. 15. Variations of MCC-1 Test with (a) $\mathrm{MCC} 76-68$ and (b) C31-3 Glass in $\mathrm{MgCl}_{2}$ Solution: (O) B in Tests in which Layer is Removed after 57 Days, (०) B in Tests in which Layer is Not Removed, ( $\square$ ) Si in Tests in which Layer is Removed after 57 Days, and ( $\mathbf{\square}) \mathrm{Si}$ in Tests in which Layer is Not Removed (after [152]). 
The observed effect depends on the relative rates of sepiolite precipitation, which consumes silicic acid, and glass corrosion, which supplies silicic acid. The observation that the silicic acid concentration also increased upon removal of the layer implies that both glasses corrode faster than sepiolite precipitated. The effect of the silicic acid concentration on the glass corrosion rate is summarized in the next section.

Similar tests were conducted on the glass SM58LW11 in a $\mathrm{MgCl}_{2}$-dominated brine by Conradt and Roggendorf [156,157]. They periodically interrupted MCC-2 type tests (conducted with monolithic samples at 120 and $200^{\circ} \mathrm{C}$ and an SA $/$ ratio of $3.4 \mathrm{~m}^{-1}$ ) and removed the corroded sample. Next they manipulated the sample by one of the following methods:

(A) remove the alteration layer from the glass and replace layer and glass into same leachate;

(B) remove the alteration layer, dry it at $110^{\circ} \mathrm{C}$, weigh it, and then replace layer and glass into same leachate; (C) remove sample and place it in fresh leachant, with layer intact; (D) remove layer and place the sample in fresh leachant; or (E) replace the sample without further manipulation. Other tests were conducted without interruption for comparison. The results of tests conducted at $120^{\circ} \mathrm{C}$ are shown in Fig. 16. The one test conducted following method $\mathrm{E}$ was consistent with uninterrupted tests and showed that cooling the test vessels and reheating them did not affect the results. Tests following methods $A$ and $B$, where the layers were removed then the samples replaced in the same leachate, both showed slightly greater corrosion than untreated tests. This result implies the layers had a slight protective effect. The results of manipulations $A$ and $B$ were deemed indistinguishable by the authors. Tests following methods $C$ and $D$, where the samples were periodically placed in fresh leachant) both showed significantly greater corrosion than the untreated tests. The presence of the layer in $\mathrm{C}$ and the absence of the layer in $\mathrm{D}$ had little effect on the extent of corrosion, however. This finding demonstrates that the physical removal of the layer does not measurably affect the continued corrosion of the glass.

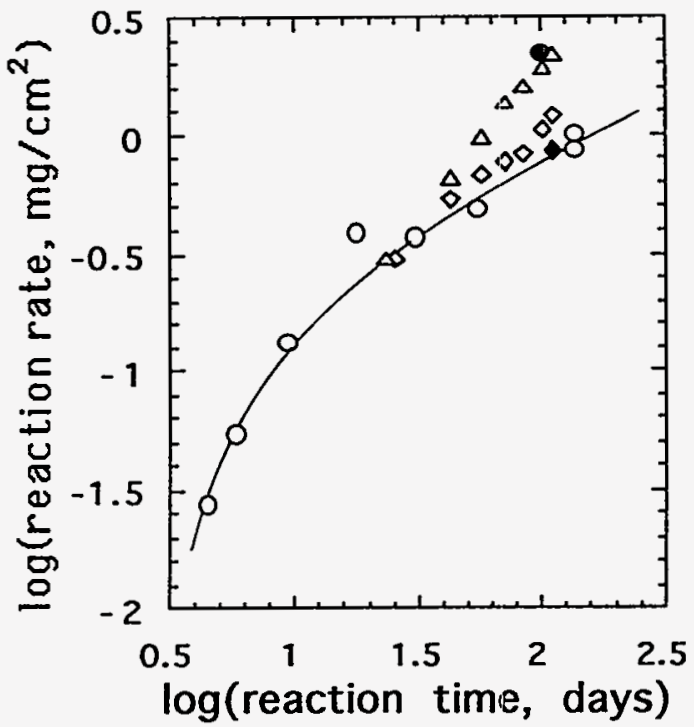

Fig. 16. Measured Extent of Corrosion vs. Time for Tests with Variously Treated Samples: (O) Uninterrupted Static Tests, ( () Tests with Manipulations A or B, (0) Tests with Manipulation $C,(\Delta)$ Tests with Manipulation D, and $(\diamond)$ Tests with Manipulation E (see text, after [156]). 
The results of tests with similar manipulations at $200^{\circ} \mathrm{C}$ lead to the same conclusions, except the removal of the layers in treatments A and B had no measurable effect. These results demonstrate that the layer has little or no effect on the corrosion rate of this glass in a brine solution, while the corrosion products that build up in the leachate slow the corrosion.

The effects of the $\mathrm{MgCl}_{2}$ brine solutions observed by Grambow and Strachan and by Conradt and Roggendorf at first appear contradictory. While the tests conducted by Grambow and Strachan are similar to methods A and B used by Conradt and Roggendorf, Conradt and Roggendorf replaced both the removed layer and the glass back into the solution, while Grambow and Strachan replaced only the glass. Therefore, while the mass transport effects are eliminated in both methods, chemical interactions continue between the layer and the solution in the Conradt and Roggendorf methods A and B tests. If a secondary phase such as sepiolite is present in the layer, then its influence on the solution chemistry and the silicic acid activity will continue after being separated from the glass. That is, the perturbation of the equilibrium proposed by Grambow and Strachan will not occur if the sepiolite phase remains in contact with the solution. The different glass compositions tested by Grambow and Strachan and by Conradt and Roggendorf may also affect the comparison of the test results. (C31-3 glass has a higher content of aluminum and barium and a lower content of boron and alkali.) The difference in reaction temperature probably also affected the tests. Conradt and Roggendorf found the effect of layer separation to decrease as the temperature increased.

The results of Chick and Pederson, Grambow and Strachan, and Conradt and Roggendorf all show the solution chemistry to dominate the glass corrosion rate during the initial stages of glass corrosion (Stages I and $I$ as described in Section 2.4). Alteration layers formed during glass corrosion have only a minor mitigating effect in these tests. The importance of this with regard to the effects of the SA/V on glass corrosion is that the effects of the SA/V occur solely through the solution chemistry. That is, the SA/V affects the solution chemistry and the solution chemistry is the primary controlling influence on the glass corrosion rate. However, differences in the solution chemistries generated in tests at different $S A / V$ will affect the alteration layers formed on the glass surface as the glass corrodes.

It is important to recognize that the test methods used to assess the effects of the layer all maintained dilute conditions such that solutions did not approach saturation during the test period. Very different effects of the layers on glass corrosion have been observed in various tests that generated saturated solutions. An understanding of the corrosion mechanism under these conditions is essential, since these conditions are expected to dominate the long-term corrosion behavior. Unfortunately, the very slow kinetics that occur under saturated conditions make it difficult to characterize the corrosion mechanism or measure the corrosion rate under these conditions. Some test results have been interpreted to suggest that the effect of the alteration layers on the glass corrosion rate become more significant as the leachate solution becomes saturated and the hydrolysis reaction to release silicic acid slows to a negligible rate. Some researchers have suggested that ion exchange reactions determine the long-term glass corrosion rate [158-160], while Grambow et al. have suggested that water diffusion through the alteration layers controls the corrosion rate under silica-saturated conditions [161]. This issue is discussed in Sec. 3.5.

Barkatt and co-workers have observed transient increases in tests with several glass compositions during periodic replenishment tests that they attributed to cracking or sloughing of the alteration layer to expose fresh glass beneath the layer $[127,162,163]$. However, such transient excursions have only been observed in periodic replenishment tests, and may be an artifact of that test method (see also Section 2.5.2.3). Clear evidence of cracking or spalling has not been demonstrated. Also, possible effects of decreasing particle size need to be accounted for in long-term tests. In fact, examination of the data presented by Sang et al. show no obvious 
"spike" of boron release beyond the range of experimental error. What is observed is an initially higher rate at the beginning of the test, which may be due to the presence of fines and reaction of high energy sites such as points and sharp edges on the crushed glass.

\subsection{Observed Effects of Silicic Acid Concentration on Glass Corrosion}

Since most waste glasses contain roughly $50 \mathrm{wt} \% \mathrm{SiO}_{2}$, it is not surprising that leach tests have shown the silicic acid solution concentration to strongly affect the glass corrosion rate. For example, Pederson et al. [72] conducted MCC-1 tests $\left(28\right.$ days, $\left.10 \mathrm{~m}^{-1}, 90^{\circ} \mathrm{C}\right)$ with PNL 76-68 glass in solutions having various silicic acid concentrations, including two groundwaters. Figure 17 shows the normalized boron release from PNL 76-68 glass plotted against the silicic acid concentration. The glass corrosion rate, as measured by the boron solution concentration, decreases as the silicic acid concentration increases up to about $100 \mathrm{ppm}$. At silicic acid concentration higher than that, the glass corrodes at a very low and approximately constant rate. The open symbols represent silicic acid solutions, while the two solid symbols represent groundwaters. The observation that all data fit the same trend indicates that other components in the groundwater have only a minor affect on the corrosion rate.

The same dependence of the rate on the silicon concentration is shown in Fig. 18, where the corrosion rate of JSS-A glass, as measured by the boron release, is plotted against the ion activity product of silica in 28 -day leach tests at $90^{\circ} \mathrm{C}$ at SA $/ \mathrm{V}$ ratios of $10 \mathrm{~m}^{-1}$ [154,164]. From these results, it is clear that the maximum corrosion rate occurs when the solution contains no silicic acid and the minimum rate occurs when the silicic acid concentration attains a so-called "saturation" concentration. Further increases in the ion activity product (or silicic acid concentration) do not affect the corrosion rate.

The presence of silicic acid in the leachant solution has also been shown to affect the corrosion rates of high level waste glasses using a flow-through apparatus [71]. Some results are discussed in Section 3.2.

The effect of the concentration of silicic acid in the leachant solution becomes insignificant in tests at high SA/V, since silicic acid released from the glass dominates the solution after very short time periods. This is shown in tests conducted to assess the effects of $\mathrm{SA} / \mathrm{V}$ on the corrosion of glasses relevant to the DWPF (see Sec. and Appendix IV B) in the boron concentrations in tests conducted at $90^{\circ} \mathrm{C}$ and at SA/V of about 340 and $20,000 \mathrm{~m}^{-1}$ in deionized water and in a tuff groundwater solution containing about 45 ppm silicon [66]. For example, the boron concentrations in tests in deionized water and in groundwater at $340 \mathrm{~m}^{-1}$ conducted for 14 days were about 10 and $3 \mathrm{ppm}$, respectively, while the corresponding silicon concentrations are about $65 \mathrm{ppm}$ in tests in deionized water and about $75 \mathrm{ppm}$. This shows the higher initial silicon concentration results in less corrosion in tests in groundwater than in deionized water. In contrast, the boron concentrations are about $77 \mathrm{ppm}$ and silicon concentrations are about $280 \mathrm{ppm}$ both in tests in deionized water and in groundwater at $20,000 \mathrm{~m}^{-1}$ after only 3 days. This shows the silica released from the glass during the test quickly dominates the solution concentration, and the extent of corrosion in these tests is independent of the initial leachant composition.

A model in which the glass corrosion rate is controlled by the amount of silicic acid in solution has been developed by Grambow $[153,154]$. This model is based on an equation derived to represent the dissolution rates of silicate minerals. The rate limiting step is modeled to be the hydrolysis of a silane bond to release silicic acid into solution and form a silanol group in the glass. The net reaction given earlier in Eq. $6 \mathrm{~b}$, can be written to include the activated complex as 


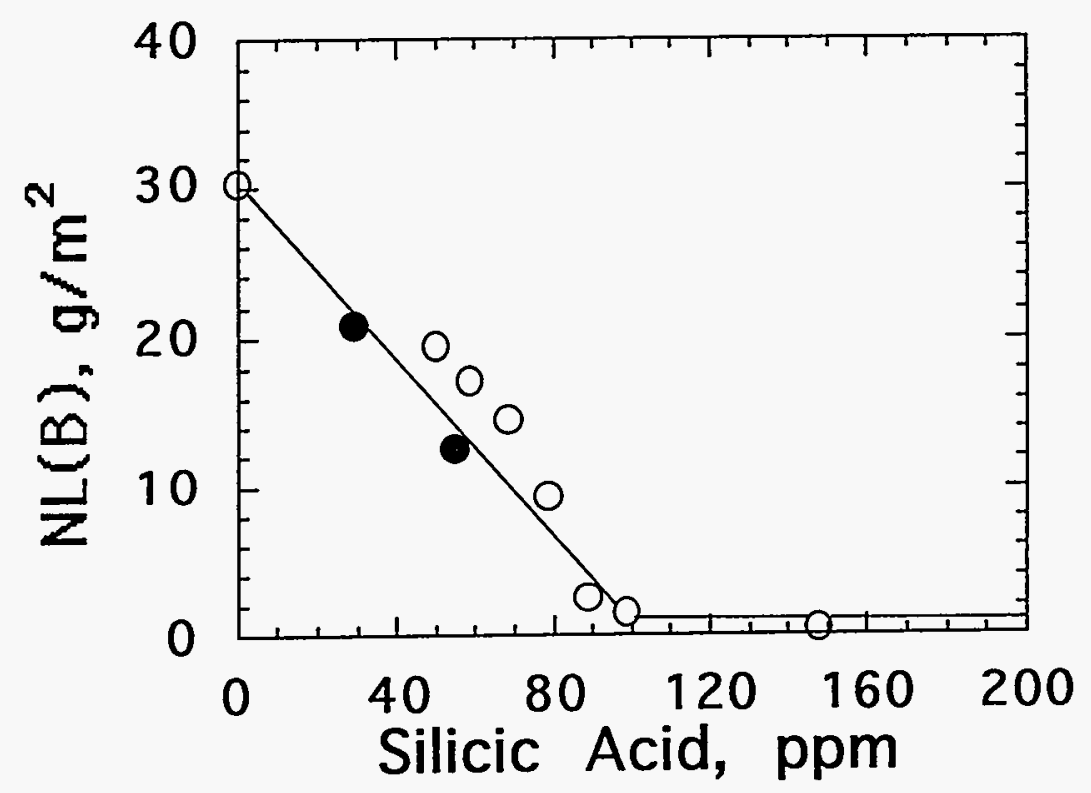

Fig. 17. Boron Release from PNL 76-68 Glass vs. Silicic Acid Concentration (O) Synthetic Solutions and (๑) Groundwaters (after [72])

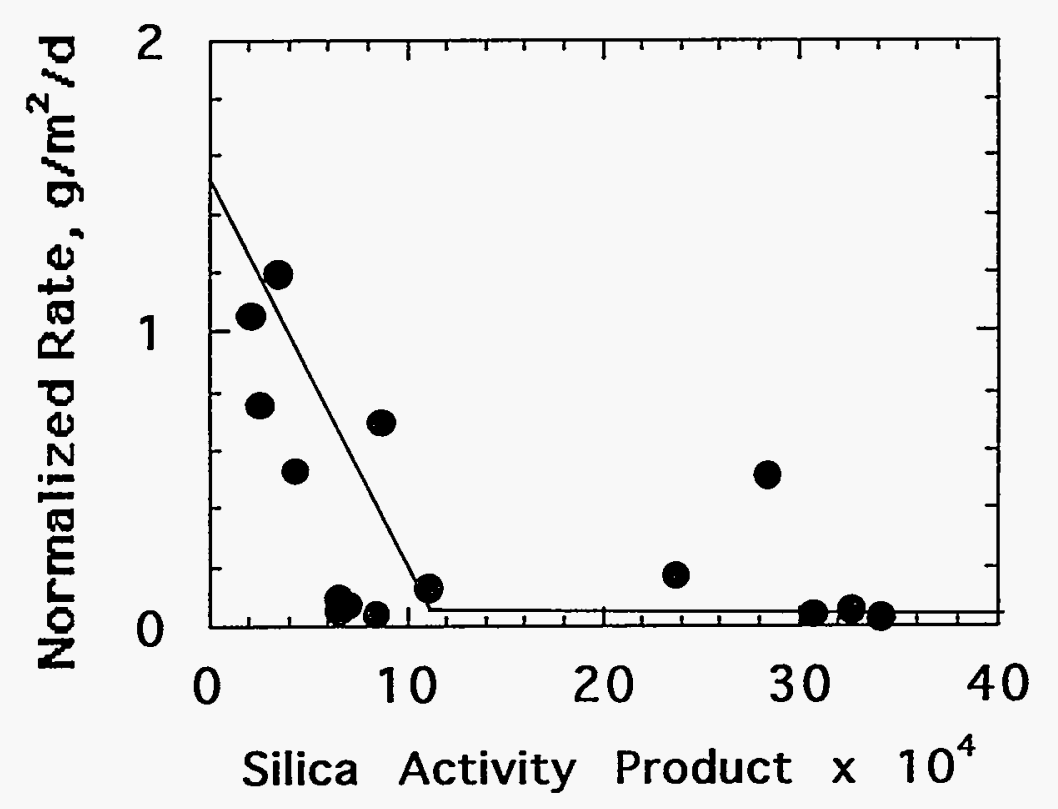

Fig. 18. Glass Corrosion Rate (as Measured by Boron Release) vs. the Ion Activity Product of Silicic Acid (after [154]) 


$$
\equiv \mathrm{SiO}-\mathrm{Si}(\mathrm{OH})_{3}+\mathrm{H}_{2} \mathrm{O}=\left\{\equiv \mathrm{Si}-\mathrm{O}-\mathrm{Si}(\mathrm{OH})_{3}\right\} \bullet \mathrm{OH}_{2} \rightarrow \equiv \mathrm{SiOH}+\mathrm{H}_{4} \mathrm{SiO}_{4(\mathrm{aq})}
$$

where the species in brackets represents the activated complex. The formation of the activated complex involves the adsorption of a water molecule onto the terminal silicon atom, which then becomes coordinated to five oxygen atoms, and the formation of a hydrogen bond between one of the hydrogen atoms of the adsorbed water molecule and the bridging oxygen. The mechanics of this reaction have been described by Lasaga and Gibbs [55], who point out that five-fold coordinated silicon is a stable structure. In addition, they show that adsorption of water to silicon through electron donation by the oxygen is a stable configuration, though less stable than sorption to silanol groups through electron acceptance of the hydrogen [55]. The breaking of the bond between the terminal silicon and bridging oxygen atoms shown in the activated complex (and dissociation of the water molecule) results in the release of silicic acid.

An expression describing the rate of this reaction was derived by Aagaard and Helgeson [54]. (A discussion of the derivation is presented in Appendix B.) The rate of the hydrolysis reaction to release silicic acid given in Eq. 6a has been modeled on the basis of irreversible thermodynamics; the rate is expressed as the product of a forward rate term and a term representing the chemical affinity for the reaction to release silicic acid from the glass [54]. The chemical affinity can be expressed in terms of the difference between the actual silicic acid concentration and a "saturation" concentration of silicic acid. Expressed this way, the reaction affinity decreases as the solution concentration of silicic acid approaches the "saturation" concentration [153]. In its simplest form, the rate of silicic acid release can be expressed as

$$
\frac{\frac{\mathrm{d}\left(\mathrm{n}\left(\mathrm{H}_{4} \mathrm{SiO}\right)\right)}{\mathrm{dt}}}{\mathrm{S}}=\mathrm{k}_{+}\left(1-\frac{\left[\mathrm{H}_{4} \mathrm{SiO}_{4}\right]}{\left[\mathrm{H}_{4} \mathrm{SiO}_{4}\right]_{\text {sat }}}\right)
$$

where $\mathrm{n}\left(\mathrm{H}_{4} \mathrm{SiO}_{4}\right)$ is the number of moles of silicic acid released from the glass into solution, $\mathrm{S}$ is the surface area of the glass, $\mathrm{k}_{4}$ is the rate coefficient for the hydrolysis reaction, $\left[\mathrm{H}_{4} \mathrm{SiO}_{4}\right]$ is the molar silicic acid concentration in solution, and $\left[\mathrm{H}_{4} \mathrm{SiO}_{4}\right]_{\text {sat }}$ is the molar "saturation" concentration of silicic acid. The term $\left(1-\left[\mathrm{H}_{4} \mathrm{SiO}_{4}\right] /\left[\mathrm{H}_{4} \mathrm{SiO}_{4}\right]_{\mathrm{sat}}\right)$ is the reaction affinity. Note that the rate as written in Eq. 13 has the units of moles silicic acid per time per area. Variations of Eq. 13 are often written in which the surface area term, S, is moved to the right-hand side of the equation. This change facilitates the use of experimental data for which the silicic acid concentration is measured after various reaction times (see below). It is important to remember that such an expression (with $S$ on the right) gives the rate at which the solution composition changes, but not the corrosion rate of the glass. The corrosion rate of the glass is given in Eq. 13.

In general, the solubility limits of dissolved species are established by solid phases with measurable thermodynamic equilibrium constants. Glasses are thermodynamically metastable and cannot equilibrate with solutions. Instead, they appear to approach an apparent "saturation" concentration at which the dissolution rates become very low. While no solid phase that establishes the "saturation" concentration has been identified, empirically determined "saturation" concentrations are usually found to be intermediate between the solubilities of quartz and amorphous silica. This model and the concept of silica "saturation" have been found to describe the observed effects of the silicic acid concentration on the glass corrosion rate and the "roll over" behavior seen in Fig. 4 well. 
On the basis of observations in dynamic tests, another term is sometimes included to account for the diffusion of material through the alteration layer that forms on the glass surface during corrosion $[154,165]$. This term may be important for glass compositions with relatively large values of $k_{+}$but will not be further considered here.

Two limiting cases to Eq. 13 exist: when the affinity term is near one (when $\left[\mathrm{H}_{4} \mathrm{SiO}_{4}\right] \ll\left[\mathrm{H}_{4} \mathrm{SiO}_{4}\right]_{\text {sat }}$ ), and when the affinity term is near zero (when $\left[\mathrm{H}_{4} \mathrm{SiO}_{4}\right] \approx$ $\left.\left[\mathrm{H}_{4} \mathrm{SiO}_{4}\right]_{\text {sat }}\right)$. When the affinity term is one, the rate is given simply by $\mathrm{k}_{+}$. When the affinity term is zero, the corrosion rate predicted by Eq. 13 is also zero. Experiments conducted under these limiting conditions have demonstrated the need to modify Eq. 13. First, tests under conditions for which the affinity term remains near one have shown the corrosion rate to be sensitive to the solution $\mathrm{pH}$. Second, tests under conditions for which the affinity term approached zero suggest that the rate does not reach zero even after very long reaction times; rather it approaches a minimum value that may be two or three orders of magnitude less than $\mathrm{k}_{+}$ [67,116,166-168]. These observations and the modifications of the rate equation made to account for them are discussed below.

In a slightly different approach, Bourcier has represented the reaction affinity in terms of all elements found in the alteration layer which forms on the reacted glass, instead of only silicon. The silicic acid concentration in the affinity term in Eq. 13 is replaced by the activity product of components which remain in the layer, while the silicic acid saturation value is replaced by a term computed using the solubilities of several phases modeled to comprise the layer [143]. The layer composition is measured experimentally, but is well approximated by simply excluding soluble alkali metals and boron from the glass composition. Layers typically include silicon, aluminum, iron, calcium, and magnesium. The solubility products calculated using this approach are similar to those regressed from experiments, since the calculated values are dominated by the contribution of silica. This approach identifies the phase controlling the "saturation" of the solution to be the alteration layer. That is, the terms [ $\left.\mathrm{H}_{4} \mathrm{SiO}_{4}\right]$ and $\left[\mathrm{H}_{4} \mathrm{SiO}_{4}\right]_{\text {sat }}$ are replaced by terms including other components in addition to silicic acid. The approach is appealing since the alteration phases are more likely to be equilibrated with the solution than is the glass. In fact, in the computer model used by Bourcier, the alteration layer is comprised of phases that have equilibrated with the solution. However, the reaction that the affinity term is meant to represent occurs between the glass and the solution.

\subsection{Observed Effects of the Leachate pH on Glass Corrosion}

The effect of the leachate $\mathrm{pH}$ on the corrosion of waste glasses has been measured under conditions where the silicic acid concentration remained low throughout the test. From Eq. 13, the corrosion rate under these conditions can be expressed as rate $=k_{+}$. To express its $\mathrm{pH}$-dependence, the rate coefficient can be written as $\mathrm{k}_{+}=\mathrm{k}_{\mathrm{f}}\left(\mathrm{H}^{+}\right)^{\mathrm{h}}$, where $\mathrm{k}_{\mathrm{f}}$ is the $\mathrm{pH}$-independent rate coefficient and $\mathrm{h}$ is an experimentally determined value. In the limit where the silicic acid concentration is zero, the rate can be written as rate $=k_{+}=k_{f}\left(H^{+}\right)^{h}$ or as $\log ($ rate $)=\log \left(\mathrm{k}_{\mathrm{f}}\right)-\mathrm{h}(\mathrm{pH})$. Workers at Lawrence Livermore National Laboratory have measured the effects of the solution chemistry on the corrosion rate of high-level waste glasses by using a single-pass flow-through device. In these experiments, $\mathrm{pH}$ buffers were added to the leachant solution to measure the effects of the leachant $\mathrm{pH}$ on the glass corrosion rate. The solution leaving the reaction cell was sampled periodically until constant solution concentrations of key glass components were measured. The constant concentrations indicated that apparent "steady state" conditions had been reached, where it could be assumed that the effects of fines and surface stresses were eliminated (see below). 
Figures $19 \mathrm{a}$ and $19 \mathrm{~b}$ show the release of several elements from the CSG glass in tests conducted at $70^{\circ} \mathrm{C}$ at $\mathrm{pH}$ values of about 6 and 10 [112]. The CSG glass is a simple, five-component glass that was designed to simulate a compositionally more complex DWPF glass. It contains, in oxide weight percent, $11.7 \mathrm{Al}_{2} \mathrm{O}_{3}, 8.4 \mathrm{~B}_{2} \mathrm{O}_{3}, 6 \mathrm{CaO}, 18.2 \mathrm{Na}_{2} \mathrm{O}$, and $55.7 \mathrm{SiO}_{2}$. At pH 6, boron, calcium, and sodium are released stoichiometrically and at a higher rate than are silicon or aluminum. At $\mathrm{pH} \mathrm{10,} \mathrm{all} \mathrm{five} \mathrm{elements} \mathrm{are} \mathrm{released} \mathrm{stoichiometrically.}$ This is because the solubility of silicon is greater at pH 10 (see below). Notice that the release of boron, calcium, and sodium is similar at $\mathrm{pH} 6$ and $\mathrm{pH} 10$. The increased release rate observed for aluminum is not due to an increase in its solubility. Instead, aluminum probably is removed from the glass stoichiometrically with silicon as the surface disintegrates and it is flushed by the solution flow. Aluminum is commonly observed to be retained on the glass surface as insoluble residue in static tests, many of which attain high pH (e.g., see Appendix D).

Figure 20 shows the corrosion rate of CSG glass at $70^{\circ} \mathrm{C}$ (measured by the solution concentrations of glass components) as a function of the leachant $\mathrm{pH}$, as measured in a singlepass flow-through apparatus [70]. The logarithm of the rate, as measured by the silica release, increases linearly with the $\mathrm{pH}$ over the range $6>\mathrm{pH}>13$. The same $\mathrm{pH}$ effects have been observed in tests with DWPF reference glasses [71]. The increase in the rate with decreasing $\mathrm{pH}$ below about $\mathrm{pH} 6$ is probably due to reactions involving aluminum (see below).

A similar $\mathrm{pH}$ dependence was observed in modified $\mathrm{MCC}-1$ tests $\left(\mathrm{SA} / \mathrm{V}=2 \mathrm{~m}^{-1}\right.$ ) with monolithic samples of a defense waste reference glass (DWRG) glass in buffer solutions at $90^{\circ} \mathrm{C}$ [169]. The measured silica concentration had a minimum between $\mathrm{pH}$ values of $\mathrm{pH} 4$ and $\mathrm{pH} 7$ and increased at both lower and higher $\mathrm{pH}$ values.

Figure 21 shows the corrosion rate of R7T7 glass in static tests similar to MCC-1 tests performed at $90^{\circ} \mathrm{C} \mathrm{[92].} \mathrm{The} \mathrm{reaction} \mathrm{times} \mathrm{varied} \mathrm{with} \mathrm{the} \mathrm{pH}$ to measure the rate under dilute conditions. Short testing times (a few hours) were used at high $\mathrm{pH}$ values and longer testing times (up to three weeks) were used at low $\mathrm{pH}$ values. These results also show the corrosion rate to increase linearly with the $\mathrm{pH}$ in neutral and alkaline solutions. The slope of the $\mathrm{pH}$ dependence (h) has a value of about 0.4 for both the simulated DWPF glass at $70^{\circ} \mathrm{C}$ [70] and the French glass R7T7 at $90^{\circ} \mathrm{C}$ [92]. We note that the tests with R7T7 glass did not show the increase in the corrosion rate at low $\mathrm{pH}$ values observed in tests with CGS glass. This is probably due to differences in the glass compositions, the reaction temperatures, or other test conditions.

A similar $\mathrm{pH}$ effect was reported by Barkatt et al. in tests with Pyrex and a high-silica glass conducted at $70^{\circ} \mathrm{C}$ [136]. In these tests, the dissolution rate of the high-silica glass was about 2.5 orders of magnitude higher in tests at $\mathrm{pH} 9$ than at $\mathrm{pH}$, while the dissolution rates were similar at $\mathrm{pH} 2$ and $\mathrm{pH} 6$.

Insight into the $\mathrm{pH}$ dependence of waste glass corrosion can be drawn from the study of the release of various components from simple glasses. Tests were conducted by reacting $2 \mathrm{~g}$ of crushed glass in $100 \mathrm{~mL}$ of various aqueous buffer solutions having $\mathrm{pH}$ values between about 1 and 13 at a constant temperature. Different size fractions, test temperatures, and reaction times were used in different studies. The release rates of alkali metals from several binary alkali silicate glasses are shown in Figs. 22a-c as a function of the solution $\mathrm{pH}$ [170]. Tests were conducted at $35^{\circ} \mathrm{C}$ using $-30+52$ mesh glass. Tests were conducted for up to 4 hours. Over this time period, the release of alkali metal ions was found to increase with the square root of time, which indicates that water diffusion and ion exchange are the dominant reactions. The extent of extraction is generally insensitive to the solution $\mathrm{pH}$ for values of about $\mathrm{pH} 9$ or lower, but decreases precipitously as the $\mathrm{pH}$ was increased to $\mathrm{pH} 10$ or higher [170]. The decreased extraction with increasing $\mathrm{pH}$ can be explained as a shift in the ion exchange equilibrium (see 
(a)

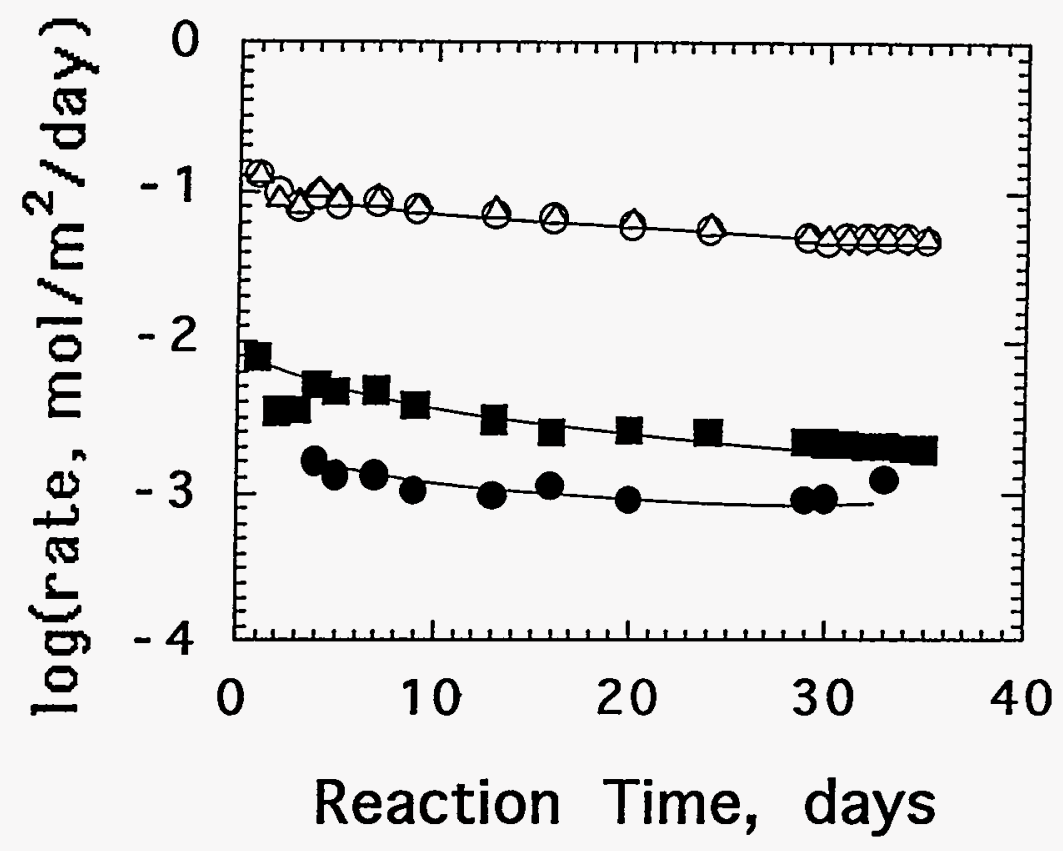

(b)

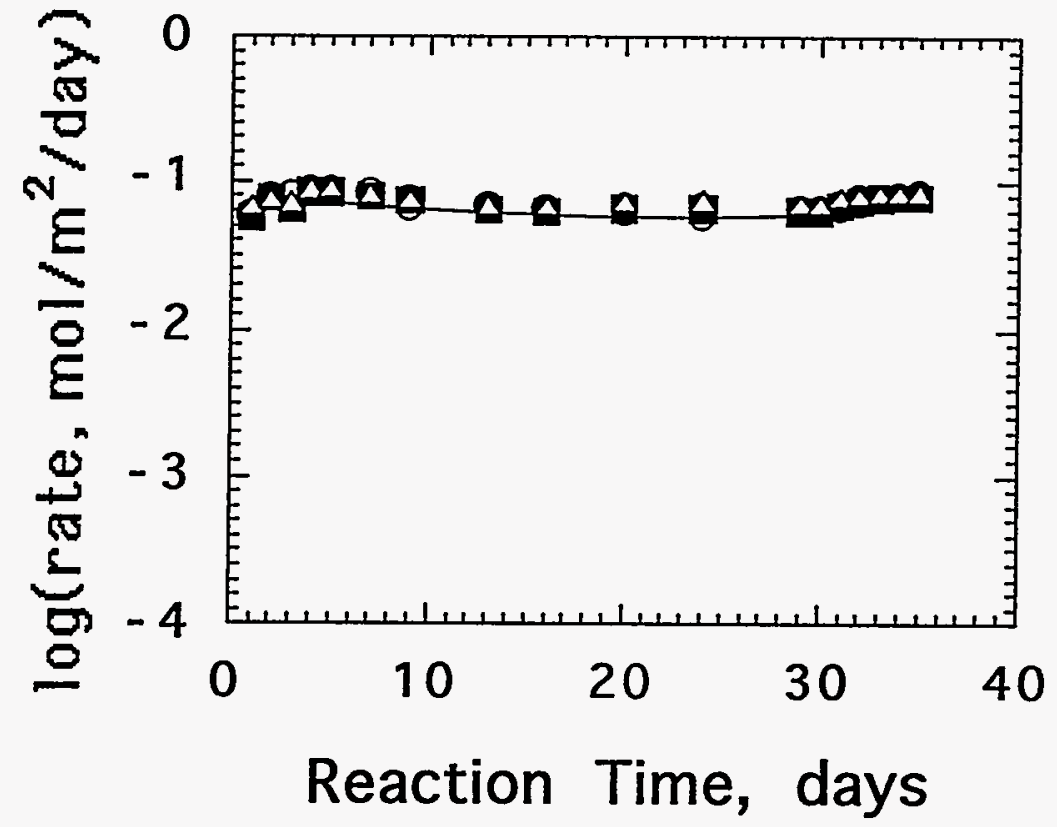

Fig. 19. Release of $\mathrm{B}, \mathrm{Na}, \mathrm{C}, \mathrm{Al}$, and $\mathrm{Si}$ from CSG Glass as a Function of Time at (a) $\mathrm{pH} 6$ and (b) $\mathrm{pH}$ 10: (৫) $\mathrm{Si},(\bullet) \mathrm{Al},(0) \mathrm{B},(\Delta) \mathrm{Na}$, and (O) $\mathrm{Ca}$ (after [112]). 


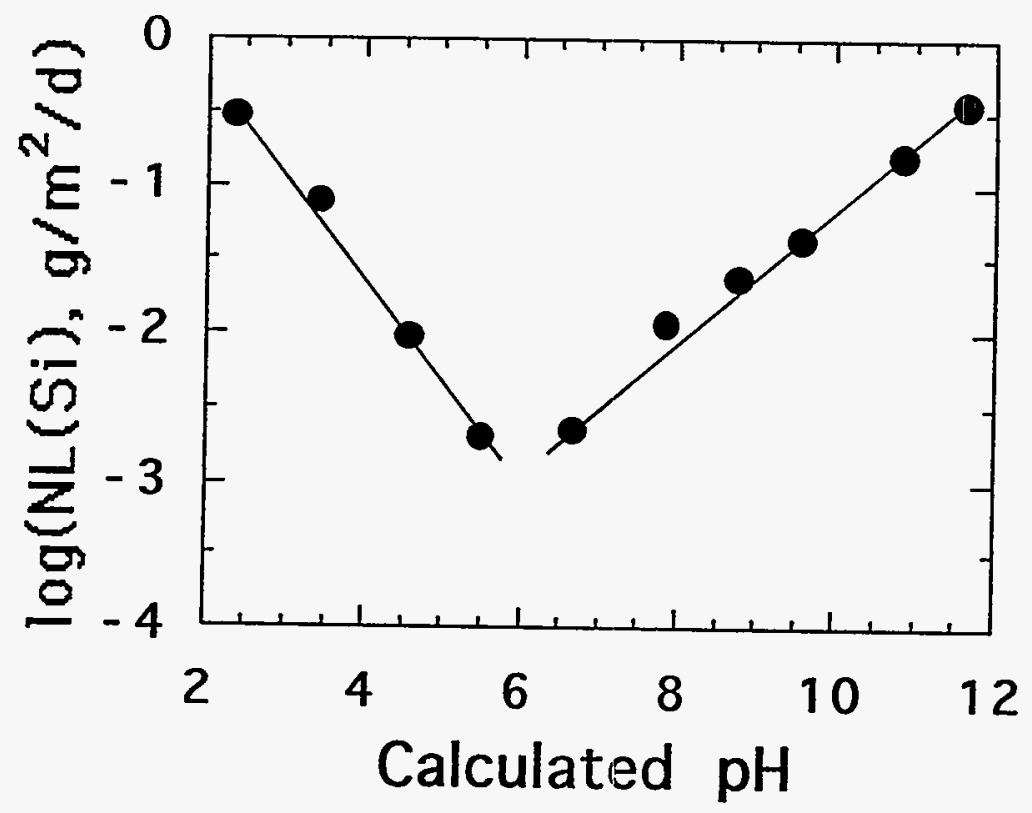

Fig. 20. Corrosion Rate of CSG Glass vs. pH (after [70])

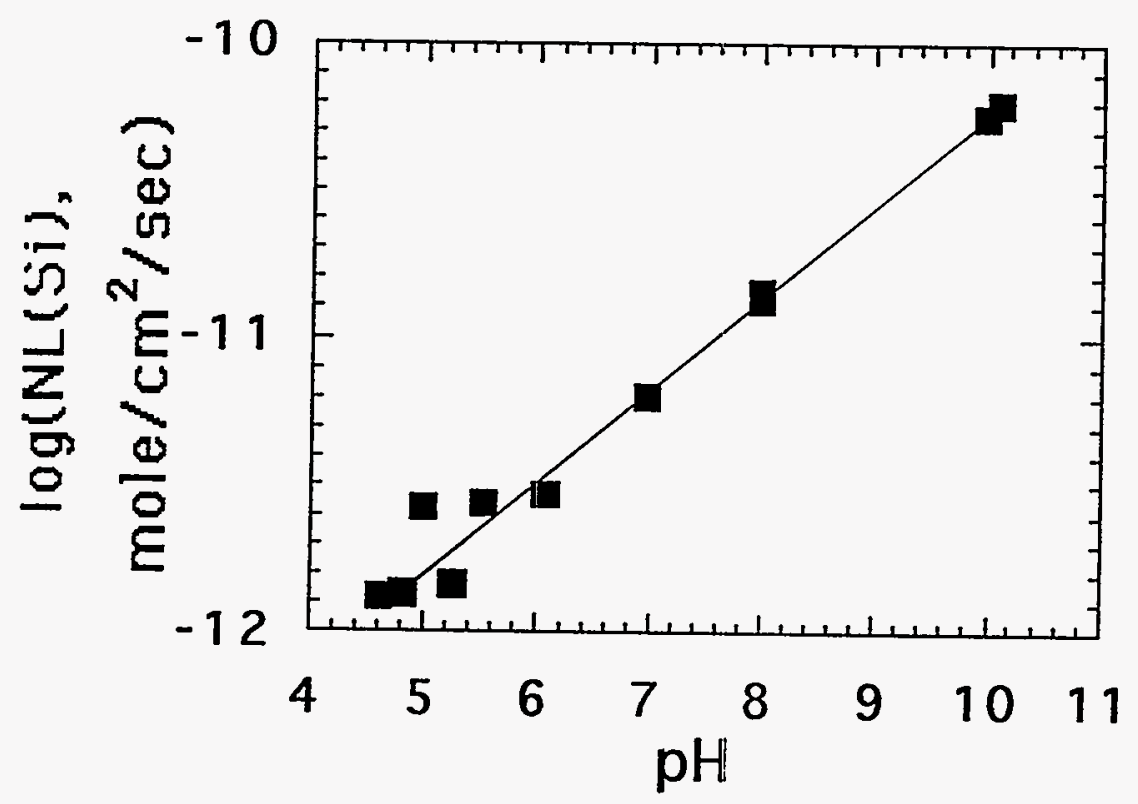

Fig. 21. Corrosion Ratio of R7T7 Glass vs. pH (after [92]) 
(a)

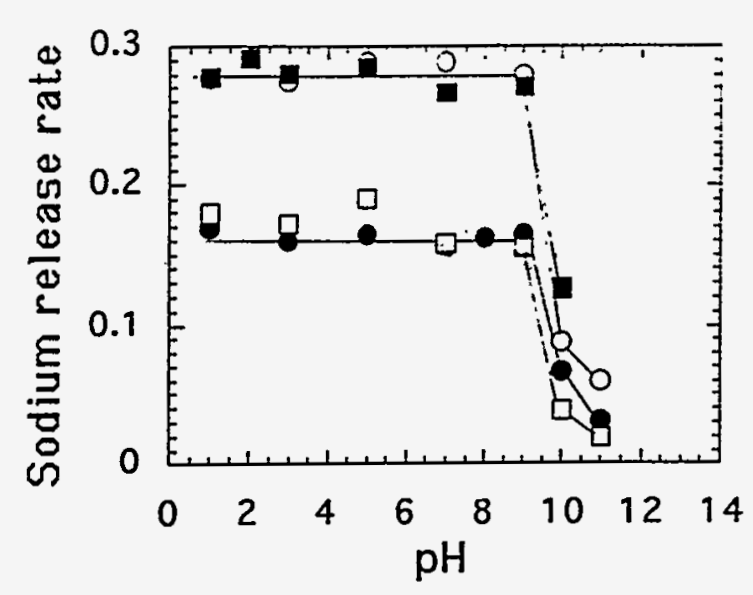

(b)

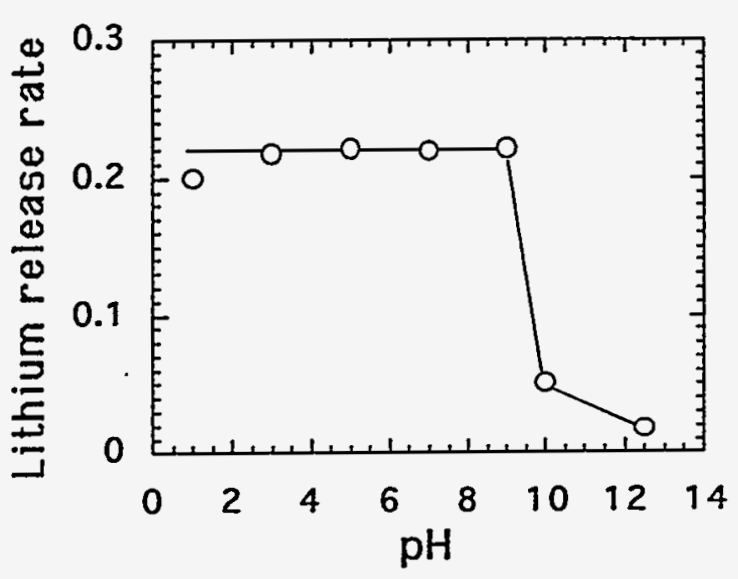

(c)

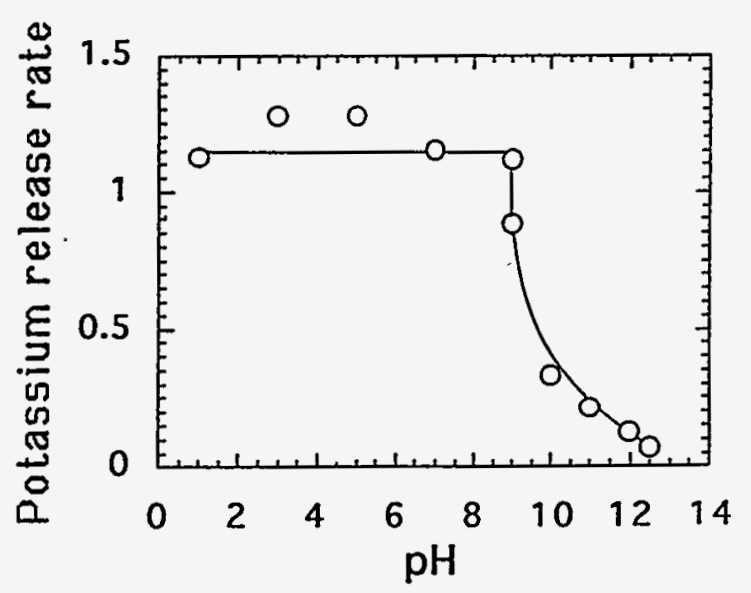

Fig. 22. Alkali Release Rates from Simple Glasses vs. pH: (a) Sodium Release Rate from (•) $15 \mathrm{Na}_{2} \mathrm{O} \cdot 85 \mathrm{SiO}_{2}$, (O) $25 \mathrm{Na}_{2} \mathrm{O} \cdot 5 \mathrm{CaO} \cdot 70 \mathrm{SiO}_{2}$, (a) $25 \mathrm{Na}_{2} \mathrm{O} \cdot 10 \mathrm{CaO} \cdot 65 \mathrm{SiO}_{2}$, (D) $22 \mathrm{Na}_{2} \mathrm{O} \cdot 6 \mathrm{CaO} \cdot 72 \mathrm{SiO}_{2}$; (b) Lithium Release Rate from $33 \mathrm{Li}_{2} \mathrm{O} \cdot 85 \mathrm{SiO}_{2}$; and (c) Potassium Release Rate from $15 \mathrm{~K}_{2} \mathrm{O} \cdot 85 \mathrm{SiO}_{2}$ (after [170]). 
Eq. 2 in Section 2.1). It is interesting to note that, although the critical pH values for alkali extraction are similar for lithium, sodium, and potassium, the exchange equilibrium constants measured by Dugger et al. [6] suggest that the critical $\mathrm{pH}$ should increase slightly as $\mathrm{Li}<\mathrm{Na}<\mathrm{K}$ (see also Fig. 1 from [7]).

The extraction of silicon from fused silica powder and from some simple glasses is shown in Figs. 23a and 23b as a function of the solution $\mathrm{pH}[170,171]$. Tests in Fig. 23a were conducted at $80^{\circ} \mathrm{C}$ and tests in Fig. $23 \mathrm{~b}$ were conducted at $35^{\circ} \mathrm{C}$ using $-30+52$ mesh glass. Tests were conducted for up to two hours. Over this time period, the release of alkali metal ions was found to increase linearly with time, which indicates that hydrolysis is the dominant reaction. In all cases, the amount extracted is insensitive to the $\mathrm{pH}$ for $\mathrm{pH}$ values below about 9 but increases as the $\mathrm{pH}$ is increased above about 9 . The increased extent of extraction is due to the dissociation of silicic acid. While the solubility of nondissociated silicic acid is itself insensitive to changes in the $\mathrm{pH}$, the extent of its dissociation to form $\mathrm{H}_{3} \mathrm{SiO}_{4}^{-}, \mathrm{H}_{2} \mathrm{SiO}_{4}{ }^{2-}, \mathrm{HSiO}_{4}{ }^{3-}$, and $\mathrm{SiO}_{4}{ }^{4}$ increases with the solution $\mathrm{pH}$.

Figure 24 shows the extraction of sodium, calcium, and silicon from a glass composed of $20 \mathrm{~mol} \%$ each of soda and lime and $60 \mathrm{~mol} \%$ silica at $70^{\circ} \mathrm{C}$ [172]. Both sodium and calcium release increases with decreasing $\mathrm{pH}$, while the silicon release is insensitive to the $\mathrm{pH}$ at values below about $\mathrm{pH} 9$.

The extraction of aluminum and sodium from two sodium glasses was measured at different solution $\mathrm{pH}$ in tests conducted at $70^{\circ} \mathrm{C}$ using $-25+36$ mesh glass [173]. Tests were conducted for 20 minutes. Glass I contained $12.9 \mathrm{~mol} \% \mathrm{Na}_{2} \mathrm{O}$ and $16.1 \mathrm{~mol} \% \mathrm{Al}_{2} \mathrm{O}_{3}$ and glass $I I$ contained about $13.0 \mathrm{~mol} \% \mathrm{Na}_{2} \mathrm{O}$ and $5.0 \mathrm{~mol} \% \mathrm{Al}_{2} \mathrm{O}_{3}$, with the balance being $\mathrm{SiO}_{2}$. Figure 25 shows the extent of extraction of aluminum and sodium from two sodium glasses at different solution $\mathrm{pH}$ values. The extraction of aluminum is insensitive to the leachant $\mathrm{pH}$ at $\mathrm{pH}$ values greater than about 6 but increases as the $\mathrm{pH}$ decreases below about 3 for both glasses. The sodium release, while insensitive to $\mathrm{pH}$ changes below about 9 in soda lime silicate glasses, shows increased extraction below about 3 in glass I and below about 8 in glass $I$. The difference in the sodium release behavior of the two glasses can be understood in terms of the relative amounts of sodium and aluminum in the two glasses. Glass I has a sodium-to-aluminum mole ratio less than one, so all sodium are fixed in $\left[\mathrm{NaAlO}_{4 / 2}\right]$ groups. Glass II has a sodium-toaluminum mole ratio much greater than one, so most sodium atoms are fixed in nonbridging $\equiv \mathrm{SiO}-\mathrm{Na}$ groups. Release of sodium from $=\mathrm{SiO}-\mathrm{Na}$ groups in glass $\Pi$ occurs preferentially to and at higher $\mathrm{pH}$ values than the release of sodium from $\left[\mathrm{NaAlO}_{4 / 2}\right]$ groups in glass $\mathrm{I}$.

A similar $\mathrm{pH}$-dependence has been observed for the dissolution rate of silicate minerals, for which the slope of the $\log$ rate versus $\mathrm{pH}$ plot is 0.3 at $70^{\circ} \mathrm{C}$ and about 0.5 at $90^{\circ} \mathrm{C}[174,175]$. The $\mathrm{pH}$-dependence was attributed to the formation of negatively charged silica groups at the surface, and the temperature effect was attributed to the temperature dependence of the dissociation constant of silanol.

The importance of the solution $\mathrm{pH}$ to both the rate and mechanism of the corrosion of simple glasses is clearly evidenced by a variety of experiments and has been well known for many years. The similar $\mathrm{pH}$ effects seen in tests with simple silicate glasses and with high-level waste glasses demonstrate that similar reactions control their corrosion behaviors, namely ion exchange to release alkali metal and alkaline earth ions and acid-catalyzed hydrolysis to release aluminum and base-catalyzed hydrolysis to release silicon. Based on knowledge gained from tests with simple glasses, the effects of $\mathrm{pH}$ on the corrosion of high-level waste glasses can be summarized as follows: Dealkalization occurs at a high rate that is independent of the $\mathrm{pH}$ in acidic and near neutral solutions, but is quenched at $\mathrm{pH}$ values above about $\mathrm{pH} 9$. Aluminum release increases as the $\mathrm{pH}$ decreases below about $\mathrm{pH} 4$, and silicon release is greatly enhanced 
(a)

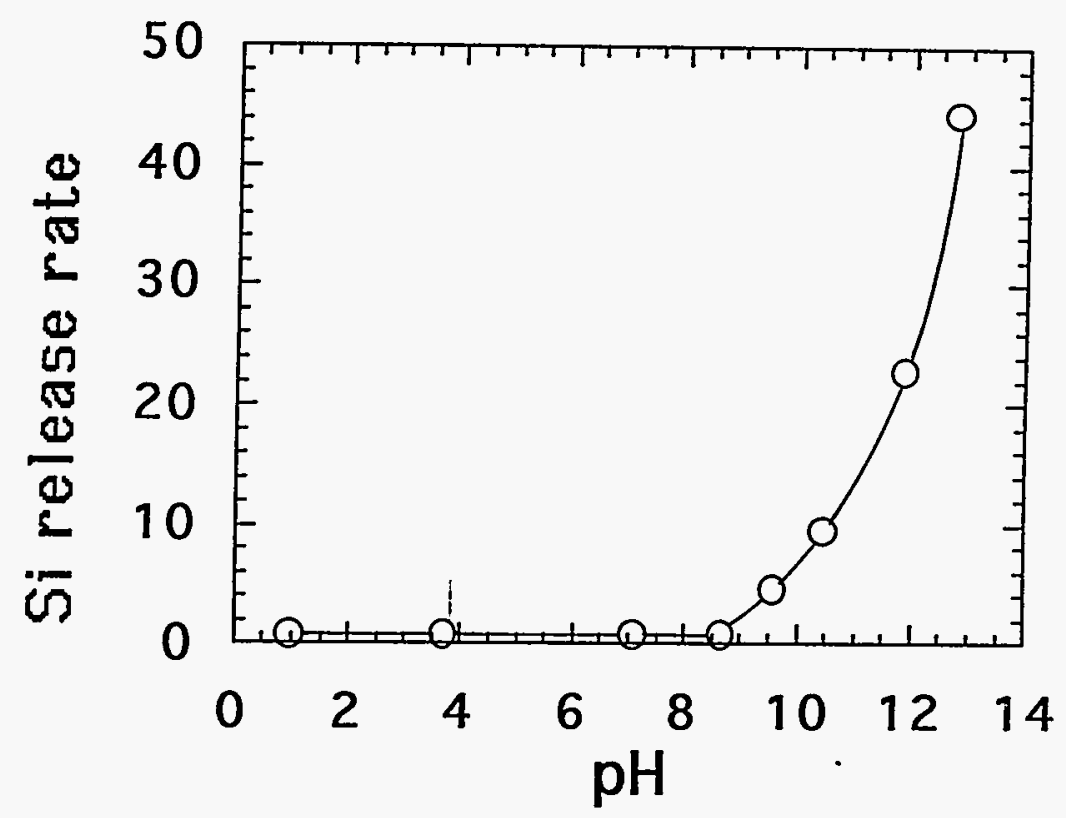

(b)

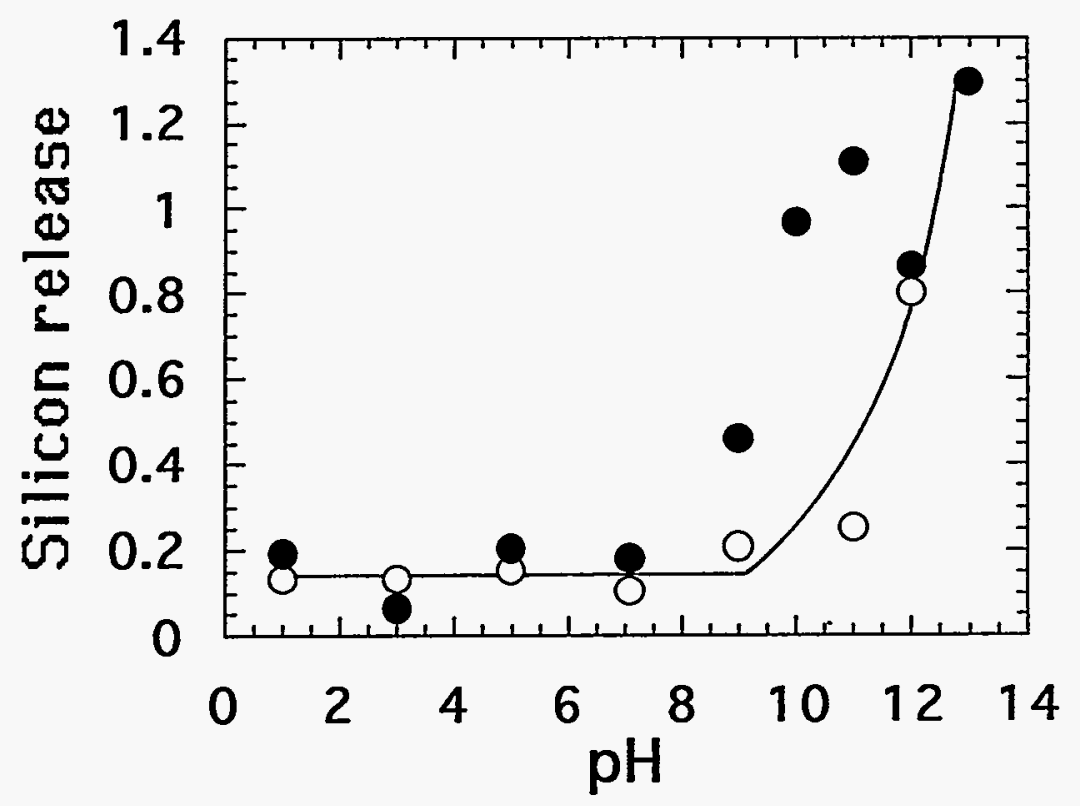

Fig. 23. (a) Silicon Release from Fused Silica vs. $\mathrm{pH}$ (after [172]) and (b) Silicon Release vs. $\mathrm{pH}$ from (O) $33 \mathrm{Li}_{2} \mathrm{O} \cdot 67 \mathrm{SiO}_{2}$ and $(\bullet) 15 \mathrm{~K}_{2} \mathrm{O} \cdot 85 \mathrm{SiO}_{2}$ Glasses (after $[170,171]$ ). 


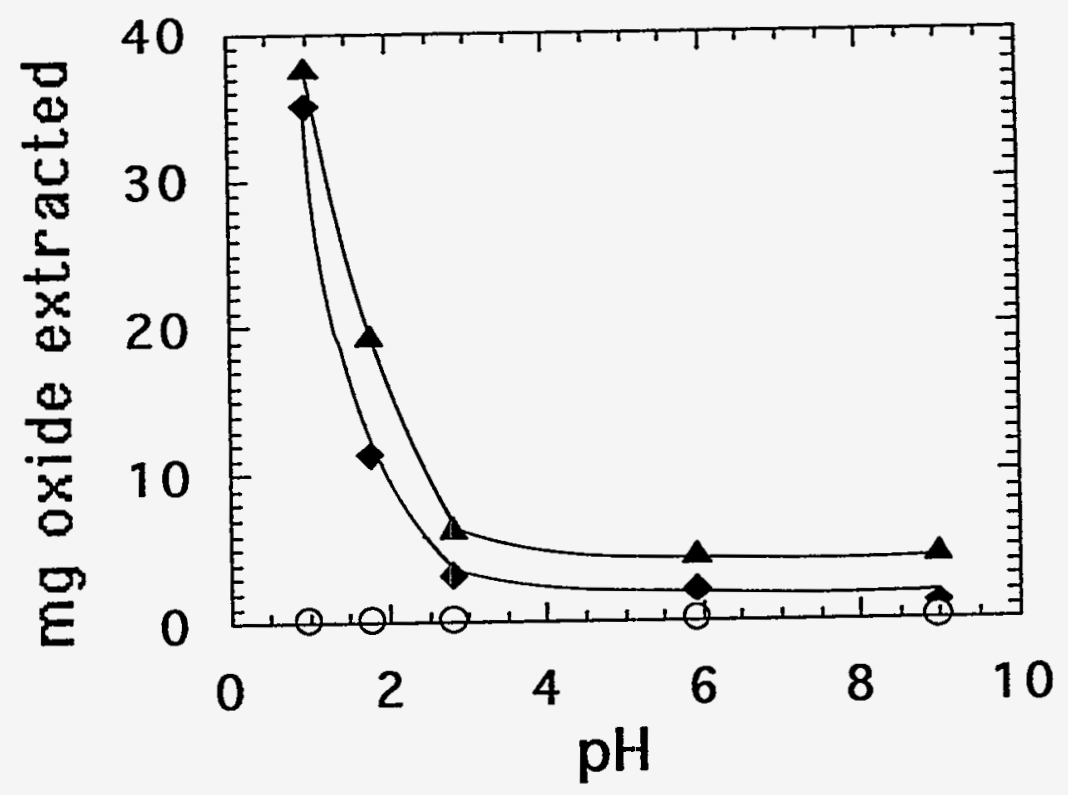

Fig. 24. Release of Sodium, Calcium, and $\mathrm{Si}$ from $20 \mathrm{Na}_{2} \mathrm{O} \cdot 20 \mathrm{CaO} \cdot 60 \mathrm{SiO}_{2}$ vs. pH: (ム) $\mathrm{Na}$, $(\bullet) \mathrm{Ca}$, and (O) Si (after [172]). 
(a)

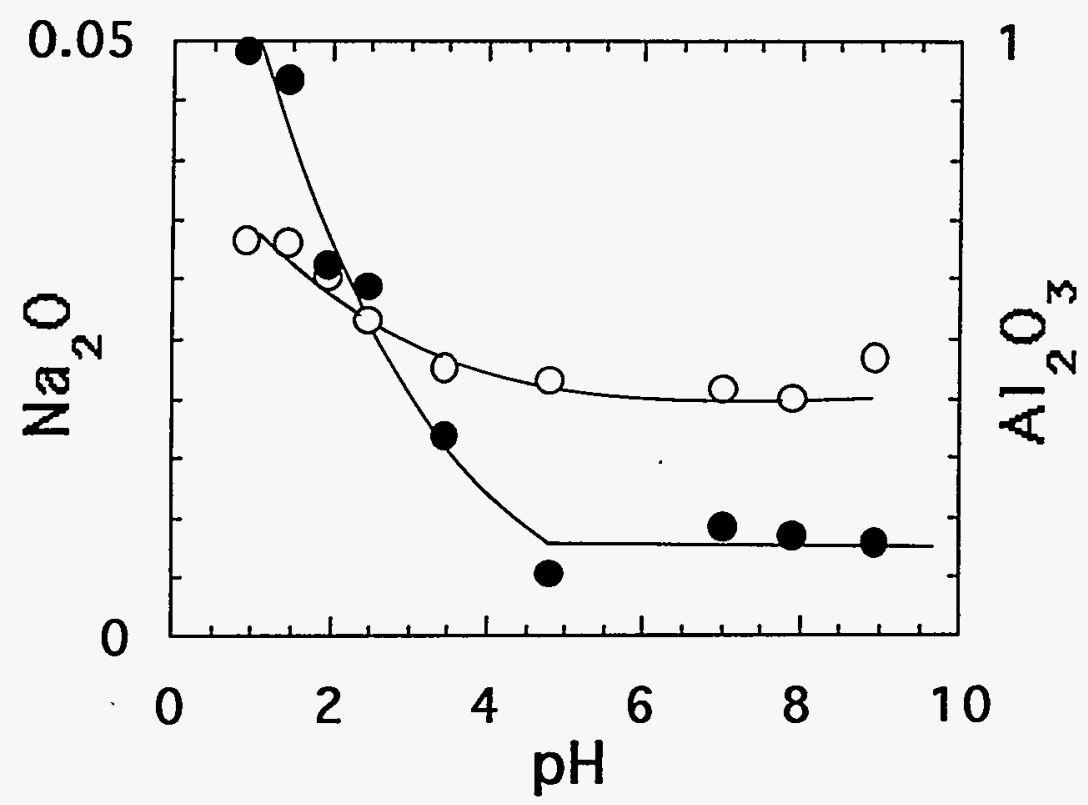

(b)

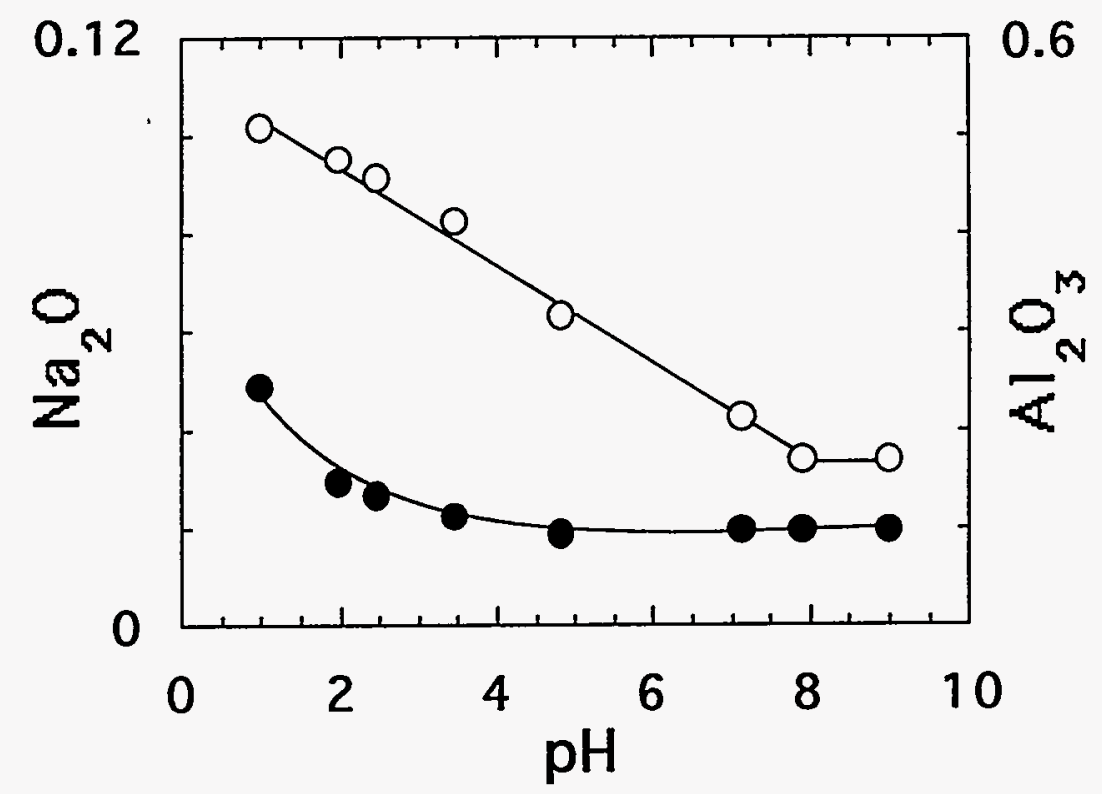

Fig. 25. Release of Aluminum and Sodium vs. $\mathrm{pH}$ (a) from Glass 1 and (b) from Glass 2: (O) $\mathrm{Na}$ and (O) Al (after [173]) 
above about $\mathrm{pH} 9$ (see also Section 3.3). Dealkalization of waste glasses generally results in basic conditions, so the waste glass corrosion is controlled by the release of silicon. (Radiolysis may result in acidic leachates, in which case waste glass corrosion will be controlled by the release of aluminum.) The similarity of values for the $\mathrm{pH}$-dependence of silica release that have been measured for simple silicate glasses and for high-level waste glasses support the model that detachment of silica groups controls the glass corrosion rate under alkaline $\mathrm{pH}$ values and that the effects of other constituents are negligible.

Models designed to simulate the long-term corrosion behavior of high-level waste glasses have only recently included terms to account for the effects of the solution $\mathrm{pH}$ on glass corrosion $[176,177]$. As will be shown in later sections, the $\mathrm{pH}$ effects are very important in understanding the effect of the SA/V ratio.

\subsection{Observed Effects of Other Species on Glass Corrosion}

Various test methods have been used to assess the effects of specific solution species on the glass corrosion rate. The effects observed in these different tests are related to the reaction that is highlighted in the test method. That is, the effect of a species in a test that generates a highly concentrated leachate solution may be very different than the effect measured in test that maintains a dilute leachate solution. That is, the measured effect may involve different rateaffecting reactions in the different tests, and the effect of a particular species may be very different when measured in the different tests. Thus, it is important to understand the corrosion conditions when discussing the effects of different species.

Flow-through tests with doped leachants have been used to assess the effects of various solution components on the corrosion rate of high-level waste glasses. These tests maintain highly dilute conditions and measure the effects on the forward reaction rate. The measured effects of silicon, aluminum, magnesium, and calcium on the forward reaction rate of CGS glass at different $\mathrm{pH}$ values are shown in Fig. 26 [71,112]. The dashed lines show the limiting slopes

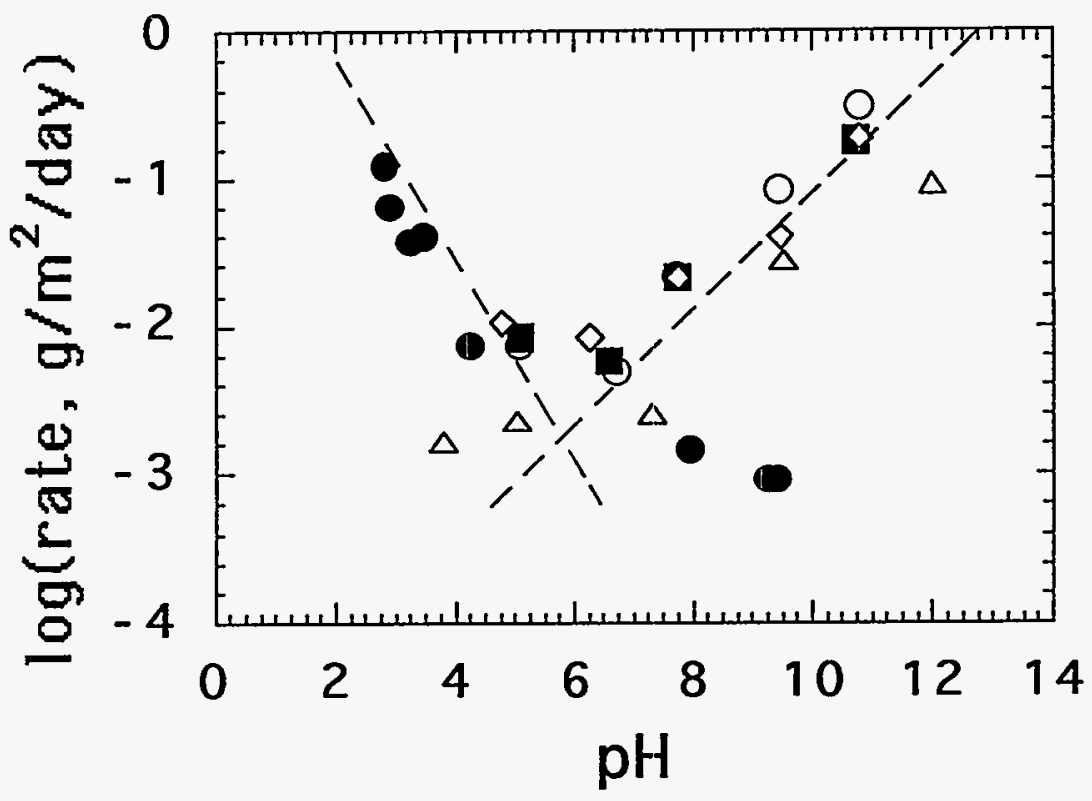

Fig. 26. Effects of $\mathrm{Si}, \mathrm{Al}, \mathrm{Mg}$, and $\mathrm{Ca}$ on CSG Dissolution: (O) Undoped Leachant, $(\bullet)$ Si-Doped Leachant, $(\Delta)$ Al-Doped Leachant, ( $\square$ ) Mg-Doped Leachant, and $(\diamond)$ Ca-Doped Leachant (after 71,112]) 
of the data of Knauss et al. in tests with the same glass [70]. Tests conducted by Bourcier et al. with undoped leachant show good agreement with the results of Knauss et al. The presence of calcium and magnesium in the leachant did not affect the corrosion rate of the glass. The presence of boron in the solution also did not have an effect. The presence of silicon in the leachant solution decreased the corrosion rate at solution $\mathrm{pH}$ values greater than about $\mathrm{pH} 6$, while the presence of aluminum decreased the corrosion rate at $\mathrm{pH}$ values less than about $\mathrm{pH} 6$. The data in Fig. 26 suggest that the $\mathrm{pH}$ dependence of glass corrosion in silicon- and aluminumcontaining solutions follows the limiting slopes found by Knauss et al. This suggests that glass corrosion in acidic solutions is dominated by reactions affected by the aluminum concentration and corrosion in alkaline solutions is dominated by reactions affected by the silicon concentration. As shown in Fig. 25, the $\mathrm{pH}$ at which the aluminum release increases depends on the glass composition. (This is probably why R7T7 glass did not show increased corrosion at low $\mathrm{pH}$ values [166].)

The lack of effect of magnesium determined in the flow-through tests by Bourcier et al. is contrary to the severe quenching effect of magnesium in brine solutions observed by Barkatt et al. in highly saturated environments [14,163,178]. Barkatt et al. attributed the observed effect of magnesium to its attachment to silicate sites and its tendency to reduce the solubility of silica. These effects are not observed in flow-through tests. Therefore, while a solution species may not directly affect the reaction leading to the release of glass components, it may indirectly affect the glass corrosion rate through its effect on solubilities or interactions with the glass surface. The test method used to assess the effects of species controls which effects may or may not be observed, so that several tests may be required to fully characterize the effects of a species over a range of conditions.

The above results indicate that in alkaline solutions, it is valid to model the forward corrosion rate to depend primarily on the $\mathrm{pH}$ and silicic acid concentrations. In acidic solutions, however, the effects of aluminum may also have to be taken into account. Acidic conditions may exist when large volumes of brines contact the glass [179] or when small volumes of water undergo radiolysis [114]. The validity of representing the corrosion of a multicomponent glass based only on the solution concentration of silicic acid rests primarily on the general success of the model when applied to waste glasses. (See $[92,94,154,164,165,180,181-184]$ for some applications.) It is likely that any error due to omission of the possible minor effects of other glass components on the corrosion rate in the analytical equation is corrected by the fact that experimental data are used to define various model parameters (see Section 6.1). That is, even though the effects of species other than silicic acid are not specifically accounted for in the model, their influence is included by the use of experimentally derived model parameters.

\subsection{Glass Corrosion under Silicon Saturated Conditions: The Residual Rate}

Many workers have emphasized the importance of knowing the long-term corrosion behavior of waste glasses in order to model waste glass performance in disposal sites $[185,186]$. McGrail et al. state that "it is impossible to accurately predict the solubility-limited, masstransfer rate without accurate knowledge of the kinetics of glass reaction" [185]. Their model calculations show that the transmissivity of radionuclides through a geologic site is proportional to the rate of glass corrosion.

In contrast to Eq. 13, in which the hydrolysis rate is predicted to become nil as silicic acid approaches its saturation concentration and the affinity approaches zero, experiments show the glass corrosion rate to approach a very low, yet non zero value after reaction times of about six months. It is important to note that the residual rate may not represent the long-term corrosion rate (see below). For example, Fig. 27 shows the release of boron and silicon from C-31-3EC 


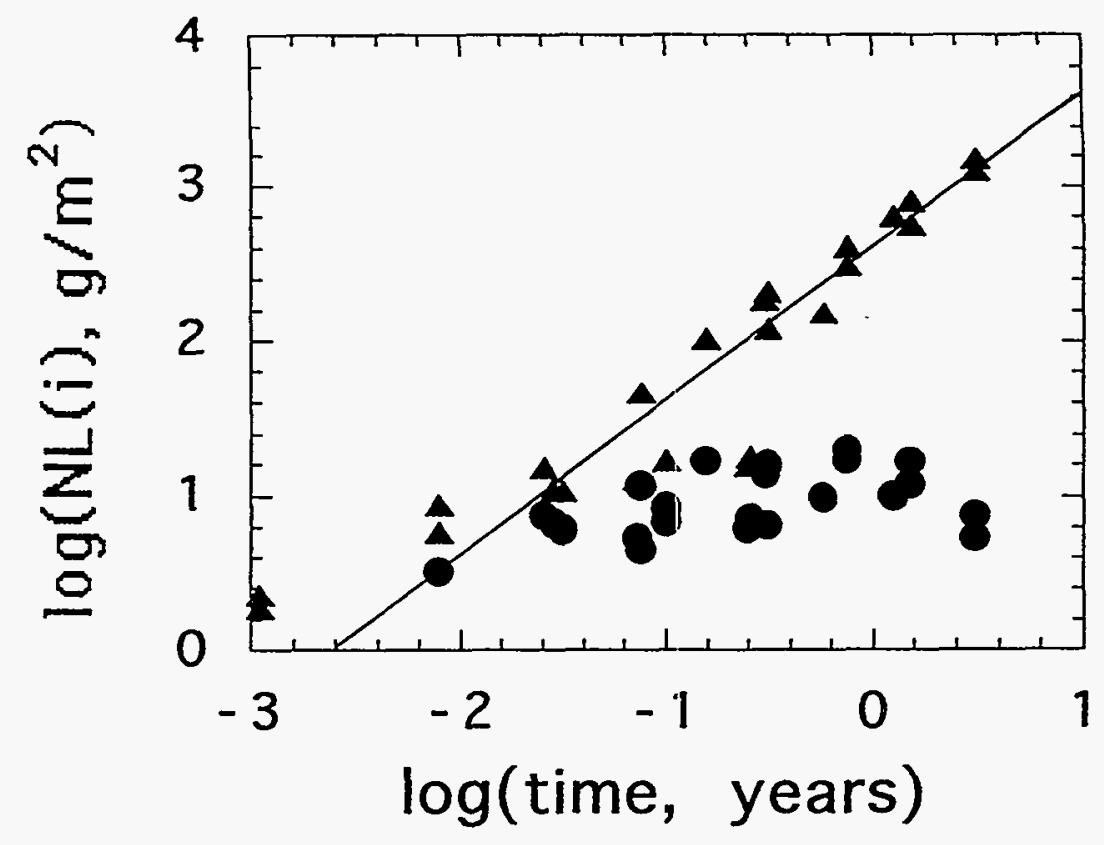

Fig. 27. Normalized Mass Loss of (A) Boron and (1) Silicon from C-31-3EC Glass in $\mathrm{NaCl}$ Solutions at $200^{\circ} \mathrm{C}$ (after [165])

glass in static leach tests ( $\mathrm{SA} / \mathrm{V}$ from 10 to $100 \mathrm{~m}^{-1}$ ) conducted in saturated $\mathrm{NaCl}$ solutions at $200^{\circ} \mathrm{C}$. The constant increase in the normalized boron mass loss at a slope of one on the log-log plot indicates that boron is being released to solution at a constant rate (see also Section 6). Fruede et al. report the boron release rate to be $1 \mathrm{~g} / \mathrm{m}^{2} / \mathrm{d}$ after several months [168]. The normalized boron mass loss data at short reaction times lie above the line, indicating a high corrosion rate occurs at short time periods. Fruede et al. report the forward rate to be $6 \mathrm{~g} / \mathrm{m}^{2} / \mathrm{d}$ [168]. In contrast, the normalized silicon mass loss remains essentially constant between about 5 and $16 \mathrm{~g} / \mathrm{m}^{2}$. This indicates that additional silicon is not being released to solution as the reaction progresses, and that the solution has become saturated with silica after short reaction times. An ad hoc, constant "residual rate" or "contextual affinity" term is sometimes added to the rate expression to account for the continued reaction under "saturated" conditions $[153,166,168,181]$. This is shown in Eq. 14, where the first term on the right hand side is from Eqn. 13 and $R_{\infty}$ is the residual rate.

$$
\frac{\frac{\mathrm{d}\left(\mathrm{n}\left(\mathrm{H}_{4} \mathrm{SiO}_{4}\right)\right)}{\mathrm{dt}}}{\mathrm{S}}=\mathrm{k}_{+}\left(1-\frac{\left[\mathrm{H}_{4} \mathrm{SiO}_{4}\right]}{\left[\mathrm{H}_{4} \mathrm{SiO}_{4}\right]_{\text {sat }}}\right)+\mathrm{R}_{\infty}
$$

Values of the $\mathrm{R}_{\infty}$ term are generally derived from experimental results, and often from tests conducted at high SA/V. High SA/V conditions are used to generate highly concentrated leachates such that the silicic acid concentration approaches the saturation limit and the affinity term approaches zero within short test periods. Under these conditions, the instantaneous rate measured after long reaction times corresponds to the residual rate. However, the corrosion rate measured and the value of $R_{\infty}$ have been found to depend on the SA/V of the test $[67,167]$, probably because of differences in the solution $\mathrm{pH}$ in tests at different SA/V. Vernaz and 
coworkers have suggested that the stability of the phase controlling the silicic acid concentration may vary with the SA/V, or other, more stable phases may form under different test conditions. The attainment of high solution $\mathrm{pH}$ values occurs through the same $\mathrm{SA} / \mathrm{V}$ effect of a low degree of dilution as the attainment of high silicic acid concentrations, and is a natural consequence of using $\mathrm{SA} / \mathrm{V}$ to attain concentrated solutions. The high solution $\mathrm{pH}$ values result in an increase in the forward rate coefficient, $\mathrm{k}_{+}$, and increased silicon concentrations due to dissociation of silicic acid. Both the increase in $\mathrm{k}+$ and the $\mathrm{pH}$-induced increase in the measured silicic acid concentration must be accounted for when computing the residual rate. The residual rate should be evaluated based on the results of tests conducted at several different SA/V to account for the effects of $\mathrm{pH}$ on corrosion and on the residual rate. Estimates of the residual rate made to date are based on corrosion under a single test condition [165,180] (see also Section 6). Modeling the long-term glass corrosion behavior requires an understanding of the effects of test conditions and solution chemistry on the corrosion behavior of glass in general, and on the residual rate, or contextual affinity, in particular.

The concept of a "residual reaction affinity" for glass corrosion based on silicon saturation has been questioned by some researchers who point out that other glass components besides silicon continue to be released from the glass at measurable rates even when the solution is "saturated" with silicon [24]. They suggest that the concept of residual affinity should not be limited to silicon, but extended to include effects of other glass components.

Although the process responsible for continued glass corrosion remains uncertain, continued corrosion in solutions that are saturated with silicic acid has recently been attributed to diffusion-limited ion exchange [187] or water diffusion into the glass [161]. While these reactions are known to occur during glass corrosion, they are generally assumed not to be involved in the rate controlling reaction. Although diffusion through surface layers is known not to significantly affect the corrosion rate of borosilicate waste glasses at early stages of corrosion (see Section 3.1), these tests do show a minor influence of the layer. The effects of the layer may become more significant as the solution effects become negligible. That is to say, the tests conducted to distinguish the effects of the solution and the layer were conducted under conditions relevant to the early stages of corrosion. Other tests relevant to the long-term stages of corrosion must be used to distinguish the relative importance of the alteration layers on the long-term corrosion rate.

The uncertainty surrounding the "residual rate" is due to the fact that the tests used to derive the expression for the corrosion rate focus on the approach of the solution chemistry to saturation, while the residual rate occurs under saturated conditions. Grambow et al. have speculated that residual rate is controlled by diffusion based on their observation that the corrosion rate appears to be proportional to the square root of the reaction time in tests conducted in brines at high SA/V [161]. They also show that the apparent diffusion coefficient for the residual rate is similar to those of several obsidians. Grambow suggested both affinity terms and diffusion terms be utilized in the corrosion rate expression, where one or the other term dominates the corrosion rate, depending on the reaction conditions.

The conclusion of Grambow et al. that the long-term corrosion rate is controlled by the diffusion of water through an alteration layer is contrary to several experimental findings regarding the effects of alteration layers on the glass corrosion rate. First, thicker layers are known to form in tests at lower SA/V than in tests at high SA/V, so that the long-term rate would be expected to be lower in tests at lower SA/V than in tests at higher SA/V. The opposite is observed [167]. Characterization of the glass/layer interfaces developed on some high-level waste glasses shows the layer to crystallize and separate from the glass, such that the corroding solution directly contacts the glass surface [87]. No diffusion layers exist on these glasses under saturated conditions. (Other glasses may behave differently.) Inspection of the data of 
Grambow et al. does not show an obvious root time dependence. Their interpretation would be considerably strengthened by tests at common $(\mathrm{SA} / \mathrm{V}) \mathrm{t}^{1 / 2}$. Finally, the same corrosion behavior is not observed by Grambow et al. in different brine solutions. The behavior is seen to be affected by the solution $\mathrm{pH}$.

Inagaki et al. have included such a term in their reaction path calculations to describe test results with a borosilicate glass identified as P0798 [188]. Tests were conducted with crushed glass $(-100+200 \mathrm{mesh})$ in deionized water at a SA/V of about $1200 \mathrm{~m}^{-1}$ at $90^{\circ} \mathrm{C}$ for up to 130 days. Modeling the test results with the reaction path model and without the diffusion term resulted in an underestimation of the release rates by a factor of two. Apparently, Inagaki et al. used the forward rate, saturation concentration and residual affinity terms determined in tests with other glasses. Certainly, determination of values for these constants using test data for P0798 would improve the fit. Instead, the authors fit the data using a diffusion term and observed a much improved fit to the data. (The contribution of the reaction path term was significantly reduced by halving the forward rate coefficient.) This is due to the fact that the data being fitted were used to determine the fitting coefficients. We note that the test data are constrained to the initial stages of corrosion which can be fit equally well by a diffusion equation and by an affinity-based equation, if appropriate model parameters are used [143].

The the need to understand the long-term reaction mechanism and rate-determining reaction is underscored by the importance of the long-term rate to projecting glass corrosion behavior. The time dependence of corrosion under saturated conditions, whether it is linear or root time, will have a significant impact on projections of glass corrosion behavior to long time periods.

Experimental evidence suggests that most high-level waste glasses do not continue to corrode at the "residual rate." Rather, the corrosion accelerates because of the effects other phases that form during glass corrosion have on the silicic acid activity. Attainment of the "residual rate" is known to be prevented in the presence of silicon-consuming phases that may be present as part of the test system, such as clay [189-192] and iron [193,194], and upon the formation of secondary phases form the leachate solution as the glass corrodes $[65,81,116]$. The effect of secondary phase formation were discussed by Grambow and Strachan when accounting for corrosion in Mg-brines in MCC-1 type tests [152] (see Sec. 3.1). Increased corrosion rates have been observed for several glasses upon formation of secondary phases in tests conducted at high SA/V ratios under PCT conditions [65] and under vapor conditions [81,105,111].

The formation of secondary phases is an unavoidable result of glass corrosion. They form as the glass converts to a more stable assemblage of phases to reduce the free energy of the system. However, because the material for these phases originates in the glass, the opportunity exists to formulate waste forms to reduce the impact of secondary phase formation on glass corrosion by controlling which phases form. For example, analcime $\left(\mathrm{NaAlSiO}_{4}\right)$ is a siliconconsuming phase that is typically formed early in the corrosion process. However, its formation has less effect on the corrosion rate in tests with glasses having low aluminum contents, such as PNL 76-68, than in tests with glasses having high aluminum contents, such as SRL 131, because much higher silicic acid concentrations can be maintained in the presence of analcime if the aluminum concentration is low [104]. In other words, the solution concentration of aluminum limits the formation of analcime. The response of the glass corrosion process to the formation of secondary phases such as analcime also depends on the solution chemistry: under the alkaline conditions common during waste glass corrosion, the glass corrosion rate can accelerate to supply silicon. On the other hand, the glass corrosion rate does accelerate under alkaline conditions to supply aluminum for secondary phase formation. Therefore, the effects of 
analcime formation on the glass corrosion rate are less in glasses with low aluminum concentrations, and waste forms could be formulated to reduce the aluminum concentration to improve the long-term durability.

However, the aluminum content is not the only factor affecting the durability of waste glasses. The formation of secondary phases in vapor hydration tests conducted at ANL using the R7T7 glass, which contains about 5 wt $\% \mathrm{Al}_{2} \mathrm{O}_{3}$, has been found not to greatly increase the corrosion rate. The durability demonstrated in vapor hydration tests and the durability under acidic conditions observed by Advocat et al. [92] (see Fig. 21) suggests that aluminum is quite stable in the R7T7 glass structure. Of course, the formation of kinetically favored phases, such as clays, will also affect the evolution of the solution chemistry and the formation of precipitated phases.

\subsection{Summary}

Laboratory tests with high-level waste glasses have shown the solution chemistry to strongly affect the corrosion rate. Mass transport of water species and corrosion products through alteration layers has only a minor affect on the corrosion rate during the early stages of glass corrosion, although mass transport may affect the corrosion rates of some glasses under saturated solution conditions. The aspects of the solution chemistry that have the most effect on the corrosion rate are the silicic acid concentration and the $\mathrm{pH}$. The $\mathrm{pH}$ affects the reactions responsible for the release of glass components: the release of alkali metals and alkaline earths are promoted at low $\mathrm{pH}$ values (below about $\mathrm{pH} 9$ ), while the release of silicon is promoted at high pH (above about pH 9). The release of silicon is slowed by the presence of silicic acid in the solution. The glass corrosion rate is well-represented by an equation describing the silicic acid release rate that accounts for both the $\mathrm{pH}$ and silicic acid solution effects. This equation also accounts for the effects of other phases on the silicic acid concentration and $\mathrm{pH}$. These phases may be present in the system initially or may be formed as secondary phases as the glass corrodes. An additional term may be required to describe the nonzero corrosion rate observed under saturated conditions prior to secondary phases formation. Such a term can presently only be determined experimentally, and the effects of test conditions on the value of the term are not clear. The physical meaning of the term is likewise unclear, and remains a shortcoming of our ability to model and predict long-term glass behavior. 


\subsection{OBSERVED EFFECTS OF THE GLASS SURFACE AREA- TO-SOLUTION VOLUME RATIO IN STATIC TESTS}

Most studies of the effects of the SA/V used in a test on the corrosion rate of high-level waste glasses have focused on changes in the solution concentration. While the release of glass components into solution is of prime interest, since transport of waste components requires their initial release into the leachant, the effects of the SA/V on the alteration of the glass surface are important to understanding the effects on the corrosion mechanism and the long term corrosion behavior. Knowledge regarding the effects of SA/V on the alteration of the glass surface is needed to determine if tests conducted at high SA/V can be used to characterize advanced stages of corrosion. Thus, both the effects of SA/V on the leachate chemistry and the effects of SA/V on the alteration of the glass surface must be understood to support modeling of the long-term corrosion behavior. Laboratory tests have shown the chemical and physical alteration of the glass surface to have only a minor effect on the corrosion rate (see Section 3.1). However, the alteration phases that accumulate at the glass surface do interact with the leachate solution and affect the corrosion behavior at advanced stages of corrosion (Stage III in Fig. 6). The model used by Bourcier utilizes the composition of the layer to determine the saturation concentration in the affinity term [143].

\subsection{Effects of SA/V on the Solution Chemistry}

The current model of glass corrosion considers the glass surface to be a reservoir for reactants, and the glass surface area can be assumed to be proportional to the amount of each glass constituent (including alkali metals, silicon, and radioelements) that is available for reaction. The solution volume acts both as a reservoir for dissolved glass components and as a source of reactants, including molecular water and hydroxide involved in hydrolysis reactions, alkali metal ions involved in ion exchange, and silicic acid. The glass surface area and the solution volume are not phenomenologically related. Rather, their coupling into a single test parameter is derived from the rate expression describing the hydrolysis of silicon-oxygen bonds at the glass surface. If all silicic acid released from the glass remains in solution, then the number of moles of silicic acid released from the glass, $\mathrm{n}\left(\mathrm{H}_{4} \mathrm{SiO}_{4}\right)$ in Eq. 13, can be replaced with the molar concentration of silicic acid as $\mathrm{n}\left(\mathrm{H}_{4} \mathrm{SiO}_{4}\right)=\left[\mathrm{H}_{4} \mathrm{SiO}_{4}\right] \cdot \mathrm{V}$, where $\mathrm{V}$ is the solution volume, and Eq. 13 can be written as

$$
\frac{\mathrm{d}\left[\mathrm{H}_{4} \mathrm{SiO}_{4}\right]}{\mathrm{dt} \cdot}=\left(\frac{\mathrm{SA}}{\mathrm{V}}\right) \mathrm{k}_{+}\left(1-\frac{\left[\mathrm{H}_{4} \mathrm{SiO}_{4}\right]}{\left[\mathrm{H}_{4} \mathrm{SiO}_{4}\right]_{\text {sat }}}\right)
$$

The observed "effect" of the glass surface area/solution volume ratio (SA/V) used in a static test on the glass corrosion rate is the net effect of the glass surface area, which establishes the amount of glass-phase reactant initially available, and of the solution volume, which establishes the extent to which reaction products are diluted. The solution concentrations of key glass components then affect the rates of reactions critical to glass dissolution, including ion exchange and hydrolysis reactions. As discussed earlier, the left hand side of Eq. 15 no longer represents the rate at which silicic acid is released from the glass (i.e., the corrosion rate); rather, it represents the rate at which the silicic acid concentration in the leachate changes. The rate of change is proportional to the SA/V of the test.

Equation 15 predicts that the SA/V used in a corrosion test can be increased to accelerate the saturation of the solution, that is, to drive $\left[\mathrm{H}_{4} \mathrm{SiO}_{4}\right]$ to $\left[\mathrm{H}_{4} \mathrm{SiO}_{4}\right]_{\text {sat }}$. Several series of tests have been conducted to verify the predicted effect of SA/V for various glass composition. The results of a few representative tests are discussed below to assess the utility of Eq. 15. In the 
presented results, the solution concentrations of glass components other than silicon are sometimes used to quantify the extent of corrosion. The qualitative effect of the SA/V is the same, regardless of which glass component is used, and, as discussed in Section 2.2, highly soluble components, such as boron and alkali metals, provide the best measure of corrosion as the solution becomes saturated.

Clark et al. performed tests at SA/V ratios of about 7.7, 77, and $770 \mathrm{~m}^{-1}$ for up to ten days at $100^{\circ} \mathrm{C}[135]$. They used deionized water and various masses of the $-45+60$ mesh fraction of crushed glass and deionized water to attain the different SA/V ratios. The glass used contained $14 \mathrm{~mol} \% \mathrm{Na}_{2} \mathrm{O}, 10 \mathrm{~mol} \% \mathrm{CaO}, 72 \mathrm{~mol} \% \mathrm{SiO}_{2}, 1.8 \mathrm{~mol} \% \mathrm{Al}_{2} \mathrm{O}_{3}, 0.7 \mathrm{~mol} \% \mathrm{MgO}$, $0.5 \mathrm{~mol} \% \mathrm{~K}_{2} \mathrm{O}$, and about $1 \mathrm{~mol} \%$ "other" oxides. The normalized sodium mass losses and the measured $\mathrm{pH}$ values for tests at different $\mathrm{SA} / \mathrm{V}$ are plotted in Figs. 28a and 28b, which show that tests at lower $\mathrm{SA} / \mathrm{V}$ attain higher sodium releases and higher corrosion rates, based on sodium release. Tests at higher SA/V generate solutions with higher $\mathrm{pH}$ values and higher sodium concentrations, but normalization to the $\mathrm{SA} / \mathrm{V}$ shows tests at higher $\mathrm{SA} / \mathrm{V}$ to have lower glass corrosion rates when expressed as mass glass dissolved per unit area.

Shade and Strachan studied the corrosion of PNL 76-68 glass in deionized water and in a silicate/bicarbonate solution at $90^{\circ} \mathrm{C}$. They used different size fractions of crushed glass to attain $\mathrm{SA} / \mathrm{V}$ of about 670,1780 , and $>1780 \mathrm{~m}^{-1}$ (calculated on the assumption that the grains were cubes with an edge length equal to the average mesh opening) [132]. Figure 29a shows the $\mathrm{pH}$ values to be slightly higher for the tests conducted at higher SA/V. (Similar $\mathrm{pH}$ values were measured in tests conducted in the silicate/bicarbonate solution under the same test conditions.) This indicates that the glass corrosion products overwhelm the initial leachant chemistry in these tests, and that the initial leachant does not significantly affect the corrosion rates of these tests. The $\mathrm{pH}$ increase occurs over the initial few days and reaches a nearly constant value at longer times. The leveling off of the $\mathrm{pH}$ occurs sooner for tests at higher $\mathrm{SA} / \mathrm{V}$ ratios.

Figure $29 \mathrm{~b}$ shows the leachate concentrations of silicon in the same tests in deionized water. The silicon concentrations increases initially, more so for tests at higher SA $/ \mathrm{V}$, and then increases at a constant rate for longer reaction times. Notice that the results of tests conducted for 1 year are above the trend shown by the shorter term tests. Similar behavior has been seen in tests with other glasses, which has been attributed to the effects of secondary phase formation (see below). Filtration of the leachate revealed that the amount of dissolved silicon (that passing through a 1.8-nm filter) was similar at all SA/V and that colloidal silicon accounted for the differences in the leachate concentrations at different $S A / V$ beyond about 90 days. (This is surprising, in light of the different $\mathrm{pH}$ values in tests at different SA/V.) The silicon concentrations measured in tests in the silicate/bicarbonate solution were similar to those measured in corresponding tests in deionized water. This behavior demonstrates that the glass corrosion products (rather than the initial leachant chemistry) dominate the solution chemistry in these tests.

Tests with the same glass were conducted at $150^{\circ} \mathrm{C}$ to accelerate the glass corrosion [132]. Similar solution $\mathrm{pH}$ values (between about 9.9 and 10.1) and similar silicon concentrations (about $500 \mathrm{ppm}$ ) were measured in the leachates of tests conducted at all SA/V. The $\mathrm{pH}$ increased slightly with time, while the silicon concentration did not. The measured boron concentrations increased slightly with time, and the concentrations were consistently higher in tests at higher SA/V for all times tested. The continued boron release indicates that the glass continues to corrode even after silicon becomes saturated. The silicon concentration was likely set by the solution $\mathrm{pH}$, which was similar at all SA/V. The glass components that have saturated the solution become incorporated in secondary phases, while those that have not saturated the solution continue to dissolve (see Section 2.2). 
(a)

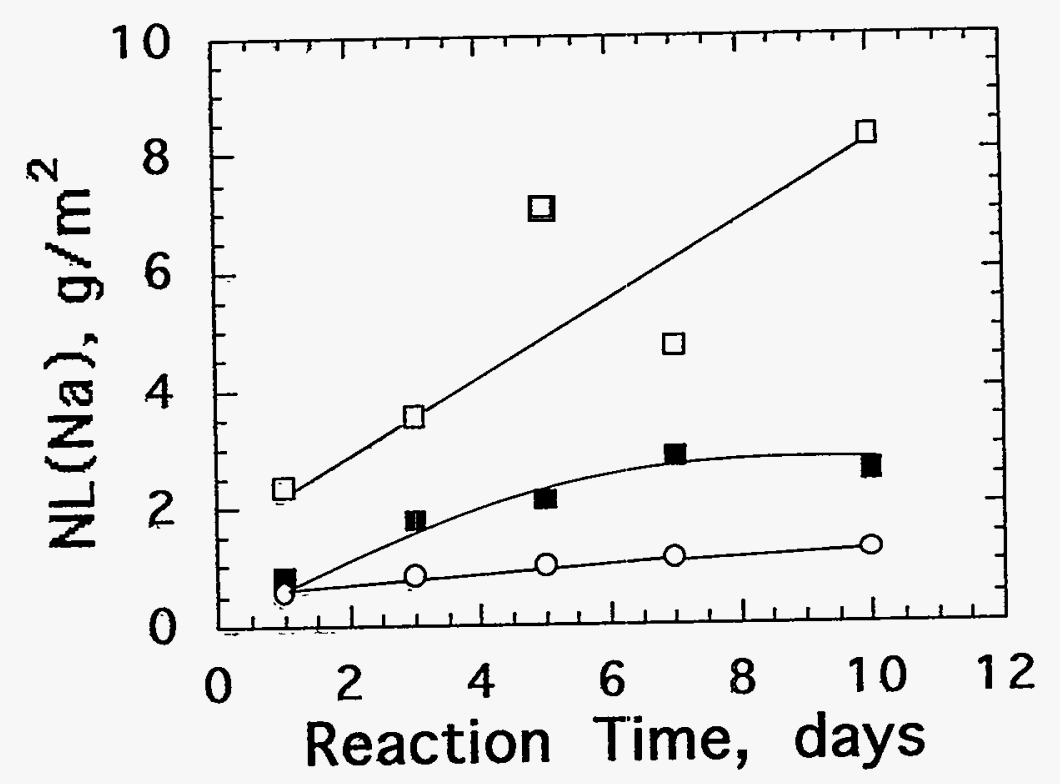

(b)

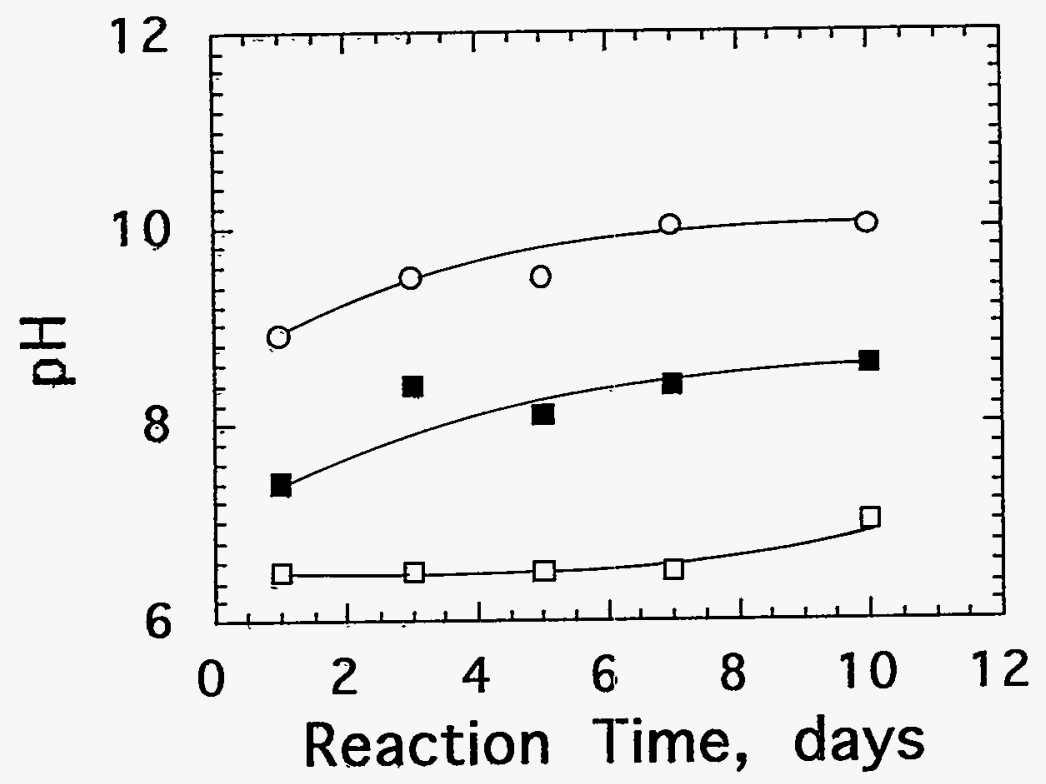

Fig. 28. (a) Normalized Sodium Mass Loss and (b) $\mathrm{pH}$ vs. Time at Different SA/V Ratios: (ㅁ) $7.7 \mathrm{~m}^{-1}$, (匹) $77 \mathrm{~m}^{-1}$, and (O) $770 \mathrm{~m}^{-1}$ (after [135]) 
(a)

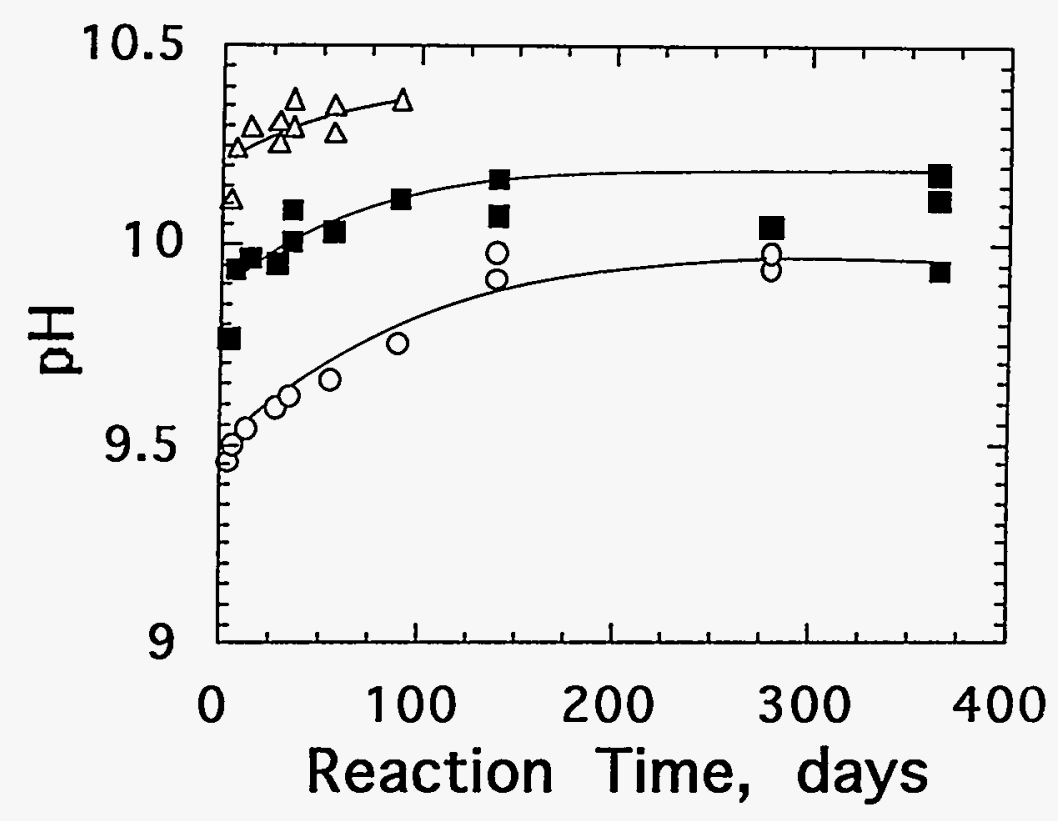

(b)

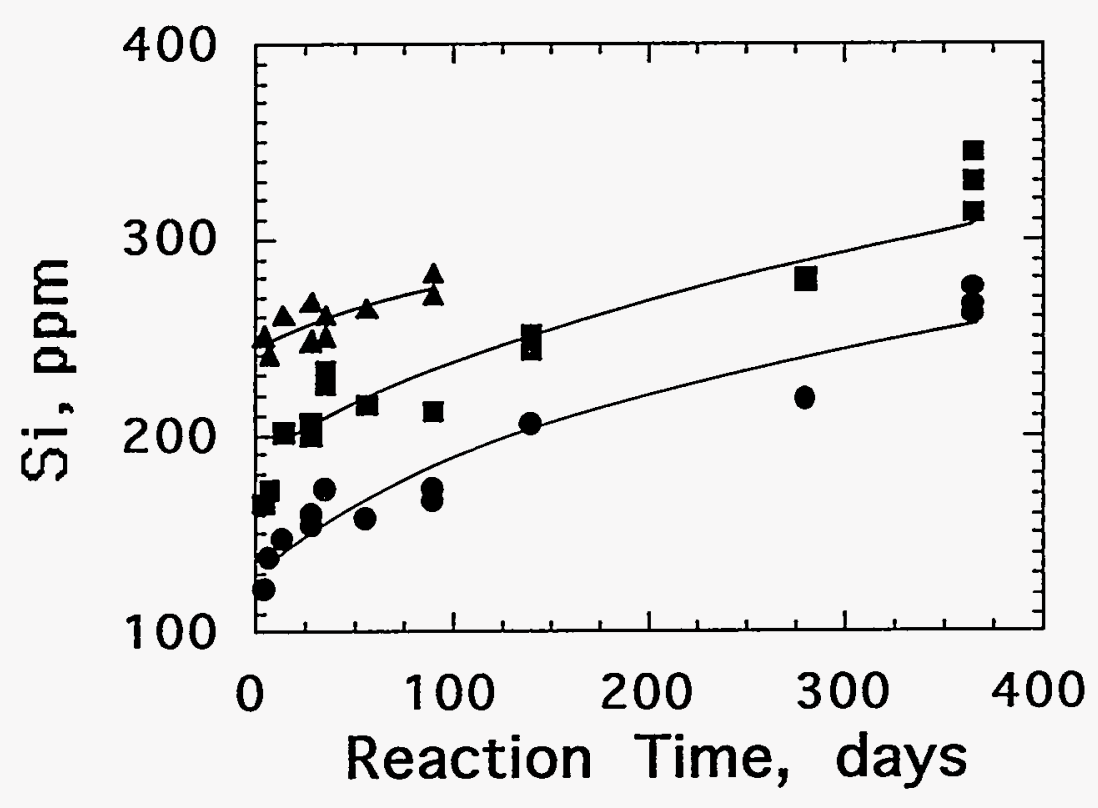

Fig. 29. Measured (a) $\mathrm{pH}$ and (b) Si in Tests with PNL 76-68 Glass Conducted in Deionized

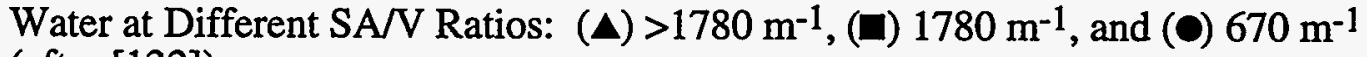
(after [132]) 
The tests conducted by Clark et al. and by Shade and Strachan show that solution $\mathrm{pH}$ and the solution concentrations of glass components increase with the SA/V used in a static leach test, and that high SA/V tests may result in the saturation of silicic acid. The latter observation suggests that results from high $\mathrm{SA} / \mathrm{V}$ tests will provide insight into the advanced stages of glass corrosion.

\subsection{Applicability of (SA/V)t as a Scaling Factor}

Before test at high $\mathrm{SA} / \mathrm{V}$ can be used to characterize advanced stages of glass corrosion, the relationship between tests at different SA/V and between tests using monolithic and crushed glass samples must be established. The relationship between tests at different $S A / V$ can be determined by integration of Eq. 15, which leads to the form [153]

$$
\left[\mathrm{H}_{4} \mathrm{SiO}_{4}\right]=\left[\mathrm{H}_{4} \mathrm{SiO}_{4}\right]_{\text {sat }}\left(1-\exp \left\{\frac{-(\mathrm{SA} / \mathrm{V}) \mathrm{t} \mathrm{k}_{+}}{\left[\mathrm{H}_{4} \mathrm{SiO}_{4}\right]_{\text {sat }}}\right\}\right)
$$

Eq. 16 predicts that the silicic acid concentration will approach the limiting value $\left[\mathrm{H}_{4} \mathrm{SiO}_{4}\right]_{\text {sat }}$ at a rate that is a function of the product $(\mathrm{SA} / \mathrm{V}) \mathrm{tk} \mathrm{k}_{+}$. If $\mathrm{k}_{+}$remains constant for tests at all $\mathrm{SA} / \mathrm{V}$, then tests performed for the same (SA/V)t are expected to attain the same silicic acid concentrations in the solution. Therefore, the silicic acid concentration will approach the limiting value $\left[\mathrm{H}_{4} \mathrm{SiO}_{4}\right]_{\text {sat }}$ as the product $(\mathrm{SA} / \mathrm{V}) \mathrm{t}$ increases. Tests conducted for larger products of $(\mathrm{SA} / \mathrm{V}) \mathrm{t}$ will attain a more advanced stage of corrosion.

The effects of SA/V on the dissolution of PNL, 76-68 glass have been studied in tests conducted at $90^{\circ} \mathrm{C}$ with monolithic samples run at SA/V of 1,10 , and $100 \mathrm{~m}^{-1}[72,73]$. In Fig. 30, the boron and silicon concentrations are shown plotted against the product of the SA/V and the reaction time. Both the boron and silicon concentrations increase linearly with the product $(\mathrm{SA} / \mathrm{V}) \mathrm{t}$, and data from tests run at different $\mathrm{SA} / \mathrm{V}$ can be represented by a single line. As evidenced by the linearity of data at each SA/V over the times tested, these test conditions maintain rather dilute solutions and corrosion is dominated by ion exchange. These tests were conducted for a maximum of $(\mathrm{SA} / \mathrm{V}) \mathrm{t}=2800 \mathrm{~d} / \mathrm{m}$, and are not relevant to the use of the $\mathrm{SA} / \mathrm{V}$ to attain saturated solution conditions.

Static leach tests were conducted using monolithic and crushed SRL 165 glass at $90^{\circ} \mathrm{C}$ [195]. Tests with monolithic samples were conducted at SA/V of 10,30 , and $50 \mathrm{~m}^{-1}$, and tests with crushed glass $\left(-40+80\right.$ mesh) were conducted at $S A / V$ of 30,50 , and $100 \mathrm{~m}^{-1}$. The surface area of the crushed glass was calculated assuming the grains were spheres with a diameter of $298.5 \mu \mathrm{m}$ and the surface area of the monoliths were measured geometrically. The measured boron concentrations are plotted against the product (SA/V)t in Fig. 31. The open symbols represent tests with crushed glass samples and the filled symbols represent tests with monolithic samples. Notice that the tests with monolithic samples have lower measured boron concentrations than tests with crushed samples at common (SA/V)t. This may be due to the higher reactivity of the crushed glass or to the actual surface area of the crushed glass being greater than estimated. Instead of the measured solution concentrations, the authors plotted the normalized leach rates against the product (SA/V)t. This has the effect of canceling the estimated surface area of the samples from the plot.

Chandler et al. conducted tests to assess the applicability of (SA/V)t as a scaling factor to relate tests with monolithic samples and tests with crushed glass [196]. Tests were conducted in deionized water at $90^{\circ} \mathrm{C}$ for times up to 28 days using SRP black frit waste glass. Monolithic samples were tested at $\mathrm{SA} / \mathrm{V}$ ratios of 10 and $100 \mathrm{~m}^{-1}$, and crushed samples of the $-40+60$ mesh 


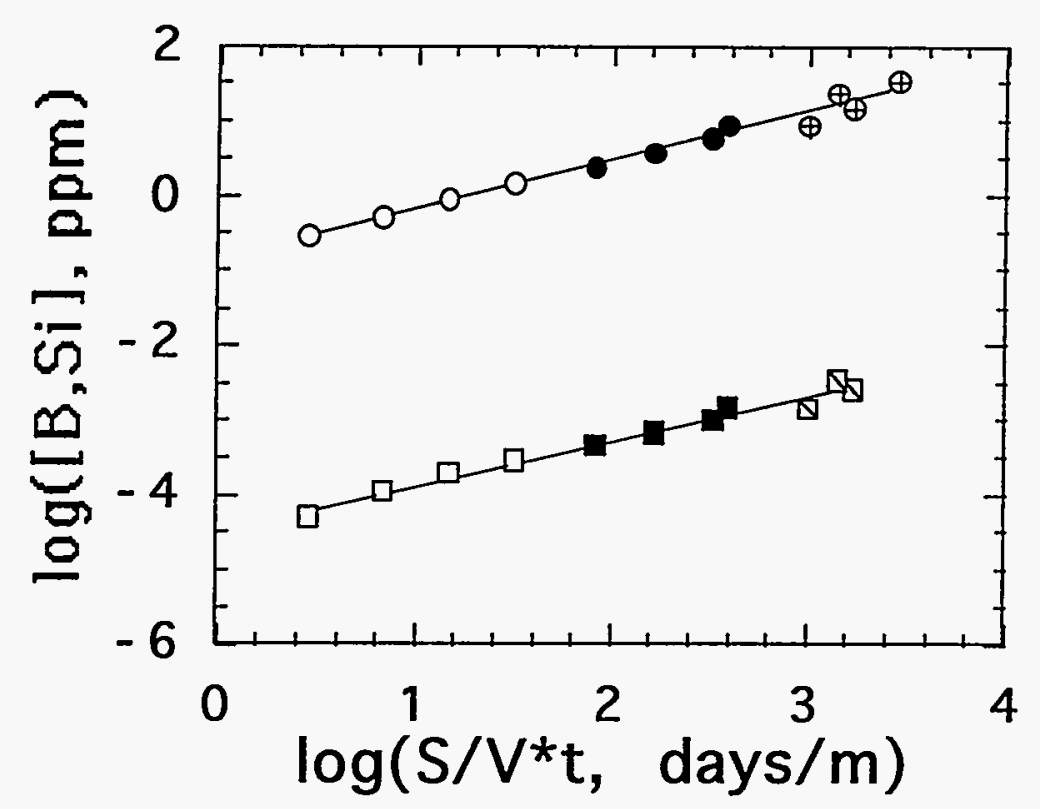

Fig. 30. Concentration vs. (SA/V)t for Release of Boron and Silicon from PNL 76-68 Glass at

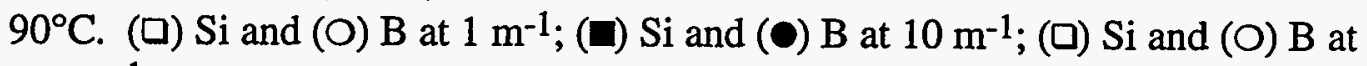
$100 \mathrm{~m}^{-1}$ (after [72,73]).

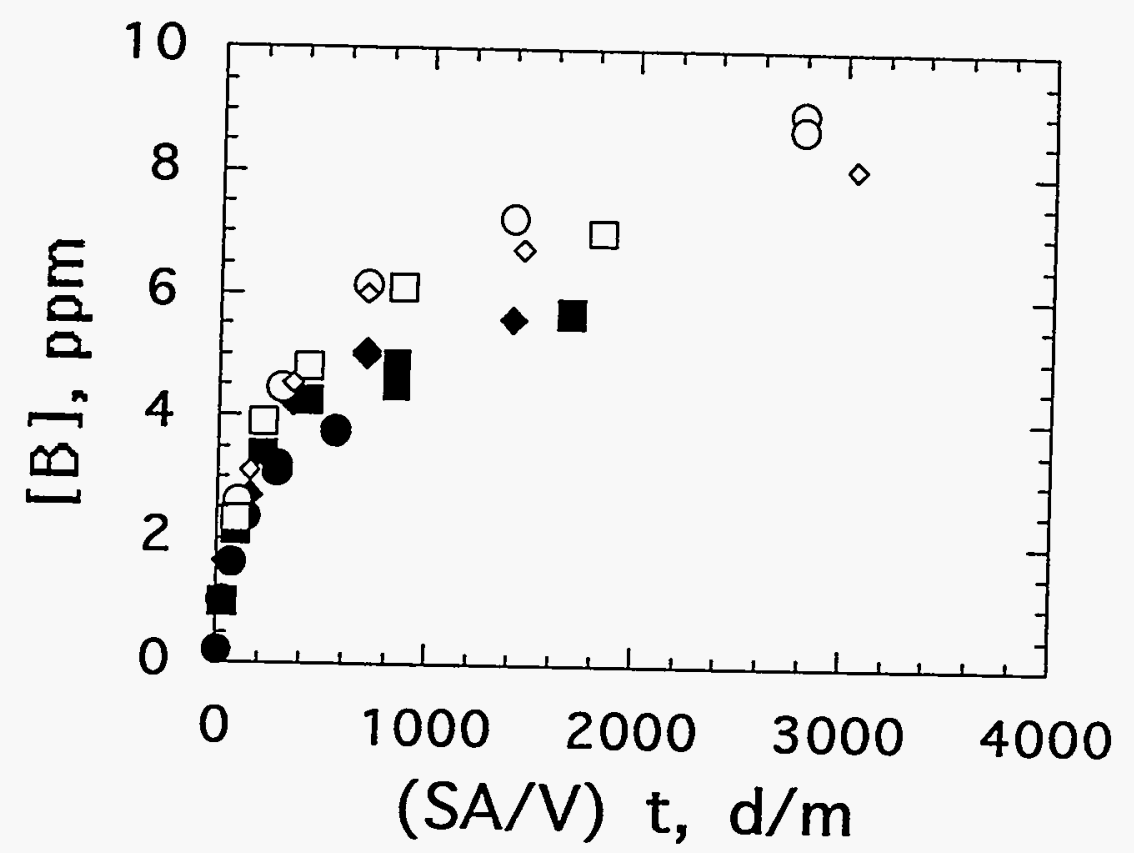

Fig. 31. Measured Boron Concentrations in Tests with SRL 165 Glass vs. (SA/V)t: Tests with Monolithic Samples at $(\bullet)$ 10, (৫) 30, and $(\bullet) 50 \mathrm{~m}^{-1}$ and Tests with Crushed Samples at (口) $30,(\diamond) 50$, and (O) $100 \mathrm{~m}^{-1}$ (after [195]) 
size fraction were tested at $\mathrm{SA} / \mathrm{V}$ ratios of 100 and $1000 \mathrm{~m}^{-1}$. Figure 32 shows the boron concentration plotted against the product (SA/V)t for these test data. Data from both tests with monolithic samples and tests with crushed samples can be described using a single curve. The curve is not a straight line, as was seen in the data of Pederson et al. This indicates that the affinity term affected the corrosion to a small degree, but the data are not relevant to nearsaturated conditions. These tests were conducted for a maximum of $(\mathrm{SA} / \mathrm{V}) \mathrm{t}=2.8 \times 10^{4} \mathrm{~d} / \mathrm{m}$.

Note in Fig. 32 that tests with monolithic samples and with crushed samples at $100 \mathrm{~m}^{-1}$ give almost identical results. This implies that the estimated surface area of the crushed glass is accurate and that the monolithic and crushed glass reacts at a similar rate. Of course, the similarity depends on the surface area assigned to the crushed glass. The authors mentioned that the surface area of the crushed glass was measured using gas adsorption, but did not report the measured area. Neither were the mass of crushed glass or the mass of leachant solution reported. It is possible that the surface area of the crushed glass was determined by matching the data to that from tests with monolithic samples. The greatest difference between tests with monolithic samples and those with crushed samples occurs after 1 day of reaction, when the boron concentration in the test with the monolith is $2.54 \mathrm{ppm}$ and in the test with the crushed glass is $1.50 \mathrm{ppm}$. The boron concentration is higher in tests with crushed glass than in corresponding tests with monoliths at all other test times, however. The authors ascribe the difference in the 1-day tests to differences in surface morphology, which are expected to become less significant as the glasses corrode. However, while the morphologies may become similar, changes in the solution chemistry due to the initial corrosion will significantly affect the continued corrosion behavior. This is evidenced in tests with crushed samples, which consistently had slightly higher $\mathrm{pH}$ values than corresponding tests with monoliths, which indicates that more dealkalization occurred early in the tests with crushed glass.

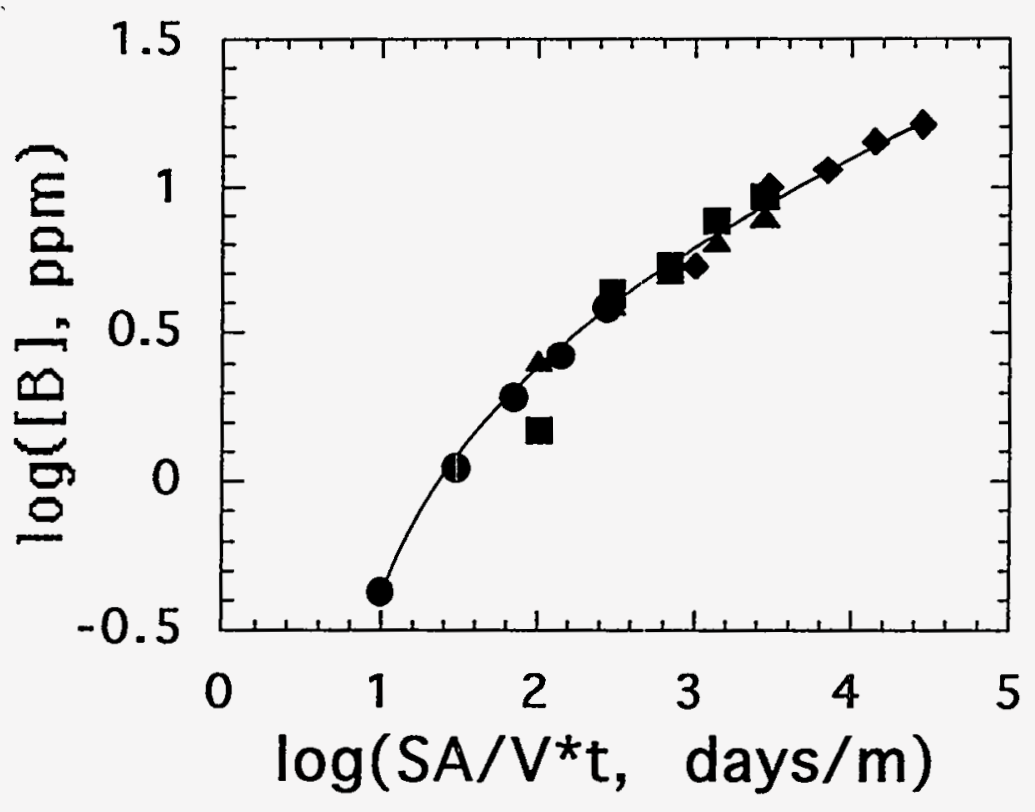

Fig. 32. Boron Release vs. (SA/V)t in Tests of SRP Black Frit Glass at Various SA/V Ratios:

(•) $10 \mathrm{~m}^{-1}$ and (A) $100 \mathrm{~m}^{-1}$ with Monolithic Samples and (ש) $100 \mathrm{~m}^{-1}$ and

(•) $1000 \mathrm{~m}^{-1}$ with Powdered Samples (after [196]) 
Monolithic samples of SRL 165 glass were corroded in deionized water at $90^{\circ} \mathrm{C}$ at SA/V of 7.9 and $85 \mathrm{~m}^{-1}$ for times up to 56 days [197]. The silica concentrations are plotted against the product $(\mathrm{SA} / \mathrm{V}) \mathrm{t}$ in Fig. 33. Although the authors fit all the data using the single curve shown in the figure, it is obvious that test results at the lower $\mathrm{SA} / \mathrm{V}$ deviate from the curve as $(\mathrm{SA} / \mathrm{V}) \mathrm{t}$ increases. (The tests at the lower SA/V were only progressed to a $(\mathrm{SA} / \mathrm{V}) \mathrm{t}$ value of $442.4 \mathrm{~d} / \mathrm{m}$.) The deviation can be attributed to the difference in the $\mathrm{pH}$ values attained in the tests at different $\mathrm{SA} / \mathrm{V}$ : the $\mathrm{pH}$ values in tests at $85 \mathrm{~m}^{-1}$ were consistently about one half unit higher than those in tests at $7.9 \mathrm{~m}^{-1}$.

Hench and coworkers have studied the corrosion of simple alkali silicate glasses to determine the kinetics of the dealkalization process [198,199]. As a part of their investigations, the corrosion of monolithic and crushed glass samples was studied. Figure 34 reproduces the measured silica concentrations in tests conducted with monolithic and crushed $\mathrm{Li}_{2} \mathrm{O} \cdot 2 \mathrm{SiO}_{2}$ glass at $50^{\circ} \mathrm{C}$ [198]. Tests with monolithic samples were conducted at a SA/V of $77 \mathrm{~m}^{-1}$, while tests with crushed glass samples were conducted at a reported SA/V of $43 \mathrm{~m}^{-1}$. The authors fit the data for tests with monolithic samples to two lines, as shown in Fig. 34 , and interpreted the change in slope after about 300 days to indicate a change in the kinetics from $\mathrm{t}^{1 / 2}$ to $\mathrm{t}^{1}$ dependence. They drew a single curve through the data for tests with crushed glass and implied that the tests with powders required longer reaction times to change kinetics. However, as shown in Fig. 34 , the results of tests with crushed glass can be fit by lines having the same slope and the same change in slope as those drawn through the data from tests with monolithic samples. Thus, the monolithic and crushed glass react identically. The difference in measured silica concentrations is due to the different SA/V of the tests. The differences suggest that the $\mathrm{SA} / \mathrm{V}$ of the tests with crushed glass is about one third that of the monolithic tests.

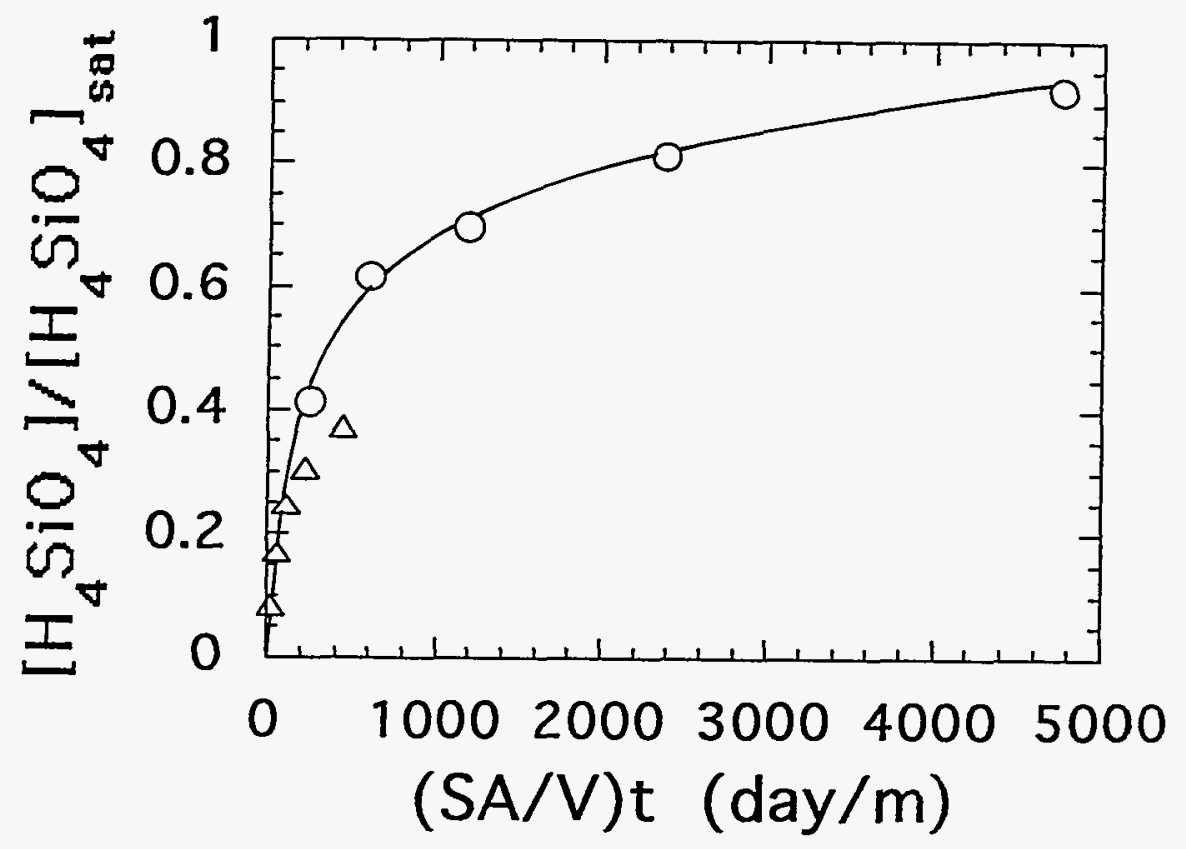

Fig. 33. Measured Silicic Acid Concentration vs. (SA/V)t for Tests with SRL 165 Glass at SA/V Ratios of ( $\triangle$ ) 7.9 and (O) $85 \mathrm{~m}^{-1}$ (after [197]) 


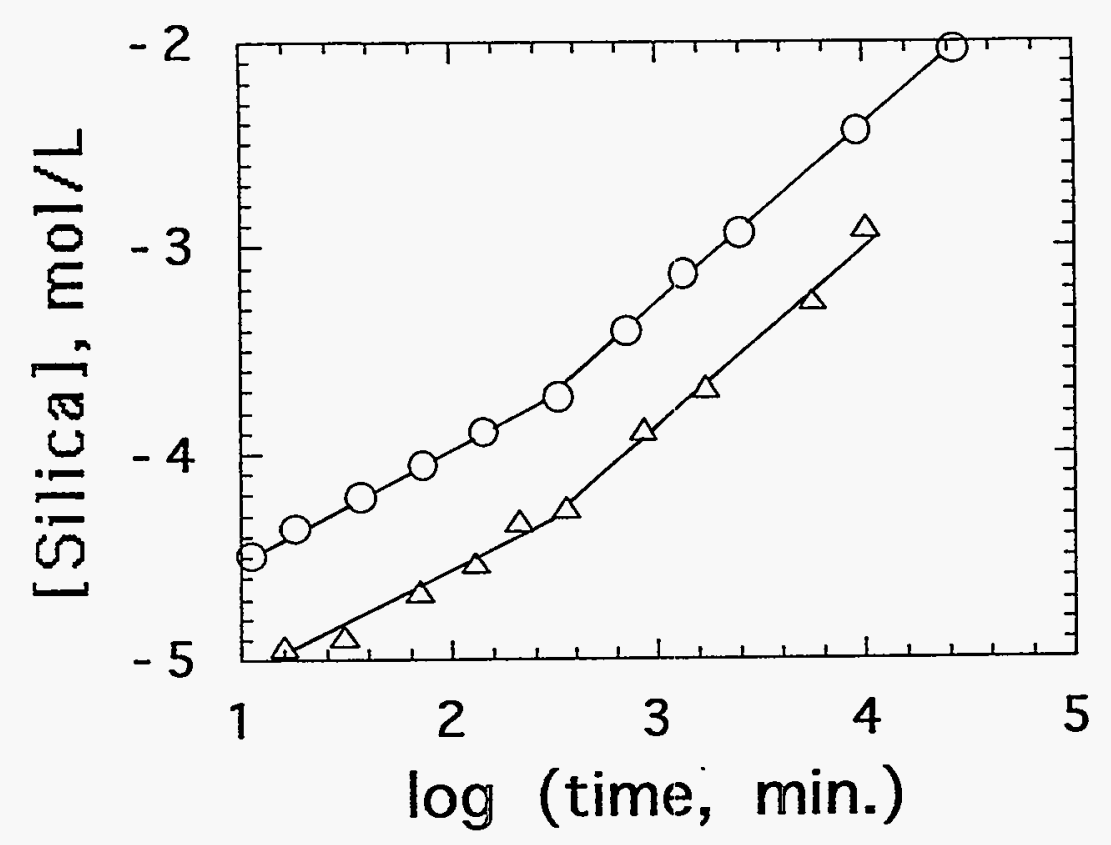

Fig. 34. Measured Silica Concentration vs. $\mathrm{Log}$ Time for Tests with $\mathrm{Li}_{2} \mathrm{O} \cdot 2 \mathrm{SiO}_{2}$ Glass: (O) Monolithic Samples at $77 \mathrm{~m}^{-1}$ and $(\Delta)$ Crushed Samples at $43 \mathrm{~m}^{-1}$ (after [198])

Another study to determine the usefulness of (SA/V)t as a scaling factor for tests with monolithic samples and crushed glass samples was carried out by S. Bates et al., who performed static leach tests in deionized water at $90^{\circ} \mathrm{C}$ using $H W-39$ glass at $S A / V$ ratios of $10,530,2000$, and $20,000 \mathrm{~m}^{-1}$ [133]. Tests at $10 \mathrm{~m}^{-1}$ were performed using monolithic glass samples following the MCC-1 protocol, while tests at 530,2000 and $20,000 \mathrm{~m}^{-1}$ were performed using crushed glass in the $-100+200$ mesh size fraction following the PCT protocol. The surface area of the crushed glass was approximated by assuming the grains were spheres with a diameter equal to the average mesh size. The leachates from tests with crushed glass were filtered using $450 \mathrm{~nm}$ filters to remove suspended glass particles, while the leachates from tests with monolithic samples were not filtered.

The boron concentrations in the leachates and the solution $\mathrm{pH}$ values are plotted against the product (SA/V)t in Figs. 35a and b. The data from tests at 10,530 , and $2000 \mathrm{~m}^{-1}$ may be represented by a common curve, within experimental error, but the tests at $20,000 \mathrm{~m}^{-1}$ clearly deviate from this curve. Again, the curvature of data from tests at common SA/V conducted for various time periods indicates that the silicic acid released to solution is affecting the continued glass corrosion rate. These tests were conducted for a maximum of $(\mathrm{SA} / \mathrm{V}) \mathrm{t}=5.6 \times 10^{5} \mathrm{~d} / \mathrm{m}$. The test matrix use by Bates et al. includes tests at different SA/V but overlapping (SA/V)t which allow direct comparison of the reaction progress. Previously discussed tests did not have overlapping data. Although the data from tests conducted at SA/V of $2000 \mathrm{~m}^{-1}$ or less show the same general trend, the tests conducted at higher SA/V consistently have a higher boron release than tests at lower SA/V but at the same $(\mathrm{SA} / \mathrm{V}) \mathrm{t}$. The difference is greatest between tests at 2000 and $20,000 \mathrm{~m}^{-1}$. These data suggest that the deviations from (SA/V)t scaling increases with the $\mathrm{SA} / \mathrm{V}$ and with the product $(\mathrm{SA} / \mathrm{V}) \mathrm{t}$, and show that tests should be conducted for large values 
(a)

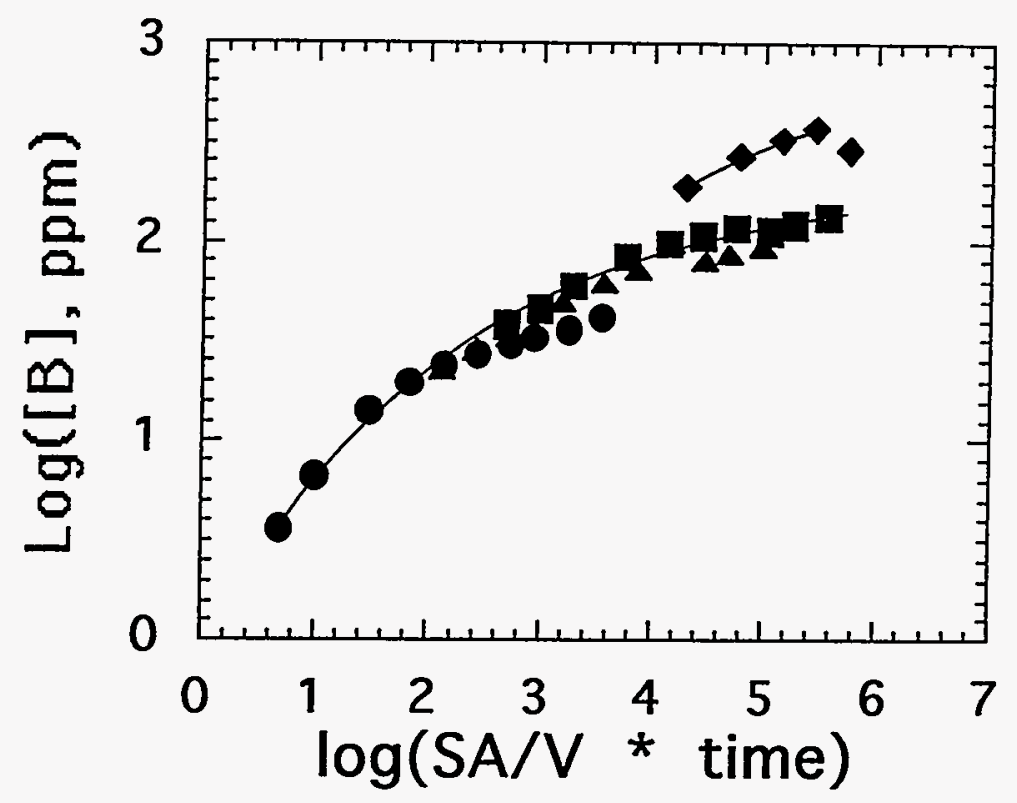

(b)

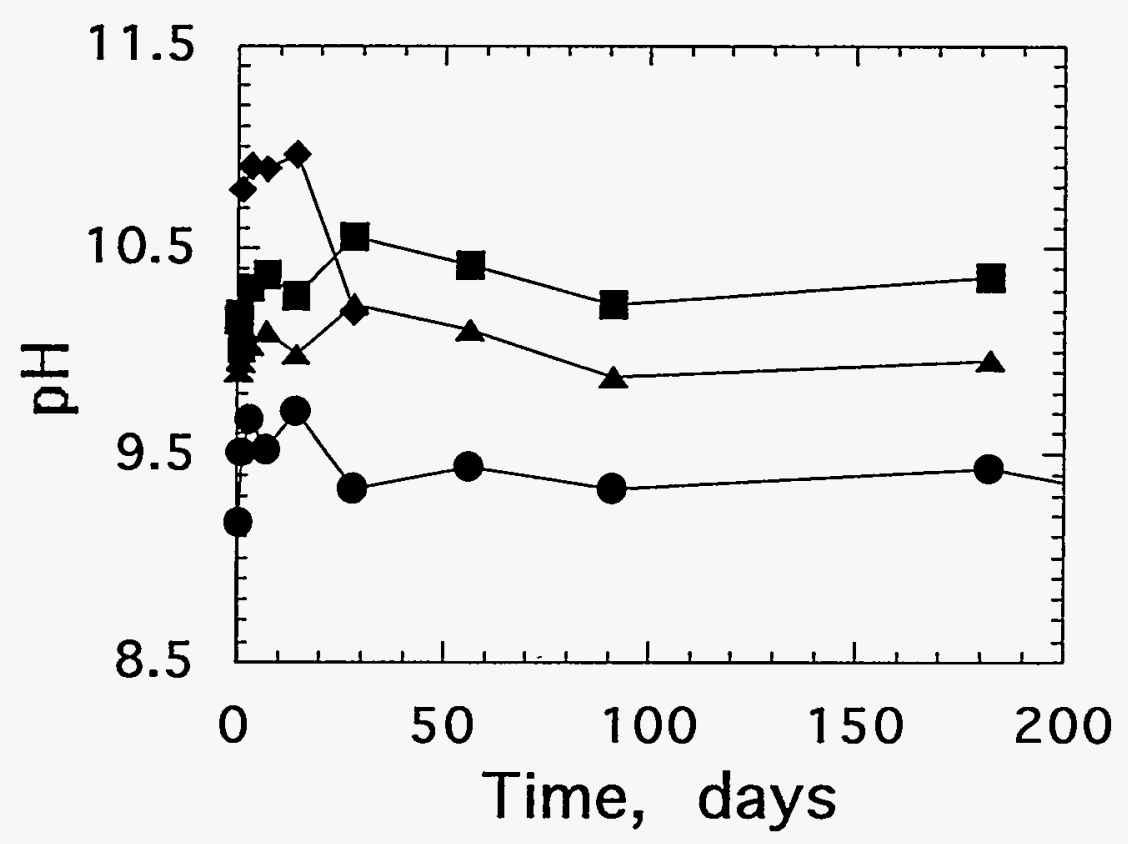

Fig. 35. Corrosion of HW-39 Glass at Various SA/V Ratios: (a) B, ppm, vs. (SA/V)t and

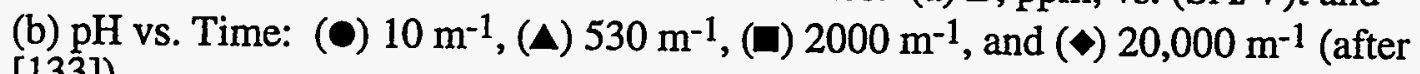
[133]) 
of (SA/V)t to determine the effects of SA/V on the corrosion behavior and to determine if tests at high $S A / V$ can be used to accelerate the corrosion and to characterize advanced corrosion

behavior. Note that the boron concentrations in tests with monolithic samples at $530 \mathrm{~m}^{-1}$ and the tests with crushed glass at $2000 \mathrm{~m}^{-1}$ differ by an extent similar to the difference between the tests at $10 \mathrm{~m}^{-1}$ and $530 \mathrm{~m}^{-1}$. This indicates that the surface area of the crushed glass is well approximated by assuming the grains are spheres with a diameter equal to the average mesh size.

Some insight into the effects of SA/V is gained by considering the solution $\mathrm{pH}$ values attained in the tests conducted by $\mathrm{S}$. Bates et al. The $\mathrm{pH}$ values measured at room temperature for these tests are seen to increase with the $S A / V$ of the test and with the reaction time, shown in Fig. 35b. (The pH values shown in Fig. 35b were tabulated by S. Bates et al., although values that were calculated for $90^{\circ} \mathrm{C}$ are plotted in [133]. The trends of the data for tests at different $\mathrm{SA} / \mathrm{V}$ are the same, with the $90^{\circ} \mathrm{C} \mathrm{pH}$ values being about $2 \mathrm{pH}$ units lower than those measured at room temperature.) Both the leachate $\mathrm{pH}$ and the boron concentration are consistently higher in the tests at higher SA/V at all (SA/V)t tested. The difference is greatest at high SA/V. S. Bates et al. recognized that the higher solution $\mathrm{pH}$ values promote glass dissolution and suggested that test data may scale better under buffered conditions.

The effects of the SA/V on the corrosion of R7T7 glass was investigated by Vernaz et al. by reacting R7T7 glass in deionized water for reaction times up to 364 days [167]. Tests at $400 \mathrm{~m}^{-1}$ were performed using monolithic samples, while tests at higher SA/V were attained by using crushed glass of various size fractions: $2000 \mathrm{~m}^{-1}$ using the $40-50 \mu \mathrm{m}$ size fraction, $8000 \mathrm{~m}^{-1}$ using the $100-125 \mu \mathrm{m}$ size fraction, and $20,000 \mathrm{~m}^{-1}$ using the $400-500 \mu \mathrm{m}$ size fraction. The SA/V of tests with crushed glass was based on assuming the grains to be spheres with a diameter equal to the average mesh size. The results are plotted in Figs. 36a-36d. The lines drawn through the boron data were interpreted by Vernaz et al. to represent the residual rates at each SA/V.

In tests at $400 \mathrm{~m}^{-1}$, the silicon concentration increases at the same rate as the boron concentration through about 273 days, as seen be drawing a line through the silicon data having the same slope as the line drawn by Vernaz et al. through the boron data. This indicates that corrosion is not occurring under silica saturation conditions and that the rate quoted is not the residual rate. Note that the data at 364 days suggest a slowing of the corrosion rate. Longer reaction times are needed to characterize the corrosion rate at $400 \mathrm{~m}^{-1}$.

Tests at $2000 \mathrm{~m}^{-1}$ also show the silicon concentration to increase at the same rate as the boron concentration through the longest times tested, as seen be drawing a line through the silicon data having the same slope as the line drawn by Vernaz et al. through the boron data. This indicates that corrosion is not occurring under silica saturation conditions in these tests either, and that the rate quoted is not the residual rate. Longer reaction times are needed to characterize the corrosion rate at $2000 \mathrm{~m}^{-1}$.

The silicon concentration increases at a lower rate than the boron concentration in the tests conducted at $8000 \mathrm{~m}^{-1}$ also show, as seen be drawing a line through the silicon data. The observation that the silicon concentration continues to increase, albeit slower than the boron concentration, indicates that corrosion is not occurring under silica saturation conditions in these tests, and, again, the rate quoted is not the residual rate. Longer reaction times are needed to characterize the corrosion rate at $8000 \mathrm{~m}^{-1}$. 

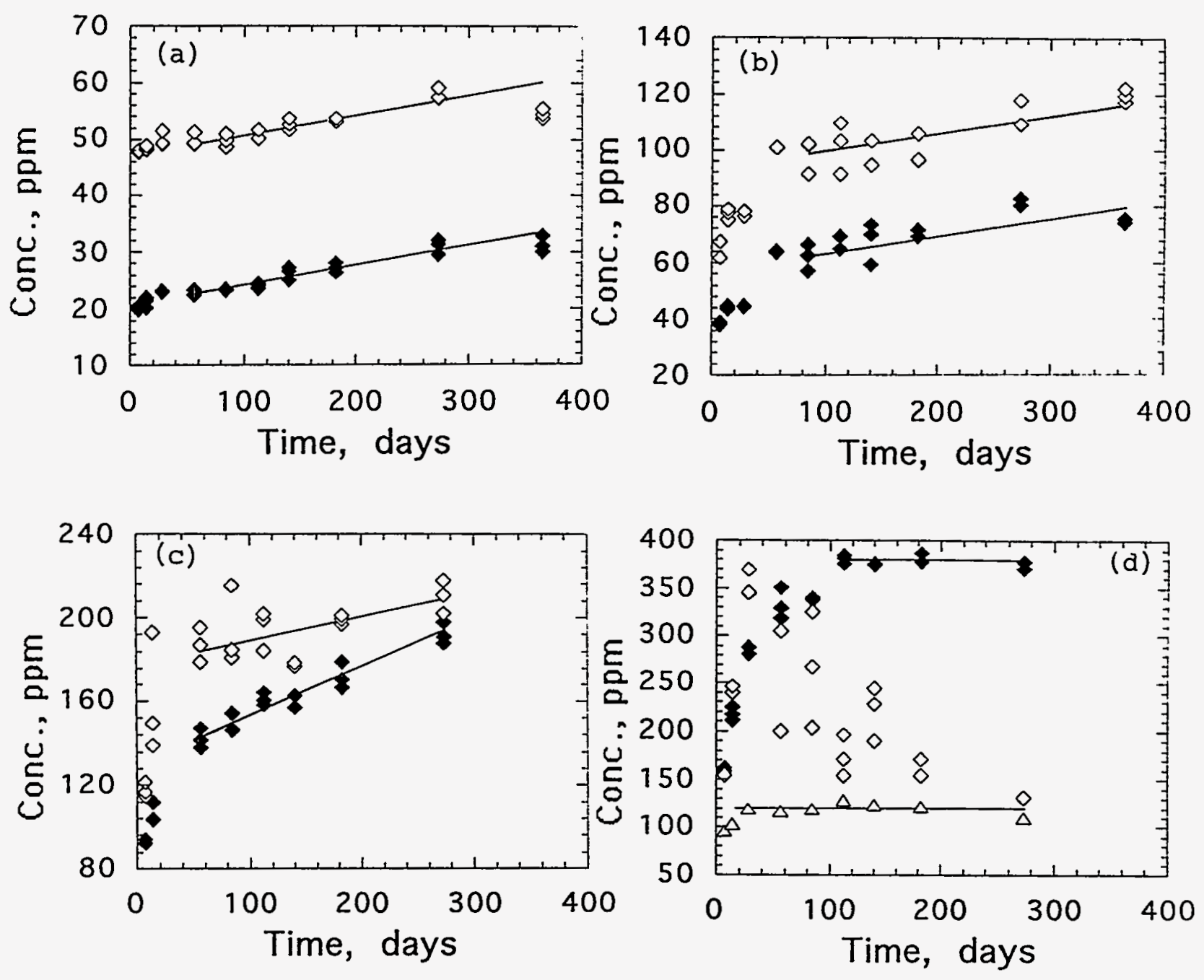

Fig. 36. Measured Concentrations of $(\diamond)$ Boron and ( $\bullet$ Silica in Tests with R7T7 Glass: (a) at $400 \mathrm{~m}^{-1}$ with Monolithic Samples, (b) at $2000 \mathrm{~m}^{-1}$ with Crushed Samples, (c) at $8000 \mathrm{~m}^{-1}$ with Crushed Samples, and (d) at $20,000 \mathrm{~m}^{-1}$ with Crushed Samples (after [167])

Tests conducted at $20,000 \mathrm{~m}^{-1}$ show the boron concentration to be essentially constant beyond about 112 days. The total silicon concentration increases through about 28 days, then decreases at longer time periods. Vernaz et al. showed that the decrease was due to the settling of iron silicate colloids from solution. They also filtered the leachate solutions through an approximately $1.8 \mathrm{~nm}$ filter to remove colloidal material, and found that the concentration of dissolved silicon (i.e., that which is not removed by filtration) to remain constant at about $120 \mathrm{ppm}$ beyond 28 days. The boron concentrations in these tests do represent the residual rate under silicon saturation, and suggest that the rate is immeasurably low. Vernaz et al. reported the rate to be $<1.5 \times 10^{-4} \mathrm{~g} / \mathrm{m}^{2} / \mathrm{d}$. 
Although the authors concluded that the residual rate decreased with the SA/V of the test, consideration of their data strongly suggests that tests conducted at SA/V less than $20,000 \mathrm{~m}^{-1}$ did not attain silica saturation and do not represent the residual rate. The observation that higher rates were measured at lower SAV simply indicates that the corrosion is occurring under unsaturated conditions. The authors do note that higher solution $\mathrm{pH}$ values are attained in tests at higher SA/V. The "mean leachate $\mathrm{pH}$ " values measured at $25^{\circ} \mathrm{C}$ are: 8.91 in tests at $400 \mathrm{~m}^{-1}$, 9.28 in tests at $2000 \mathrm{~m}^{-1}, 9.61$ in tests at $8000 \mathrm{~m}^{-1}$ and 9.77 in tests at $20,000 \mathrm{~m}^{-1}$. They also conclude that "the significant slowdown of the final rate is not due to diffusion of leached species through a growing surface layer, since the altered glass thickness diminishes as the SA/V ratio increases" [167], although they do not present measured layer thicknesses in the paper.

Short-term corrosion tests conducted by Feng et al. using several different glasses have shown various correlations between the extent of glass corrosion and the product (SA/V)t [159]. Glasses were reacted using a modified MCC-3 procedure (similar to the PCT procedure) wherein a small fraction of the solution was sampled periodically and replaced with fresh leachant. As discussed in Section 2.5.2.3, such a periodic replenishment of the leachate solution will greatly complicate interpretation of the test results. Continued dilution of the leachate solution will cause a cycling of the corrosion rate between a high value after replenishment and a much lower value after the solution reconcentrates. As the key effect of the SA/V on test results is through dilution of corrosion products, test results will be affected by both the effects of the SA/V and the effects of the leachate replacement. Despite the inappropriateness of the test method, departure from $(\mathrm{SA} / \mathrm{V}) \mathrm{t}$ scaling was most evident in tests conducted at large values of $\mathrm{SA} / \mathrm{V}$, as seen in static leach tests. The authors suggested that the observed deviation from (SA/V)t scaling may be due to $\mathrm{pH}$ effects and to mass transport effects or may arise form the formation of protective alteration layers. Although tests at higher SA/V attain higher solution concentrations, the release rates per unit area of glass are lower in tests at higher SA/V than in tests at lower $\mathrm{SA} / \mathrm{V}$. Therefore, the differences in the layer thickness generated in tests at high and low SA/V cannot account for observed effects of SA/V.

Long-term tests were conducted at ANL that attained larger values of (SA/V)t than had been attained previously [67]. (The testing program is summarized in Section 9.) Tests were performed using glasses SRL 131 and SRL 202 that vvere doped with technetium and transuranics (and are referred to as SRL 131A and SRL 202A) at 10, 340, 2000, and 20,000 m-1. Tests were conducted in both deionized water and in a tuff groundwater. Tests at 10 and $340 \mathrm{~m}^{-1}$ were performed following a modified MCC-1 procedure and tests at 2000 , and $20,000 \mathrm{~m}^{-1}$ were performed following a modified PCT procedure. Differences in the extent of corrosion of SRL 202 glass in deionized water and tuff groundwater were negligible at reaction times beyond a few days [66]. Figure 37a shows the boron concentrations plotted against the product (SA/V)t for tests with SRL 202A glass in a tuff groundwater solution. These data clearly cannot be fitted by a single curve. Note that the deviations from a common curve are greater at larger values of $(\mathrm{SA} / \mathrm{V}) \mathrm{t}$, as seen earlier in the data of S. Bates et al. [133]. Figure $37 \mathrm{~b}$ shows the measured $\mathrm{pH}$ values plotted against the reaction time for tests conducted at the different $S A / V$ ratios. In these tests, the increase in the solution $\mathrm{pH}$ is a consequence of the natural corrosion process.

In other tests, radiolysis of the air in the test vessel by radioactive glass has been seen to affect the glass corrosion behavior. A sudden increase in the corrosion rate of a glass made from SRL 200 frit (referred to as SRL 200S glass) similar to that observed by Ebert and Bates was observed in static tests performed by Feng et al. [200]. Tests were conducted at $90^{\circ} \mathrm{C}$ following the PCT method B protocol in tuff groundwater and at a SA/V ratios of 2000 and $20,000 \mathrm{~m}^{-1}$. Tests were performed using the $-100+200$ mesh size fraction. Tests conducted under analogous conditions using a glass made from the same frit plus added radioactive sludge (referred to as SRL 200R glass) did not show a corresponding sudden increase, however. The two glasses reacted 
(a)

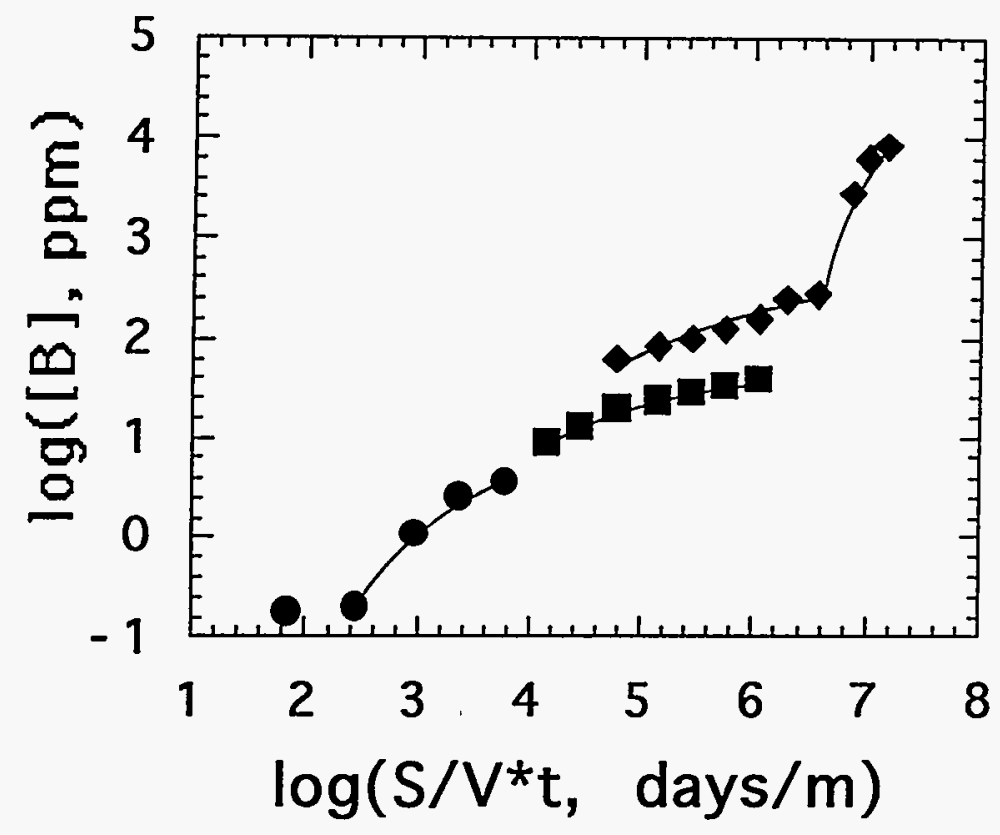

(b)

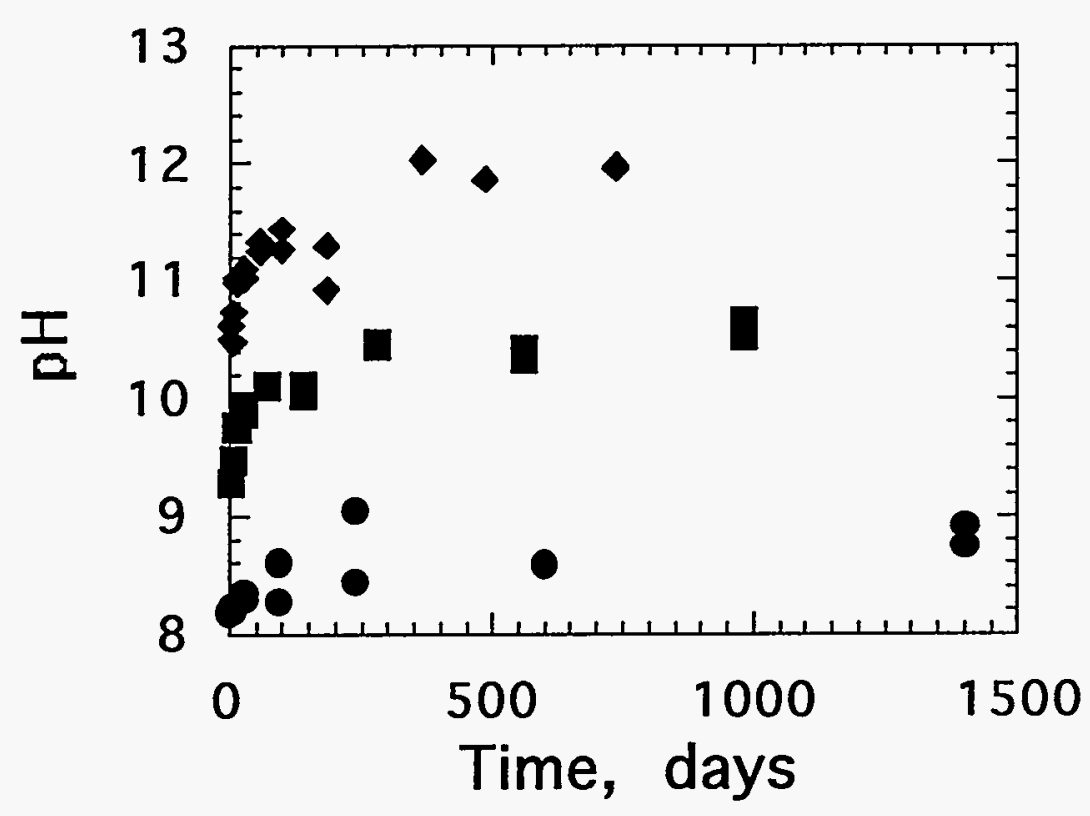

Fig. 37. (a) Measured Boron Concentration vs. (SA/V)t and (b) Measured $\mathrm{pH}$ vs. Time for Tests with SRL 202 Glass at SA/V Ratios of (•) 10, (घ) 2000, and ( $\bullet) 20,000 \mathrm{~m}^{-1}$ (after [67]) 
identically in all tests at $2000 \mathrm{~m}^{-1}$ (results were presented through 364 days) and in through about 182 days in tests at $20,000 \mathrm{~m}^{-1}$. Beyond 182 days in tests at $20,000 \mathrm{~m}^{-1}$, tests with the nonradioactive glass showed a significant increase in the release rate of soluble glass components. The normalized mass losses of boron, lithium, sodium, and silicon in tests conducted at $20,000 \mathrm{~m}^{-1}$ with SRL 200R and SRL 200S glasses are shown in Figs. 38a and 38b. (Data for the release of sodium from the SRL 200R glass are not distinguishable in the plots published by Feng et al.)

All four elements are released suddenly (lithium, boron, and sodium after 330 days and silicon after 364 days), but that the release at the next longest test period is different for the different elements: lithium is released to a slightly greater extent after 364 days than it is after 330 days, silicon is released to a similar extent after 390 days as before the increased release, slightly less sodium and boron is released after 390 days than after 364 days. Also, the release is not stoichiometric. The normalized mass losses decrease as $\mathrm{B}>\mathrm{Na} \gg \mathrm{LI}>\mathrm{Si}$; this shows the increase is not a testing artifact (such as including glass grains in the analyzed solutions). The boron mass loss after 330 days is very near the maximum normalized mass loss for tests with the $-100+200$ mesh size fraction, which is $50 \mathrm{~m}^{-1}$. This indicates that very little unreacted glass remains and that mass losses at longer reaction times are not expected to show much increase. The slight decrease in the boron and sodium mass losses after 364 days may indicate that the corrosion rate was not accelerated at the same time in tests at 330 and 364 days, or may be due to analytical uncertainties. The decreased silicon mass loss after 390 days may indicate a settling of colloidal silicon.

The authors attributed the rate increase to the formation of clinoptilolite, $(\mathrm{Na}, \mathrm{K})_{6}\left(\mathrm{Al}_{6} \mathrm{Si}_{30} \mathrm{O}_{72}\right) \cdot 20 \mathrm{H}_{2} \mathrm{O}$, which was abundant in the tests with the nonradioactive glass but not found in the tests with the radioactive glass. The authors concluded that the lower solution $\mathrm{pH}$ values in the tests with the radioactive glass prevented the formation of clinoptilolite and the concomitant increase in the corrosion rate. Radiolysis of the air in the test vessel resulted in the lowering of the $\mathrm{pH}$ value in tests with the radioactive glass. Figure $38 \mathrm{c}$ shows the solution $\mathrm{pH}$ values measured in the tests at $20,000 \mathrm{~m}^{-1}$ with SRL 200R and SRL 200S glasses. The $\mathrm{pH}$ values (which were measured at room temperature) remain below about 11.65 in tests with SRL 200R glass, but rise above 12 in tests with SRL 200S glass. The authors speculated that the formation of clinoptilolite occurred in solutions having high $\mathrm{pH}$ values under the test conditions. Thus, the key effect of the $\mathrm{pH}$ in these tests was on the formation of secondary phases, which then affected the glass corrosion rate.

\subsection{Modeling the Effects of the Solution $\mathrm{pH}$}

The differences in the solution concentrations attained in tests run at different SA/V can be attributed to the effect of the $\mathrm{pH}$ on the corrosion rate. Tests under conditions where the affinity term is maintained near one have clearly shown that the corrosion rate is sensitive to the solution $\mathrm{pH}$ (see Figs. 16 and $17[70,92]$ ). The rate coefficient $\mathrm{k}_{+}$has a $\mathrm{pH}$ dependency that can be written explicitly as $\mathrm{k}_{+}=\mathrm{k}_{\mathrm{f}}\left(\mathrm{H}^{+}\right)^{-0.4}$, where $\mathrm{k}_{\mathrm{f}}$ is the $\mathrm{pH}$-independent reaction coefficient and the exponent was determined experimentally and is valid for $\mathrm{pH}$ values greater than about $\mathrm{pH} 7$. Substituting this expression for $\mathrm{k}_{+}$into Eq. 16 yields

$$
\left[\mathrm{H}_{4} \mathrm{SiO}_{4}\right]=\left[\mathrm{H}_{4} \mathrm{SiO}_{4}\right]_{\text {sat }}\left(1-\exp \left\{\frac{-(\mathrm{SA} / \mathrm{V}) \mathrm{tk}_{\mathrm{f}}\left(\mathrm{H}^{+}\right)^{-0.4}}{\left[\mathrm{H}_{4} \mathrm{SiO}_{4}\right]_{\text {sat }}}\right\}\right)
$$


(a)

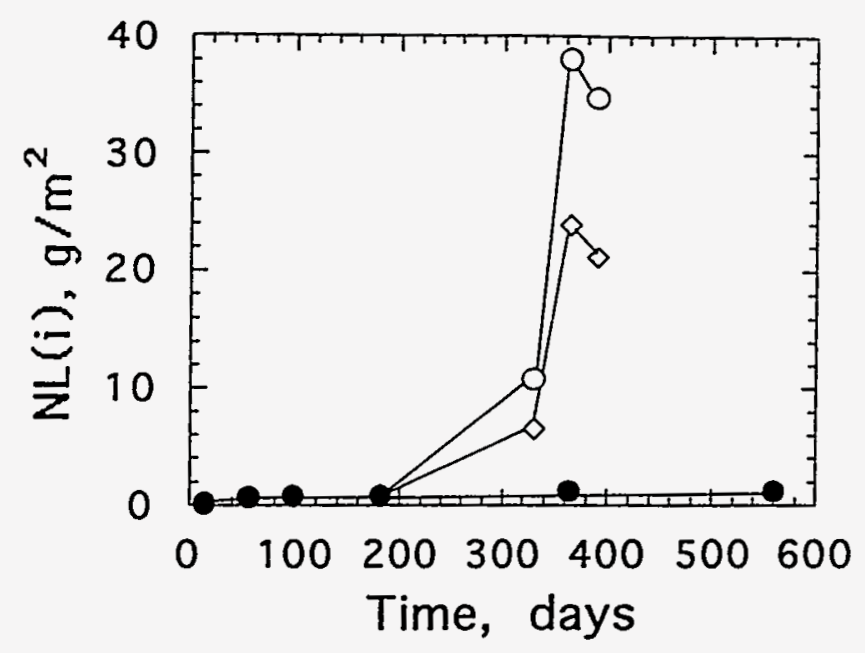

(b)

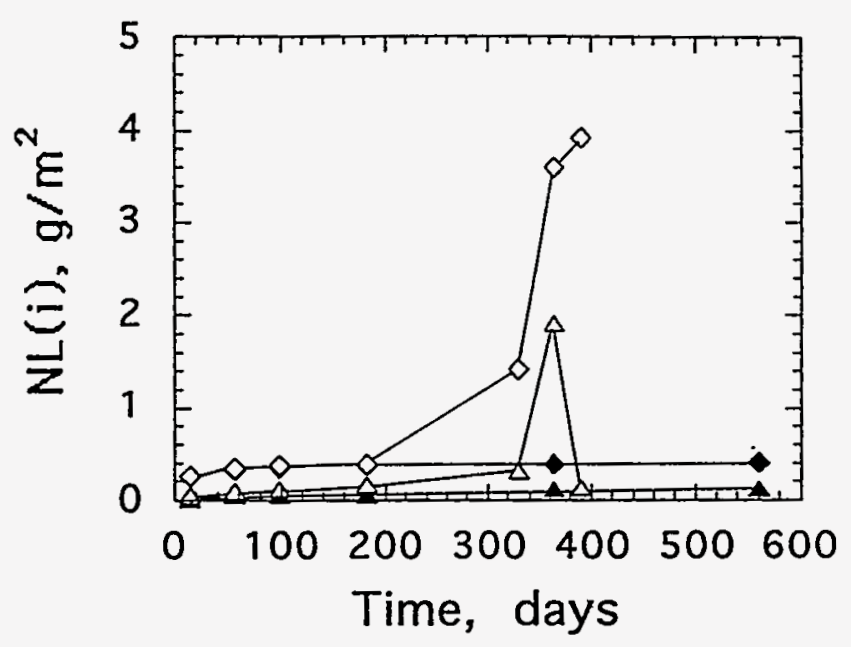

(c)

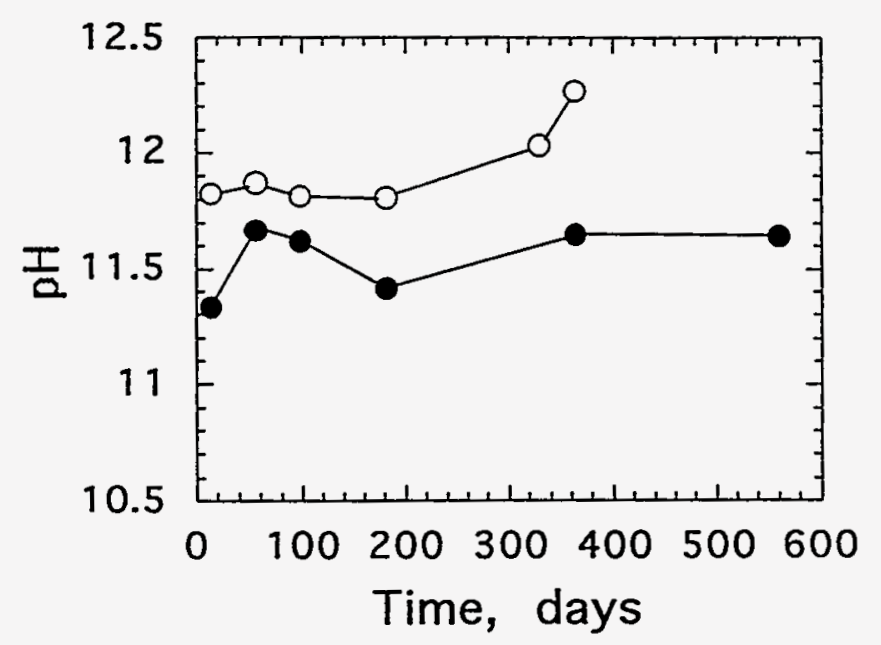

Fig. 38. Normalized Mass Loss vs. Reaction Time for Tests with SRL 200R and SRL 200S

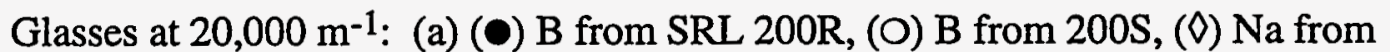
200S; (b) ( $\bullet$ ) Li from SRL 200R, ( () Li from 200S, (A) Si from 200R, ( $\Delta$ ) Si from 200S; and (c) Measured pH for Tests with (৫) SRL 200R and (O) SRL 200S Glass (after [200]) 
From this expression, the coefficient for scaling static test results is $(\mathrm{SA} / \mathrm{V}) \mathrm{t}\left(\mathrm{H}^{+}\right)^{-0.4}\left(\mathrm{k}_{\mathrm{f}}\right.$ and $\left[\mathrm{H}_{4} \mathrm{SiO}_{4}\right]_{\text {sat }}$ are constant with respect to time and solution $\left.\mathrm{pH}\right)$. Therefore, tests performed for common values of (SA/V)t will generate the same silicic acid concentrations only if the solution $\mathrm{pH}$ values are the same. Since the SA/V of a static test also determines the extent of dilution of hydroxide generated during dealkalization, tests at different $\mathrm{SA} / \mathrm{V}$ will generally attain different $\mathrm{pH}$ values. Since dealkalization affects the solution chemistry primarily during the initial stage of corrosion, the solution $\mathrm{pH}$ usually approaches a constant value as corrosion proceeds (see Fig. $37 \mathrm{~b}$, for example). Of course, the change in the $\mathrm{pH}$ depends strongly on the amounts of alkali metals in the glass and the buffering capacity of silicic and boric (and perhaps other) acids released from the glass as it corrodes. The $\mathrm{pH}$ of the solution is expected to scale with the SA/V of the test better at short times and at small values of $\mathrm{SA} / \mathrm{V}$, for which the corrosion rate is not strongly affected by the affinity term.

The use of tests at high $\mathrm{SA} / \mathrm{V}$ to evaluate advanced stages of corrosion requires solution to a rate equation that depends on both the silicic acid concentration and the $\mathrm{pH}$, such as that given by Eq. 15 , where $\mathrm{k}_{+}=\left(\mathrm{H}^{+}\right)^{\mathrm{h}}$. The results of tests conducted at different $\mathrm{SA} / \mathrm{V}$ can only be directly compared when the effects of either the silicic acid concentration or the $\mathrm{pH}$ on the rate can be isolated. That is, the $\mathrm{pH}$ must be constant to compare the effects of the silicic acid concentration on the rate and the silicic acid concentration must be constant to compare the effects of the $\mathrm{pH}$. Tests in which the $\mathrm{pH}$ and silicic acid concentrations are free to vary cannot be compared directly. Instead, a rate equation that accounts for both effects must be used to compare the data. For example, the results of tests being conducted at ANL have been utilized to demonstrate how the $\mathrm{pH}$ can be incorporated into the model being developed at LLNL [176]. Therefore, the deviations observed to occur in plots of solution concentrations against the product ( $\mathrm{SA} / \mathrm{V}) \mathrm{t}$ can be attributed to differences in the $\mathrm{pH}$, and these differences can be accounted for in the rate equation used to describe glass corrosion. Tests at high SA/V can be used to characterize advanced stages of corrosion only if differences in the solution chemistry are accounted for by modeling.

First, the differences in $\mathrm{pH}$ must be accounted for when comparing the results of tests conducted at different $\mathrm{SA} / \mathrm{V}$. The high $\mathrm{pH}$ values attained in tests at high $\mathrm{SA} / \mathrm{V}$ are not an artifact of the test method, they are due to the limited volume of water available to dilute hydroxide generated during glass corrosion. We also note that solution $\mathrm{pH}$ values are usually measured at room temperature while tests are conducted at much higher temperatures. Cooling the solution from the test temperature to room temperature can change the solution $\mathrm{pH}$ significantly. The $\mathrm{pH}$ values at the test temperature can be calculated from the measured solution chemistry using thermodynamic codes. The $\mathrm{pH}$ values at the test temperature should be used in the rate equation. The solution concentrations of dissolved components are not expected to be affected by cooling the solution, although in concentrated solutions some components may be removed from solution by precipitation of saturated phases. Solutions are generally acidified to maintain components in solution.

Second, the alteration layers formed on the glass surface may vary with the SAVV of the test. The differences may affect the disposition of some glass components and waste components as the glass corrodes. The amount of glass that must dissolve to saturate the solution decreases as the SA/V increases, so thicker layers form at lower SA V. While the layers do not significantly affect the glass corrosion rate, they do interact with released materials, including radionuclides, to affect their solution concentrations. Thus, the behavior of waste components may not track the behavior of the glass matrix. (For example, the release of transuranic elements and other components from SRI 202A glass does not follow the increased release of boron and alkali metals shown in Fig. 37a. See also Section 7.) 
Third, utilization of the results obtained in tests at high SA/V for predicting advanced corrosion behavior should be related to anticipated disposal conditions, and other environmental effects on the solution chemistry should be taken into account. For example, radiolysis of the air and groundwater to generate nitric acid will lower the $\mathrm{pH}$ but not affect the silicic acid concentration. Thus, the $\mathrm{pH}$ and silicic acid levels attained in tests at high SA/V will not accurately simulate the corrosion environment. Such effects may greatly affect the glass corrosion behavior $[200,201]$.

\subsection{Effects of SA/V on Alteration Layers}

Differences in the alteration layers generated when a glass is corroded in tests at different SA/V occur because (1) more glass must react in a test at low SA/V than in a test at high SA/V to attain the same level of solution saturation, and (2) the quicker approach to saturation in tests at high SA/V means the surface is exposed to more concentrated solution chemistries during the test period. For glasses that dissolve nonstoichiometrically, thicker layers are generated in tests at low SA/V than are generated in tests at high SA/V. However, the layers formed on glasses reacted in tests at high SA/V mature faster because they are exposed to more concentrated solutions. Tests at low SA/V can be used to characterize the initial development of the alteration layers because the layers are relatively thick and form in the presence of a solution that remains relatively dilute for long test periods. Interactions between the developing layer and the solution can therefore be tracked as a function of time. Interactions between the layers formed at high SA/V occur more rapidly due to the quick saturation of the solution. While tests conducted at low SA/V can be used to characterize the early stages of layer formation and tests conducted at high SA/V can be used to characterize the later stages of layer formation, differences in the solution chemistry must be accounted for. Specifically, the solution $\mathrm{pH}$ will be significantly higher in tests conducted at high SA/V than in tests conducted at low $\mathrm{SA} / \mathrm{V}$, so that the layer will develop under different conditions. Alteration phases formed in highly basic solutions in tests at high SA/V may not form in less basic conditions in tests at low SA/V.

Houser et al. analyzed the films formed on monolithic samples of three different waste glasses after corrosion at SA/V of 10 and $0.1 \mathrm{~m}^{-1}\left(10^{-1}\right.$ and $\left.10^{-3} \mathrm{~cm}^{-1}\right)$ for up to 100 hours at $90^{\circ} \mathrm{C}$ [202]. While the film thicknesses formed on all glasses increased with time, the films formed in tests conducted at $\mathrm{SA} / \mathrm{V}$ of $10 \mathrm{~m}^{-1}$ were slightly thicker than those formed in tests as $0.1 \mathrm{~m}^{-1}$ after the longest test time. Although solution concentrations were not reported, the observation of thinner layers in tests at lower $\mathrm{SA} / \mathrm{V}$ indicates that more surface dissolution occurred in tests at $0.1 \mathrm{~m}^{-1}$, which maintained more dilute solutions.

Oversby and Phinney characterized the layers that formed on SRL 165 glass in MCC-1 tests using secondary ion mass spectrometry. Tests were conducted for up to 91 days at $90^{\circ} \mathrm{C}$ in either deionized water or a solution containing $60 \mathrm{ppm}$ silica, $183 \mathrm{ppm}$ sodium, $200 \mathrm{ppm}$ bicarbonate, and $250 \mathrm{ppm}$ nitrate [88]. The layers formed on the "as cast" faces were analyzed to avoid any effects of mechanical preparation on the layer development. Analysis showed the alkali metals and boron to be depleted in the layer, and alkaline earths, iron and manganese to be enriched in the layer, relative to the glass. Layers were found to develop in "an orderly process" in the silicate leachant, while the results of layer formation in deionized water were "more complex and less reproducible" [88]. The authors attributed the difference to the faster dissolution that occurs in the deionized water. The corrosion in the silicate solution decreased with the reaction time, as evidenced by both solution results and the layer thickness as defined by the depletion depth of lithium. A layer about $1.3 \mu \mathrm{m}$ thick had formed after 91 days. Oversby and Phinney noted that the structure of the layer developed over time as more stable alteration phases formed. 
Tests conducted using the same SRL 165 glass as used by Oversby and Phinney had been conducted earlier under modified MCC-1 conditions (monolithic samples reacted in J-13 groundwater at $90^{\circ} \mathrm{C}$ and at a SA $/$ of $30 \mathrm{~m}^{-1}$ ) [95]. The groundwater solution had slightly less silica than the solution used by Oversby and Phinney. Analysis by transmission electron microscopy of the layers formed as a function of the reaction time reveal how the layer structure evolves [87]. Tests conducted for short times resulted in the formation of an amorphous layer that was depleted of soluble glass components, such as alkali metals and boron, consistent with the composition determined by Oversby. After longer corrosion times, the layer was seen to crystallize and separate from the underlying glass. The layer is comprised of small laths of clay that have formed on a "backbone" of an iron-rich amorphous phase. The layer is seen to have completely crystallized and separated from the glass after about 278 days. The underlying glass continues to dissolve nearly congruently beneath the layer. Soluble material released from the glass is presumed to enter solution and insoluble material to be incorporated into the clay.

Analysis of the alteration layers formed in modified MCC-1 tests with SRL 165 glass by Kamizono et al. by secondary ion mass spectrometry showed boron to be depleted to a depth of about $1.5 \mu \mathrm{m}$ in tests at $8.5 \mathrm{~m}^{-1}$ and about $3 \mu \mathrm{m}$ in tests at $7.9 \mathrm{~m}^{-1}$ after 28 days of reaction [197]. The normalized mass loss of boron after 28 days was about 3 times higher in tests at $7.9 \mathrm{~m}^{-1}$ than in tests at $85 \mathrm{~m}^{-1}$. The difference between the thickness predicted based on boron release and that measured is due to dissolution of the glass surface, which is expected to be greater in the tests at $7.9 \mathrm{~m}^{-1}$ than in tests at $85 \mathrm{~m}^{-1}$.

Modified MCC- 1 tests conducted in deionized water at $90^{\circ} \mathrm{C}$ using monolithic samples of SRL 131 glass at SA/V of 10,50 , and $100 \mathrm{~m}^{-1}$ for 100,20 , and 10 days, respectively [203]. All tests were conducted for the same product $(\mathrm{SA} / \mathrm{V}) \mathrm{t}$, namely $1000 \mathrm{~d} / \mathrm{m}$. The solution $\mathrm{pH}$ and the solution concentrations of most glass components increased slightly with the $S A / N$ (results were about $10 \%$ higher in tests at $100 \mathrm{~m}^{-1}$ than those at $10 \mathrm{~m}^{-1}$ ). The thickness of the layers were measured to be 40,3 , and $1 \mu \mathrm{m}$ for tests conducted at 10,50 , and $100 \mathrm{~m}^{-1}$. Theoretical maximum layer thicknesses calculated from the measured boron concentrations, assuming the weight fraction of boron in the glass is 0.04 (see Appendix B) and the glass to have a density of $2.7 \mathrm{~g} / \mathrm{cm}^{3}$, are $22.5,4.6$, and $2.6 \mu \mathrm{m}$ for tests conducted at 10,50 , and $100 \mathrm{~m}^{-1}$, respectively. The measured values are in good agreement at the higher SA/V, but about a factor of 2 too large at $10 \mathrm{~m}^{-1}$. The error is likely due to the layer not being cross-sectioned normal to the glass surface. The layers formed at 50 and $100 \mathrm{~m}^{-1}$ may be thinner than predicted due to dissolution of the outer surface.

The layer formed at $10 \mathrm{~m}^{-1}$ was found to be more complex than those formed in tests at the higher $\mathrm{SA} / \mathrm{V}$. It included more phases and sublayers than the layers formed at $50 \mathrm{~m}^{-1}$ and $100 \mathrm{~m}^{-1}$, which were found to be structurally similar. The similarity of the solution chemistries imply that the differences are due to the different reaction times used in the tests. The authors conclude that phases observed in tests conduced at $10 \mathrm{~m}^{-1}$ were absent from layers formed at $50 \mathrm{~m}^{-1}$ and $100 \mathrm{~m}^{-1}$ due to insufficient time for the phases to be precipitated [203]. However, the thinness of the layers formed at 50 and $100 \mathrm{~m}^{-1}$ may have prevented resolution of similar sublayers. The alteration phases observed on the sample reacted for 100 days at $10 \mathrm{~m}^{-1}$ are consistent with those formed on SRL 131 glass under the same test conditions after longer reaction times [62]. Analysis of samples reacted for shorter time periods showed the layer to evolve from a single clay phase to complex layer containing several distinct phases. 
Tests conducted with SRL 165 glass under modified MCC-1 conditions (monolithic samples reacted in $\mathrm{J}-13$ groundwater at $90^{\circ} \mathrm{C}$ and at a SA/V of $30 \mathrm{~m}^{-1}$ ) show a less complicated layer to form [87]. Tests conducted for short times resulted in the formation of an amorphous layer that was depleted of soluble glass components, such as alkali metals and boron. After longer corrosion times, the layer was seen to crystallize and separate from the underlying glass. The layer formed on SRL 165 glass at $30 \mathrm{~m}^{-1}$ is different than that formed on SRL 131 glass in tests at 10,50 , or $100 \mathrm{~m}^{-1}$ in that the layer formed on SRL 165 glass completely crystallizes and separates from the glass. The underlying glass continues to dissolve nearly congruently beneath the layer. In contrast, the layer formed on SRL 131 glass does not completely crystallize and does not separate from the glass. While the compositions of SRL 131 and SRL 165 glasses are quite similar (see Appendix B), the alteration layers formed are quite different.

The alteration layers formed on SRL 202 glass in tests at $340 \mathrm{~m}^{-1}$ using monolithic samples and in tests at $2000 \mathrm{~m}^{-1}$ using crushed glass were found to develop similarly [64]. (See Sec. 9.3 for a more detailed discussion of layer formed during these tests.) The layers formed in tests at $20,000 \mathrm{~m}^{-1}$ through 182 days were the same as those formed in tests at $2000 \mathrm{~m}^{-1}$. The layers are comprised of laths of clay formed parallel to the glass surface. In tests at $20,000 \mathrm{~m}^{-1}$ conducted for 364 days or longer, a thick layer of the same clay material forms between the layer of clay laths and the glass. The inner layer contains smaller crystallites of the same clay that forms the outer layer. The sudden increase in the layer thickness corresponds to a sudden increase in the release of soluble glass components from the glass [65]. The increased corrosion rate has been attributed to the formation of secondary phases from the leachate solutions. The thickness of the outer layer of clay is about the same after 182 days (before the corrosion is accelerated) and after 364 days (after the corrosion has been accelerated). This indicates that the outer clay layer is unaffected by the significant changes in the solution chemistry that lead to a significant increase in the glass corrosion rate. The clay layer formed on the glass surface does not affect either the solution chemistry or the glass corrosion rate. It is also interesting to note that the highest corrosion rate occurs when the alteration layer is the thickest. This indicates that the layer does not act as a diffusion barrier, and that sloughing of the layer off the glass is not likely to affect the corrosion rate of SRL 202 glass.

The appearance of the layers formed after acceleration of the corrosion rate is very similar to that formed in tests with SRL 131 at $100 \mathrm{~m}^{-1}$ in the work of Mazer et al. [203], though the layer formed on SRL 202 glass is much thicker than that formed on SRL 131. As SRL 131 glass is known to be less durable than SRL 202 glass [67], this implies that the evolution of the alteration layer is affected by the reaction time and the glass composition. While tests conducted with SRL 202 glass at different SA/V attained very different solution chemistries, similar layers developed at all SA/V. This indicates that the development of the layers on SRL 202 glass is not affected by the solution chemistry.

\subsection{Tests Conducted to Attain Highly Concentrated Solutions}

Because the solution composition controls the glass reaction rate and because the SA/V ratio of a static test determines the extent of dilution of the solution, the SA/V used in a test provides a valuable means of attaining highly concentrated solution conditions. As discussed earlier, the reaction affinity can be kept near one, and the forward reaction rate can be determined by maintaining dilute solutions at very low SA/V in static tests or in dynamic tests. Conversely, the reactivities of glasses in highly concentrated leachate solutions where the reaction affinity is very small can be characterized by performing tests at very high SA/V. Such tests provide valuable insight into glass behavior in highly concentrated solutions, such as those that might eventually contact the waste form in a repository. 
Crushed glass is used routinely to achieve high SA/V ratios. Such tests provide for analyses of both the leachates and corroded solids, although the very large number of reacted grains complicates the cataloging of all secondary phases that may be generated. Very high $\mathrm{SA} / \mathrm{V}$ ratios have also been attained by reacting monolithic samples in vapor at elevated temperatures [104,106-108,111,139]. In a vapor environment, reaction occurs in a thin film of condensed water, and highly concentrated solutions are generated after very little glass has reacted [111]. Secondary phases that form in the leachate film remain on the sample surface, where they can be readily identified and cataloged. Leachate analyses cannot be performed with this method because the water evaporates during test termination; however, residual salts can be rinsed from the sample or redissolved in fresh condensate and analyzed [81].

The results of tests at very high SA/V ratios are consistent with the effects of SA/V predicted by Eq. 15, namely, that highly concentrated solutions are quickly attained and the reaction rate quickly becomes very low. Because glass is metastable and cannot equilibrate with the leachate, the glass corrodes at a "final reaction rate" even after the solution becomes saturated $[151,168]$; the continued corrosion has been attributed to a "residual" or "contextual" reaction affinity [166]. Continued glass reaction under saturated conditions (where the reaction affinity becomes small), even though the reaction rate may be immeasurably low $[24,161,166,168]$.

Tests performed in highly concentrated solutions have shown that the low residual rate attained under saturated conditions does not necessarily represent the long-term reaction rate. An increase in the glass reaction rate has been observed in tests where secondary phases have precipitated from the fluid after long reaction times $[65,67,116]$. For example, in Fig. 37a, the boron concentration suddenly increases after 182 days of reaction in tests conducted at $20,000 \mathrm{~m}^{-1}$. This change is indicative of an increase in the reaction rate coincident with secondary phase formation. Such increases can be explained by the effects of secondary phase formation on the solution chemistry, which will result in an increased reaction rate if the solubility limit of silicic acid is maintained at a value less than the saturation concentration, or if the $\mathrm{pH}$ increases [143]. Enhanced reaction has also been observed in the presence of clays [204]: silicon is removed from solution as more clay is formed, so the solution chemistry is affected. The effect of added clays is different from that of clays which form during glass corrosion. Clays formed as alteration layers on the glass surfaces during corrosion do not affect the corrosion rate. Although silicic acid has been identified as the dominant species affecting the reaction $[153,154,205]$, recent results suggest that other solution species may also influence the observed reaction rates under some conditions [24,71]. The relative importance of the silicic acid concentration on the glass corrosion rate may depend on the glass composition and the reaction conditions. Thus, the effect of secondary phase formation on other solution components may also affect the glass corrosion rate.

\subsection{Summary}

The observed effects of the SA/V ratio used in static leach tests can be accounted for by the differences in the extent of dilution of corrosion products, especially the $\mathrm{pH}$ and the silicic acid concentration. While the $\mathrm{pH}$ and silicic acid concentrations will be affected at all SA/V, the effects of the $\mathrm{pH}$ on the corrosion rate become experimentally measurable only when the $\mathrm{pH}$ exceeds about $\mathrm{pH} 9$. Below $\mathrm{pH} 9$, the SA/V primarily affects only the silicic acid concentration. The results of tests conducted at different $S A / V$ can be directly related through the product of $\mathrm{SA} / \mathrm{V}$ and the reaction time only if the $\mathrm{pH}$ remains below about $\mathrm{pH}$ 9. If the $\mathrm{pH}$ exceeds about $\mathrm{pH} 9$, then the difference in $\mathrm{pH}$ values of the tests being related must be accounted for analytically. 


\subsection{OBSERVED EFFECTS OF THE FLOW RATES IN DYNAMIC LEACH TESTS}

The difference between static and dynamic tests is that in dynamic tests, the solution concentrations of dissolved glass components do not become concentrated in the solution in contact with the corroding glass as quickly (or as completely) as in static tests because the leachate solution is continuously or periodically replaced with fresh leachant. The effect of the leachant flow rate in dynamic tests is like that of the SA/V in static tests: it primarily affects the silicic acid concentration and the $\mathrm{pH}$ at the glass surface. As described in Section 2.5.2, dynamic conditions can be achieved by either pumping solution through a reaction cell or periodically removing a portion of the leachate solution an replacing it with an equal volume of fresh leachant. The rate at which solution is pumped through the cell or the extent of leachant replenishment, which can be described as an effective flow, determines how the solution chemistry changes as the glass dissolves. Higher flow rates and more frequent and more complete replenishments maintain lower concentrations of all released glass components. Solutions contacting the glass in tests at high flow rates maintain the initial leachant conditions, while tests at low flow rates allow reaction products to accumulate in the solution. The effect of flow rate on glass corrosion is well illustrated by the results of tests performed using SRL 131 glass [206]. Figure 39 shows the measured mass loss of the sample as a function of reaction time for tests conducted using deionized water at various flow rates. Tests run using flow rates less than $1.0 \mathrm{~mL} / \mathrm{h}$ show reaction rates that continually decrease with the reaction time, similar to static tests, while tests at higher flow rates show reaction rates that are linear in time and increase with the flow rate up to a rate of about $50 \mathrm{~mL} / \mathrm{h}$. Tests at $100 \mathrm{~mL} / \mathrm{h}$ react at the same rate as tests $50 \mathrm{~mL} / \mathrm{h}$, which is the maximum rate under these temperature and $\mathrm{pH}$ conditions (see below).

From this data, the forward rate is about $2.5 \mathrm{~g} / \mathrm{m}^{2} /$ day.

The glass corrosion rate can be related to the leachant flow rate by a simplified mass balance equation written in terms of the flux of silicic acid from the glass surface. Consider the reaction of a glass in a well-mixed reactor having a cell volume $V$ with a solution that passes through the cell at a flow rate $\phi$. The change in the silicic acid concentration in the cell over time depends on the amount added to the cell as it is released from the glass and the amount removed from the cell due to solution flow. These amounts are given by Eq. 18

$$
\frac{\mathrm{d}\left[\mathrm{H}_{4} \mathrm{SiO}_{4}\right]}{\mathrm{dt}}=\frac{\mathrm{SA}}{\mathrm{V}} \mathrm{k}_{+}\left(1-\frac{\left[\mathrm{H}_{4} \mathrm{SiO}_{4}\right]}{\left[\mathrm{H}_{4} \mathrm{SiO}_{4}\right]_{\text {sat }}}\right)-\frac{\phi\left[\mathrm{H}_{4} \mathrm{SiO}_{4}\right]}{\mathrm{V}}
$$

where the first term on the right-hand side represents the release of silicic acid from the glass (from Eq. 14), and the second term represents the removal of silicic acid from the reaction cell via flow. An additional term is needed if the leachant contains silicic acid. Equation 18 will not apply under very low flow conditions, in which mass transport through the solution will become important. Under constant flow conditions, a steady state may be attained, in which the silicic acid concentration within the cell remains constant. The steady-state silicic acid concentration will depend on the flow rate, and can be determined from Eq. 18 by setting $d\left[\mathrm{H}_{4} \mathrm{SiO}_{4}\right] / \mathrm{dt}=0$ and solving for $\left[\mathrm{H}_{4} \mathrm{SiO}_{4}\right]$. In this context, "steady state" refers to the condition where the rate at which silicic acid is released from the glass is equal to the rate at which it is removed from the reaction cell. The expression for the steady-state silicic acid concentration, which is represented by the term $\left[\mathrm{H}_{4} \mathrm{SiO}_{4}\right]_{s s}$, can then be substituted into Eq. 13 to obtain 
(a)

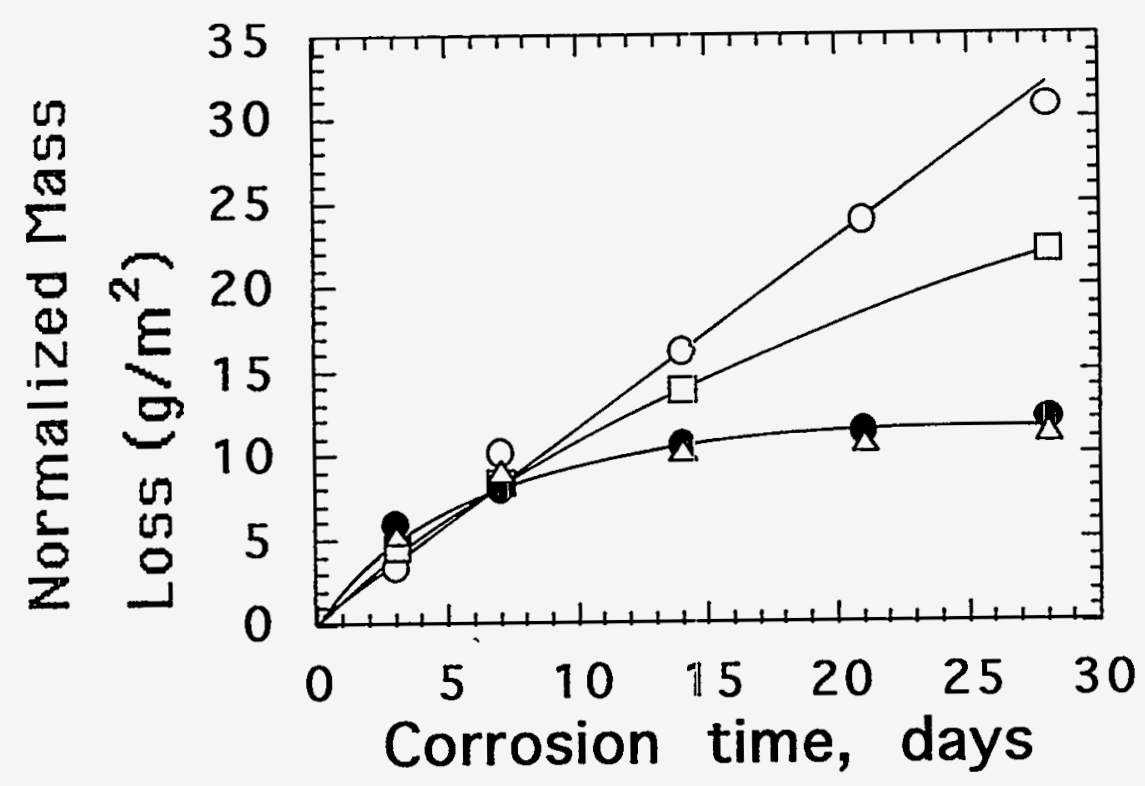

(b)

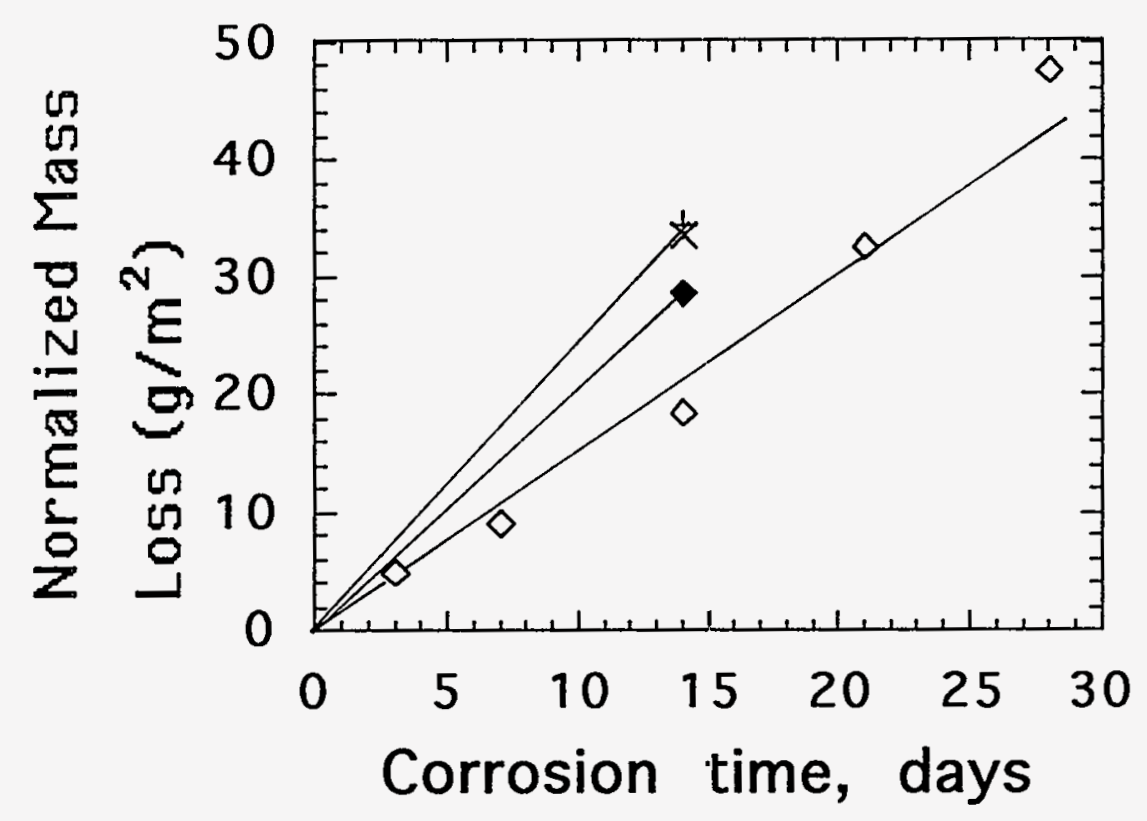

Fig. 39. Mass Loss vs. Corrosion Time for SRL 131 Glass at $90^{\circ} \mathrm{C}$ at Various Flow Rates:

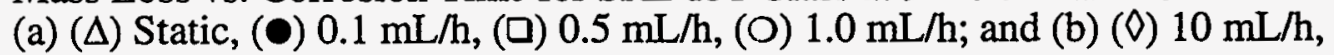

(४) $25 \mathrm{~mL} / \mathrm{h},(+) 50 \mathrm{~mL} / \mathrm{h},(x) 100 \mathrm{~mL} / \mathrm{h}$ (after [206]) 


$$
\frac{\mathrm{dn}\left[\mathrm{H}_{4} \mathrm{SiO}_{4}\right]_{\mathrm{ss}}}{\mathrm{dt}}=\frac{\mathrm{SA} \mathrm{k}_{+} \phi}{\left(\frac{\mathrm{SA}}{\left[\mathrm{H}_{4} \mathrm{SiO}_{4}\right]_{\text {sat }}}\right) \mathrm{k}_{+}+\phi}
$$

where $\mathrm{n}\left(\mathrm{H}_{4} \mathrm{SiO}_{4}\right)_{\mathrm{ss}}$ is the number of moles of silicic acid released from the glass at steady state. Under sufficiently high flow rate conditions such that $\phi \gg\left(\mathrm{SA} /\left[\mathrm{H}_{4} \mathrm{SiO}_{4}\right]_{\text {sat }}\right) \mathrm{k}_{4}$, the glass corrosion rate becomes

$$
\frac{\mathrm{dn}\left[\mathrm{H}_{4} \mathrm{SiO}_{4}\right]}{\mathrm{dt}}=\mathrm{SA} \mathrm{k}_{+}
$$

and is independent of the flow rate. Under low flow rate conditions, such that $\phi<<$ $\left(\mathrm{S} /\left[\mathrm{H}_{4} \mathrm{SiO}_{4}\right]_{\text {sat }}\right) \mathrm{k}_{4}$, the glass corrosion rate becomes

$$
\frac{\mathrm{dn}\left[\mathrm{H}_{4} \mathrm{SiO}_{4}\right]}{\mathrm{dt}}=\left[\mathrm{H}_{4} \mathrm{SiO}_{4}\right]_{\text {sat }} \phi
$$

and is proportional to the flow rate. At very low flow rates, the glass corrodes faster than the flow rate, a steady-state concentration is not maintained, and Eq. 18 becomes equivalent to Eq. 14 . It is important to note that it is not the actual flow rate that determines whether a system is in the low or high flow limit, but the difference between the solution flow and the intrinsic corrosion rate. This means that the response of a glass to changes in the solution flow will depend on its composition.

The results obtained by Adiga et al. [206] are replotted in Fig. 40 as the glass reaction rate (i.e., the slopes of the lines in Figs. 39a and 39b) against the leachant flow rate. This plot shows the behavior predicted by Eqs. 20 and 21: at high flow rates the glass corrodes at a constant rate, and at lower flow rates it corrodes at a rate that depends on the flow rate. The constant rate achieved at high flow rates is characteristic of the glass composition, the leachant composition, and the reaction temperature. From Eq. 20, the maximum rate is proportional to the rate coefficient for glass dissolution. From further analysis of these test results, Apted and Adiga concluded that all flow rates tested were high relative to the glass dissolution rate and that the test conditions did not simulate expected repository conditions [142]. They also pointed out that the crossover from transport control to surface reaction control occurs at flows higher than expected in a repository and that radionuclide release rates would be overestimated at high flows. Finally, Apted and Adiga noted that the thickness of a static layer of solution at the glass surface is expected to decrease at higher flow rates. Because this layer provides a diffusion barrier to the transport of released glass components away from the glass surface, they suggested that a decrease in the layer thickness is expected to result in and increase the glass dissolution rate. The effect of such a static fluid layer on the glass corrosion rate is analogous to the effect of the flow rate: thicker barriers would lead to increased concentrations of glass corrosion products at the glass surface and are expected to have the same effect as lower flow rate, namely they are expected to slow the corrosion.

The high flow rates (relative to glass corrosion rates) attained in continuous flow apparatuses are not relevant to any anticipated repository environments, which are usually engineered to prevent water from contacting waste packages. Difficulties associated with attaining low flow rates with liquid pumps in laboratory tests have led to the use of partialreplenishment tests (sometimes called pulsed-flow tests), in which some or all of the leachate is periodically removed from the reaction vessel and replaced with fresh leachant. Of coursc, periodic replenishment only becomes strictly equivalent to continuous flow in the limit where the 


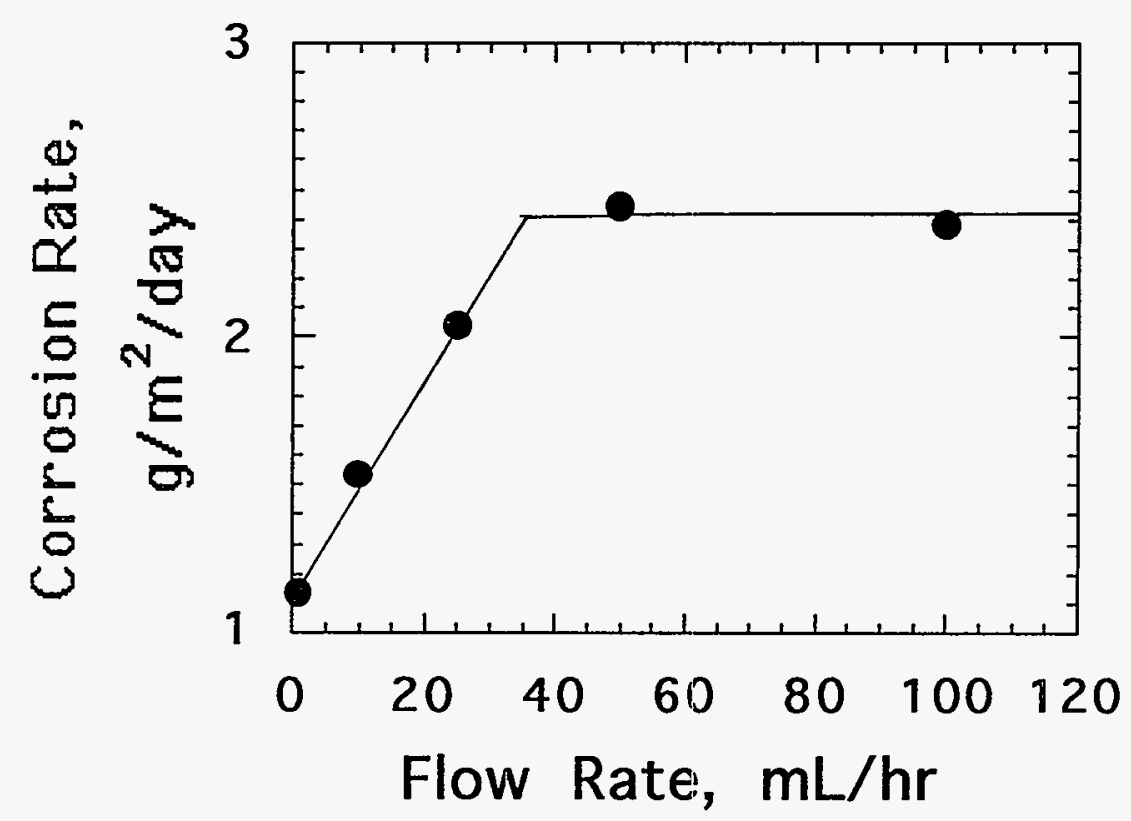

Fig. 40. Results Obtained by Adiga et al. Plotted as Glass Corrosion Rate vs. Flow (after [164])

replacement period becomes infinitesimal. The frequency and extent of replacement can be related to an effective flow rate through the number of exchanged volumes in the reaction cell per year [207,208]. (The fact that an effective flow can be defined for periodic replenishment does not address the issue of whether or not the glass corrodes in the same manner in flow tests and in periodic replenishment tests.) If the volume of the reaction cell is $\mathrm{V}$, and the amount of leachate removed and replaced with an equal amount of fresh leachant is $\Delta \mathrm{V}$, and the time between replacements is $\Delta t$, then a residence time can be defined as $T_{r}=\Delta t(\Delta V / V)$. The residence time is the time required for complete replacement of the vessel volume. For example, if one-fourth of the volume is replaced every 28 days, then the entire volume will be replaced after four exchanges and a residence time of 112 days. Tests have been conducted to determine the dependence of the extent of corrosion on $\Delta t$ and $\Delta V / V$ at a constant value of $T_{r}$ [127]. The results, which are shown in Fig. 41, show that larger replacement volumes and longer replacement times lead to greater corrosion up to about 168 days. The scatter in the data beyond 168 days is similar to the differences measured at shorter times. Barkatt et al. interpreted these results as demonstrating that the corrosion rate depends on $T_{r}$ after long reaction times, but not on the exchange period $\Delta t$ or the exchange fraction $\Delta V / V$ independently [127]. However, the scatter in the data weakens this conclusion. The assumed equivalence of common products of $T_{r}$ from different $\Delta \mathrm{t}$ and $\Delta \mathrm{V}$ is tantamount to assuming the equivalence of common products of $(\mathrm{SA} / \mathrm{V}) \mathrm{t}$, an assumption that was demonstrated earlier to fail in most cases because it neglects a $\mathrm{pH}$-dependence of the glass corrosion. In these tests, $t$ is replaced with $\mathrm{T}_{\mathrm{r}}$ and $\mathrm{V}$ with $\Delta \mathrm{V}$. The same arguments against using (SA/V)t as a scaling factor apply to $(\mathrm{SA} / \Delta \mathrm{V}) \mathrm{T}_{\mathrm{r}}$ as a scaling factor . In practice, the effects of $\mathrm{pH}$ may be inconsequential at near-neutral values and under dilute conditions, as seen in the results of Pederson et al. [72]. It may be expected that the effects of variations in the exchange volume fraction and time that are observed in partial replenishment tests will depend on the SA/V of the test system and will become more important as the SA/V increases. The test results shown in Fig. 7 were obtained at an SA/V of about $970 \mathrm{~m}^{-1}$. The $\mathrm{pH}$ 


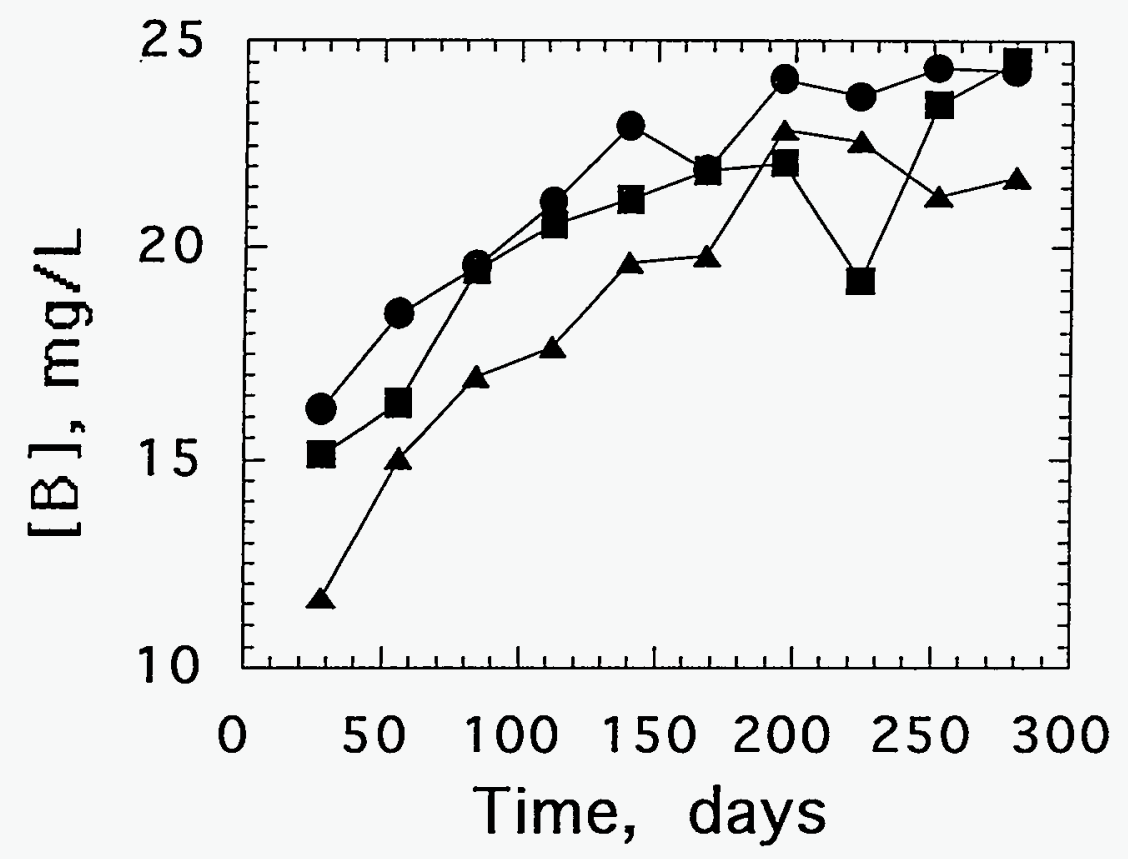

Fig. 41. Measured Boron Concentration in Partial Replenishment Tests Conducted at Constant $\mathrm{T}_{\mathrm{r}}=112$ Days: $(\bullet) \Delta \mathrm{t}=28$ days, $\Delta \mathrm{V} / \mathrm{V}=0.25 ;(\boldsymbol{\Pi}) \Delta \mathrm{t}=7$ days, $\Delta \mathrm{V} / \mathrm{V}=0.0625$

(A) $\Delta \mathrm{t}=3.5$ days, $\Delta \mathrm{V} / \mathrm{V}=0.0312($ after [127])

values were not reported, although the measured differences in the boron concentrations as a function of $\Delta \mathrm{V}$ suggest the $\mathrm{pH}$ was greater than 9 . It should be pointed out that the volume of leachate that reacts with the sample is different than the volume of leachate in the test cell at any time. This distinction is not accounted for in the treatment of the data. Likewise, the dilution of the leachate that occurs upon every replenishment cycle is not accounted for. This change may be important when the solution is near saturation.

The cycling of the solution between dilute and near saturation conditions may affect the alteration of the glass surface. While the alteration layers formed on nuclear waste glasses do not significantly retard the glass corrosion, they do interact with the solution and may affect the solution chemistry, which does affect the glass corrosion rate. The impact of cycling the solution chemistry may affect the evolution of the surface alteration phases and continued glass corrosion rate.

Despite questions of the equivalence of different replacement volumes and periods, the results of partial-replenishment tests can be described by the effects of flow predicted in Eqs. 20 and 21. Figure 42 shows the results of partial-replenishment tests with SRL 131 glass conducted in deionized water at $90^{\circ} \mathrm{C}$ [124]. These tests show the corrosion rate to increase with the flow until a maximum rate of about $2.5 \mathrm{~g} / \mathrm{m}^{2} /$ day occurs at sufficiently high flow rates. This maximum rate is the same as that found in continuous flow tests conducted with SRL 131 glass [206]. Appropriate selection of exchange volumes and periods is seen to mimic the expected solution behavior at very low effective flow rates not attainable in continuous flow apparatuses. The results show the corrosion rate to continually decrease as the flow decreases. 


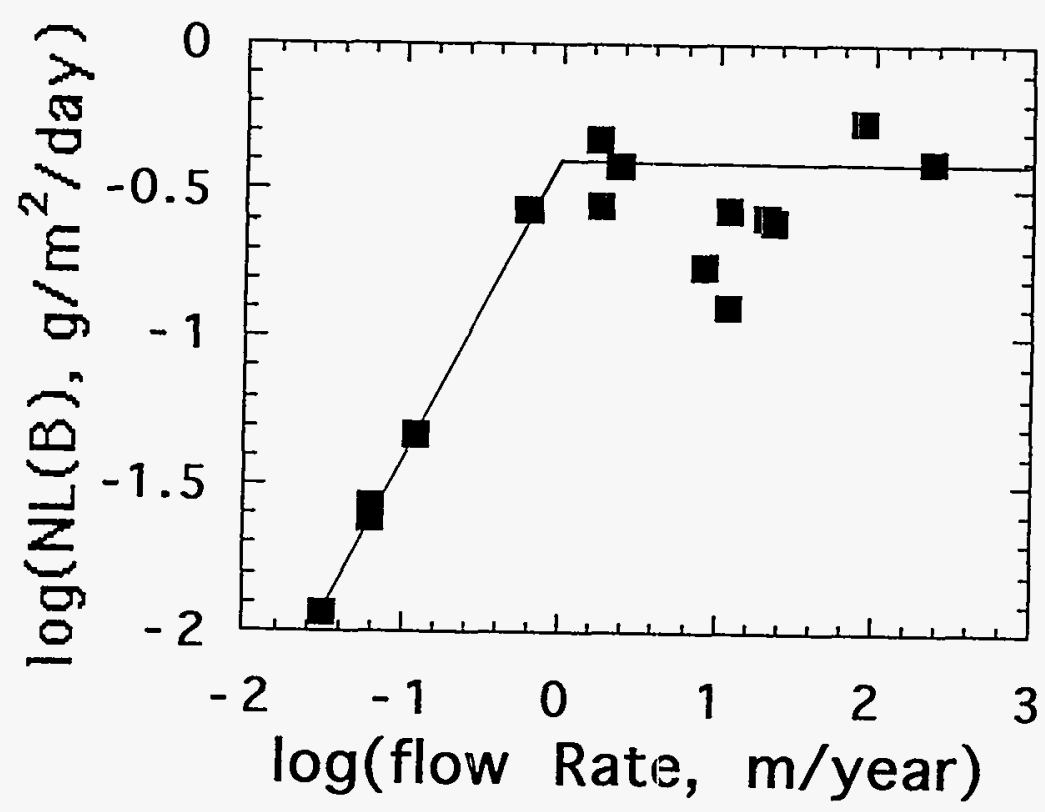

Fig. 42. Boron Release from SRL 131 Glass as a Function of Flow Rate as Simulated by Periodic Replenishment of the Solution (after [124])

While the expected trend toward Stage II of corrosion (apparent saturation) is observed in these tests, uncertainties remain regarding the effects of the cycling of the leachate between saturated and diluted conditions on the corrosion behavior. That is, while the average solution volume over the test period can be related to that of a test conducted at a low flow rate the sample has been subjected to very different solution chemistries than those of a low flow rate test. Probably as important as comparing the results of periodic replacement tests at high effective flow rates to tests using a constant flow are their comparison at low effective flow rates to static tests. The effects of varying the solution chemistry through periodic replenishments are more significant under these corrosion conditions, where the periodic dilution hinders the nucleation of secondary phases from the saturated solution by periodically diluting the solution and lowering the $\mathrm{pH}$. These effects prevent the natural evolution of the corrosion process and result in a misrepresentation of the long-term corrosion rate. That is, the corrosion rate prior to the formation of secondary phases does not represent the long-term corrosion rate.

The complexities of periodic dilution of the solution have also prevented the use of test data in corrosion models. The primary usefulness of the test results appears to be verification of the effects of the flow rate and the solution chemistry on glass corrosion behavior at intermediate flow rates. While the forward rate determined at high effective flows is consistent with that measured using a constant flow devise, the test is not well-suited for measuring the forward rate. In the limit of replenishment schedules the test reduces to an MCC-1 or PCT type test, depending on if monolithic or crushed glass is used. Replenishment of the solution would not benefit the measurement of the forward rate. The forward rate is better measured using single-pass flow through or Soxhlet tests. 
Although static tests may most faithfully simulate repository environments where groundwater flow will be extremely low, tests performed at high leachant flows provide conditions in which waste forms corrode free from interactions with corrosion products. These tests allow much-needed characterization of the effects of various solution species and the solution $\mathrm{pH}$ on the corrosion process. A knowledge of the forward rate coefficient and how it depends on the solution chemistry is of key importance to defining the corrosion behavior of waste glasses and predicting their long-term behavior. Tests in flowing leachants used to determine the effects of solution chemistry on glass corrosion have been discussed previously (see Sections 3.2-3.4). 


\subsection{DETERMINATION OF MODEL PARAMETERS FROM LABORATORY TESTS}

The observed effects of the solution chemistry, the results of static tests conducted at different $\mathrm{SA} / \mathrm{V}$, and the flow rates used in dynamic tests on glass corrosion have lead to a mechanistic description of the processes that result in glass corrosion. By utilizing different tests and different experimental conditions, various reactions have been highlighted and characterized. Analytical models based on descriptions of these processes can be used to predict corrosion behavior of high-level waste glasses over a wide range of environmental conditions and over long time periods. Models are needed because it is not possible to measure experimentally the durabilities of high-level waste glasses for the many tens of thousands of years that they must control the release of radionuclides or under the range of environments that may exist in different disposal systems over time.

The corrosion of most high-level waste glasses can be described as occurring in three stages. These stages correspond to limiting conditions in the analytical description of the glass corrosion rate given in Eq. 14.

$$
\frac{\mathrm{d}\left[\mathrm{H}_{4} \mathrm{SiO}_{4}\right]}{\mathrm{dt}}=\left(\frac{\mathrm{SA}}{\mathrm{V}}\right) \mathrm{k}_{+}\left(1-\frac{\left[\mathrm{H}_{4} \mathrm{SiO}_{4}\right]}{\left[\mathrm{H}_{4} \mathrm{SiO}_{4}\right]_{\text {sat }}}\right)
$$

The first stage occurs when the solution concentrations of glass components (primarily silicic acid for borosilicate waste glasses) are so low that they do not affect the corrosion reactions. The glass corrodes at its forward rate under these conditions, which depends only on the solution $\mathrm{pH}$ and the temperature. Experiments have shown that alkali metals and boron are preferentially leached from the glass under these conditions and that the solution $\mathrm{pH}$ increases. The increased $\mathrm{pH}$ results in a higher corrosion rate. The second stage occurs as the solution concentration of silicic acid increases and begins to affect the glass corrosion rate. The increased silicic acid concentration slows the glass corrosion rate through the affinity term $\left(1-\left[\mathrm{H}_{4} \mathrm{SiO}_{4}\right] /\left[\mathrm{H}_{4} \mathrm{SiO}_{4}\right]_{\text {sat }}\right)$. The rate continually slows during the second stage and approaches an apparent minimum rate as the affinity term approaches zero. The minimum rate depends on the glass composition, temperature, and the solution $\mathrm{pH}$. The third stage of corrosion occurs when secondary phases precipitate from the leachate solution to establish solubility limits for important glass components (primarily silicic acid for borosilicate waste glasses). Changes in the solution chemistry due to secondary phase formation then affect the glass corrosion rate through the affinity term as $\left[\mathrm{H}_{4} \mathrm{SiO}_{4}\right]$ is maintained at a value less than $\left[\mathrm{H}_{4} \mathrm{SiO}_{4}\right]_{\text {sat }}$ by the secondary phases. In some systems, materials that are present throughout the test may affect the solution chemistry in such a way that the minimum rate is never reached. This situation occurs in the presence of clays that may be present in backfill and ductile iron, which consume silicon from the solution as it is released from the glass.

Given this description of the solution control of the corrosion rate, corrosion models must account for the intrinsic corrosion rate in highly dilute solutions, the effects of the solution $\mathrm{pH}$, the decrease in the corrosion rate as the silicic acid solution concentration increases, and the effects of secondary phases and other materials on the corrosion rate. Most current models of waste glass corrosion are based on variations of the model developed by Grambow $[153,154]$ (see Section 3). Implementation of these models requires the determination of several model parameters through the regression of experimental data. It is important to understand how information obtained from the experiments or tests performed relate to the corrosion mechanism and to the corrosion model to ensure that the data are being used correctly. 
A simple form of the rate equation is used in current simulations of glass corrosion; it can be written in a form similar to that of Eq. 14:

$$
\frac{\mathrm{d}[\mathrm{B}]}{\mathrm{dt}}=(\mathrm{SA} / \mathrm{V}) \mathrm{k}_{+}\left(1-\frac{\left[\mathrm{H}_{4} \mathrm{SiO}_{4}\right]}{\left[\mathrm{H}_{4} \mathrm{SiO}_{4}\right]_{\text {sat }}}\right)+\mathrm{R}_{\infty}
$$

where the rate is expressed in terms of the solution concentration of boron rather than silicic acid and the term $R_{\infty}$ has been added to account for the nonzero reaction rate under "saturated" conditions. The rate is expressed in terms of the measured solution concentration of boron rather than the measured solution concentration of silicon because silicon is commonly incorporated into secondary phases so that the solution concentration of silicon often does not represent the extent of glass corrosion (see also Section 2.2). The affinity term is nevertheless determined by the solution concentration of silicon, regardless of whether or not secondary phases form. Note that this is the rate of change of the solution concentration, and the rate of glass corrosion is given by this rate divided by the surface area. If other phases are present in the system or if secondary phases form as the glass corrodes that limit the silicic acid concentration in solution such that the affinity term remains nonzero, then the rate will likely be determined by the first term on the right side. Otherwise, the first term will approach zero as the solution becomes saturated with respect to silicon, and the long-term rate will be given by $\mathrm{R}_{\infty}$.

To solve Eq. 22 as the glass corrodes, one must know $\mathrm{k}_{+},\left[\mathrm{H}_{4} \mathrm{SiO}_{4}\right]_{\text {sat }}$, and $\mathrm{R}_{\infty}$. The values of these parameters are determined experimentally either by regression to the test data or by conducting separate experiments designed to highlight and measure the values of specific parameters. Separate tests are required to determine the parametric values for different glass compositions, and under different temperature and $\mathrm{pH}$ conditions. The $\mathrm{pH}$ dependence of the rate coefficient can be written as $\mathrm{k}_{+}=\mathrm{k}_{\mathrm{f}}\left[\mathrm{OH}^{-}\right]^{\mathrm{h}}$, where $\mathrm{k}_{\mathrm{f}}$ and $\mathrm{h}$ must be determined in separate tests (see Section 3.3). The term $R_{\infty}$ is an ad hoc corrective value to account for the difference between the long-term rate predicted in Eq. 22 and experimental observations, and is defined so as to improve the fit of the equation to specific long-term data. Tests at high SAN are used to characterize $R_{\infty}$ [167]. The values of model parameters determined by using experimental results include both measurement errors and errors in the model. Thus, the value determined for $\mathrm{R}_{\infty}$ includes error in the experimental measurement of the corrosion rate and error in the definition of $\mathrm{R}_{\infty}$ and expressing the rate in terms of Eq. 22. The values of $\left[\mathrm{H}_{4} \mathrm{SiO}_{4}\right]_{\text {sat }}$ and $\mathrm{R}_{\infty}$ are usually found to depend on the test conditions and the $\mathrm{pH}$.

Two limiting cases with regard to Eq. 22 can be defined: highly dilute solutions for which the affinity is near one (i.e., where $\left[\mathrm{H}_{4} \mathrm{SiO}_{4}\right] /\left[\mathrm{H}_{4} \mathrm{SiO}_{4}\right]_{\text {sat }}$ is near zero), and highly concentrated or "saturated" solutions, for which the affinity is near zero (i.e., where $\left[\mathrm{H}_{4} \mathrm{SiO}_{4}\right] /\left[\mathrm{H}_{4} \mathrm{SiO}_{4}\right]_{\text {sat }}$ is near one). Under the limit where $\left[\mathrm{H}_{4} \mathrm{SiO}_{4}\right]$ approaches zero, the rate equation can be written as

$$
\frac{\mathrm{d}[\mathrm{B}]}{\mathrm{dt}}=(\mathrm{SA} / \mathrm{V}) \mathrm{k}_{+}+\mathrm{R}_{\infty}
$$

Dynamic flow-through tests provide an excellent measure of the forward reaction rate. The use of these tests to measure the corrosion rates under various leachant chemistries has been discussed previously (Sections 3.2 and 3.3). Tests performed at very low values of (SA/V)t, which maintain dilute solutions, provide another measure of the forward reaction rate. These tests approach a maximum rate as $(\mathrm{SA} / \mathrm{V}) \mathrm{t}$ decreases, which represents the forward reaction rate. (The value of $R_{\infty}$ is generally several orders of magnitude less than $k_{+}$and can be ignored under the test conditions represented by Eq. 23.) 
Under the limit where $\left[\mathrm{H}_{4} \mathrm{SiO}_{4}\right]$ approaches $\left[\mathrm{H}_{4} \mathrm{SiO}_{4}\right]_{\text {sat }}$, the rate equation can be written as

$$
\frac{\mathrm{d}[\mathrm{B}]}{\mathrm{dt}}=\mathrm{R}_{\infty}
$$

Solutions can be saturated by doping the leachant with silicic acid or by conducting experiments under conditions in which the solution rapidly approaches "saturation" conditions. The latter method will generate solutions with high concentrations of other glass components that may also affect the corrosion rate. The increase in solution concentrations can be accelerated by conducting tests at high $\mathrm{SA} / \mathrm{V}$ ratios, although higher solution $\mathrm{pH}$ values will also occur in tests at high SA/V that will also accelerate the corrosion. The effects of the $\mathrm{pH}$ and the silicic acid concentration on the measured reaction rate cannot be separated based on static test data. Tests conducted in silicic acid-doped solutions likely will not provide a representative measure of the long-term corrosion rate because the secondary phases which control the long-term rate require other glass components be present in solution as well as silicic acid. Unnatural solution chemistries may result in secondary phase formation, but the resulting solution chemistries will be different than those that will result be dissolution of the glass and will affect the glass corrosion rates differently. That is, the silicic acid concentration established in the silicic aciddoped leachant will be different than that established in the leachate generated by dissolution of the glass. The different silicic acid concentrations will result in different long-term corrosion rates.

Reaction parameters can be determined from the results of static leach tests by plotting the corrosion rate against the silicic acid activity (or concentration). In such a plot, $\mathrm{k}_{+}$is the rate when the silicic acid activity is zero, and the saturation concentration and residual rate are estimated from the solution chemistry and corrosion rate measured at high silicic acid activities. These limits are illustrated in Fig. 43 [154,164]: the rate at a silicic acid activity of zero gives the forward rate, which is about $1.5 \mathrm{~g} / \mathrm{m}^{2} /$ day, and the constant rate at silicic acid activities greater than about $10^{-4}$ gives the residual rate, which is about $0.0017 \mathrm{~g} / \mathrm{m}^{2} /$ day. Scatter in test data leads to large uncertainties in the regressed values in this approach. The values determined by this method are probably not sufficiently reliable for use in performance assessment calculations, which are highly sensitive to the long-term corrosion rate [185].

Determination of the rate coefficient, $\mathrm{k}_{+}$, is straightforward from static leach tests conducted for short time periods, since these are likely to have the lowest silicic acid concentrations and so are likely to have affinity terms nearest to one. The rate coefficient can be obtained from static leach test data by combining the forward rate expression in Eq. 23 and the normalized mass loss function in Eq. 10 as

$$
\mathrm{k}_{+}=\text {rate }=\frac{\mathrm{NL}_{\mathrm{i}}}{\mathrm{t}}=\frac{\mathrm{c}_{\mathrm{i}}}{(\mathrm{SA} / \mathrm{V}) \mathrm{f}_{\mathrm{i}} \mathrm{t}}
$$

where $t$ is the reaction time and the other variables have the same meaning as in Eq. 10. Tests should be conducted for several time periods to ensure that the solution remains dilute enough that the affinity term remains near one. Separate tests in $\mathrm{pH}$-buffered solutions are required to ascertain the $\mathrm{pH}$ dependence of the rate coefficient $\mathrm{k}_{+}$.

The regression of residual rate from test data is well described by Curti [180], and the data and methodology are reproduced here. Data shown in Fig. 44 were obtained in static leach tests using a British glass referred to as MW and deionized water at $90^{\circ} \mathrm{C}$ and at an SA/V ratio of $1320 \mathrm{~m}^{-1}[180]$. The residual rate is determined from the measured release of highly soluble 


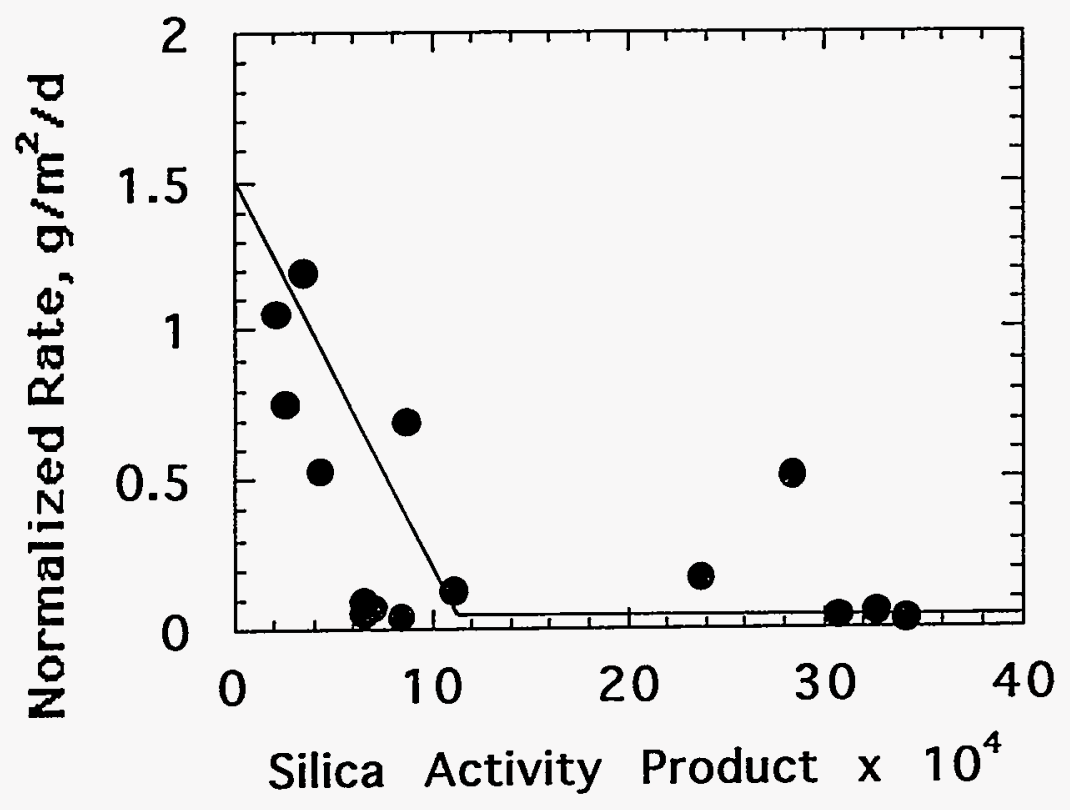

Fig. 43. Corrosion Rate (as Measured by Boron Release) vs. Ion Activity Product of Silicon (after [154])

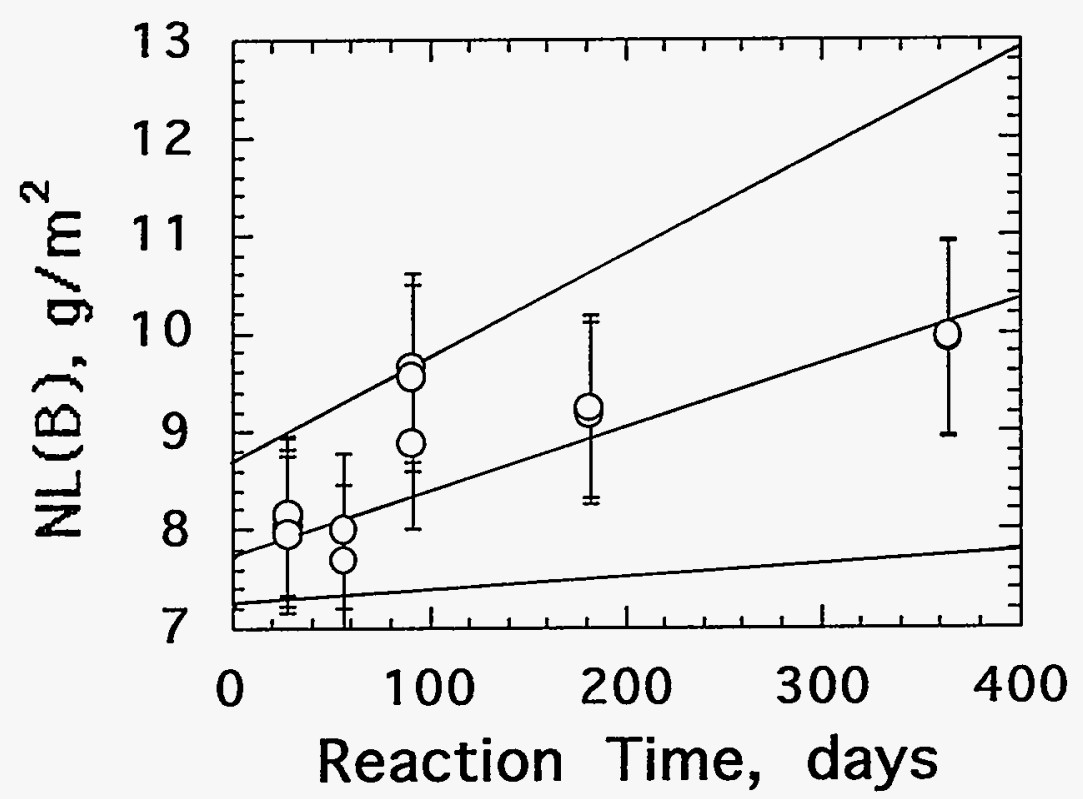

Fig. 44. Normalized Boron Mass Loss from MW Glass vs. Reaction Time (after [180]) 
glass components after silicic acid has become saturated. This is best approximated using tests conducted for the longest reaction times. Curti used test data from tests conducted longer than 28 days to determine the residual rate for corrosion of MW glass, as shown in Fig. 44. The slope of the fitted line gives the residual rate, which is reported to be $0.006 \mathrm{~g} / \mathrm{m}^{2} /$ day from Fig. 44 . However, as reported by Curti, this value represents the mean value while the data include a range of between 0.0014 and $0.0105 \mathrm{~g} / \mathrm{m}^{2} /$ day at the $95 \%$ confidence level. Clearly, a much lower residual rate would be obtained by considering only data from tests conducted for 91 days or longer (the three longest time periods tested). In fact, the residual rate according to these data is essentially zero. This variation demonstrates the high degree of subjectivity in this approach. Again, the wide range of rates is probably not acceptable for conducting performance assessments. The silicic acid saturation concentration that is needed in the rate equation is given by the measured silicic acid concentration in the tests used to determine the residual rate, corrected for dissociation via

$$
\left[\mathrm{H}_{4} \mathrm{SiO}_{4}\right]^{\circ}=\frac{[\mathrm{Si}]}{\left(1+10^{\mathrm{pH}-\mathrm{pK} 1}+10^{2 \mathrm{pH}-\mathrm{pK2}}+10^{3 \mathrm{pH}-\mathrm{pK} 3}\right)}
$$

where $\left[\mathrm{H}_{4} \mathrm{SiO}_{4}\right]^{\circ}$ is the concentration of undissociated silicic acid, [Si] is the measured amount of silica in the solution, $\mathrm{pH}$ is the measured $\mathrm{pH}$ corrected to the test temperature, and $\mathrm{pK} 1, \mathrm{pK} 2$, and $\mathrm{pK} 3$ are the dissociation constants for silicic acid at the test temperature.

For conditions under which the glass corrodes at a constant rate, Eq. 22 predicts that a plot of $\log [\mathrm{B}]$ versus $\log \{(\mathrm{SA} / \mathrm{V}) \mathrm{t}\}$ will be a line with a slope equal to one and a $y$-intercept that is proportional to the rate $[67,209,210]$. The glass is expected to react at a constant rate in the limits (1) when the silicic acid concentration is near zero, where the y-intercept corresponds to the forward rate, and (2) when silicic acid has attained its saturation concentration, where the yintercept corresponds to the residual rate. Experimental data are expected to converge to these lines at both low and high values of $(\mathrm{SA} / \mathrm{V}) \mathrm{t}$. Note that the values of $\mathrm{k}_{+}$and $\mathrm{R}_{\infty}$ determined from the data will both depend on the solution $\mathrm{pH}$ of the tests under the limiting conditions of low and high $(\mathrm{SA} / \mathrm{V}) \mathrm{t}$, respectively. The solution $\mathrm{pH}$ is expected to be different in the two limits for most alkali borosilicate waste glasses. Hence, the differences in the rates will be due to both the different solution concentrations of silicic acid and the different $\mathrm{pH}$ values at the two limits.

The results of MCC-1 (at $10 \mathrm{~m}^{-1}$ ) and MCC-3 (at $2000 \mathrm{~m}^{-1}$ ) tests with four glasses produced during development of a waste glass for West Valley Demonstration Project and the DWPF glass SRL 165 were plotted as $\log [B]$ vs $\log [(\mathrm{SA} / \mathrm{V}) \mathrm{t}]$ by Strachan [211]. Strachan actually used (SA/V)t/10 for the $\mathrm{x}$-axis so that the data were plotted as "equivalent to $10 \mathrm{~m}^{-1}$ ". The plot is reproduced in Fig. 45. The forward rate coefficient $\mathrm{k}_{+}$determined from limiting slope at small equivalent time, and the "saturated rate," $\mathrm{R}_{\infty}$ (represented as $\mathrm{k}+$ in [211]) determined from the limiting slope at large equivalent time are included in the plot. The forward rates of the five different glasses are the same, within a factor of two, while the "saturated rates" differ by more than a factor of 15. Strachan pointed out that the "saturation rates" of all glasses were higher than those needed to conform to the maximum allowable release according to criterion of the Nuclear Regulatory Commission 10 CFR 60.

Note that Strachan ignored the data point for WV205 glass at the longest equivalent time tested (3920 d equivalent), which had a significantly higher boron concentration than the previous test. While tests at longer equivalent times are needed to verify the long-term behavior of WV205 glass, tests with SRL 202 glass have shown an identical sudden increase at long 


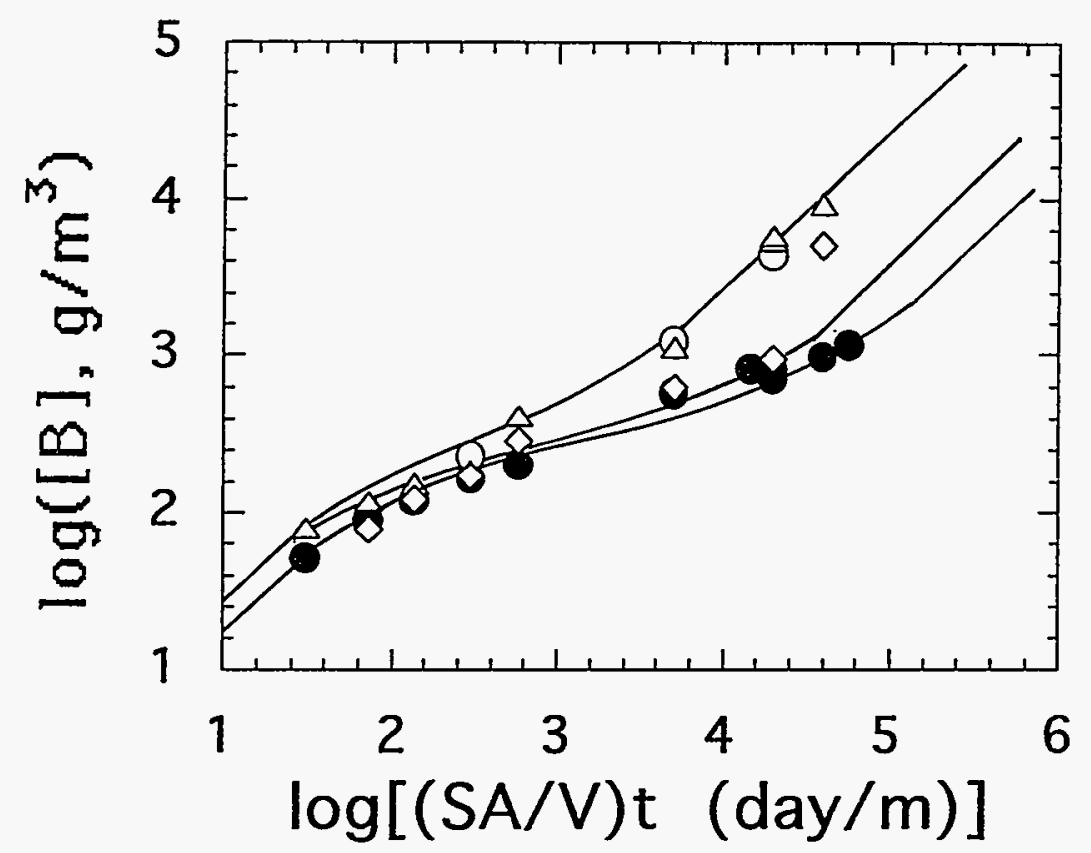

Fig. 45. Log of Normalized Boron Mass Loss vs. $\log (\mathrm{SA} / \mathrm{V}) \mathrm{t}$ for Tests with $(\mathrm{O})$ WV205A.01, $(\Delta)$ ECM85-13, $(\diamond)$ WV205, and $(\bullet)$ SRL 165 Glasses (after [211])

reaction progress (see below). Long-term tests with West Valley Reference glass 6, which is compositionally similar to WV205, are currently in progress at ANL. These tests will clarify the long-term behavior of that glass.

Application of the log-log plot to determine the forward and "saturation" rates is demonstrated using the data presented in Fig. 46, where lines with slopes of one have been drawn through data for SRL 202A glass reacted at $20,000 \mathrm{~m}^{-1}$ [67]. The line with a slope of one drawn through the data point from the test run for the shortest time, which was three days, yields a $y$-intercept of $\log [\mathrm{B}]=-3$. Note that these tests are expected to react at a rate significantly lower than the forward rate because of the very high SA/V used. Tests at very low SA/V are appropriate for measuring the forward rate. These data are used only to demonstrate application of the technique. About $1 \times 10^{-3} \mathrm{~g}$ of boron per cubic meter of solution (units of $[\mathrm{B}] \mathrm{are} \mathrm{mg} / \mathrm{L}$ ) has been released after a reaction progress of $(\mathrm{SA} / \mathrm{V}) \mathrm{t}=1 \mathrm{day} / \mathrm{m}$. The rate calculated by this method is the integrated rate, that is, the average rate over the entire time tested to that point. The average release rate over the first three days is therefore $1 \times 10^{-3} \mathrm{gboron} / \mathrm{m}^{2} /$ day. Since the weight fraction of boron in SRL 202 glass is 0.025 gglass/gglass, the rate of glass corrosion given in terms of the mass of glass dissolved is about 0.04 gglass $/ \mathrm{m}^{2} /$ day. Note that this value is the average rate over three days and that the solution $\mathrm{pH}$ has increased from about 8.2 to about 10.5 over this period. Similar calculations using data obtained in static tests run at $2000 \mathrm{~m}^{-1}$ gives a forward rate of $0.025 \mathrm{gglass} / \mathrm{m}^{2} /$ day on the basis of data for 3 days of reaction. The lower rate measured in tests at $2000 \mathrm{~m}^{-1}$ probably results from the lower $\mathrm{pH}$ attained in these tests. The rate is known to be very sensitive to the $\mathrm{pH}$ [70]. Measurement of the solution $\mathrm{pH}$ value is usually reliable to within about 0.02 units at room temperature with aqueous solutions, though measurement of the $\mathrm{pH}$ values of solutions having high ionic strength is less reliable. The $\mathrm{pH}$ 
values measured at room temperature can be used to estimate the $\mathrm{pH}$ values at the test temperature using solution equilibration codes. The $\mathrm{pH}$ values at $90^{\circ} \mathrm{C}$ are typically about $1 \mathrm{pH}$ unit lower than those measured at room temperature due to the differences in the dissociation constant of water.

The minimum rate attained in the experiments with SRL 202 glass at $20,000 \mathrm{~m}^{-1}$ occurred after about 182 days (at $\log [(\mathrm{SA} / \mathrm{V}) \mathrm{t}]=6.56)$. The line of slope one drawn through this point gave a rate of $0.0025 \mathrm{~g}$ glass $/ \mathrm{m}^{2} /$ day. This is 16 times lower than the rate measured after 3 days of reaction. The observed decrease in the rate between 3 and 180 days is due to the approach of the solution to saturation, although the higher solution $\mathrm{pH}$ after 180 days of reaction compared to the $\mathrm{pH}$ attained after 3 days of reaction (about 11.2 compared to about 10.5) probably accelerated the reaction. It is important to remember that the solution conditions change continuously with the reaction time prior to secondary phase formation, and that the evolution of the solution chemistry is different for tests conducted at different SA/V. This is clearly demonstrated by the difference in boron concentrations in tests conducted at 2000 and $20,000 \mathrm{~m}^{-1}$ at the same values of $(\mathrm{SA} / \mathrm{V}) \mathrm{t}$.

Tests at $20,000 \mathrm{~m}^{-1}$ run longer than 182 days yield reaction rates that are higher than that measured after 182 days. The increase is due in part to the higher $\mathrm{pH}$ values (about 12) that are reached in tests run longer than 182 days. The increase in $\mathrm{pH}$ is expected to increase the value of $\mathrm{k}_{+}$and accelerate the reaction. The cause of the $\mathrm{pH}$ increase is uncertain; it may be due to the formation of secondary phases or a resurgence of ion exchange as the glass corrosion rate increases. The observed increase in the corrosion rate indicates that the "saturation rate" does not represent the long-term corrosion rate for this glass under these conditions and that the longterm rate may be significantly higher than the "saturation rate." Notice that the lowest rate observed in tests performed at $2000 \mathrm{~m}^{-1}$ can be seen in Fig. 46 to be lower than the lowest rate

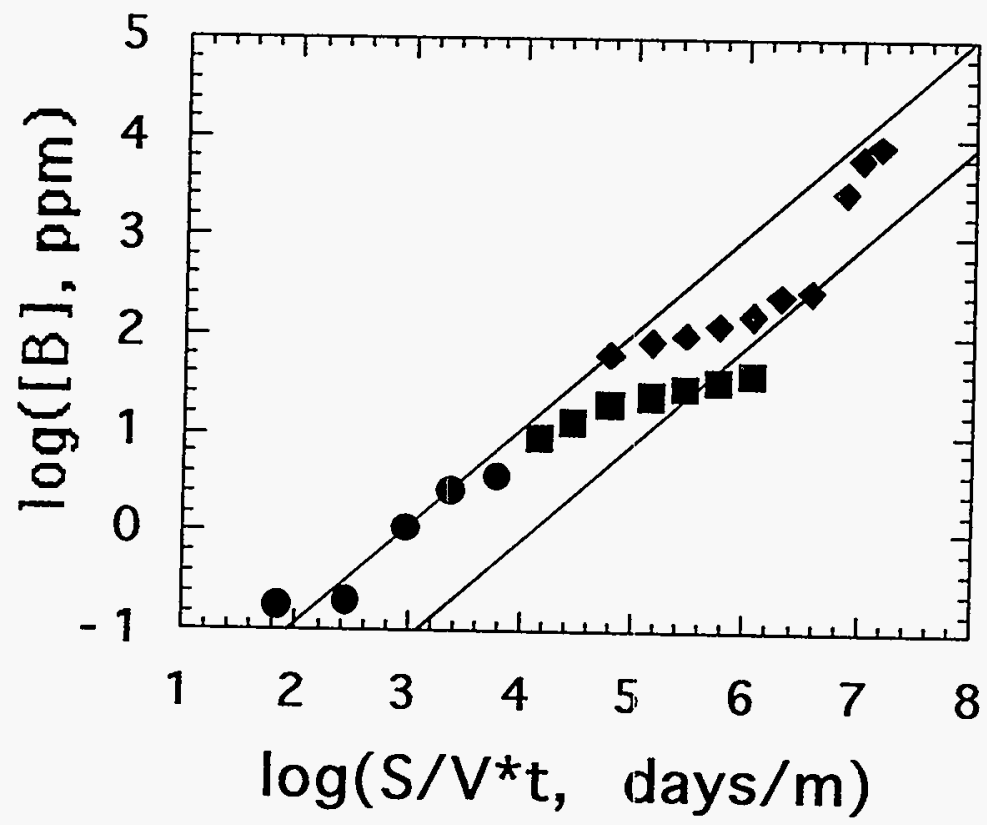

Fig. 46. Release of Boron from SRL 202A Glass as $\log [B]$ vs. (SA/V)t for Tests at SA/V: (•) 10 , (⿴囗十) 2000 , and ( $\bullet$ ) $20,000 \mathrm{~m}^{-1}$. Lines having a slope of one are drawn through data showing the maximum and minimum corrosion rates in tests at $20,000 \mathrm{~m}^{-1}$ (after [67]). 
attained in tests performed at $20,000 \mathrm{~m}^{-1}$. (Data points for tests at $2000 \mathrm{~m}^{-1}$ conducted for large $(\mathrm{SA} / \mathrm{V}) \mathrm{t}$ lie to the right of the line of slope one drawn through the data point showing the lowest rate in tests at $20,000 \mathrm{~m}^{-1}$.) Because of the different $\mathrm{pH}$ values in tests at 2000 and $20,000 \mathrm{~m}^{-1}$, the saturation concentrations will different in tests conducted at different $S A / V$. Differences in the solution $\mathrm{pH}$ affect both the forward rate and the solution saturation conditions.

A similar increase in the corrosion rate at long reaction times has been observed in other tests upon formation of clinoptilolite [200,201]. The acceleration can be interpreted based on the effects of clinoptilolite (and possibly other secondary phases) on the dissolution affinity and the effects of the solution $\mathrm{pH}$ on the precipitation of secondary phases. That is, secondary phase formation controls the glass corrosion rate and the solution $\mathrm{pH}$ controls the formation of the secondary phases.

The long-term behavior measured in tests conducted at different SA/N and in tests with radioactive and nonradioactive glasses is very different. The corrosion rate in tests conducted at $2000 \mathrm{~m}^{-1}$ are seen to continually decrease as corrosion proceeds, while the rates in tests conducted at $20,000 \mathrm{~m}^{-1}$ decrease initially then increase. The difference in behavior can be attributed to the different solution chemistries attained under the different test conditions: higher $\mathrm{pH}$ values and higher solution concentrations are attained in tests conducted at higher $\mathrm{SA} / \mathrm{V}$, and the higher $\mathrm{pH}$ values support precipitation of secondary phases. Faced with two very different long-term corrosion rates from tests at different $\mathrm{SA} / \mathrm{V}$, one must relate the anticipated disposal conditions to the test conditions to determine which rate is relevant. In most cases, the tests at higher SA/V are more relevant to conditions where the amount of water contacting the glass is minimized.

The value of $\left[\mathrm{H}_{4} \mathrm{SiO}_{4}\right]_{\text {sat }}$ can also be determined from experimental data when the minimum rate occurs. Values are usually found to vary slightly for different glass and leachant compositions, but they are typically on the order of $1 \times 10^{-3} \mathrm{M}$ and are intermediate between the solubilities of quartz and amorphous silica [161,166,167,205,212,213].

Reaction rates determined by using the methods described above are presented in Table 5 for several glass compositions. The forward reaction rates are generally between 1 and 2 gglass $/ \mathrm{m}^{2} /$ day at $90^{\circ} \mathrm{C}$, although some measurements yield much lower rates. The forward rates measured under dynamic conditions are probably more accurate than those measured under static conditions because of the build up of reaction products in the leachate affects the results of static tests. The build up of reaction products in static tests and in dynamic tests at low flow rates severely reduces the reaction rate, such that the average rates measured over even short time periods represent lower limits of the forward rates.

The rates measured under "saturated" conditions are typically two to three orders of magnitude smaller than the forward reaction rates. However, "saturated" rates measured for the same glass reacted in different leachants or at different SA/V ratios may indicate that a unique "saturation" rate may not exist for different glass compositions [167] or that the solution has not become "saturated" in the tests, perhaps because secondary phases have formed. In addition, the corrosion rate may increase as more stable secondary phases form over very long reaction times [64,200,201]. For example, the instantaneous long-term rates of SRL 202 and SRL 131 glasses in tests at $20,000 \mathrm{~m}^{-1}$ are about 0.03 and $0.04 \mathrm{~g} / \mathrm{m}^{2} /$ day after secondary phases form [67]. 
Table 5. Experimentally Determined Reaction Rates at $90^{\circ} \mathrm{C}$, in g glass $/ \mathrm{m}^{2} /$ day

\begin{tabular}{|c|c|c|c|c|}
\hline Glass/Leachant & $\mathrm{SA} / \mathrm{V}\left(\mathrm{m}^{-1}\right)$ & Forward Rate & Saturation Rate & Reference \\
\hline \multicolumn{5}{|c|}{ Static Tests } \\
\hline PNL 76-69/DIW & 2000 & 1.6 & $0.08^{\mathrm{a}}$ & 209 \\
\hline SRL 165/DIW & 2000 & 0.80 & $0.024^{\mathrm{a}}$ & 211 \\
\hline EMS-11/DIW & 2000 & 0.083 & $0.0016^{a}$ & 211 \\
\hline SRL 165/DIW & & 1.7 & $0.016^{a}$ & 211 \\
\hline WV205 & & 3.4 & $0.035^{\mathrm{a}}$ & 211 \\
\hline JSS-A/DIW & $10^{\mathrm{b}}$ & 1.5 & 0.0025 & 165 \\
\hline PNL 76-68/DIW & $10^{\mathrm{b}}$ & 1.8 & 0.0075 & 165 \\
\hline SRL 131/DIW & $10^{\mathrm{b}}$ & 3.0 & 0.033 & 165 \\
\hline SRL 131/J-13c & 10 & 0.14 & & 67 \\
\hline SRL 131/J-13 & 2000 & 0.27 & 0.021 & 67 \\
\hline SRL $131 / J-13$ & 20000 & 0.84 & 0.053 & 67 \\
\hline SRL 202/J-13 & 10 & 0.099 & & 67 \\
\hline SRL $202 / \mathrm{J}-13$ & 2000 & 0.025 & 0.0016 & 67 \\
\hline SRL $202 / \mathrm{J}-13$ & 20000 & 0.040 & 0.0025 & 67 \\
\hline R7T7/DIW & 5 & $4.9\left(100^{\circ} \mathrm{C}\right)$ & & 166 \\
\hline R7T7/DIW & 50 & & 0.0083 & 167 \\
\hline R7T7/Volvic ${ }^{d}$ & 50 & & 0.0133 & 167 \\
\hline R7T7/DIW & 400 & & 0.0045 & 167 \\
\hline R7T7/Volvic & 400 & & 0.025 & 167 \\
\hline R7T7/Volvic & 2000 & & 0.0006 & 167 \\
\hline R7T7/Volvic & 8000 & & 0.0006 & 167 \\
\hline R7T7/Volvic & 20000 & & $<0.0001$ & 167 \\
\hline MW/DIW & 1320 & 1.1 & 0.01 & 180 \\
\hline MW/DIW & 10 & 1.2 & 0.009 & 164 \\
\hline MW/DIW & 10 & 1.1 & & 214 \\
\hline \multicolumn{5}{|c|}{ Dynamic Tests } \\
\hline SRL 202/pH 7 & & $0.28\left(80^{\circ} \mathrm{C}\right)$ & & 215 \\
\hline SRL $165 \mathrm{e} / \mathrm{pH} 10.5$ & & $0.05^{\mathrm{a}}$ & & 70 \\
\hline SRL $165 \% / p H ~ 10$ & & $0.08\left(70^{\circ} \mathrm{C}\right)$ & & 112 \\
\hline R7T7/DIW & & 1.03 & & 69 \\
\hline SRL 131/DIW & & 2.5 & & 206 \\
\hline \multicolumn{5}{|c|}{ 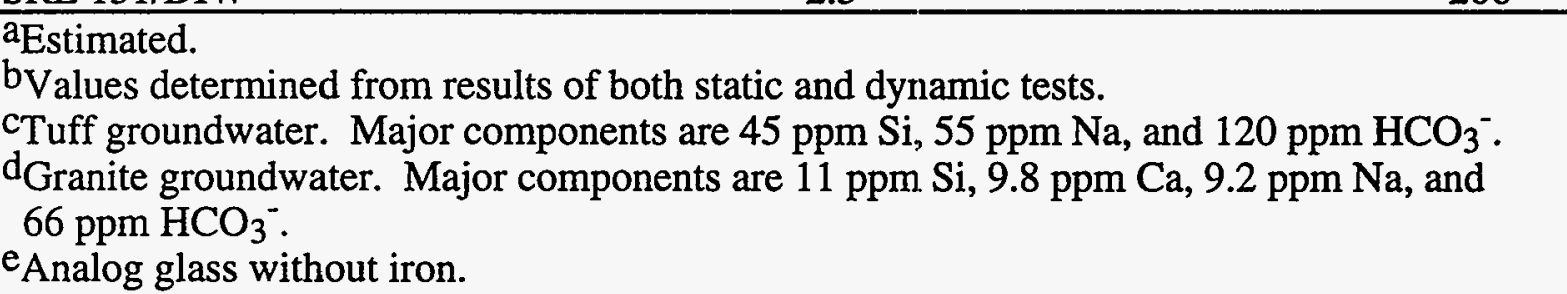 } \\
\hline
\end{tabular}




\subsection{EFFECTS OF SA/V ON RADIONUCLIDE DISPOSITION}

The vast majority of laboratory tests have focused on the corrosion behavior of the glass matrix rather than the release of radionuclides because the glass corrosion rate is usually assumed to provide an upper limit of radionuclide release, because of difficulties associated with handling radioactive materials, and difficulties in monitoring radionuclides present in low concentrations. While the chemical behavior of radionuclides in well-constrained environments is known, very few tests have been conducted to characterize their behavior in the presence of glass corrosion products. The few results available regarding the chemistries of actinides and long-lived fission products have not yet been incorporated into glass corrosion models. While the intrinsic resistance of borosilicate waste glasses towards corrosion is important in stabilizing the radionuclides and establishes a bounding upper limit on their release rates, several factors affect the subsequent mobilization and transport of radionuclides away from the waste form. These include the solubilities of the radionuclides and their complexes, colloid formation, and sorption onto other phases.

Knowledge of the behavior of radioactive waste components under various conditions over long time periods will allow realistic source terms representing the release of specific radionuclides to be incorporated into performance assessment calculations. While the solubilities of many radionuclides are known to be very low in neutral and alkaline groundwaters, laboratory tests are required to determine the effects of complexation, sorption, and colloid and secondary phase formation on the behavior of radioelements in a geologic environment.

All radionuclides are stabilized when incorporated into a glass matrix because their mobilization can only occur as the silicate network of the glass corrodes, and so their release from the waste form is limited by the glass corrosion rate. Actinides exist in borosilicate highlevel waste glass as $\mathrm{U}(\mathrm{VI}), \mathrm{Np}(\mathrm{IV}), \mathrm{Pu}(\mathrm{IV})$, and $\mathrm{Am}(\mathrm{III})$ [2], where the oxidation states are controlled primarily by redox couples within the melt. Technetium has been found to be present as Tc(IV) in borosilicate glass produced under oxidizing conditions [216]. (Both Tc(IV) and metallic Tc was found in glasses produced under reducing conditions.) The oxidation states of these radionuclides in the leachate will be determined by other redox couples in the surrounding geology and will be more strongly affected by the atmosphere above the solution [217]. For example, the dominant oxidation states in tuff groundwater under slightly oxidizing conditions have been determined to be $\mathrm{Np}(\mathrm{V}), \mathrm{Pu}(\mathrm{V}$ and $\mathrm{VI})$, and $\mathrm{Am}(\mathrm{III})[218,219]$. Uranium will be present as U(VI) and technetium as Tc(VII) under these conditions. The oxidation states in the solution will determine the disposition of the radionuclides.

While the intrinsic resistance of borosilicate waste glasses towards corrosion is important in stabilizing the radionuclides and establishes a bounding upper limit on their release rates, several factors affect the subsequent mobilization and transport of radionuclides away from the waste form. These include the solubilities of the radionuclides and their complexes, colloid formation, and sorption onto other phases. The solubility of a particular element is given by the sum of the concentrations of all species of that element which exist in solution, including free ions, hydrolyzed species, and complexes. Solubility products have been measured for many actinidebearing solids, although the phases which control actinide solubilities during glass corrosion are often difficult to identify. Maximum concentrations of complexed radionuclides are established by their individual equilibria. In the absence of complexation, the solubilities of most actinides are generally quite low. Complexation generally leads to increased actinide solubilities, but may also result in decreased solubilities, depending on the solubility controlling phase. Since the extent of hydrolysis and the concentrations of several ligands depend on the $\mathrm{pH}$, the solubility of radioelements will also be $\mathrm{pH}$-dependent. Complexation by ligands which are the conjugate bases 
of weak acids is not significant at $\mathrm{pH}$ values below the $\mathrm{pK}_{\mathrm{a}}$ values of those acids. For example, carbonate is expected to be present in appreciable quantities only at $\mathrm{pH}$ values greater than about 10 , bicarbonate above about $\mathrm{pH} 6$, biphosphate above about $\mathrm{pH} 7$, etc. The solubility may be affected by changes in the ionic strength, composition, and temperature of the solution [220,221]. Most laboratory investigations of actinide and technetium hydrolysis, complexation, and solubility have been performed at or near room temperature. Radionuclide behavior at elevated temperatures must currently be extrapolated from room temperature data or estimated from the behavior of nonradioactive homologues. Much thermodynamic information is still needed before the solubilities and complexation of transuranium elements can be utilized to reliably model long-term geologic interactions [222,223].

Technetium and the actinide elements may exist in solution in different oxidation states depending on the chemistry of the contacting solution. The effect of the redox potential of the solution (i.e., whether the groundwater is oxidizing or reducing) on the preferred oxidation state(s) of an individual actinide is related to the reduction potential of that actinide, which depends on the temperature and ionic strength of the solution. The few studies conducted at different temperatures show that the solubilities are sometimes affected by temperature changes [e.g., 224] and sometimes not affected [e.g., 218,219]. Certainly, more experimental work is needed to properly account for temperature changes in performance assessment of potential repositories.

The chemistry of natural groundwaters and aquatic systems that may eventually contact high-level waste glasses can vary significantly depending on the surrounding environment. The redox potentials of various groundwaters are established by the presence of redox pairs, such as $\mathrm{Fe}^{3+} / \mathrm{Fe}^{2+}$ and $\mathrm{Mn}^{4+} / \mathrm{Mn}^{2+}$, and by dissolved oxygen. Measured potentials of shallow groundwaters, mine waters, and fresh waters are generally found to range between about -200 and $600 \mathrm{mV}$ [141]. The maximum Eh of oxic (oxidizing) groundwaters may be limited by the oxygenhydrogen peroxide equilibrium $[141,225]$, while the lower limit of anoxic (reducing) groundwaters may be established by the hematite-magnetite equilibrium $[225,226]$. The actual Eh of the groundwater will reflect its interaction with the surrounding mineralogy, primarily with ironbearing phases and carbonate minerals. The partial pressure of carbon dioxide in equilibrium with the groundwater also affects the $\mathrm{pH}$ and Eh. Typical $\mathrm{CO}_{2}$ partial pressures in contact with groundwaters may range from $0.03 \%$ up to $10 \%$, and $\mathrm{pH}$ values typically vary between about 4 and $9[141,217]$. Acidic groundwaters typically have higher Eh values than basic groundwaters.

The chemistry of the fluids contacting the waste glass in the near-field environment will differ from the initial groundwater due to corrosion of waste glass and other materials in the engineered barrier system and radiolysis of the air and groundwater $[212,227]$. The $\mathrm{pH}$ of the fluid contacting the waste glass is expected to increase as the glass corrodes due to the release of alkali (although the $\mathrm{pH}$ of some brine solutions have been found to decrease during corrosion due to secondary phase formation [179]), but decrease due to the generation of nitric and nitrous acids through the radiolysis of moist air. The $\mathrm{pH}$ of the fluid contacting the waste glass will depend on the contributions of several simultaneous interactions. Likewise, the redox potential of the solution will be affected by the release of redox-sensitive elements such as iron and the radioelements themselves from the glass and other materials during corrosion, and radiolysis of the air and groundwater. Therefore, the stabilities of actinide species in the near-field environment must be considered under a range of conditions much wider than those defined by potential groundwaters to interpret laboratory experiments and to model the long-term repository behavior in computer simulations.

While the concept of thermodynamically controlled radionuclide solubilities is useful in discussing actinide release, other interactions may significantly affect the distribution of radionuclides as the glass corrodes. Radionuclides may also sorb onto colloids occurring naturally in the groundwater or colloids generated during glass corrosion. These colloids may 
remain mobile and lead to solution concentrations that far exceed the thermodynamic solubility limitations, or they may flocculate and settle out of solution. In addition, radionuclides that are released from the glass into the groundwater may become incorporated in secondary phases that are generated as the glass corrodes, including altered layers at the glass surface and precipitated mineral phases, or they may sorb onto these phases or other materials in the vicinity of the waste form.

The potential distribution of radionuclides between mobile and immobile phases in the immediate vicinity of the corroding waste form is discussed below. Because the solubility and sorptive properties of the radioelements are strongly dependent on their oxidation states and complexation, the chemistry of technetium, uranium, neptunium, plutonium, and americium is first summarized to assist in the interpretation of various test results. Experimental observations of the interactions of these radionuclides with the altered glass layers, secondary mineral phases, and colloids near the surface of the corroding glass are then discussed.

Uranium, neptunium, plutonium, and americium readily undergo hydrolysis as the solution $\mathrm{pH}$ increases, with the ease of hydrolysis increasing with the atomic number. Most are complexed by a variety of ligands that are commonly present in groundwaters, including carbonate, fluoride, chloride, sulfate, and phosphate. Other ligands may be generated by radiolysis of air, such as nitrate and nitrite. In general, complexation decreases in strength as $\mathrm{OH}^{-}>\mathrm{CO}_{3}{ }^{2-}>\mathrm{PO}_{4}{ }^{3-}>\mathrm{F}^{-}>\mathrm{SO}_{4}{ }^{2-}>\mathrm{HPO}_{4}{ }^{2-}>\mathrm{Cl}^{-}, \mathrm{NO}_{3}^{-}$, and $\mathrm{NO}_{2}{ }^{-}$[228]. Because of its high complexing strength and natural occurrence in most groundwaters, carbonate is usually the dominant ligand for actinides dissolved in natural groundwaters [217]. Other metals released during waste form corrosion will also be complexed by these ligands.

Anions present in the waste form that are released as it corrodes will also be available to complex actinides. The anion contents of high-level waste glasses are seldom reported. Sulfur, nitrogen, chlorine, and fluorine are poorly soluble in borosilicate glasses and are usually assumed to be lost as off-gasses during vitrification. Phosphorus is poorly soluble in silicate glasses and phosphorus-bearing phases have been found to precipitate in borosilicate waste glasses $[229,230]$. Except in highly reducing solutions, technetium exists as the pertechnetate ion, $\mathrm{Tc}_{2} \mathrm{O}_{7}^{-}$, and is not complexed.

\subsection{Summary of the Chemistries of $\mathrm{Tc}, \mathrm{U}, \mathrm{Np}, \mathrm{Pu}$, and $\mathrm{Am}$}

The chemistry of the fluid in contact with the waste form, including the redox state, $\mathrm{pH}$, and available complexants, strongly affects the solubility of multivalent glass components, including the radioelements $\mathrm{Tc}, \mathrm{U}, \mathrm{Np}, \mathrm{Pu}$, and $\mathrm{Am}$. The fluid chemistry will be significantly altered due to corrosion of the glass and other materials in the near-field environment. Changes in the $\mathrm{Eh}, \mathrm{pH}$, and ionic strength due to the release of glass components into the solution during corrosion will affect the solubilities and sorptive properties of the radioelements.

The dominant oxidation states in solution under oxidizing conditions are $\mathrm{Tc}(\mathrm{VII}), \mathrm{U}(\mathrm{VI})$, $\mathrm{Np}(\mathrm{V}), \mathrm{Pu}(\mathrm{V})$, and $\mathrm{Am}(\mathrm{III})$, while under reducing conditions Tc(IV), U(IV), Np(IV), Pu(III,IV), and $A m(I I I)$ are dominant. Other oxidation states may be stabilized by complexation. Important complexants include naturally occurring carbonate and humic acids in the groundwater, and may include phosphate, sulfate, and fluoride released from the waste form during corrosion. Complexation may increase or decrease the soluble amounts of radionuclides, depending on what solid phase controls the solubility. 
The extent of sorption of actinides onto mineral surfaces generally decreases as the oxidation state increases, as $A n(I I I, I V)>A n(V)>A n(V I)$. Uranium in the hexavalent state and neptunium in the pentavalent state are poorly sorbed onto most mineral surfaces, while tetravalent plutonium and trivalent americium are strongly adsorbed onto most geologic minerals. Cationic complexes are sorbed much more strongly than are anionic complexes.

The behavior of uranium is expected to be dominated by the chemistry of the uranyl ion, $\mathrm{UO}_{2}{ }^{2+}$. It is strongly hydrolyzed and complexed by several ligands which may increase the uranium solubility. Carbonate and phosphate complexes are expected to be present. Reduction to $\mathrm{U}^{4+}$ may occur under mildly reducing conditions. $\mathrm{U}^{4+}$ is more strongly complexed than $\mathrm{UO}_{2}{ }^{2+}$, and is more strongly sorbed by mineral surfaces. Uranium is sparingly soluble under reducing conditions with the species $\mathrm{U}(\mathrm{OH})_{4}$ being the dominant solution species [231]. Uranium is much more soluble under oxidizing conditions, where the species $\mathrm{UO}_{2}(\mathrm{OH})_{3}\left(\mathrm{CO}_{3}\right)^{-}$ and $\mathrm{UO}_{2}\left(\mathrm{CO}_{3}\right)_{3}{ }^{4-}$ are dominant solution species at basic $\mathrm{pH}$ values [232-234]. The solubility limiting phase is expected to be $\mathrm{UO}_{2}(\mathrm{OH})_{2}$ under oxidizing conditions and $\mathrm{UO}_{2}$ under reducing conditions [235].

The behavior of neptunium is expected to be controlled by the chemistry of pentavalent neptonyl as $\mathrm{NpO}_{2}{ }^{+}$under mildly oxidizing conditions [236] or $\mathrm{Np}(\mathrm{OH})_{4}$ under mildly reducing conditions $[237,238]$. Neptunium(V) as $\mathrm{NpO}^{+}$is more soluble and less strongly hydrolyzed and complexed than $\mathrm{Np}(\mathrm{IV}) ; \mathrm{NpO}_{2}\left(\mathrm{CO}_{3}\right)_{3}{ }^{5-}$ and $\mathrm{NpO}_{2}\left(\mathrm{CO}_{3}\right)_{2}{ }^{3-}$ become important above about $\mathrm{pH} 8$ in oxidizing solutions [239]. The solubility limiting phase is expected to be $\mathrm{NpO}_{2}$ under both oxidizing and reducing conditions [235].

The behavior of plutonium may be controlled by the chemistry of $\mathrm{Pu}^{4+}, \mathrm{PuO}_{2}{ }^{+}$, or $\mathrm{PuO}_{2}{ }^{2+}$ depending on the redox potential of the solution. Under slightly reducing conditions where $\mathrm{Pu}(\mathrm{IV}, \mathrm{V})$ dominate, important species will include $\mathrm{Pu}(\mathrm{OH})_{4}$ or $\mathrm{PuO}_{2}\left(\mathrm{CO}_{3}\right)_{3}{ }^{5-}$ and $\mathrm{PuO}_{2}\left(\mathrm{CO}_{3}\right)_{2}{ }^{3-}$ at high carbonate contents [217]. Higher solubilities are expected at $\mathrm{pH}$ values above about 9 where the $\mathrm{PuO}_{2}\left(\mathrm{CO}_{3}\right)_{3}{ }^{5-}$ species dominates the solution. The solubility limiting phase is expected to be $\mathrm{PuO}_{2}(\mathrm{OH})_{2}$ or $\mathrm{PuO}_{2} \mathrm{CO}_{3}$ under oxidizing conditions [235] and $\mathrm{PuO}_{2}$, $\mathrm{PuO}_{2} \mathrm{CO}_{3}$, or $\mathrm{Pu}(\mathrm{OH})_{4}[240]$ under reducing conditions.

The behavior of americium is expected to be controlled by the chemistry of the trivalent $\mathrm{Am}^{3+}$ species. The $\mathrm{Am}(\mathrm{OH})^{4+}[241]$ and $\mathrm{AmCO}_{3}{ }^{+}$species [242] and organic complexes are expected to be important. The solubility limiting phase is expected to be $\operatorname{Am}(O H)_{3}$ under high $\mathrm{pH}(>10)$ conditions and $\mathrm{Am}\left(\mathrm{CO}_{3}\right)_{3}$ under low $\mathrm{pH}$ conditions [235].

The behavior of technetium is expected to be dominated by the chemistry of the highly soluble pertechnetate ion, $\mathrm{TcO}_{4}^{-}$, which is not hydrolyzed [243,244], complexed, or sorbed onto mineral surfaces. Tc(IV) species may dominate under reducing conditions. The behavior of $\mathrm{Tc}(\mathrm{IV})$ species is very different from Tc(VII), and Tc(IV) may become complexed and strongly sorbed onto mineral surfaces [245]. The solubility limiting phase is expected to be $\mathrm{TcO}_{2}$ under most conditions [246]. 


\subsection{Experimental Measurement of Radionuclide Release}

The disposition of individual radionuclides between mobile and immobile phases as glass corrodes is strongly influenced by the solution chemistry and the presence of complexants and various sorbents. As presented above, technetium, uranium, and neptunium are generally more soluble than plutonium or americium in oxic solutions at the basic $\mathrm{pH}$ values expected in most repositories and attained in most tests, and are expected to attain higher solution concentrations in leach tests. Laboratory tests do show technetium, uranium, and neptunium to be released into solution to a greater extent than either plutonium or americium during corrosion of several glass compositions, including PNL 76-68 [247], R7T7 [248,249], ATM-8 [95,250], SRL 131 [67], SRL 165 [95], and SRL 202 [67,112]. Likewise, higher solution concentrations of Cs than the less soluble Pu or Am were observed during leaching of SRP glass [251], JSS-A glass [252], and TOKAI glass [253]. In other tests, technetium was found to be released congruently with boron under oxidizing conditions [3]. Actinides which do not enter solution due to low solubilities may be retained in the surface layers of the altered glass, either by sorption or incorporation into secondary phases, or may become sorbed on the surfaces of other materials that may be present in the vicinity of the corroding glass.

The conditions of the test will strongly influence the behavior of the radionuclides observed during the test. Tests which maintain dilute solution conditions highlight the reactions releasing the radionuclides from the glass matrix and their solubilities in the leachant. Tests which result in the accumulation of glass corrosion products in the corroding fluid will show effects of colloids and secondary phases on the disposition of the released radionuclides, in addition to changes in the fluid chemistry resulting from glass corrosion, such as higher $\mathrm{pH}$ values.

\subsubsection{Tests Conducted under Dilute Solution Conditions}

Monolithic samples of the borosilicate glass I 117 were reacted in deionized water at a SA/V of $25 \mathrm{~m}^{-1}$ at $90^{\circ} \mathrm{C}$ for $7,10,20$, and 40 days in the presence of oxygen/argon, carbon dioxide/oxygen/argon, carbon dioxide/hydrogen/argon, and hydrogen/argon mixtures [254]. The presence of carbonate ions was found not to affect the mass loss of any glass component, including uranium, while the extent of glass corrosion was greater under oxidizing conditions. Uranium was found to be depleted in the alteration layers.

The retention of uranium in the surface layer was greater after leaching in an anoxic atmosphere than in air [254]. The uranium solubility is expected to be greater in the presence of air due to formation of carbonato species [255]. Deaeration of deionized water, a bicarbonate solution, and a salt brine was previously seen to decrease the solution concentrations of $\mathrm{Np}, \mathrm{Pu}$, and $\mathrm{U}$ in tests with PNL 76-68 glass [256].

The effect of the redox state on the solubility and chemical behavior of released actinides was studied using dynamic leach tests with crushed borosilicate glass I 117 doped with $2.4 \mathrm{wt} \% \mathrm{~Np}, 0.01 \mathrm{wt} \% \mathrm{Pu}$, and $0.03 \mathrm{wt} \%$ Am under oxidizing and reducing conditions [257]. The 0.95-1.4 mm size fraction was used in the tests. The glass was reacted in flowing solutions of bicarbonate groundwater $\left(55.2 \mathrm{ppm} \mathrm{Na}, 159 \mathrm{ppm}^{-\mathrm{HCO}_{3}}\right.$ ) and in a salt brine $(78 \mathrm{ppm} \mathrm{K}$, $164 \mathrm{ppm} \mathrm{Ca}, 538 \mathrm{ppm} \mathrm{SO}_{4}{ }^{2-}$ ) under both oxidizing (air-saturated) and reducing (Eh $\leq-200 \mathrm{mV}$ measured against $\mathrm{Pt}$ electrodes) conditions. The flow rate was $1.5 \mathrm{~cm}^{3} / \mathrm{h}$ and the tests were conducted at $25^{\circ} \mathrm{C}$. The glass corrosion rate (based on the sodium release rate) was about $1 \times 10^{-6} \mathrm{~g} / \mathrm{m}^{2} / \mathrm{d}$. The steady-state solution concentration of Am was found to be the same under both oxidizing and reducing conditions because $\mathrm{Am}(\mathrm{III})$ is the dominant oxidation state under both test conditions. The measured release rates were $3 \times 10^{-7} \mathrm{~g} / \mathrm{m}^{2} / \mathrm{d}$ in the bicarbonate water 
under both oxidizing and reducing conditions The release of americium was about an order of magnitude higher in the bicarbonate water than in the brine, under oxic conditions. On the other hand, $\mathrm{Np}(\mathrm{IV})$ and $\mathrm{Pu}(\mathrm{IV})$ were the dominant solution species under reducing conditions while $\mathrm{Np}(\mathrm{V})$ and $\mathrm{Pu}(\mathrm{V})$ were the dominant solution species under oxidizing conditions. The release rates of $\mathrm{Np}$ were $1 \times 10^{-6} \mathrm{~g} / \mathrm{m}^{2} / \mathrm{d}$ in both the bicarbonate solution and the brine under oxidizing conditions, and $6 \times 10^{-8} \mathrm{~g} / \mathrm{m}^{2} / \mathrm{d}$ in the bicarbonate solution under reducing conditions. The release of neptunium was about an order of magnitude lower in the brine under oxic conditions than under reducing conditions. The release rates of Pu were $4 \times 10^{-7}$ and $3 \times 10^{-7} \mathrm{~g} / \mathrm{m}^{2} / \mathrm{d}$ in the bicarbonate solution and the brine under oxidizing conditions, and $9 \times 10^{-8} \mathrm{~g} / \mathrm{m}^{2} / \mathrm{d}$ in the bicarbonate solution under reducing conditions. The release of neptunium was about an order of magnitude lower in the brine, under oxic conditions.

The behavior observed in these tests is dominated by the solubilities of the different actinide elements. Because the solubilities of the An(V) species are higher than those of the $A n(I I I)$ and $A n(I V)$ species, the solution concentrations of both $\mathrm{Np}$ and $\mathrm{Pu}$ were found to be higher under oxidizing conditions, where they exist preferentially in the An(V) state, than under reducing conditions, where they exist in the $\mathrm{An}(\mathrm{VI})$ state. Some reduction of $\mathrm{Pu}(\mathrm{V})$ at the glass surface was suggested to occur under oxidizing conditions [257]. Americium exists in the An(III) state except under highly oxidizing conditions, and its release behavior was not affected under either of the conditions tested. The actinides not released from the glass likely accumulated in the alteration layers that formed on the glass surface.

The release of $\mathrm{Sr}, \mathrm{Cs}, \mathrm{Tc}, \mathrm{Np}, \mathrm{Pu}$, and Am from glass UK 189 into distilled water at $60^{\circ} \mathrm{C}$ was measured in flow-through tests at various flow rates [258]. The measured leach rates are given in Table 6 (data converted from $\mathrm{g} / \mathrm{cm}^{2} / \mathrm{d}$ to $\mathrm{g} / \mathrm{m}^{2} / \mathrm{d}$ by multiplying data by $10^{4} \mathrm{~cm}^{2} / \mathrm{m}^{2}$ ). The corrosion rate of the glass UK 189 at high flow rates was reported to be $0.65 \mathrm{~g} / \mathrm{m}^{2} / \mathrm{d}$. The authors interpreted the finding that the technetium release rate was independent of the flow rate as evidence that its release is limited by its rate of escape from the glass rather than its solubility. The release of the other radionuclides decreased as the flow rate decreased, indicating that their release was affected by their solubilities. The release rates of glass matrix components were not reported. It is expected that the corrosion rate of the glass matrix would also decrease with the flow rate due to saturation effects. The results of Hall et al. may indicate that the release of technetium is not affected by the corrosion of the glass matrix.

After 270 days of corrosion, the alteration layers formed on the samples were removed and analyzed. The fractions of each isotope retained in the layer was calculated, and is included in Table 6 . The high degree of retention of the actinides is consistent with other test results. The authors pointed out that cesium was retained, while lithium and sodium were readily released, and attributed the difference to the different ionic radii. The lower ion exchange rate of cesium probably also contributes to the observed retention. Notice also that the technetium is not completely depleted in the alteration layers. The retained technetium may have been reduced to the less soluble $\mathrm{Tc}^{4+}$ state during formation of the glass, though this is only speculation.

The corrosion of R7T7 glasses doped with ${ }^{237} \mathrm{~Np},{ }^{239} \mathrm{Pu}$, or ${ }^{241} \mathrm{Am}$ has been measured in leach tests with R7T7 glass run 70 days in deionized water at room temperature [249]. These tests were conducted with daily renewal of the leachant, which prevented accumulation of glass corrosion products The extent of corrosion was small in these tests, and only thin surface layers were observed. The measured leach rates were $6 \times 10^{-3} \mathrm{~g} / \mathrm{m}^{2} / \mathrm{d} \mathrm{Np}$, $6 \times 10^{-3} \mathrm{~g} / \mathrm{m}^{2} / \mathrm{d} \mathrm{Pu}$, and $3 \times 10^{-3} \mathrm{~g} / \mathrm{m}^{2} / \mathrm{d} \mathrm{Am}$. The corrosion rate of the glass matrix was reported to be $12 \times 10^{-3} \mathrm{~g} / \mathrm{m}^{2} / \mathrm{d}$. Thus, the actinide elements were released at rates slightly lower than the rate at which the glass was corroding. 
Table 6. Leach Rates from UK 208 Glass in Distilled Water at $60^{\circ} \mathrm{C}\left(\mathrm{g} / \mathrm{m}^{2} / \mathrm{d}\right)$ [258]

\begin{tabular}{llllc} 
& \multicolumn{4}{c}{ Flow Rate } \\
\cline { 2 - 5 } & $9 \mathrm{~mL} /$ day & $1 \mathrm{~mL} /$ day & $1 \mathrm{~mL} /$ week & $\begin{array}{c}\text { \% Retained } \\
\text { in Layer }\end{array}$ \\
\hline${ }^{99} \mathrm{Tc}$ & 0.7 & 0.7 & 0.6 & 15 \\
${ }^{90} \mathrm{Sr}$ & 0.5 & 0.2 & 0.05 & 62 \\
${ }^{137} \mathrm{Cs}$ & 0.6 & 0.4 & 0.2 & 31 \\
${ }^{237} \mathrm{~Np}$ & 0.18 & 0.1 & 0.03 & 91 \\
${ }^{239} \mathrm{Pu}^{\mathrm{a}}$ & 0.005 & 0.0013 & 0.00015 & 99.4 \\
${ }^{241} \mathrm{Am}$ & 0.00004 & 0.000006 & 0.000006 & 99.8 \\
\hline
\end{tabular}

aIsotope not reported. Pu data reported as uncertain.

In 28-day Soxhlet tests of the same actinide-doped R7T7 glass at $100^{\circ} \mathrm{C}$, release rates layers about $6 \mathrm{~g} / \mathrm{m}^{2} / \mathrm{d} \mathrm{Np}, 5 \times 10^{-3} \mathrm{~g} / \mathrm{m}^{2} / \mathrm{d} \mathrm{Pu}$, and $3 \times 10^{-3} \mathrm{~g} / \mathrm{m}^{2} / \mathrm{d}$ Am. The release rate of boron was reported to be $2 \mathrm{~g} / \mathrm{m}^{2} / \mathrm{d}$, which is taken to be the corrosion rate of the glass matrix. The corrosion rate of the glass increased about 150 times and the release of neptunium increased by a factor of 1000 , while the plutonium and americium release rates were similar at 25 and $100^{\circ} \mathrm{C}$. The low release rates of plutonium and americium were attributed to their retention in the alteration layer formed on the glass surface during the tests [249]. Layers about $20 \mathrm{~mm}$ thick were formed during the Soxhlet tests. These test conditions likewise maintain dilute solutions and minimize the effects of dissolved corrosion products while maintaining a high glass dissolution rate. It was concluded from these tests that the alteration layer formed on R7T7 glass effectively retained $\mathrm{Pu}, \mathrm{Am}$, and $\mathrm{Cm}$, although $\mathrm{Np}$ was not significantly retained by the layers under these test conditions [249]. This is consistent with the higher solubility of neptunium.

The retention of any element $i$ by an alteration layer during glass corrosion can be quantified by comparing the amount of that element in solution to the amount of boron in solution, as $R F(i)=N L(B) / N L(i)$, where $N L(i)$ is the normalized mass loss of element $i$ $[249,259]$. Boron is assumed to be totally released into solution and to represent the extent of glass corrosion. High values of $\mathrm{RF}(\mathrm{An})$ indicate that actinides remain largely immobile as the glass corrodes, while low values indicate that mobilization of the actinides may occur as the glass corrodes.

It should be emphasized that the retention factor is a measure of the release of actinides (and other elements) to solution relative to the release of boron and does not indicate the extent of glass corrosion. A low RF(i) value simply means that element $i$ is released from the glass at a rate similar to boron, which may be fast or slow. Indeed, changes in the release factor under different experimental conditions is as much a measure of the affect of the test conditions on the boron release as it is a measure of the release of less soluble elements.

Actinide retention factors for test results reported by Vernaz et al. are quite low for the tests at $25^{\circ} \mathrm{C}$, $(\mathrm{RF}(\mathrm{Np})=2, \mathrm{RF}(\mathrm{Pu})=2$, and $\mathrm{RF}(\mathrm{Am})=4)$, but higher for corrosion in the Soxhlet tests $(\mathrm{RF}(\mathrm{Np})=3.7, \mathrm{RF}(\mathrm{Pu})=412$, and $\mathrm{RF}(\mathrm{Am})=469)$. 
The release of uranium, neptunium, plutonium, and americium from R7T7 glasses into distilled water was measured in static leach tests with monolithic samples at $\mathrm{SA} / \mathrm{V}$ of $50 \mathrm{~m}^{-1}$ at 50 and $90^{\circ} \mathrm{C}[167,248]$. Both the normalized boron release and the normalized actinide releases increased with the reaction time. Boron, uranium, and neptunium were dissolved in the leachates, while most of the plutonium and americium in the leachate was present primarily as suspended material that was removed using a $25 \mathrm{~nm}$ filter. The suspended material was considered to be released from the glass and was included in the released fraction. The normalized release of boron and uranium from R7T7 glass doped with natural uranium and boron and neptunium from R7T7 glass doped with ${ }^{237} \mathrm{~Np}$ in tests at $90^{\circ} \mathrm{C}$ are shown in Fig.47a. The boron release is similar from the R7T7 glasses doped with either natural uranium or with neptunium, and the normalized releases of uranium and neptunium are similar. The boron release is rapid initially, then levels off at longer reaction times. The normalized release of boron and plutonium from R7T7 glasses doped with ${ }^{238} \mathrm{Pu}$ or ${ }^{239} \mathrm{Pu}$ and boron and americium from R7T7 glass doped with ${ }^{241} \mathrm{Am}$ in tests at $90^{\circ} \mathrm{C}$ are shown in Fig. $47 \mathrm{~b}$. The boron release is similar from the R7T7 glasses doped with either plutonium or americium through 91 days, but the boron release from glasses doped with $238 \mathrm{Pu}$ or ${ }^{241} \mathrm{Am}$ increases significantly after longer reaction times. The normalized releases of plutonium and americium remain low and are not affected by the increased glass corrosion rate at 182 days or longer. Vernaz et al. ascribed the differences in reactivity to radiolysis and the resulting differences in the solution $\mathrm{pH}$. The measured solution $\mathrm{pH}$ values for tests at $90^{\circ} \mathrm{C}$ are shown in Fig. $47 \mathrm{c}$. Tests conducted with the $\mathrm{U},{ }^{237} \mathrm{~Np}$, or ${ }^{239} \mathrm{Pu}$ doped glasses attain higher $\mathrm{pH}$ values than the glasses with the ${ }^{238} \mathrm{Pu}$ or ${ }^{241}$ Am doped glasses, which have higher specific activities. The solution $\mathrm{pH}$ reflects the effects of glass corrosion, which raise the $\mathrm{pH}$, and radiolysis of the air in the test vessel, which lower the $\mathrm{pH}$.

The measured releases of the same elements in identical tests conducted at $50^{\circ} \mathrm{C}$ are shown in Figs.48a and $\mathrm{b}$. Notice that the boron continues to be released from the glasses doped with $\mathrm{U},{ }^{237} \mathrm{~Np}$, and ${ }^{239} \mathrm{Pu}$ through 364 days, but reaches a nearly constant value in tests with glasses doped with ${ }^{238} \mathrm{Pu}$ and ${ }^{241} \mathrm{Am}$. The normalized mass losses of ${ }^{237} \mathrm{~Np}$ increases slightly with the reaction time, but the mass losses of the other actinides remain low at all test times. The lower corrosion rates measured in tests at $50^{\circ} \mathrm{C}$ (as measured by the boron release) correspond to the much lower solution $\mathrm{pH}$ values attained in tests with ${ }^{238} \mathrm{Pu}$ and ${ }^{241} \mathrm{Am}$ compared to tests with $\mathrm{U},{ }^{237} \mathrm{~Np}$, and ${ }^{239} \mathrm{Pu}$. The measured $\mathrm{pH}$ values are shown in Fig. $48 \mathrm{c}$. The relative effects of glass corrosion and radiolysis on the solution $\mathrm{pH}$ are different in tests at 50 and $90^{\circ} \mathrm{C}$ because of the lower glass corrosion rate at $50^{\circ} \mathrm{C}$. The effect of radiolysis is less sensitive to the temperature than the glass corrosion rate, and so the effects of radiolysis lower the solution $\mathrm{pH}$ more in tests at $50^{\circ} \mathrm{C}$ than in tests at $90^{\circ} \mathrm{C}$.

The logarithms of the measured retention factors for tests conducted at $90^{\circ} \mathrm{C}$ are plotted in Fig. 49a. The data show that all actinides are retained in the layers to some extent $(\log (\mathrm{RF})>0$ for all actinides at all times), with americium and plutonium being retained to the highest degree. The increase in the boron release from the ${ }^{238} \mathrm{Pu}$ and ${ }^{241} \mathrm{Am}$ doped glasses results in a slight decrease in the retention factors of these elements. Overall, the retention factors remain essentially constant over all times tested. As the glass corrodes under these conditions, only about $10 \%$ of the uranium and neptunium, $3 \%$ of the plutonium, and $0.3 \%$ of the americium are released into the solution. The balance accumulates in alteration layers on the glass surfaces. 
(a)

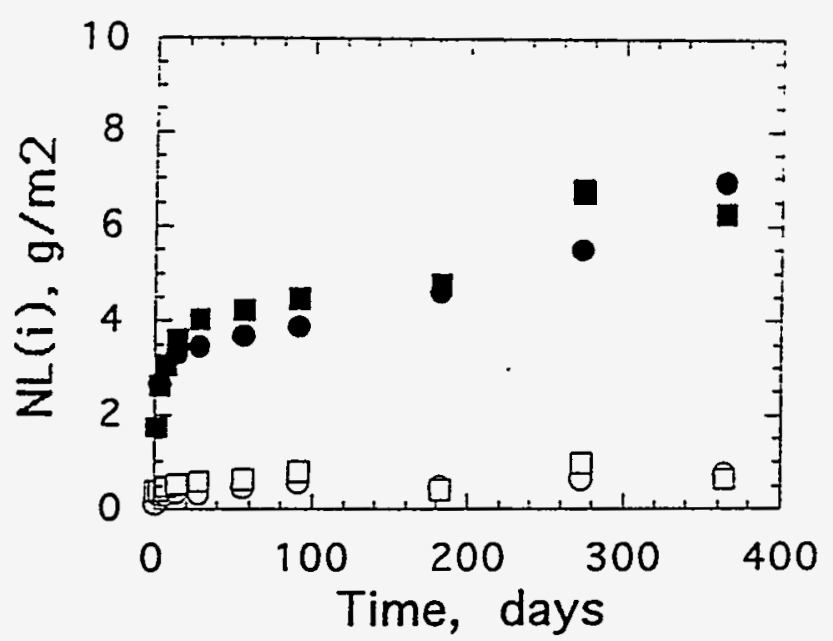

(b)

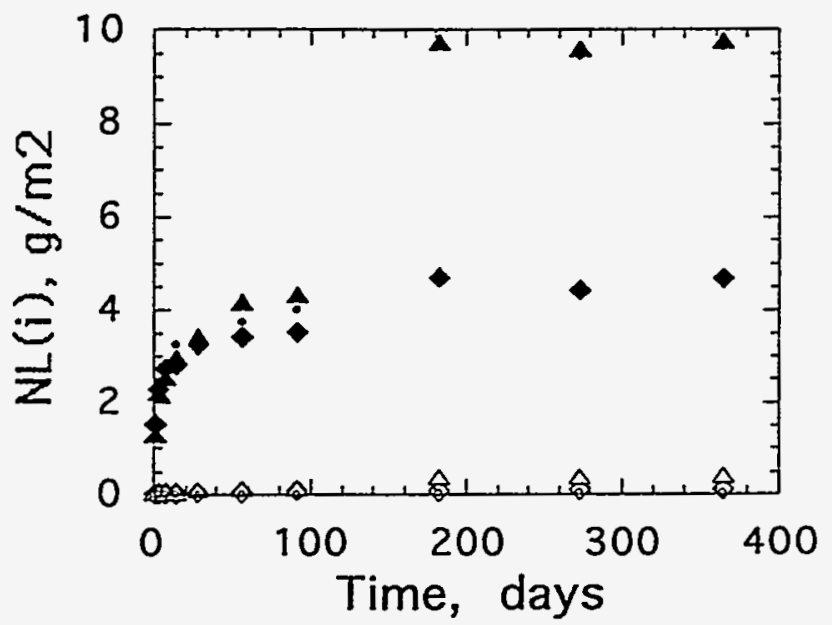

(c)

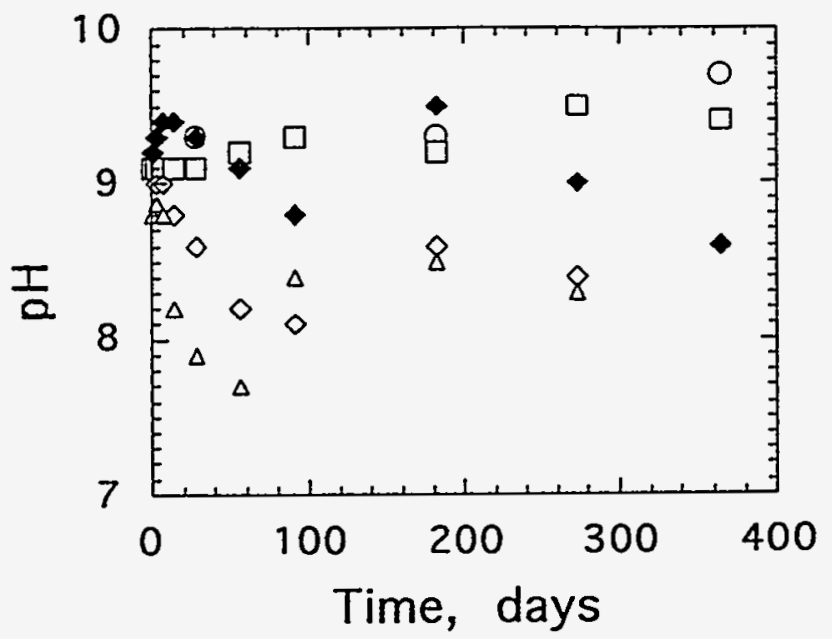

Fig. 47. Measured Normalized Mass Losses in Tests with Actinide-Doped R7T7 Glasses in Tests at $90^{\circ} \mathrm{C}$ and $50 \mathrm{~m}^{-1}$ : (a) (O) U and (๑) B from U-Doped R7T7 Glasses and

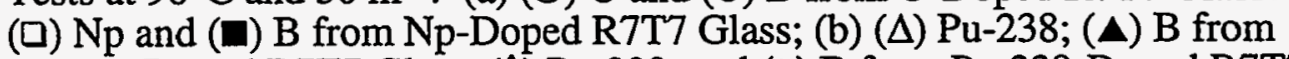
Pu-238-Doped R7T7 Glass, ( $\diamond)$ Pu-239, and $(\bullet)$ B from Pu-239-Doped R7T7 Glass; and $(\mathrm{O}) \mathrm{Am}-241$ and $(\bullet) \mathrm{B}$ from Am-241-Doped R7T7 Glass; and (c) Measured pH

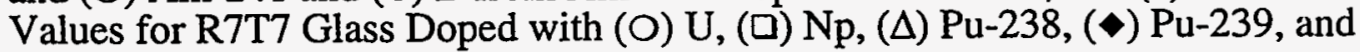
$(\Delta)$ Am-241 (after [249]) 
(a)

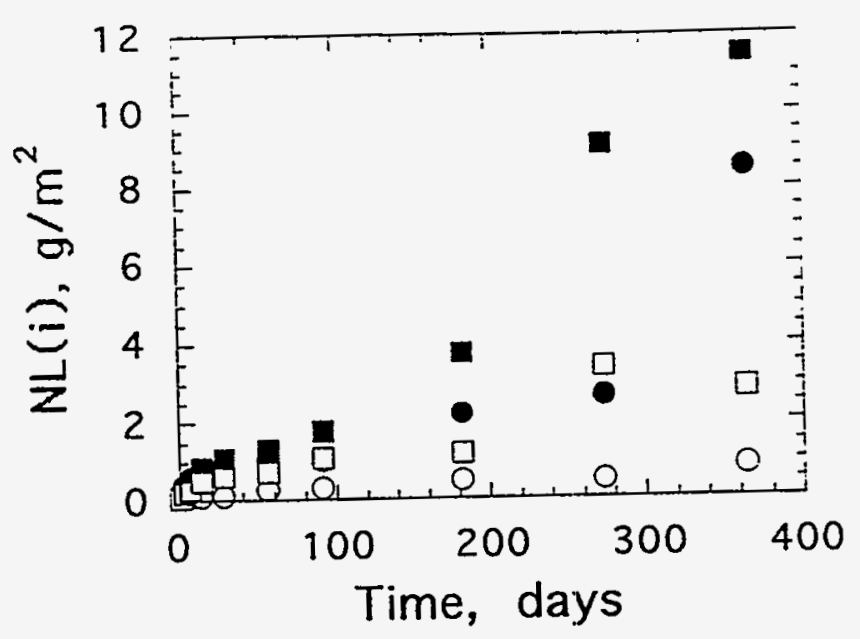

(b)

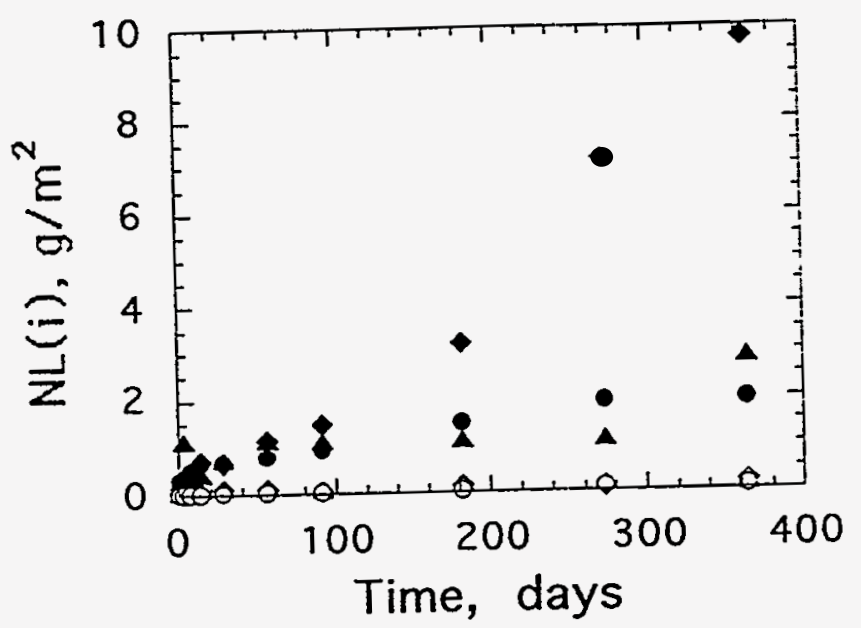

(c)

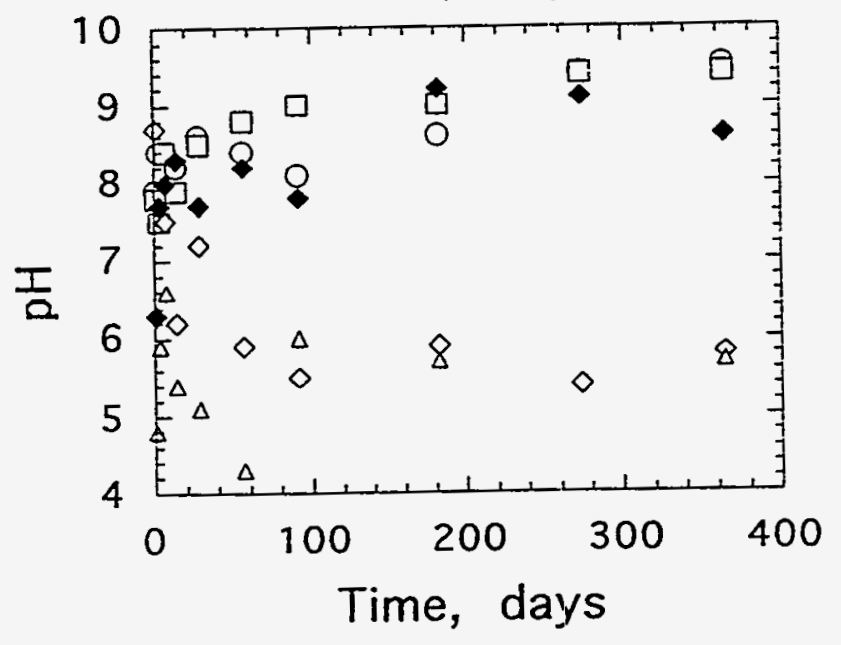

Fig. 48. Measured Normalized Mass Losses in Tests with Actinide-Doped R7T7 Glasses in Tests at $50^{\circ} \mathrm{C}$ and $50 \mathrm{~m}^{-1}$ : (a) (O) U and (O) B from U-Doped R7T7 Glasses and

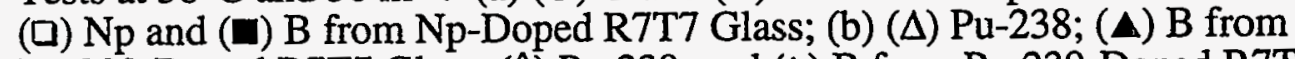
$\mathrm{Pu}$-238-Doped R7T7 Glass, ( $\diamond) \mathrm{Pu}-239$, and $(\bullet) \mathrm{B}$ from Pu-239-Doped R7T7 Glass; and (O) Am-241 and (०) B from Am-241-Doped R7T7 Glass; and (c) Measured pH

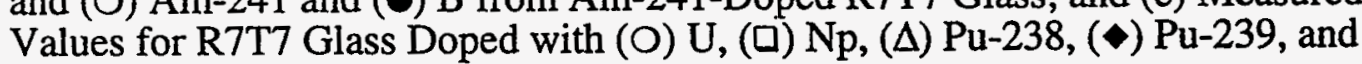
(A) Am-241 (after [249]) 
(a)

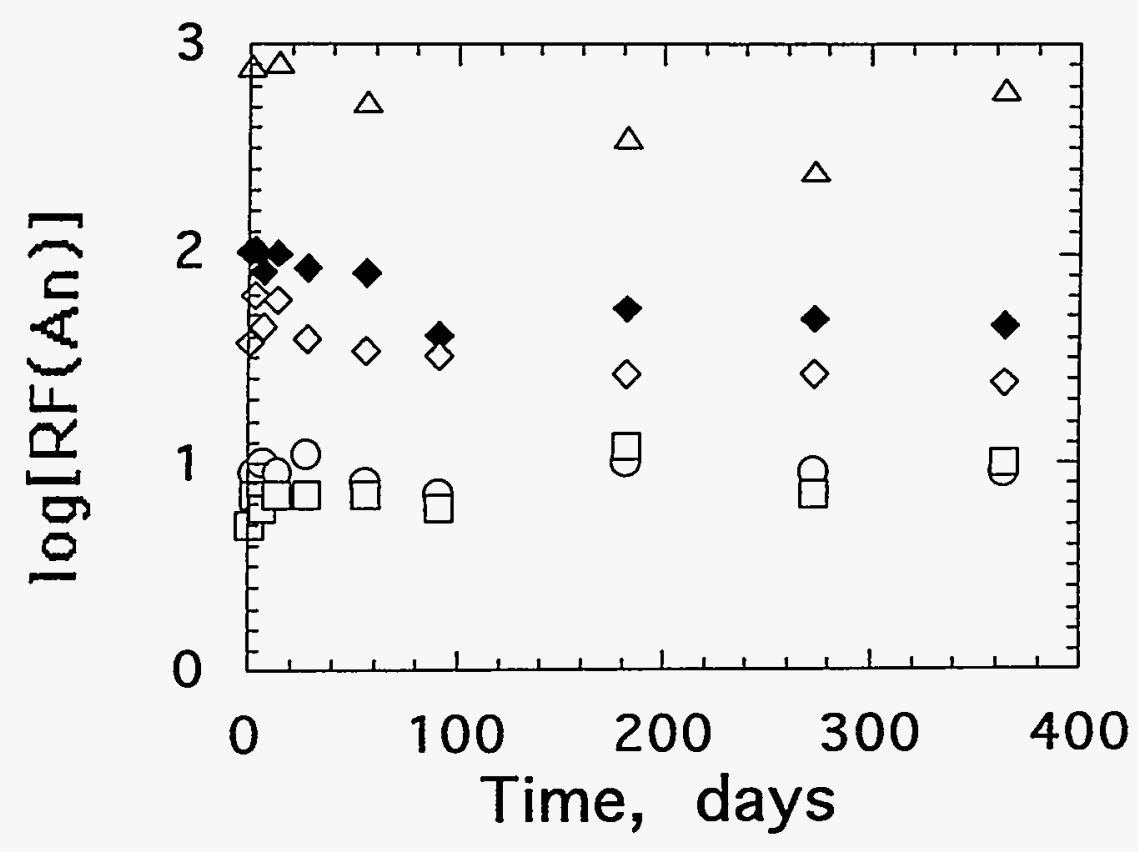

(b)

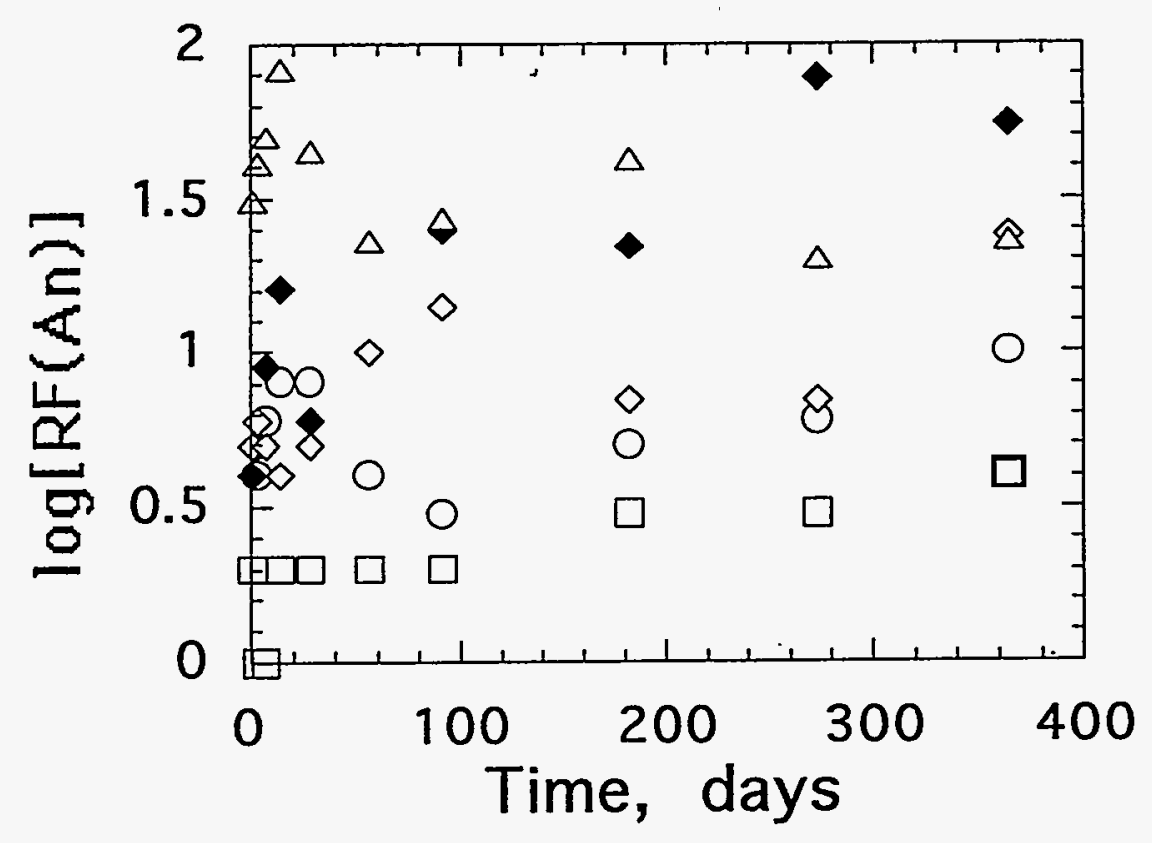

Fig. 49. Calculated Retention Factors vs. Reaction Time for Tests at (a) $90^{\circ} \mathrm{C}$ and (b) $50^{\circ} \mathrm{C}$ with R7T7 Glass Doped with (O) U, (口) Np, ( () Pu-238, ( $\bullet$ Pu-239, and ( $\Delta$ ) Am-241 (after [249]) 
The retention factors calculated from the normalized mass losses in tests at $50^{\circ} \mathrm{C}$ are shown in Fig. 49b. The retention factors measured in tests conducted at $50^{\circ} \mathrm{C}$ are about half those measured in tests conducted at $90^{\circ} \mathrm{C}$. Part of the difference is due to the different reactivities of the glasses at 50 and $90^{\circ} \mathrm{C}$ and the differences in the solution $\mathrm{pH}$. (The dopant oxides were present at $0.85 \mathrm{w} \%$ in all glasses, and probably didn't affect the intrinsic reactivity of the R7T7 glass.)

A Savannah River Laboratory glass generated by mixing SRL 165 with radioactive waste was corroded in a tuff groundwater solution [251]. The glass composition differed from SRL 165 mainly in the aluminum and iron contents, which were measured to be $9.8 \mathrm{wt} \% \mathrm{Al}_{2} \mathrm{O}_{3}$ and $6.0 \mathrm{wt} \% \mathrm{Fe}_{2} \mathrm{O}_{3}$ in the glass. The groundwater solution contained about $42 \mathrm{ppm}$ sodium and $27 \mathrm{ppm}$ silica [260] and typically $120 \mathrm{ppm}$ bicarbonate and $30 \mathrm{ppm}$ sulfate. Tests were conducted using monolithic samples at an SA/V of $100 \mathrm{~m}^{-1}$ and at $90^{\circ} \mathrm{C}$ for up to 134 days. Figure 50 shows the normalized mass loss of $238 \mathrm{Pu}$ calculated based on the amount of plutonium measured in the leachate (circles) and based on the total amount found in the leachate plus fixed to the steel vessel (squares). Most of the plutonium becomes fixed to the steel vessel during the test.

Tests were conducted using actinide-doped SRL 165 glass in tuff groundwater at an SA/V of $30 \mathrm{~m}^{-1}$ and at $90^{\circ} \mathrm{C}$ for up to 278 days [95]. Figure 51 shows the average retention factors calculated for uranium, neptunium, plutonium, and americium. All the uranium and most of the neptunium were dissolved or suspended in the leachate solution, while most of the plutonium and americium were fixed to the steel reaction vessel. Material fixed to the vessel

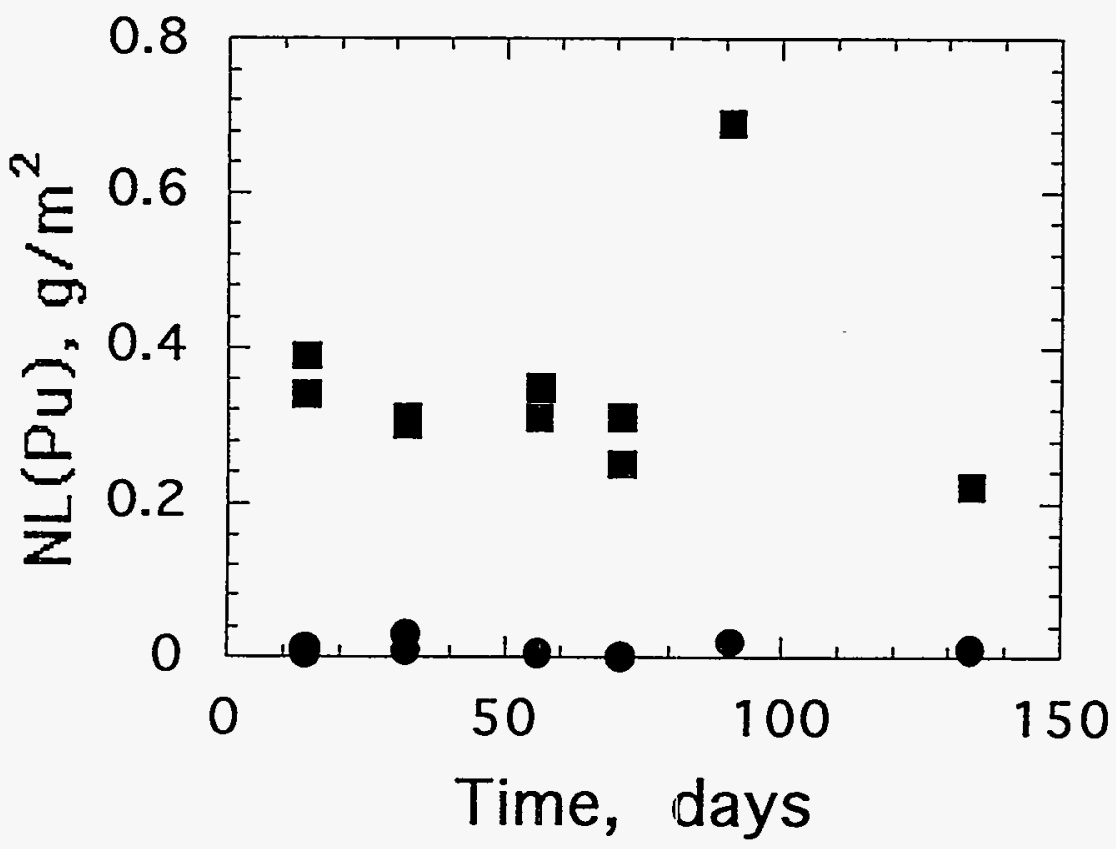

Fig. 50. Normalized Mass Loss of Plutonium-238 from SRL 165 Glass in Tests at $100 \mathrm{~m}^{-1}$ Based on (๑) ${ }^{238} \mathrm{Pu}$ in Solution and (汭 ${ }^{238} \mathrm{Pu}$ in Solution Plus on Vessel Walls (after [251]) 


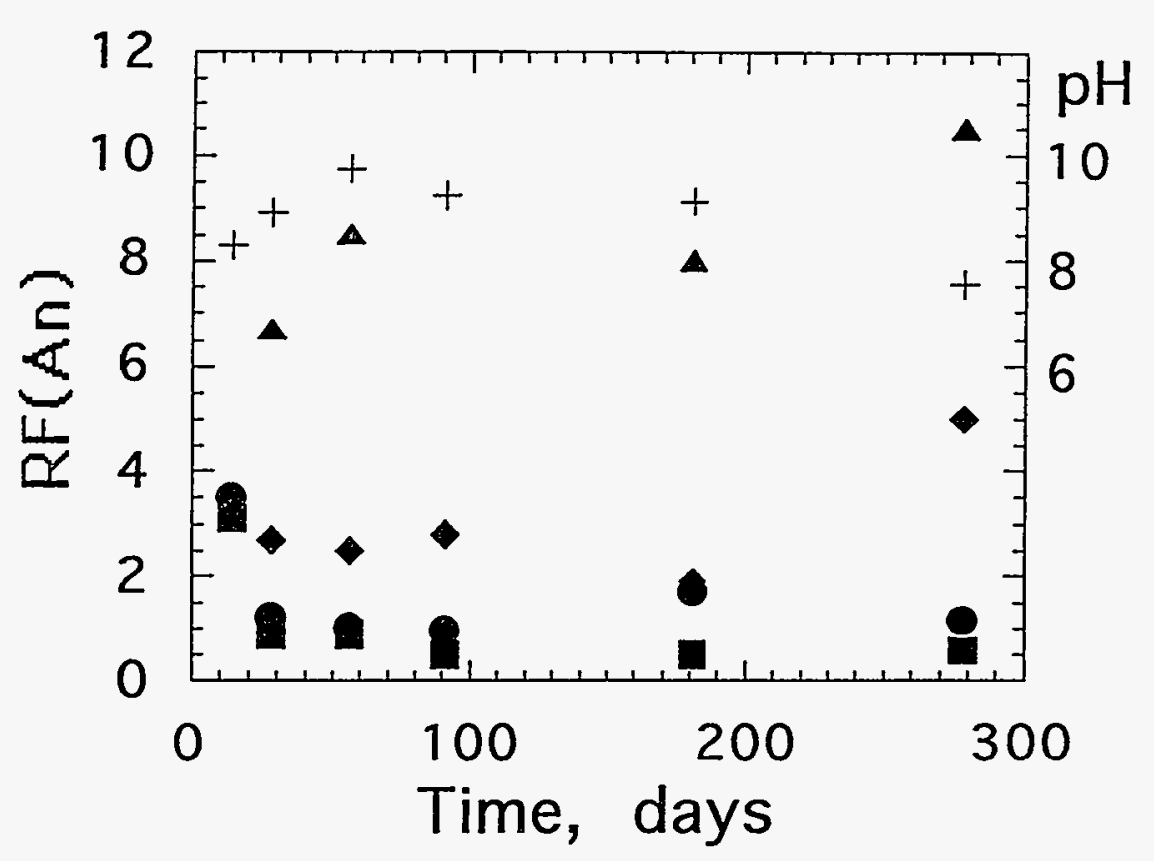

Fig. 51. Calculated Retention Factors and Measured $\mathrm{pH}$ Values for Tests with Actinide-Doped

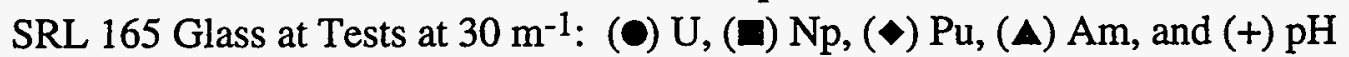
(after [95])

was counted as being released form the glass. The average solution $\mathrm{pH}$ values measured at room temperature are included in the plot. The relative retention of the different actinides is consistent with their solubilities: americium is retained to the highest degree and is the least soluble, uranium and neptunium have relatively soluble and are only slightly retained. Surface analyses verified the relative concentrations of the actinides in the surface layers [95]. The relatively low $\mathrm{SA} / \mathrm{V}$ of these tests maintained sufficiently dilute conditions that the disposition of the actinides was not significantly affected by glass corrosion products in the solution. However, the release was sensitive to the solution $\mathrm{pH}$.

\subsubsection{Tests under Concentrated Solution Conditions}

The disposition of actinides in tests conducted in flowing solutions and at low $\mathrm{SA} / \mathrm{V}$ is dominated by the actinide solubilities. While the disposition may be affected by interactions with species in the groundwater, it is only weakly affected by interactions with glass components in the solution. The effects of dissolved glass components have been shown to be very important in understanding the long-term behavior of the glass matrix, and are likely to be just as important to the disposition of released radionuclides. Tests conducted at high SA/V highlight the interactions between the leachate solution and the dissolving glass. The effects of the formation of alteration layers and secondary phases are highlighted in these tests. These effects will be important under disposal conditions, where only small volumes of water are likely to contact the waste glass.

The corrosion of two Savannah River glasses (SRL $165 / 42$ and SRL 200R) was measured using the PCT test. The glasses were crushed and the $-100+200$ mesh size fractions were reacted in deionized water at $90^{\circ} \mathrm{C}$ for 7 days [261]. Tests were conducted in two laboratories to assess the reproducibility of the test method. The release of components from the 
glass matrix (B, $\mathrm{Li}, \mathrm{Na}, \mathrm{Si}$ ) agreed within $10 \%$. The $\mathrm{SRL} 165 / 42$ glass was measured to be less corroded than the SRL 202R glass (the average normalized concentrations of boron were $0.37 \mathrm{~g} / \mathrm{L}$ for SRL $165 / 42$ glass and $1.1 \mathrm{~g} / \mathrm{L}$ for SRL 202R). The release of radioactive components agreed within a factor of about 2 . The larger variation in the radionuclide results may be attributed to greater analytical difficulties. The average retention factors of radioactive elements (calculated from the ratio of the normalized concentrations of boron and the radionuclides of interest) measured by the different laboratories are: $\mathrm{RF}\left({ }^{137} \mathrm{Cs}\right)=5$, $\mathrm{RF}\left({ }^{90} \mathrm{Sr}\right)=12, \mathrm{RF}\left({ }^{238} \mathrm{Pu}+{ }^{239} \mathrm{Pu}+{ }^{244} \mathrm{Cm}\right)=5$ for $\mathrm{SRL} 165 / 42$, and $\mathrm{RF}\left({ }^{137} \mathrm{Cs}\right)=6$, $\mathrm{RF}\left({ }^{90} \mathrm{Sr}\right)=264, \mathrm{RF}\left({ }^{238} \mathrm{Pu}+{ }^{239} \mathrm{Pu}+{ }^{244} \mathrm{Cm}\right)=15$ for $\mathrm{SRL} 200 \mathrm{R}$. By comparison to the results of a nonradioactive glass having a composition similar to SRL 202R, the authors determined that radiolysis did not affect the test results. This is probably due to the relatively high solution concentrations attained in PCT tests compared to those attained in tests at $50 \mathrm{~m}^{-1}$ (cf. discussion of [249] above, where radiolysis effects were observed).

Tests conducted at higher SA/V ratios and for longer time periods have shown that behavior of the radioactive components becomes more or less independent of the glass corrosion behavior at advanced stages of the corrosion process. For example, the increased corrosion rate observed in tests with actinide-doped SRL 202 glass (see Fig. 37) did not affect the disposition of released actinides [67]. That is, the amounts of $\mathrm{Np}, \mathrm{Pu}$, and $\mathrm{Am}$ found either suspended in solution as colloids, dissolved in solution, or fixed to steel vessel were not affected by the increased glass corrosion rate at reaction times longer than 182 days. (These results are discussed in more detail in Section 9.2.3.) These results imply that, while the corrosion rate of the glass matrix provides an upper boundary to the release of actinides, other factors limit their release rates at much lower levels.

\subsection{Actinide-Bearing Secondary Phases}

Identification of the solid phases which control radionuclide solubilities is required to accurately predict the radionuclide behavior during glass corrosion in repository setting. While phases controlling solubilities in pure systems may be identified by precipitation from supersaturated solutions (e.g., [219]), solubility-controlling phases are not easily identified in tests with waste glasses because most laboratory tests are performed using simulated glasses with low concentrations of radioelements, and the phases formed with radioelements are typically very small and interspersed with several other phases. Identification of phases containing radionuclides is complicated by the difficulties associated with handling radioactive samples and the limited sensitivity of analytical methods. Nevertheless, progress has been made in identifying phases formed which contain radionuclides.

Leach tests with relatively high uranium contents have lead to the identification of several uranium-bearing phases which form during glass corrosion. Weeksite, a uranium silicate, is the most commonly formed uranium-bearing mineral during glass corrosion $[61,111,108]$. Other uranium silicates have been found to form, including uranophanes and haiweeite $[112,113,262,263]$. These phases are generally formed separate from the glass, although they may nucleate on the glass surface, and crystallites may remain suspended in the leachate. A uranium- and titanium-bearing phase (suggested to be brannerite) was also observed to form on SRL 131 glass [62] and, with thorium, within the altered layer of WVCM 50 glass [111]; a similar phase was also found suspended in solution [263].

Less is known about the phases affecting the solubilities of $\mathrm{Np}, \mathrm{Pu}$, and Am or their incorporation into secondary phases due to the low levels of these elements in glasses used in laboratory tests and limitations of analyzing these elements. In addition, the relatively high solubility of neptunium and the fact that plutonium and americium are preferentially fixed onto metal surfaces results in only small amounts being available for secondary phase formation in 
leach tests. Plutonium and actinide-bearing phases are typically very small and may be distributed on the altered glass surface or may be suspended in the leachate as colloids. Characterization of plutonium and actinide-bearing phases has been achieved by filtering solutions through holey carbon grids and analyzing the filtrant in an electron microscope [262-264]. Microcrystallites of brockite $\left[(\mathrm{Ca}, \mathrm{Th}) \mathrm{PO}_{4} \cdot \mathrm{H}_{2} \mathrm{O}\right]$ which have incorporated plutonium and americium have been observed both isolated and supported on colloidal clay particles removed from solution [262]. The similarity between clay colloids removed from solution and clays at the surfaces of reacted glass suggests that material may slough from the reacted glass surface and become suspended in solution in a mobile state [262]. Thus, radionuclides found immobilized during short-term tests may become mobilized due to mechanical separation at longer exposure times.

The chemical similarity of radioelements to nonactive elements will result in the substitution of radioelements into other mineral phases which precipitate out of solution, and minerals may incorporate some radioelements via ion exchange. For example, ${ }^{137} \mathrm{Cs}$ may become incorporated into analcime, a sodium aluminosilicate which commonly forms during glass corrosion $[61,112,191]$. Other zeolites may also incorporate radionuclides. Crystalline lanthanoid phases such as powellite and cerianite-type materials, which may host Am and Cm, were found during the reaction of R7T7 glass in salt solutions [265]. Incorporation of radioelements into mineral phases or their sorption onto mineral phases may be immobilize for different durations, depending on the stability of the host phase. Aging of the mineral phases formed due to glass corrosion will result in thermodynamically more stable phases, which may have a reduced affinity for radionuclides than previous phases.

Colloid formation will also affect the disposition of radioelements over long time periods. Laboratory tests show that aluminum and iron colloids are formed during glass corrosion [167], and that these may sorb radionuclides $[67,266]$. Depending on the colloid size, solution chemistry, groundwater flow and the characteristics of the surrounding geology, these colloids may or may not be transportable, and their transportability may change over time. Colloids which settle out of solution during static leach tests may behave differently under dynamic conditions. Likewise, incorporation into alteration phases on the glass surface may only temporarily immobilize the radionuclides. Actinide-bearing colloids may be formed by exfoliation of alteration phases from corroded surfaces under stress or over long time periods [262].

\subsection{Effects of Other Materials on Actinide Disposition}

The extent of accumulation of actinides in the altered layer will depend on the presence of other materials in the vicinity of the corroding glass onto which released actinides may sorb, such as clay backfills or metal barrier components. These materials may act as sinks to remove dissolved actinides from solution and provide additional barriers against radionuclide migration. Tests with R7T7 glass showed the amounts of $\mathrm{Np}$ and $\mathrm{Pu}$ retained in surface layers varied when other materials, including sand, granite, bentonite, smectite, illite, Boom clay, and salt were included in the test vessel [249]. The presence of these materials also affects the solution chemistry and the glass corrosion rate [267]. In general, the solutions in tests with the added materials contained more $\mathrm{Np}$ than those in tests with distilled water alone, but less Pu. Smectite, illite, and Boom clays sorbed $\mathrm{Np}$ (weakly) and $\mathrm{Pu}$ (strongly), granite and bentonite sorbed $\mathrm{Pu}$ (strongly), and salt sorbed Pu (weakly) [249]. It was also found that the amount of plutonium in the altered layer remained high if the smectite was not in direct contact with the glass $(R F(P u)=80)$, but was significantly reduced if the clay directly contacted the glass $(R F(P u)=6)$ [249]. This implies that diffusion of plutonium through the solution limits the amount sorbed onto the clay surface. 
Leach tests in a slurry of Boom clay showed retention of $\mathrm{Np}$ and $\mathrm{Pu}$ in layers formed on R7T7 glass and on a Pamela glass SM513, but a third glass, Pamela SM527, did not generate an alteration layer and did not retain these elements [268]. Technetium was not retained by the layers. The Pu and Am released from the glass was strongly sorbed onto the clay, while the Tc and $\mathrm{Np}$ were only partially sorbed onto the clay. The plutonium and americium had attained steady-state concentrations after two years of reaction (both below $2 \times 10^{-9} \underline{\mathrm{M}}$ ), while neptunium was present at about $2 \times 10^{-6} \underline{\mathrm{M}}$ and technetium at about $4 \times 10^{-7} \underline{\mathrm{M}}$. Further, addition of $\mathrm{Fe}_{2} \mathrm{O}_{3}$ to the tests slowed the corrosion of all three glasses by at least a factor of 68 [268].

Released radionuclides may be immobilized by incorporation into or sorption onto mineral phases present naturally or generated by corrosion of repository materials. Immobilization by sorption onto repository components or geologic minerals may be transient, depending on the stabilities of the host phases. Conversely, sorption onto colloids present in the groundwater or generated during glass corrosion may mobilize radionuclides in excess of their solubilities. Sorption of radionuclides onto colloids generated during glass corrosion will strongly affect the distribution of actinides. Sorption is specific to the host mineral phase and the oxidation state of the radionuclide: sorption of lower oxidation states is generally stronger than that of higher oxidation states. Sorption may result in a change in the oxidation state of the radionuclide. Competition between actinides and other metal ions in complexation and sorption reactions results in complex chemistries in real systems, and will, in general, decrease the amounts of radionuclides removed from solution.

Colloidal materials that occur naturally in the groundwater or are generated during corrosion of engineered barrier materials will be available to sorb radionuclides [193,269]. This material may remain suspended in solution, or may eventually be sorbed by immobile phases, may be filtered from the solution by microprobes, or may flocculate and settle out of solution. Sorption is generally stronger for actinides in lower oxidation states, so that $\mathrm{Am}(\mathrm{III})$ and $\mathrm{Pu}(\mathrm{IV})$ are expected to be more strongly sorbed to colloids and mineral phases than $U(V I)$ or $N p(V)$ [270]. The highly soluble pertechnetate ion is not expected to be sorbed onto colloids.

Migration of radionuclides released from the glass will be affected by the many materials present in the geologic formations or engineered barriers surrounding the waste form, as well as interactions involving the corrosion products of the waste glass itself. Various clays, zeolites, and other mineral phases are known to form as glass waste forms corrode [112,271], and interactions between released radionuclides and these secondary phases may either enhance or reduce their release from the immediate vicinity of the waste form, depending on the mobilities of the particular phases. Species having low valences, such as Am(III) and Pu(IV), generally interact more strongly with sorbents than species with higher valences, such as $\mathrm{Np}(\mathrm{V})$ and U(VI). Technetium (VII) does not sorb onto mineral phases, but sorption may occur on some surfaces after reduction to Tc(IV). Reliable long-term predictions of waste behavior must account for these interactions.

Colloidal material formed by naturally occurring inorganic and/or organic materials or microorganisms in the groundwater will be available to sorb radionuclides from the solution to form pseudocolloids that may remain suspended in the groundwater or may flocculate and settle out of solution $[269,272,273]$. Sorption may occur through dispersive interactions (physisorption), coulombic interactions (electrostatic adsorption and ion exchange), or electron exchange (chemisorption). The presence of colloids bearing radionuclides may lead to increased aqueous concentrations which far exceed the solubility limits of pure phases. Depending on the size and transportability of these colloids, radionuclides may become available for dispersal away from a repository site by groundwater flow or may become immobilized as the colloids settle out of solution. Colloids initially suspended in the groundwater may eventually interact 
with geologic materials after longer times or as they migrate away from the waste form so that the colloids become filtered by micropores or as radioelements become more strongly sorbed onto mineral surfaces present in the host rock [274,275].

The sorption energy of the actinides onto mineral surfaces generally decreases as $A n(I I I, I V)>A n(V)>A n(V I)$. Uranium in the hexavalent state and neptunium in the pentavalent state are poorly sorbed onto most mineral surfaces, while tetravalent plutonium and trivalent americium are strongly adsorbed onto most geologic minerals. Sorption of radionuclides that exist in high valance states in solution may occur on sites capable of reducing the radionuclide to a lower oxidation state. Several studies have been performed to characterize the sorption of radionuclides onto mineral surfaces, both onto monolithic samples and onto mineral colloids. Some are discussed below.

In addition to actinides, sorption sites on mineral surfaces have an affinity for other solution species, including metal ions, complexants, and dissolved humic materials. Competition between different sorbants for surface sites and complexation of actinides in solution will affect the amount of actinides that may become sorbed onto mineral colloids in solution or minerals fixed in rocks.

\subsection{Summary}

The disposition of individual radionuclides between mobile and immobile phases as glass corrodes is strongly influenced by the solution chemistry and the presence of complexants and various sorbents. Technetium, uranium, and neptunium are generally more soluble than plutonium or americium in oxic solutions at the basic $\mathrm{pH}$ values expected in most repositories and attained in most tests, and are expected to attain higher solution concentrations in leach tests. Laboratory tests show technetium, uranium, and neptunium to be released into solution to a greater extent than either plutonium or americium during corrosion of several glass compositions. Actinides which do not enter solution due to low solubilities may be retained in the surface layers of the altered glass, either by sorption or incorporation into secondary phases, or may become sorbed on the surfaces of other materials that may be present in the vicinity of the corroding glass.

Identification of the phases controlling the solubilities of the actinides remains an important information need for predicting long-term behavior of high-level waste glasses. Tests conducted under conditions leading to saturation of the corroding fluids and the precipitation of phases affecting the solubility of released radionuclides are needed to identify important phases. Also, the effects of colloid formation is a critical uncertainty that must be addressed in performance models. 


\subsection{SUMMARY OF CRITICAL REVIEW OF EFFECTS OF SA/V ON GLASS CORROSION AND DISCUSSION OF REMAINING UNCERTAINTIES}

This report has summarized the present state of knowledge regarding the effects of the glass surface area/solution volume $(\mathrm{SA} / \mathrm{V})$ ratio on the corrosion behavior of borosilicate waste glasses. The $\mathrm{SA} / \mathrm{V}$ ratio affects the rate of glass corrosion through the extent of dilution of corrosion products released from the glass into the leachate solution. The observed effects of the $\mathrm{SA} / \mathrm{V}$ used in a static leach test were interpreted in terms of differences in solution chemistries attained in tests at different SA/V. A detailed discussion of the effects of the solution chemistry on the glass corrosion rate and mechanism was provided to support interpretations of the effects of the SA/V on a mechanistic scale. Glass corrosion was discussed in some detail by first presenting a brief summary of glass structure and then discussing the processes leading to the alteration and dissolution of the glass structure. Laboratory tests performed to elucidate the corrosion process and the relevance of the information they provide to the corrosion behavior and the effects of the solution chemistry on glass corrosion. The results of static leach tests conducted specifically to characterize the effects of the SA/V on glass corrosion are then discussed based on what is known about the effects of the solution chemistry on glass corrosion. Observed effects of the SA/V, solution chemistry, and test procedure on the disposition of radionuclides during glass corrosion was discussed. Key findings are summarized below.

\subsection{Observed Corrosion Behavior}

Laboratory tests conducted to distinguish the effects of the fluid chemistry and glass alteration layers on glass dissolution has shown that the effects of the fluid chemistry dominate the glass corrosion rate in tests conducted at relatively low SA/V where the solution does not become saturated with silicic acid. The silicic acid concentration and the $\mathrm{pH}$ affect the rate most strongly, with the reaction to release silicic acid usually being modeled as the key corrosion step. The rate of silicic acid release from the glass depends on the temperature, glass composition, silicic acid concentration in solution, and the solution $\mathrm{pH}$. For a given glass composition at a given temperature, any test conditions that affect the silicic acid concentration or the solution $\mathrm{pH}$ will affect the glass corrosion rate. The SA/V used in the test affects the corrosion rate through dilution of the silicic acid and $\mathrm{pH}$. That is, tests at low SA/V dilute the silicic acid more than tests at high $\mathrm{SA} / \mathrm{V}$ and also maintain lower $\mathrm{pH}$ values (assuming that corrosion of glass results in an increased $\mathrm{pH}$ ).

The silicic acid concentration and the solution $\mathrm{pH}$ are also affected by the release of other glass components as the glass corrodes, the volume of solution available to dilute released glass components, the refreshment of the leachate solution due to solution flow, if any, and the formation of secondary phases. The relative importance of these effects in a particular experiment will be determined by the glass and initial fluid compositions, and by the test method and test conditions utilized. Glasses having large amounts of alkali metals will generate higher solution $\mathrm{pH}$ values as the glass corrodes than glasses with less alkali, and the effect of the SA/V. on the $\mathrm{pH}$ will be greater in tests generating higher $\mathrm{pH}$ values. Less durable glasses will attain more concentrated solutions after shorter corrosion times than more durable glasses, and the effects of the SA/V and flow on the corrosion rate will be greater in tests with less durable glasses. In addition, the extent of corrosion of less durable glasses will be greater than that of more durable glasses in a common laboratory test, so that the effects of SA $/ \mathrm{V}$ and flow occur at a more advanced stage of corrosion in tests with the less durable glass. Species in the leachant may affect the corrosion through interactions with the glass surface, such as ion exchange and precipitation, or by affecting the solubilities of released glass components, such as the formation of transuranic carbonates. Of course, the silicic acid concentration in the fluid contacting the glass and the solution $\mathrm{pH}$ will also affect the glass corrosion. In general, the glass and solution 
compositions are determined by the waste form and the disposal site, while the test conditions may be selected to simulate particular environmental conditions, highlight a particular reaction step, or characterize the general corrosion behavior.

Past efforts to describe glass corrosion behavior has been mostly empirical, where sets of data were fit by simple analytic expressions, such as diffusion equations. However, reliable predictions of long-term glass corrosion behavior cannot rely on empirical correlations to short term data due to the complex nature of glass corrosion and changing conditions of temperature, solution chemistry, etc. anticipated to occur over the service life of a waste repository. That rates measured in short-term laboratory tests cannot be extrapolated to long times is clearly demonstrated by the observation that the glass corrosion rate varies by about three orders of magnitude as corrosion proceeds, even under static reaction conditions. Instead a mechanistic model of the corrosion processes is needed to account for the effects of varying solution chemistries and flow rates, as well as varying temperatures over the long time periods that radioactive wastes must be contained.

\subsection{Modeling}

Glass corrosion is well described by a rate equation that combines a rate coefficient that depends on the glass composition, a pH-dependence, and a reaction affinity term expressed as the difference between the actual silicic acid concentration and a "saturation" value. Use of this equation requires the experimental determination of (1) the rate coefficient and its temperature dependence, (2) the $\mathrm{pH}$ dependence of the rate coefficient, and (3) the saturation value. Laboratory testing has shown that, under some conditions, a residual affinity remains under saturated conditions so that a final rate term must be measured experimentally. In other tests, secondary phases that precipitate from highly concentration solutions have shown to control the silicic acid concentration after long corrosion progress. These phases must be identified to predict the long-term corrosion behavior. Other phases present in a disposal system may likewise affect the solution chemistry, particularly the solution $\mathrm{pH}$ and silicic acid concentration, and influence the corrosion rate. These effects must also be accounted for.

The corrosion of borosilicate waste glasses can be described as occurring in three stages, which are related to the solution chemistry. The first stage occurs when the solution concentration of silicic acid is very low and the glass corrodes at a relatively high rate that depends on the glass composition and the solution $\mathrm{pH}$. This is sometimes referred to as the "intrinsic" rate, since it depends on the glass composition and is not affected by the affinity term. The second stage occurs as the silicic acid concentration increases to a maximum level, the socalled "saturation" concentration and the glass corrosion rate decreases to a minimum value. The decrease in the corrosion rate is due to a decrease in the reaction affinity. A third stage occurs when secondary phases precipitate from solution to establish a silicic acid solubility limit that may be different than the "saturation" concentration. This change affects the rate through the affinity term, because the presence of secondary phases causes the silicic acid concentration to be maintained below the apparent saturation value imposed by the glass.

\subsection{Testing}

Different laboratory tests can be used to highlight particular aspects of the corrosion process. An understanding of how different test conditions affect the solution conditions and the glass corrosion process is required to correctly utilize the test results to describe the corrosion behavior and determine values for model parameters. Tests with large solution volumes relative to the exposed glass surface area (low effective SA/V), such as MCC-1 and flow-through tests, will maintain dilute conditions and the glass corrosion rate will be unaffected or only weakly affected by the minor accumulation of silicic acid in solution. These tests are used to determine the intrinsic corrosion rate and the $\mathrm{pH}$-dependence of the corrosion rate. Tests that allow silicic 
acid to accumulate in the solution, such as the MCC-3 test and the Product Consistency Test, show the effects of the affinity term on the corrosion rate and the approach to saturation. If these tests are conducted for sufficiently long time periods, secondary phases will precipitate from the saturated solutions and affect the corrosion rate. The corrosion process may be affected by secondary phase formation under some test conditions, although reaction times of several months or years may be required before secondary phases precipitate. The Vapor Hydration Test developed at ANL was designed to quickly attain saturation of the solution and drive the corrosion process to the point where the solution chemistry was controlled by secondary phases within a short time period.

While many laboratory tests have been developed to investigate the corrosion process, these are rarely related to modeling efforts either with regard to elucidating corrosion processes or deriving values of model parameters. This divergence has resulted in a situation where many studies are done on the basis of a test procedure that provides little or no information relevant to describing the corrosion behavior. A need exists to clearly relate test procedures and test results to a mechanistic description of the glass corrosion process that can be used to predict long-term glass durability and conduct performance assessments of disposal systems.

\section{$8.4 \quad \underline{\mathrm{SA} / \mathrm{V}}$}

The $\mathrm{SA} / \mathrm{V}$ ratio used in static leach tests affects the dilution of the reaction products, including the silicic acid concentration and the $\mathrm{pH}$, both of which affect the glass corrosion rate. The SA/V affects how fast the solution chemistry changes as the glass occurs, and a value can be selected to highlight the early stages of the reaction under dilute conditions (at low SA/V) or the more advanced stages of corrosion under more concentrated conditions (at high SA/V). The solution results of tests conducted at different $\mathrm{SA} / \mathrm{V}$ ratios are conveniently plotted as a function of the product of the SA/V and the corrosion time to include data on a single plot. The product $(\mathrm{SA} / \mathrm{V}) \mathrm{t}$ can be used to estimate the reaction progress. That is, tests conducted for greater $(\mathrm{SA} / \mathrm{V}) \mathrm{t}$ attain a greater extent of reaction, as measured by the release of glass components. Plotting solution concentrations as a function of $(\mathrm{SA} / \mathrm{V}) \mathrm{t}$ is based on the different dilution effects of tests at different $\mathrm{SA} / \mathrm{V}$, assuming the glass corrodes at the same rate at both $\mathrm{SA} / \mathrm{V}$. However, two caveats exist regarding the comparison of data measured at different $\mathrm{SA} / \mathrm{V}$ but common $(\mathrm{SA} / \mathrm{V}) \mathrm{t}$ :

(1) The glass corrosion rates are also affect by the solution $\mathrm{pH}$ value. A simple correlation variables such as $(\mathrm{SA} / \mathrm{V}) \mathrm{t}$ cannot be used to quantitatively relate tests run at different $\mathrm{SA} / \mathrm{V}$ unless the effects of the $\mathrm{pH}$ are taken into account. While the solution $\mathrm{pH}$ affects the rates of reactions to release most glass components, most important is the increased release of aluminum at $\mathrm{pH}$ values below about $\mathrm{pH}$ 5 and the increased release of silicon at $\mathrm{pH}$ values above about $\mathrm{pH} 9$. Solutions in tests conducted at higher SA/V generally attain higher $\mathrm{pH}$ values than tests conducted at lower $\mathrm{SA} / \mathrm{V}$. The higher $\mathrm{pH}$ values accelerate the corrosion rate, so that the glass reacts faster in tests at higher SA/V than in tests at lower SA/V. Thus, more glass will have reacted and more glass components will have been released into solution in a tests at a higher SA/V than in a test at a lower SA/V conducted for the same product of (SA/V)t. This is observed in tests with highlevel waste glasses, where tests at high $\mathrm{SA} / \mathrm{V}$ consistently have higher solution concentrations of soluble glass components than tests at low SA/V at common $(\mathrm{SA} / \mathrm{V}) \mathrm{t}$. Higher concentrations of some glass components, including silicon and boron, can occur due to dissociation at high $\mathrm{pH}$ values.

(2) The alteration of the glass surface will not scale as (SA/V)t due to the effects of different solution chemistries and due to the kinetics of secondary phase formation. Thicker layers are formed in tests at low SA/V than at high SA/V 
when a glass dissolves nonstoichiometrically, so a larger volume of alteration phases is formed in tests at lower SA/V. While tests conducted at high SA/V are interpreted to represent advanced stages of corrosion, it must be recognized that the alteration phases form in a leachate solution having a higher $\mathrm{pH}$ than those formed at lower SA $\mathrm{V}$. The solution $\mathrm{pH}$ strongly affect the stabilities of most secondary phases. For example, the solubility of analcime, a zeolite commonly formed during corrosion of high-level waste glasses, is given as $\mathrm{K}_{\mathrm{sp}}=$ $\left[\mathrm{Na}^{+}\right]\left[\mathrm{Al}^{3+}\right] /\left[\mathrm{H}^{+}\right]^{4}$. Thus, the formation of analcime is expected to occur after less glass has corroded in tests at high $\mathrm{SA} / \mathrm{V}$ which attain a higher $\mathrm{pH}$ value than in tests at lower $\mathrm{SA} / \mathrm{V}$ which attain lower $\mathrm{pH}$ values.

\section{$8.5 \quad$ Flow}

The flow rate used in dynamic tests also affects the concentration of silicic acid at the glass surface. The corrosion rate will be proportional to the flow rate at intermediate flow rates, but a maximum corrosion rate is attained at high flow rates. At low flow rates, the corrosion rate is limited by the accumulation of glass corrosion products in the fluid and the resulting low reaction affinity. The glass corrosion behavior under very low flow conditions approaches that in a static test. Tests at higher flow rates are generally used to measure the forward reaction rate. Tests using $\mathrm{pH}$ buffered leachants that are performed at high flow rates can be used to measure the forward reaction rate as a function of $\mathrm{pH}$. These tests are also well suited to measuring the effects of glass composition on the corrosion behavior, since they minimize the effects of reaction products on the continued glass corrosion.

\subsection{Remaining Issues}

Based on this review of the literature regarding the effects of SA/V on glass corrosion, several key issues identified in the initial literature review that impact waste management remain unresolved. These include:

- How can tests at different SA/V be related?

Tests have shown (SA/V)t scaling of solution concentrations to be affected by the solution $\mathrm{pH}$. Current models of the glass corrosion rate account for the effects of the $\mathrm{pH}$ and may be used to improve the scaling approach, although this remains to be done.

- $\quad$ Can tests at high SA/V be used to characterize advanced stages of corrosion with regard to alteration of the glass?

Tests conducted at high SA/V attain saturated solution conditions after shorter reaction times than tests conducted at lower $\mathrm{SA} / \mathrm{V}$, but also attain higher solution $\mathrm{pH}$ values. The higher solution $\mathrm{pH}$ values will likely influence the formation of secondary phases. The effects of solution chemistry on secondary phases formation can be predicted using solution equilibration computer codes.

- Are tests conducted using monolithic and crushed glass samples at the same SA/V equivalent?

Several investigations have compared the corrosion rates of monolithic and crushed glass samples. The inability to accurately measure the effective surface area of crushed glass samples limits the direct comparison of test data. Geometric approximation based on sieve sizes are more realistic than surface areas measured using adsorption techniques. It is generally assumed that the corrosion rates of monolithic and crushed glass are the same, and comparison of 
solution concentrations generated by monoliths and crushed glass samples in tests maintaining dilute leachate solutions is often used to determine the effective surface area of the crushed glass. Comparison of tests at different estimated SA/V using the same size fraction of crushed glass is probably more reliable than comparison of tests using different size fractions to attain different SA/V.

- How can the loss of surface area by accounted for in long-term tests with crushed glass?

The reduction in the surface area available for reaction is currently not accounted for when calculating the corrosion rate. An analytical method to correct the calculated normalized mass loss and normalized corrosion rate for changes in the surface area of crushed glass is needed.

- Does the SA/V used in a test affect the disposition of released radionuclides?

Very few tests have been conducted to assess the effects of released glass matrix components on the disposition of radionuclides. Most of these have been conducted at low SA/V or under flowing conditions that are not relevant to repository disposal conditions. Tests at high SA $\mathrm{V}$ are needed to measure the effects of the higher solution $\mathrm{pH}$, secondary phase formation, and colloid formation.

- Which secondary phases control the solubility of released radionuclides?

Little is known regarding the phases controlling the solubilities of radionuclides in the presence of glass corrosion products. Determination of the solubility controlling phases is complicated by difficulties associated with work with radioactive materials, analytical limitations for detecting low concentrations of some elements and the low solubilities of some radionuclides $(\mathrm{Np}, \mathrm{Pu})$, difficulties in isolating the small phases that form.

- What is the effect of long corrosion times in tests at high SAN?

Tests conducted at high SA/V show deviations from the trends observed in tests at lower SA/V, such as resumption of high corrosion rates after secondary phase formation. It needs to be determined if the high corrosion rate is a transient phenomenon or if it represents the long-term corrosion rate. Tests in which a high corrosion rate is observed need to be conducted to determine which rate should be used in the corrosion model.

- What process controls the glass corrosion rate under silica saturation prior to secondary phase formation?

Prior to secondary phase formation, the corrosion slows to a negligible rate as the solution approaches apparent saturation with respect to the glass phase. However, the glass is metastable and continues to corrode, even under apparent saturated conditions. Because the glass may continue to corrode under these conditions for very long times prior to secondary phase formation, the process controlling the corrosion rate must be understood. Experimental measurement of the rate is difficult, due to its low value and the relatively large uncertainties of experimental measurements. Of course, the resumption of higher corrosion rates after secondary phases form will overwhelm the importance of this process to continued glass corrosion. 
- Which $\mathrm{SA} / \mathrm{V}$ ratios are relevant to repository storage?

The SA/V representative of repository storage conditions will vary with time as water may slowly reenter the repository and contact the glass. (In fact, corrosion of glass in the repository and laboratory tests at various $\mathrm{SA} / \mathrm{V}$ are readily related through the effects of the solution chemistry.) Initial contact will likely occur between glass and condensed water vapor, with small volumes of liquid water eventually collecting on the glass. These conditions are represented by an initially extremely high then slowly decreasing SA/V. Hence, the conditions in the repository are not well represented by commonly performed laboratory tests, which exposed the glass to large volumes of water throughout the test period. (An exception is the Unsaturated Test Method developed at ANL.) These tests are conducted to characterized the corrosion behavior of the glass, but do not directly simulate glass corrosion under service conditions. Glass corrosion models must be correctly integrate both laboratory test results and site characterization results to model glass corrosion under service conditions. Other tests, such as the Unsaturated Test Method, must be developed to simulate changes in environmental conditions, such as the effective $\mathrm{SA} / \mathrm{V}$, to verify model predictions.

- What other repository-relevant conditions will impact the glass corrosion behavior, the solution chemistry, and the disposition of radionuclides and should be tested?

Conditions specific to a particular repository may impact the corrosion behavior of waste glasses. For a repository in an unsaturated environment, cycling of wet and dry conditions and episodic contact by relatively large volumes of water may significantly affect the performance of waste glasses. Periodic evaporation of water from the glass may result in cracking and peeling of alteration layers. These layers may be flushed from the glass at later times. Evaporation may also leave hygroscopic residues that will attract water entering at later times. Changing temperatures will affect the solubilities of most glass components and released radionuclides, which may result in the precipitation of secondary phases, formation of colloids, and other effects that will affect the corrosion of the glass and the transportability of radionuclides. 


\subsection{ANL TASK "RELATIONSHIP BETWEEN HIGH SA/V TESTS AND MCC-1"}

The DOE sponsored an experimental task designed to clarify the effects of the SA/V ratio used in static leach tests on the corrosion mechanism and corrosion rate of borosilicate waste glasses relevant to the DWPF, and to assess the effects of the SA/V on the release on disposition of important radionuclides. The task was designed to include MCC-1 tests at SA/V of $10 \mathrm{~m}^{-1}$ and PCT tests at SA/V of $2000 \mathrm{~m}^{-1}$, plus tests following the MCC-1 and PCT protocols at SA/V of 340 and $20,000 \mathrm{~m}^{-1}$. Both solutions and corroded solids were analyzed to completely characterize the reacted system. Although several long-term tests remain in progress, enough tests have been completed to address the issues identified regarding the effects of the SA/V used in a static leach test on the solution chemistry, glass alteration, and disposition of actinide components. The results of this task have been published as data has become available [64-67,112,276,277]. Some results have also been discussed in earlier sections of this report. The technical approach and the data collected to date are summarized in this chapter. Spreadsheets containing all available data are also included in Appendix D. Tests being conducted for long time periods are still in progress, and a report describing the task and all test results will be issued upon completion of the task.

\subsection{Technical Approach}

Frit material for preparing SRL 131 and SRL 202 glasses was supplied by the Savannah River Technology Center and doped with ${ }^{99} \mathrm{Tc},{ }^{238} \mathrm{U},{ }^{237} \mathrm{~Np},{ }^{239} \mathrm{Pu}$, and ${ }^{241} \mathrm{Am}$ at ANL. Glasses doped with the full compliment of radionuclides are referred to as SRL 131A and SRL 202A. Another glass was prepared by doping SRL 202 with natural uranium only. This glass is referred to as SRL 202U. The SRL 202 composition is the current "Design-Basis" glass composition for blended Savannah River waste [278]. The SRL 131 composition was an earlier reference composition known to be less durable than the SRL 202 composition. It is similar to the "Purex" glass, which was a possible "worst-case" composition [278] prior to specification of the "Environmental Assessment" glass for the DWPF (SRL EA) [101]. It was tested to demonstrate the effects of SA/V on a glass having a relatively poor durability, and to assure that the corrosion would advance to a large extent within the testing time. In addition, both the MCC-1 and PCT tests were designed to distinguish the relative durabilities of glasses having different compositions. Testing two glasses provides insight regarding the ability of different tests to distinguish the long-term corrosion behavior. The SRL 131A and SRL 202A compositions are given in Appendix B. The SRL 202U composition is identical to the SRL 202A composition, except for the absence of Tc, $\mathrm{Np}, \mathrm{Pu}$, and Am.

Both monolithic disk samples and crushed glass samples were prepared for testing. The faces and circumference edges of the monolithic disks were ground to a 600 -grit finish for use in MCC-1 type tests at SA/V ratios of about 10 and $340 \mathrm{~m}^{-1}$. All surfaces on the monoliths were ground to the same finish to assure uniformity in the surfaces of all samples to minimize the effects of the surface finish on corrosion (see Section 2.7). The crushed glass was sieved to isolate the $-100+200$ mesh fraction and washed for use in PCT type tests at SA/V ratios of about 2000 and $20,000 \mathrm{~m}^{-1}$. Tests with SRL 131A and SRL 202A glass were performed in type 304L stainless steel vessels having a volume of approximately $22 \mathrm{~mL}$. Tests with SRL 202U glass were performed in PFA Teflon vessels having a similar volume to provide data free from any effects of stainless steel for use in computer modeling. All tests were conducted at $90 \pm 2^{\circ} \mathrm{C}$ in either a constant-temperature oven (tests in steel vessels) or water bath (tests in Teflon vessels). Teflon vessels were placed in a water bath such that the vessel bottoms were submerged but the vessel tops were not submerged to prevent possible leakage of water from the water bath into the 
vessel. A water bath was used to minimize loss of leachate from the vessels over long test periods, and to reduce the diffusion of carbon dioxide into the vessels. Other tests have shown carbon dioxide to penetrate teflon vessels and affect the solution $\mathrm{pH}[102,279]$.

The leachant was a tuff groundwater solution that was prepared by reacting pulverized caliche-free tuff rock with actual groundwater from well J-13 on the Nevada Test Site at $90^{\circ} \mathrm{C}$ for about 28 days. This was done to simulate the interaction of groundwater with tuff near the repository where the rock temperature is anticipated to be near $90^{\circ} \mathrm{C}$, since the solution concentrations of ions in the groundwater will change as the temperature increases from 25 to $90^{\circ} \mathrm{C}$. The resulting groundwater solution was then cooled to room temperature and filtered through $0.1 \mu \mathrm{m}$ filters to remove any suspended tuff particles. The resulting solution is referred to as EJ-13 water. The composition of the J-13 groundwater and the EJ-13 water are given in Table 7. The major changes in the J-13 chemistry due to reaction with tuff are an increase in the sodium and silicon concentrations, an increase in the $\mathrm{pH}$, and a decrease in the alkaline earth concentrations resulting from their retrograde solubilities. The actual groundwater that might eventually contact the waste glass in a repository will be affected by interaction with the waste package components and by radiolysis, as well as by interaction with the tuff. These interactions may affect the $\mathrm{pH}$ and all solute concentrations. Because the engineered barrier system has not yet been specified, only the effects of interactions with the tuff were considered in this Task. The effects of radiation on the corrosion behavior of high-level waste glasses have been measured previously [95,113,114,280-283]. SRL 202U glass was tested in both EJ-13 and in deionized water to assess effects of the leachant composition on the corrosion.

MCC-1 type tests were conducted by placing either one or four monolithic disks in either 16 or $2 \mathrm{~mL}$ of leachant to achieve SA/V ratios of 10 and $340 \mathrm{~m}^{-1}$, respectively. PCT type tests were conducted by adding either 1 or $5 \mathrm{~g}$ of crushed glass to either 10 or $5 \mathrm{~mL}$ of leachant to achieve SA/V ratios of 2000 or $20,000 \mathrm{~m}^{-1}$, respectively. The surface area of the grains was approximated by assuming they were spheres having a diameter equal to the average mesh opening (which was about $112 \mu \mathrm{m}$ ). The surface area of the monolithic disks was measured geometrically. All tests were conducted in duplicate at $90 \pm 2^{\circ} \mathrm{C}$. Test durations were selected to provide both a temporal description of the corrosion of both glass types at all SA/V. Tests were also scheduled to provide results at different SA/V but common products of (SA/V)t. This was done to compare solution and solids analyses and to assess the usefulness of (SA/V)t as a measure of the corrosion progress. The test matrix is summarized in Table 8, which shows the overlap in (SA/V)t of tests performed at different SA/V.

Table 7. Compositions of Tuff Groundwater from Well J-13a and EJ-13 Water

\begin{tabular}{lclllr}
\hline & $\mathrm{J}-13$ & $\mathrm{EJ}-13$ & & $\mathrm{~J}-13$ & $\mathrm{EJ}-13$ \\
\hline $\mathrm{Al}$ & 0.37 & 1.1 & $\mathrm{HCO}_{3}-$ & $\mathrm{N} / \mathrm{A}$ & 120 \\
$\mathrm{~B}$ & 0.15 & 0.16 & $\mathrm{~F}^{-}$ & $\mathrm{N} / \mathrm{A}$ & 3 \\
$\mathrm{Ca}$ & 11.7 & 5.1 & $\mathrm{Cl}^{-}$ & $\mathrm{N} / \mathrm{A}$ & 9 \\
$\mathrm{~K}$ & 6.6 & 7.3 & $\mathrm{NO}_{3-}^{-}$ & 10.1 & 16 \\
$\mathrm{Li}$ & 0.035 & 0.05 & $\mathrm{SO}^{2-}$ & $\mathrm{N} / \mathrm{A}$ & 35 \\
$\mathrm{Mg}$ & 1.8 & 0.39 & & & \\
$\mathrm{Na}$ & 42 & 54 & & & \\
$\mathrm{Si}$ & 28 & 46 & $\mathrm{pH}$ & 6.9 & 8.2 \\
\hline
\end{tabular}

aFrom [195]. 
Table 8. Summary of Test Matrix for ANL Task

\begin{tabular}{|c|c|c|c|c|}
\hline \multirow{2}{*}{$\begin{array}{l}(\mathrm{SA} / \mathrm{V}) \mathrm{t} \\
(\mathrm{days} / \mathrm{m})\end{array}$} & \multicolumn{4}{|c|}{ Test Length (days) } \\
\hline & $10 m^{-1 a, b}$ & $340 \mathrm{~m}^{-1 \mathrm{c}}$ & $2000 \mathrm{~m}^{-1 a, b, c}$ & $20,000 \mathrm{~m}^{-1 \mathrm{a}, \mathrm{b}, \mathrm{c}}$ \\
\hline 70 & 7 & & & \\
\hline 280 & 28 & & & \\
\hline 940 & 94 & & & \\
\hline 2380 & 238 & & & \\
\hline 4760 & & 14 & & \\
\hline 6000 & 600 & & 3 & \\
\hline 9520 & & 28 & & \\
\hline 14,000 & 1400 & & 7 & \\
\hline 19,040 & & 56 & & \\
\hline 28,000 & $(2800)^{\mathrm{d}}$ & & 14 & \\
\hline 30,940 & & 91 & & \\
\hline 60,000 & & & 30 & 3 \\
\hline 61,200 & & 180 & & \\
\hline 122,400 & & 360 & & \\
\hline 140,000 & & & 70 & 7 \\
\hline 183,000 & & 540 & & \\
\hline 224,800 & & 720 & & \\
\hline 280,000 & & & 140 & 14 \\
\hline 560,000 & & & 280 & 28 \\
\hline $1,120,000$ & & & 560 & 56 \\
\hline $1,960,000$ & & & 980 & 98 \\
\hline $3,640,000$ & & & $(1820)$ & 182 \\
\hline $7,280,000$ & & & & 364 \\
\hline $14,560,000$ & & & & 728 \\
\hline $20,000,000$ & & & & 1000 \\
\hline $\mathrm{TBD}^{\mathrm{e}}$ & & & TBD & TBD \\
\hline
\end{tabular}

aDuplicate tests performed with SRL 202A glass.

bDuplicate tests performed with SRL 131A glass

cDuplicate tests performed with SRL 202U glass.

dTime in parentheses indicate tests in progress.

eTBD = test time "to be determined." 
After the predetermined test duration, the vessel was removed from the oven and opened. The leachate solution and reacted glass were removed for analysis. The leachates were removed while still hot to avoid thermal effects, such as precipitation of solids or flocculation of colloids. To isolate suspended solids, some of the leachate solution was filtered through a holey carbon grid used in a transmission electron microscope (TEM). In this procedure, a small volume of leachate is placed on a holey carbon grid and then wicked through the grid using alcohol. Suspended solids are filtered from the solution as it passes through the grid and remain on top of the grid where they can be analyzed using the TEM. An aliquot of the leachate solution was also removed for actinide analysis. The remaining solution was then filtered through a preheated $0.45-\mu \mathrm{m}$ cartridge filter. A portion of this solution was further filtered through an Amicon model CF50A filter, which is assumed to have an effective filter size of about $6 \mathrm{~nm}$. Solutions were analyzed for $\mathrm{pH}$ (using a combination electrode), anions (by ion chromatography), cations [by inductively coupled plasma-atomic emission or inductively coupled plasma-mass spectrometry (ICP-MS)], and actinides (by alpha spectrometry). Additional aliquots of the unfiltered leachate solutions or the solutions produced by filtering through $0.45-\mu \mathrm{m}$ filters were taken for analysis of the radionuclides using ICP-MS. These aliquots were evaporated to dryness then redissolved and acidified with nitric acid for analysis. The measured $\mathrm{pH}$ values are expected to be affected by the temperature (they were measured at room temperature) and the high ionic strengths of the leachate solutions. Nevertheless, the $\mathrm{pH}$ values provide a qualitative measure of the effects of the SA/V ratio and reaction time on the solution chemistry.

The reacted glass was gently rinsed with deionized water then removed from the vessel and allowed to dry. The vessel was then filled with a nitric acid solution and placed in a $90^{\circ} \mathrm{C}$ oven overnight to dissolve any actinides that had become fixed to the stainless steel surface. An aliquot of this acid soak solution was stippled onto stainless steel planchets and analyzed by alpha spectroscopy. Some of the more saline solutions were electroplated onto planchets and then analyzed. The solution results are tabulated in spreadsheets included in Appendix D.

Solids were analyzed by using an optical microscope, scanning electron microscope (SEM), and transmission electron microscope (TEM) to characterize the glass alteration and identify secondary phases formed during glass corrosion. Reacted glass was analyzed by using analytical electron microscopy (AEM), which combines high-resolution TEM with energydispersive X-ray spectroscopy (EDS). Representative samples of the reacted glass were fixed in epoxy, and thin-sections were prepared by using an ultramicrotome [284]. Grains of powdered glass were fixed in resin directly, while sample chips were removed from reacted monoliths using a diamond knife. Secondary phases found distributed among the reacted grains were isolated and analyzed separately by using optical microscopy, SEM, TEM, and X-ray diffraction for phase identification.

\subsection{Observed Effects of the SA/V on the Leachate Chemistry}

\subsubsection{Leachate $\mathrm{pH}$}

The aliquots of the leachates from tests at 10 and $340 \mathrm{~m}^{-1}$ and the filtrates from tests at 2000 and $20,000 \mathrm{~m}^{-1}$ taken for $\mathrm{pH}$ analysis were allowed to cool to room temperature prior to analysis. The $\mathrm{pH}$ measured at room temperature is expected to be about one $\mathrm{pH}$ unit higher than the $\mathrm{pH}$ at the reaction temperature because of the difference in the ionization constant of water at 90 and $25^{\circ} \mathrm{C}$. The measured $\mathrm{pH}$ values from duplicate tests with SRL 202A, SRL 202U, and SRL 131A reacted in EJ-13 water or in deionized water are plotted versus the reaction time in Fig. 52a-c. Differences in duplicate tests with crushed glass were generally small, but large differences were measured in some tests with monolithic samples, both at 10 and $340 \mathrm{~m}^{-1}$. (The solution concentrations of glass components also differ significantly in these tests, as shown below.) The measured $\mathrm{pH}$ values in tests with all glasses are higher than the $\mathrm{pH}$ 
(a)

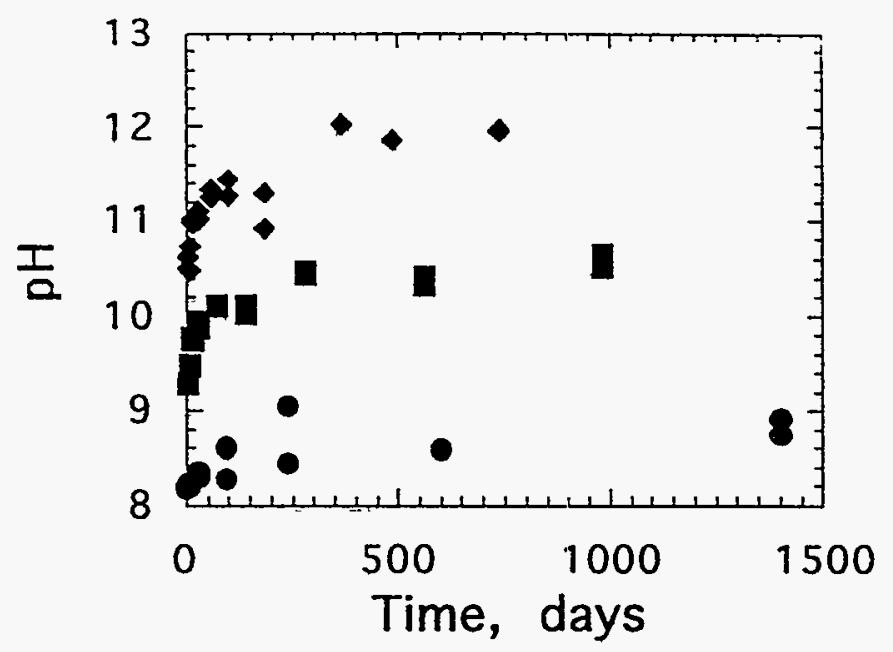

(b)
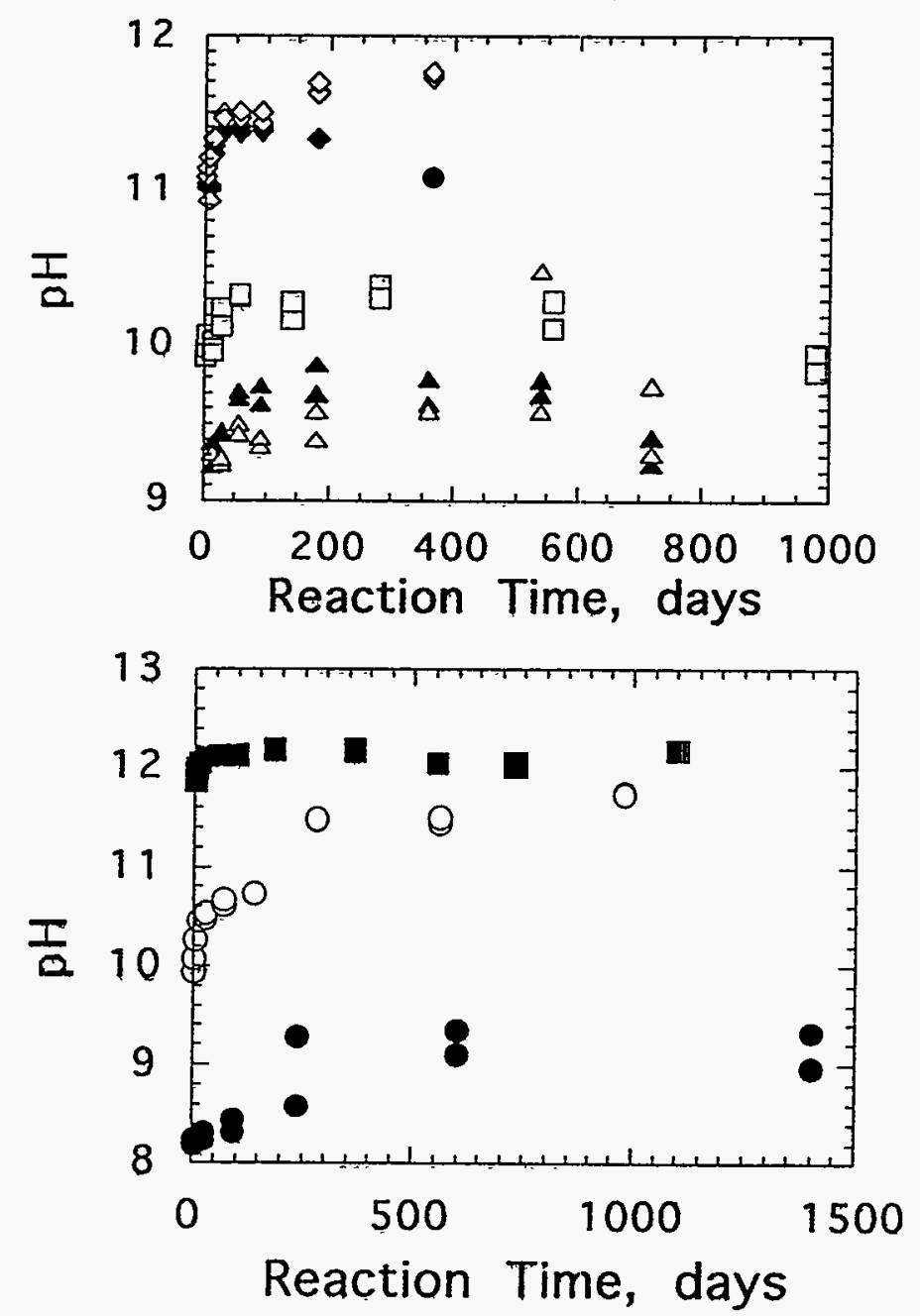

Fig. 52. Measured pH Values in ANL Tests with (a) SRL 202A Glass, (b) SRL 202U Glass, and (c) SRL 131A Glass in Tests at $90^{\circ} \mathrm{C}$ at (O) 10, (A) 340, (iii) 2000, and

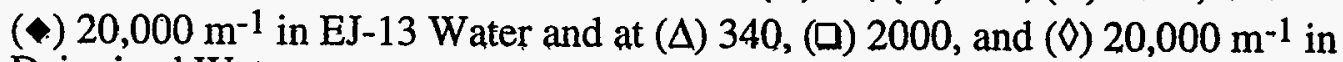
Deionized Water 
value of the initial leachant, which was 8.2 for the EJ-13 leachant and about 5.5 for the deionized water, at all reaction times. Higher $\mathrm{pH}$ values were attained in tests conducted at higher $\mathrm{SA} / \mathrm{V}$ for all glasses and at all reaction times. The $\mathrm{pH}$ is increased only about one $\mathrm{pH}$ unit above the initial leachant $\mathrm{pH}$ in tests at $10 \mathrm{~m}^{-1}$, even after 1400 days. Tests conducted with SRL 202U glass in deionized water had slightly higher $\mathrm{pH}$ values than tests conducted in EJ-13 water in tests at $20,000 \mathrm{~m}^{-1}$ (larger differences were measured after 182 and 364 days), but slightly lower pH values than tests conducted in EJ-13 water in tests at $340 \mathrm{~m}^{-1}$ at each time tested. Tests with SRL 131A generally had higher leachate $\mathrm{pH}$ values than tests with SRL 202A conducted at the same SA/V and for the same reaction time.

\subsubsection{Glass Matrix Components}

The leachate and filtrate solutions were analyzed by using inductively coupled plasma-atomic emission spectroscopy, inductively coupled plasma-mass spectroscopy, and fluorescence spectroscopy. Figures 53, 54, and 55 show the normalized elemental mass losses of several glass components from SRL 202A and SRL 131A glass at 10, 2000, and $20,000 \mathrm{~m}^{-1}$ and from SRL 202U glass at 340 and $20,000 \mathrm{~m}^{-1}$ in tests conducted in EJ-13 tuff groundwater solution, and from SRL 202U in tests in deionized water. The average values of duplicate tests are plotted. The data for each test is included in Table 2 of Appendix D. Difference in the results of duplicate tests are generally within the size of the symbol for tests with crushed glass, but slightly larger for tests with monolithic samples. The difference in the error ranges of tests with crushed glass or monolithic glass samples is due in large part to the normalization of the solution concentrations by dividing them by the SA/V of the test. Similar analytical errors in the measured concentrations are more significant when divided by 10 or 340 than they are when divided by 2000 or 20,000 . The normalized elemental mass losses were computed directly from the leachate results for tests with monolith samples, and from the results of solution filtered through $0.45-\mu \mathrm{m}$ filters for tests with crushed glass.

The release of different glass components from SRL 202A, SRL 131A, and SRL $202 \mathrm{U}$ in tests at different SA/V can be compared directly using the normalized mass loss of each element. Figures 53, 54, and 55 show the release of $\mathrm{Li}, \mathrm{Na}, \mathrm{K}, \mathrm{B}, \mathrm{Si}$, and $\mathrm{U}$ into solution to be nonstoichiometric (with respect to the glass composition) for all glasses and at all SA/V tested. (Stoichiometric release would result in the data points for all elements falling on the same curve.) The general order of elemental release is $\mathrm{Li}>\mathrm{Na}>\mathrm{B}>\mathrm{K}>\mathrm{Si}>\mathrm{U}$, though at $10 \mathrm{~m}^{-1}$ the sodium release is greater than the lithium release from both SRL 202A and SRL 131A glass. The alkali metal and boron concentrations increase at high rates initially, but the release slows as the reaction progresses at intermediate reaction times. As the reaction continues for longer times under several test conditions, the boron and alkali metal release rates suddenly increase and become nearly linear in time. This change occurs in tests with SRL 202A, SRL 202U, and SRL 131A glasses at $20,000 \mathrm{~m}^{-1}$ beyond about 182 days, and in tests with SRL 131A glass at $2000 \mathrm{~m}^{-1}$ beyond about 140 days. The time at which the corrosion accelerated can be estimated by extrapolating the constant rate observed in tests conducted for the longest times back to where it intersects the rate curve determined by tests run for shorter times. The acceleration is estimated to occur shortly beyond 182 days in tests with SRL 202A glass at $2000 \mathrm{~m}^{-1}$, after about 182 days in tests with SRL131A glass at $2000 \mathrm{~m}^{-1}$, and after about 90 days in tests with SRL 131A glass at $2000 \mathrm{~m}^{-1}$. Data are not yet available to determine when tests with SRL 202U glass at $2000 \mathrm{~m}^{-1}$ are accelerated, although the similarity between tests with SRL 202U and SRL $202 \mathrm{~A}$ glasses suggest that they, too, were accelerated shortly after about 182 days. Other tests with SRL 202U glass are in progress that are expected to show the same corrosion rate as tests run for 364 days. 
(a)

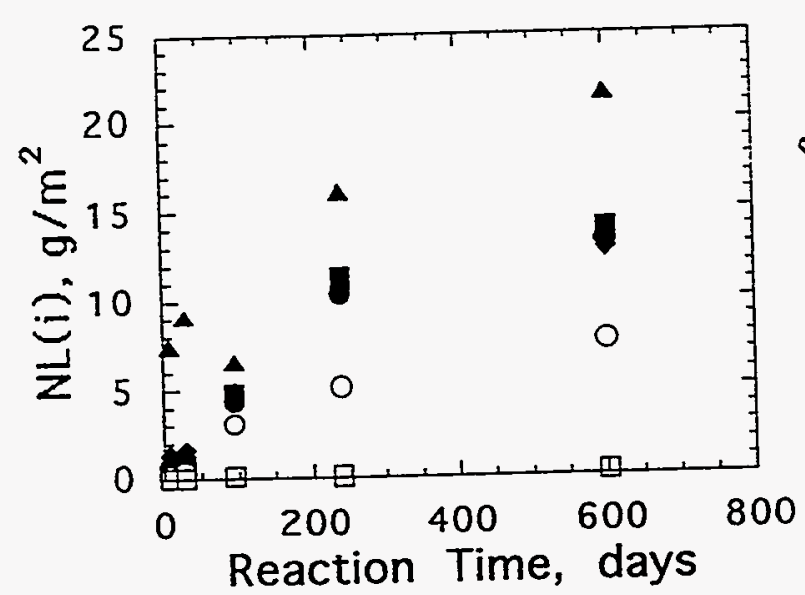

(c)

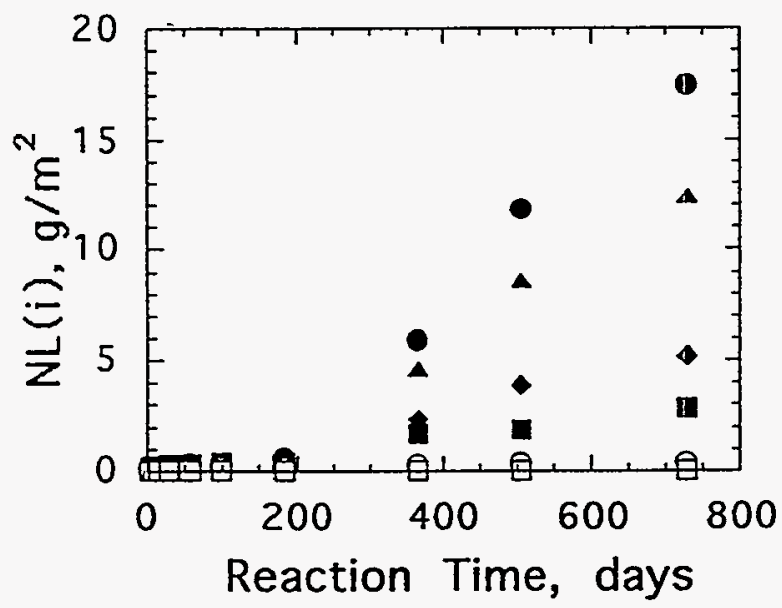

(b)

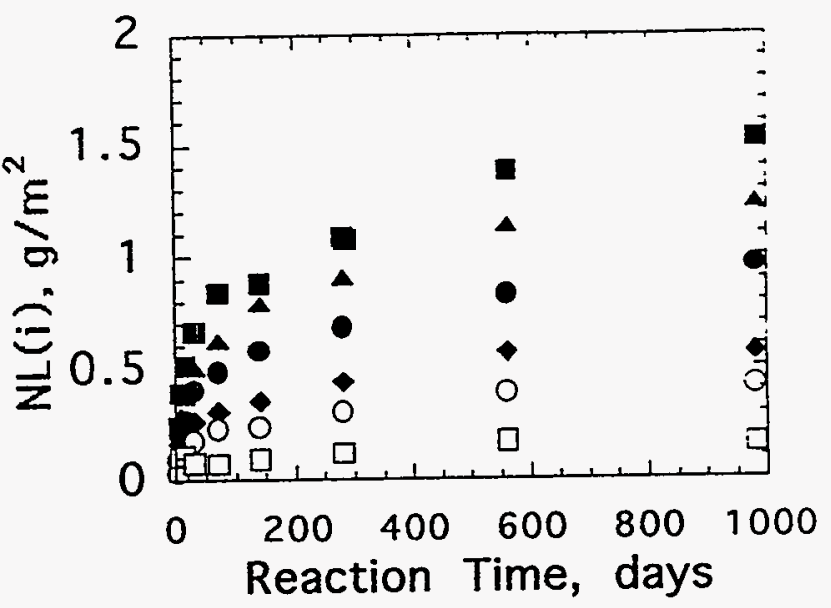

(d)

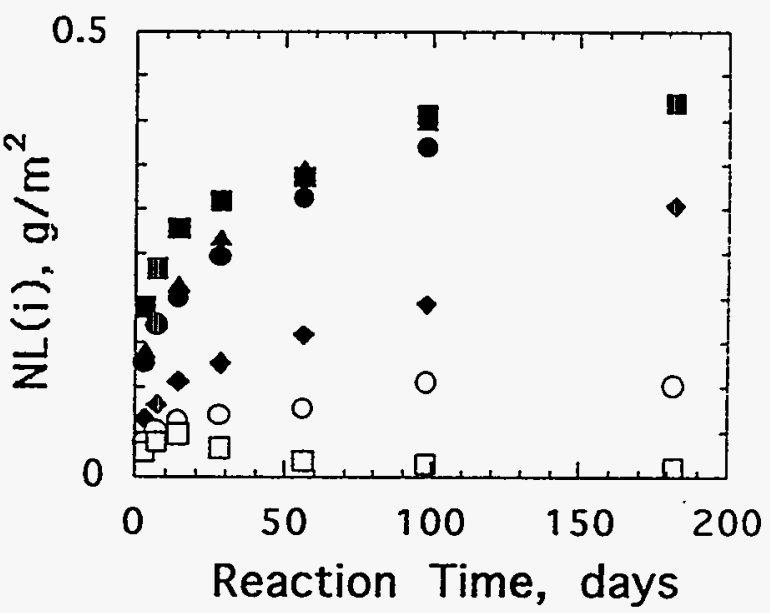

Fig. 53. Measured Cation Concentrations in ANL Tests with SRL 202A Glass Conducted at

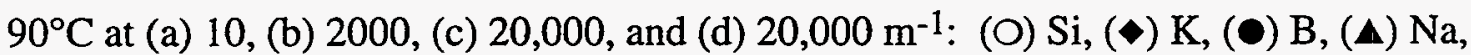
(a) $\mathrm{Li}$, and (a) U 
(a)

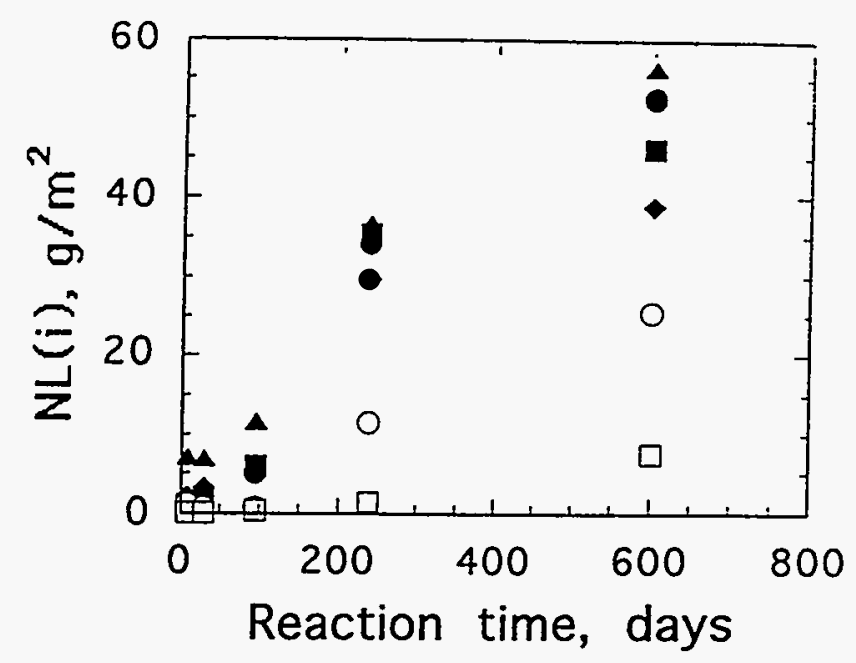

(b)

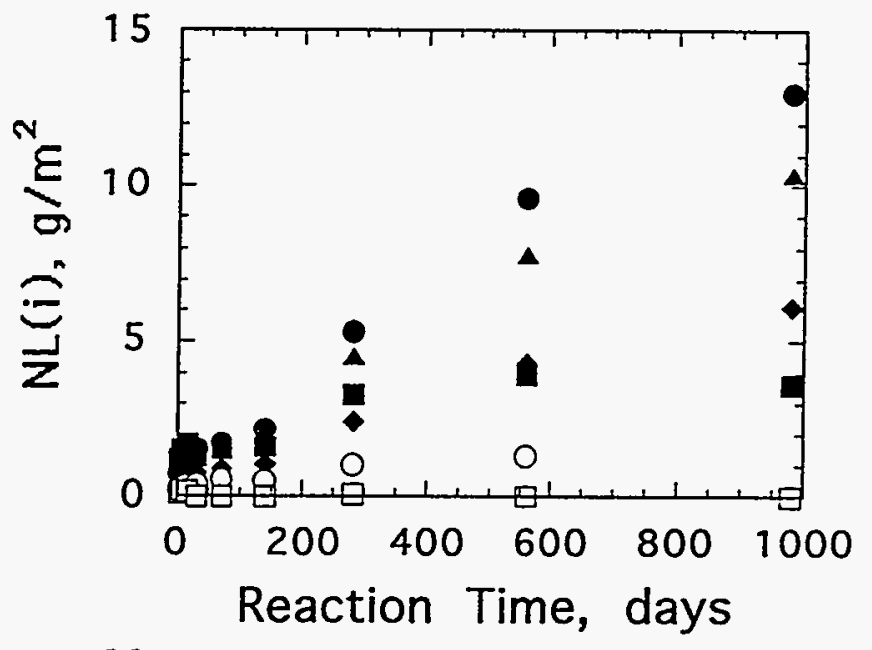

(c)

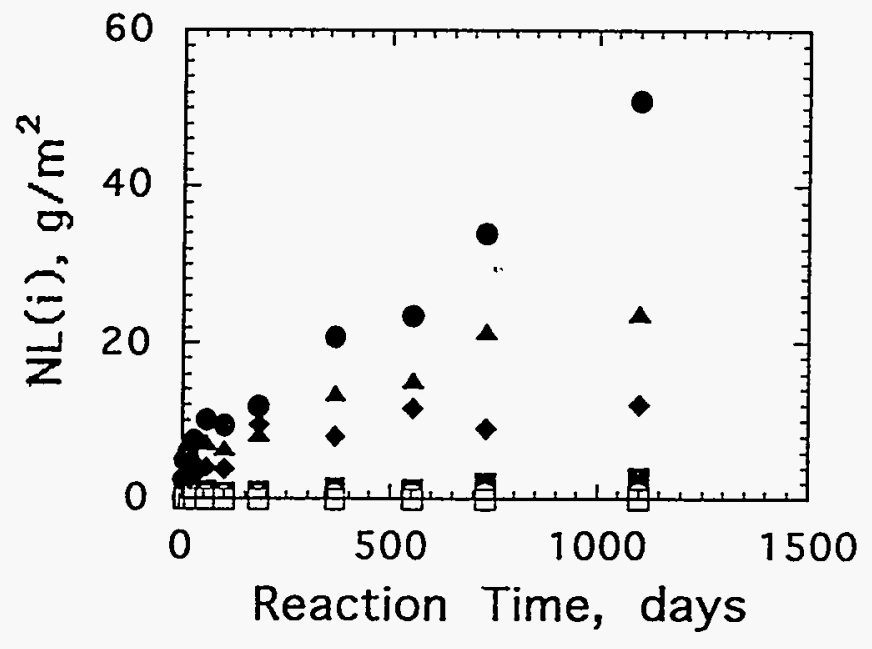

Fig. 54. Measured Cation Concentrations in ANL Tests with SRL 131A Glass Conducted at $90^{\circ} \mathrm{C}$ at (a) 10 , (b) 2000 , and (c) $20,000 \mathrm{~m}^{-1}$ : (O) $\mathrm{Si},(\bullet) \mathrm{K},(\bullet) \mathrm{B},(\boldsymbol{\Lambda}) \mathrm{Na},(\boldsymbol{\square}) \mathrm{Li}$, and (ㅁ) $\mathrm{U}$ 
(a)

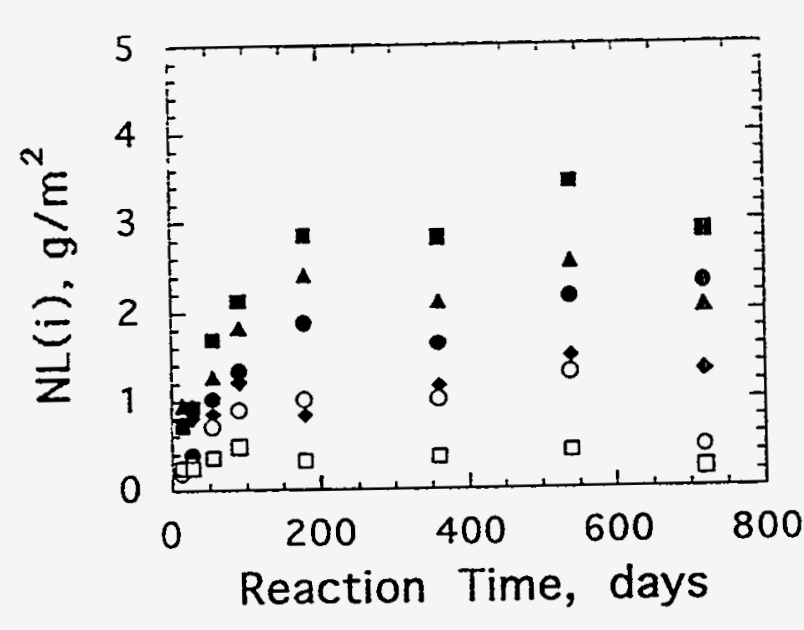

(c)

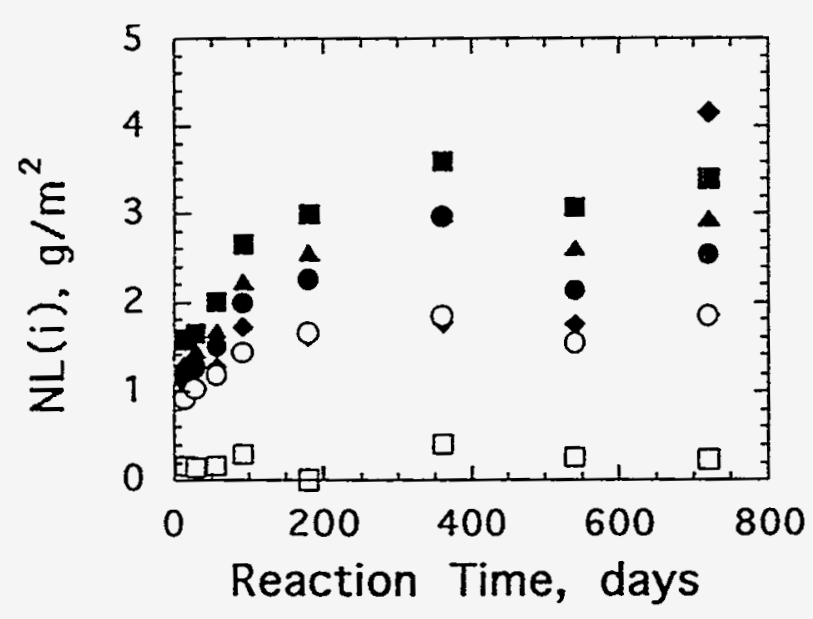

(e)

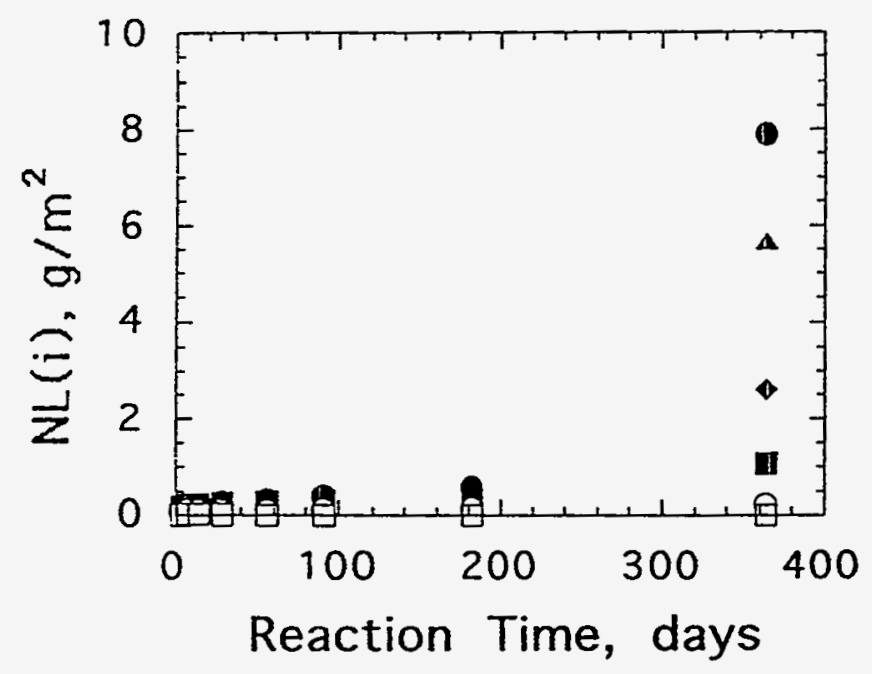

(b)

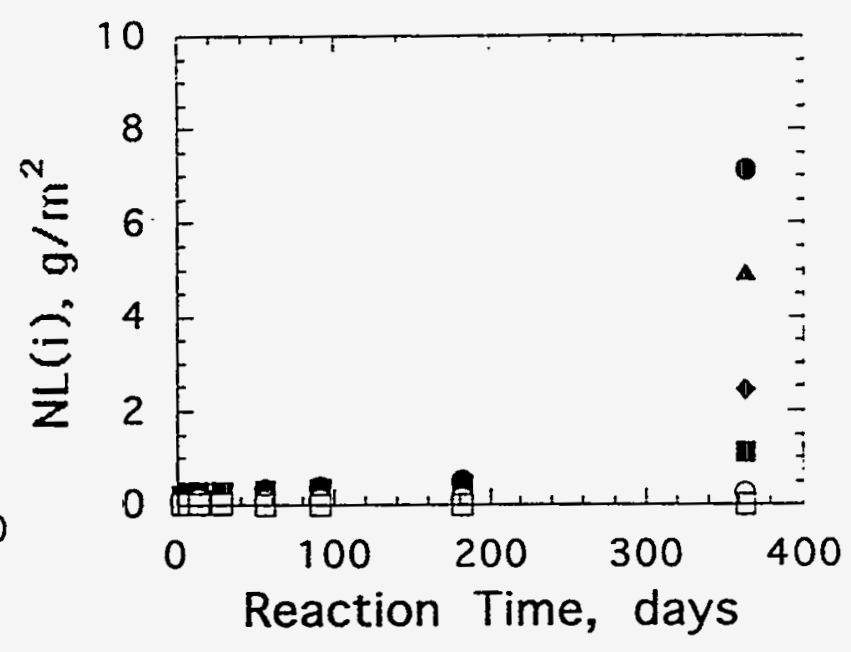

(d)

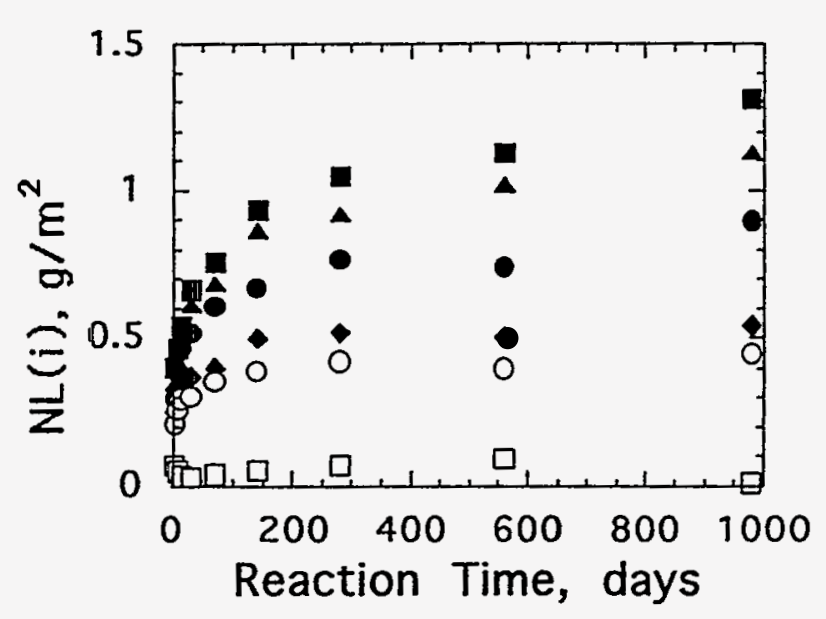

Fig. 55.

Measured Cation Concentrations in ANL Tests with SRL 202U Glass Conducted at $90^{\circ} \mathrm{C}$ at (a) 340 and (b) $20,000 \mathrm{~m}^{-1}$ in EJ-13 and at (c) 340 , (d) 2000 , and (e) $20,000 \mathrm{~m}^{-1}$

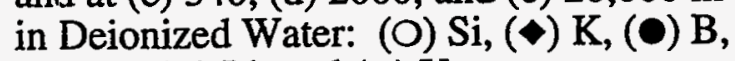

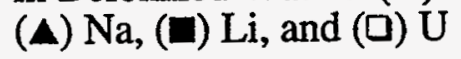


When the corrosion is accelerated, the boron release to solution becomes greater than the release of the alkali metals. The normalized silicon mass loss is only slightly increased when boron and the alkali metals show accelerated release in tests at $20,000 \mathrm{~m}^{-1}$ (refer to Figs. 53d, 54d, and 55c). The normalized uranium mass losses remain well below the normalized silicon mass loss in all tests.

Both suspended and dissolved material were included in the calculation of the normalized mass loss to represent the total amount of glass that had dissolved. Leachate solutions were further filtered through 6-nm filters to remove suspended material and measure the amounts of various glass components that were dissolved. Table 9 shows the measured concentrations of aluminum, iron, silicon, and boron in the $0.45-\mu \mathrm{m}$ and $6-\mathrm{nm}$ filtrates of tests with SRL 202U glass reacted at $20,000 \mathrm{~m}^{-1}$. The aluminum and iron concentrations in the $0.4-\mu \mathrm{m}$ filtrates increase with the reaction times through 14 days, but then decrease to low values at longer times. Filtration through 6-nm filters removed most of the aluminum and iron from solution. The silicon and boron concentrations increase with the reaction time at all times tested. Further filtration through 6-nm filters did not significantly reduce these concentrations; this result indicates that these components are dissolved. Large increases in the boron and silicon concentrations are measured in both the $0.45-\mu \mathrm{m}$ and $6-\mathrm{nm}$ filter solutions after 364 days of reaction. Similar trends were seen upon filtration of the leachates of tests with SRL 202U, SRL 202A, and SRL 131A glass at all SA/V ratios tested. That is, the concentrations of soluble alkali metals, boron, and silicon are not reduced by filtration (the reduction in the silicon concentration is less than the assumed analytical error range of about 15\%), and aluminum, iron, and other components with low solubilities are further removed from the leachate by $6 \mathrm{~nm}$ filters.

Table 9. Results of Filtering Leachate Solutions from Tests with SRL 202U Glass through $6 \mathrm{~nm}$ Filters

\begin{tabular}{|c|c|c|c|c|c|c|c|c|c|c|}
\hline \multirow[b]{2}{*}{ Test No. } & \multirow{2}{*}{$\begin{array}{c}\text { Time } \\
\text { (days) }\end{array}$} & \multirow[b]{2}{*}{$\mathrm{pH}$} & \multicolumn{2}{|c|}{$\mathrm{Al}, \mathrm{ppm}$} & \multicolumn{2}{|c|}{$\mathrm{Fe}, \mathrm{ppm}$} & \multicolumn{2}{|c|}{$\mathrm{Si}, \mathrm{ppm}$} & \multicolumn{2}{|c|}{$\mathrm{B}, \mathrm{ppm}$} \\
\hline & & & $0.45 \mu \mathrm{m}$ & $6 \mathrm{~nm}$ & $0.45 \mu \mathrm{m}$ & $6 \mathrm{~nm}$ & $0.45 \mu \mathrm{m}$ & $6 \mathrm{~nm}$ & $0.45 \mu \mathrm{m}$ & $6 \mathrm{~nm}$ \\
\hline 1 & 3 & 10.86 & 5.27 & 1.26 & 25.9 & 0.38 & 279 & 243 & 75.9 & 73.5 \\
\hline 2 & 3 & 10.89 & 5.42 & 1.25 & 26.8 & 0.38 & 283 & 243 & 77.0 & 75.0 \\
\hline 3 & 7 & 11.03 & 9.18 & 1.31 & 44.2 & 0.30 & 351 & 267 & 93.8 & 85.9 \\
\hline 4 & 7 & 11.01 & 9.33 & ..a & 38.2 & - & 338 & - & 92.8 & -- \\
\hline 5 & 14 & 11.23 & 11.5 & - & 51.7 & - & 411 & - & 111 & - \\
\hline 6 & 14 & 11.28 & 12.1 & $<2.5^{\mathrm{b}}$ & 55.0 & 0.75 & 417 & 323 & 111 & 107 \\
\hline 7 & 28 & 11.37 & 6.05 & 1.80 & 18.5 & 0.28 & 361 & 360 & 119 & 127 \\
\hline 8 & 28 & 11.38 & 3.85 & 1.93 & 8.37 & $<0.19$ & 350 & 362 & 121 & 128 \\
\hline 9 & 56 & 11.37 & 1.55 & - & 0.50 & -- & 379 & - & 148 & - \\
\hline 10 & 56 & 11.41 & 1.52 & -- & 0.37 & -- & 385 & -- & 149 & -- \\
\hline 11 & 91 & 11.4 & $<1.3$ & $<1.1$ & 0.66 & $<0.23$ & 429 & 440 & 177 & 182 \\
\hline 12 & 91 & 11.38 & 2.33 & 1.70 & 0.24 & $<0.23$ & 436 & 446 & 180 & 181 \\
\hline 13 & 182 & 11.63 & 1.12 & $<1.64$ & 0.173 & $<0.328$ & 562 & 532 & 246 & 230 \\
\hline 14 & 182 & 11.66 & 1.21 & $<1.72$ & 0.173 & $<0.345$ & 567 & 539 & 244 & 229 \\
\hline 15 & 364 & 11.75 & $<2.86$ & 1.3 & 1.43 & 0.602 & 1060 & 971 & 3490 & 3400 \\
\hline
\end{tabular}

aSolution not analyzed.

${ }^{b}$ Below detection limit, value given is adjusted for dilution. 
The results plotted in Figs. 53, 54, and 55 showed that the amounts of glass components released into solution are also affected by the $S A / V$ ratio of the test. In plotting the normalized mass losses, the measured solution concentrations are divided by the SA/V ratio to account for differences in dilution at different $S A / V$. If the reaction rates are the same in tests at different $\mathrm{SA} / \mathrm{V}$, then the normalized released rates should also be the same. Comparing the results at different SA/V ratios in Figs. 53, 54, and 55, the normalized release rates of all elements plotted are different in tests at different $S A / V$. For example, Fig. 56 shows the normalized boron mass losses from SRL 202A and SRL 202U glasses in tests at 10, 340, 2000, and $20,000 \mathrm{~m}^{-1}$ on the same plot. (Figures $56 \mathrm{a}$ and $56 \mathrm{~b}$ show the same results on different scales.) At short reaction times ( $<200$ days), the normalized mass loss of boron decreases with the SA/V of the test. The long-term tests at different SA/V show very different reaction trends. Tests at 10 and $2000 \mathrm{~m}^{-1}$ release boron at a rate that decreases as the reaction progresses, with tests at $2000 \mathrm{~m}^{-1}$ reaching a low rate after short reaction times. The release rate of boron is lowest at $20,000 \mathrm{~m}^{-1}$ at short reaction times, but the reaction is accelerated after about 182 days to a high rate that remains constant with time thereafter. This implies that the glass reaction rate and/or reaction mechanism is affected differently by the different solution chemistries attained in tests at differe nt $\mathrm{SA} / \mathrm{V}$.

Instead of plotting the normalized elemental mass loss against time, as in Fig. 56, the measured concentrations can be plotted against the product of the reaction time and the SA/V of the test. The product (SA $/ \mathrm{V}$ )t has been suggested as a single parameter that can be used to measure the extent of glass reaction, meaning that tests at different $\mathrm{SA} / \mathrm{V}$ but equivalent $(\mathrm{SA} / \mathrm{V}) \mathrm{t}$ should yield the same extent of glass reaction $[72,285,286]$. The boron data from replicate tests with SRL 202A, SRL 131A, and SRL 202U glasses are plotted against(SA/V)t in Fig. 57. Tests at 2000 and $20,000 \mathrm{~m}^{-1}$ performed for equivalent (SA/V)t clearly do not generate equivalent solution compositions in tests with either SRL 202A, SRL 131A, or SRL 202U glass. Tests performed at higher $\mathrm{SA} / \mathrm{V}$ but shorter reaction times consistently have greater boron releases. Tests with SRL 131A show a larger difference than tests with SRL 202A. Although duplicate tests at $10 \mathrm{~m}^{-1}$ show significant scatter, some tests at $10 \mathrm{~m}^{-1}$ have boron concentrations similar to those in tests at $2000 \mathrm{~m}^{-1}$ at equivalent (SA/V)t .

The results of these tests show that the reaction is characterized by an initially high forward rate, an intermediate stage where a minirnum rate is approached, and a long-term rate that may be greater than the minimum rate. The progression of the reaction from the initial rate to the intermediate rate to the long-term rate is controlled primarily by the leachate solution composition. Under dilute solution compositions, the observed rate will likely depend on the glass composition and the solution $\mathrm{pH}$. The leachate $\mathrm{pH}$ will generally increase as the glass reacts to release alkali metals, and the glass reaction rate will increase with the $\mathrm{pH}$. However, the rate decreases as the leachate becomes more concentrated in glass components (primarily in silicic acid). The rate-decreasing effects of the buildup of solution concentrations overwhelms the rate-increasing effect of the higher $\mathrm{pH}$ values, and a minimum reaction rate is approached as the leachate approaches saturation. The glass may react at the minimum rate for very long times. The reaction may eventually be accelerated if secondary phases form to affect the solution composition. As the assemblage of secondary phases changes as the reaction progresses, the reaction rate will likely also change. A characteristic "final dissolution rate" may not exist because of the influence of the $\mathrm{pH}$ and the secondary phases on the reaction rate. 
(a)

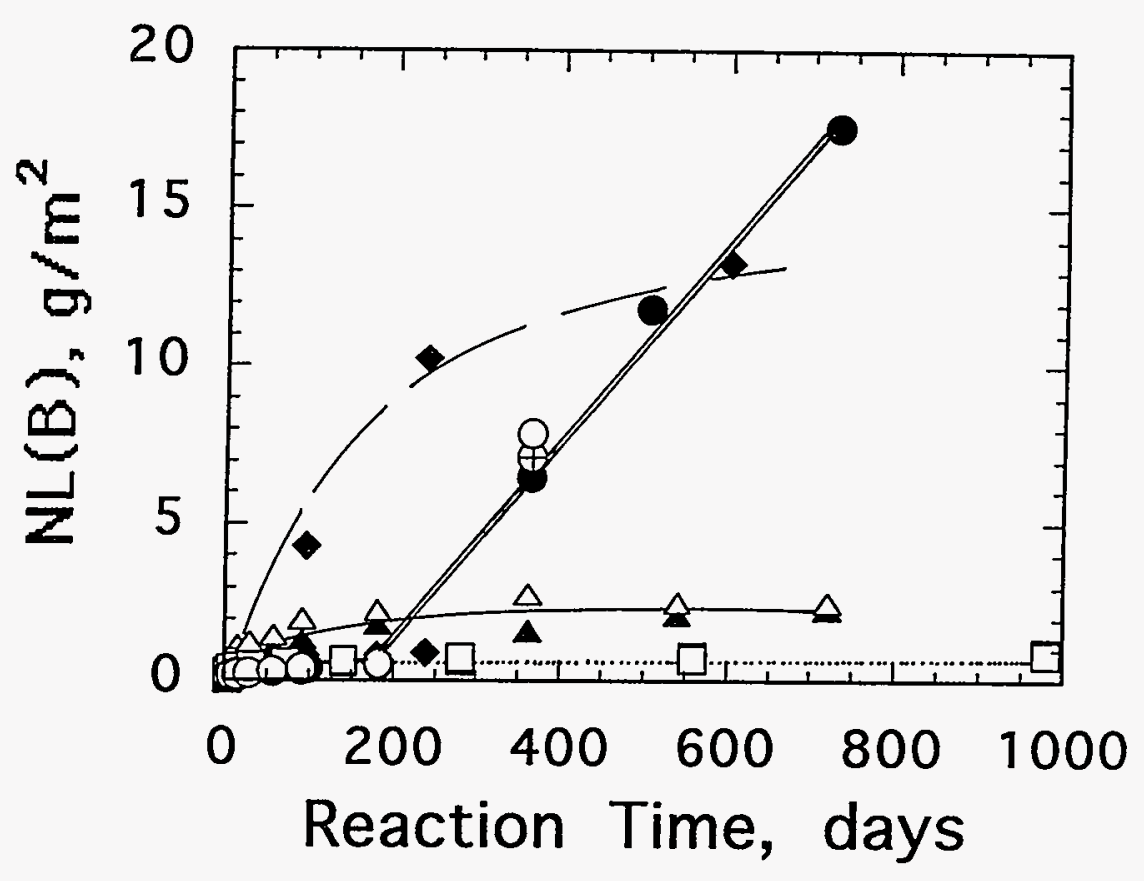

(b)

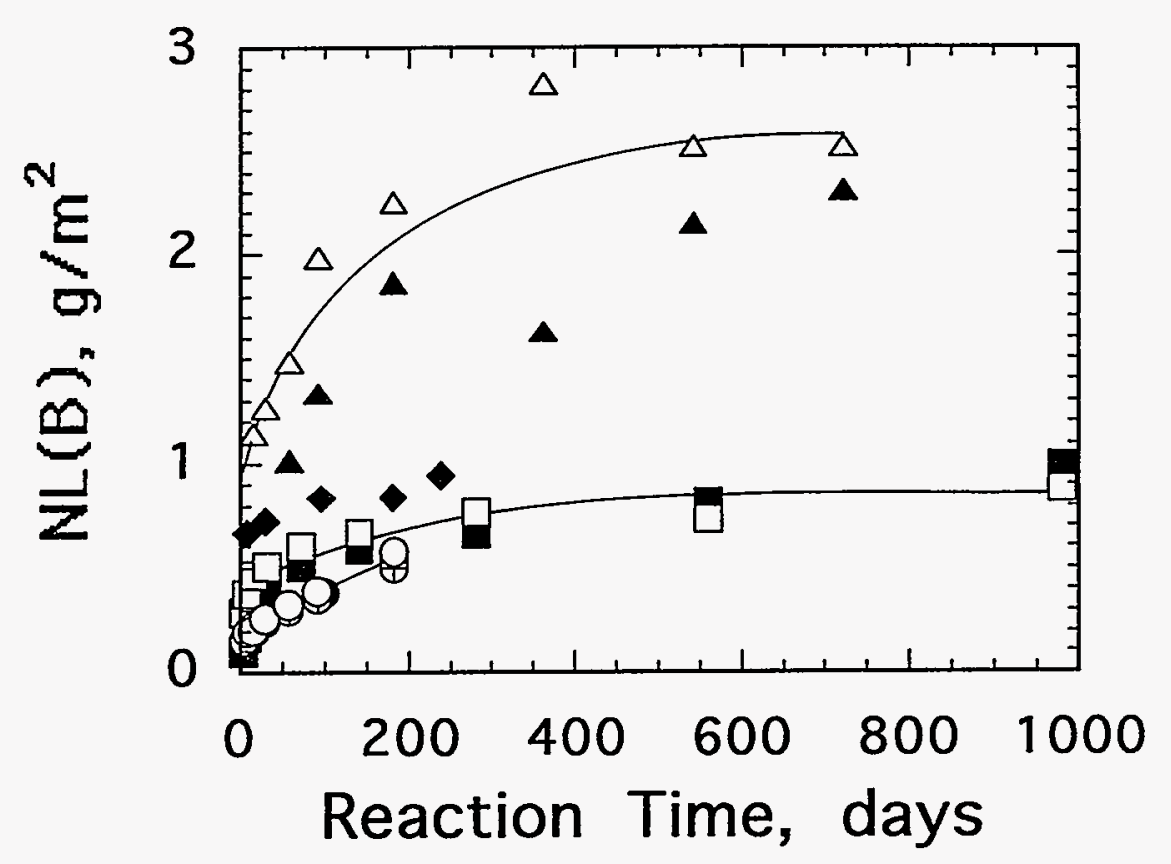

Fig. 56. Normalized Boron Mass Loss from Glasses vs. Reaction Time in Tests Conducted at

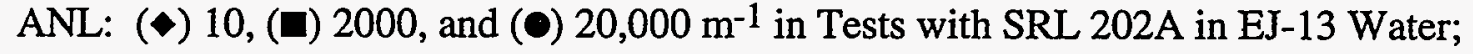

(A) 340 and $(\oplus)$ 20,000 $\mathrm{m}^{-1}$ in Tests with SRL 202U in EJ-13 Water; $(\Delta) 340$, (D) 2000, and (O) 20,000 $\mathrm{m}^{-1}$ in Tests with SRL 202U in Deionized Water. (a) All data plotted and (b) data for $\mathrm{NL}(\mathrm{B})<3 \mathrm{~g} / \mathrm{m}^{2}$. 
(a)

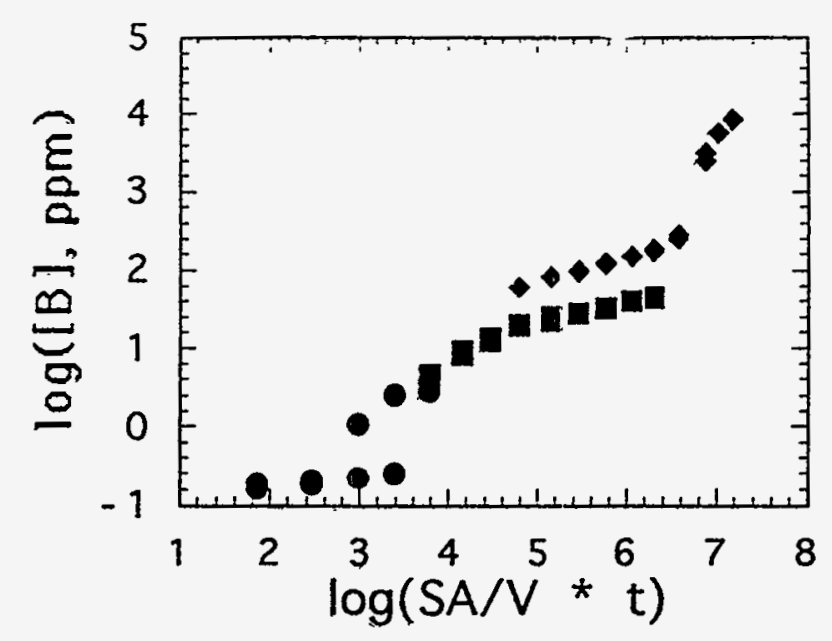

(b)

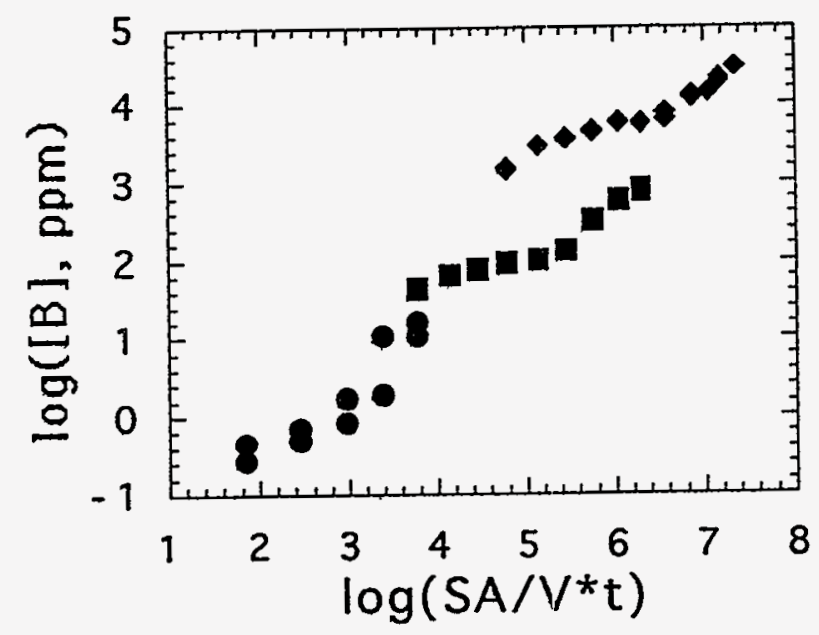

(c)

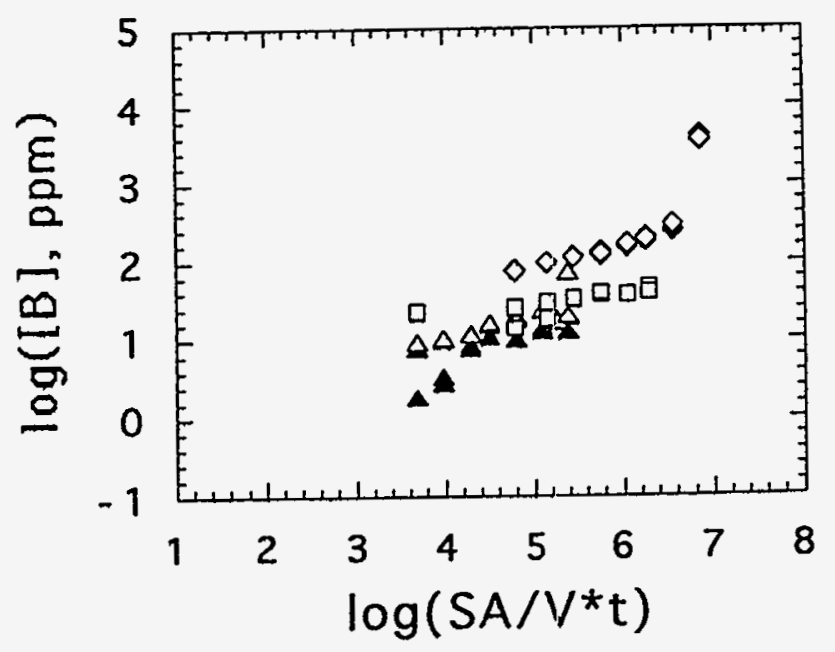

Fig. 57. Logarithm of Measured Boron Concentration vs. Logarithm of (SA/V)t for Tests with (a) SRL 131A, (b) SRL 202A, and (c) SRL 202U Glasses Conducted at SA/V of:

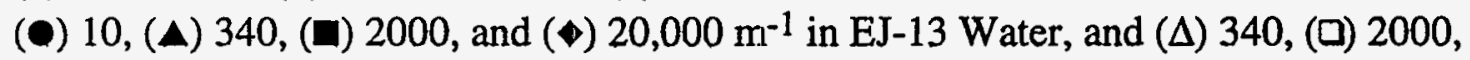
and $(0) 20,000 \mathrm{~m}^{-1}$ in Deionized Water 
Tests conducted at different $\mathrm{SA} / \mathrm{V}$ ratios generate very different solution chemistries which affect the glass corrosion rate and will affect the disposition of radioelements. The $\mathrm{pH}$ and anion concentrations are expected to affect the behavior of released actinides. The $\mathrm{pH}$ values and anion concentrations measured in the leachate solutions of tests with SRL 131A and SRL 202A glasses at 2000 and $20,000 \mathrm{~m}^{-1}$ are listed in Table 10 . High leachate pH values are reached after only a few days tests with SRL 131A and SRL 202A because of alkali release as the glasses corrode. Different $\mathrm{pH}$ values are attained in tests at different $\mathrm{SA} / \mathrm{V}$ ratios because of the different volumes of solution available to dilute the hydroxide and other corrosion products.

Quantification of fluoride in some samples was complicated by the overlap of the fluoride and formate $\left(\mathrm{HCO}_{2}^{-}\right)$peaks in the ion exchange column. Most tests showed the fluoride concentration to be approximately the same as its initial concentration in the leachant, about $3 \mathrm{ppm}$. Fluoride levels were assumed to remain near $3 \mathrm{ppm}$ for all tests, and the formate levels were calculated after subtracting this amount of fluoride from overlapped peaks. At these low concentrations, neither fluoride nor formate is expected to affect the solubilities of the actinides. Relatively high concentrations of oxalate $\left(\mathrm{C}_{2} \mathrm{O}_{2}{ }^{2-}\right)$ were measured in tests with SRL 202A glass, but most tests with SRL 131A glass had oxalate concentrations below the detection limit of $0.5 \mathrm{ppm}$. This suggests that the organics were released from the glasses as they corroded, and that the SRL 202 frit used to produce SRL 202A and SRL 202U glasses had more carbon than the SRL 131 frit used to produce SRL 131A glass.

The chloride content remained near its concentration in the EJ-13 leachant for most tests at $2000 \mathrm{~m}^{-1}$, but increased to more than $200 \mathrm{ppm}$ in tests with both glasses at $20,000 \mathrm{~m}^{-1}$. Nitrate concentration in all tests remained near the leachant level of $16 \mathrm{ppm}$. Both chloride and nitrate are weak complexants and these levels are not expected to significantly increase the actinide solubilities. The hydrogen phosphate level increased in all tests with both glasses: tests with SRL $131 \mathrm{~A}$ glass attain concentrations exceeding $400 \mathrm{ppm}$, and tests with SRL 202A glass attain concentrations exceeding $100 \mathrm{ppm}$ after two years at $20,000 \mathrm{~m}^{-1}$. Very high sulfate concentrations were also measured in tests with SRL $131 \mathrm{~A}$ glass at $20,000 \mathrm{~m}^{-1}$ at all reaction times and with SRL 202A glass in tests beyond two years. Both plutonium and americium form phosphates having lower solubilities than the oxides, and the release of hydrogen phosphate and sulfate is expected to lower the solubilities of plutonium and americium in these leachates.

The organic and inorganic carbon contents of the leachates were also measured, and the results are included in Table 10 as the total organic carbon (TOC) and total inorganic carbon (TIC) concentrations. The EJ-13 leachant contains about $3 \mathrm{ppm}$ organic carbon and $22 \mathrm{ppm}$ inorganic carbon (as the bicarbonate ion). Corrosion of the glass increases both the organic and inorganic carbon levels. In general, tests with SRL 202A glass at $2000 \mathrm{~m}^{-1}$ attained slightly higher inorganic carbon levels than tests with SRL 131A glass at the same SA/V ratio. Tests at $20,000 \mathrm{~m}^{-1}$ had greater amounts of total carbon than tests at $2000 \mathrm{~m}^{-1}$, a result that is consistent with carbon being released from the glass. Also, tests at $20,000 \mathrm{~m}^{-1}$ show a decrease in the inorganic content after 364 days or longer. This may be due to the formation of various carbonate secondary phases (see below). Formation of such phases will decrease the amounts of carbonate available to complex actinides. 
Table 10. Anion Concentrations in Tests with SRL 131 and SRL 202 Glasses $^{\mathrm{a}}$

\begin{tabular}{|c|c|c|c|c|c|c|c|c|c|c|}
\hline Time & $\mathrm{pH}$ & $\mathrm{F}^{-}$ & $\mathrm{HCO}_{2}^{-}$ & $\mathrm{Cl}^{-}$ & $\mathrm{NO}_{3}^{-}$ & $\mathrm{HPO}_{4}{ }^{2-}$ & $\mathrm{SO}_{4}^{2-}$ & $\mathrm{C}_{2} \mathrm{O}_{2}^{2-}$ & TOC & TIC \\
\hline EJ-13 & 8.20 & 3 & $<0.5$ & 9 & 16 & $<0.5$ & 25 & $<0.5$ & 3 & 22 \\
\hline \multicolumn{11}{|c|}{$\mathrm{SRL} 202$ at $2000 \mathrm{~m}^{-1}$} \\
\hline 14 & 9.77 & 3 & $<0.5$ & 11 & 16 & $<0.5$ & 26 & $<0.5$ & 11 & 64 \\
\hline 140 & 10.11 & 3 & $<0.5$ & 11 & 11 & 6 & 25 & 2 & 13 & 38 \\
\hline 560 & 10.42 & 4 & $<0.5$ & 10 & 9 & 6 & 31 & 3 & 32 & 130 \\
\hline 980 & 10.65 & 5 & $<0.5$ & 18 & 22 & 10 & 68 & 5 & 27 & 130 \\
\hline \multicolumn{11}{|c|}{ SRL 202 at $20,000 \mathrm{~m}^{-1}$} \\
\hline 14 & 11.01 & 3 & 17 & 12 & 16 & 12 & 37 & 7 & 70 & 72 \\
\hline 98 & 11.26 & $3^{b}$ & $29 \mathrm{~b}$ & 15 & 22 & 28 & 42 & 14 & 160 & 92 \\
\hline 182 & 11.29 & $3^{b}$ & $23^{b}$ & 18 & -- & 71 & 39 & 18 & 220 & 120 \\
\hline 364 & 12.03 & $3^{b}$ & $28^{\mathrm{b}}$ & $<10$ & 17 & 121 & 95 & 17 & 80 & 83 \\
\hline 736 & 11.98 & $3^{b}$ & $30^{\mathrm{b}}$ & 215 & 26 & 103 & 293 & 56 & 98 & 40 \\
\hline \multicolumn{11}{|c|}{ SRL 131 at $2000 \mathrm{~m}^{-1}$} \\
\hline 14 & 10.45 & 3 & $<0.5$ & 10 & 16 & $<0.5$ & 27 & $<0.5$ & 12 & 27 \\
\hline 140 & 10.72 & 3 & $<0.5$ & 12 & 11 & $<2$ & 28 & $<0.5$ & 14 & 34 \\
\hline 560 & 11.46 & $3^{b}$ & $<0.5^{b}$ & 12 & 9 & 9 & 32 & 1 & 13 & 55 \\
\hline 980 & 11.72 & $3^{b}$ & $3^{b}$ & 50 & 16 & $<40$ & 170 & $<40$ & 24 & 71 \\
\hline \multicolumn{11}{|c|}{ SRL 131 at $20,000 \mathrm{~m}^{-1}$} \\
\hline 14 & 12.05 & 3 & $<0.5$ & 66 & 21 & 66 & 160 & $<0.5$ & 91 & 14 \\
\hline 98 & 12.12 & $3^{b}$ & $<0.5$ & 90 & 20 & 99 & 190 & $<0.5$ & 160 & 20 \\
\hline 182 & 12.19 & $<3$ & $<5$ & 150 & 27 & 190 & 780 & $<0.5$ & 29 & 68 \\
\hline 364 & 12.18 & $\mathrm{c}$ & $c$ & 210 & 12 & 244 & 1300 & $<1$ & 100 & 8 \\
\hline 729 & 12.03 & $<3$ & $<5$ & 240 & 18 & 460 & 1710 & $<40$ & 120 & 4 \\
\hline
\end{tabular}

aUnits given as: $\mathrm{S} / \mathrm{V}\left(\mathrm{m}^{-1}\right)$, Time (days), anions (ppm), Total Organic Carbon (TOC, ppm), Total Inorganic Carbon (TIC, ppm).

bFormate values estimated based on $3 \mathrm{ppm} \mathrm{F}^{-}$.

cValues not reported. 
Specific analysis of the formate and oxalate concentrations by ion chromatography shows relatively high formate levels in tests with SRL 202A glass at $2000 \mathrm{~m}^{-1}$ but only small or undetectable levels in other tests. In many instances, the formate peak had to be resolved from the fluoride peak by assuming that only $3 \mathrm{ppm}$ fluoride was present. Significant levels of oxalate were measured only in tests with SRL 202A and SRL $202 \mathrm{U}$ glasses at 2000 and $20,000 \mathrm{~m}^{-1}$. (The oxalate peaks are fully resolved in the ion chromatograms. The presence of oxalate supports the values assigned to the formate levels in these same tests. That is, formate and oxalate levels appear to be correlated.) The measured formate and oxalate levels are consistent with the measured TOC concentrations, though the TOC concentrations are significantly higher. Although humic acids are known to complex actinides [269,287], the simple organics presence in these tests are not expected to significantly affect the solubilities of the transuranics.

\subsubsection{Observed Effects of SAVV on the Behavior of Radionuclides}

Of key interest in this Task is how the SA/V used in a static test affects the behavior of released radionuclides. Tests conducted in this Task were designed to address several questions regarding the behavior of radionuclides as the glass corrodes: Is the amount of radionuclides sensitive to the different solution chemistries attained under different SA/V? Does the presence of other glass components in the leachate affect the release, solubility, and stability of released radionuclides? Does the corrosion rate of the glass matrix affect the disposition of the radionuclides. Are the amounts of radionuclides in solution controlled by their solubilities? Is the disposition of the radionuclides affected by the formation of secondary phases? Is the behavior observed in short-term tests representative of the long-term behavior of radionuclides?

The amounts of technetium and actinides in the leachate solutions were measured using ICP-MS, and the calculated normalized mass losses based on the measured concentrations of technetium, uranium, and neptunium are compiled in Table 11. The normalized mass losses based on the boron release is included for comparison. Analyses were performed on the unfiltered leachates or the $0.45-\mu \mathrm{m}$ filtrate solutions. The boron release represents the extent of glass corrosion. The results of the test having the highest boron release of the duplicate tests conducted at each SA/V and reaction time are tabulated. In most cases, the results of duplicate tests are within the expected analytical error (see Tables 2 and 4 in Appendix D). The normalized mass losses for tests conducted at 2000 or $20,000 \mathrm{~m}^{-1}$ with both glasses are plotted against the reaction time in Figs. 58a-d. Lines are drawn to show the trends in the data, but do not represent analytical fits to the data.

The boron data show the glass to corrode at a continuously decreasing rate in tests with SRL 202A glass at $2000 \mathrm{~m}^{-1}$. Technetium is released to solution stoichiometrically with boron, while uranium and neptunium are released to lesser extents than boron and technetium. In tests with SRL 202A glass at $2000 \mathrm{~m}^{-1}$, boron is released at a low rate through 182 days, but is released at a high rate after 182 days. Technetium is released at a low rate similar to boron through 182 days, and at a higher rate after 182 days, but the release of technetium after 182 days is less than that of boron. Of course, data from tests run for longer times are needed to determine the long-term behavior of technetium. Analysis of these solutions is in progress. Uranium and neptunium are released at low rates at all times tested, and are not affected by the increased glass corrosion rate after 182 days. 
Table 11. Normalized Mass Losses for Radionuclides

\begin{tabular}{|c|c|c|c|c|}
\hline Time, days & $\overline{N L(B)}$ & $\mathrm{NL}(\mathrm{Tc})$ & NL(U) & NL(Np) \\
\hline \multicolumn{5}{|c|}{ SRL 202A, $10 \mathrm{~m}^{-1}$} \\
\hline 7 & 0.69 & 2.7 & 0.045 & 0.67 \\
\hline 28 & 0.75 & $<1 \mathrm{a}$ & 0.04 & $<0.2$ \\
\hline 94 & 4.3 & $<1$ & 0.18 & $<0.2$ \\
\hline 238 & 0.95 & $<1$ & 0.11 & $<0.1$ \\
\hline 600 & 15 & $<1$ & 0.29 & 0.33 \\
\hline \multicolumn{5}{|c|}{ SRL 202A, $2000 \mathrm{~m}^{-1}$} \\
\hline 3 & 0.096 & 0.056 & 0.046 & 0.049 \\
\hline 7 & 0.19 & 0.12 & 0.11 & 0.099 \\
\hline 14 & 0.27 & 0.19 & 0.11 & 0.14 \\
\hline 30 & 0.41 & 0.28 & 0.077 & 0.14 \\
\hline 140 & 0.57 & 0.44 & 0.095 & 0.20 \\
\hline 280 & 0.67 & 0.87 & 0.12 & -b \\
\hline 560 & 0.83 & 0.87 & 0.17 & - \\
\hline 980 & 0.95 & 1.1 & 0.16 & - \\
\hline \multicolumn{5}{|c|}{ SRL 202A, $20,000 \mathrm{~m}^{-1}$} \\
\hline 3 & 0.13 & 0.074 & 0.028 & 0.025 \\
\hline 7 & 0.17 & 0.095 & 0.039 & 0.037 \\
\hline 14 & 0.20 & 0.12 & 0.046 & 0.041 \\
\hline 28 & 0.24 & 0.17 & 0.036 & 0.031 \\
\hline 56 & 0.31 & 0.26 & 0.019 & 0.021 \\
\hline 98 & 0.36 & 0.29 & 0.013 & 0.014 \\
\hline 182 & 0.57 & 1.5 & 0.010 & 0.072 \\
\hline 364 & 5.2 & 2.7 & 0.0038 & 0.0041 \\
\hline 484 & 12 & -- & 0.0023 & - \\
\hline 728 & 18 & -- & 0.0013 & - \\
\hline \multicolumn{5}{|c|}{ SRL 131A, $10 \mathrm{~m}^{-1}$} \\
\hline 7 & 1.0 & $<0.8$ & 0.16 & 0.59 \\
\hline 28 & 1.8 & 3.2 & 0.19 & 2.6 \\
\hline 94 & 5.2 & $<0.8$ & 0.45 & 1.2 \\
\hline 238 & 34 & 7.3 & 1.6 & 3.5 \\
\hline 601 & 53 & 34 & 7.7 & 15 \\
\hline \multicolumn{5}{|c|}{ SRL 131A, $2000 \mathrm{~m}^{-1}$} \\
\hline 3 & 0.76 & 0.35 & 0.12 & 0.80 \\
\hline 7 & 1.1 & 0.51 & 0.20 & 0.12 \\
\hline 14 & 1.3 & 0.61 & 0.19 & 0.12 \\
\hline 30 & 1.5 & 0.68 & 0.12 & 0.11 \\
\hline 70 & 1.7 & 0.81 & 0.081 & 0.041 \\
\hline 140 & 2.2 & 0.96 & 0.083 & 0.041 \\
\hline 280 & 5.4 & 0.96 & 0.082 & -- \\
\hline 560 & 9.6 & 5.4 & 0.036 & -- \\
\hline 980 & 13 & 7.5 & -- & - \\
\hline \multicolumn{5}{|c|}{ SRL $131 \mathrm{~A}, 20,000 \mathrm{~m}^{-1}$} \\
\hline 3 & 2.6 & 1.3 & 0.0033 & 0.00081 \\
\hline 7 & 4.8 & 2.5 & 0.0043 & 0.00096 \\
\hline 14 & 6.0 & 3.3 & 0.0036 & 0.0011 \\
\hline 28 & 7.5 & 4.5 & 0.0052 & 0.0013 \\
\hline 56 & 9.7 & 5.4 & 0.0054 & 0.0012 \\
\hline 98 & 9.5 & 9.7 & 0.0050 & 0.0017 \\
\hline 182 & 11 & 8.5 & 0.0055 & 0.0041 \\
\hline 364 & 20 & 12 & 0.0039 & 0.0016 \\
\hline 552 & 23 & -- & 0.0036 & -- \\
\hline 728 & 36 & - & 0.0068 & -- \\
\hline
\end{tabular}

aMaximum value based on analyzed radionuclide concentration below detection limit. bAnalysis not yet performed. 
(a)

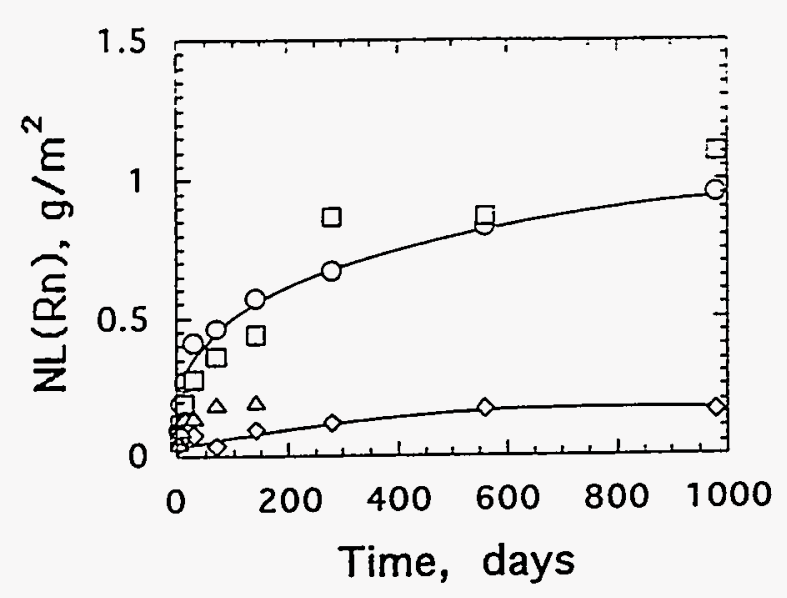

(c)

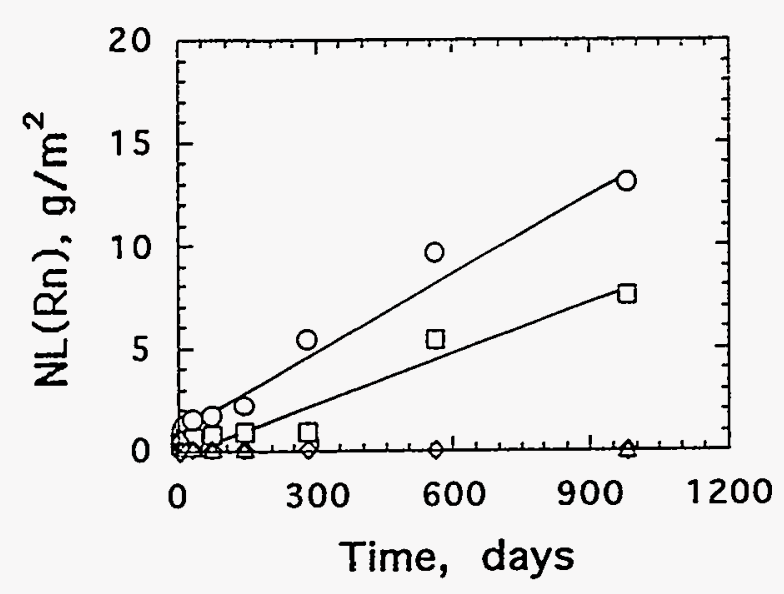

(b)

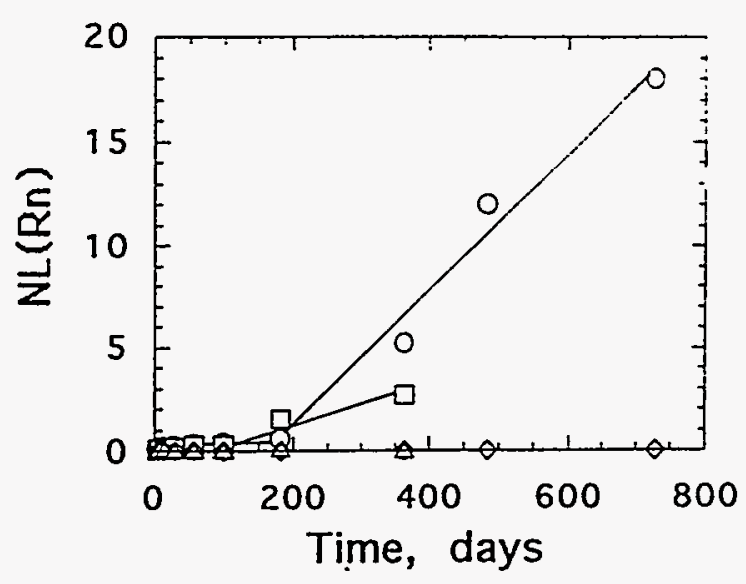

(d)

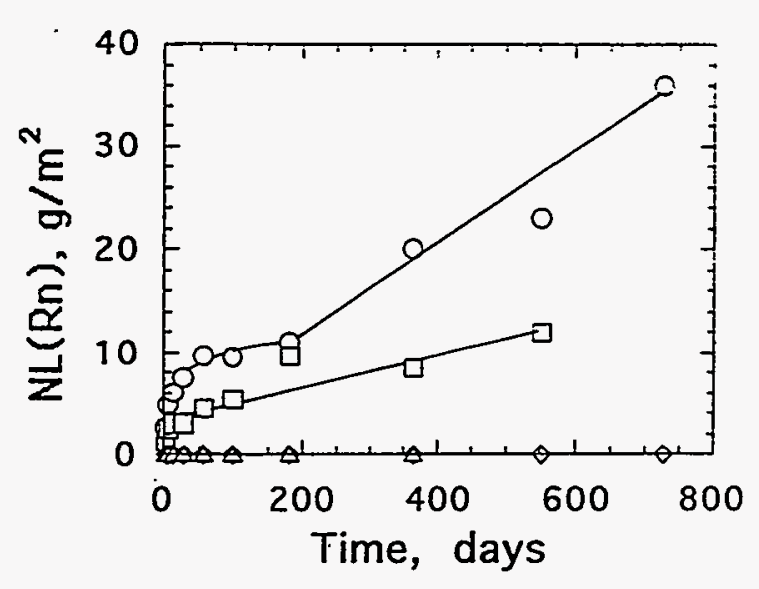

Fig. 58. Normalized Mass Loss vs. Reaction Time for (a) Tests with SRL 202A Glass at $2000 \mathrm{~m}^{-1}$, (b) Tests with SRL 202A Glass at $20,000 \mathrm{~m}^{-1}$, (c) Tests with SRL 131A Glass at $2000 \mathrm{~m}^{-1}$, and (d) Tests with SRL $131 \mathrm{~A}$ Glass at $20,000 \mathrm{~m}^{-1}:(\mathrm{O}) \mathrm{B},(\mathrm{D}) \mathrm{Tc}$, $(\mathrm{O}) \mathrm{U}$, and $(\Delta) \mathrm{Np}$. 
Tests with SRL 131A glass at $2000 \mathrm{~m}^{-1}$ show the glass to corrode at a high rate after about 140 days, as shown by the boron release. Technetium shows a similar constant release rate, but the normalized mass loss of technetium is about half that of boron. Uranium and neptunium are released to much lower extents than boron or technetium. The same behavior is seen in tests at $20,000 \mathrm{~m}^{-1}$, where boron and technetium are released at nearly constant rates, with boron being released a little faster than technetium, and uranium and neptunium are released at low rates at all times tested.

The behavior of technetium, uranium, and neptunium in the presence of glass corrosion products is of key interest due to their known high solubilities in oxidizing groundwaters. These tests show that technetium is released nearly congruently with boron as the glass corrodes, and that an increase in the glass corrosion rate due to secondary phase formation likely results in an increase in the release of technetium, although more analyses are needed to confirm this behavior in tests with SRL $202 \mathrm{~A}$ at $20,000 \mathrm{~m}^{-1}$, it is clearly demonstrated in tests with SRL $131 \mathrm{~A}$ at both 2000 and $20,000 \mathrm{~m}^{-1}$. The observation that $\mathrm{NL}(\mathrm{B})$ is consistently higher than NL(Tc) in tests with SRL 131A may be attributed to the large difference in the amounts of boron and technetium in the glass, although similar amounts are in SRL 202A. The thick alteration layers formed on SRL 131A and SRL 202A glasses after long reaction times may attenuate the release of technetium slightly.

Other analyses were performed to determine the disposition of the sparingly soluble actinide elements. The amounts of neptunium, plutonium, and americium that were suspended in solution (that fraction which passed through a $0.45-\mu \mathrm{m}$ filter but did not pass through a 6-nm filter), dissolved in solution (that fraction which passed through a 6-nm filter), or became fixed to the stainless steel reaction vessel (the fraction in the acid soak solution) were measured using alpha spectroscopy. The results of these analyses in terms of the amounts suspended in solution, dissolved in solution, and fixed to the steel are presented in Table 12 for several, representative tests with SRL 202A and SRL 131A glasses under columns labeled "sus," "dis," and "ads." Actinides not found in these fractions are assumed to remain associated with the altered glass or to have settled out of solution during the test.

Although there is scatter in the results, several general trends appear in the distributions of the actinides between various fractions in tests conducted at different SA/V and for different time periods. On average, neptunium was evenly distributed between the suspended and dissolved fractions in tests with both glasses. Neptunium was fixed to the vessel in tests at $20,000 \mathrm{~m}^{-1}$ but not in tests at $2000 \mathrm{~m}^{-1}$. About four times more plutonium was present in the suspended fraction than in the dissolved fraction in tests at $2000 \mathrm{~m}^{-1}$, but plutonium was found in only small amounts in the suspended fractions in tests at $20,000 \mathrm{~m}^{-1}$. Plutonium was preferentially fixed to the steel vessel in all tests. Only small amounts of dissolved americium are present in all tests. Similar amounts of americium are suspended in the leachate and fixed to the vessel in tests at $2000 \mathrm{~m}^{-1}$, but americium was only detected fixed to the vessel in tests at $20,000 \mathrm{~m}^{-1}$.

Between 75 and $90 \%$ of the released uranium was dissolved and the balance was suspended in all tests. The uranium distribution between the dissolved and suspended phases was similar for all time periods beyond a few days. Uranium was not found fixed to the stainless steel. 
Table 12. Mass of Actinides in Various Fractions. Reaction time in days; fractions that are suspended (sus), dissolved (dis), and fixed to the steel vessel (ads) in ng

\begin{tabular}{|c|c|c|c|c|c|c|c|c|c|}
\hline \multirow[b]{2}{*}{ Time, $d$} & \multicolumn{3}{|c|}{$\mathrm{Np}, \mathrm{ng}$} & \multicolumn{3}{|c|}{$\mathrm{Pu}, \mathrm{ng}$} & \multicolumn{3}{|c|}{ Am, ng } \\
\hline & sus & dis & ads & sus & dis & ads & sus & dis & ads \\
\hline \multicolumn{10}{|c|}{ SRL 202 at $2000 \mathrm{~m}^{-1}$} \\
\hline 14 & 420 & 96 & 51 & 34 & 0.75 & 8.6 & 0.87 & 0.017 & 0.24 \\
\hline 30 & 440 & 100 & 56 & 50 & 1.4 & 23 & 1.4 & 0.029 & 0.51 \\
\hline 70 & 110 & 290 & 15 & 60 & 12 & 30 & 1.3 & 0.23 & 1.2 \\
\hline 140 & 0 & 670 & 0 & 68 & 38 & 33 & 1.6 & 0.61 & 1.3 \\
\hline 280 & 540 & 160 & 0 & 140 & 3.1 & 170 & 2.1 & 0.074 & 3.6 \\
\hline 560 & 0 & 550 & 0 & 130 & 34 & 110 & 3.2 & 0.79 & 2.6 \\
\hline 980 & 87 & 170 & 0 & 74 & 19 & 150 & 1.8 & 0.39 & 3.0 \\
\hline \multicolumn{10}{|c|}{ SRL 202 at $20,000 \mathrm{~m}^{-1}$} \\
\hline 14 & 3000 & 180 & 120 & 180 & 5.5 & 17 & 3.7 & 0.12 & 0.59 \\
\hline 28 & 380 & 150 & 160 & 65 & 11 & 50 & 1.4 & 0.28 & 1.9 \\
\hline 56 & 180 & 68 & 270 & 10 & 0.97 & 38 & 0.22 & 0.02 & 1.6 \\
\hline 98 & 75 & 89 & 290 & 1.3 & 0.28 & 52 & 0.026 & 0.002 & 1.8 \\
\hline 182 & 21 & 76 & 360 & 0 & 1.7 & 58 & 0.02 & 0.003 & 2.1 \\
\hline 364 & 28 & 6.8 & 1100 & 0.11 & 0 & 88 & 0.01 & 0 & 2.6 \\
\hline 504 & 0.43 & 1.5 & 860 & 0 & 0.01 & 60 & 0 & 0 & 2.7 \\
\hline 728 & 0 & 69 & 660 & 0 & 0.37 & 203 & 0 & 0.004 & 6.9 \\
\hline \multicolumn{10}{|c|}{ SRL 131 at $2000 \mathrm{~m}^{-1}$} \\
\hline 14 & 221 & 125 & 580 & 5.4 & 0.65 & 4.5 & 0.07 & 0.004 & 0.097 \\
\hline 30 & 87 & 130 & 170 & 4.2 & 1.5 & 41 & 0.032 & 0.007 & 0.35 \\
\hline 70 & 64 & 230 & 0 & 13 & 4.7 & 46 & 0.20 & 0.036 & 0.93 \\
\hline 140 & 127 & 180 & 0 & 12 & 7.0 & 97 & 0.14 & 0.088 & 2.3 \\
\hline 280 & 41 & 35 & 0 & 2.4 & 11 & 92 & 0.022 & 0.009 & 1.0 \\
\hline 560 & 18 & 17 & 0 & 0.25 & 0.22 & 109 & 0.013 & 0 & 1.7 \\
\hline 980 & 46 & 0 & 0 & 0 & 0 & 122 & 0 & 0 & 1.9 \\
\hline \multicolumn{10}{|c|}{ SRL 131 at $20,000 \mathrm{~m}^{-1}$} \\
\hline 14 & 2.4 & 1.9 & 140 & 0.78 & 0.002 & $\overline{15}$ & 8.6 & $\overline{0}$ & 0.48 \\
\hline 28 & 3.0 & 1.5 & 260 & 0.003 & 0.001 & 53 & 0 & 0 & 1.4 \\
\hline 56 & 75 & 2.2 & 230 & 4.6 & 0.003 & 32 & 94 & 0 & 1.3 \\
\hline 98 & 1.3 & 0.95 & 330 & 0 & 0.002 & 140 & 0 & 0 & 3.9 \\
\hline 182 & 0 & 7.7 & 25 & 0 & 0.014 & 33 & 0 & 0 & 1.2 \\
\hline 364 & 0 & 2.7 & 383 & 0 & 0.012 & 24 & 0.05 & 0 & 0.78 \\
\hline 552 & 4.6 & 0 & 290 & 0.11 & 0 & 81 & 0.004 & 0 & 2.2 \\
\hline 728 & $13^{\circ}$ & 0 & 330 & 1.4 & 0 & 100 & 0.006 & 0 & 2.8 \\
\hline
\end{tabular}


The amounts of plutonium and americium in the suspended and dissolved fractions generally decreased with the reaction time, most noticeably in tests at $20,000 \mathrm{~m}^{-1}$. The amounts of each found fixed to the vessel generally increased with the reaction time. Temporal changes are probably due to increased glass corrosion, changes in the solution chemistry, and flocculation of colloids.

The highest levels of neptunium, plutonium, and americium measured in the dissolved fractions under each test condition are summarized in Table 13. Also shown are the solubilities of these actinides measured in J-13 groundwater at room temperature at $\mathrm{pH} 8.5$ [221]. These solubility concentrations are several orders of magnitude greater than the amounts measured to be dissolved in these tests. This discrepancy may be due to competing equilibria among dissolved, colloidal, and sorbed or plated phases, or to different solubility-controlling phases. The amounts of actinides released from either SRL 131A or SRL 202A in these tests were much smaller than the amounts released from R7T7 glass in tests conducted at lower SA/V ratios $[248,249]$. Except for weeksite, which may control the solubility of uranium, solubilitycontrolling phases remain to be identified.

These tests are relevant to glass performance because they indicate the amounts of each transuranic element that can be transported by groundwater flow. The dissolved fraction is assumed to be fully mobile, while the suspended fraction may or may not be fully mobile, depending on the surroundings. Although the fractions of actinides sorbed to the vessel or remaining with the glass are immobile during the test, actinides may be mobilized as the metal corrodes, as corrosion products slough off the glass, or as the solution chemistry changes. Of course, subsequent interactions may also affect the mobility of the dissolved fraction.

The amounts of actinides that remain associated with the glass during static leach tests can be estimated by comparing the amounts found to be suspended, dissolved, or fixed to the vessel to the extent of glass corrosion in each test. A "retention factor" has been used to describe ability of the corroded glass to mitigate the release of actinides to the solution [249]. The retention factor is defined as the ratio of the normalized boron mass loss (which defines the extent of corrosion) to the normalized actinide mass loss, where the boron release represents the extent of glass corrosion. Retention factors for technetium, uranium, and neptunium were calculated from the ICP-MS results. The sum of the amounts in the suspended, dissolved, and fixed fractions measured by alpha spectroscopy was used to compute the normalized mass losses of neptunium, plutonium, and americium. The amounts adsorbed to the steel vessel were included in the total amount released because the adsorbed material was not retained by the

Table 13. Highest Actinide Concentrations Measured in Leach Tests

\begin{tabular}{lcll}
\hline Test & Np (M) & Pu (M) & Am (M) \\
\hline SRL 202 at 2000 m-1 & $2 \times 10^{-7}$ & $1 \times 10^{-8}$ & $1 \times 10^{-9}$ \\
SRL 202 at 20,000 m-1 & $2 \times 10^{-7}$ & $5 \times 10^{-9}$ & $1 \times 10^{-10}$ \\
SRL 131 at 2000 m-1 & $1 \times 10^{-7}$ & $5 \times 10^{-9}$ & $1 \times 10^{-11}$ \\
SRL 131 at 20,000 m-1 & $7 \times 10^{-9}$ & $6 \times 10^{-12}$ & 0 \\
Solubility in J-13 $\left(25^{\circ} \mathrm{C}\right)^{\mathrm{a}}$ & $4.4 \times 10^{-5}$ & $2.9 \times 10^{-7}$ & $2.4 \times 10^{-4}$ \\
\hline
\end{tabular}

aFrom Nitsche [221]. 
altered glass and was available for transport upon its release. The calculated retention factors for each radionuclide, $R F(R n)$, where $R n$ is Tc, $U, N p, P u$, or $A m$, are given for the tests in Table 14. A value of $R F(R n)$ near one indicates that the actinide is released into solution to a similar extent as boron, while large values of $R F(R n)$ indicate that the actinide is retained by the glass as it corrodes. Retention may result from several phenomena, including secondary phase formation, sorption, flocculation of colloidal material, and simply the limited solubilities of the actinides.

Note that the difference in the RF(Np) calculated from the ICP-MS results and from the alpha spectroscopy results is due to the neptunium sorbed to the vessel walls. This fraction is counted as not being retained in the RF(Np) calculated from the alpha spectroscopy results, but is counted as being retained in the $R F(N p)$ calculated from the ICP-MS results. As seen in Table 12, the neptunium adsorbed onto the vessel walls is the dominant fraction in tests conducted at $20,000 \mathrm{~m}^{-1}$ for both glasses. Technetium and uranium are not adsorbed onto the vessel walls.

In general, these tests show (1) actinides are retained by the glass much more than technetium (2) similar techentium retention factors are calculated for both glasses, at all SA/V ratios, and at all test durations, (3) the retention factors of the actinides are greater for the less durable SRL 131A glass than for the SRL 202A glass, increase with the SAV ratio of the test, and increase with the test durations. The first finding can be explained as a result of the much higher solubilitiy of technetium than any of the actinides. The differences in the solubilities of the actinides are also correlated with their retention: of the actinides, uranium and neptunium are more soluble and have lower retention factors than plutonium and americium. The observation that the retention factor of technetium is independent of the glass composition, SA $/$ ratio, and duration of the test simply shows that technetium is released congruent with boron, and that the effects of glass composition, SA/V ratio, and time on the glass corrosion rate similarly effect the technetium release. Conversely, this shows that technetium cannot be released any faster than the glass corrodes (as measured by the release of boron to solution).

The observation that the retention of the actinides varies with the glass composition, SA/V ratio, and duration of the test shows that their retention is affected by glass corrosion products, and can be explained as an effect of the formation of alteration layers, secondary phases, and colloids. This is seen in the difference of the dissolved and suspended fractions in Table 12. At short reaction times and at low SA/V ratios, most of the plutonium and americium are found suspended in solution. At longer times, very little is found suspended in solution. The change is attributed to the settling of colloids at longer reaction times. Analysis of the leachate solution before and after filtration through $6 \mathrm{~nm}$ filters has indicated that material containing aluminum, iron, and probably silicon becomes suspended the leachates as the glasses corrode. This material remains suspended in the leachates of tests conducted at $2000 \mathrm{~m}^{-1}$, but settles out of solution after 28 days in tests at $20,000 \mathrm{~m}^{-1}$. Actinides that become associated with suspended material during the initial stages of corrosion are removed from solution as the material flocculates and settles out of solution.

The higher retention of actinides by SRL 131 glass is due to its poorer durability. (The NL(B) values for tests with SRL 131 glass are about an order of magnitude higher than those for tests with SRL 202 glass under the same test conditions.) Colloids containing actinides form in, and then settle out of, solution faster in tests with SRL 131A than in tests with SRL 202A glass. In addition, thicker alteration layers form on SRL 131A glass as it corrodes, which provides much more surface area for sorption of actinides than the thinner layers formed on SRL 202A glass. 
Table 14. Radionuclide Retention Factors for Tests with SRL 202A and SRL 131A Glasses ${ }^{\mathrm{a}}$

\begin{tabular}{|c|c|c|c|c|c|}
\hline Time, days & $\mathrm{RF}(\mathrm{Tc})$ & $\mathrm{RF}(\mathrm{U})$ & $R F(\mathrm{~Np})$ & $\mathrm{RF}(\mathrm{Pu})$ & $\mathrm{RF}(\mathrm{Am})$ \\
\hline \multicolumn{6}{|c|}{ SRL 202 at $10 \mathrm{~m}^{-1}$} \\
\hline 7 & 0.45 & 16 & 1.2 & $\overline{1.5}$ & 0.07 \\
\hline 28 & $<0.8^{\mathrm{b}}$ & 18 & $<4$ & $<4$ & $<0.5$ \\
\hline 94 & $<1$ & 31 & $<$ & $<$ & $<0.5$ \\
\hline 238 & $<6$ & 9.1 & 4.5 & $<27$ & $<$ \\
\hline 600 & 26 & 59 & 42 & 60 & $<15$ \\
\hline \multicolumn{6}{|c|}{ SRL 202 at $2000 \mathrm{~m}^{-1}$} \\
\hline 14 & 1.4 & 2.4 & 1.9 & $\overline{12}$ & $\overline{19}$ \\
\hline 30 & 1.5 & 5.2 & 2.9 & 11 & 16 \\
\hline 70 & 1.3 & 7.5 & 2.4 & 9.2 & 14 \\
\hline 140 & 1.3 & 6.1 & 2.8 & 8.5 & 13 \\
\hline 280 & 0.9 & 5.9 & 2.0 & 4.5 & 9.7 \\
\hline 560 & 0.95 & 5.9 & 3.0 & 6.1 & 9.9 \\
\hline 980 & 0.85 & 6.0 & 12 & 8.3 & 15 \\
\hline \multicolumn{6}{|c|}{ SRL 202 at $20,000 \mathrm{~m}^{-1}$} \\
\hline 14 & $\overline{1.7}$ & 4.0 & 4.8 & 1.9 & 3.6 \\
\hline 28 & 1.2 & 7.0 & 8.0 & 3.8 & 5.3 \\
\hline 56 & 1.1 & 15 & 15 & 13 & 14 \\
\hline 98 & 1.1 & 27 & 25 & 14 & 17 \\
\hline 182 & 0.63 & 58 & 7.9 & 18 & 19 \\
\hline 364 & 2.0 & 1400 & 9.0 & 120 & 160 \\
\hline 484 & $-^{c}$ & 9000 & 28 & 400 & 360 \\
\hline 736 & - & 8000 & 54 & 180 & 210 \\
\hline \multicolumn{6}{|c|}{ SRL 131 at $10 \mathrm{~m}^{-1}$} \\
\hline 7 & $<0.5$ & 5.5 & 1.7 & 2 & $<02$ \\
\hline 28 & 0.75 & 9.7 & 1.4 & 0.7 & 4.3 \\
\hline 94 & $<2.8$ & 8.5 & 6.0 & $<11$ & $<14$ \\
\hline 238 & 2.8 & 7 & 1.4 & 30 & 14 \\
\hline 601 & 1.7 & 8.5 & 3.4 & $<340$ & $<460$ \\
\hline \multicolumn{6}{|c|}{ SRL 131 at $2000 \mathrm{~m}^{-1}$} \\
\hline 14 & 2.3 & 7.0 & 10 & 120 & 570 \\
\hline 30 & 2.3 & 13 & 15 & 65 & 310 \\
\hline 70 & 2.1 & 19 & 42 & 120 & 120 \\
\hline 140 & 2.2 & 26 & 52 & 37 & 70 \\
\hline 280 & 5.5 & 63 & 59 & 110 & 380 \\
\hline 560 & 1.8 & 270 & 550 & 180 & 460 \\
\hline 980 & 1.7 & 1000 & 600 & $\underline{220}$ & 580 \\
\hline \multicolumn{6}{|c|}{ SRL 131 at $20,000 \mathrm{~m}^{-1}$} \\
\hline 14 & 1.7 & 4.0 & 40 & 800 & 1000 \\
\hline 28 & 1.7 & 3.2 & 58 & 280 & 430 \\
\hline 56 & 1.8 & 4.0 & 64 & 520 & 600 \\
\hline 98 & 1.1 & 4.4 & 55 & 130 & 190 \\
\hline 182 & 1.2 & 5.9 & 710 & 670 & 730 \\
\hline 364 & 1.6 & 12 & 100 & 1700 & 2100 \\
\hline 552 & - & 12 & 160 & 570 & 840 \\
\hline 728 & - & 15 & 210 & 720 & 1000 \\
\hline
\end{tabular}

aValues in bold type calculated from alpha-counting analyses. Values in regular type calculated from ICP-MS analyses.

bMaximum value based on analyzed radionuclide concentration below detection limit.

cAnalysis not yet performed. 
Colloids that have been identified to be suspended in the leachates of these tests include smectite and kaolinite clays, nagelschmidtite, calcite, dolomite, magnesium oxide, titanium oxide, weeksite, uranophane, boltwoodite, and phases rich in uranium-titanium-, iron-, and aluminum [263]. Smectite clays are the most ubiquitous and abundant phases found suspended in the solution. Smectite clays are known to be an abundant alteration phase of the hydrated surfaces of these glasses [64,65]. All the other phases have been found to be either associated with alteration layers formed on individual glass grains or existing as separate phases mixed with the reacted glass. These phases may nucleate both in the solution and on the reacted glass surface, and then settle out of solution as separate solids as they increase in size. The suspended phases allow mobile fractions of actinide elements to exist in the leachate at levels far above their solubilities either through their being sorbed onto or incorporated into the phases. While several uranium-bearing phases have been identified, the low concentrations of transuranic elements used in testing make it difficult to identify the secondary phases which sequester them and/or affect their solubilities using electron beam techniques. Autoradiography has been used to identify particles containing plutonium and americium oxides associated with colloidal clay material in other tests [262]. The same clay colloids have been found to form in the present tests, and it is likely that actinides become associated with them. Work is in progress to identify which actinide phases are formed during glass corrosion.

\subsection{Effects of the SA/V on the Alteration of the Glass Surface}

The alteration of the glass surface as the glass corrodes is of interest because (1) alteration layers provide valuable insight into the corrosion mechanism of the glass, particularly the glass/layer interface. The relation between the processes leading to glass dissolution (i.e., water diffusion, ion exchange, and hydrolysis) will be revealed at this interface. For example, whether or not the interrelation of water diffusion and ion exchange observed in the early stages of corrosion in work by Dran et al. (see Section 2.1.3) persists throughout the corrosion process needs to be known to describe the long-term corrosion behavior. An understanding of the glass corrosion mechanism and rate limiting process is important for characterizing the long-term corrosion rate needed in performance assessment models. (2) Alteration layers provide insight regarding the control the solubilities of key glass matrix elements during Stage II of the corrosion (prior to the formation of secondary phases). One approach to projecting the glass corrosion rate requires analysis of the alteration layer to define the saturation conditions [143]. Also, secondary phases formed after the solution becomes saturated may nucleate on or in the alteration layer. These phases must be identified to project long-term glass corrosion behavior. (3) Alteration layers commonly retain radionuclides as the glass corrodes, and layer analysis is needed to characterize their disposition.

Alteration layers that formed in tests in this Task that were conducted at different SA/V ratios are being characterized and compared to determine how the SA/V ratio of the test affected the composition, structure, and thickness of the layers. Crystalline layers were observed to form at the glass surface in tests conducted at SA/V ratios of 340,2000 , and $20,000 \mathrm{~m}^{-1}$ at all reaction times tested. (Layers formed in tests at $10 \mathrm{~m}^{-1}$ have not yet been analyzed. Layers formed after short reaction times are expected to be amourphous, as was seen in tests with SRL 165 glass at an SA/V ratio of $30 \mathrm{~m}^{-1}$ conducted for 56 days or less [87].) Fibrous clay crystals, oriented perpendicular to the glass surface, formed at the shortest reaction times tested at all SA/V ( 3 days in the PCT tests and 7 days in the MCC-1 tests). After longer reaction times, these crystals became consolidated into laths of clay that were generally oriented perpendicular to the glass surface. Figures 59a-f show the TEM images of alteration layers that formed on SRL 202U glass in tests in either deionized water or EJ-13 solution at SA/V ratios of 340,2000 , or $20,000 \mathrm{~m}^{-1}$. The alteration layers are on the top halves of Figs. 59a-e and on the left side of Fig. 59f. The layers have formed separated from the glass in Figs. 59b-e, but not in Figs. 59a and 59f. The 
(a)

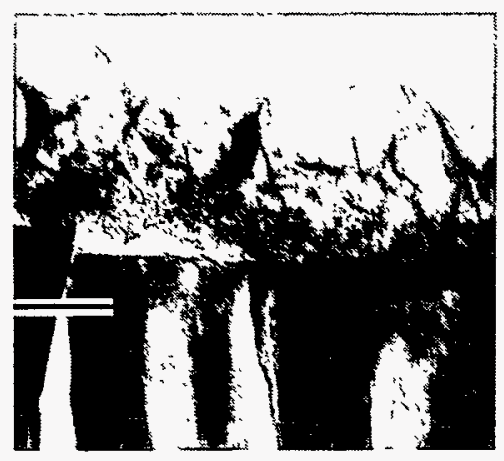

(c)

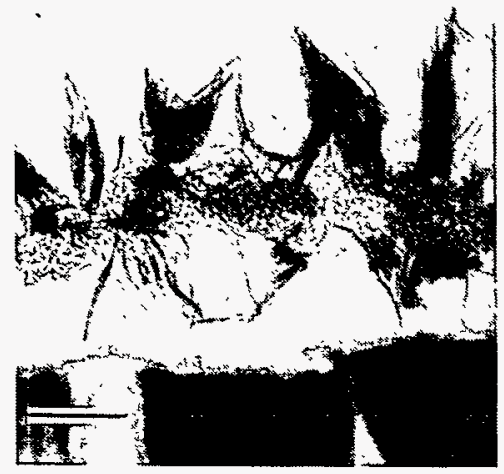

(e)

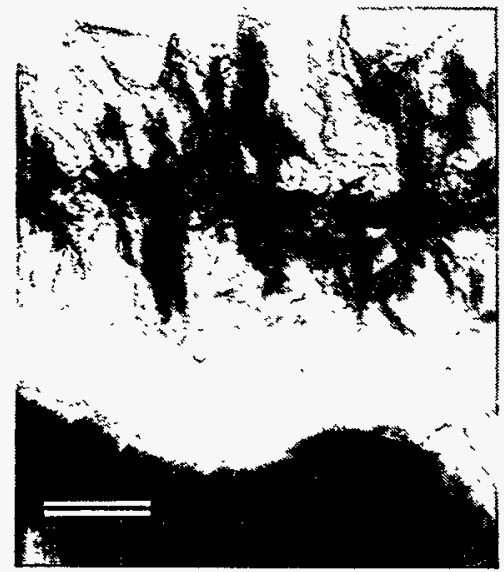

(b)

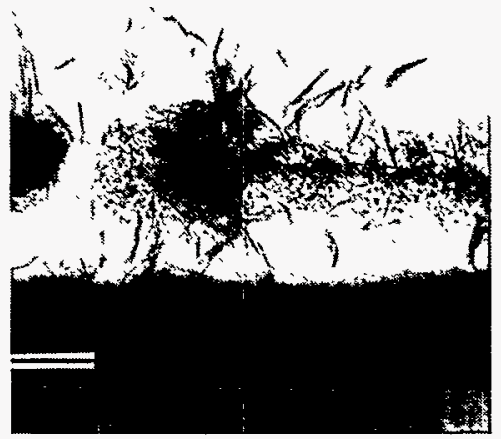

(d)

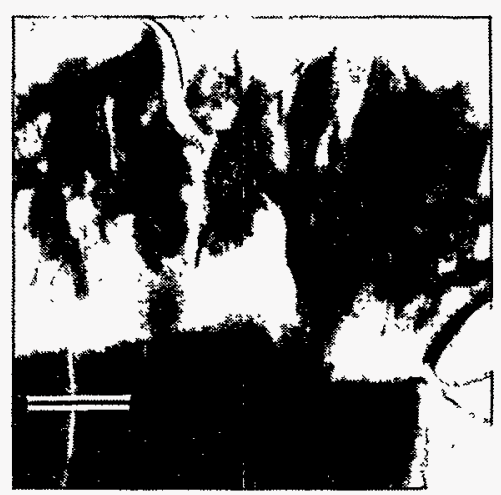

(f)

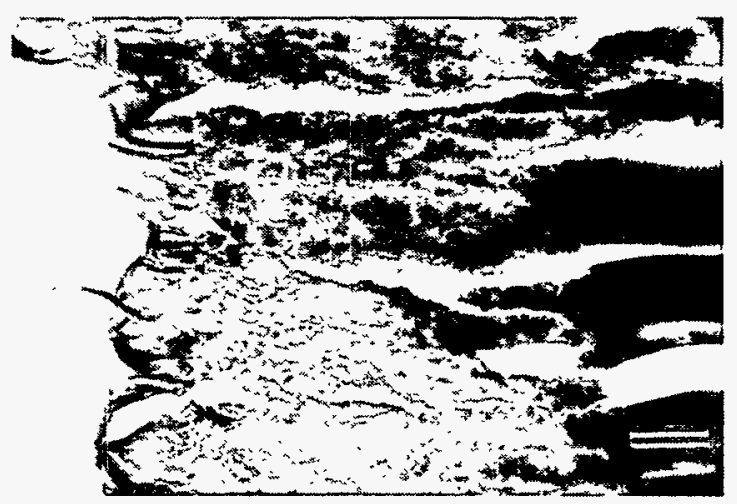

Fig. 59. Photomicrographs of Alteration Layers Formed on SRL 202U Glass:

(a) $340 \mathrm{~m}^{-1} / \mathrm{DIW}$ after 28 Days (bar $=100 \mathrm{~nm}$ ), (b) $340 \mathrm{~m}^{-1} / \mathrm{DIW}$ after 360 Days (bar $=100 \mathrm{~nm}$ ), (c) $340 \mathrm{~m}^{-1} / \mathrm{DIW}$ after 360 Days (bar $=100 \mathrm{~nm}$ ), (d) $2000 \mathrm{~m}^{-1} / \mathrm{EJ}-13$ after 360 Days (bar $=100 \mathrm{~nm}$ ), (e) 20,000 m-1/EJ-13 after 180 Days (bar $=100 \mathrm{~nm}$ ), and (f) $20,000 \mathrm{~m}^{-1} / \mathrm{EJ}-13$ after 364 Days (bar $=400 \mathrm{~nm}$ ). Distance represented by bars are given in parentheses (after [64]). 
unreacted glass was chattered during sample preparation, but the alteration layers that had formed on the glass during testing were not chattered. The layers remained intact because epoxy used to fix the particles during sample preparation had penetrated the layers but not the glass.

Figure 59a shows the layer formed in a test conducted at an SA/V ratio of $340 \mathrm{~m}^{-1}$ after 28 days of corrosion in deionized water. Laths of crystalline material radiate perpendicular to the surface, and would have projected into the leachate solution during the test. A layer of finegrained material can be seen beneath the layer of coarse crystals. The fine-grain material appears to remain in contact with the underlying glass, though the layer has been mechanically separated from the glass in some areas during sample preparation. Figure 59b shows that, after 360 days in tests in deionized water, small, randomly oriented crystallites have formed within the initially amorphous layer, and discrete laths of crystalline material identical to that formed at shorter times has formed between the amourphous layer and the glass surface. This results in the formation of a twinned layer of coarse-grained crystalline laths that separates from the underlying glass. A thin band of fine-grained material remains between the layers of lath-like crystals in most samples. Notice that another amorphous layer does not form on the glass surface exposed as the layer separates from the glass. The glass beneath the layer appears to dissolve stoichiometrically as corrosion continues, though the erosion of the glass is not uniform and the glass eventually looks pitted.

The layer formed after corrosion in deionized water at $2000 \mathrm{~m}^{-1}$ for 360 days is shown in Fig. 59c. This layer is very similar to that formed in tests at $340 \mathrm{~m}^{-1}$ for 360 days shown in Fig. 59b. Both have a twinned appearance with coarse-grained crystalline laths protruding inward towards the glass and outward into the resin with an inner band of fine-grained material in the center. The layers in both tests have separated from the underlying glass and over-all thicknesses are similar. The layer in tests in EJ-13 solution at $2000 \mathrm{~m}^{-1}$ run for 360 days is shown in Fig. 59d. This layer is similar to that shown in Fig 59c, except the inner band of finegrain material is thinner in layers formed on glasses reacted in EJ-13 solution than on those formed in deionized water. A similar layer is formed after reaction for 182 days in EJ-13 solution at $20,000 \mathrm{~m}^{-1}$, as shown in Fig. 59e.

The layer formed in a test conducted for 364 days in EJ-13 solution at $20,000 \mathrm{~m}^{-1}$ is shown in Fig. 59f. This test had shown a sudden release of soluble glass components indicative of an increased corrosion rate (see Section 4.1). The increased corrosion rate results in the formation of a much thicker alteration layer than seen prior to the increase in the rate. The outer portion of the layer is identical to that formed previously. It consists of an outer layer of coarsegrained crystals oriented perpendicular to the surface that has a thickness similar to that of the layer formed after 182 days. A thicker inner layer of fine-grain material is present between the outer layer of coarse-grain material and the unreacted glass. The fine-grain material interfaces with both the outer layer and the underlying glass, i.e., it does not show the separation between layer and glass the way the coarse-grain layers did (compare to Figs. 59b-e).

Compositional analysis of the layers by means of EDS and structural analysis by means of selected area electron diffraction (SAED) showed that the outer and inner layers were composed of the same polycrystalline material, which is identified as a smectite clay. Table 15 gives a representative composition of the clay laths in the oriented outer layers and the random inner layer formed in deionized water. The compositions of the inner and outer layers are similar; both are depleted of soluble glass components and enriched in aluminum and chloride. Clays with a similar composition are formed in EJ-13 [64]. Table 16 gives the lattice spacings measured from several representative SAED patterns and those of some smectite clays. 
Table 15. Measured Composition of Alteration Layers Formed on SRL 202 Glass Reacted in DIW at $20,000 \mathrm{~m}^{-1}$ for 364 Days $^{\mathrm{a}}$

\begin{tabular}{lccc}
\hline Element & Outer Layer & Inner Layer & Unreacted Glass \\
\hline $\mathrm{Al}$ & 8.5 & 10 & 4.9 \\
$\mathrm{Ca}$ & 0.1 & 0.1 & 2.2 \\
$\mathrm{Cl}$ & 3.2 & 3.2 & $<0.5$ \\
$\mathrm{Fe}$ & 20 & 16 & 21 \\
$\mathrm{~K}$ & 0.6 & 0.9 & 7.5 \\
$\mathrm{Mg}$ & 1.1 & 1.5 & 0.2 \\
$\mathrm{Mn}$ & 4.4 & 4.7 & 4.3 \\
$\mathrm{Na}$ & 0.5 & 0.1 & 8.2 \\
$\mathrm{Ni}$ & 2.5 & 2.9 & 2.1 \\
$\mathrm{Si}$ & 57.8 & 59.5 & 48 \\
$\mathrm{Ti}$ & 1.3 & 1.1 & 1.6 \\
\hline
\end{tabular}

aln elemental weight percent, normalized to $100 \%$ total.

Table 16. Measured d-Spacings from Selected Area Electron Diffraction Analysis of SRL 202 Glass Reacted at $20,000 \mathrm{~m}^{-1}$ for 364 Days, in Angstroms

\begin{tabular}{|c|c|c|c|c|c|}
\hline Layer I & Layer II & Layer III & $\begin{array}{l}\text { Nontronite } \\
(29-1497)^{\mathrm{a}}\end{array}$ & $\begin{array}{l}\text { Montmorillonite } \\
(12-219)\end{array}$ & $\begin{array}{c}\text { Saponite } \\
(29-1491)\end{array}$ \\
\hline $9.0^{\mathrm{b}}$ & & & 15.2 & $\begin{array}{l}17.6 \\
9.00\end{array}$ & 15.5 \\
\hline & & & 7.44 & 599 & $\begin{array}{l}7.73 \\
5.11\end{array}$ \\
\hline 4.48 & 4.48 & $4.42 \pm 0.12^{c}$ & 4.48 & 4.49 & 4.57 \\
\hline & & $\begin{array}{l}3.20 \pm 0.07 \\
2.58 \pm 0.05\end{array}$ & $\begin{array}{l}3.58 \\
3.05 \\
2.564\end{array}$ & $\begin{array}{l}3.58 \\
2.99 \\
2.57\end{array}$ & $\begin{array}{l}3.834 \\
3.07 \\
2.61\end{array}$ \\
\hline 2.57 & 2.53 & $2.58 \pm 0.05$ & 2.564 & $\begin{array}{l}2.37 \\
2.242\end{array}$ & \\
\hline $\begin{array}{l}1.50 \\
1.29 \\
\end{array}$ & $\begin{array}{l}1.50 \\
1.29 \\
\end{array}$ & $\begin{array}{l}1.70 \pm 0.03 \\
1.50 \pm 0.02 \\
1.30 \pm 0.02\end{array}$ & $\begin{array}{l}1.51 \\
1.336 \\
\end{array}$ & $\begin{array}{l}1.699 \\
1.504 \\
1.294\end{array}$ & $\begin{array}{l}1.731 \\
1.533 \\
1.321 \\
\end{array}$ \\
\hline
\end{tabular}

Layer I: outer layer/EJ-13; II: inner layer/DIW; III: outer layer/DIW.

aJCPDS file number of representative smectite clay.

bMeasured from lattice fringes in image.

'Estimate measurement errors common to all experimental values. 
The structure and composition of isolated secondary phases were analyzed by AEM and SEM. Phases identified include the uranium-bearing phases weeksite and boltwoodite, zeolites in the chabazite and heulandite groups, clinoptilolite, and nagelschmidtite. A noncrystalline feature bearing uranium and titanium was also identified within the clay phase or between the clay layer and the underlying glass.

\subsection{Summary}

A series of static leach tests is being conducted to address remaining issues regarding the effect of the SA/V ratio on the glass corrosion behavior and the disposition of radionuclides during glass corrosion. These results show that the SA/V ratios used in static leach tests affect both the dominant reaction step and the long-term rate of glass reaction. The reaction can be characterized in terms of three stages: reaction at a forward rate characteristic of the glass composition and solution $\mathrm{pH}$, at an intermediate rate that may become very low, and at a longterm rate, which may be the same as the intermediate rate or may be accelerated by the effects of secondary phase formation. The SA/V used in a test influences which reaction stage is observed during the test. Tests at low SA/V (10 and $340 \mathrm{~m}^{-1}$ ) maintain dilute leachate conditions, and the observed glass reaction proceeds at near the forward reaction rate. The glass reaction is dominated by the leaching of alkali metals and boron from the glass, but the silicon release and dissolution of the glass network is low under these conditions. The release of alkali metals leads to an increase in the solution $\mathrm{pH}$ value, which facilitates the silicon release and network dissolution. As the silicic acid concentration increases as the glass reacts, the reaction slows and approaches a minimum rate. The higher $\mathrm{pH}$ values attained in tests at $2000 \mathrm{~m}^{-1}$ promote silica dissolution and lead to high solution concentrations of several glass components. The high silicic acid concentrations eventually slow the glass corrosion to a low rate. At an even higher $\mathrm{SA} / \mathrm{V}\left(20,000 \mathrm{~m}^{-1}\right)$, the glass reaction slows initially as the silicic acid level increases, but then it accelerates as secondary phases form to consume silicic acid and other solution components. Because the reaction step controlling the observed glass dissolution rate may differ in tests at different $\mathrm{SA} / \mathrm{V}$, the measured long-term rate is sensitive to the test conditions, including the $\mathrm{pH}$ that is attained and secondary phase formation, and the SA/V cannot be used to quantitatively or uniformly accelerate the glass reaction progress.

Actinide elements become distributed between dissolved, suspended, and immobile phases as glass waste forms corrode. The disposition of actinides measured in laboratory tests may be affected by their interaction with glass corrosion products, complexants in the leachate, and the reaction vessel. Specific solubilities and interactions of the actinides are complicated by chemical and physical changes that may occur during the test. These include changes in the solution chemistry as the glass corrodes, especially the ligand concentrations and the $\mathrm{pH}$; in the abundance of colloidal material; and in the reaction vessel. Corresponding effects will be present during storage of waste forms in geologic repositories and must be accounted for when assessing the performance of a disposal site. The present tests at ANL demonstrate that (1) the concentrations of complexants and the disposition and solubilities of actinide elements are sensitive to the SA/V of the test, (2) association of actinides with suspended corrosion products may increase their solution contents, (3) the abundance of suspended material depends on the solution chemistry and the reaction time, and (4) the amounts of actinide materials available for transport are affected by their solubilities and the stabilities of suspended phases with which they become associated.

The release of transuranics increases as the glass reacts during this stage, but their low solubilities limit the solution concentrations to low values. The release of transuranics into solution increases initially, but secondary phase and colloid formation eventually removes them from solution at longer reaction times. The release of transuranics is not affected by the accelerated glass reaction at high $\mathrm{SA} / \mathrm{V}$, but is controlled by their low solubilities. 


\section{ACKNOWLEDGMENTS}

This work was supported by the U.S. Department of Energy, Office of Environmental Restoration and Waste Management, under Contract W-31-109-ENG-38. Technical review by John $\mathrm{K}$. Bates is gratefully acknowledged. Data review and verification performed by Seymour Vogler. Solution analyses performed by Edmund A. Huff, Alice M. Essling, Meiling Gong, Stephen F. Wolf, Delbert L. Bowers, Florence P. Smith, Lohman D. Hafenrichter, and Mark T. Surchik. Analytical electron microscopy samples prepared by Nancy L. Dietz. AEM microscopic analyses performed by Edgar C. Buck, Neil R. Brown, and Charles R. Bradley. XRD performed by Benjamin S. Tani. This manuscript was prepared by Roberta Riel. 


\section{REFERENCES}

1. S. F. Kerrisk, An Assessment of the Important Radionuclides in Nuclear Waste, Los Alamos National Laboratory Report LA-10414-MS (1985).

2. B. W. Veal, J. N. Mundy, and D. J. Lam, "Actinides in Silicate Glasses" in Handbook on the Physics and Chemistry of the Actinides, A. J. Freeman and G. H. Lander, eds., Elsevier Science Publishers B.V., pp. 271-309 (1987).

3. N. E. Bibler and A. R. Jurgensen, "Leaching Tc-99 from SRP Glass in Simulated Tuff and Salt Groundwaters," Mater. Res. Soc. Symp. Proc. 112, 585-593 (1988).

4. E. Freude, W. Lutze, C. Russel, and H. A. Schaeffer, "Investigation of the Redox Behavior of Technetium in Borosilicate Glass Melts by Voltammetry," Mater. Res. Soc. Symp. Proc. 127, 199-204 (1989).

5. F. M. Ernsberger, "The Role of Molecular Water in the Diffusive Transport of Protons in Glasses," Phys. Chem. Glasses 21(4), 146-149 (1980).

6. D. L. Dugger, J. H. Stanton, B. N. Irby, B. L. McConnel, W. W. Cummings, and R. W. Maatman, "The Exchange of Twenty Metal Ions with the Weakly Acidic Silanol Group of Silica Gel," J. Phys. Chem. 68, 757-760 (1964).

7. B. C. Bunker, "Waste Glass Leaching: Chemistry and Kinetics," Mater. Res. Soc. Symp. Proc. 84, 493-507 (1987).

8. R. M. Barrer and J. Klinowski, "Hydrogen Mordenite and Hydronium Mordenite. Ion Exchange and Thermal Stability," J. Chem. Soc. Farad. 71, 690-698 (1975).

9. B. C. Bunker, G. W. Arnold, D. E. Day, and P. J. Bray, "The Effect of Molecular Structure on Borosilicate Glass Leaching," J. Non-Cryst. Solids 87, 226-253 (1986).

10. B. M. J. Smets and T. P. A. Lommen, "The Leaching of Sodium Aluminosilicate Glass Studied by Secondary Ion Mass Spectrometry," Phys. Chem. Glasses 23(3), 83-87 (1982).

11. C. Q. Buckwalter and L. R. Pederson, "Inhibition of Nuclear Waste Glass Leaching by Chemisorption," J. Am. Ceram. Soc. 65(9), 431-436 (1982).

12. R. L. Lehman and F. A. Kuchinsky, 'The Effect of Various Lead Species on the Leaching Behavior of Borosilicate Waste Glass," Mater. Res. Soc. Symp. Proc. 44, 179-186 (1985).

13. C. T. Lee and D. E. Clark, "Effects of Solution Cations on Waste Glass Leaching," Adv. Ceram. 20, 541-550 (1986).

14. Aa. Barkatt, E. E. Saad, R. B. Adiga, W. Sousanpour, Al. Barkatt, X. Feng, J. A. O'Keefe, and S. Alterescu, "Interactions of Silicate Glasses with Aqueous Environments under Conditions of Prolonged Contact and Flow," Mater. Res. Soc. Symp. Proc. 125, 129-142 (1988).

15. W. G. Spitzer and J. R. Ligenza, "Oxygen Exchange Between Silica and High Pressure Steam," J. Phys. Chem. Solids 17(3/4) 196-202 (1961). 
16. G. J. Roberts and J. P. Roberts, "An Oxygen Tracer Investigation of the Diffusion of 'Water' in Silica Glass," Phys. Chem. Glasses Z(3), 82-89 (1966).

17. R. Pfeffer, "Network Oxygen Exchange During Water Diffusion in $\mathrm{SiO}_{2}$," J. Appl. Phys. $\underline{52}(2), 777-784$ (1981).

18. B. C. Bunker, G. W. Arnold, E. K. Beuchamp, and D. E. Day, "Mechanisms for Alkali Leaching in Mixed-Na-K Silicate Glasses," J. Non-Cryst. Solids 묘, 295-322 (1983).

19. B. C. Bunker and G.W. Arnold, "The Effect of Solution $\mathrm{pH}$ and Ion Concentrations on Leaching of Silicate Glass," Mater. Res. Soc. Symp. Proc. 15, 151-158 (1983).

20. I. Friedman and W. Long, "Hydration Rate of Obsidian," Science 191, 347-352 (1976).

21. K. E. Lapham, J. R. Holloway, and J. R. Delany, "Diffusion of $\mathrm{H}_{2} \mathrm{O}$ and $\mathrm{D}_{2} \mathrm{O}$ in Obsidian at Elevated Temperatures and Pressures," J. Non-Crystal. Solids $\underline{67}$, 179-191 (1984).

22. J. J. Mazer, C. M. Stevenson, W. L. Ebert, and J. K. Bates, "The Experimental Hydration of Obsidian as a Function of Relative Humidity and Temperature," Amer. Antiq. 56(3), 504-513 (1991).

23. J. J. Mazer, J. K. Bates, C. M. Stevenson, and C. R. Bradley, "Obsidians and Tektites: Natural Analogues for Water Diffusion in Nuclear Waste Glasses," Mater. Res. Soc. Symp. Proc. 257, 513-520 (1992).

24. J.-C. Petit, M. C. Magonthier, J.-C. Dran, and G. Della Mea, "Long-Term Dissolution Rate of Nuclear Waste Glasses in Confined Environments: Does a Residual Affinity Exist?," J. Mater. Sci. 25, 3048-3052 (1990).

25. G. N. Greaves, "EXAFS, Glass Structure and Diffusion," Philos. Mag. B $\underline{60}$ (6), 793-800 (1989).

26. M. D. Ingram, M. A. Mackenzie, W. Muller, and M. Torge, "Structural Granularity and Ionic Conduction Mechanism in Glass," Solid State Ionics 40-41, 671-675 (1990).

27. M. C. Magontheir, C. Brousse, J.-C. Petit, J.-C. Dran, G. Della Mea, and A. Paccagnella, "Hydration Rates of Natural and Nuclear Glasses Investigated by a Resonant Nuclear Reaction," Proc. of the Second Internat. Conf. on Natural Glasses, Prague, pp. 57-64 (1987).

28. M. A. Rana and R. W. Douglas, "The Reaction between Glass and Water. Part I. Experimental Methods and Observations," Phys. Chem. Glasses 22(6), 179-195 (1961).

29. M. A. Rana and R. W. Douglas, "The Reaction between Glass and Water. Part II. Discussion of the Results," Phys. Chem. Glasses 2(6), 196-205 (1961).

30. R. H. Doremus, "Interdiffusion of Hydrogen and Alkali Ions in a Glass Surface," J. Non-Cryst. Sol. 19, 137-144 (1975).

31. R. H. Doremus, "Diffusion in Glasses and Melts," J. Non-Cryst. Solids 25, 263-292 (1977). 
32. R. H. Doremus, "Interdiffusion of Alkali and Hydronium Ions in Glass: Partial Ionization," J. Non-Cryst. Solids $\underline{48}$, 431-436 (1982).

33. R. H. Doremus, "Reactions of Glasses with Aqueous and Nonaqueous Environments," Mater. Res. Soc. Symp. Proc. 125, 177-188 (1988).

34. K. H. Schnatter, R. H. Doremus, and W. A. Lanford, "Hydrogen Analysis of Soda-Lime Silicate Glass," J. Non-Cryst. Solids 102, 11-18 (1988).

35. I. S. T. Tsong, C. A. Houser, W. B. White, L. L. Power, and S. S. C. Tsong, "Glass Leaching Studies by Sputter-Induced Photon Spectroscopy (SIPS)," J. Non-Cryst. Solids $\underline{38}, 649-654$ (1980).

36. I. S. T. Tsong, C. A. Houser, W. B. White, A. L. Wintenberg, P. D. Miller, and C. D. Moak, "Evidence for Interdiffusion of Hydrogen and Alkali Ions in Leached Glasses," Appl. Phys. Lett. $\underline{39}(8), 669-670$ (1981).

37. W. A. Lanford, K. Davis, P. Lamarche, T. Laursen, and R. Groleau, "Hydration of SodaLime Glass," J. Non-Cryst. Sol. 33, 249-266 (1979).

38. I. Shapiro and H. G. Weiss, "Bound Water in Silica Gel," J. Phys. Chem. 57, 219-221 (1953).

39. C.-K. Wu, "Nature of Incorporated Water in Hydrated Silicate Glasses," J. Am. Ceram. Soc. $\underline{63}, 453-457$ (1980).

40. C. G. Armistead, A. J. Tyler, F. H. Hambleton, S. A. Mitchell, and J. A. Hockey, "The Surface Hydroxylation of Silica," J. Phys. Chem. 73, 3947-3952 (1969).

41. W. L. Ebert, R. F. Hoburg, and J. K. Bates, "The Sorption of Water on Obsidian and a Nuclear Waste Glass," Phys. Chem. Glasses 32(4), 133-137 (1991).

42. B. M. J. Smets and T. P. A. Lommen, "The Role of Molecular Water in the Leaching of Glass," Phys. Chem. Glasses 24(1), 35-36 (1983).

43. B. M. J. Smets, M. G. W. Tholen, and T. P .A. Lommen, "The Effects of Divalent Cations on the Leaching of Glass," J. Non-Cryst. Solids 65, 319-332 (1984).

44. F. M. Ernsberger, "Molecular Water in Glass," J. Amer. Ceram. Soc. $\underline{60}(1,2), 91-92$ (1977).

45. Y. Moriya and M. Nogami, "Hydration of Silicate Glass in Steam Atmosphere," J. NonCryst. Solids $\underline{38 \& 39}$, 667-672 (1980).

46. R. D. Aines, H. C. Weed, and J. K. Bates, "Hydrogen Speciation in Hydrated Layers on Nuclear Waste Glass," Mater. Res. Soc. Symp. Proc. 84 , 547-558 (1987).

47. L. R. Pederson, D. R. Baer, G. L. McVay, and M. H. Engelhard, "Reaction of Soda Lime Silicate Glass in Isotopically Labelled Water," J. Non-Cryst. Solids $\underline{86}, 369-380$ (1986).

48. L. R. Pederson, "Comparison of Sodium Leaching Rates from a $\mathrm{Na}_{2} \mathrm{O} \cdot 3 \mathrm{SiO}_{2}$ Glass in $\mathrm{H}_{2} \mathrm{O}$ and $\mathrm{D}_{2} \mathrm{O}$," Phys. Chem. Glasses 28(1), 17-21 (1987). 
49. L. R. Pederson, D. R. Baer, G. L. McVay, K. F. Ferris, and M. H. Engelhard, "Reaction of Silicate Glasses in Water Labeled with D and ${ }^{18} \mathrm{O}$," Phys. Chem. Glasses $\underline{31}$, 177-182 (1990).

50. J.-C. Dran, G. Della Mea, A. Paccagnella, J.-C. Petit, and L. Trotignon, "The Aqueous Dissolution of Alkali Silicate Glasses: Reappraisal of Mechanisms by $\mathrm{H}$ and $\mathrm{Na}$ Depth Profiling with High Energy Beams," Phys. Chem. Glasses 29(6), 249-255 (1988).

51. L. L. Hench, "Physical Chemistry of Glass Surfaces," J. Non-Cryst. Sol. $\underline{5}$, 343-369 (1977).

52. J.-C. Dran, J.-C. Petit, L. Trotignon, A. Paccagnella, and G. Della Mea, "Hydration Mechanisms of Silicate Glasses, Respective Role of Ion Exchange and Water Permeation," Mater. Res. Soc. Symp. Proc. 127, 25-32 (1989).

53. B. C. Bunker, D. R. Tallant, T. J. Headley, G. L. Turner, and R. J. Kirkpatrick, "The Structure of Leached Sodium Borosilicate Glass," Phys. Chem. Glasses 29(3), 106-120 (1988).

54. P. Aagaard and H. C. Helgeson, "Thermodynamics and Kinetic Constraints on Reaction Rates Among Minerals and Aqueous Solutions. I. Theoretical Considerations," Amer. J. Sci. 282, 237-285 (1982).

55. A. C. Lasaga and G. V. Gibbs, "Ab-Initio Quantum Mechanical Calculations of WaterRock Interactions: Adsorption and Hydrolysis Reactions," Amer. J. Sci. 290, 263-295 (1990).

56. P. Schindler and H. R. Kamber, "Die Aciditat von Silanolgruppen," Helvetica Chemica Acta $\underline{51}(7), 1781-1786(1968)$.

57. J. Schwartzentruber, W. Furst, and H. Renon, "Dissolution of Quartz into Dilute Alkaline Solutions at $90^{\circ} \mathrm{C}$ : A Kinetic Study," Geochim. Cosmochim. Acta 51, 1867-1874 (1987).

58. R. Keren, R. G. Gast, and B. Bar-Yosef, "pH Dependent Boron Adsorption by Na-Montmorillonite," Soil Sci. Soc. Amer. J. 45, 45-48 (1981).

59. R. E. Mesmer, C. F. Baes, Jr., and F. H. Sweeton, "Acidity Measurements at Elevated Temperatures: VI. Boric Acid Equilibria," Inorg. Chem. 11(3), 537-543 (1972).

60. C. C. Allen, D. L. Lane, R. G. Johnston, A .D. Marcy, and R. R. Adee, "Hydrothermal Studies of Simulated Defense Waste Glass Plus Basalt," Mater. Res. Soc. Symp. Proc. 44, 451-458 (1985).

61. A. B. Harker and J. F. Flintoff, "The Formation of Surface Layers and Reaction Products in the Leaching of Defense Borosilicate Nuclear Waste Glass," Nucl. Technol. 76, 263-275 (1987).

62. T. A. Abrajano, J. K. Bates, A. B. Woodland, J. P. Bradley, and W. L. Bourcier, "Secondary Phase Formation during Nuclear Waste-Glass Dissolution," Clays and Clay Miner. $\underline{38}, 537-548$ (1990).

63. T. A. Abrajano, J. K. Bates, and J. P. Bradley, "Analytical Electron Microscopy of Leached Nuclear Waste Glasses," Ceram. Trans. 2, 211-228 (1990). 
64. W. L. Ebert, J. K. Bates, C. R. Bradley, E. C. Buck, N. L. Dietz, and N. R. Brown, "The Long-Term Alteration of Borosilicate Waste Glasses," Ceram. Trans. 39, 333-340 (1993).

65. W. L. Ebert, J. K. Bates, E. C. Buck, and C. R. Bradley, "Accelerated Glass Reaction Under PCT Conditions," Mater. Res. Soc. Symp. Proc. 294, 569-576 (1993).

66. W. L. Ebert, J. K. Bates, and E. C. Buck, "The Reaction of SRL 202 Glass in J-13 and DIW," Mater. Res. Soc. Symp. Proc. 294, 137-144 (1993).

67. W. L. Ebert and J. K. Bates, "A Comparison of Glass Reaction at High and Low Surface Area to Volume," Nucl. Technol. 104(3), 372-384 (1993).

68. B. M. Scheetz, W. P. Freeborn, D. K. Smith, C. Anderson, M. Zolensky, and W. B. White, "The Role of Boron in Monitoring the Leaching of Borosilicate Glass Waste Forms," Mater. Res. Soc. Symp. Proc. 44, 129-134 (1985).

69. F. Delage and J. L. Dussossoy, "R7T7 Glass Initial Dissolution Rate Measurements Using a High-Temperature Soxhlet Devise," Mater. Res. Soc. Symp. Proc. 212, 41-47 (1991).

70. K. G. Knauss, W. L. Bourcier, K. D. McKeegan, C. I. Merzbacher, S. N. Nguyen, F. J. Ryerson, D. K. Smith, and H. C. Weed, "Dissolution Kinetics of a Simple Analogue Nuclear Waste Glass as a Function of $\mathrm{pH}$, Time, and Temperature," Mater. Res. Soc. Symp. Proc. 176, 371-381 (1990).

71. W. L. Bourcier, H. C. Weed, S. N. Nguyen, J. K. Nielsen, L. Morgan, L. Newton, and K. G. Knauss, "Solution Compositional Effects on the Dissolution Kinetics of Borosilicate Glass," Proceedings of the Seventh International Symposium on Water-Rock Interactions, 81-84 (1992).

72. L. R. Pederson, C. Q. Buckwalter, G. L. McVay, and B. L. Riddle, "Glass Surface Area to Solution Volume Ratio and its Implications to Accelerated Leach Testing," Mater. Res. Soc. Symp. Proc. 15 , 47-54 (1983).

73. L. R. Pederson, C. Q. Buckwalter, and G. L. McVay, "The Effects of Surface Area to Solution Volume on Waste Glass Leaching," Nucl. Technol. 62, 151-158 (1983).

74. C. M. Jantzen, N. E. Bibler, D. C. Beam, W. G. Ramsey, and B. J. Waters, Nuclear Waste Glass Product Consistency Test (PCT) Version 5.0(U), Westinghouse Savannah River Company Report WSRC-TR-90-539, Rev. 2 (1992).

75. D. E. Clark, L. Urwongse, and C. Maurer, "Application of Glass Corrosion Concepts to

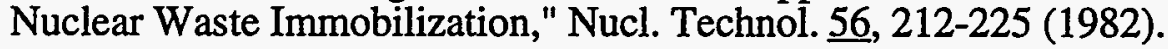

76. B. K. Zoitos and D. E. Clark, "Role of Surface Layers in the Leaching Behavior of Glass," Mater. Res. Soc. Symp. Proc. 125, 169-176 (1988).

77. L. L. Hench, A. Lodding, and L. Werme, "Nuclear Waste Glass Interfaces after One Year Burial in Stripa - Part 1: Glass/Glass," J. Nucl. Mater. 125, 273-279 (1984).

78. L. L. Hench, L. Werme, and A. Lodding, "Nuclear Waste Glass Interfaces after One Year Burial in Stripa. Part 3: Glass/Granite," J. Nucl. Mater. 126, 226-233 (1984). 
79. C. G. Namboodri, Jr., G. G. Wicks, A. R. Lodding, L. L. Hench, and R. G. Newton, "Surface Analysis of SRS Waste Glass Buried for up to Two Years in Limestone in the United Kingdom," Ceram. Trans. 23, 653-662 (1991).

80. A. Lodding, L. L. Hench, and L. Werme, "Nuclear Waste Glass Interfaces after One Year Burial in Stripa, Part 2: Glass/Bentonite," J. Nucl. Mater. 125, 280-286 (1984).

81. W. L. Ebert and J. K. Bates, "The Importance of Secondary Phases in Glass Corrosion," Mater. Res. Soc. Symp. Proc. 212, 89-97 (1991).

82. Standard Practice for Prediction of the Long-Term Behavior of Waste Package Materials Including Waste Forms Used in the Geologic Disposal of High-Level Nuclear Waste, ASTM Standard C1174-91, ASTM Philadelpha, PA (1991).

83. International Atomic Energy Agency, Chemical Durability and Related Properties of Solidified High-Level Waste Forms, Report No. 257 (1985).

84. Nuclear Waste Materials Handbook, DOE/TIC-11400 (1982).

85. D. M. Strachan, "Effect of Gamma Irradiation on Simulated Waste Glass Leaching and on the Leach Vessel," J. Am. Ceram. Soc. 66(9), C-158-160 (1983).

86. D. M. Strachan, B. O. Barnes, and R. P. Turcotte, "Standard Leach Tests for Nuclear Waste Materials," Sci. Basis for Nucl. Waste Mgmt. III, 347-354 (1981).

87. J. K. Bates, W. L. Ebert, J. P. Bradley, and W. L. Bourcier, "Mechanistic Interpretation of Glass Reaction: Input to Kinetic Model Development," Proc. of the Second Annual Internation High-Level Radioactive Waste Management Topical Meeting, American Nuclear Society, Las Vegas, NV, Vol. 1, 720-727 (1991).

88. V. M. Oversby and D. L. Phinney, "The Structure of Alteration Layers on Cast Glass Surfaces," Mater. Res. Soc. Symp. Proc. 257, 99-108 (1992).

89. V. M. Oversby and D. L. Phinney, "The Development of Surface Alteration Layers on SRL-165 Nuclear Waste Glass," J. Nucl. Mater. 190, 247-268 (1992).

90. H. M. Kingston, D. J. Cronin, and M. S. Epstein, "Investigation of a Precise Static Leach Test for the Testing of Simulated Nuclear Waste Materials," Nucl. Chem. Waste Mgmt. 5, 3-15 (1984).

91. J. W. Johnston and J. L. Daniel, Summary Repost for the Interlaboratory Round Robin on the MCC-1 Static Leach Test Method, Pacific Northwest Laboratory Report PNL-4249 (1982).

92. T. Advocat, J. L. Crovisier, E. Vernaz, G. Ehret, and H. Charpentier, "Hydrolysis of R7T7 Nuclear Waste Glass in Dilute Media: Mechanisms and Rate as a Function of pH," Mater. Res. Soc. Symp. Proc. 212, 57-64 (1991).

93. D. M. Strachan, "Results from Long-Term Use of the MCC-1 Static Leach Test Method," Nucl. Chem. Waste Mgmt. 4 , 177-188 (1983).

94. D. M. Strachan, L. R. Pederson, and R. O. Lokken, "Results from the Long-Term Interaction and Modeling of SRL-131 Glass with Aqueous Solutions," Mater. Res. Soc. Symp. Proc. 50, 195-202 (1985). 
95. W. L. Ebert, J. K. Bates, and T. J. Gerding, The Reaction of Glass During Gamma Irradiation in a Saturated Tuff Environment. Part 4: SRL 165, ATM-1c, and ATM-8 Glasses at $1 E 3 R / h$ and $0 R / H$, Argonne National Laboratory Report ANL-90/13 (1990).

96. T. A. Abrajano, J. K. Bates, T. J. Gerding, and W. L. Ebert, The Reaction of Glass during Gamma Irradiation in a Saturated Tuff Environment, Part 3: Long-Term Experiments at $1 \times 10^{4} \mathrm{rad} /$ hour, Argonne National Laboratory Report ANL-88/14 (1988).

97. J. K. Bates, W. L. Ebert, J. J. Mazer, J. P. Bradley, C. R. Bradley, and N. L. Dietz, "The Role of Surface Layers in Glass Leaching Performance," Mater. Res. Soc. Symp. Proc. $\underline{212}, 77-87$ (1991).

98. Test Method for Static Leaching of Monolithic Waste Forms for Disposal of Radioactive Waste, ASMT Standard C1220-92, ASTM Philadelphia, PA (1992).

99. W.T. Goldston and M.J. Plodinec, "The DWPF Strategy for Producing an Acceptable Product," Ceramic Trans. 23, 443-452 (1991).

100. M. J. Plodinec, "The DWPF Product, and its Qualification Program," Proc. of the Second Annual Internation High-Level Radioactive Waste Management Topical Meeting, American Nuclear Society, Las Vegas, NV, Vol. 1, 1124-1131 (1991).

101. C. M. Jantzen, N. E. Bibler, D. C. Beam, C. L. Crawford, and M. A. Pickett, Characterization of the Defense Waste Processing Facility (DWPF) Environmental Assessment (EA) Glass Standard Reference Material, Westinghouse Savannah River Company Report WSRC-TR-92-346, Rev. 1 (1993).

102. S. Xing and I. L. Pegg, "Effects of Container Material on PCT Leach Test Results for High-Level Nuclear Waste Glasses," Mater. Res. Soc. Symp. Proc. 333, 557-564 (1994).

103. Standard Test Methods for Determining Chemical Durability of Nuclear Waste Glasses: The Product Consistency Test (PCT), ASTM Standard C1285-94, ASTM Philadelpha, PA (1994).

104. J. K. Bates, L. J. Jardine, and M. J. Steindler, The Hydration Process of Nuclear Waste Glass: An Interim Report, Argonne National Laboratory Report ANL-82-11 (1982).

105. J. K. Bates and M. J. Steindler, "Alteration of Nuclear Waste Glass by Hydration," Mater. Res. Soc. Symp. Proc. 15, 83-90 (1983).

106. J. K. Bates, M. G. Seitz, and M. J. Steindler, "The Relevance of Vapor Phase Hydration Aging to Nuclear Waste Isolation," Nucl. Chem. Waste Mgmt. $\underline{5}, 63-73$ (1984).

107. T. A. Abrajano, Jr., J. K. Bates, and C.D. Byers, "Aqueous Corrosion of Natural and Nuclear Waste Glasses, I. Comparative Rates of Hydration in Liquid and Vapor Environments at Elevated Temperatures," J. Non-Cryst. Solids 4ㅜ, 251-257 (1986).

108. W. L. Ebert and J. K. Bates, "The Reaction of Synthetic Nuclear Waste Glass in Steam and Hydrothermal Solution," Mater. Res. Soc. Symp. Proc. 176, 339-346 (1990).

109. J. K. Bates, W. L. Ebert, and T. J. Gerding, "Vapor Hydration and Subsequent Leaching of Transuranic-Containing SRL and WV Glasses," Proc. of the Internat. High-Level Radioactive Waste Mgmt. Conf., American Nuclear Society, Las Vegas, NV, pg. 1095 (1990). 
110. W. L. Bourcier, "Overview of Chemical Modeling of Nuclear Waste Glass Dissolution," Mater. Res. Soc. Symp. Proc. 211, 3-18 (1991).

111. W. L. Ebert, J. K. Bates, and W. L. Bourcier, "The Hydration of Borosilicate Waste Glass in Liquid Water and Steam at $200^{\circ} \mathrm{C}$," Waste Mgmt. 11, 205-221 (1991).

112. J. K. Bates, W. L. Ebert, X. Feng, and W. L. Bourcier, "Issues Affecting the Prediction of Glass Reactivity in an Unsaturated Environment," J. Nucl. Mater. 190, 198-227 (1992).

113. D. J. Wronkiewicz, L. M. Wang, J. K. Bates, and B. S. Tani, "Effects of Radiation Exposure on Glass Alteration in a Steam Environment," Mater. Res. Soc. Symp. Proc. 294, 183-206 (1993).

114. D. J. Wronkiewicz, C. R. Bradley, J. K. Bates, and L. M. Wang, "Effects of Radiation Exposure on SRL 131 Composition Glass in a Steam Environment," Mater. Res. Soc. Symp. Proc. 333 , 259-267 (1994).

115. J. Hagymassy, S. Brunauer, and R. Sh. Mikhail, "Pore Strucutre Analysis by Water Vapor Adsorption. Part 1. T-Curves for Water Vapour," J. Coll. Interf. Sci. 29, 485-493 (1969).

116. P. Van Iseghem and B. Grambow, "The Long-Term Corrosion and Modeling of Two Simulated Belgian Reference High-Level Waste Glasses," Mater. Res. Soc. Symp. Proc. $\underline{112}, 631-639$ (1988).

117. I. Tovena, T. Advocat, D. Ghaleb, E. Vernaz, and F. Larche, "Thermodynamic and Structural Models Compared with the Initial Dissolution Rates of 'SON' Glass Samples," Mater. Res. Soc. Symp. Proc. 333, 595-602 (1994).

118. B. T. Kenna, "Analysis of Long-Term Soxhlet Tests," Nucl. Chem. Waste Mgmt. $\underline{3}$, 69-78 (1982).

119. T. Murakami and T. Banba, "The Leaching Behavior of a Glass Waste Form - Part I: The Characteristics of Surface Layers," Nucl. Technol. 67, 419-428 (1984).

120. H. C. Weed and D. D. Jackson, Design of a Variable-Flow-Rate, Single-Pass Leaching System, Lawrence Livermore Laboratory Report UCRL-52785 (1979).

121. D. G. Coles, Single-Pass Continuous-Flow Leach Test of PNL 76-68 Glass: Some Selected Bead Leach I Results, Lawrence Livermore Laboratory Report UCRL-85405 (1981).

122. D. G. Coles, "A Continuous-Flow Leach Testing Method for Various Nuclear Waste Forms," Nucl. Chem. Waste Mgmt. 2, 245-252 (1981).

123. Aa. Barkatt, P. B. Macedo, W. Sousanpour, Al. Barkatt, M. A. Boroomand, C. F. Fisher, J. J. Shirron, P. Szoke, and V. L. Rogers, "The Use of a Flow Test and a Flow Model in Evaluating the Durability of Various Nuclear Waste-Form Materials," Nucl. Chem. Waste Mgmt. $\underline{4}$, 153-169 (1983).

124. Aa. Barkatt, W. Sousanpour, Al. Barkatt, M. A. Boroomand, and P. B. Macedo, "Leach Behavior of SRL TDS-131 Defense Waste Glass in Water at High/Low Flow Rates," Mater. Res. Soc. Symp. Proc. 26, 643-653 (1984). 
125. E. D. Hespe, "Leach Testing of Immobilized Radioactive Waste Solids: A Proposal for a Standard Method," in Atomic Energy Review, Vol. 9, No. 1, International Atomic Energy Agency, Vienna, pp. 195-207 (1971).

126. M. Fuhrmann, P. F. Pietrzak, J. Heiser, E. M. Franz, and P. Colombo, Accelerated Leach Test for Diffusive Releases from Solidified Waste and a Computer Program to Model Diffusive, Fractional Leaching from Cylindrical Waste Forms, Brookhaven National Laboratory Report PNL-44881 (1990).

127. Aa. Barkatt, S. A. Olszowka, W.Sousanpour, T. Choudhury, Y. Guo, Al. Barkatt, and R. Adiga, "The Use of Partial-Replenishment Tests in Modeling the Leach Behavior of Glasses," Mater. Res. Soc. Symp. Proc. 212, 133-139 (1991).

128. A.E. Ogard and E.A. Bryant, "The Misused and Misleading IAEA Leach Test," Nucl. Chem. Waste Mgmt. $\underline{3}, 79-81$ (1982).

129. Aa. Barkatt, S. A. Olszowka, W. Sousanpour, M. A. Adel-Hadadi, R. Adiga, A. Barkatt, G. S. Marbury, and S. Li, "Leach Rate Excursions in Borosilicate Glasses: Effects of Glass and Leachant Composition," Mater. Res. Soc. Symp. Proc. 212, 65-76 (1991).

130. J. C. Sang, Aa. Barkatt, I. G. Talmy, and M. K. Norr, "Effects of Cracking on the Kinetics of the Interaction of Silicate Glasses with Water," Mater. Res. Soc. Symp. Proc. 294, 583-589 (1993).

131. V. M. Oversby, Leach Testing of Waste Forms: Interrelationship of ISO-and MCC-Type Tests, Lawrence Livermore National Laboratory Report UCRL-87621 (1982).

132. J. W. Shade and D. M. Strachan, "Effect of High Surface Area to Solution Volume Ratios on Waste Glass Leaching," Amer. Ceram. Bull. 65(12), 1568-1573 (1986).

133. S. O. Bates, G. F. Piepel, and J. W. Johnston, Leach Testing of Simulated Hanford Waste Vitrification Plant Reference Glass HW-39, Pacific Northwest Laboratory Report PNL-6884 (1989).

134. G. L. Pine and C.M. Jantzen, Implications of One-Year Basalt Weathering/Reactivity Study for a Basalt Repository Environment, Savannah River Laboratory Report DP-1742 (1987).

135. D. E. Clark, C. G. Pantano, Jr., and L. L. Hench, Corrosion of Glass, Magazines for Industry, Inc., New York (1979).

136. Aa. Barkatt, A. Barkatt, P. E. Pehrsson, P. Szoke, and P. B. Macedo, "Static and Dynamic Tests for the Chemical Durability of Nuclear Waste Glass," Nucl. Chem. Waste Mgmt. 2, 151-164 (1981).

137. C. Q. Buckwalter, L. R. Pederson, and G. L. McVay, "The Effects of Surface Area to Solution Volume Ratio and Surface Roughness on Glass Leaching," J. Non-Cryst. Solids 49, 397-412 (1982).

138. J. L. Dussossoy, C. Dubois, E. Vernaz, and A. Chambaudet, "Effect of Surface Finish on Nuclear Glass Dissolution Rate," Mater. Res. Soc. Symp. Proc. 257, 109-115 (1992).

139. J. K. Bates, M. J. Steindler, and P. L McDaniel, "Hydration of Stressed Nuclear Waste Glass," Mater. Lett. 2 , 296-300 (1984). 
140. J. M. Perez, Jr., and J. H. Westsik, Jr., "Effects of Cracks on Glass Leaching," Nucl. Chem. Waste Mgmt. 2, 165-168 (1981).

141. L. G. M. Baas-Becking, I. R. Kaplan, and D. Moore, "Limits of the Natural Environment in Terms of pH and Oxidation-Reduction Potentials," J. Geol. 68, 243-284 (1960).

142. M. J. Apted and R. Adiga, "The Effect of Groundwater Flow on the Release Behavior of Borosilicate Glass," Mater. Res. Soc. Symp. Proc. 44, 163-170 (1985).

143. W. L. Bourcier, D. W. Peiffer, K. G. Knauss, K. D. McKeegan, and D. K. Smith, "A Kinetic Model for Borosilicate Glass Dissolution Based on the Dissolution Affinity of a Surface Alteration Layer," Mater. Res. Soc. Symp. Proc. 176, 209-216 (1990).

144. G. Malow, "The Mechanisms for Hydrothermal Leaching of Nuclear Waste Glasses: Properties and Evaluation of Surface Layers," Mater. Res. Soc. Symp. Proc. 11, 25-33 (1982).

145. R. M. Wallace and G. G. Wicks, "Leaching Chemistry of Defence Borosilicate Glass," Mater. Res. Soc. Symp. Proc. 15, 23-28 (1983).

146. T. Banba and T. Murakami, "The Leaching Behavior of a Glass Waste Form - Part II: The Leaching Mechanisms," Nucl. Technol. 70, 243-248 (1985).

147. T. Banba, T. Murakami, and H. Kimura, "Moving Boundary Model for Leaching of Nuclear Waste Glass," Mater. Res. Soc. Symp. Proc. 44, 113-120 (1985).

148. T. Banba, "Leaching Model of Nuclear Waste Glass," J. Nucl. Sci. Technol. 233(12), 1075-1082 (1986).

149. A. J. Machiels and C. Pescatore, "The Influence of Surface Processes in Waste Form Leaching," Scientific Basis for Nuclear Waste Management III, 371-378 (1981).

150. K. B. Harvey and C. D. Litke, "Model for Leaching Behavior of Aluminosilicate Glasses Developed as Matrices for Immobilizing High-Level Wastes," J. Am. Ceram. Soc. 67(8), 553-556 (1984).

151. B. Grambow, Ein Physikalish-Chemisches Modell fur den Mechanismus der Glaskorrosion Unter Besonderer Beruecksichtigung Simulierter Radioaktiver Abfallglaser, Doctoral Thesis, Freien Universitat Berlin, Berlin, Federal Republic of Germany (1984).

152. B. Grambow and D. M. Strachan, "Leach Testing of Waste Glasses Under Near Saturation Conditions," Mater. Res. Soc. Symp. Proc. 26, 623-634 (1984).

153. B. Grambow, "A General Rate Equation for Nuclear Waste Glass Corrosion," Mater. Res. Soc. Symp. Proc. 44, 15-24 (1985).

154. B. Grambow, Nuclear Waste Glass Dissolution: Mechanism, Model, and Application, JSS Report 87-02 (1987).

155. L. A. Chick and L. R. Pederson, "The Relationship Between Reaction Layer Thickness and Leach Rate for Nuclear Waste Glasses," Mater. Res. Soc. Symp. Proc. 26, 635-642 (1984). 
156. R. Conradt and H. Roggendorf, Determination of the Corrosion Mechanisms of High Level Waste Containing Glass, Fraunhofer-Institut Fur Silicatforschung Report DE88 754321 (1985).

157. R. Conradt, H. Roggendorf, and H. Scholze, "A Contribution to the Modeling of the Corrosion Process for HLW Glass," Mater. Res. Soc. Symp. Proc. 44, 155-162 (1985).

158. S. Fillet, J. Nogues, E. Vernaz, and N. Jacquet-Francillon, "Leaching of Actinides from the French LWR Glass," Mater. Res. Soc. Symp. Proc. 50, 211-218 (1985).

159. X. Feng, I. L. Pegg, Y. Guo, Aa. Barkatt, and P. B. Macedo, "Effects of Surface Area-toSolution Volume Ratio on Chemical Durability of Nuclear Waste Glasses," Mater. Res. Soc. Symp. Proc. 176, 383-392 (1990).

160. K. Lemmens and P. Van Iseghem, "The Long-Term Dissolution Behavior of the Pamela Borosilicate Glass SM 527 Application of SA/V as Acceleration Parameter," Mater. Res. Soc. Symp. Proc. 257, 49-56 (1992).

161. B. Grambow, W. Lutze, and R. Muller, "Empirical Dissolution Rate Law for the Glass R7T7 Contacting Halite and Silica Saturated Brines," Mater. Res. Soc. Symp. Proc. 257, 143-150 (1992).

162. Aa. Barkatt, J. C. Sang, S. B. Xing, Y. Guo, I. L. Pegg, and Al. Barkatt, "Laboratory Testing of the Corrosion of Waste Glasses in Aqueous Environments - Effects of Experimental Parameters," Mater. Res. Soc. Symp. Proc. 333, 123-132 (1994).

163. J. C. Sang, Y. Guo, M. A. Adel-Hadadi, G. S. Marbury, and Aa. Barkatt, "Dissolution Mechanism of Soda-Lime Silicate Glass and of PNL 76-68 in the Presence of Dissolved Mg," Mater. Res. Soc. Symp. Proc. 333, 519-524 (1994).

164. L. O. Werme, I. K. Bjorner, G. Bart, H. U. Zwicky, B. Grambow, W. Lutze, R. C. Ewing, and C. Mazrabi, "Chemical Corrosion of Highly Radioactive Borosilicate Nuclear Waste Glass Under Simulated Repository Conditions," J. Mater. Res. $\underline{5}, 1130-1146$ (1990).

165. B. Grambow and D. M. Strachan, A Comparison of the Performance of Nuclear Waste Glass by Modeling, Pacific Northwest Laboratory Report PNL-6698 (1988).

166. T. Advocat, J. L. Crovisier, B. Fritz, and E. Vernaz, "Thermochemical Model of Borosilicate Glass Dissolution: Contextual Affinity," Mater. Res. Soc. Symp. Proc. 176, 241-248 (1990).

167. E. Vernaz, T. Advocat, and J. L. Dussossoy, "Effects of the SA/V Ratio on the LongTerm Corrosion Kinetics of R7T7 Glass," Ceram. Trans. 2, 175-185 (1990).

168. E. Freude, B. Grambow, W. Lutze, H. Rabe, and R. C. Ewing, "Long-Term Release from High Level Waste Glass - Part IV: The Effect of Leaching Mechanism," Mater. Res. Soc. Symp. Proc. 44, 99-105 (1985).

169. L. L. Hench and D. E. Clark, Surface Properties and Performance Prediction of Alternative Waste Forms, Final Report, U.S. Nuclear Regulatory Commission Report NUREG/CR-3472 (1986).

170. T. M. El-Shamy, J. Lewins, and R. W. Douglas, "The Dependence on the $\mathrm{pH}$ of the Decomposition of Glass by Aqueous Solutions," Glass Technol. 13(3), 81-87 (1972). 
171. R. W. Douglas and T. M. El-Shamy, "Reactions of Glasses with Aqueous Solutions," J. Am. Ceram. Soc. 50(1), 1-8 (1967).

172. T. M. El-Shamy, S. E. Morsi, H. D. Taki-Eldin, and A. A. Ahmed, "Chemical Durability of $\mathrm{Na}_{2} \mathrm{O}-\mathrm{CaO}-\mathrm{SiO}_{2}$ Glasses in Acidic Solutions," J. Non-Crystal. Solids $\underline{19}, 241-250$ (1975).

173. T. M. El-Shamy, S. E. Morsi, and S. A. Melibari, "Chemical Durability of $\mathrm{Na}_{2} \mathrm{O}-\mathrm{Al}_{2} \mathrm{O}_{3}$ $\mathrm{SiO}_{2}$ Glasses in Relation to their Membrane Potentials," J. Non-Crystal. Solids 19 , 201-211 (1975).

174. A. Blum and A. Lasaga, "Role of Surface Speciation in the Low-Temperature Dissolution of Minerals," Nature $\underline{31}$, 431-433 (1988).

175. P. V. Brady and J. V. Walther, "Controls on Silicate Dissolution Rates in Neutral and Basic pH Solutions at $25^{\circ} \mathrm{C}$," Geochim. Cosmochim. Acta $\underline{53}, 2823-2830$ (1989).

176. W. L. Bourcier, W. L. Ebert, and X. Feng, "Modeling Surface Area to Volume Effects on Borosilicate Glass Dissolution," Mater. Res. Soc. Symp. Proc. 294, 577-582 (1993).

177. D. M. Strachan, W. L. Bourcier, and B. P. McGrail, "Towards a Consistent Model for Glass Dissolution," Radioact. Waste Mgmt. and Environ. Res. 19, 129-145 (1994).

178. Aa. Barkatt, E. E. Saad, R. B. Adiga, W. Sousanpour, Al. Barkatt, X. Feng, J. A. O'Keefe, and S. Alterescu, "Interactions of Silicate Glasses with Aqueous Environments under Conditions of Prolonged Contact and Flow," Mater. Res. Soc. Symp. Proc. 125 , 129-142 (1988).

179. B. Grambow and R. Muller, "Chemistry of Glass Corrosion in High Saline Brines," Mater. Res. Soc. Symp. Proc. 176, 229-240 (1990).

180. E. Curti, Modeling the Dissolution of Borosilicate Glasses for Radioactive Waste Disposal with the PHREEQE/GLASSOL Code: Theory and Practice, Paul Scherrer Inst., PSI-BERICHT Report Nr. 86 (1991).

181. B. Grambow, H. P. Hermansson, I. K. Bjorner, H. Christensen, and L. Werme, "Reaction of Nuclear Waste Glass with Slowly Flowing Solutions," Adv. Ceram. 20, (1986).

182. B. Grambow, H. U. Zwichy, G. Bart, I. K. Bjorner, and L. O. Werme, "Modeling of the Effect of Iron Corrosion Products on Nuclear Waste Glass Performance," Mater. Res. Soc. Symp. Proc. $\underline{84}$, 471-481 (1987).

183. E. Y. Vernaz and J. L. Dussossoy, "Current State of Knowledge of Nuclear Waste Glass Corrosion Mechanisms: The Case of R7T7 Glass," Appl. Geochem. Suppl. Issue 1, 13-22 (1992).

184. L. Michaux, E. Mouche, J.-C. Petit, and B. Fritz, "Geochemical Modelling of the LongTerm Dissolution Behaviour of the French Nuclear Glass R7T7," Appl. Geochem. Suppl. Issue 1, 41-52 (1992). 
185. B. P. McGrail, M. J. Apted, D. W. Engel, and A. M. Liebetrau, "A Coupled ChemicalMass Transport Submodel for Predicting Radionuclide Release from and Engineered Barrier System Containing High-Level Waste Glass," Mater. Res. Soc. Symp. 176, 785-792 (1990).

186. B. P. McGrail and D. W. Engel, "Coupled Process Modeling and Waste-Package Performance," Mater. Res. Soc. Symp. Proc. 294, 215-223 (1993).

187. P. Van Iseghem, T. Amaya, Y. Suzuki, and $\mathrm{H}$. Yamamoto, "The Role of $\mathrm{Al}_{2} \mathrm{O}_{3}$ in the Long-Term Corrosion Stability of Nuclear Waste Glasses," J. Nucl. Mater. 190, 269-276 (1992).

188. Y. Inagaki, H. Furuya, K. Idemitsu, and S. Yonezawa, "Corrosion Behavior of a Powdered Simulated Nuclear Waste Glass: A Corrosion Model Including Diffusion Process," J. Nucl. Mater. 208, 27-34 (1994).

189. N. Godon, Z. Andriambololona, and E. Vernaz, "Effect of a Siliceous Additive in a Clay Engineered Barrier on Aqueous Corrosion of R7T7 Nuclear Waste Glass," Mater. Res. Soc. Symp. Proc. 257, 135-141 (1992).

190. P. Van Iseghem and R.DeBatist, "The Interaction Between Nuclear Waste Glass and Clay," Adv. Ceram. 20, 627-637 (1986).

191. P. Van Iseghem, K. Berghman, and W. Timmermans, "The Interaction Between Nuclear Waste Glasses and Clay-II," Mater. Res. Soc. Symp. Proc. 176, 291-298 (1990).

192. E. Curti and P. A. Smith, "Enhancement of Borosilicate Glass Dissolution by Silica Sorption and Diffusion in Compacted Bentonite," Mater. Res. Soc. Symp. Proc. 212 , 31-39 (1991).

193. G. L. McVay and C. Q. Buckwalter, "Effect of Iron on Waste-Glass Leaching," J. Am. Ceram. Soc. $\underline{66}(3), 170-174$ (1983).

194. G. Bart, H. U. Zwicky, E. T. Aerne, Th. Graber, D. Z'Berg, and M. Tokiwai, "Borosilicate Glass Corrosion in the Preesence of Steel Corrosion Products," Mater. Res. Soc. Symp. Proc. $\underline{84}$, 459-470 (1987).

195. F. Bazan and J. Rego, Parametric Testing of a DWPF Glass, Lawrence Livermore National Laboratory Report UCRL-53606 (1985).

196. G. T. Chandler, G. G. Wicks, and R. M. Wallace, "Effects of SA/V and Saturation on the Chemical Durability of SRP Waste Glass," Adv. Ceram. 20, 455-463 (1986).

197. H. Kamizono, T. Sagawa, and S. Tashiro, "Continuous-Flow Leach Tests of Simulated High-Level Waste Glass in Synthetic Basalt Groundwater," Waste Mgmt. 9, 189-194 (1989).

198. L. L. Hench and D. E. Clark, "Physical Chemistry of Glass Surfaces," J. Non-Cryst. Sol. 28, 83-105 (1978).

199. E. C. Ethridge, D. E. Clark, and L. L. Hench, "Effects of Glass Surface Area to Solution Volume Ratio on Glass Corrosion," Phys. Chem. Glasses 20(2), 35-40 (1979). 
200. X. Feng, J. K. Bates, E. C. Buck, C. R. Bradley, and M. Gong, "Long-Term Comparison of Dissolution Behavior Between Fully Radioactive and Simulated Nuclear Waste Glasses," Nucl. Technol. 104(2), 193-206 (1993).

201. X. Feng, J. K. Bates, C. R. Bradley, and E. C. Buck, "Does Fully Radioactive Glass Behave Differently Than Simulated Waste Glass?" Mater. Res. Soc. Symp. Proc. 294, 207-214 (1993).

202. C. A. Houser and C. J. Pantano, "Early Stages of Film Formation during the Leaching of Radioactive Waste Glasses," Mater. Res. Soc. Symp. Proc. 44, 205-212 (1985).

203. J. J. Mazer, J. K. Bates, B. M. Biwer, and C. R. Bradley, "AEM Analysis of SRL 131 Glass Altered as a Function of SA/V," Mater. Res. Soc. Symp. Proc. 257, 73-81 (1992).

204. N. Godon and E. Vernaz, "R7T7 Nuclear Waste Glass Behavior in Moist Clay: Role of the Clay Mass/Glass Surface Area Ratio," Mater. Res. Soc. Symp. Proc. 176, 319-326 (1990).

205. B. Grambow and W. Lutze, "Performance Assessment of Glass as a Long-Term Barrier to the Release of Radionuclides into the Environment," Mater. Res. Soc. Symp. Proc. $112,713-724$ (1988).

206. R. B. Adiga, E. P. Akomer, and D. E. Clark, "Effects of Flow Parameters on the Leaching of Nuclear Waste Glass," Mater. Res. Soc. Symp. Proc. 44, 45-54 (1985).

207. P. B. Macedo, Aa. Barkatt, and J. H. Simmons, "A Flow Model for the Kinetics of Dissolution of Nuclear Waste Glasses," Nucl. Chem. Waste Mgmt. 3 , 13-21 (1982).

208. P. B. Macedo, Aa. Barkatt, and J. H. Simmons, "A Flow Model for the Kinetics of Dissolution of Nuclear Waste Forms; A Comparison of Borosilicate Glass, Synroc, and High-Silica Glass," Mater. Res. Soc. Symp. Proc. 11, 57-69 (1982)

209. L. R. Bunnell, G. D. Maupin, and K. H. Oma, "High-Temperature Glasses for Nuclear Waste Isolation," Adv. Ceram. 20, 167-173 (1986).

210. D. M. Strachan, B. P. McGrail, M. J. Apted, D. W. Engle, and P. W. Eslinger, Preliminary Assessment of the Controlled Release of Radionuclides from Waste Packages Containin gBorosilicate Waste Glass, Pacific National Laboratory Report PNL-7591 (1990).

211. D. M. Strachan, "Leach Testing and Evaluation of WV-205 Glass from a Repository Perspective," Appendix, in Laboratory Work in Support of West Valley Glass Development, L. R. Bunnell, ed., Pacific Northwest Laboratory Report PNL-6539 (1988).

212. B. Grambow, What Do We Know About Nuclear Waste Glass Performance in the Repository Near Field?, SKB Technical Report 91-59 (1991).

213. F. Delage, D. Ghaleb, J. L. Dussossoy, O. Chevalier, and E. Vernaz, "A Mechanistic Model for Understanding Nuclear Waste Glass Dissolution," J. Nucl. Mater. 190, 191-197 (1992).

214. H. U. Zwicky, B. Grambow, C. Magrabi, E. T. Aerne, R. Bradley, B. Barnes, Th. Graber, M. Mohos, and L. O. Werme, "Corrosion Behaviour of British Magnox Waste Glass in Pure Water," Mater. Res. Soc. Symp. Proc. 127, 129-137 (1989). 
215. B. P. McGrail and S. O. Bates, Aqueous Dissolution of Laboratory and Field Samples from the In-Situ Vitrification Process, Pacific Northwest Laboratory Report SA-19786 (1991).

216. M. Antonini, A. E. Merlini, and R. F. Thornley, "EXAFS Structures of Technetrium in Glasses Prepared under Different Redox Conditions," J. Non-Cryst. Sol. 71, 219-225 (1985).

217. J. I. Kim, "Chemical Behaviour of Transuranic Elements in Natural Aquatic Systems," in Handbook on the Physics and Chemistry of the Actinides, A. J. Freeman and C. Keller, eds., Elsevier Science Publishers B.V., pp. 413-455 (1986)

218. H. Nitsche, Temperature Effects on the Solubility and Speciation of Selected Actinides, Lawrence Berkeley Laboratory Report LBL-20387 (1985).

219. H. Nitsche and N. M. Edelstein, "Solubilities and Speciation of Selected Transuranium Ions. A Comparison of a Non-Complexing Solution with a Groundwater from the Nevada Tuff Site," Radiochim. Acta 39, 23-33 (1985).

220. R. J. Lemire and F. Garisto, "The Effect of Ionic Strength, Groundwater Composition and Temperature on Calculated Radionuclide Solubilities," Radiochim. Acta $\underline{58 / 59}$, 37-44 (1992).

221. H. Nitsche, A. Muller, E. M. Standifer, R. S. Deinhammer, K. Becraft, T. Prussin, and R. C. Gatti, "Dependence of Actinide Solubility and Speciation on Carbonate Concentration and Ionic Strength in Groundwater," Radiochim. Acta 58/59, 27-32 (1992).

222. J. Fuger, "Thermodynamic Properties of Actinide Aqueous Species Relevant to Geochemical Problems," Radiochim. Acta 58/59, 81-91 (1992).

223. B. Grambow, "Remaining Uncertainties in Predicting Long-Term Performance of Nuclear Waste Glass from Experiments," Mater. Res. Soc. Symp. Proc. $\underline{333}$, 167-180 (1994).

224. R. J. Lemire, G. D. Boyer, and A. B. Campbell, "The Solubilities of Sodium and Potassium Dioxoneptunium(V) Carbonate Hydrates at 30,50 , and $75^{\circ} \mathrm{C}$," Radiochim. Acta 61, 57-63 (1993).

225. M. Sato, "Oxidation of Sulfide Ore Bodies, 1. Geochemical Environments in Terms of Eh and pH," Econ. Geol. 55, 928 (1960).

226. J. Paquette and R. J. Lemire, "A Description of the Chemistry of Aqueous Solutions of Uranium and Plutonium to $200^{\circ} \mathrm{C}$ Using Potential-pH Diagrams," Nucl. Sci. Eng. 79 , 26-48 (1981).

227. W. Lutze and B. Grambow, "The Effect of Glass Corrosion on Near Field Chemistry," Radiochim. Acta 58/59, 3-7 (1992).

228. International Atomic Energy Agency, J. Fuger, I. L Khodakovsky, E. I. Sergeyeva, V. A. Medvedev, and J. D. Navratil, The Chemical Thermodymics of Actinide Elements and Compounds. Part 12. The Actinide Aqueous Inorganic Complexes, IAEA, Vienna (1991). 
229. A. C. Buechele, X. Feng, H. Gu, and I. L. Pegg, "Alteration of Microstructure of West Valley Glass by Heat Treatment," Mater. Res. Soc. Symp. Proc. 176, 393-402 (1990).

230. A. C. Buechele, X. Feng, H. Gu, I. S. Muller, W. Wagner, and I. Pegg, "Effects of Composition Variations on Microstructure and Chemical Durability of West Valley Reference Glass," Mater. Res. Soc. Symp. Proc. 212, 141-152 (1991).

231. D. Rai, A. R. Felmy, and J. L. Ryan, "Uranium(IV) Hydrolysis Constants and Solubility Product of $\mathrm{UO}_{2} \times \mathrm{xH}_{2} \mathrm{O}(\mathrm{am})$," Inorg. Chem. 29, 260-264 (1990).

232. F. Weigel, "The Carbonates, Phosphates and Arsenates of the Hexavalent and Pentavalent Actinides," in Handbook on the Physics and Chemistry of the Actinides, A. J. Freeman and C. Keller, eds., Elsevier Science Publishers B.V., pp. 243-288 (1985).

233. T. Kimura, J. Serrano, S. Nakayama, K. Takahashi, and H. Takeishi, "Speciation of Uranium in Aqueous Solutions and in Precipitates by Photoacoustic Spectroscopy," Radiochim. Acta 58/59, 173-178 (1992).

234. L. Maya, "Hydrolysis and Carbonate Complexation of Dioxouranium(VI) in the NeutralpH Range at $25^{\circ} \mathrm{C}$," Inorg. Chem. 21, 2895-2898 (1982).

235. Nuclear Energy Agency Organisation for Economic Co-Operation and Development, "The Geochemistry of Actinides," Chapter 3 in Geological Disposal of Radioactive Waste, Geochemical Processes, pp. 49-68 (1982).

236. Ch. Lierse, W. Treiber, and J. I. Kim, "Hydrolysis Reactions of Neptunium(V)," Radiochim. Acta $\underline{38}$, 27-28 (1985).

237. M. I. Pratopo, H. Moriyama, and K. Higashi, "Carbonate Complexation of Neptunium(IV) and Analogous Complexation of Ground-Water Uranium," Radiochim. Acta 51, 27-31 (1990).

238. M. I. Pratopo, T. Yamaguchi, H. Moriyama, and K. Higashi, "Adsorption of Np(IV) on Quartz in Carbonate Solutions," Radiochim. Acta 55, 209-213 (1991).

239. G. Bidoglio, G. Tanet, and A. Chatt, "Studies on Neptunium(V) Carbonate Complex under Geologic Repository Conditions," Radiochim. Acta $\underline{38}$, 21-26 (1985).

240. J. I. Kim and B. Kanellakopulos, "Solubility Products of Plutonium(IV) Oxide and Hydroxide," Radiochim. Acta 48, 145-150 (1989).

241. S. Stadler and J. I. Kim, "Hydrolysis Reactions of Am(III) and Am(V)," Radiochim. Acta. $44 / 45,39-44$ (1988).

242. A. R. Felmy, D. Rai, and R. W. Fulton, "The Solubility of $\mathrm{AmOHCO}_{3}(\mathrm{c})$ and the Aqueous Thermodynamics of the System $\mathrm{Na}^{+}-\mathrm{Am}^{3+}-\mathrm{HCO}_{3}{ }^{2-}-\mathrm{OH}^{-}-\mathrm{H}_{2} \mathrm{O}$," Radiochim. Acta 50, 193-204 (1990).

243. K. H. Lieser and Ch. Bauscher, "Technetium in the Hydrosphere and in the Geosphere. Part I," Radiochim Acta. 42, 205-213 (1987).

244. K. H. Lieser and Ch. Bauscher, "Technetium in the Hydrosphere and in the Geosphere. Part II," Radiochim. Acta 44/45, 125-128 (1988). 
245. T. T. Vandergraaf, K. V. Ticknor, and I. M. George, "Reactions between Technetium in Solution and Iron-Containing Minerals under Oxic and Anoxic Conditions," in Geochemical Behavior of Disposed Radioactive Waste, ACS Symp. Ser. 246, G. S. Barney, J. D. Navratil, and W. W. Schulz, eds., pp. 25-43 (1984).

246. K. H. Lieser, Ch. Bauscher, and T. Nakashima, "Dissolution of $\mathrm{TcO}_{2}$ in Aqueous Solutions under Various Conditions," Radiochim. Acta 42, 191-200 (1987).

247. D. J. Bradley, C. O. Harvey, and R. P. Turcotte, Leaching of Actinides and Technetium from Simulated High-Level Waste Glass, Pacific Northwest Laboratory Report PNL-3152 (1979).

248. S. Fillet, J. Nogues, E. Vernaz, and N. Jacquet-Francillon, "Leaching of Actinides from the French LWR Glass," Mater. Res. Soc. Symp. Proc. 50, 211-218 (1985).

249. E. Y. Vernaz and N. Godon, "Leaching of Actinides from Nuclear Waste Glass: French Experience," Mater. Res. Soc. Symp. Proc. 257, 37-48 (1992).

250. F. Bazan, J. Rego, and R. D. Ewing, "Leaching of Actinide-Doped Nuclear Waste Glass in a Tuff-Dominated System," Mater. Res. Soc. Symp. Proc. $\underline{84}$, 447-458 (1987).

251. N. E. Bibler, "Leaching Fully Radioactive SRP Nuclear Waste Glass in Tuff Groundwater in Stainless Steel Vessels," Adv. Ceram. 20, 619-626 (1986); Savannah River Laboratory Report DP-MS-85-141 (1986).

252. I.-K. Bjorner, H. Christensen, H. P. Hermansson, M. Tsukamoto, and L. Werme, "Corrosion of Radioactive, Crushed Waste Glass," Mater. Res. Soc. Symp. Proc. 127, 113-120 (1988).

253. K. Miyahara, T. Ashida, Y. Yusa, N. Sasaki, and N. Tsunoda, "Static Leaching of Actinides and Fission Products from Fully Radioactive Waste Glass of HLLW Generated in Tokai Reprocessing Plant," Mater. Res. Soc. Symp. Proc. 127, 121-128 (1988).

254. A. Manara, F. Lanza, G. Ceccone, G. Della Mea, and G. Salvagno, "Application of XPS and Nuclear Technique to the Study of the Gel Layers Formed under Different Redox Conditions on Leached Glasses," Mater. Res. Soc. Symp. Proc. 44, 63-71 (1985).

255. A. Manara, F. Lanza, G. Ceccone, and T. Visani, Influence of Bicarbonate Ions and Redox Conditions on the Surface Composition of a Leached Borosilicate Glass, Commission of the European Communities Report EUR 12022 EN (1989).

256. R. D. Peters and H. Diamond, Actinide Leaching fron Waste Glass: Air-Equilibrated Versus Deaerated Conditions, Pacific Northwest Laboratory Report PNL-3971 (1981).

257. G. Bidoglio, P. Offermann, A. DePlano, and G. P. Lazzari, "Influence of Groundwater Composition on Glass Leaching and Actinide Speciation," Mater. Res. Soc. Symp. Proc. $112,621-630(1988)$.

258. A. R. Hall, A. Hough, and J. A. C. Marples, "Leaching of Vitrified High-Level Radioactive Waste," Mater. Res. Soc. Symp. Proc. 11, 83-92 (1982). 
259. L. Trotignon, J.-C. Petit, G. Della Mea, and J.-C. Dran, "The Compared Aqueous Corrosion of Four Simple Borosilicate Glasses: Influence of $\mathrm{Al}, \mathrm{Ca}$, and $\mathrm{Fe}$ on the Formation and Nature of Secondary Phases," J. Nucl. Mater. 190, 228-246 (1992).

260. N. E. Bibler, G. G. Wicks, and V. M. Oversby, "Leaching Savannah River Plant Nuclear Waste Glass in a Saturated Tuff Environment," Mater. Res. Soc. Symp. Proc. 44, 247-256 (1985).

261. N. E. Bibler and J. K. Bates, "Product Consistency Leach Tests of Savannah River Site Radioactive Waste Glasses," Mater. Res. Soc. Symp. Proc. 176, 327-338 (1990).

262. J. K. Bates, J. P. Bradley, A. Teetsov, C. R. Bradley, and M. Buchholtz ten Brink, "Colloid Formation During Waste Form Reaction: Implications for Nuclear Waste Disposal," Science 256, 649-651 (1992).

263. E. C. Buck, J. K. Bates, J. C. Cunnane, W. L. Ebert, X. Feng, and D. J. Wronkiewicz, "Analytical Electron Microscopy Study of Colloids from Nuclear Waste Glass Reaction," Mater. Res. Soc. Symp. Proc. 294, 199-206 (1993).

264. J. C. Cunnane and J. K. Bates, "Identification of Colloids in Nuclear Waste Glass Reaction," Ceram. Trans. 233, 65-72 (1991).

265. A. Rother, W. Lutze, and P. Schubert-Bischoff, "Characterization of Lanthanoid Phases Formed upon Glass Dissolution in Salt Solutions," Mater. Res. Soc. Symp. Proc. 257, 57-64 (1992).

266. J. W. Shade, L. L. Ames, and J. E. McGarrah, "Actinide and Technetium Sorption on Iron-Silicate and Dispersed Clay Colloids," in Geochemical Behavior of Disposed Radioactive Waste, ACS Symp. Ser. 246, G. S. Barney, J. D. Navratil, and W. W. Schulz, eds., pp. $67-77$ (1984).

267. N. Godon, E. Vernaz, J. H. Thomassin, and J. C. Touray, "Effects of Environmental Materials on Aqueous Corrosion of R7T7 Glass," Mater. Res. Soc. Symp. Proc. 127, 97-104 (1989).

268. K. Lemmens, P. Van Iseghem, and L. Wang, "The Leaching of Pu, Am, Np and Tc from High-Level Waste Glasses in Clay Media," Mater. Res. Soc. Symp. Proc. 294, 147-154 (1993).

269. V. Moulin, J. Tits, and G. Ouzounian, "Actinide Speciation in the Presence of Humic Substances in Natural Water Conditions," Radiochim. Acta 58/59, 179-190 (1992).

270. B. Torstenfelt, "Migration of the Fission Products Strontium, Technetium, Iodine and Cesium in Clay," Radiochim. Acta 39, 97-104 (1986).

271. H. Roggendorf, "Corrosion of the Borosilicate Glass R7T7 in a Concentrated NaClBrine- Experimental Data and Surface Characterization," Ceram. Trans. 23, 675-684 (1991).

272. J. D. F. Ramsey, "The Role of Colloids in the Release of Radionuclides from Nuclear Waste," Radiochim. Acta 44/45, 165-170 (1988).

273. J. I. Kim, "Actinide Colloid Generation in Groundwater," Radiochim. Acta 52/53, 71-81 (1991). 
274. K. H. Lieser, B. Gleitsmann, S. Peschke, and Th. Steinkopff, "Colloid Formation and Sorption of Radionuclides in Natural Systems," Radiochim. Acta 40, 39-47 (1987).

275. L. Righetto, G. Bidoglio, B. Marcandalli, and I. R. Bellobono, "Surface Interactions of Actinides with Alumina Colloids," Radiochim. Acta 44/45, 73-75 (1988).

276. W. L. Ebert, "The Effects of the Leachate $\mathrm{pH}$ and the Ratio of Glass Surface Area to Leachant Volume on Glass Reactions," Phys. Chem. Glasses 34(2), 58-65 (1993).

277. W. L. Ebert, J. K. Bates, E. C. Buck, M. Gong, and S. F. Wolf, "Disposition of Actinides Released From High-Level Waste Glass," Ceram. Trans. 45, 231-241 (1994).

278. J. R. Fowler and M. J. Plodinec, "Projected Compositions and Radiogenic Properties of DWPF Glasses," Proc. of Third Internat. High-Level Radioactive Waste Mgmt. Conf., American Nuclear Society, Las Vegas, NV, 904-910 (1992).

279. Aa. Barkatt, Al. Barkatt, P. E. Pehrsson, P. B. Macedo, and J. H. Simmons, "The Importance of $\mathrm{CO}_{2}$ Buffering and of the Total Ionic Balance in Measurements on the Durability of Glasses," Nucl. Technol. ㅌ, 271-277 (1982).

280. J. K. Bates, W. L. Ebert, D. F. Fischer, and T. J. Gerding, "The Reaction of Reference Commercial Nuclear Waste Glasses during Gamma Irradiation in a Saturated Tuff Environment," J. Mater. Res. $\underline{3}(3), 576-597$ (1988).

281. N. E. Bibler, M. H. Tosten, and D. C. Beam, "Recent Results on the Effect of Gamma Radiation on the Durability and Microstructure of DWPF Glass," Proc. of the Second Annual Internat. High-Level Radioactive Waste Mgmt. Topical Meeting, American Nuclear Society, Las Vegas, NV, Vol. 1, 1103-1109 (1991).

282. W. L. Ebert, J. K. Bates, T. A. Abrajano, and T. J. Gerding, "The Influence of Penetrating Gamma Radiation on the Reaction of Simulated Nuclear Waste Glass in Tuff Groundwater," Ceram. Trans. 9, 155-164 (1990).

283. W. L. Ebert, J. K. Bates, T. J. Gerding, and R. A. Van Konynenburg, "The Effects of Gamma Radiation on Groundwater Chemistry and Glass Reaction in a Saturated Tuff Environment," Mater. Res. Soc. Symp. Proc. $\underline{84}$, 613-622 (1987).

284. C. R. Bradley, N. L. Dietz, and J. K. Bates, "Shaping Particles for Ultramicrotomy," Mater. Res. Soc. Symp. Proc. 254, 279-284 (1992).

285. J. E. Mendel, compiler, Final Report of the Defense High-Level Waste Leaching Mechanisms Program, Pacific Northwest Laboratory Report PNL-5157 (1984).

286. E. D. Spinoza and J. L. Means, "Progress Report on Experimental Evaluation of a Nuclear Waste Glass Corrosion Model," Adv. Ceram. 20, 531-539 (1986).

287. V. Moulin, J. Tits, C. Moulin, P. Decambox, P. Mauchien, and O. de Ruty, "Complexation Behaviour of Humic Substances towards Actinides and Lanthanides Studied by Time-Resolved Laser-Induced Spectrofluorometry," Radiochim. Acta 58/59, 121-128 (1992). 
288. L. R. Eisenstatt, Description of the West Valley Demonstration Project Reference HighLevel Waste Form and Canister, U.S. Department of Energy Report DOE/NE/44139-26 (1986). 


\section{APPENDIX A. DEFINITIONS}

Accelerated Test - a test in which the values of independent test parameters used result in increased alteration rates of the specimen relative to rates under expected service conditions. Any change in the corrosion mechanism must be accounted for in the use of test data.

Alteration - a change in the chemical or physical properties of a material.

Characterization Test - any test performed to provide information to support the mechanistic description of the corrosion process.

Corrosion Model - a set of chemical reactions and/or physical processes that can be used to describe observed phenomena.

Diffusion - the process of mass transfer of a reactant or product species through either a liquid or solid phase as a result of chemical potential gradient to maintain corrosion. A diffusion coefficient is defined in some corrosion models to characterize the release of silicic acid and other glass components during corrosion via partial retention by the altered surface.

Dynamic Test - a laboratory test in which the corroding fluid is continuously or periodically refreshed during the test.

Effective Flow Rate - the equivalent velocity of leachant passing the glass surface simulated by the periodic removal of leachate and addition of fresh leachant in a reaction cell. Associated with the effective flow rate is a characteristic replacement time, which is the period required for complete refreshment of the solution within the sample chamber or corrosion cell.

Empirical Model - a model based exclusively on experimental observations, independent of model or theory.

Extent of Corrosion - a measure of the amount of glass that is chemically or physically altered as a result of interactions with a fluid. Material may be dissolved from the glass and either enter solution or become separated from the glass structure and form a secondary solid phase.

Flow Rate - the velocity of the leachant passing the glass surface. The flow rate affects the corrosion by changing the fluid chemistry at the corroding surface.

Glass Surface Area - the area of glass available for corrosion reactions. The total surface area includes free surfaces as well as surfaces within cracks and pores that are accessible to water.

Glass Surface Area-to-Solution Volume Ratio (SA/V) - the ratio of the surface area of the glass available for reaction and the leachant solution volume available to dilute the reaction products. The SA/V ratio is a system parameter and not a property of either the glass or the fluid. Because they occur in most reaction rate equations as a ratio, the glass surface area and leachant volume are conveniently represented as a ratio and discussed as a single parameter.

Hydrolysis - the reaction in which molecular water is inserted into a bond, resulting in the rupture of that bond. The hydrolysis reaction may be catalyzed by the nucleophilic attack by hydroxide ions (base-catalyzed) or electrophilic attack by hydronium ions (acid-catalyzed) on the glass. Hydrolysis of an oxygen-alkali metal or alkaline earth bond is also referred to as ion exchange. 
Instantaneous Corrosion Rate - the rate at a specific time during corrosion, usually determined over a specific time interval.

Integrated Corrosion Rate - the rate determined by dividing the measured extent of corrosion by the total reaction time.

Intrinsic Corrosion Rate - the rate at which a glass reacts in the absence of corrosion products. The intrinsic corrosion rate depends on the glass composition, temperature, and solution $\mathrm{pH}$.

Ion Exchange - the reaction in which an ion fixed in the glass is replaced by an ion previously associated with a mobile species. The hydrolysis reactions responsible for the release of alkali metals and alkaline earths from the glass are often described as occurring through ion exchange reactions, in which a proton from molecular water or hydronium replaces the alkali metal or alkaline earth ion in the glass structure. The alkali or alkaline earth ion is then free to be solvated and migrate into solution.

Leachant - the fluid that has not yet interacted with the glass. In laboratory tests, the leachant is the fluid added to the tests.

Leachate - the fluid that has contacted and interacted with the glass and contains dissolved glass components. The leachate solution may be analyzed to provide a measure of the extent of glass corrosion.

Mechanistic Model - a model based on chemical and physical laws and processes.

Model - a representation of a process as a set of chemical reactions and processes.

Nuclear Waste Glass - the fusion product of radioactive waste and glass-forming additives.

Response Test - any test performed primarily to provide information for comparative purposes independent of a corrosion mechanism.

Simulated Waste Glass -the fusion product of glass-forming additives and chemicals intended to represent the chemical behavior of radioactive elements.

Simulation - an implementation of a mechanistic model described in a mathematical algorithm, usually in a computer code.

Solution Volume - the volume of fluid contacting the glass. In static tests, this is the volume of leachant included in the test less the amount lost to vessel leakage and evaporation. In dynamic tests, the solution volume may refer to the integrated volume contacting the glass throughout the test duration, or to the volume of the corrosion cell.

Static Test - a laboratory test in which the corroding fluid is not refreshed during the test.

Waste Form - the radioactive waste materials and any stabilizing matrix prepared for long-term storage or disposal.

Vitrification - the process of fusing chemicals to generate a glass. 


\section{APPENDIX B. GLASS COMPOSITIONS}

The compositions of glasses used in tests described in this report are tabulăted for reference. Because compositional variations occur between different laboratories and even between different testing programs within a laboratory, the compositions presented should be used as a guide for differences in major components. Compositions were obtained from the following references.

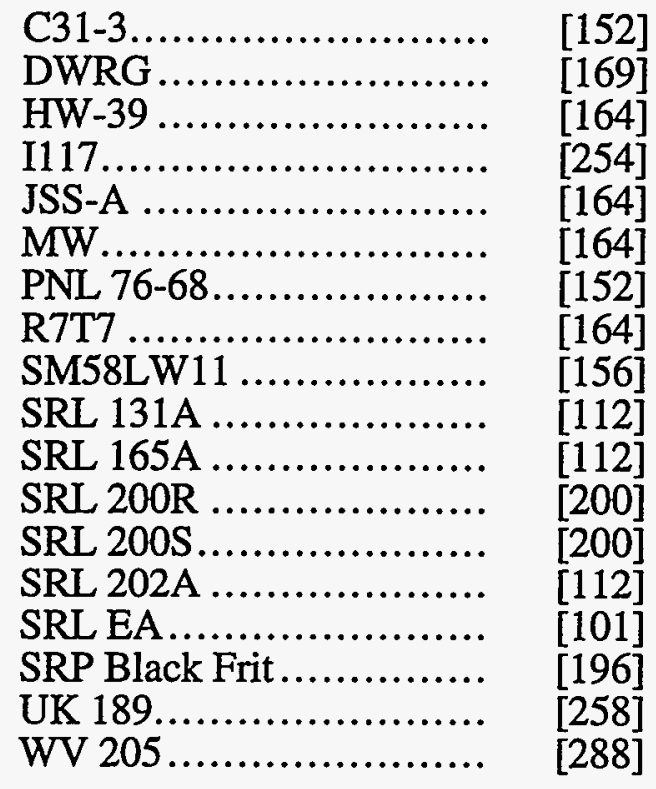




\begin{tabular}{|c|c|c|c|c|c|c|c|c|c|}
\hline$O \times I D E$ & C31-3 & DWRG & $\mathrm{HW}-39$ & I 117 & JSS-A & Min & PNL 76-68 & R7T7 & SMS8LW11 \\
\hline AgO & & & & & 0.03 & 0.02 & & 0.03 & \\
\hline $\begin{array}{r}\mathrm{A} 1203 \\
\mathrm{B2} 203\end{array}$ & $\begin{array}{r}10.28 \\
+4.14\end{array}$ & 6.58 & 4.3 & 5 & 4.87 & 5.33 & 0.6 & 4.91 & 2.1 \\
\hline $\begin{array}{r}\text { B203 } \\
\text { BoO }\end{array}$ & $\begin{array}{r}4.14 \\
15.28\end{array}$ & 6.77 & $\begin{array}{l}9.6 \\
0.2\end{array}$ & $\begin{array}{r}15 \\
0.33\end{array}$ & 14.4 & 16.65 & 8.81 & 14.02 & $\begin{array}{r}12.3 \\
0.1\end{array}$ \\
\hline CoO & 3.84 & 1.95 & 2.9 & 0.33 & $\begin{array}{l}0.61 \\
4.01\end{array}$ & 0.57 & $\begin{array}{l}0.53 \\
2.36\end{array}$ & $\begin{array}{l}0.06 \\
4.04\end{array}$ & $\begin{array}{l}0.1 \\
3.8\end{array}$ \\
\hline $\begin{array}{r}\mathrm{CdO} \\
\mathrm{CeO2}\end{array}$ & 1.38 & & & & 0.03 & 0.02 & 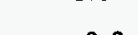 & 0.03 & \\
\hline $\begin{array}{r}C_{e} 02 \\
\text { CoO }\end{array}$ & & 0.02 & & & $\begin{array}{l}0.96 \\
0.12\end{array}$ & 1.02 & 0.9 & $\begin{array}{l}0.98 \\
0.03\end{array}$ & 0.18 \\
\hline Cr203 & & 0.06 & 1.3 & & 0.5 & 0.41 & & 0.51 & 0.28 \\
\hline $\begin{array}{r}\text { Cs20 } \\
\text { CuO }\end{array}$ & 1.18 & $\begin{array}{l}0.31 \\
0.01\end{array}$ & $\begin{array}{l}0.2 \\
0.1\end{array}$ & $\begin{array}{l}0.76 \\
0.04\end{array}$ & 1.41 & 1.13 & 1.02 & 1.42 & 0.16 \\
\hline $\begin{array}{l}\text { Eu203 } \\
\text { Fez03 }\end{array}$ & & & & & & 0.04 & 04 & 797 & 1.71 \\
\hline $\begin{array}{r}\text { Feo } \\
\text { Feo }\end{array}$ & 0.33 & 10.05 & 11.1 & 3.25 & 2.89 & 2.69 & 9.4 & 2.91 & 1.71 \\
\hline $\begin{array}{r}6 d 203 \\
K 20\end{array}$ & & & & & & 0.02 & & & \\
\hline $\operatorname{lo} 203$ & $\begin{array}{l}0.67 \\
0.98\end{array}$ & & 0.5 & 0.63 & 0.89 & 0.52 & 4.06 & 0.9 & \\
\hline $\begin{array}{l}\operatorname{Liz}_{\mathrm{MgO}} \\
\text { M }\end{array}$ & $\begin{array}{l}0.98 \\
1.44\end{array}$ & $\begin{array}{r}4.85 \\
0.074\end{array}$ & $\begin{array}{l}3.8 \\
0.8\end{array}$ & & $\begin{array}{l}1.97 \\
0.04\end{array}$ & $\begin{array}{l}3.79 \\
5.85\end{array}$ & $\begin{array}{r}0 \\
0.15\end{array}$ & 1.98 & $\begin{array}{l}3.7 \\
2.1\end{array}$ \\
\hline $\mathrm{MnOZ}$ & & 3.09 & & 1.35 & 0.71 & & & 0.72 & 0.67 \\
\hline $\mathrm{MoO}_{3}$ & 2.36 & & 0.3 & 0.16 & 2.69 & 1.76 & 1.93 & 1.7 & 0.28 \\
\hline $\mathrm{Na} 2 \mathrm{O}$ & 3.12 & 9.2 & 10.4 & 18.49 & 9.79 & 8.1 & 11.53 & 9.86 & 8 \\
\hline $\begin{array}{r}\mathrm{Nd} 203 \\
\mathrm{NiO}\end{array}$ & & 2.01 & $\begin{array}{l}0.5 \\
0.6\end{array}$ & $\begin{array}{l}0.99 \\
0.32\end{array}$ & $\begin{array}{l}1.58 \\
0.74\end{array}$ & $\begin{array}{l}1.61 \\
0.27\end{array}$ & 1.38 & $\begin{array}{l}1.59 \\
0.74\end{array}$ & $\begin{array}{l}0.57 \\
0.43\end{array}$ \\
\hline $\begin{array}{r}\text { P205 } \\
\text { Pbo }\end{array}$ & & & & & 0.48 & 0.18 & 0.7 & 0.28 & \\
\hline Pr6011 & & & 0.1 & 0.31 & 0.44 & 1.02 & & 0.14 & \\
\hline Rb20 & & & & & & 0.13 & & & \\
\hline $\begin{array}{r}\text { Ru02 } \\
503\end{array}$ & 1.28 & & 0.4 & & & 0.06 & & & \\
\hline 56203 & & & & & 0.01 & 0.01 & & 0.01 & \\
\hline $\begin{array}{l}\mathrm{SeO2} \\
\mathrm{SiO} 02\end{array}$ & 34.75 & 49.9 & 51.3 & 48 & 45.12 & $\begin{array}{l}0.03 \\
46.2\end{array}$ & 40.84 & 45.48 & 56.8 \\
\hline $5 \times 203$ & & & & 1.12 & & 0.32 & & & 36.8 \\
\hline $\begin{array}{r}\text { SnOZ } \\
\text { Sro }\end{array}$ & 0.45 & 0.46 & & $\begin{array}{l}0.03 \\
0.22\end{array}$ & 0.02 & 0.02 & & 0.02 & \\
\hline $\begin{array}{r}\text { Sro } \\
\text { Te02 }\end{array}$ & & & & 0.22 & 0.33 & $\begin{array}{l}0.36 \\
0.18\end{array}$ & 0.42 & $\begin{array}{l}0.33 \\
0.23\end{array}$ & 0.05 \\
\hline $\begin{array}{r}\text { Tc207 } \\
\text { Th02 }\end{array}$ & & & & & 0.16 & & & 0.33 & \\
\hline $\begin{array}{r}\mathrm{TiO} \\
\text { Yo2 }\end{array}$ & $\begin{array}{r}2.8 \\
1.37\end{array}$ & 0.17 & & & 95 & & 3.13 & & 4.5 \\
\hline U308 & & 2.61 & & 1.38 & 0.51 & & & 0.52 & 0.11 \\
\hline $\begin{array}{r}\mathrm{Y203} \\
\mathrm{ZnO}\end{array}$ & 4.9 & & & 0.03 & & & & 0.2 & \\
\hline $2 \mathrm{rOz}$ & 3.08 & 0.94 & 0.6 & 1.06 & & $\begin{array}{l}0.21 \\
1.71\end{array}$ & $\begin{array}{l}4.72 \\
1.68\end{array}$ & $\begin{array}{r}2.5 \\
2.65\end{array}$ & $\begin{array}{l}0.02 \\
0.81\end{array}$ \\
\hline OXIDE & SRL 131A & SRL 165A & SRL 200R & SRL 2005 & SRL 202A & SRP Black & SRL EA & UK 189 & WV 205 \\
\hline AgO & & & & & & & & & \\
\hline Al203 & 3.27 & $\begin{array}{l}4.08 \\
6.76\end{array}$ & 5.9 & $\begin{array}{l}5.5 \\
9.6\end{array}$ & 3.84 & 4.54 & 3.6 & 5.03 & 3.31 \\
\hline $\begin{array}{r}8203 \\
800\end{array}$ & $\begin{array}{l}9.65 \\
8.16\end{array}$ & $\begin{array}{l}6.76 \\
0.06\end{array}$ & $\begin{array}{r}9.7 \\
0.02\end{array}$ & 0.63 & $\begin{array}{l}7.97 \\
0.22\end{array}$ & 7.35 & 11.16 & 21.87 & $\begin{array}{l}9.97 \\
0.06\end{array}$ \\
\hline $\begin{array}{l}\mathrm{CaO} \\
\mathrm{CdO}\end{array}$ & 0.93 & 1.62 & 0.9 & 0.9 & 1.2 & & 1.23 & & 0.61 \\
\hline $\mathrm{CeOZ}$ & & & & & & & & & 0.18 \\
\hline $\mathrm{Cr} 203$ & 0.13 & & 0.3 & 0.2 & 0.03 & & & 0.55 & 0.22 \\
\hline $\begin{array}{r}\text { Cs20 } \\
\text { CuO }\end{array}$ & 0.02 & & 0.1 & 0.1 & 0.4 & 0.07 & & & 0.11 \\
\hline Eu203 & & & & & & & & & \\
\hline $\begin{array}{r}\mathrm{Fe} 203 \\
\mathrm{FeO}\end{array}$ & 12.66 & 11.74 & 9 & 8.8 & 11.41 & 11.4 & $\begin{array}{l}7.58 \\
1.59\end{array}$ & 2.68 & 11.83 \\
\hline $\begin{array}{r}G d 203 \\
K 20\end{array}$ & 3.86 & 0.19 & & & 371 & & & & \\
\hline $\operatorname{La} 203$ & 5.86 & 0.19 & $\begin{array}{l}3.5 \\
0.06\end{array}$ & $\begin{array}{r}3.3 \\
0.01\end{array}$ & 3.71 & & $\begin{array}{l}0.04 \\
0.28\end{array}$ & & $\begin{array}{l}3.62 \\
0.12\end{array}$ \\
\hline Lizo & $3_{3}^{3}$ & 4.18 & 3.4 & 3.3 & 4.23 & & 4.21 & 3.69 & \\
\hline $\mathrm{MgO}_{\mathrm{MOO}}$ & $\begin{array}{l}\frac{1.31}{2.43} \\
2.43\end{array}$ & 2.79 & $\begin{array}{l}1.6 \\
1.6\end{array}$ & 1.7 & 1.32 & 0.7 & 1.79 & 6.23 & 1.24 \\
\hline Mo03 & & & $\begin{array}{l}1.6 \\
0.01\end{array}$ & $\begin{array}{r}1.5 \\
0.02\end{array}$ & $\begin{array}{l}2.21 \\
0.05\end{array}$ & 1.98 & 1.36 & & 1.73 \\
\hline $\begin{array}{r}\mathrm{Na} 20 \\
\mathrm{Nd} 2 \mathrm{O3}\end{array}$ & 12.08 & 10.85 & 0.1 & 16 & 8.92 & 10.2 & 16.88 & 7.68 & 11.05 \\
\hline $\begin{array}{r}\text { NiO } \\
\text { P205 }\end{array}$ & 1.24 & 0.85 & 0.9 & 0.9 & 0.82 & & 0.53 & 0.36 & 0.7 \\
\hline $\mathrm{PbO}$ & & & 0.03 & 0.05 & 0.01 & & & & 2.52 \\
\hline $\begin{array}{l}\text { Pr6011 } \\
\text { Pu02 }\end{array}$ & & 0.01 & & & & & & & \\
\hline Rb20 & & & & & & & & & \\
\hline $\begin{array}{r}\mathrm{RuO2} \\
\mathrm{S03}\end{array}$ & & & & & & & & & 0.07 \\
\hline $\begin{array}{r}\mathrm{Sb} 203 \\
\mathrm{Se} 02\end{array}$ & & & & & & & & & \\
\hline $\begin{array}{r}S i 02 \\
S=203\end{array}$ & 43.76 & 52.86 & 45.5 & 45.4 & 48.95 & 53.5 & 48.76 & $\$ 1.51$ & 45.25 \\
\hline $\begin{array}{r}5 n 02 \\
5 r 0\end{array}$ & 0.01 & a. & a a) & 0 & $\theta_{0}$ & & & & 0.13 \\
\hline Te0z & 0.01 & 0.11 & 0.01 & 0.02 & 0.03 & 0.09 & & & 0.13 \\
\hline $\begin{array}{r}\text { Tc207 } \\
\text { Th02 }\end{array}$ & 0.02 & 0.02 & & & $\begin{array}{l}0.02 \\
0.26\end{array}$ & & & & \\
\hline $\begin{array}{r}\mathrm{riO} \\
\text { yo2 }\end{array}$ & $\begin{array}{l}0.65 \\
2.73\end{array}$ & 0.14 & $\begin{array}{r}0.08 \\
1.8\end{array}$ & $\begin{array}{l}0.1 \\
1.9\end{array}$ & $\begin{array}{l}0.91 \\
1.93\end{array}$ & & 0.65 & & 0.99 \\
\hline U308 & & 0.92 & 1.8 & 1.9 & 1.93 & 1.19 & & 0.06 & \\
\hline Y203 & & & & & & & & & 0.03 \\
\hline $\begin{array}{r}2 n 0 \\
2 \mathrm{r} 02\end{array}$ & $\begin{array}{l}0.02 \\
0.22\end{array}$ & $\begin{array}{l}0.04 \\
0.66\end{array}$ & $\begin{array}{l}0.02 \\
0.05\end{array}$ & & $\begin{array}{r}0.02 \\
0.1\end{array}$ & & $\begin{array}{l}0.26 \\
0.48\end{array}$ & 0.44 & 2.99 \\
\hline
\end{tabular}




\section{APPENDIX C. DERIVATION OF RATE EQUATION}

The following derivation is based on that of Aagaard and Helgeson [54]; deviations and comments are noted.

In general, the net rate of a chemical reaction is given as the difference of the forward and reverse rates

$$
\text { net rate }=\text { rate }_{\mathrm{f}}-\text { rate }_{\mathrm{r}}
$$

This expression can be rearranged as

$$
\text { net rate }=\text { rate }_{\mathrm{f}}\left(\frac{1-\text { rate }_{\mathrm{r}}}{\text { rate }_{\mathrm{f}}}\right)
$$

For a system at equilibrium,

$$
\frac{\text { rate }_{\mathrm{r}}}{\text { rate }_{\mathrm{f}}}=1
$$

For a system not at equilibrium,

$$
\frac{\text { rate }_{\mathrm{r}}}{\text { rate }_{\mathrm{f}}}=\exp \left(\frac{\mathrm{A}}{\mathrm{RT}}\right)
$$

where $\mathrm{R}$ is the gas constant, $\mathrm{T}$ is the absolute temperature, and $\mathrm{A}$ is the reaction affinity, which is defined as the negative of the partial derivative of the Gibbs free energy with respect to the reaction progress at constant temperature and pressure (where $d$ indicates the partial derivative):

$$
A=-\left(\frac{d G}{d \xi}\right)_{T, P}
$$

where $G$ is the Gibbs free energy of the reaction and $\xi$ is the reaction progress variable. The Gibbs free energy is a function of temperature, pressure, and the number of moles of each species, and its derivative can be written

$$
\mathrm{dG}=\left(\frac{d \mathrm{G}}{d \mathrm{t}}\right)_{\mathrm{P}, \mathrm{N}} \mathrm{dT}+\left(\frac{d \mathrm{G}}{d \mathrm{P}}\right)_{\mathrm{T}, \mathrm{N}} \mathrm{dP}+\Sigma_{\mathrm{i}}\left(\frac{d \mathrm{G}}{d \mathrm{~N}_{\mathrm{i}}}\right)_{\mathrm{T}, \mathrm{P}, \mathrm{N} \neq \mathrm{i}} \mathrm{dN}_{\mathrm{i}}
$$

At constant $\mathrm{T}$ and $\mathbf{P}$

$$
\mathrm{dG}=\Sigma_{\mathrm{i}}\left(\frac{d \mathrm{G}}{d \mathrm{~N}_{\mathrm{i}}}\right)_{\mathrm{T}, \mathrm{P}, \mathrm{N} j \neq \mathrm{i}} \mathrm{dN}_{\mathrm{i}}
$$


The partial derivative of the free energy with respect to the extent of reaction, $\xi$, is

$$
\left(\frac{d \mathrm{G}}{d \xi}\right)_{\mathrm{T}, \mathrm{P}}=\Sigma_{\mathrm{i}} \mu_{\mathrm{i}} v_{\mathrm{i}}
$$

where $\mu_{\mathrm{i}}$ is the chemical potential, and $v_{\mathrm{i}}$ is the stoichiometric coefficient of species $\mathrm{i}$ in the reaction. Substituting

$$
\mu_{i}=\mu_{i}^{\circ}+R T \ln a_{i}
$$

where $\mathrm{R}$ is the gas constant, into Eq. $\mathrm{C}-8$ gives

$$
\left(\frac{d \mathrm{G}}{d \xi}\right)_{T, \mathrm{P}}=\Sigma_{\mathrm{i}}\left(\mu_{\mathrm{i}}^{\circ}+\mathrm{RT} \ln \mathrm{a}_{\mathrm{i}}\right) v_{\mathrm{i}}
$$

or

$$
\left(\frac{\mathrm{dG}}{\mathrm{d} \xi}\right)_{T, P}=\Sigma_{\mathrm{i}} \mu_{\mathrm{i}}^{0} v_{\mathrm{i}}+\Sigma_{\mathrm{i}} \mathrm{RT} \ln \left(\mathrm{a}_{\mathrm{i}}\right)^{v_{\mathrm{i}}}
$$

By definition,

$$
\Sigma_{\mathrm{i}} \mu_{\mathrm{i}}^{\circ} v_{\mathrm{i}}=\Delta \mathrm{G}^{\circ}=-\mathrm{RT} \ln \mathrm{K}
$$

where $\mathrm{K}$ is the equilibrium constant, so

$$
\left(\frac{d \mathrm{G}}{d \xi}\right)_{\mathrm{T}, \mathrm{P}}=-\mathrm{A}=-\mathrm{RT} \ln \mathrm{K}+\mathrm{RT} \ln \mathrm{Q}=\mathrm{RT} \ln \left(\frac{\mathrm{Q}}{\mathrm{K}}\right)
$$

where $\mathrm{Q}$ is the ion activity product. Substituting this expression for the affinity, A, into Eqs. C-4 and $\mathrm{C}-2$,

$$
\text { net rate }=\operatorname{rate}_{\mathrm{f}}\left(1-\exp \left(\frac{\ln \mathrm{Q}}{\mathrm{K}}\right)\right)=\operatorname{rate}_{\mathrm{f}}\left(1-\frac{\mathrm{Q}}{\mathrm{K}}\right)
$$

Now consider the application of Eq. C-14 to the release of silicic acid from a glass phase. The net reaction can be written as

$$
\Rightarrow \mathrm{Si}-\mathrm{O}-\mathrm{Si}(\mathrm{OH})_{3}+\mathrm{OH}^{-}=\Rightarrow \mathrm{SiO}^{-}+\mathrm{H}_{4} \mathrm{SiO}_{4}
$$

where the fact that silicic acid is a weaker base than silanol is accounted for in the reaction. The rate of this reaction can be written as

$$
\text { net rate }=\frac{\mathrm{d}\left[\mathrm{H}_{4} \mathrm{SiO}_{4}\right]}{\mathrm{dt}}
$$


We note that when applying Eq. C-14 to a glass, the substitution made in Eq. C-12 is inexact because the standard states of all species $i$ in the glass and in solution are not the same. The inexactness of this substitution may the reason an ad hoc residual rate is required in the rate expression (see Eq. 21).

The forward rate is conveniently expressed by using activated complex theory. Consider the reaction to release silicic acid by nucleophilic attack of hydroxide at the underscored silicon atom to form the activated complex

$$
=\underline{\mathrm{Si}}-\mathrm{O}-\mathrm{Si}(\mathrm{OH})_{3}+\mathrm{OH}^{-}=\left\{\equiv \mathrm{Si}(\mathrm{OH})-\underline{O}-\mathrm{Si}(\mathrm{OH})_{3}\right\}^{-} \dagger
$$

Equation C-17 differs from previous derivations, where molecular water was the nucleophile. Hydroxide is indicated as the nucleophile in Eq. C-17 in response to the observed effects of the $\mathrm{pH}$ on glass corrosion. If molecular water is the reactant rather than hydroxide, then the activated complex is uncharged and its formation is $\mathrm{pH}$-independent. In addition, molecular water is likely to form a hydrogen bond with the underscored oxygen atom. The activated complex can decompose by breaking the bond between the underscored silicon and the reactant hydroxide, in which case no net reaction occurs, or by breaking the bond between the underscored silicon and the underscored oxygen as

$$
\left\{\equiv \underline{\mathrm{Si}}(\mathrm{OH})-\underline{\mathrm{O}}-\mathrm{Si}(\mathrm{OH})_{3}\right\}^{-} \dagger \rightarrow \equiv \underline{\mathrm{SiO}}^{-}+\mathrm{H} \underline{\mathrm{O}}-\mathrm{Si}(\mathrm{OH})_{3}
$$

The proton on the nucleophilic hydroxide may also form a hydrogen bond with the underscored oxygen atom. The silicate species (either $\mathrm{H}_{4} \mathrm{SiO}_{4}$ or $\mathrm{H}_{3} \mathrm{SiO}_{4}^{-}$) formed during decomposition is shown to be in the reprotonated form to form silicic acid. In activated complex theory, the forward reaction rate is given by the rate of the decomposition step in Eq. C-18. If the concentration of the activated complex is given as $\mathrm{AC}^{\dagger}$, the first-order decomposition reaction rate can be written as

$$
\text { rate }_{\mathrm{f}}=\alpha_{\mathrm{i}} \mathrm{fAC}^{\dagger}
$$

where $\alpha_{i}$ and $f$ are the usual transmission coefficient and frequency factor. Activated complex theory assumes an equilibrium exists between the reactants and the activated complex, so we can write

$$
\mathrm{K}^{\dagger}=\frac{\mathrm{AC}^{\dagger}}{\left(\equiv \mathrm{Si}-\mathrm{O}-\mathrm{Si}(\mathrm{OH})_{3}\right)\left(\mathrm{OH}^{-}\right)}
$$

where $\mathrm{K}^{\dagger}$ is the equilibrium constant. Note that the $\left(\mathrm{OH}^{-}\right)$term does not occur if molecular water is the nucleophile in the reaction. Solving Eq. C-20 for $\mathrm{AC}^{\dagger}$ and substituting into Eq. C-19 gives

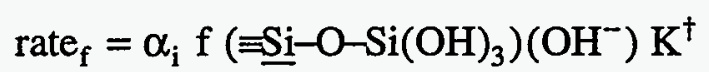

Now, the concentration of terminal $\left(=\mathrm{Si}-\mathrm{O}-\mathrm{Si}(\mathrm{OH})_{3}\right)$ groups can be considered proportional to the total surface area:

$$
\left(\equiv \underline{\mathrm{Si}}-\mathrm{O}-\mathrm{Si}(\mathrm{OH})_{3}\right)=\beta_{\mathrm{i}} \mathrm{SA}
$$


where $S A$ is the total surface area and $\beta_{i}$ is a proportionality constant. Substituting this expression in Eq. $\mathrm{C}-19$ gives

$$
\operatorname{rate}_{\mathrm{f}}=\alpha_{\mathrm{i}} \mathrm{f} \beta_{\mathrm{i}} \mathrm{SA}\left(\mathrm{OH}^{-}\right) \mathrm{K}^{\dagger}
$$

The Gibbs free energy of formation of the activated complex can be expressed as

$$
\Delta \mathrm{G}^{\dagger^{\circ}}=-\mathrm{RT} \ln \mathrm{K}^{\dagger}
$$

Also,

$$
\Delta G^{\dagger^{\circ}}=\Delta H^{\dagger^{\circ}}+T \Delta S^{\dagger^{\circ}}
$$

and

$$
\Delta H^{\dagger^{\circ}}=\mathrm{E}_{\mathrm{a}}-\mathrm{RT}
$$

so the equilibrium constant can be written as

$$
\mathrm{K}^{\dagger}=\exp \left(\frac{-\mathrm{E}_{\mathrm{a}}}{\mathrm{RT}}\right) \exp \left(\frac{\mathrm{RT}}{\mathrm{RT}}\right) \exp \left(\frac{\Delta \mathrm{S}^{\mathrm{t}^{\circ}}}{\mathrm{R}}\right)
$$

Substituting Eq. C-27 into Eq. C-23 gives

$$
\text { rate }_{\mathrm{f}}=\alpha_{\mathrm{i}} \mathrm{f} \beta_{\mathrm{i}} \mathrm{SA}(\mathrm{OH})^{-} \exp \left(\frac{-\mathrm{E}_{\mathrm{a}}}{\mathrm{RT}}\right) \exp \left(\frac{1+\Delta \mathrm{S}^{t^{\circ}}}{\mathrm{R}}\right)
$$

Substituting Eq. C-28 into Eq. C-14 gives

$$
\text { net rate }=\alpha_{i} f \beta_{i} S A\left(O^{-}\right) \exp \left(\frac{-E_{a}}{R T}\right) \exp \left(\frac{1+\Delta S^{\circ}}{R}\right)\left(1-\frac{Q}{K}\right)
$$

Equation C-29 can be simplified by defining the reaction coefficient as

$$
\mathrm{k}_{+}=\alpha_{\mathrm{i}} \mathrm{f} \beta_{\mathrm{i}}\left(\mathrm{OH}^{-}\right) \exp \left(\frac{-\mathrm{E}_{\mathrm{a}}}{\mathrm{RT}}\right) \exp \left(\frac{1+\Delta \mathrm{S}^{\circ}}{\mathrm{R}}\right)
$$

which gives the simplified expression for the corrosion rate as

$$
\text { net rate }=\mathrm{SA} \mathrm{k}_{+}\left(1-\frac{\mathrm{Q}}{\mathrm{K}}\right)
$$


The values of $\mathrm{k}_{+}$and $\mathrm{K}$ are determined experimentally. Equation $\mathrm{C}-30$ explicitly predicts the Arrhenius behavior of the rate coefficient The $\mathrm{pH}$ dependence is also accounted for by the $k_{+}$term. Equations $\mathrm{C}-31$ and $\mathrm{C}-16$ can be combined as

$$
\frac{\mathrm{d}\left[\mathrm{H}_{4} \mathrm{SiO}_{4}\right]}{\mathrm{dt}}=\mathrm{SA} \mathrm{k}_{+}\left(1-\frac{\mathrm{Q}}{\mathrm{K}}\right)
$$




\section{APPENDIX D. DATA GENERATED IN THE STUDY OF THE RELATIONSHIP BETWEEN HIGH SA/V TESTS AND MCC-1 TESTS}

This appendix contains spreadsheets presenting data generated in tests conducted in the ANL Task "Relationship between High SA/V Tests and MCC-1." Tests within the Task were organized into series having a common glass, common leachant, and common $S A / V$, as shown in Table D.1 below. The results are presented in four tables: Table D.2, test matrices; Table D.3, cation results; Table D.4, anion results; and Table D.5, actinide results. Each table has a cover sheet that explains the spreadsheet headings. Calculations performed in Tables D.2 through D.5 are summarized in Table D.6, where sample calculations are also presented.

Table D.1. Test Series Description

\begin{tabular}{llcr}
\hline Test Series & Glass Type & Leachant & SA/V (m-1) \\
\hline BX1 - BX16 & SRL 202A & EJ-13 & 10 \\
BW1 - BW16 & SRL 202A & EJ-13 & 340 \\
BY1 - BY22 & SRL 202A & EJ-13 & 2,000 \\
BZ1 - BZ20 & SRL 202A & EJ-13 & 20,000 \\
& & & \\
PX1 - PX16 & SRL 131A & EJ-13 & 10 \\
PW1 - PW16 & SRL 131A & EJ-13 & 340 \\
PY1 - PY22 & SRL 131A & EJ-13 & 2,000 \\
PZ1 - PZ20 & SRL 131A & EJ-13 & 20,000 \\
& & & \\
TW1 - TW18 & SRL 202U & DIW & 340 \\
TW31 - TW48 & SRL 202U & EJ-13 & 340 \\
TY1 - TY20 & SRL 202U & DIW & 2,000 \\
TZ1 - TZ18 & SRL 202U & DIW & 20,000 \\
TZ31 - TZ48 & SRL 202U & EJ-13 & 20,000 \\
\hline
\end{tabular}


Table D.2. Test Matrices

Column Heading

TEST No.

TARGET TIME

VESSEL No.

DATE IN

DATE OUT

RXN TIME, d

$\mathrm{SA}, \mathrm{mm} 2$

EJ-13, g

DIW, $g$

$\mathrm{SA} / \mathrm{V}, / \mathrm{m}$

VESSEL IN

VESSEL OUT

CHANGE, $g$

$\mathrm{pH}$

UF LPE

+UF SOLN

$+\mathrm{DIW}+\mathrm{HNO} 3$

UF DF

F50 LPE

+ F50 SOLN

+DIW+HNO9

F50 DF
Comment

Test number in task matrix (Table D.1).

Scheduled reaction time, in days.

Vessel number in which test was performed.

Calendar date test was initiated.

Calendar date test was terminated.

Actual reaction time, in days.

Measured geometric surface area of monolithic sample(s), in square millimeters.

Mass of EJ-13 solution added as leachant, in grams.

Mass of deionized water added as leachant, in grams.

Calculated glass surface area/leachant volume ratio, in inverse meters.

Vessel mass at test initiation, in grams.

Vessel mass at test termination, in grams.

Change in vessel mass during test, as VESSEL OUT-IN, in grams.

Leachate $\mathrm{pH}$ measured at room temperature.

Mass of empty solution bottle used for unfiltered leachate, in grams.

Mass of solution bottle plus unfiltered leachate, in grams.

Mass of deionized water and nitric acid added to leachate, in grams.

Dilution factor for unfiltered leachate.

Mass of empty solution bottle used for filtrate, in grams.

Mass of solution bottle plus filtrate, in grams.

Mass of deionized water and nitric acid added to filtrate, in grams.

Dilution factor for filtrate. 


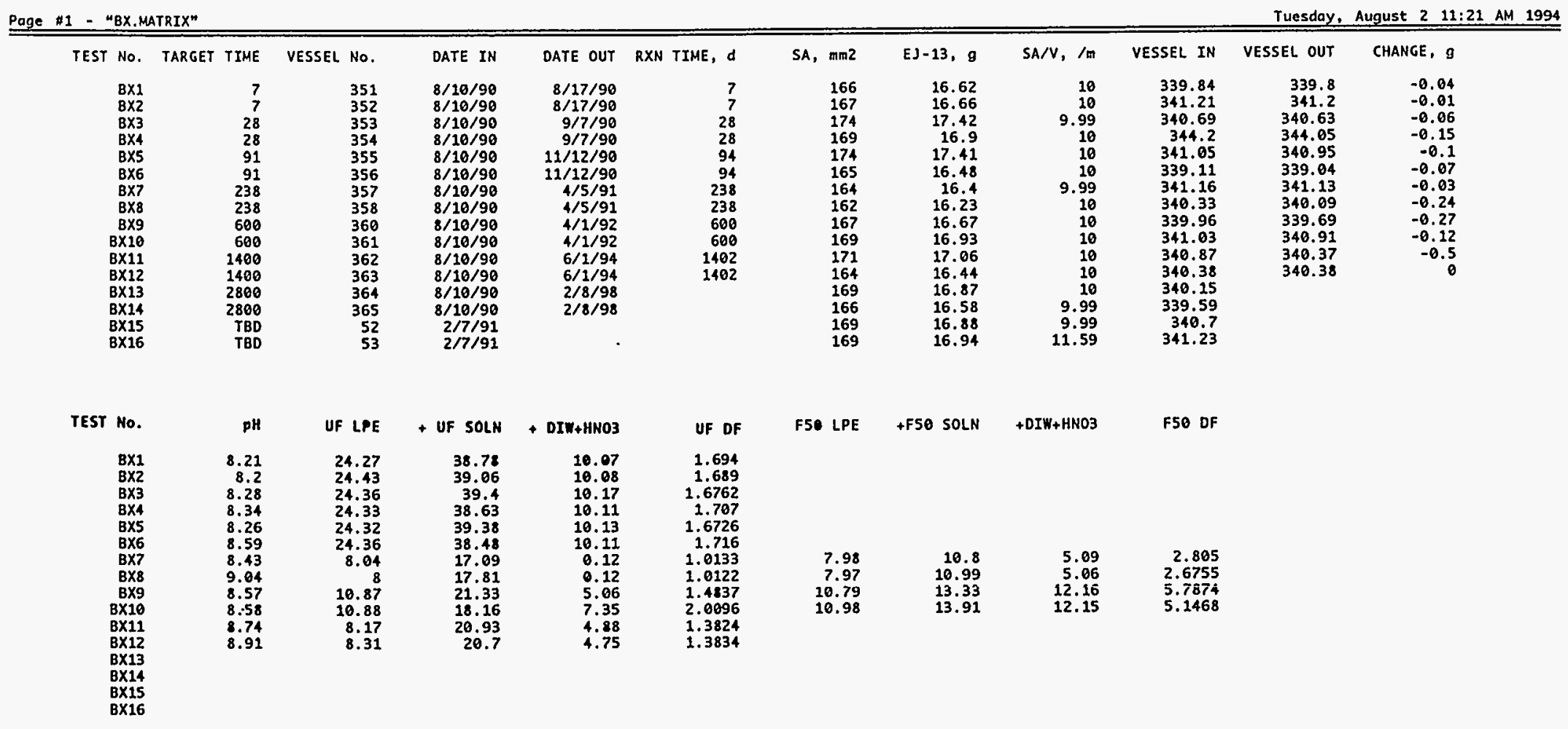


Page \#1 - "BW.MATRIX"

test no. target time vessel no.

DATE IN

DATE OUt RXN time, d

SA, $m m^{2}$

EJ-13, g

SA/v, / I

Fridoy, December $30 \quad 1: 21$ PM 1994

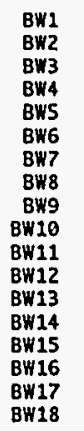

14
14
28
28
56
56
91
91
780
780
360
360
540
540
720
720
180
180

not run

not run
not run

not run

not run

not run

not run

$\begin{array}{rr}0 & \text { not run } \\ 183 & 7 / 1 / 91 \\ 202 & 7 / 1 / 91\end{array}$

$\begin{array}{rrr}242 & 7 / 1 / 91 & 6 / 25 / 92 \\ & 7 / 1 / 91 & 6 / 25 / 92\end{array}$

$\begin{array}{rr}6 / 25 / 92 \\ 7 / 1 / 91 & 12 / 22 / 92\end{array}$

$12 / 22 / 92$

$7 / 1 / 91 \quad 6 / 20 / 93$

TEST No.

not run

683
680
674
677

686
668
679
675

$\begin{array}{llll}675 & 2.05 & 331 & 326.84 \\ 683 & 2.05 & 333 & 324.65\end{array}$

$\begin{array}{llll}683 & 2.05 & 333 & 324.65 \\ 672 & 2.05 & 328 & 297.49 \\ 682 & 2.05 & 333 & 294.91\end{array}$

540
540
715

297.12
294
299.89

289.35

-1.81
-0.33

683
674
677

293.89
327.37

293.56
327.29

-0.33
-0.08

BW1

PH F.45 LPE +F.45 SOLN +DIW+HNO3

F. 45 DF

F5O LPE +FSO SOLN

+OIW+HNO3

F50 DF

BW2
BW3
BW4

$8 W 5$
$8 W 6$

BW7

BWF
BW9
BW10

$\begin{array}{lrrrrr}\text { BW11 } & 8.03 & 10.78 & 11.45 & 15.28 & 23.806 \\ \text { BW12 } & 8.14 & 11 & 11.89 & 15.15 & 18.022\end{array}$

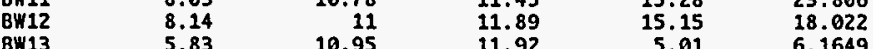

$\begin{array}{llllll}\text { BW14 } & 6.42 & 10.99 & 11.91 & 9.77 & 11.62 \\ \text { BW15 } & 6.42 & 10.94 & 12.12 & 9.82 & 9.322 \\ \text { BW16 } & 3.67 & & \end{array}$

BW17 


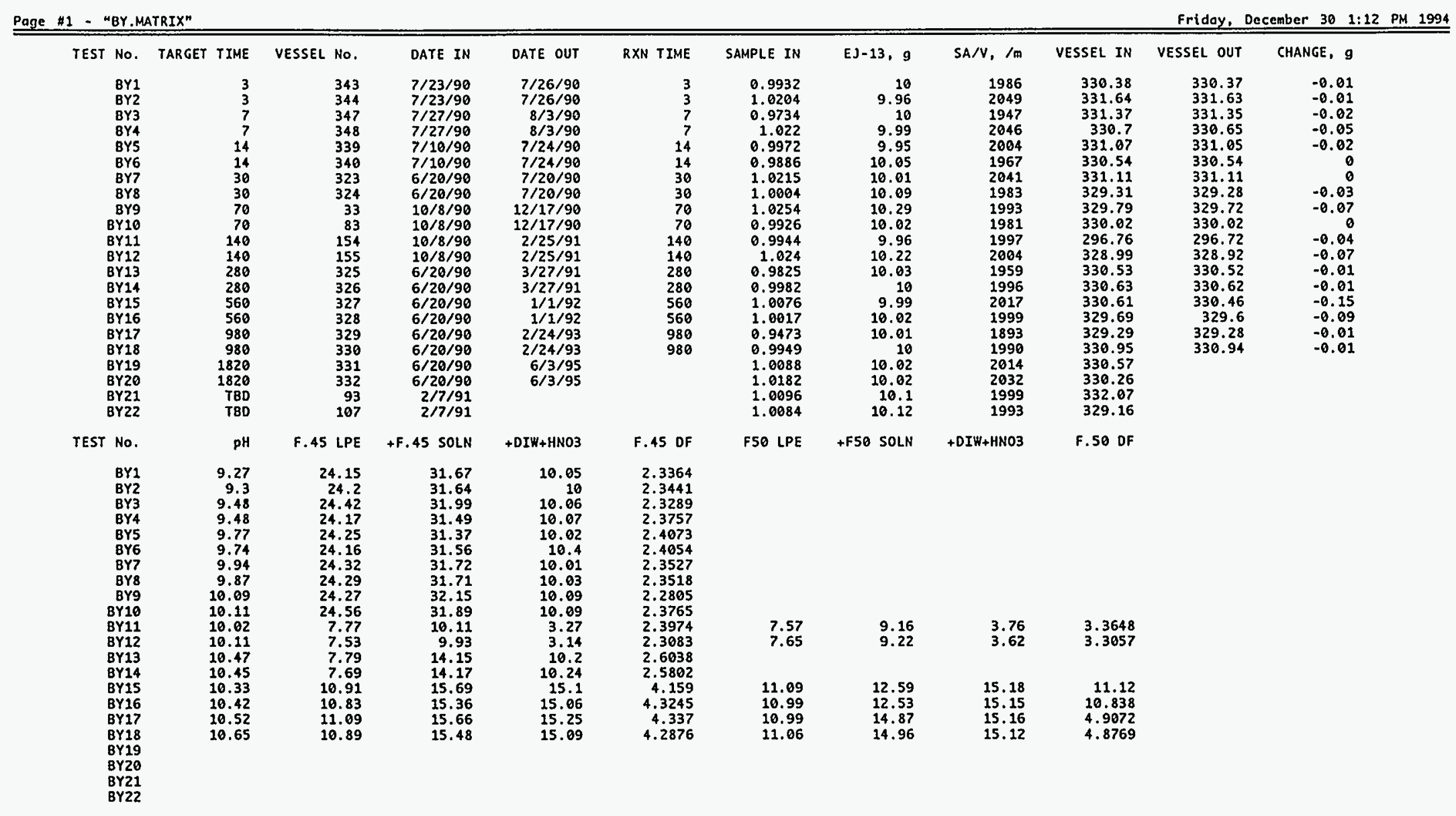


Page \#1 - "BZ.MATRIX"

test No. TARGet tIME VESSEl No.

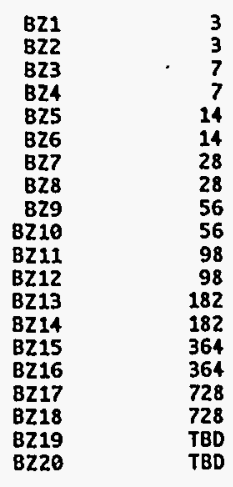

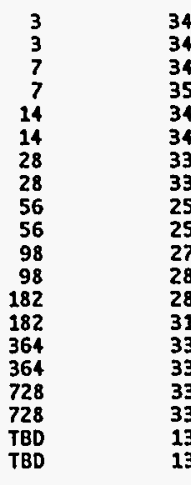

$\begin{array}{rrr}345 & 7 / 23 / 90 & 7 / 26 / 90 \\ 346 & 7 / 23 / 90 & 7 / 26 / 90 \\ 349 & 7 / 27 / 90 & 8 / 3 / 90 \\ 350 & 7 / 2790 & 8 / 3 / 90 \\ 341 & 7 / 10 / 90 & 7 / 24 / 90 \\ 342 & 7 / 10 / 90 & 7 / 24 / 90 \\ 337 & 6 / 2290 & 7 / 20 / 90 \\ 338 & 6 / 22 / 90 & 7 / 20 / 90 \\ 250 & 10 / 8 / 90 & 122 / 9 / 90 \\ 257 & 10 / 8990 & 122 / 3 / 90 \\ 270 & 10 / 8 / 90 & 1 / 14 / 91 \\ 281 & 10 / 8 / 90 & 1 / 14 / 91 \\ 283 & 10 / 8990 & 1 / 8 / 91 \\ 318 & 10 / 8 / 90 & 4 / 8 / 91 \\ 333 & 6 / 20 / 90 & 6 / 19 / 91 \\ 334 & 6 / 20990 & 619 / 91 \\ 335 & 6 / 20 / 90 & 6 / 25 / 92 \\ 336 & 6 / 20 / 90 & 6 / 25 / 92 \\ 135 & 2 / 7991 & 6 / 25 / 92 \\ 139 & 2 / 7 / 91 & \end{array}$

$\begin{array}{rr}\text { IME, d } & \text { SAMPLE IN } \\ 3 & 5.0123 \\ 3 & 4.9312 \\ 3 & 5.018 \\ 7 & 4.965 \\ 7 & 4.9457 \\ 14 & 4.9793 \\ 14 & 5.0235 \\ 28 & 58 \\ 28 & 5.0319 \\ 56 & 4.9792 \\ 56 & 5.0185 \\ 98 & 5.0027 \\ 98 & 4.9837 \\ 182 & 4.9708 \\ 182 & 5.0419 \\ 364 & 5.0021 \\ 364 & 5.0221 \\ 736 & 4.9692 \\ 736 & 4.9337 \\ 484 & 5.0224 \\ & 5.0552\end{array}$

EJ-13,
4.99
5.93
5.01
4.97
4.98
4.95
5.02
4.99
5.9
4.97
4.98

5.
5.92
5.92
5.

A $/ \mathrm{N}, \mathrm{m}$

ESSEL IN

328.34
327.85

327.85
329.63

329.08
328.51

328.51
329.64

329.24
329.62

329.31

330.5

329.63
330.21

328.59

336.09

328.48
330.07

329.53

329.16
328.12

Tuesday, Auqust 30 5:42 PM 1994

ESSEL OUT CHANGE, $g$

$\begin{array}{ll}328.32 & -0.02 \\ 327.84 & -0.01 \\ 329.62 & -0.01 \\ 329.07 & -0.01 \\ 328.5 & -0.01 \\ 329.61 & -0.03 \\ 329.22 & -0.02 \\ 332.6 & -0.02 \\ 329.25 & -0.06 \\ 330.49 & -0.01 \\ 329.59 & -0.04 \\ 330.14 & -0.07 \\ 328.4 & -0.19 \\ 328.38 & -0.02 \\ 330.03 & -0.06 \\ 328.28 & -0.2 \\ 329.94 & -0.13 \\ 329.47 & -0.06 \\ 329.11 & -0.05\end{array}$


TEST NO. TARGET TIME

VESSEL No.

337
338
339
340
341
342

$\begin{array}{lrr}\text { PX2 } & 7 & 337 \\ \text { PX2 } & 7 & 338 \\ \text { PX3 } & 28 & 339 \\ \text { PX4 } & 28 & 340 \\ \text { PX5 } & 91 & 341 \\ \text { PX6 } & 91 & 342 \\ \text { PX7 } & 238 & 343 \\ \text { PX8 } & 238 & 344 \\ \text { PX9 } & 690 & 345 \\ \text { PX19 } & & \text { 14 }\end{array}$

$P \times 10$
$P \times 10$
$P \times 11$
$P \times 12$
$P \times 13$
$P X 14$

$P \times 12$
PX14
$P X 15$

PX14
PX15
PX16

600
140
140
280
280
$T B 0$
$T$

$-$

TEST No.

$P X 1$
$P X 2$
$P X 3$
$P X 4$
$P X 5$
$P X 6$
$P X 6$
$P X 7$
$P X 8$
$P X 9$
$P X 10$
$P X 11$
$P X 12$
$P X 13$
$P X 14$
$P X 15$
$P X 16$
DATE IN

$8 / 28 / 90$

$8 / 28 / 90$

$8 / 28 / 90$

$8 / 28 / 90$

$1 / 28 / 90$

$8 / 28 / 90$

$8 / 28 / 90$

$8 / 28 / 90$

$8 / 28 / 96$

$8 / 28 / 90$

$2 / 7 / 91$

$9 / 4 / 90$
$9 / 4 / 90$
$9 / 25 / 90$
$9 / 25 / 90$
$11 / 30 / 90$
$11 / 30 / 90$
$4 / 23 / 91$
$1 / 23 / 91$
$4 / 20 / 92$
$1 / 20 / 92$
$6 / 21 / 94$
$6 / 21 / 94$
$2 / 26 / 98$
$2 / 26 / 98$

DAE OUT RXN TIME,

$\begin{array}{rl}7 & 17 \\ 7 & 17 \\ 28 & 168 \\ 28 & 168 \\ 94 & 168 \\ 94 & 168 \\ 238 & 165 \\ 238 & 172 \\ 601 & 18 \\ 601 & 17 \\ 1404 & 16 \\ 1404 & 169 \\ & 169 \\ & 16 \\ & 173 \\ & 167\end{array}$

172
172
168
168
168
168
165
172
185
171
166
169
169
166
173
167

EJ-13,

17.19
17.1
16.78
16.78
16.79
16.79
16.51
17.17
18.51
17.11
16.61
16.91
16.93
16.63
17.3
16.8

UF DF

F50 LPE

+ +F5O SOLN +DIW+HNO3

$39.21 \quad 10.08 \quad 1.6729$

39.15
38.66
38.95

38.96

15.19
19.7

22.15

21.62
18.46
20.57

10.13

1.6771
1.699
1.6945

1.6945

$\begin{array}{rl}9.45 & 1.6473 \\ 10.06 & 1.6962\end{array}$

$6.99 \quad 1.9308$

1.583
1.4965
$i .472$

7.71
7.76
10.92
10.81
11.01
11.1

9.5
9.67
13.64
15
14.63
14.81

5.1
5.08
10.2
11.07
7.45
7.46

$\begin{array}{ll}5.14 & 1.472 \\ 7.51 & 2.6094 \\ 7.19 & 1.7917\end{array}$

3.8492

3.8492
3.6597
4.75

Tucsday, August 30 5:17 PM 1994 
Page "1 - "PW.MATRIX"

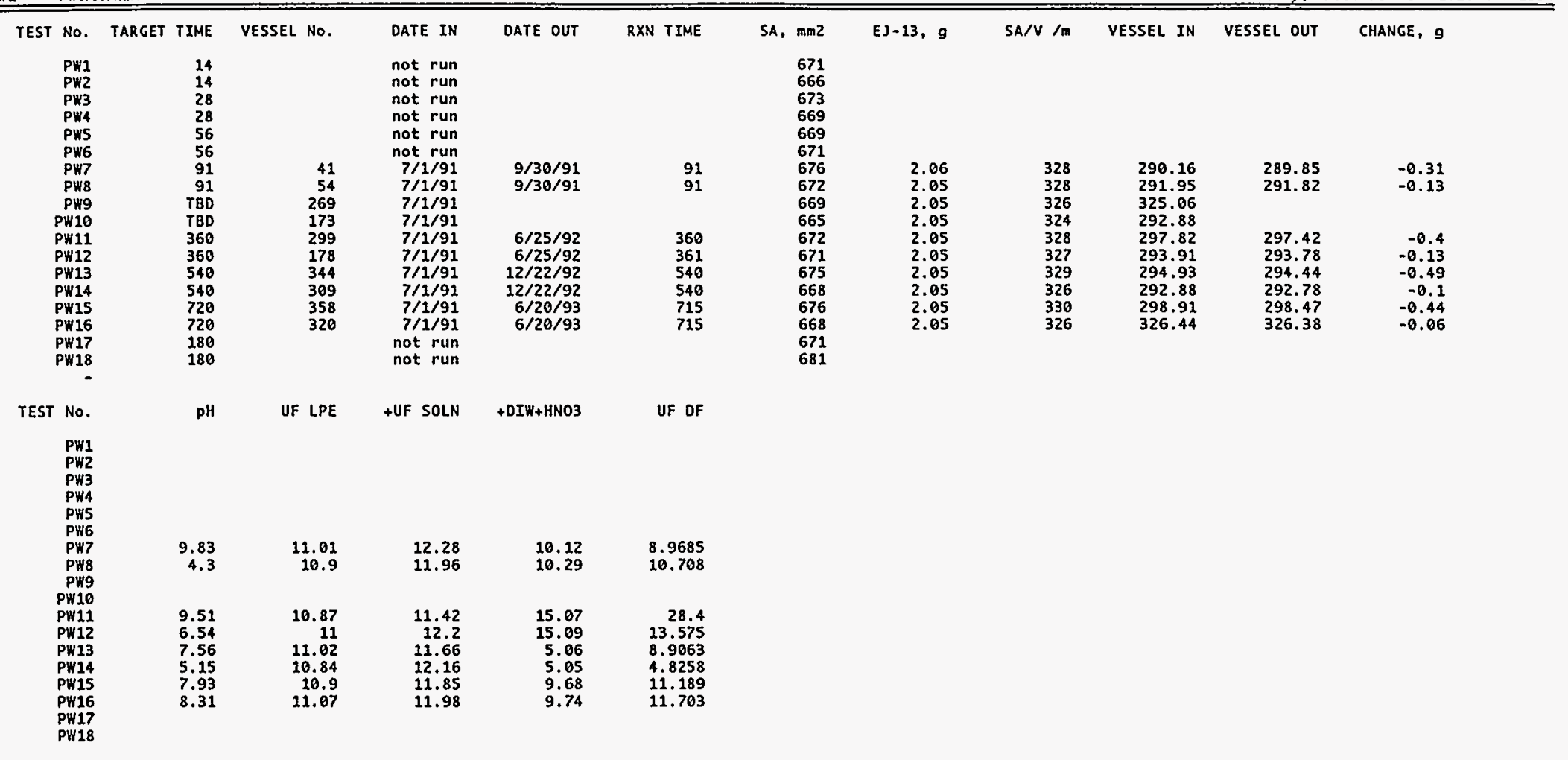




\section{Page \#1 - "PZ.MATRIX"}

test no. target time vessel no.

$\begin{array}{lr}\text { PZ1 } & 3 \\ \text { P22 } & 3 \\ \text { P23 } & 7 \\ \text { PZ4 } & 7 \\ \text { P25 } & 14 \\ \text { PZ6 } & 14 \\ \text { PZ7 } & 28 \\ \text { P28 } & 28 \\ \text { P29 } & 56 \\ \text { PZ10 } & 56 \\ \text { P211 } & 98 \\ \text { P212 } & 98 \\ \text { PZ13 } & 182 \\ \text { P214 } & 182 \\ \text { P215 } & 364 \\ \text { P216 } & 364 \\ \text { P217 } & 728 \\ \text { PZ18 } & 728 \\ \text { PZ19 } & \text { TBD } \\ \text { P220 } & \text { TBD }\end{array}$

$\begin{array}{rr}3 & 359 \\ 3 & 380 \\ 7 & 381 \\ 7 & 382 \\ 74 & 383 \\ 14 & 384 \\ 28 & 145 \\ 28 & 156 \\ 56 & 353 \\ 56 & 354 \\ 98 & 383 \\ 98 & 384 \\ 182 & 366 \\ 182 & 367 \\ 364 & 368 \\ 364 & 369 \\ 728 & 370 \\ 728 & 371 \\ \text { TBD } & 297 \\ \text { TBD } & 244\end{array}$

DATE IN

DATE OUT RXN tIME, d SAMPLE IN

EJ-13, 9

SA/V, /m

$\begin{aligned} & 8 / 28 / 90 \\ & 8 / 28 / 90\end{aligned} \quad 8 / 31 / 90$

$8 / 28 / 90$

$8 / 31 / 90$
$9 / 4 / 90$
$9 / 4 / 90$

$8 / 28 / 90 \quad 9 / 11 / 90$

$8 / 28 / 90 \quad 9 / 11 / 90$

$10 / 8 / 90 \quad 9 / 27 / 90$

$10 / 8 / 90 \quad 12 / 3 / 90$

$10 / 8 / 90$

$12 / 3 / 90$

$10 / 8 / 90 \quad 1 / 14 / 91$

$8 / 15 / 90 \quad 2 / 13 / 91$

$8 / 15 / 90 \quad 8 / 14 / 91$

$8 / 15 / 90 \quad 8 / 14 / 91$

$8 / 15 / 90 \quad 8 / 12 / 92$

$\begin{array}{rr}8 / 15 / 90 & 8 / 12 / 92 \\ 2 / 791 & 8 / 12 / 92 \\ 2 / 7 / 91 & 2 / 11 / 94\end{array}$

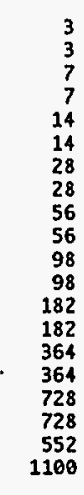

5.0469
5.0583
5.023
5.0365
4.9886
5.0328
4.9982
4.9922
5.0169
5.0138
4.998
5.0046
4.9839
4.9914
4.9962
5.0273
5.0247
5.0703
5.0131
4.9938

$\begin{array}{ll}5.05 & 19988 \\ 5.05 & 20073 \\ 5.02 & 20012 \\ 5.05 & 19947 \\ 5 & 19954 \\ 5.09 & 19775 \\ 5.01 & 19953 \\ 4.99 & 20009 \\ 5.03 & 19948 \\ 5.03 & 19936 \\ 4.99 & 20032 \\ 4.99 & 20059 \\ 4.98 & 20016 \\ 5.01 & 19926 \\ 5.02 & 19905 \\ 5.02 & 20029 \\ 5.05 & 19900 \\ 5.09 & 19923 \\ 4.99 & 20093 \\ 5 & 19975\end{array}$

VESSEL IN VESSEL OUT

334.4
328.7
328.9
328.14
330.5
328.8
326.38
327.67
328.64
328.82
329.25
328.4
330.14
328.3
298.9
329.54
331.5
337.17
328.49
331.4

334.48
328.77
328.97
328.14
330.56
328.89
326.38
327.67
328.64
328.82
329.25
328.4
330.14
328.32
298.9
329.54
331.55
337.17
328.49
331.44

334.16
328.76

328.94

-0.02
-0.01
-0.01

$328.82 \quad-0.07$

$\begin{array}{ll}327.62 & -0.06 \\ 327.65 & -0.02\end{array}$

328.13

328.17

328.29
329.91

328.27

298.38

330.86

336.87

328.41
331.38

-0.51
-0.65
-0.05

-0.05
-0.11

-0.23
-0.05

-0.52
-0.25

$-0.3$

-0.03
-0.06

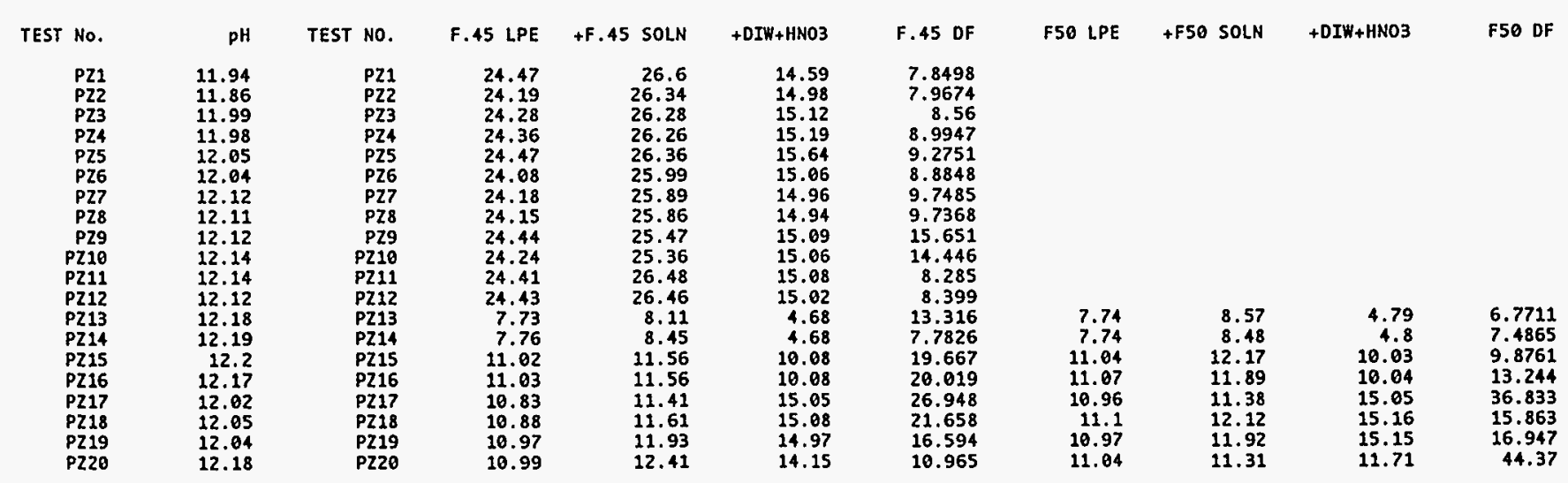




\begin{tabular}{|c|c|c|c|c|c|c|c|c|c|}
\hline TEST NO. & TARGET TIME & VESSEL NO. & DATE IN & DATE OUT & RXN TIME, $d$ & SA, $\mathrm{mm} 2$ & EJ-13, 9 & $S A / v, / m$ & VESSEL IN \\
\hline 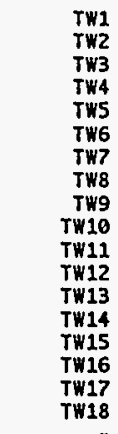 & $\begin{array}{l}14 \\
14 \\
28 \\
28 \\
56 \\
56 \\
91 \\
91 \\
180 \\
180 \\
360 \\
360 \\
540 \\
540 \\
720 \\
720 \\
780 \\
T B D\end{array}$ & $\begin{array}{r}1 \\
2 \\
3 \\
4 \\
5 \\
6 \\
7 \\
8 \\
9 \\
10 \\
11 \\
12 \\
13 \\
14 \\
15 \\
19 \\
136 \\
137\end{array}$ & $\begin{array}{l}6 / 26 / 91 \\
6 / 26 / 91 \\
6 / 26 / 91 \\
6 / 26 / 91 \\
6 / 26 / 91 \\
6 / 26 / 91 \\
6 / 26 / 91 \\
6 / 26 / 91 \\
6 / 26 / 91 \\
6 / 26 / 91 \\
6 / 26 / 91 \\
6 / 26 / 91 \\
6 / 29 / 91 \\
6 / 26 / 91 \\
6 / 26 / 91 \\
6 / 26 / 91 \\
7 / 16 / 92 \\
7 / 16 / 92\end{array}$ & $\begin{array}{r}7 / 10 / 91 \\
7 / 10 / 91 \\
7 / 24 / 91 \\
7 / 24 / 91 \\
8 / 21 / 91 \\
8 / 21 / 91 \\
9 / 25 / 91 \\
9 / 25 / 91 \\
12 / 23 / 91 \\
12 / 23 / 91 \\
6 / 26 / 92 \\
6 / 20 / 92 \\
12 / 18 / 92 \\
12 / 18 / 92 \\
6 / 15 / 93 \\
6 / 15 / 93\end{array}$ & $\begin{array}{r}14 \\
14 \\
28 \\
28 \\
56 \\
56 \\
91 \\
91 \\
181 \\
181 \\
360 \\
360 \\
540 \\
540 \\
720 \\
720\end{array}$ & $\begin{array}{l}703 \\
710 \\
704 \\
699 \\
705 \\
699 \\
707 \\
708 \\
708 \\
693 \\
706 \\
701 \\
700 \\
710 \\
698 \\
698 \\
713 \\
699\end{array}$ & $\begin{array}{l}2.05 \\
2.03 \\
2.04 \\
2.05 \\
2.05 \\
2.06 \\
2.06 \\
2.06 \\
2.05 \\
2.05 \\
2.05 \\
2.05 \\
2.05 \\
2.06 \\
2.05 \\
2.05 \\
2.02 \\
2.02\end{array}$ & $\begin{array}{l}343 \\
350 \\
345 \\
341 \\
344 \\
339 \\
343 \\
344 \\
345 \\
338 \\
344 \\
342 \\
341 \\
345 \\
340 \\
340 \\
353 \\
346\end{array}$ & $\begin{array}{l}36.77 \\
36.44 \\
36.95 \\
36.5 \\
36.48 \\
36.14 \\
37.03 \\
37.05 \\
36.69 \\
36.11 \\
37.11 \\
36.67 \\
36.59 \\
36.47 \\
36.93 \\
36.35 \\
36.17 \\
36.16\end{array}$ \\
\hline TEST No. & $\mathrm{pH}$ & UF LPE & + UF SOLN & $+\mathrm{DIW}+\mathrm{HNOB}$ & UF DF & F5O LPE & +F50 SOLN & +DIW+HNO3 & FSO DF \\
\hline $\begin{array}{l}\text { TW1 } \\
\text { TW2 } \\
\text { TW3 } \\
\text { TW4 } \\
\text { TW5 } \\
\text { TW6 } \\
\text { TW7 } \\
\text { TW8 } \\
\text { TW9 } \\
\text { TW10 } \\
\text { TW11 } \\
\text { TW12 } \\
\text { TW12 } \\
T W 13 \\
T W 14 \\
\text { TW15 } \\
\text { TW16 } \\
\text { TW16 } \\
\text { TW17 } \\
\text { TW18 }\end{array}$ & $\begin{array}{r}8.95 \\
9.31 \\
9.24 \\
9.28 \\
9.5 \\
9.44 \\
9.36 \\
9.41 \\
9.4 \\
9.58 \\
9.63 \\
9.59 \\
10.48 \\
9.58 \\
9.31 \\
9.75\end{array}$ & $\begin{array}{r}11 \\
11.08 \\
11.03 \\
11.04 \\
11 \\
11.08 \\
11.15 \\
8.02 \\
10.88 \\
11.04 \\
10.87 \\
10.87 \\
10.93 \\
10.86 \\
11.08 \\
11.04\end{array}$ & $\begin{array}{r}12.22 \\
11.71 \\
11.48 \\
11.58 \\
12.44 \\
12.53 \\
11.52 \\
9.55 \\
11.4 \\
11.58 \\
12.28 \\
12 \\
12.33 \\
12.35 \\
12.74 \\
12.67\end{array}$ & $\begin{array}{r}5.23 \\
5.14 \\
10.06 \\
10.06 \\
10.04 \\
10.04 \\
10 \\
10 \\
14.98 \\
15.09 \\
15.28 \\
15.36 \\
5.04 \\
5.09 \\
9.62 \\
9.77\end{array}$ & $\begin{array}{r}5.2869 \\
9.1587 \\
23.356 \\
19.63 \\
7.9722 \\
7.9241 \\
28.027 \\
7.5359 \\
29.808 \\
28.944 \\
11.837 \\
14.593 \\
4.6 \\
4.4161 \\
6.7952 \\
6.9939\end{array}$ & $\begin{array}{r}7.8 \\
10.87 \\
11.06\end{array}$ & $\begin{array}{r}8.53 \\
11.7 \\
11.93\end{array}$ & $\begin{array}{l}10.09 \\
14.93 \\
14.99\end{array}$ & $\begin{array}{r}12.614 \\
17 \\
13.444 \\
\\
14.822 \\
18.988 \\
18.23\end{array}$ \\
\hline
\end{tabular}




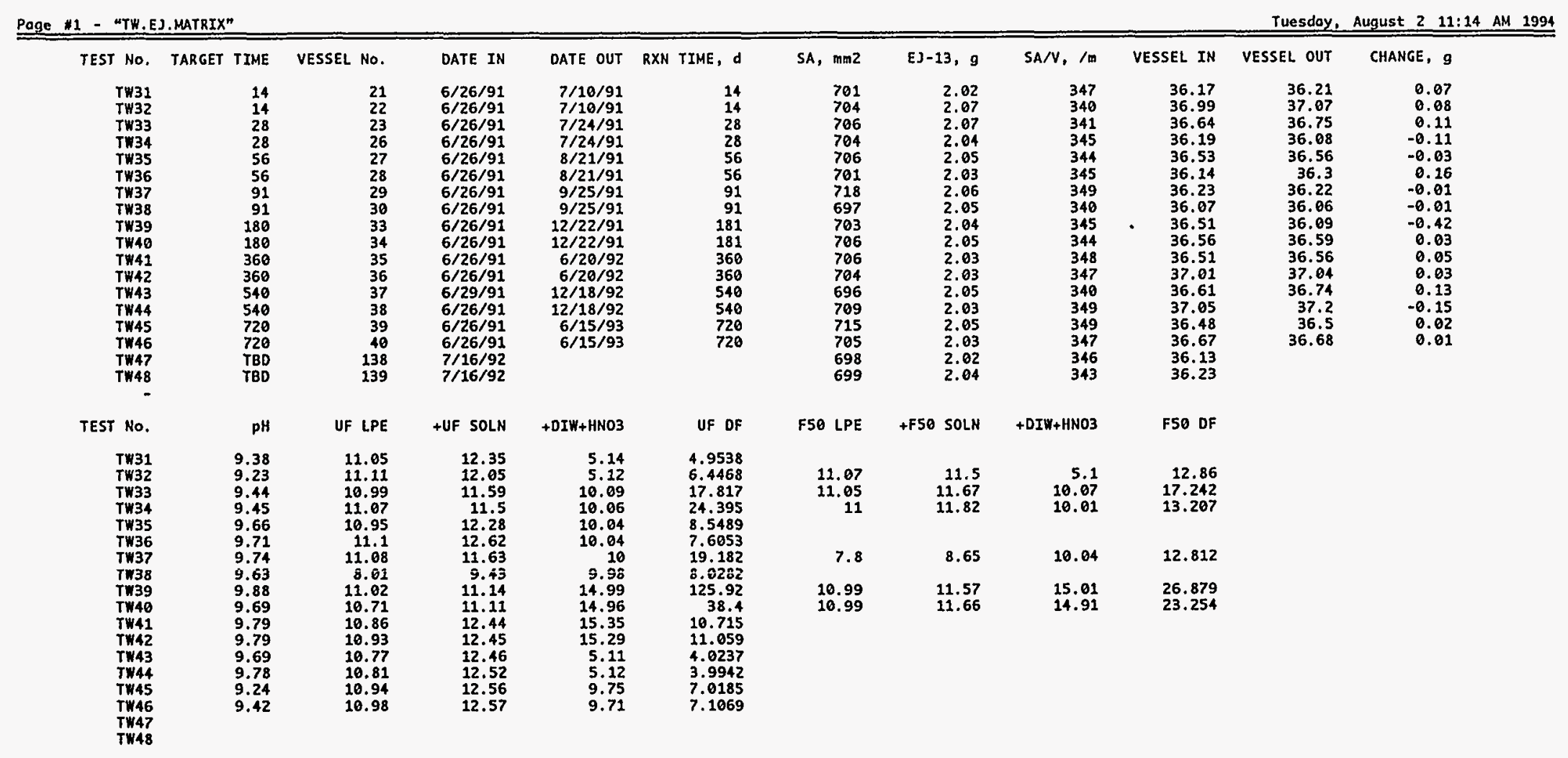




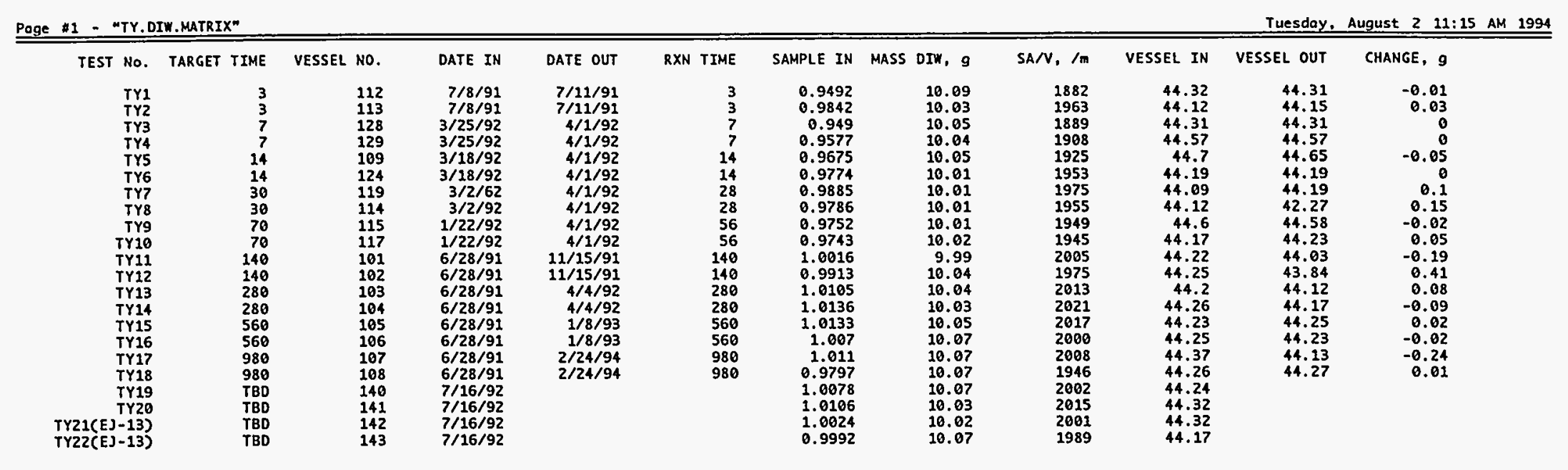

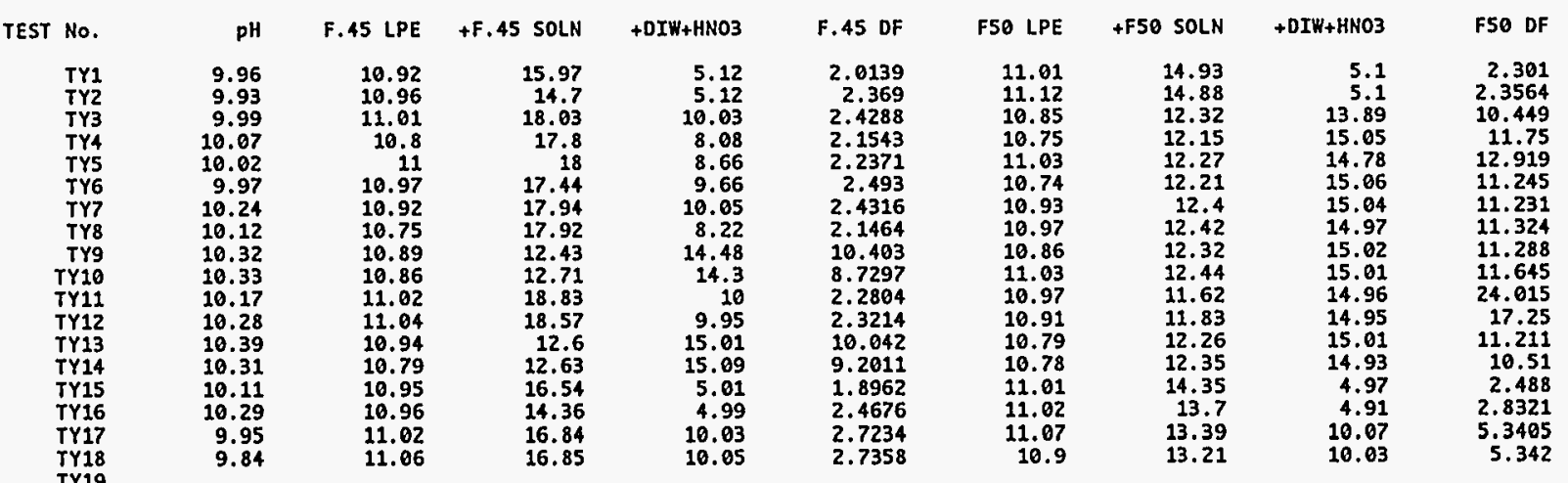


Page \#1 - "T2. DIW.MATRIX"

TEST NO. TARGET TIME

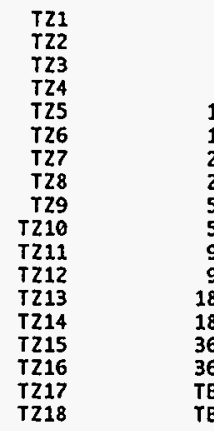

$\begin{array}{rrrr}3 & 41 & 7 / 8 / 91 & 7 / 11 / 91 \\ 3 & 42 & 7 / 8 / 91 & 7 / 11 / 91 \\ 7 & 43 & 6 / 26 / 91 & 7 / 3 / 91 \\ 7 & 44 & 6 / 26 / 91 & 7 / 3 / 91 \\ 14 & 45 & 6 / 26 / 91 & 7 / 10 / 91 \\ 14 & 46 & 6 / 26 / 91 & 7 / 10 / 91 \\ 28 & 47 & 6 / 26 / 91 & 7 / 24491 \\ 28 & 48 & 6 / 26 / 91 & 7 / 24 / 91 \\ 56 & 49 & 6 / 26 / 91 & 8 / 21 / 91 \\ 56 & 50 & 6 / 26 / 91 & 8 / 21191 \\ 91 & 51 & 6 / 26 / 91 & 9 / 25 / 91 \\ 91 & 52 & 6 / 26 / 91 & 9 / 25 / 91 \\ 182 & 53 & 6 / 26 / 91 & 12 / 23 / 91 \\ 182 & 54 & 6 / 26 / 91 & 12 / 23 / 91 \\ 364 & 55 & 6 / 26 / 91 & 6 / 24492 \\ 364 & 56 & 6 / 26 / 91 & 6 / 24 / 92 \\ \text { TBD } & 132 & 7 / 16 / 92 & \\ \text { TBD } & 133 & 7 / 16 / 92 & \end{array}$

$\begin{array}{rr}3 & 4.9913 \\ 3 & 4.9969 \\ 7 & 5.0338 \\ 7 & 4.9978 \\ 14 & 5.0089 \\ 14 & 4.9973 \\ 28 & 5.0202 \\ 28 & 4.9914 \\ 56 & 5.013 \\ 56 & 5.0052 \\ 91 & 5.0083 \\ 91 & 4.9979 \\ 181 & 5.0157 \\ 181 & 5.0057 \\ 364 & 4.9949 \\ 364 & 4.9962 \\ & 4.9915 \\ & 5.0145\end{array}$

DIW, $g$

5.03
5.01
5.03
4.98
4.99
4.99
4.99
4.98
4.99
4.99
4.97
4.99
4.98
4.98
4.99
5.02
5.02
5.03

$\begin{array}{rrrr}\text { SA/N, /m } & \text { VESSEL IN } & \text { VESSEL OUT } & \text { CHANGE, g } \\ 19846 & 43.73 & 43.73 & 0 \\ 19948 & 43.65 & 43.69 & 0.04 \\ 20015 & 43.62 & 43.58 & -0.04 \\ 20071 & 43.62 & 43.61 & -0.01 \\ 20076 & 44.1 & 44.28 & 0.18 \\ 20029 & 43.45 & 43.56 & 0.11 \\ 20121 & 44.29 & 44.37 & 0.18 \\ 20046 & 43.16 & 43.25 & 0.09 \\ 20092 & 43.59 & 43.81 & 0.22 \\ 20061 & 43.77 & 43.79 & 0.02 \\ 20154 & 43.02 & 43.09 & 0.07 \\ 20032 & 44.24 & 44.35 & 0.11 \\ 20143 & 44.1 & 44.22 & 0.12 \\ 20103 & 43.93 & 44.08 & 0.15 \\ 20020 & 44.07 & 44.08 & 0.01 \\ 19985 & 43.7 & 43.87 & 0.17 \\ 19886 & 43.52 & & \\ 19938 & 43.56 & & \end{array}$

\begin{tabular}{|c|c|c|c|c|c|c|c|c|c|}
\hline TEST No. & $\mathrm{pH}$ & F.45 LPE & $+F .45$ SOLN & $+\mathrm{DIH}_{+}+\mathrm{HNO}$ & F.45 OF & F50 LPE & +F5O SOLN & $+\mathrm{OIW}+\mathrm{HNO} 3$ & F5O DF \\
\hline $\begin{array}{l}T Z 1 \\
\text { TZ2 }\end{array}$ & $\begin{array}{l}11.09 \\
11.14\end{array}$ & $\begin{array}{l}11.05 \\
10.95\end{array}$ & $\begin{array}{l}12.96 \\
12.91\end{array}$ & $\begin{array}{l}10.12 \\
10.14\end{array}$ & $\begin{array}{l}6.2984 \\
6.1735\end{array}$ & $\begin{array}{r}11.1 \\
10.96\end{array}$ & $\begin{array}{l}12.02 \\
11.92\end{array}$ & $\begin{array}{l}5.11 \\
5.09\end{array}$ & $\begin{array}{l}6.5543 \\
6.3021\end{array}$ \\
\hline T23 & 10.93 & 10.98 & 14.01 & 10.01 & 4.3036 & & & & \\
\hline T24 & 11.21 & 10.98 & 12.81 & 10.06 & 6.4973 & 10.95 & 12.03 & 5.02 & 5.6481 \\
\hline$\tau 25$ & 11.34 & 11.05 & 13.82 & 10.13 & 4.657 & & & & \\
\hline TZ6 & 11.33 & 10.07 & 13.56 & 10.12 & 3.8997 & 10.95 & 11.38 & 5.12 & 12.907 \\
\hline 127 & 11.49 & 11.06 & 12.52 & 10.03 & 7.8699 & 10.92 & 11.99 & 10.05 & 10.393 \\
\hline TZ8 & 11.46 & 10.96 & 11.6 & 10.07 & 16.734 & 11.13 & 13.21 & 10.04 & 5.8269 \\
\hline TZ9 & 11.46 & 11.13 & 14.26 & 10.04 & 4.2077 & & & & \\
\hline TZ10 & 11.5 & 10.92 & 13.66 & 10.02 & 4.6569 & & & & \\
\hline TZ11 & 11.43 & 11.04 & 12.15 & 10 & 10.009 & 7.7 & 9.16 & 10 & 7.8493 \\
\hline TZ12 & 11.5 & 11.09 & 12.27 & 10 & 9.4746 & 8.03 & 9.47 & 10.02 & 7.9583 \\
\hline TZ13 & 11.62 & 10.77 & 12.64 & 14.9 & 8.9679 & 11.05 & 11.98 & 14.96 & 17.086 \\
\hline T214 & 11.69 & 10.91 & 12.84 & 15.03 & 8.7876 & 10.87 & 11.82 & 14.95 & 16.737 \\
\hline$T 215$ & 11.72 & 11 & 11.43 & 15.31 & 36.605 & 10.97 & 12.72 & 15.22 & 9.6971 \\
\hline TZ & 11.76 & 10.88 & 11.65 & 15.24 & 20.792 & 10.88 & 12.41 & 15.32 & 11.013 \\
\hline$T 217$ & & & & & & & & & \\
\hline
\end{tabular}


TEST No. TARGET TIME

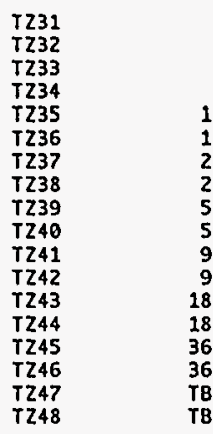

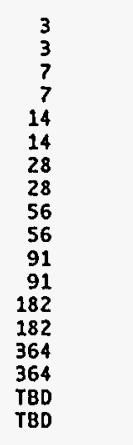

DATE IN

$7 / 8 / 91$

$7 / 8 / 91$
$6 / 26 / 91$
$6 / 26 / 91$

$6 / 26 / 91$

$6 / 26 / 91$

$6 / 26 / 91$

$6 / 26 / 91$

$6 / 26 / 91$

$6 / 26 / 9$

$6 / 26 / 9$

$6 / 26 / 91$

$6 / 26 / 91$

$6 / 26 / 91$

$7 / 166 / 92$

$$
\begin{gathered}
7 / 11 / 91 \\
7 / 1191 \\
7 / 3 / 91 \\
7 / 3191 \\
7 / 1091 \\
7 / 10 / 91 \\
7 / 24 / 91 \\
7 / 2491 \\
8 / 21 / 91 \\
8 / 2191 \\
9 / 25 / 91 \\
9 / 25 / 91 \\
12 / 2391 \\
12 / 23 / 91 \\
6 / 24 / 92 \\
6 / 24 / 92 \\
\end{gathered}
$$

TIME, d

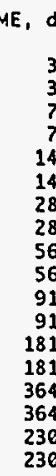

SAMPLE IN

5.0103
5.00162
5

5.0162
5.0152
5
5.0156
5.036

5.0151
5.0156
5.0136

5.0014
5.001
4.9993

5.001
4.9999

4.9999
5.015
5.0058

5.6058
4.9975

4.9975
5.0035

5.0035
5.0038

5.0038
5.0069
5.006

5.006
4.9911
EJ-13, 9

5.06
5.02
5.04
5.11
5.01
4.99
5.01
5
5.04
5.05
5.05
5.04
5.06
5.06
5.07
5.06
5.01
4.99
SA/V, /m

19804

19985
19901

19631
20014

20046

19964

19845

19802
19861

19864

19753
19777

19777
19739

19739

19984
20004
Friday, December 30 10:44 AM 1994

44.08
43.62
43.67
44.14
43.45
44.04
43.72
43.79
43.58
43.23
43.91
44.29
43.8
43.6
43.22
44.01
43.13
43.18

44.08
43.62
43.67
44.14
43.45
44.04
43.72
43.79
43.58
43.23
43.91
44.29
43.8
43.6
43.22
44.01
43.13
43.18

\begin{tabular}{|c|c|c|c|c|c|c|c|c|c|}
\hline TEST No. & pH & F.45 LPE & $+F .45$ SOLN & $+\mathrm{DIW}+\mathrm{HNO3}$ & F. $45 \mathrm{DF}$ & F50 LPE & +F50 SOLN & $+\mathrm{OIH}+\mathrm{HNO} 3$ & F50 DF \\
\hline TZ31 & 11.02 & 11.02 & 12.94 & 10.13 & 6.276 & 11.11 & 11.98 & 5.08 & 6.8391 \\
\hline TZ32 & 11.04 & 11.04 & 12.86 & 10.06 & 6.5275 & 10.96 & 11.92 & 5.09 & 6.3021 \\
\hline$T 233$ & 11.03 & 10.92 & 12.71 & 10.03 & 6.6034 & 10.89 & 11.99 & 5.02 & 5.5636 \\
\hline TZ34 & 21.01 & 10.98 & 14.01 & 10.05 & 4.3168 & & & & \\
\hline$T Z 35$ & 11.23 & 10.98 & 13.52 & 10.09 & 4.9724 & & & & \\
\hline$T 236$ & 11.28 & 10.87 & 13.32 & 10.14 & 5.1388 & 11.09 & 11.51 & 5.11 & 13.167 \\
\hline T237 & 11.37 & 11.02 & 12.04 & 10.21 & 11.01 & 11.13 & 12.91 & 10.05 & 6.6461 \\
\hline TZ38 & 11.38 & 10.98 & 11.94 & 10.11 & 11.531 & 11.09 & 12.83 & 10.09 & 6.7989 \\
\hline TZ39 & 11.37 & 10.94 & 13.84 & 10.02 & 4.4552 & & & & \\
\hline$T 240$ & 11.41 & 11.01 & 13.96 & 10.04 & 4.4034 & & & & \\
\hline TZ41 & 11.4 & 10.97 & 12.19 & 9.97 & 9.1721 & 7.75 & 9.18 & 10 & 7.993 \\
\hline$T 242$ & 11.38 & 11.07 & 12.4 & 9.96 & 8.4887 & 8.05 & 9.5 & 9.97 & 7.8759 \\
\hline$T 243$ & 11.63 & 11.03 & 12.99 & 15 & 8.6531 & 11.04 & 12.01 & 14.95 & 16.412 \\
\hline TZ44 & 11.66 & 10.93 & 12.89 & 14.98 & 8.6429 & 10.94 & 11.86 & 14.94 & 17.239 \\
\hline T245 & 11.09 & 10.86 & & & & 10.88 & 12.41 & 15.32 & 11.013 \\
\hline TZ46 & 11.75 & 10.97 & 11.52 & 15.18 & 28.6 & 10.88 & 12.58 & 15.35 & 10.029 \\
\hline
\end{tabular}


Table D.3. Cation Results

Column Heading Comment

TEST NUMER Test number in test matrix (Table D.1). Corresponds to unfiltered leachate in tests with monolithic samples and to leachates filtered through

$0.45 \mu \mathrm{m}$ filters in tests with crushed glass samples.

-UF Unfiltered solution.

$-\mathrm{F} 25$

Solution filtered using Amicon model CF25A filter.

$-\mathrm{F} 50$

Solution filtered using Amicon model CF50A filter.

RXN TIME

Actual reaction time, in days.

$\mathrm{pH}$

Leachate $\mathrm{pH}$ measured at room temperature.

DIL FACTOR

Dilution factor for solutions.

$\mathrm{SA} / \mathrm{V}$

Surface area-to-leachant volume ratio, in inverse meters.

LEACHANT

Mass of EJ-13 solution or deionized water added as leachant, in grams.

ACL NUMBER

Solution identification number.

i, ACL

Analytical result for species $i$ in submitted solution, in parts per million.

i, ppm

Concentration of species i corrected for dilution, in parts per million.

NL(i), g/m2

Normalized mass loss based on element $i$, in grams per square meter.

f(i)

Elemental weight fraction of species $i$ in the glass.

BKGND

Average concentration of species measured in blank tests, in parts per million. 


\begin{tabular}{|c|c|c|c|c|c|c|c|c|c|c|c|}
\hline TEST NUMBER & RXN TIME & $\mathrm{pH}$ & OIL FACTOR & SAN & LEACHANT VO & ACL NUMBER & Al, ACL & Al. $p p m$ & $N L(A L), g / m$ & B. $\mathrm{ACl}$ & B, ppm \\
\hline $\begin{array}{r}B \times 1 \\
B \times 2 \\
B \times 3 \\
8 \times 4 \\
8 \times 5 \\
8 \times 6 \\
8 \times 7 \\
8 \times 7-F 50 \\
B \times 8 \\
B \times 8-F 50 \\
B \times 9 \\
B \times 9-F 50 \\
B \times 10 \\
B \times 10-F 50\end{array}$ & $\begin{array}{r}7 \\
7 \\
28 \\
28 \\
94 \\
94 \\
238 \\
238 \\
600 \\
600\end{array}$ & $\begin{array}{l}8.21 \\
8.2 \\
8.28 \\
8.34 \\
8.26 \\
8.59 \\
8.43 \\
9.04 \\
8.57 \\
8.58\end{array}$ & $\begin{array}{r}1.694 \\
1.689 \\
1.6762 \\
1.707 \\
1.6726 \\
1.716 \\
1.5657 \\
2.805 \\
1.5219 \\
2.6755 \\
1.4838 \\
5.7874 \\
2.0096 \\
5.1468\end{array}$ & $\begin{array}{r}10.01 \\
10 \\
9.99 \\
10 \\
10 \\
10 \\
9.99 \\
10.02 \\
10.01 \\
10.01\end{array}$ & $\begin{array}{r}16.62 \\
16.66 \\
17.42 \\
16.9 \\
17.41 \\
16.48 \\
164 \\
15.99 \\
16.67 \\
16.93\end{array}$ & $\begin{array}{l}90-0561-01 \\
90-0561-02 \\
90-0561-03 \\
90-0561-04 \\
91-0093-05 \\
91-0093-06 \\
91-0346-01 \\
91-0346-03 \\
91-0346-02 \\
91-0346-04 \\
92-0353-01 \\
92-0353-02 \\
92-0353-03 \\
92-0353-04\end{array}$ & $\begin{array}{l}0.54 \\
0.52 \\
0.49 \\
0.42 \\
0.2 \\
0.3 \\
0.35 \\
0.21 \\
0.37 \\
0.22 \\
0.33 \\
-0.1 \\
0.26 \\
-0.1\end{array}$ & $\begin{array}{r}0.91476 \\
0.87828 \\
0.82134 \\
0.71694 \\
0.33452 \\
0.5148 \\
0.548 \\
0.58905 \\
0.5631 \\
0.58861 \\
0.48965 \\
-0.57874 \\
0.5225 \\
-0.51468\end{array}$ & $\begin{array}{r}1.7458 \\
1.5679 \\
1.2887 \\
0.7731 \\
-1.1107 \\
-0.22266 \\
-0.059197 \\
0.015255 \\
-0.34619 \\
-0.18456\end{array}$ & $\begin{array}{l}0.11 \\
0.1 \\
0.12 \\
0.11 \\
0.65 \\
0.13 \\
0.16 \\
0.13 \\
1.69 \\
1.06 \\
2.49 \\
0.62 \\
1.44 \\
0.55\end{array}$ & $\begin{array}{r}0.18634 \\
0.1689 \\
0.20114 \\
0.18777 \\
1.0872 \\
0.22308 \\
0.25051 \\
0.36465 \\
2.572 \\
2.836 \\
3.6947 \\
3.5882 \\
2.8938 \\
2.8307\end{array}$ \\
\hline $\begin{array}{r}\text { BKGND } \\
f(i)\end{array}$ & & & $\begin{array}{l}1 \\
1\end{array}$ & & & & $\begin{array}{r}0.56 \\
0.0203\end{array}$ & $\begin{array}{r}0.56 \\
0.0203\end{array}$ & & $\begin{array}{r}0.15 \\
0.0248\end{array}$ & $\begin{array}{r}0.15 \\
0.0248\end{array}$ \\
\hline TEST NO. & $N L(B), g / m 2$ & $\mathrm{Ba}, \mathrm{ACL}$ & Ba, ppm & $N L(B a), g / m$ & $\mathrm{Ca}, \mathrm{ACL}$ & $\mathrm{Ca}, \mathrm{ppm}$ & $N L(C a), g / m$ & $\mathrm{Cr}, \mathrm{ACL}$ & $\mathrm{cr}, \mathrm{ppm}$ & $\mathrm{NL}(\mathrm{Cr}), \mathrm{g} / \mathrm{m}$ & $\mathrm{Cu}, \mathrm{ACL}$ \\
\hline $\begin{array}{r}\text { BX1 } \\
\text { BX2 } \\
\text { BX3 } \\
8 \times 4 \\
\text { BX5 } \\
\text { BX6 } \\
\text { BX7 } \\
\text { BX7-F50 } \\
\text { BX8 } \\
\text { BX8-F50 } \\
\text { BX9 } \\
\text { BX9-F50 } \\
\text { BX10 } \\
\text { BX10-F50 }\end{array}$ & $\begin{array}{r}0.14639 \\
0.07621 \\
0.20643 \\
0.1523 \\
3.779 \\
0.29468 \\
0.4057 \\
9.7467 \\
14.279 \\
11.053\end{array}$ & $\begin{array}{l}-0.02 \\
-0.02 \\
-0.02 \\
-0.02 \\
-0.02 \\
-0.02 \\
-0.02 \\
-0.02 \\
-0.02 \\
-0.02 \\
0.02 \\
-0.02 \\
-0.02 \\
-0.02\end{array}$ & $\begin{array}{r}-0.03388 \\
-0.03378 \\
-0.033524 \\
-0.03414 \\
-0.033452 \\
-0.03432 \\
-0.031314 \\
-0.0561 \\
-0.030438 \\
-0.05351 \\
0.029676 \\
-0.11575 \\
-0.040192 \\
-0.10294\end{array}$ & $\begin{array}{r}-1.6923 \\
-1.689 \\
-1.6779 \\
-1.707 \\
-1.6726 \\
-1.716 \\
-1.5673 \\
0 \\
-1.5189 \\
0 \\
1.4823 \\
-2.0076\end{array}$ & $\begin{array}{l}3.16 \\
3.17 \\
3.24 \\
3.1 \\
2.65 \\
2.77 \\
2.29 \\
1.1 \\
2.09 \\
1.16 \\
1.76 \\
0.37 \\
2.05 \\
0.62\end{array}$ & $\begin{array}{r}5.353 \\
5.3541 \\
5.4309 \\
5.2917 \\
4.4324 \\
4.7533 \\
3.5855 \\
3.0855 \\
3.1808 \\
3.1036 \\
2.6115 \\
2.1413 \\
4.1197 \\
3.191\end{array}$ & $\begin{array}{r}9.096 \\
9.1178 \\
10.02 \\
8.3919 \\
-1.6001 \\
2.1316 \\
-11.46 \\
-16.122 \\
-22.751 \\
-5.2311\end{array}$ & $\begin{array}{l}-0.02 \\
-0.02 \\
-0.02 \\
-0.02 \\
-0.02 \\
-0.02 \\
-0.02 \\
-0.02 \\
-0.02 \\
-0.02 \\
-0.02 \\
-0.02 \\
-0.02 \\
-0.02\end{array}$ & $\begin{array}{r}-0.03388 \\
-0.03378 \\
-0.033524 \\
-0.03414 \\
-0.033452 \\
-0.03432 \\
-0.031314 \\
-0.0561 \\
-0.030438 \\
-0.05351 \\
-0.029676 \\
-0.11575 \\
-0.040192 \\
-0.10294\end{array}$ & $\begin{array}{r}-6.7692 \\
-6.756 \\
-6.7115 \\
-6.828 \\
-6.6904 \\
-6.864 \\
-6.2691 \\
0 \\
-6.0754 \\
0 \\
-5.9293 \\
-8.0304\end{array}$ & $\begin{array}{r}0.06 \\
0.2 \\
0.25 \\
0.06 \\
4.82 \\
0.26 \\
0.18 \\
-0.02 \\
0.34 \\
-0.02 \\
0.25 \\
-0.02 \\
0.28 \\
-0.02\end{array}$ \\
\hline $\begin{array}{l}\text { BKGND } \\
f(i)\end{array}$ & & 0.002 & 0.002 & & $\begin{array}{r}4.57 \\
0.0086\end{array}$ & $\begin{array}{r}4.57 \\
0.0086\end{array}$ & & $\begin{array}{r}0 \\
0.0005\end{array}$ & $\begin{array}{r}0 \\
0.0005\end{array}$ & & 0.0032 \\
\hline TEST NO. & $\mathrm{Cu}, \mathrm{ppm}$ & $N L(C u), g / m$ & $\mathrm{Fe}, \mathrm{ACL}$ & $\mathrm{Fe}, \mathrm{ppm}$ & $\mathrm{NL}(\mathrm{Fe}), \mathrm{g} / \mathrm{m}$ & $\mathrm{K}, \mathrm{ACL}$ & $\mathrm{K}, \mathrm{ppm}$ & $\mathrm{NL}(\mathrm{K}), g / \mathrm{mL}$ & $L i, A C L$ & Li, ppm & $N L(L i), g / m$ \\
\hline $\begin{array}{r}\text { BX1 } \\
8 \times 2 \\
\text { BX3 } \\
8 \times 4 \\
8 \times 5 \\
8 \times 6 \\
8 \times 6 \\
8 \times 7 \\
8 \times 7-850 \\
8 \times 8\end{array}$ & $\begin{array}{r}0.10164 \\
0.3788 \\
0.41905 \\
0.10242 \\
8.0619 \\
0.44616 \\
0.28183 \\
-0.0561 \\
0.51745\end{array}$ & $\begin{array}{r}3.1731 \\
10.556 \\
13.108 \\
3.2006 \\
251.94 \\
13.942 \\
8.8159 \\
0 \\
16.138\end{array}$ & $\begin{array}{r}0.03 \\
0.03 \\
0.03 \\
0.07 \\
0.21 \\
0.1 \\
0.02 \\
-0.02 \\
0.15\end{array}$ & $\begin{array}{r}0.05082 \\
0.05067 \\
0.050286 \\
0.11949 \\
0.35125 \\
0.1716 \\
0.031314 \\
-0.0561 \\
0.22829\end{array}$ & $\begin{array}{r}0.063621 \\
0.063496 \\
0.063078 \\
0.14974 \\
0.44016 \\
0.21504 \\
0.03928 \\
0.2855\end{array}$ & $\begin{array}{r}4.38 \\
4.46 \\
4.48 \\
4.42 \\
5.11 \\
4.32 \\
4.9 \\
6.78\end{array}$ & $\begin{array}{l}7.4197 \\
7.5329 \\
7.5094 \\
7.5449 \\
8.547 \\
7.4131 \\
7.6719 \\
10.318\end{array}$ & $\begin{array}{l}1.2276 \\
1.5953 \\
1.5205 \\
1.6341 \\
4.877 \\
1.2075 \\
2.0471 \\
10.589\end{array}$ & $\begin{array}{l}0.08 \\
0.08 \\
0.12 \\
0.11 \\
0.52 \\
0.15 \\
0.2 \\
\\
1.32\end{array}$ & $\begin{array}{r}0.13552 \\
0.13512 \\
0.20114 \\
0.18777 \\
0.86975 \\
0.2574 \\
0.31314 \\
2.0089\end{array}$ & $\begin{array}{r}0.43368 \\
0.43208 \\
0.768 \\
0.69934 \\
4.1612 \\
1.0528 \\
1.3371 \\
9.9238\end{array}$ \\
\hline $\begin{array}{r}8 \times 8-F 50 \\
\text { BX9 }\end{array}$ & $\begin{array}{r}-0.05351 \\
0.37095\end{array}$ & $\begin{array}{r}10.130 \\
11.581\end{array}$ & $\begin{array}{l}0.04 \\
0.18\end{array}$ & $\begin{array}{l}0.10702 \\
0.26708\end{array}$ & 0.3835 & $\begin{array}{l}6.18 \\
7.82\end{array}$ & 11.603 & $\begin{array}{l}10.389 \\
14.753\end{array}$ & $\begin{array}{l}1.32 \\
1.88\end{array}$ & $\begin{array}{l}2.6089 \\
2.7895\end{array}$ & $\begin{array}{l}9.9638 \\
13.892\end{array}$ \\
\hline $\begin{array}{r}B \times 9-F 50 \\
B \times 10 \\
B \times 10-F 50\end{array}$ & $\begin{array}{r}-0.11575 \\
0.56269 \\
-0.10294\end{array}$ & 17.566 & $\begin{array}{r}-0.02 \\
0.23 \\
-0.02\end{array}$ & $\begin{array}{r}-0.11575 \\
0.46221 \\
-0.10294\end{array}$ & 0.57863 & $\begin{array}{r}2.05 \\
5.15 \\
2.2\end{array}$ & $\begin{array}{l}11.864 \\
10.349 \\
11.323\end{array}$ & 10.699 & $\begin{array}{r}0.45 \\
1.05 \\
0.4\end{array}$ & $\begin{array}{l}2.6043 \\
2.1101 \\
2.0587\end{array}$ & 10.447 \\
\hline $\begin{array}{r}\text { BKGND } \\
f(i)\end{array}$ & 0.0032 & & $\begin{array}{r}0 \\
0.0798\end{array}$ & $\begin{array}{r}0 \\
0.0798\end{array}$ & & $\begin{array}{r}7.04 \\
0.0309\end{array}$ & $\begin{array}{r}7.04 \\
0.0309\end{array}$ & & $\begin{array}{r}0.05 \\
0.0197\end{array}$ & $\begin{array}{r}0.05 \\
0.0197\end{array}$ & \\
\hline
\end{tabular}




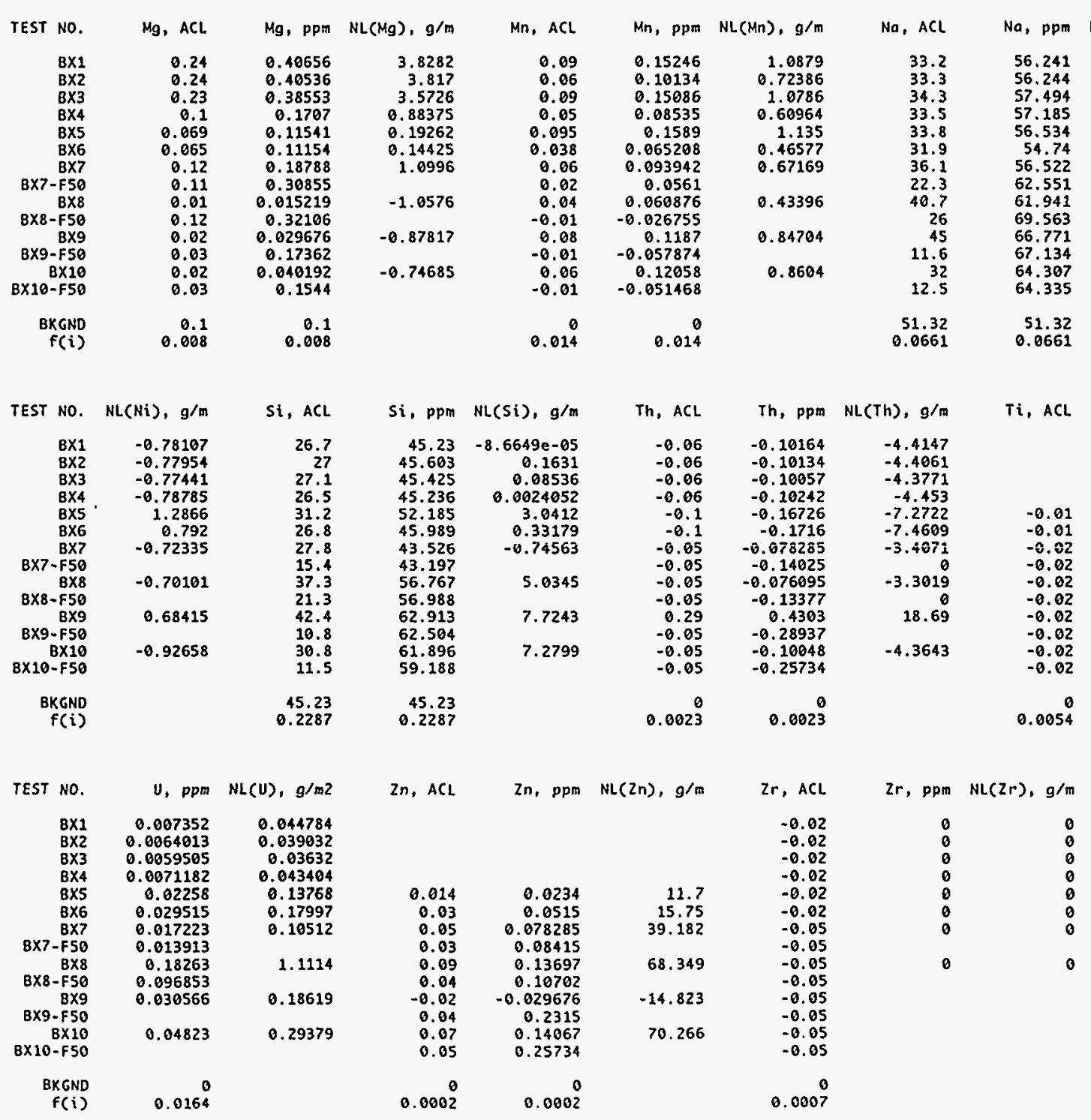

$\begin{array}{rrr}(N a), g / m & N i, A C L & N i, p p m \\ 7.437 & -0.03 & -0.05082 \\ 7.489 & -0.03 & -0.05067 \\ 9.3492 & -0.03 & -0.058286 \\ 8.8722 & -0.03 & -0.05121 \\ 7.8879 & 0.05 & 0.08363 \\ 5.1746 & 0.03 & 0.05148 \\ 7.8774 & -0.03 & -0.046971 \\ 16.037 & -0.03 & -0.08415 \\ 1637 & -0.03 & -0.045657 \\ 23.352 & -0.03 & -0.080265 \\ 19.628 & -0.03 & 0.044514 \\ & -0.03 & -0.17362 \\ & -0.03 & -0.060288 \\ & -0.03 & -0.1544 \\ & 0 & 0 \\ & 0.0065 & 0.0065\end{array}$


Friday, December $30 \quad$ 2:06 PM 1994

\begin{tabular}{|c|c|c|c|}
\hline TEST NO. & RXN TIME & pH & DIL FACTOR \\
\hline $\begin{array}{l}B Y 1 \\
B Y 2\end{array}$ & 3 & 9.27 & 2.3364 \\
\hline $\begin{array}{l}B Y 2 \\
B Y 3\end{array}$ & 3 & 9.3 & 2.3441 \\
\hline $\begin{array}{l}8 Y 3 \\
B Y 4\end{array}$ & 7 & $\begin{array}{l}9.48 \\
9.48\end{array}$ & $\begin{array}{l}2.3289 \\
2.3757\end{array}$ \\
\hline BYS & 14 & 9.77 & 2.3396 \\
\hline BY6 & 14 & 9.74 & 2.3568 \\
\hline BY7 & 30 & 9.94 & 2.3527 \\
\hline BY8 & 30 & 9.87 & 2.3518 \\
\hline BY9 & 70 & 10.09 & 2.2805 \\
\hline BY10 & 70 & 10.11 & 2.3765 \\
\hline BY11-UF & & & 9.6541 \\
\hline $\begin{array}{r}\text { BY11 } \\
\text { BY11 F50 }\end{array}$ & 140 & 10.02 & $\begin{array}{l}6.6709 \\
3.3648\end{array}$ \\
\hline $\begin{array}{c}\text { BY11-F50 } \\
\text { BY12-UF }\end{array}$ & & & $\begin{array}{l}3.3648 \\
9.6752\end{array}$ \\
\hline BY12 & 140 & 10.11 & 6.475 \\
\hline BY12-F50 & & & 3.3057 \\
\hline BY13 & 280 & 10.47 & 2.6038 \\
\hline & 280 & 10.45 & 2.5802 \\
\hline BY15-UF & & & 11.12 \\
\hline BY15 & 560 & 10.33 & 4.159 \\
\hline BY $15-F 50$ & & & 11.12 \\
\hline BY16-UF & & & 10.838 \\
\hline & 560 & 10.42 & \\
\hline BY $16-F 50$ & & & 10.838 \\
\hline $\begin{array}{r}8 Y 17 \\
\end{array}$ & 980 & 10.52 & 4.337 \\
\hline $\begin{array}{r}\text { BY17-F50 } \\
\text { BY18 }\end{array}$ & 980 & 10.65 & $\begin{array}{r}4.907 \\
4.2876\end{array}$ \\
\hline BY18-F50 & & & $\begin{array}{r}4.877 \\
4.877\end{array}$ \\
\hline $\begin{array}{c}\text { BKGND } \\
f(i)\end{array}$ & & & 1 \\
\hline
\end{tabular}

SA/V
1986
2049
1947
2046
2004
1967
2041
1983
1993
1981
1997
2004
1959
1996
2017
1999
1893
1990

$\begin{array}{rr}\text { LEACHANT } & \text { ACL NUMBER } \\ 10 & 91-0061-01 \\ 9.96 & 91-061-02 \\ 10 & 90-0513-06 \\ 9.99 & 90-0513-07 \\ 9.95 & 90-0487-18 \\ 10.05 & 90-0487-19 \\ 10.01 & 90-048-10 \\ 10.09 & 90-0487-11 \\ 10.29 & 91-0205-01 \\ 10.02 & 91-0205-02 \\ 9.96 & 91-0310-01 \\ 9.96-0310-02 \\ & 91-0310-03 \\ & 91-0310-04 \\ 10.22 & 91-0310-05 \\ & 91-0310-06 \\ 10.03 & 91-0346-05 \\ 10 & 91-0346-06 \\ & 92-0134-01 \\ 9.99 & 92-0134-02 \\ & 92-0134-03 \\ 10.02 & 92-0134-04 \\ 92-0134-05 \\ 10.01 & 92-0134-06 \\ \text { PACKET } & \text { PACKET } 1 \\ 10 & \text { PACKET. } \\ & \text { PACKET.1 }\end{array}$

Al, ACL

Al. ppm NL(Al). g/m2

B, ACL

B, ppm

0.67
0.69
1.03
1.03
1.15
1.19
1.35
1.36
0.86
1.2
0.49
0.56
0.66
0.48
0.56
0.72
1.41
1.35
0.41
0.93
0.16
0.46
0.89
0.16
0.337
0.457
0.338
0.286
0.56
0.0203

1.5654
1.6174
2.3988
2.447
2.6905
2.8046
3.1761
3.1984
1.9612
2.8518
4.7305
3.7357
2.2208
4.6441
3.625
2.3801
3.6714
3.483
4.5592
3.8679
1.7792
4.985
3.848
1.7341
1.462
2.242
1.449
1.395
0.56
0.0203

0.024938
0.025422

2.02

4.7195
4.2897

$\begin{array}{ll}3988 & 0.025422 \\ 0.046523\end{array}$

0.052372

0.056213
0.063143

0.065544

0.034634
0.05699

0.078337

0.075367

0.078238
0.072146

0.080788

0.081046

0.023473

0.022007

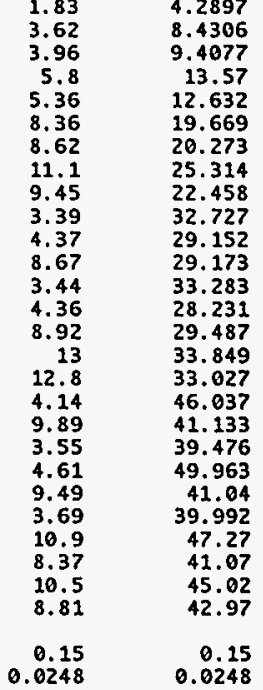


Page \#2 - "BY.CATIONS"

\begin{tabular}{|c|c|}
\hline \multicolumn{2}{|l|}{ TEST NO. } \\
\hline BYI & 09 \\
\hline BY2 & \\
\hline BY4 & 018 \\
\hline $8 Y 5$ & \\
\hline BY6 & \\
\hline 877 & \\
\hline $\begin{array}{l}\text { BY8 } \\
\text { BY9 }\end{array}$ & 56 \\
\hline BY10 & \\
\hline BY11-UF & \\
\hline $\begin{array}{r}8 Y 11 \\
\text { BY11-F50 }\end{array}$ & 0. \\
\hline BY12-UF & \\
\hline BY12 & \\
\hline $\begin{array}{r}\text { BY12-F50 } \\
\text { BY13 }\end{array}$ & \\
\hline $\begin{array}{l}\text { BY14 } \\
\text { BY13 }\end{array}$ & \\
\hline BY15-UF & \\
\hline $\begin{array}{r}\text { BY15 } \\
\text { BY15-F50 }\end{array}$ & \\
\hline BY16-UF & \\
\hline $\begin{array}{r}\text { BY16 } \\
\text { BY16-F50 }\end{array}$ & 0.8 \\
\hline & \\
\hline $\begin{array}{r}\text { BY17-F50 } \\
\text { BY18 }\end{array}$ & \\
\hline BY $18-F 50$ & \\
\hline $\begin{array}{l}\mathrm{BKGMI} \\
\mathrm{fCi}\end{array}$ & \\
\hline
\end{tabular}

Ba, ACL

Ba, pPM NL(Ba), g/m

$\begin{array}{lll}-0.02 & -0.046728 & -0.011764\end{array}$

$\begin{array}{lll}-0.01 & -0.023289 & -0.0059807 \\ -0.01 & -0.023757 & -0.0058057\end{array}$

$\begin{array}{lll}-0.01 & -0.023757 & -0.0058057 \\ -0.01 & -0.023396 & -0.0058373\end{array}$

$-0.01 \quad-0.023568 \quad-0.0059908$

$\begin{array}{lll}0.011 & 0.02588 & 0.00634 \\ 0.012 & 0.028222 & 0.0071159\end{array}$

$\begin{array}{lll}-0.02 & -0.04561 & -0.011443\end{array}$

$\begin{array}{lll}-0.02 & -0.04753 \quad-0.011996\end{array}$

$-0.02$

-0.02
-0.02

-0.02
-0.02

-0.02
-0.02
-0.02

-0.02
-0.02

-0.02
-0.02

-0.02
-0.02

-0.02
-0.02

-0.02
-0.02

-0.02
-0.00
0.00

0.009
0.004
0.01

0.003

0.002

$-0.033405$

$-0.1295$

0.06114

$-0.21676$

-0.08649
-0.21676

0.039

$0.043 \quad 0.010804$

$0.002^{\circ}$

$-0.03231$

$-0.013291$

$-0.02062$

$-0.021633$

Ca, ppm NL(CO), g/m

$\mathrm{Cr}, \mathrm{ACL}$

Cr, ppm NL(Cr), g/m

2:06 PM 1994

$\begin{array}{rrr}0.32 & 0.74765 & -0.2238 \\ 0.38 & 0.89076 & -0.20879 \\ 0.16 & 0.37262 & -0.25068 \\ 0.15 & 0.32635 & -0.23947 \\ 0.14 & 0.32754 & -0.24616 \\ 0.13 & 0.3038 & -0.25204 \\ 0.13 & 0.30585 & -0.24294 \\ 0.12 & 0.28222 & -0.25143 \\ 0.07 & 0.1593 & -0.25732 \\ 0.13 & 0.30894 & -0.25011 \\ -0.02 & -0.19388 & \\ 0.05 & 0.33354 & -0.24668 \\ 0.44 & 1.4805 & \\ -0.02 & -0.1935 & \\ 0.06 & 0.3885 & -0.24263 \\ 0.37 & 1.2231 & \\ 0.13 & 0.33849 & -0.25117 \\ 0.14 & 0.36123 & -0.24519 \\ 0.11 & 1.232 & \\ 0.34 & 1.4141 & -0.18194 \\ 0.37 & 4.1444 & \\ 0.1 & 1.0838 & \\ 0.26 & 1.1244 & -0.20043 \\ 0.73 & 7.9117 & \\ 0.884 & 3.834 & -0.045209 \\ 0.88 & 4.318 & \\ 0.929 & 3.984 & -0.034241 \\ 0.883 & 4.306 & \\ 4.57 & & \\ 0.0086 & 0.008 & \\ 0 & & \end{array}$

-0.02
-0.02
-0.02
-0.02
0.02
0.024
0.045
0.11
0.11
0.07
0.09
0.12
0.21
0.08
0.1
0.18
0.22
0.41
0.35
0.82
0.29
0.26
0.53
0.2
0.421
0.353
0.789
0.663
0.0005
0.000

$-0.046728 \quad-0.047057$

-0.047057
-0.045761
-0.047846

0.047846

0.046699

0.056563
0.10587

0.2587

0.25085
0.16635

0.057512
0.10374

0.26092

0.25174
0.16795

0.86887

0.80171

0.70661

0.77402

0.6475

0.59503

0.57284
1.0579

1.0579
3.892

3.892
3.4104
3.2248

3.2248
2.8179

2.8179
2.292
2.1676

1.826

1.732
3.383

0.80171

0.64621

0.58482

3.3816

2.2931

1.9292

3.4

0.0005

\section{$\mathrm{Cu}, \mathrm{ACL}$}

0.09
0.08

0.12

0.12
0.23
0.21

0.14
0.05

0.19
0.1

0.04

0.09

0.03

0.22
0.19
0.12

0.19
-8.02
0.19

-0.09
0.17
0.17

0.005

0.015
0.006 
Page \#3 - "BY. CATIONS"

\begin{tabular}{|c|c|c|}
\hline TEST NO. & $\mathrm{Cu}, \mathrm{ppm}$ & $N L(C u)$, \\
\hline BY1 & 0.21028 & 0.0330 \\
\hline BY2 & 0.18753 & 0.0286 \\
\hline BY3 & 0.23289 & 0.037 \\
\hline $8 Y 4$ & 0.28508 & 0.0435 \\
\hline BYS & 0.32754 & 0.0510 \\
\hline BY6 & 0.54206 & 0861 \\
\hline BY7 & 0.49407 & 0756 \\
\hline BY8 & 0.32925 & 0518 \\
\hline BY9 & 0.11402 & .0178 \\
\hline $\begin{array}{r}\text { BY10 } \\
\text { PY }\end{array}$ & 0.45153 & 0.071 \\
\hline BY11-UF & 0.96541 & \\
\hline $\begin{array}{r}\text { BY11 } \\
\text { BY11-F50 }\end{array}$ & $\begin{array}{l}0.60038 \\
0.13459\end{array}$ & \\
\hline BY12-UF & 0.77402 & \\
\hline & 0.58275 & \\
\hline $2-F 50$ & 0.099171 & \\
\hline BY13 & 0.57284 & \\
\hline $\begin{array}{r}\text { BY14 } \\
15-U F\end{array}$ & 0.49024 & \\
\hline $\begin{array}{r}15-0 F \\
\text { BY15 }\end{array}$ & $\begin{array}{l}1.3344 \\
0.79021\end{array}$ & \\
\hline BY $15-F 50$ & -0.2224 & \\
\hline BY16-UF & 0.97542 & \\
\hline & 0.73516 & 0.1 \\
\hline BY $16-F 50$ & -0.21676 & \\
\hline $\begin{array}{r}\text { BY17 } \\
\text { BY17-50 }\end{array}$ & 0.022 & 0.003 \\
\hline $\begin{array}{r}\text { BY17- } \\
\text { BY18 }\end{array}$ & $\begin{array}{l}0.074 \\
0.026\end{array}$ & 0.0046 \\
\hline BY18-F50 & 0.039 & \\
\hline BKGND & & \\
\hline$f(0$ & 0.003 & \\
\hline
\end{tabular}

$\begin{array}{rrr}\text { Fe, ACL } & \text { Fe, ppm } & \text { NL(Fe), g/m } \\ 0.18 & 0.42055 & 0.0026536 \\ 0.13 & 0.30473 & 0.0018637 \\ 0.51 & 1.1877 & 0.0076446 \\ 0.59 & 1.4017 & 0.0085848 \\ 0.88 & 2.0588 & 0.012874 \\ 0.73 & 1.7205 & 0.010961 \\ 1.35 & 3.1761 & 0.019501 \\ 1.46 & 3.4336 & 0.021698 \\ 0.33 & 0.75257 & 0.0047319 \\ 1.61 & 3.8262 & 0.024203 \\ 0.78 & 7.5302 & 0.06837 \\ 0.88 & 5.8704 & 0.036837 \\ 0.05 & 0.16824 & \\ 0.65 & 6.2889 & \\ 0.7 & 4.5325 & 0.028342 \\ 0.03 & 0.099171 & 0.034311 \\ 2.06 & 5.3638 & 0.034311 \\ 2.09 & 5.3926 & 0.033856 \\ 0.88 & 9.7856 & 0.050903 \\ 1.97 & 8.1932 & 0.05093 \\ -0.02 & -0.2224 & \\ 0.94 & 10.188 & \\ 1.84 & 7.9571 & 0.049881 \\ -0.02 & -0.21676 & \\ 0.235 & 1.019 & 0.0067456 \\ 0.009 & 0.044 & 0.0036 \\ 0.137 & 0.587 & 0.0036964 \\ -0.003 & -0.015 & \\ 0 & 0 & \\ 0.0798 & 0.0798 & \\ & & \end{array}$

X, ACL

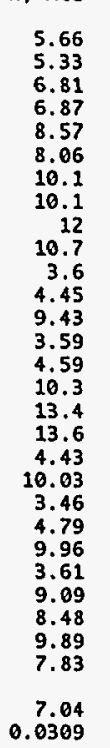

K, ppm $N L(K), g / m 2$

$13.224 \quad 0.10077$

$\begin{array}{rr}12.494 & 0.086143 \\ 15.86 & 0.1466\end{array}$

$16.321 \quad 0.1468$

$\begin{array}{rr}20.05 & 0.2101 \\ 18.996 & 0.19671\end{array}$

$\begin{array}{ll}18.996 & 0.19671 \\ 23.762 & 0.26515\end{array}$

$\begin{array}{ll}23.762 & 0.26515 \\ 23.753 & 0.27276\end{array}$

$\begin{array}{ll}27.366 & 0.33005 \\ 25.429 & 0.3004\end{array}$

$\begin{array}{ll}34.755 & \\ 29.686 & 0.36698\end{array}$

31.73
34.734

$\begin{array}{rl}39.72 & 0.36626\end{array}$

34.049
34.891

$\begin{array}{ll}34.891 & 0.46009 \\ 35.091 & 0.4548\end{array}$

$\begin{array}{ll}49.262 & \\ 41.715 & 0.55635\end{array}$

38.475

51.914

43.072
39.125

$39.42 \quad 0.5535$

$\begin{array}{ll}41.61 & 0.57521\end{array}$

38.19

7.04
0.0309
Friday, December 30 2:06 PM 2994

Li, ACl

Li, ppm NL(Li), g/m

$\begin{array}{rrr}3.6 & 8.411 & 0.21371 \\ 3.44 & 8.0637 & 0.19853 \\ 5.62 & 13.088 & 0.33993 \\ 5.83 & 13.85 & 0.34238 \\ 7.69 & 17.992 & 0.45446 \\ 7.36 & 17.346 & 0.44635 \\ 9.83 & 23.127 & 0.57395 \\ 9.85 & 23.165 & 0.59171 \\ 13.1 & 29.875 & 0.75963 \\ 12.1 & 28.756 & 0.73556 \\ 3.69 & 35.624 & \\ 4.56 & 30.419 & 0.77195 \\ 8.77 & 29.509 & \\ 3.65 & 35.314 & \\ 4.73 & 30.627 & 0.77451 \\ 9.13 & 30.181 & \\ 14.5 & 37.755 & 0.97701 \\ 14.3 & 36.897 & 0.93707 \\ 5.14 & 57.157 & 1.2024 \\ 11.5 & 47.828 & 1.2024 \\ 5.45 & 44.48 & \\ 5.56 & 60.259 & \\ 10.9 & 47.137 & 1.1957 \\ 4.08 & 44.219 & 1.35 \\ 11.7 & 50.74 & 1.3593 \\ 9.87 & 48.43 & 1.3114 \\ 12 & 51.46 & 1.3114 \\ 9.76 & 47.6 & \\ 0.05 & 0.05 & \\ 0.0197 & 0.0197 & \\ & & \end{array}$




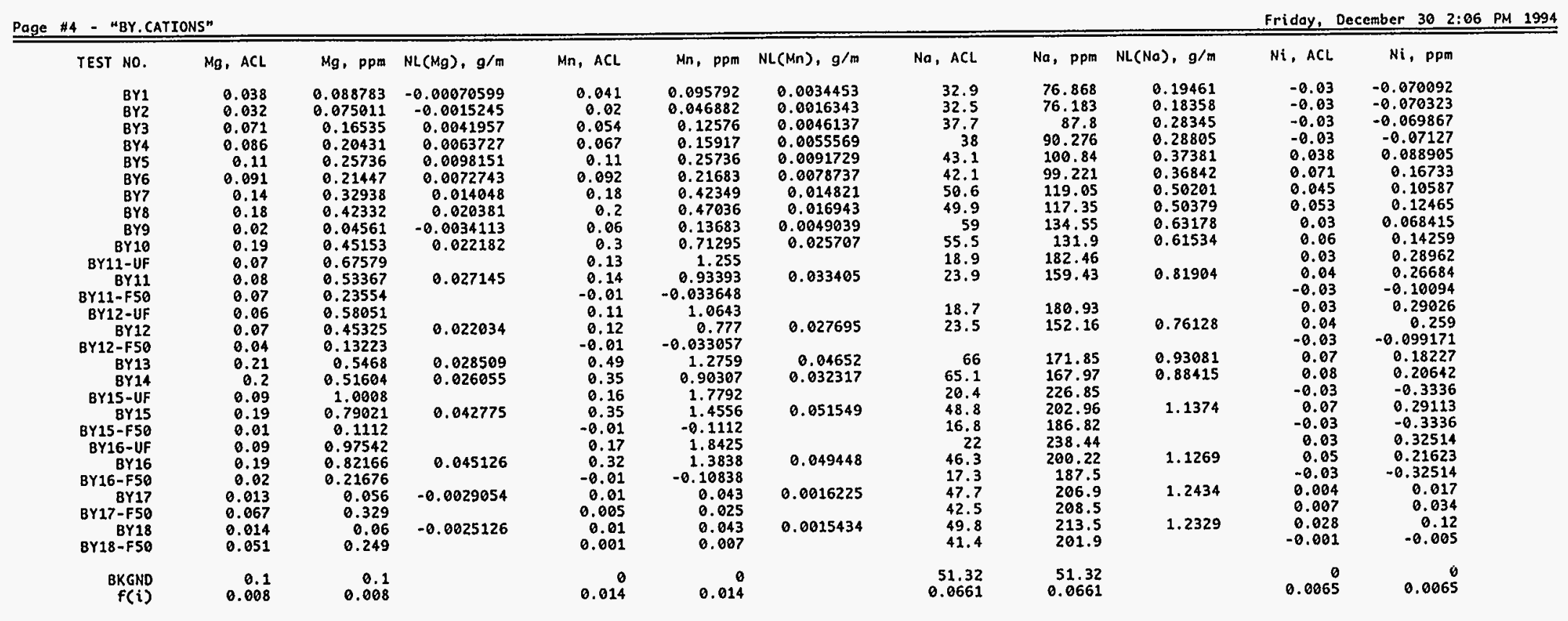


Page "

\begin{tabular}{|c|c|c|c|c|c|c|c|c|c|c|c|}
\hline TEST NO. & $N L(N i), g / m$ & Si, $\mathrm{ACL}$ & si, ppm & $N L(S i), g / m$ & Th, ACL & Th, ppm & $\mathrm{NL}(\mathrm{Th}), \mathrm{g} / \mathrm{m}$ & $T i, A C L$ & $T i, p p m$ & NL(Ti), g/m & U, ACL \\
\hline BY1 & -0.0054297 & 26.1 & 60.98 & 0.034677 & -0.1 & -0.23364 & -0.051149 & -0.01 & -0.023364 & -0.0021786 & 0.636 \\
\hline BYZ & -0.0052801 & 24.8 & 58.134 & 0.027536 & -0.1 & -0.23441 & -0.04974 & -0.02 & -0.023441 & -0.0021186 & 0.564 \\
\hline$B Y 3$ & -0.0055207 & 34.6 & 80.58 & 0.079388 & -0.2 & -0.46578 & -0.10401 & 0.027 & 0.06288 & 0.0059807 & 1.428 \\
\hline BY4 & -0.0053591 & 36 & 85.524 & 0.086114 & -0.2 & -0.47514 & -0.10097 & 0.035 & 0.083149 & 0.0075259 & 1.5 \\
\hline BYS & 0.0068252 & 43.3 & 101.3 & 0.12235 & -0.2 & -0.46792 & -0.10152 & 0.055 & 0.12868 & 0.011891 & 1.492 \\
\hline BY6 & 0.013088 & 40.6 & 95.686 & 0.11216 & -0.2 & -0.47136 & -0.10419 & 0.046 & 0.10841 & 0.010207 & 1.532 \\
\hline BY7 & 0.0079804 & 52.3 & 123.05 & 0.16671 & -0.2 & -0.47054 & -0.10024 & 0.079 & 0.18586 & 0.016864 & 1.08 \\
\hline BY8 & 0.0096703 & 52.4 & 123.23 & 0.172 & -0.2 & -0.47036 & -0.10313 & 0.091 & 0.21401 & 0.019986 & 1.066 \\
\hline BY9 & 0.0052812 & 64.8 & 147.78 & 0.22498 & 0.07 & 0.15963 & 0.034825 & 0.03 & 0.068415 & 0.006357 & 1.047 \\
\hline BY10 & 0.011074 & 62.4 & 148.29 & 0.22749 & 0.1 & 0.23765 & 0.052159 & 0.1 & 0.23765 & 0.022216 & 0.92 \\
\hline BY11-UF & & 18.6 & 179.57 & & -0.05 & -0.48271 & & 0.04 & 0.38616 & & 0.392 \\
\hline BY11 & 0.020557 & 22.8 & 152.1 & 0.23399 & -0.05 & -0.33354 & -0.072619 & 0.05 & 0.33354 & 0.03093 & 0.462 \\
\hline BY11-F50 & & 42.7 & 143.68 & & & -0.16824 & & -0.02 & -0.067296 & & 0.747 \\
\hline BY12-UF & 0.019883 & $\begin{array}{l}18 \\
23\end{array}$ & $\begin{array}{l}174.15 \\
148.93\end{array}$ & & $\begin{array}{l}-0.05 \\
-0.05\end{array}$ & $\begin{array}{l}-0.48376 \\
-0.32375\end{array}$ & & 0.04 & 0.38701 & & 0.395 \\
\hline $\begin{array}{r}\text { BY12 } \\
\text { BY12-F50 }\end{array}$ & 0.019883 & $\begin{array}{l}23 \\
45\end{array}$ & $\begin{array}{l}148.93 \\
148.76\end{array}$ & 0.22625 & $\begin{array}{l}-0.05 \\
-0.05\end{array}$ & $\begin{array}{l}-0.32375 \\
-0.16529\end{array}$ & -0.07024 & $\begin{array}{r}0.05 \\
-0.02\end{array}$ & $\begin{array}{r}0.32375 \\
-0.066114\end{array}$ & 0.029917 & 0.481 \\
\hline BY13 & 0.014314 & 69.4 & 180.7 & 0.30238 & 0.11 & 0.28642 & 0.063568 & 0.13 & $\begin{array}{r}-0.066114 \\
0.33849\end{array}$ & 0.031998 & $\begin{array}{r}0.824 \\
1.4795\end{array}$ \\
\hline BY14 & 0.01591 & 69.5 & 179.32 & 0.29375 & 0.11 & 0.28382 & 0.061824 & 0.13 & 0.33543 & 0.03112 & 1.413 \\
\hline BY15-UF & & 22.2 & 246.86 & & -0.05 & -0.556 & & 0.05 & 0.556 & & 0.597 \\
\hline BY15 & 0.022206 & 52.6 & 218.76 & 0.37619 & 0.07 & 0.29113 & 0.062756 & 0.12 & 0.49908 & 0.045822 & 1.374 \\
\hline BY15-F50 & & 18.2 & 202.38 & & -0.05 & -0.556 & & -0.02 & -0.2224 & & 0.393 \\
\hline BY16-UF & 0.036641 & $\begin{array}{l}25.6 \\
51.8\end{array}$ & $\begin{array}{l}277.45 \\
224.01\end{array}$ & 0.39105 & -0.05 & $\begin{array}{r}-0.5419 \\
0.30272\end{array}$ & 0.065841 & 0.05 & $\begin{array}{r}0.5419 \\
0.43245\end{array}$ & 0.040062 & 0.64 \\
\hline $\begin{array}{r}\text { BY16 } \\
\text { BY16-F50 }\end{array}$ & 0.010041 & $\begin{array}{l}51.8 \\
19.2\end{array}$ & $\begin{array}{l}224.01 \\
208.09\end{array}$ & & $\begin{array}{l}0.07 \\
-0.05\end{array}$ & $\begin{array}{l}0.30272 \\
-0.5419\end{array}$ & 0.065841 & $\begin{array}{c}0.1 \\
-0.02\end{array}$ & $\begin{array}{r}0.43245 \\
-0.21666\end{array}$ & 0.040062 & $\begin{array}{l}1.237 \\
0.379\end{array}$ \\
\hline $\begin{array}{r}\text { BY17 } \\
\text { BY } 17-550\end{array}$ & 0.0013816 & $\begin{array}{l}52.9 \\
44.8\end{array}$ & 229.4 & 0.4254 & 0.044 & $\begin{array}{r}0.191 \\
0.054 \\
0.054\end{array}$ & 0.043869 & $\begin{array}{l}0.02 \\
0.006\end{array}$ & $\begin{array}{r}0.087 \\
0.089 \\
0.029\end{array}$ & 0.0085109 & 1,11 \\
\hline $\begin{array}{r}\text { BY17-F50 } \\
\text { BY18 }\end{array}$ & 0.0092772 & $\begin{array}{l}44.8 \\
53.1\end{array}$ & $\begin{array}{l}219.8 \\
227.7\end{array}$ & 0.40093 & $\begin{array}{l}0.011 \\
0.043\end{array}$ & $\begin{array}{l}0.054 \\
0.187\end{array}$ & 0.040856 & $\begin{array}{r}0.006 \\
0.02\end{array}$ & $\begin{array}{l}0.029 \\
0.086\end{array}$ & 0.008003 & $\begin{array}{l}0.906 \\
1.223\end{array}$ \\
\hline B) & & 43.6 & 212.6 & & 0.009 & 0.44 & & 0.005 & 0.024 & & 1.024 \\
\hline $\begin{array}{c}\text { BKGND } \\
f(i)\end{array}$ & & $\begin{array}{r}45.23 \\
0.2287\end{array}$ & $\begin{array}{r}45.23 \\
0.2287\end{array}$ & & 0 & 00 & & $\begin{array}{r}0 \\
0.0054\end{array}$ & $\begin{array}{r}0 \\
0.0054\end{array}$ & & 0 \\
\hline
\end{tabular}




\begin{tabular}{|c|c|c|c|c|c|c|c|c|c|}
\hline Page $\mathrm{N6}-\mathrm{BY} . \mathrm{C}$ & & & & & & & & & Friday, Decembe \\
\hline TEST NO. & U, ppm & $\mathrm{NL}(U), g / m 2$ & $\mathrm{Zn}, \mathrm{ACl}$ & $z n, p p m$ & $N L(2 n), g / m$ & $\mathrm{Zr}, \mathrm{ACL}$ & $2 r, p p m$ & $N L(2 r), g / m$ & \\
\hline $8 Y_{1}$ & 1.486 & 0.045623 & -0.01 & -0.023364 & -0.058822 & -0.02 & -0.046728 & -0.033612 & \\
\hline Byz & 1.3221 & 0.039343 & -0.01 & -0.023441 & -0.057201 & -0.02 & -0.046882 & -0.032686 & \\
\hline $8 Y 3$ & 3.3257 & 0.10415 & & & & -0.02 & -0.046578 & -0.034176 & \\
\hline $8 Y 4$ & 3.5635 & 0.1662 & & & & -0.02 & -0.047514 & -0.033175 & \\
\hline BY5 & 3.4907 & 0.10621 & & & & -0.02 & -0.046792 & -0.033356 & \\
\hline BY6 & 3.6106 & 0.11193 & & & & -0.02 & -0.047136 & -0.034233 & \\
\hline BY7 & 2.5409 & 0.075911 & & & & -0.02 & -0.047054 & -0.032935 & \\
\hline BY8 & 2.507 & 0.077089 & & & & 0.02 & 0.047036 & 0.033885 & \\
\hline BY9 & 2.3877 & 0.073051 & -0.02 & -0.04561 & -0.11443 & -0.05 & -0.11402 & -0.081732 & \\
\hline BY10 & 2.2864 & 0.067297 & 0.02 & 0.04753 & 0.11996 & -0.05 & -0.11882 & -0.085689 & \\
\hline BY11-UF & 3.7844 & & -0.02 & -0.19308 & & -0.05 & -0.48271 & & \\
\hline BY11 & 3.082 & 0.094103 & -0.02 & -0.13342 & -0.33405 & -0.05 & -0.33354 & -0.2386 & \\
\hline BY11-F50 & $\begin{array}{l}2.5135 \\
3.8217\end{array}$ & & 0.05 & 0.16824 & & -0.05 & -0.16824 & & \\
\hline BY12-UF & 3.8217 & & -0.02 & -0.1935 & & -0.05 & -0.48376 & & \\
\hline $\begin{array}{r}\text { BY12 } \\
\text { BY12-F50 }\end{array}$ & $\begin{array}{l}3.1145 \\
2.7239\end{array}$ & 0.094764 & $\begin{array}{l}-0.02 \\
-0.02\end{array}$ & -0.1295 & -0.3231 & -0.05 & -0.32375 & -0.23079 & \\
\hline $\begin{array}{l}\text { BY13 } \\
0\end{array}$ & 3.8523 & 0.11991 & $\begin{array}{r}-6.02 \\
0.02\end{array}$ & $\begin{array}{r}-6.066114 \\
0.052076\end{array}$ & 0.13291 & $\begin{array}{l}-6.65 \\
-0.05\end{array}$ & $\begin{array}{l}-0.16529 \\
-0.13019\end{array}$ & -0.094939 & \\
\hline BY14 & 3.6458 & 0.11138 & $\begin{array}{l}-0.02 \\
-0.02\end{array}$ & -0.051604 & -0.12927 & -0.05 & -0.12901 & -0.092335 & \\
\hline BY15-UF & 6.6386 & & -0.02 & -0.2224 & & -0.05 & -0.556 & & \\
\hline $\begin{array}{r}\text { BY15 } \\
\text { BY15-F50 }\end{array}$ & $\begin{array}{l}5.7145 \\
4.3702\end{array}$ & 0.17275 & $\begin{array}{l}0.04 \\
0.03\end{array}$ & $\begin{array}{r}0.16636 \\
0.3336\end{array}$ & 0.41239 & -0.05 & -0.26795 & -0.14728 & \\
\hline BY16-UF & 6.9363 & & $\begin{array}{l}-0.02 \\
-0.02\end{array}$ & $\begin{array}{l}0.21676 \\
-0.21676\end{array}$ & & $\begin{array}{l}-6.63 \\
-0.05\end{array}$ & $\begin{array}{r}-0.536 \\
-0.5419\end{array}$ & & \\
\hline BY16 & 5.3494 & 0.16317 & 0.02 & 0.08649 & 0.21633 & -0.05 & -0.21623 & -0.15452 & \\
\hline BY $16-F 50$ & 4.1076 & & 0.09 & 0.97542 & & -0.05 & -0.5419 & & \\
\hline $\begin{array}{r}\text { BY17 } \\
\text { BY17-F50 }\end{array}$ & $\begin{array}{l}4.814 \\
4.446\end{array}$ & 0.15506 & $\begin{array}{r}0.019 \\
0.07\end{array}$ & $\begin{array}{l}0.082 \\
0.343\end{array}$ & 0.21659 & 0.082 & 0.006 & 0.004528 & \\
\hline BY18 & $\begin{array}{l}4.446 \\
5.244\end{array}$ & 0.16068 & $\begin{array}{r}0.07 \\
0.039\end{array}$ & $\begin{array}{l}0.343 \\
0.167\end{array}$ & 0.4196 & $\begin{array}{l}0.343 \\
0.167\end{array}$ & $\begin{array}{l}0.001 \\
0.006\end{array}$ & 0.0043073 & \\
\hline$B Y 18-F 50$ & 4.994 & & 0.03 & 0.146 & & 0.146 & 0.001 & & \\
\hline $\begin{array}{r}\text { BKGND } \\
f(i)\end{array}$ & 0.0164 & & 0.92 & 0 & & 00 & 20 & & \\
\hline
\end{tabular}


Page \#1 - "BZ.CATIONS"

\begin{tabular}{|c|c|c|c|c|c|c|c|c|c|c|c|}
\hline TEST NUMBER & RXN TIME & $\mathrm{pH}$ & DIL FACTOR & SA/V & LEACHANT VO & ACL NUMBER & $\mathrm{Al}, \mathrm{ACL}$ & Al, ppm & NL(Al), $g / m$ & B. $A C L$ & B, ppm \\
\hline 821 & 3 & 10.5 & 7.5238 & 20089 & 4.99 & $91-0061-03$ & 0.72 & 5.4171 & 0.01191 & 8.18 & 61.545 \\
\hline 822 & 3 & 10.62 & $\begin{array}{r}7.3333 \\
5\end{array}$ & 19607 & 5.03 & 91-0061-04 & 0.75 & $\begin{array}{r}5.5 \\
8.6084\end{array}$ & 0.012411 & 8.65 & 63.433 \\
\hline 823 & 7 & 10.73 & $\begin{array}{r}5.7009 \\
5.552\end{array}$ & $\begin{array}{l}20072 \\
19820\end{array}$ & 5.01 & $\begin{array}{l}90-0513-08 \\
90-0513-09\end{array}$ & 1,51 & $\begin{array}{l}8.6084 \\
8.328\end{array}$ & $\begin{array}{l}0.019752 \\
0.019307\end{array}$ & $\begin{array}{l}14.9 \\
\text { is }\end{array}$ & $\begin{array}{r}84.943 \\
83.28\end{array}$ \\
\hline $\begin{array}{l}\text { B24 } \\
\text { B25 }\end{array}$ & $\begin{array}{l}7 \\
14\end{array}$ & $\begin{array}{l}10.48 \\
10.98\end{array}$ & $\begin{array}{l}5.552 \\
7.7444\end{array}$ & $\begin{array}{l}198<6 \\
19902\end{array}$ & $\begin{array}{l}5.01 \\
4.97\end{array}$ & $90-0487-20$ & $\begin{array}{l}1.3 \\
1.5\end{array}$ & 11.617 & 0.027367 & $\begin{array}{r}15 \\
12.6\end{array}$ & $\begin{array}{r}83.28 \\
97.579\end{array}$ \\
\hline B26 & 14 & 11.01 & 7.6245 & 19997 & 4.98 & $90-0487-21$ & 1.48 & 11.284 & 0.026418 & 13.1 & 99.881 \\
\hline B27 & 28 & 11.02 & 9.3039 & 20297 & 4.95 & $90-0487-12$ & 0.25 & 2.326 & 0.004286 & 13.5 & 125.6 \\
\hline 828 & 28 & 11.1 & 11 & 20047 & 5.02 & $90-0487-13$ & 0.59 & 6.49 & 0.014572 & 11 & 121 \\
\hline 829 & 56 & 11.24 & 11.386 & 19957 & 4.99 & $91-0134-10$ & 0.16 & 1.8218 & 0.0031145 & 13.6 & 154.85 \\
\hline 8210 & 56 & 11.33 & 11.451 & 20034 & 5.01 & $91-0134-09$ & 0.2 & & 0.0042545 & 13.5 & 154.59 \\
\hline B211 & 98 & 11.26 & 10.331 & 20132 & 4.97 & $91-0205-05$ & 0.24 & 1.4464 & 0.0021689 & 18.4 & 190.1 \\
\hline BZ12 & 98 & 11.43 & 10.114 & 20015 & 4.98 & $91-0205-06$ & 0.14 & 1.416 & 0.0021067 & 17.5 & 177 \\
\hline $\begin{array}{r}B Z 13 \\
B Z 13-F 50\end{array}$ & 182 & 10.92 & & 19963 & 4.98 & 91-0346-07 & -0.1 & -1.9862 & -0.0062831 & 13.1 & 260.19 \\
\hline $\begin{array}{r}\text { BZ13-F50 } \\
\text { BZ14 }\end{array}$ & 182 & 1139 & $\begin{array}{l}4.8358 \\
16.792\end{array}$ & & & $\begin{array}{r}91-0346-09 \\
91-0346-08\end{array}$ & 0.22 & 1.0639 & & 57.9 & 279.99 \\
\hline $\begin{array}{r}B 214 \\
B 214-F 50\end{array}$ & 182 & 11.29 & $\begin{array}{l}16.0792 \\
6.0792\end{array}$ & 20168 & 5 & $\begin{array}{l}91-0346-88 \\
91-0346-10\end{array}$ & $\begin{array}{r}-0.1 \\
0.2\end{array}$ & -1.6792 & -0.0054693 & $\begin{array}{l}17.1 \\
50.5\end{array}$ & $\begin{array}{r}287.14 \\
307\end{array}$ \\
\hline BZ15 & 364 & 12.03 & 14.636 & 20008 & 5 & $91-0481-01$ & 0.11 & 1.61 & 0.0025851 & 220 & 3219.9 \\
\hline BZ15-F50 & & & 11.402 & & & $91-0481-03$ & -0.1 & -1.1402 & & 273 & 3112.7 \\
\hline $\begin{array}{r}\text { BZ16 } \\
\text { BZ16-F50 }\end{array}$ & 364 & 12.02 & $\begin{array}{r}15.89 \\
11.479\end{array}$ & 20088 & 5 & $\begin{array}{l}91-0481-02 \\
91-0481-04\end{array}$ & -0.12 & $\begin{array}{r}-1.589 \\
1.3775\end{array}$ & -0.0052699 & 164 & 2606 \\
\hline $\begin{array}{r}B 217 \\
\text { BZ17 }\end{array}$ & 728 & 11.96 & 21.658 & 20000 & 5.02 & $\begin{array}{l}91-0481-64 \\
92-0388-05\end{array}$ & $\begin{array}{l}0.12 \\
-0.1 \\
-0.1\end{array}$ & $\begin{array}{r}1.3775 \\
-2.1658\end{array}$ & -0.0067138 & $\begin{array}{l}217 \\
392\end{array}$ & $\begin{array}{l}2490.9 \\
8489.9\end{array}$ \\
\hline$B 217-F 50$ & & & 12.641 & & & $92-0388-06$ & -0.1 & -1.2641 & & 664 & 8393.6 \\
\hline$B 218$ & 728 & 11.97 & 22.153 & 20000 & 5.02 & $92-0388-07$ & -0.1 & -2.2153 & -0.0068357 & 395 & 8750.4 \\
\hline $\begin{array}{r}B Z 18-F 50 \\
B Z 19\end{array}$ & 504 & 11.86 & $\begin{array}{l}10.263 \\
20.494 \\
11.821\end{array}$ & 20000 & 5.03 & $\begin{array}{l}92-0388-08 \\
92-0388-09 \\
92-0388-10\end{array}$ & $\begin{array}{l}-0.1 \\
-0.1 \\
-0.1\end{array}$ & $\begin{array}{l}-1.0263 \\
-2.0494 \\
-1.1821\end{array}$ & -0.0064271 & $\begin{array}{l}703 \\
285 \\
491\end{array}$ & $\begin{array}{l}7214.9 \\
5840.8 \\
5804.1\end{array}$ \\
\hline $\begin{array}{c}\text { BKGND } \\
f(i)\end{array}$ & & & $\begin{array}{l}1 \\
1\end{array}$ & & & & $\begin{array}{r}0.56 \\
0.0203\end{array}$ & $\begin{array}{r}0.56 \\
0.0203\end{array}$ & & $\begin{array}{r}0.15 \\
0.0248\end{array}$ & $\begin{array}{r}0.15 \\
0.0248\end{array}$ \\
\hline
\end{tabular}




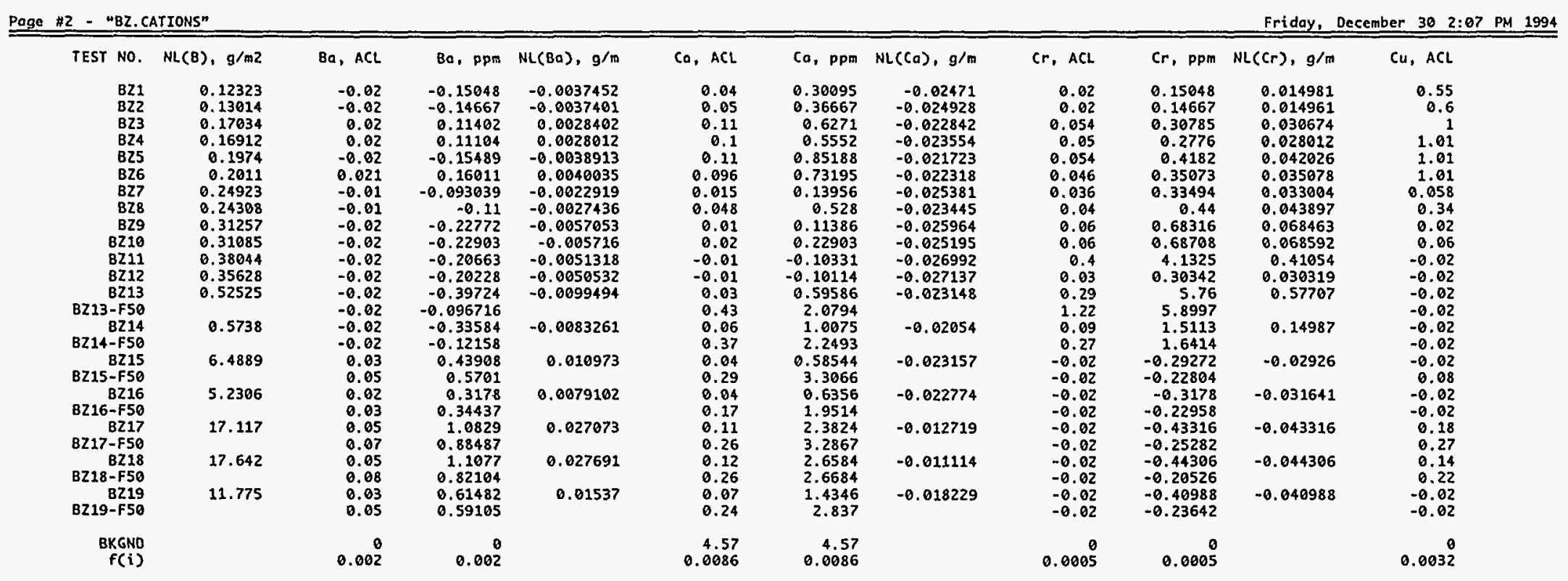


Page "3 - "BZ. CATIONS"

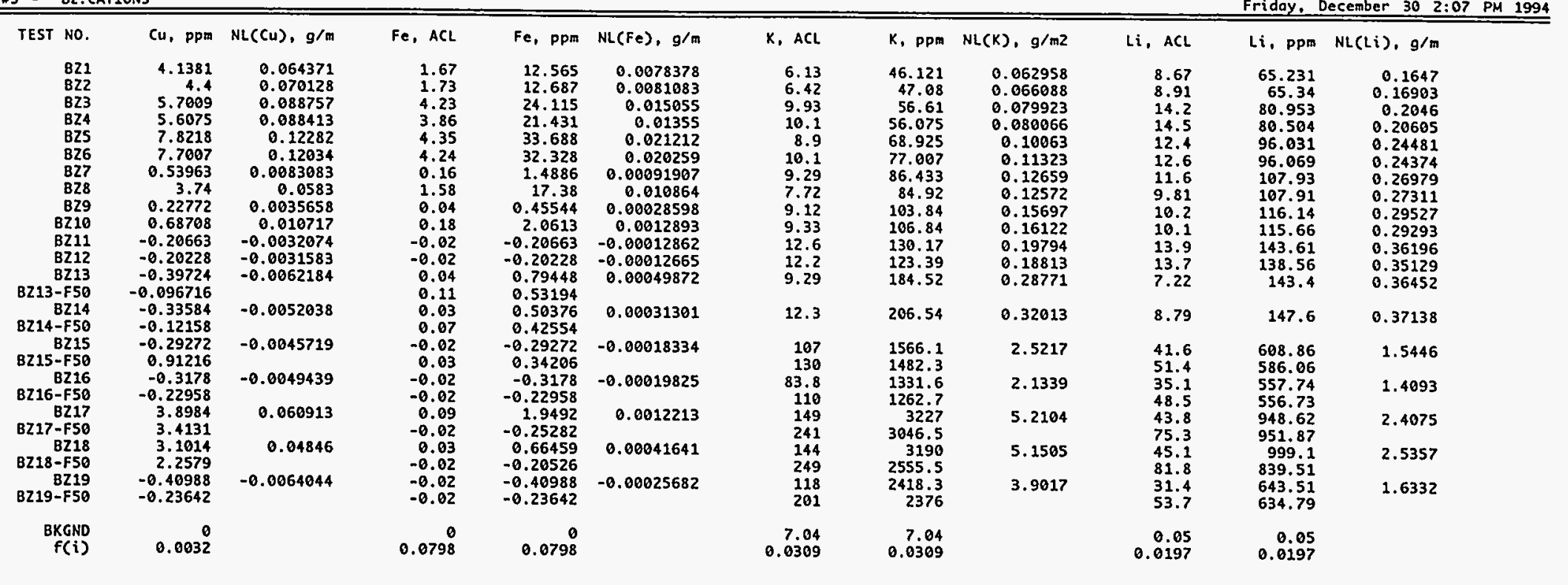




\begin{tabular}{|c|c|c|c|}
\hline TEST NO. & $\mathrm{Mg}, \mathrm{ACL}$ & $\mathrm{Mg}, \mathrm{ppm}$ & $N L(M g), g / m$ \\
\hline 82. & 0.262 & 1.9712 & 0.011643 \\
\hline $\begin{array}{l}B 22 \\
B 23\end{array}$ & 0.282 & 2.068 & 12546 \\
\hline $\begin{array}{l}B 23 \\
B 24\end{array}$ & 0.66 & $\begin{array}{l}3.7626 \\
3.7867\end{array}$ & 0.022809 \\
\hline $\begin{array}{l}824 \\
\text { BZ5 }\end{array}$ & 0.61 & 3.3867 & 0.020729 \\
\hline BZ6 & 0.63 & 4.879 & 0.030016 \\
\hline$B Z 7$ & 0.021 & $\begin{array}{r}4.8034 \\
0.19538\end{array}$ & 0.029401 \\
\hline BZ8 & 0.21 & $\begin{array}{r}0.1938 \\
2.31\end{array}$ & $\begin{array}{r}0.00058741 \\
0.01378\end{array}$ \\
\hline BZ9 & & -0.11386 & $\begin{array}{r}0.01378 \\
-0.013395\end{array}$ \\
\hline$B 210$ & & & -0.0013395 \\
\hline B211 & & -0.10331 & 0.00080506 \\
\hline BZ12 & & -0.16331 & -0.0012624 \\
\hline B213 & 0.02 & -0.16114 & -0.0012562 \\
\hline$B 213-F 50$ & $\begin{array}{l}0.02 \\
0.16\end{array}$ & $\begin{array}{l}0.39724 \\
0.77373\end{array}$ & 0.0018612 \\
\hline B214 & 0.04 & 0.67168 & 0.0035432 \\
\hline$B 214-F 50$ & 0.14 & 0.85109 & \\
\hline 8215 & -0.01 & -0.14636 & -0.0015391 \\
\hline$B Z 15-F 50$ & 0.04 & 0.45608 & \\
\hline 8216 & -0.01 & -0.1589 & -0.001611 \\
\hline $8 Z 16-F 50$ & 0.02 & 0.22958 & \\
\hline 8217 & -0.01 & -0.21658 & -0.0019786 \\
\hline $8217-F 50$ & 0.01 & 0.12641 & \\
\hline BZ18 & -0.01 & -0.22153 & -0.0020096 \\
\hline BZ18-F50 & 0.01 & 0.10263 & \\
\hline $\begin{array}{r}8219 \\
8 Z 19-F 50\end{array}$ & $\begin{array}{r}-0.01 \\
0.02\end{array}$ & $\begin{array}{r}-0.20494 \\
0.23642\end{array}$ & -0.0019059 \\
\hline & & & \\
\hline $\begin{array}{c}\text { BXGND } \\
f(i)\end{array}$ & $\begin{array}{r}0.1 \\
0.008\end{array}$ & $\begin{array}{r}0.1 \\
0.008\end{array}$ & \\
\hline
\end{tabular}

\begin{tabular}{|c|c|c|}
\hline $\mathrm{Mn}, \mathrm{ACL}$ & $M_{n}, p p m$ & $N L(M n), g / m$ \\
\hline 0.457 & 3.4384 & 0.012226 \\
\hline 0.474 & $\begin{array}{r}3.4576 \\
3.476\end{array}$ & 0.012663 \\
\hline 1.21 & 6.8981 & $\begin{array}{l}0.024548 \\
0.024\end{array}$ \\
\hline 1.13 & 6.2738 & 0.02261 \\
\hline $\begin{array}{l}1.23 \\
1.23\end{array}$ & 9.5256 & 0.034188 \\
\hline 1.24 & 9.4544 & 0.033771 \\
\hline 0.038 & 0.35355 & 0.0012442 \\
\hline 0.41 & 4.51 & 0.016069 \\
\hline 0.01 & 0.11386 & 0.00040752 \\
\hline 0.04 & 0.45806 & 0.0016331 \\
\hline-0.01 & $\begin{array}{r}-0.10331 \\
-\end{array}$ & -0.00036656 \\
\hline-0.01 & -0.10114 & -0.0003609 \\
\hline-0.01 & -0.19862 & -0.0007106 \\
\hline-0.01 & -0.048358 & \\
\hline-0.01 & -0.16792 & -0.00059 \\
\hline-0.01 & -0.060792 & \\
\hline-0.01 & -0.14636 & -0.0005225 \\
\hline-0.01 & -0.11402 & \\
\hline-0.01 & -0.1589 & -0.0005650 \\
\hline-0.01 & -0.11479 & \\
\hline-0.01 & -0.21658 & -0.000773 \\
\hline-0.01 & -0.12641 & \\
\hline-0.01 & -0.22153 & -0.00079118 \\
\hline-0.01 & -0.10263 & \\
\hline-0.01 & -0.20494 & -0.00073193 \\
\hline & -0.11821 & \\
\hline $0.014^{\circ}$ & 0.01 & \\
\hline
\end{tabular}

$\begin{array}{rr}\text { Na, ACL } & \text { Na, Ppm N } \\ 31.2 & 234.74 \\ 32 & 234.67 \\ 32 & 280.48 \\ 49.2 & 280.38 \\ 50.5 & 280.38 \\ 43.4 & 336.11 \\ 44.1 & 336.24 \\ 44.2 & 411.23 \\ 36.9 & 405.9 \\ 44.8 & 510.99 \\ 44.1 & 505.01 \\ 57.4 & 593.02 \\ 55.5 & 561.33 \\ 37.6 & 746.81 \\ 160 & 773.73 \\ 49.6 & 832.88 \\ 138 & 838.93 \\ 464 & 6791.1 \\ 605 & 6898.2 \\ 352 & 5593.3 \\ 469 & 5383.7 \\ 742 & 16970 \\ 1214 & 15346 \\ 758 & 16792 \\ 1299 & 13332 \\ 557 & 11415 \\ 923 & 10911 \\ 51.32 & 51.32 \\ 5.061 & 0.0661 \\ 0.061 & \end{array}$

Friday, Decamber 30 2:07 PM 1994

Ni, ACL Ni, PpI

$\begin{array}{rrr}0.13813 & 0.14 & 1.0533 \\ 0.14147 & 0.14 & 1.0267 \\ 0.17272 & 0.31 & 1.7673 \\ 0.17484 & 0.3 & 1.6656 \\ 0.21648 & 0.29 & 2.2459 \\ 0.21555 & 0.29 & 2.2111 \\ 0.26826 & -0.03 & -0.27912 \\ 0.26759 & 0.12 & 1.32 \\ 0.34778 & -0.03 & -0.34158 \\ 0.3426 & -0.03 & -0.34354 \\ 0.40707 & -0.03 & -0.30994 \\ 0.3855 & -0.03 & -0.30342 \\ 0.52707 & -0.03 & -0.59586 \\ 0.58627 & -0.03 & -0.14507 \\ & -0.03 & -0.50376 \\ 5.0961 & -0.03 & -0.18238 \\ 4.1737 & -0.03 & -0.43908 \\ 12.03 & -0.03 & -0.34206 \\ 12.117 & -0.03 & -0.4767 \\ 12.663 & -0.03 & -0.34437 \\ & -0.03 & -0.37974 \\ 8.5959 & -0.03 & -0.66459 \\ & -0.03 & -0.30789 \\ & -0.03 & -0.61482 \\ & 0.03 & -0.35463 \\ & 0.0065 & 0.0065\end{array}$


Page *5 - "BZ. CATIONS"

\begin{tabular}{|c|c|c|c|c|}
\hline TEST NO. & $\mathrm{NL}(\mathrm{Ni}), g / \mathrm{m}$ & $S i, A C L$ & Si, ppm & $N L\left(s_{2}\right), g$ \\
\hline BZ1 & 0.0080667 & 28.2 & .17 & 36: \\
\hline$B 22$ & 0.0080557 & 29.8 & 218.53 & 386 \\
\hline$B 23$ & 0.013546 & 49.7 & 283.33 & 0518 \\
\hline 824 & 0.012929 & 49.7 & 275.93 & 05089 \\
\hline 825 & 0.017361 & 42 & 325.26 & 0615 \\
\hline 826 & 0.017011 & 43.4 & $\begin{array}{r}330.9 \\
350.9\end{array}$ & 0.0624 \\
\hline 827 & -0.0021156 & 38.6 & 359.13 & 0.0676 \\
\hline 828 & 0.01013 & 32.8 & 360.8 & 0.068 \\
\hline B29 & -0.0026332 & 34.1 & 388.26 & 0.07515 \\
\hline BZ10 & -0.0026381 & $\begin{array}{ll}34.4 \\
50.9\end{array}$ & 393.93 & 0.07610 \\
\hline 8211 & -0.0023685 & 50.9 & 525.86 & 0.104 \\
\hline $\begin{array}{l}8712 \\
B 713\end{array}$ & -0.0023323 & 52.4 & 529.97 & 0.10 \\
\hline $\begin{array}{r}8<13 \\
8713-F 50\end{array}$ & -0.004592 & ${ }_{105}^{24.3}$ & $\begin{array}{l}486.62 \\
587.76\end{array}$ & \\
\hline $\begin{array}{r}8243-r 14 \\
B Z 14\end{array}$ & -0.0038428 & 31.7 & $\begin{array}{l}532.31 \\
532.76\end{array}$ & \\
\hline $4-F 50$ & & 92.1 & 559.89 & \\
\hline$B 215$ & -0.0033762 & 95.7 & 1400.7 & \\
\hline$B 215-F 50$ & & 117 & 1334 & \\
\hline BZ16 & -0.0036509 & 85.1 & 1352.2 & \\
\hline$B 216-F 50$ & & 112 & 1285.6 & \\
\hline BZ17 & -0.004998 & 77.8 & $\begin{array}{l}283.6 \\
1685\end{array}$ & \\
\hline$B Z 17-F 50$ & & 130 & 1643.3 & \\
\hline BZ18 & -0.0051122 & 80.5 & 1783.3 & 0.3 \\
\hline$B Z 18-F 50$ & & 141 & 1447.1 & \\
\hline $\begin{array}{r}8 Z 19 \\
8 Z 19-F 50\end{array}$ & -0.0047294 & 72.6 & 1487.9 & 0.31 \\
\hline & & & & \\
\hline $\begin{array}{l}\text { f(i) } \\
\text { f(i) }\end{array}$ & & $\begin{array}{r}45.23 \\
0.2287\end{array}$ & $\begin{array}{l}45.23 \\
0.2287\end{array}$ & \\
\hline
\end{tabular}

\begin{tabular}{|c|c|c|}
\hline Th, ACL & Th, ppm & $N L(T h), g / m$ \\
\hline-0.1 & -0.75238 & -0.01628 \\
\hline-0.1 & -0.73333 & 0162 \\
\hline-0.2 & -1.1402 & .024698 \\
\hline-0.2 & -1.1104 & -0.024358 \\
\hline-0.2 & -1.5489 & -0.033837 \\
\hline-0.2 & -1.5249 & -0.033155 \\
\hline-0.2 & -1.8608 & -0.039 \\
\hline-0.2 & -2.2 & -0.0477 \\
\hline-0.1 & -1.1386 & -0.0248 \\
\hline 0.1 & 1.1451 & $\therefore$ \\
\hline $\begin{array}{l}-0.05 \\
-0.05\end{array}$ & -0.51657 & -0.01115 \\
\hline $\begin{array}{l}-0.05 \\
-0.05\end{array}$ & -0.5057 & -0.01098 \\
\hline $\begin{array}{l}-0.03 \\
-0.05\end{array}$ & $\begin{array}{r}-0.9931 \\
-0.24179\end{array}$ & \\
\hline-0.05 & -0.8396 & 0 \\
\hline-0.05 & -0.30396 & -0.018 \\
\hline-0.05 & -0.7318 & -0.015902 \\
\hline-0.05 & -0.5701 & \\
\hline-0.05 & -0.7945 & -0.01715 \\
\hline-0.05 & -0.57395 & \\
\hline-0.05 & -1.0829 & 0235 \\
\hline-0.05 & -0.63205 & \\
\hline-0.05 & -1.1077 & -0.0240 \\
\hline-0.05 & -0.51315 & \\
\hline-0.05 & -1.0247 & -0.0222 \\
\hline & & \\
\hline 0.0023 & $0.0023^{0}$ & \\
\hline
\end{tabular}

\begin{tabular}{|c|c|c|c|}
\hline Ii, $A C L$ & $\mathrm{Ti}, \mathrm{ppm}$ & $N L(T i), g / m$ & U, ACL \\
\hline 0.1 & 0.75238 & 0.0069356 & 1.173 \\
\hline 0.1 & 0.73333 & 0.0069262 & 1.236 \\
\hline 0.25 & 1.4252 & 0.013149 & 2.318 \\
\hline 0.22 & 1.2214 & 0.011412 & 2.3 \\
\hline 0.25 & 1.9361 & 0.018015 & 2.027 \\
\hline 0.25 & 1.9061 & 0.017652 & 1.995 \\
\hline 0.011 & 0.10234 & 0.00093375 & 0.978 \\
\hline 0.094 & 1.034 & 0.0095516 & 1.088 \\
\hline-0.01 & -0.11386 & -0.0010565 & 0.493 \\
\hline 0.01 & 0.11451 & 0.0010585 & 0.545 \\
\hline-0.02 & -0.20663 & -0.0019007 & 0.481 \\
\hline-0.02 & -0.20228 & -0.0018716 & 0.409 \\
\hline-0.02 & -0.39724 & -0.003685 & 0.144 \\
\hline-0.02 & -0.096716 & & 0.582 \\
\hline-0.02 & -0.33584 & -0.0030837 & 0.199 \\
\hline-0.02 & -0.12158 & -0.0027093 & 0.514 \\
\hline $\begin{array}{l}-0.02 \\
-0.02\end{array}$ & $\begin{array}{l}-0.29272 \\
-0.22804\end{array}$ & & $\begin{array}{l}0.0741 \\
0.0821\end{array}$ \\
\hline-0.02 & -0.3178 & -0.0029297 & 0.0788 \\
\hline $\begin{array}{l}-0.02 \\
-0.02\end{array}$ & $\begin{array}{l}-0.22958 \\
-0.43316\end{array}$ & -0.0040107 & $\begin{array}{l}0.0897 \\
0.0311\end{array}$ \\
\hline-0.02 & -0.25282 & & 0.0473 \\
\hline $\begin{array}{l}-0.02 \\
-0.02\end{array}$ & $\begin{array}{l}-0.44306 \\
-0.20526\end{array}$ & -0.0041024 & 0.0336 \\
\hline $\begin{array}{l}-0.02 \\
-0.02\end{array}$ & $\begin{array}{l}-0.40988 \\
-0.23642\end{array}$ & -0.0037952 & $\begin{array}{l}0.0367 \\
0.0209 \\
0.0324\end{array}$ \\
\hline $\begin{array}{r}0 \\
0.0054\end{array}$ & $0.0054^{\circ}$ & & 00 \\
\hline
\end{tabular}




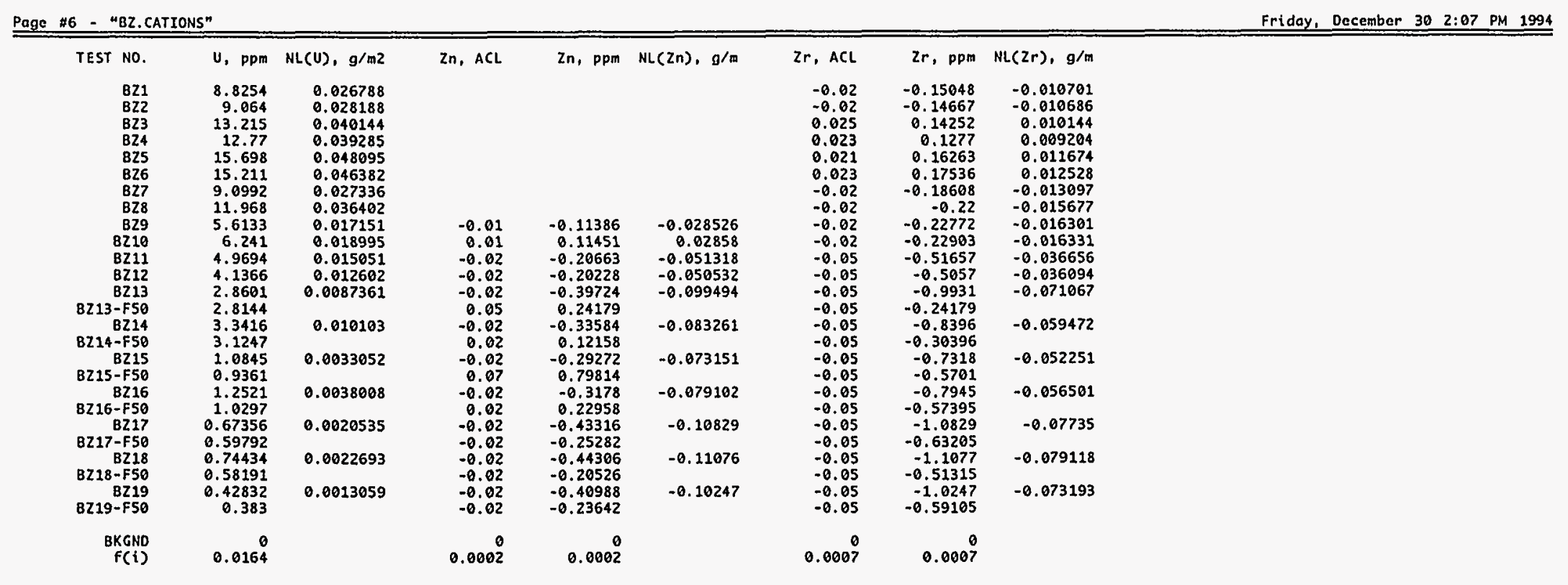


Friday, December $30 \quad 11: 16$ AM 1994

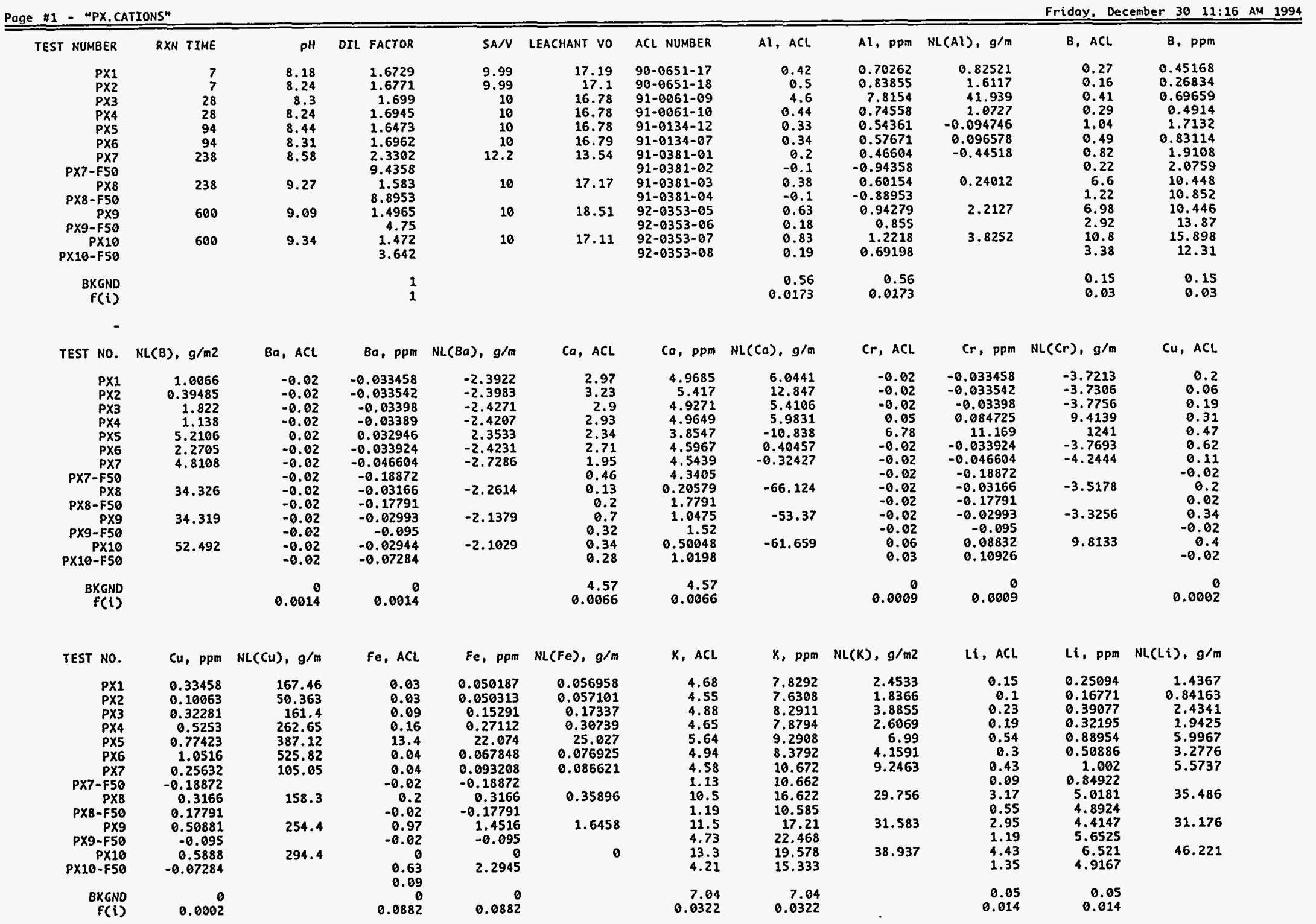




\begin{tabular}{|c|c|c|c|c|c|c|c|c|}
\hline TEST NO. & $\mathrm{Mg}, \mathrm{ACL}$ & Mg, ppm & $\mathrm{NL}(\mathrm{Mg}), g / m$ & $\mathrm{Mn}, \mathrm{ACL}$ & Mn, ppm & $N L(M n), g / m$ & $\mathrm{Na}, \mathrm{ACL}$ & $\mathrm{Na}, \mathrm{ppm}$ \\
\hline $\begin{array}{r}P X 1 \\
P \times 2 \\
P X 3 \\
P X 4 \\
P \times 5 \\
P \times 5 \\
P \times 6 \\
P X 7 \\
P X 7-F 50 \\
P X 8 \\
P \times 8-F 50 \\
P \times 9 \\
P \times 9-F 50 \\
P \times 10 \\
P X 10-F 50\end{array}$ & $\begin{array}{l}0.26 \\
0.26 \\
0.282 \\
0.276 \\
0.24 \\
0.25 \\
0.09 \\
0.03 \\
0.22 \\
0.02 \\
0.18 \\
0.03 \\
0.66 \\
0.04\end{array}$ & $\begin{array}{l}0.43495 \\
0.43605 \\
0.47912 \\
0.46768 \\
0.39535 \\
0.42405 \\
0.20972 \\
0.28307 \\
0.34826 \\
0.17791 \\
0.26937 \\
0.1425 \\
0.97152 \\
0.14568\end{array}$ & $\begin{array}{r}4.2442 \\
4.258 \\
4.799 \\
4.6542 \\
3.7366 \\
4.1019 \\
1.1384 \\
3.1425 \\
2.1439 \\
11.032\end{array}$ & $\begin{array}{r}0.02 \\
0.1 \\
0.046 \\
0.078 \\
0.69 \\
0.06 \\
0.03 \\
-0.01 \\
0.13 \\
-0.01 \\
0.19 \\
-0.01 \\
0.28 \\
-0.01\end{array}$ & $\begin{array}{r}0.033458 \\
0.16771 \\
0.078154 \\
0.13217 \\
1.1366 \\
0.16177 \\
0.069906 \\
-0.094358 \\
0.20579 \\
-0.088953 \\
0.28433 \\
-0.0475 \\
0.41216 \\
-0.03642\end{array}$ & $\begin{array}{l}0.21748 \\
1.6901 \\
0.56749 \\
0.85825 \\
7.3808 \\
0.66086 \\
0.37208 \\
1.3363 \\
1.8463 \\
2.6764\end{array}$ & $\begin{array}{l}35.1 \\
33.7 \\
34.3 \\
33.4 \\
37.4 \\
35.6 \\
31.1 \\
7.74 \\
53.1 \\
9.78 \\
59.2 \\
25.2 \\
69.1 \\
21.6\end{array}$ & $\begin{array}{l}58.719 \\
56.518 \\
58.276 \\
56.596 \\
61.609 \\
66.385 \\
72.469 \\
73.033 \\
84.057 \\
86.996 \\
88.593 \\
119.7 \\
101.72 \\
78.667\end{array}$ \\
\hline $\begin{array}{c}\text { BKGND } \\
f(i)\end{array}$ & $\begin{array}{r}0.1 \\
0.0079\end{array}$ & $\begin{array}{r}0.1 \\
0.0079\end{array}$ & & $0.0154^{\circ}$ & $0.0154^{\circ}$ & & $\begin{array}{r}51.23 \\
0.8895\end{array}$ & $\begin{array}{r}51.23 \\
0.6895\end{array}$ \\
\hline TEST NO. & $N(\mathrm{Ni}), g / m$ & $S 1, A C L$ & si, ppm & $N L(S i), g / m$ & $T h, A C L$ & Th, ppm & $N L(T h), g / m$ & $\mathrm{Ti}, \mathrm{ACL}$ \\
\hline $\begin{array}{r}P X 1 \\
P X 2 \\
P X 3 \\
P X 4 \\
P X 4 \\
P X 5 \\
P X 6 \\
P X 7 \\
P X 7-F 56 \\
P X 8 \\
P X 8-F 50 \\
P X 9 \\
P X 9-F 50 \\
P X 16 \\
P X 10-F 50\end{array}$ & $\begin{array}{r}-0.51262 \\
-0.51391 \\
0.69347 \\
0.86454 \\
90.938 \\
1.0385 \\
0.77959 \\
1.2922 \\
0.91622 \\
4.3559\end{array}$ & $\begin{array}{l}28.3 \\
28.4 \\
27.5 \\
26.8 \\
28.4 \\
26.1 \\
23.3 \\
5.75 \\
43.4 \\
7.64 \\
53.6 \\
21.4 \\
66 \\
19.8\end{array}$ & $\begin{array}{r}47.343 \\
47.63 \\
46.722 \\
45.413 \\
46.783 \\
44.271 \\
54.294 \\
54.256 \\
68.702 \\
67.96 \\
80.212 \\
101.65 \\
97.152 \\
72.112\end{array}$ & $\begin{array}{r}1.0343 \\
1.1746 \\
0.72983 \\
0.08929 \\
0.75957 \\
-0.46904 \\
3.6329 \\
11.478 \\
17.106 \\
25.39\end{array}$ & $\begin{array}{l}-0.06 \\
-0.06 \\
-0.1 \\
-0.1 \\
-0.1 \\
-0.1 \\
-0.05 \\
-0.05 \\
-0.05 \\
-0.05 \\
-0.05 \\
-0.05 \\
-0.05 \\
-0.05\end{array}$ & $\begin{array}{r}-0.10037 \\
-0.10063 \\
-0.1699 \\
-0.16945 \\
-0.16473 \\
-0.16962 \\
-0.11651 \\
-0.47179 \\
-0.07915 \\
-0.44477 \\
-0.074825 \\
-0.2375 \\
-0.0736 \\
-0.1821\end{array}$ & $\begin{array}{l}0 \\
0 \\
0 \\
0 \\
0 \\
0 \\
0 \\
0\end{array}$ & $\begin{array}{l}-0.01 \\
-0.01 \\
0.01 \\
-0.01 \\
-0.02 \\
-0.02 \\
-0.02 \\
-0.02 \\
-0.02 \\
-0.02 \\
-0.02 \\
-0.02\end{array}$ \\
\hline $\begin{array}{c}\text { BKGND } \\
f(i)\end{array}$ & & $\begin{array}{r}45.23 \\
0.2045\end{array}$ & $\begin{array}{r}45.23 \\
0.2045\end{array}$ & & $1.0000 \mathrm{e}-04^{0}$ & $1.0000-04$ & & $0.0231^{\circ}$ \\
\hline TEST NO. & u, ppm & $N L(U), g / m 2$ & $\mathrm{Zn}, \mathrm{ACL}$ & $\mathrm{Zn}, \mathrm{ppm}$ & $N L(2 n), g / m$ & $\mathrm{Zr}, \mathrm{ACL}$ & $\mathrm{Zr}, \mathrm{ppm}$ & $N L(Z r), g / m$ \\
\hline $\begin{array}{r}P X 1 \\
P X 2 \\
P X 3 \\
P X 4 \\
P X 4 \\
P X 5 \\
P X 6 \\
P X 7 \\
P X 7-F 50 \\
P X 8 \\
P X 8-F 50 \\
P X 9 \\
P X 9-F 50 \\
P X 10 \\
P X 10-F 50\end{array}$ & $\begin{array}{r}0.036637 \\
0.018951 \\
0.043155 \\
0.027281 \\
0.10444 \\
0.094309 \\
0.18898 \\
0.17645 \\
0.36726 \\
0.34096 \\
0.77668 \\
0.9025 \\
1.7664 \\
1.3038\end{array}$ & $\begin{array}{r}0.15876 \\
0.082122 \\
0.18682 \\
0.1181 \\
0.45212 \\
0.40826 \\
0.67057 \\
1.5899 \\
3.3623 \\
7.6468\end{array}$ & $\begin{array}{l}0.031 \\
0.076 \\
0.12 \\
0.02 \\
-0.02 \\
-0.02 \\
-0.02 \\
-0.02 \\
-0.02 \\
-0.02 \\
-0.02 \\
0.02\end{array}$ & $\begin{array}{r}0.052669 \\
0.12878 \\
0.19768 \\
0.033924 \\
-0.046604 \\
-0.18872 \\
-0.03166 \\
-0.17791 \\
-0.02993 \\
-0.095 \\
-0.02944 \\
0.07284\end{array}$ & $\begin{array}{r}26.335 \\
64.327 \\
98.838 \\
16.962 \\
0 \\
0 \\
0 \\
0\end{array}$ & $\begin{array}{l}-0.02 \\
-0.02 \\
-0.02 \\
-0.02 \\
-0.02 \\
-0.02 \\
-0.05 \\
-0.05 \\
-0.05 \\
-0.05 \\
-0.05 \\
-0.05 \\
-0.05 \\
-0.05\end{array}$ & $\begin{array}{r}-0.033458 \\
-0.033542 \\
-0.03398 \\
-0.03389 \\
-0.032946 \\
-0.033924 \\
-0.11651 \\
-0.47179 \\
-0.07915 \\
-0.44477 \\
-0.074825 \\
-0.2375 \\
-0.0736 \\
-0.1821\end{array}$ & : \\
\hline $\begin{array}{c}\text { BKGND } \\
f(i)\end{array}$ & 0.0231 & & 0.0002 & 0.0002 & & 0.0016 & $\begin{array}{r}0 \\
0.0016\end{array}$ & \\
\hline
\end{tabular}




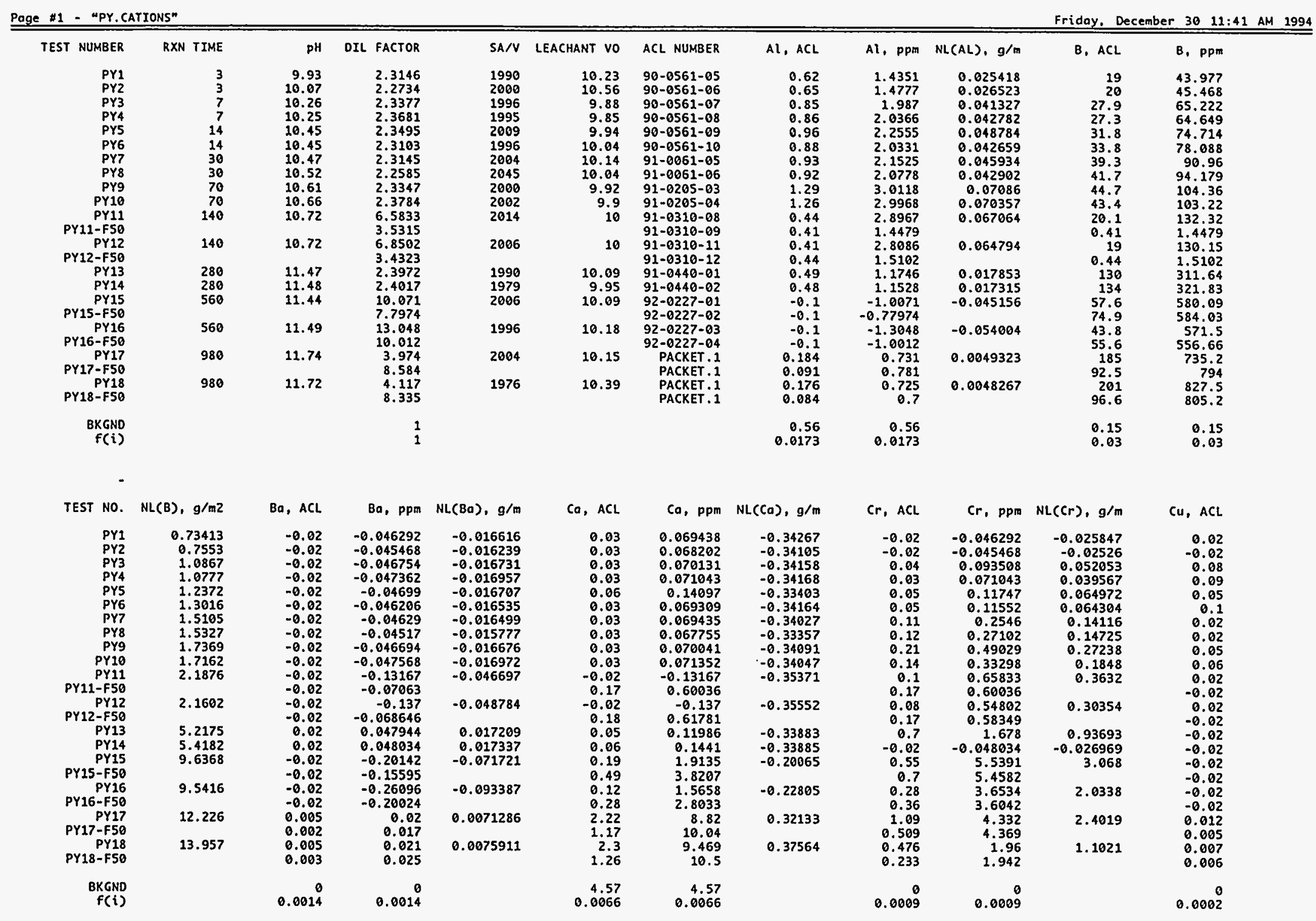




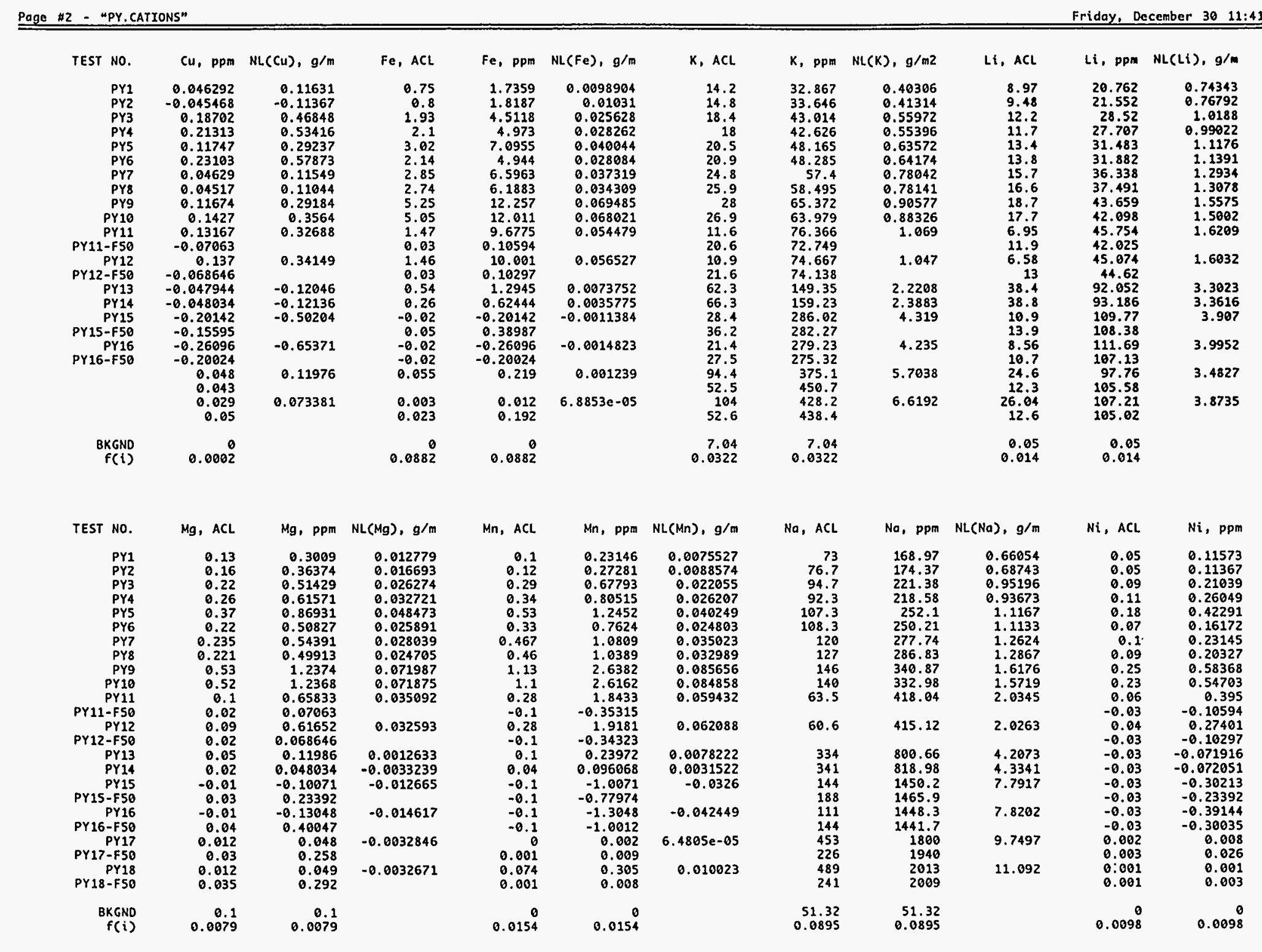




\begin{tabular}{|c|c|c|c|c|c|c|c|c|c|c|c|}
\hline TEST NO. & $N\left(N_{i}\right), g / m$ & Si, ACL & Si, ppm & $N L(S i), g / m$ & Th, ACl & Th, ppm & $\mathrm{NL}(\mathrm{Th}), \mathrm{g} / \mathrm{m}$ & $\mathrm{Ti}, \mathrm{ACL}$ & $\mathrm{Ti}, \mathrm{ppm}$ & $N L(T i), g / m$ & U, ACL \\
\hline $\begin{array}{l}\text { PY1 } \\
\text { PYZ }\end{array}$ & $\begin{array}{l}0.0059343 \\
0.0057995\end{array}$ & $\begin{array}{l}55.7 \\
58.2\end{array}$ & $\begin{array}{l}128.92 \\
132.31\end{array}$ & $\begin{array}{l}0.20566 \\
0.21291\end{array}$ & $\begin{array}{l}-0.06 \\
-0.06\end{array}$ & $\begin{array}{r}-0.13888 \\
-0.1364\end{array}$ & $: 0$ & & & & $\begin{array}{r}2.45 \\
2.5\end{array}$ \\
\hline PY3 & $\begin{array}{r}0.010756 \\
0.0105\end{array}$ & 72.5 & $\begin{array}{l}132.51 \\
169.48\end{array}$ & $\begin{array}{l}0.21291 \\
0.30441\end{array}$ & $\begin{array}{l}-6.06 \\
-0.06\end{array}$ & $\begin{array}{r}-0.1364 \\
-0.14026\end{array}$ & 0 & & & & $\begin{array}{l}2.5 \\
3.89\end{array}$ \\
\hline PY4 & 0.013324 & 71.5 & 169.32 & 0.30416 & -0.06 & -0.14209 & 0 & & & & 3.7 \\
\hline PYS & 0.02148 & 79.3 & 186.32 & 0.34341 & -0.06 & $\begin{array}{l}-0.14097 \\
\end{array}$ & 0 & & & & 3.55 \\
\hline PYG & 0.0082676 & 82.8 & 191.29 & 0.35784 & -0 & -0.13862 & 0 & & & & 3.65 \\
\hline PY7 & 0.011785 & 85.5 & 197.89 & 0.37251 & -0.01 & -0.023145 & 0 & 0.05 & 0.11573 & 0.014807 & 2.443 \\
\hline PY8 & 0.010142 & 89.2 & 201.46 & 0.37357 & -0.01 & -0.022585 & 0 & 0.05 & 0.11293 & 0.014159 & 2.527 \\
\hline PY9 & 0.029779 & 112 & 261.49 & 0.52874 & -0.05 & -0.11674 & 0 & 0.11 & 0.25682 & 0.032925 & 1.96 \\
\hline PY10 & 0.027882 & 111 & 264 & 0.53436 & -0.05 & -0.11892 & 0 & 0.11 & 0.26162 & 0.033508 & 1.9 \\
\hline PY11 & 0.020013 & 38.6 & 254.12 & 0.50717 & -0.05 & -0.32917 & 0 & 0.03 & 0.1975 & 0.025144 & 0.588 \\
\hline PY $11-F 50$ & & 68.3 & 241.2 & & -0.05 & -0.17658 & & -0.02 & -0.07063 & & \\
\hline PY12 & 0.013938 & 36.8 & 252.09 & 0.50425 & -0.05 & -0.34251 & 0 & 0. & 0.20551 & 0.026268 & \\
\hline PY12-F50 & & 74 & 253.99 & & -0.05 & -0.17162 & & -0.02 & -0.068646 & & \\
\hline PY13 & -0.0036876 & 173 & 414.72 & 0.90793 & -0.05 & -0.11986 & 0 & -0.02 & -0.047944 & -0.0061776 & 1.8 \\
\hline PY14 & -0.0037151 & 177 & 425.1 & 0.93864 & -0.05 & -0.12009 & 0 & -0.02 & -0.048034 & -0.0062236 & 1.5 \\
\hline PY15 & -0.015369 & 58.6 & 590.16 & 1.3284 & -0.05 & -0.50355 & 0 & -0 & -0.20142 & -0.025746 & 0.265 \\
\hline $\begin{array}{l}5-F 50 \\
\text { SYy1 }\end{array}$ & & 75.6 & 589.48 & & -0.05 & -0.38987 & & -0.02 & -0.15595 & & 0.184 \\
\hline $\begin{array}{r}\text { PY16 } \\
\text { PY16-F50 }\end{array}$ & -0.020011 & 43.3 & 564.98 & 1.2733 & -0.05 & -0.6524 & 0 & -0.02 & -0.26096 & -0.033523 & 0.124 \\
\hline $\begin{array}{r}\text { PY16-F50 } \\
\text { PY17 }\end{array}$ & & 57.7 & 577.68 & & -0.05 & -0.50059 & 0 & -0.02 & -0.20024 & & 0.139 \\
\hline $\begin{array}{r}\text { PY17 } \\
17-F 50\end{array}$ & 0.00040735 & 131 & 520.6 & 1.16 & $\stackrel{0}{0}$ & $\therefore$ & 0 & 0.002 & 0.008 & 0.0010236 & 0.153 \\
\hline PY18 & $5.1640 e-05$ & 146 & $\begin{array}{l}515.9 \\
601.1\end{array}$ & 1.3756 & 0 & 0 & 0 & $\begin{array}{l}0.001 \\
0.002\end{array}$ & $\begin{array}{l}0.009 \\
0.008\end{array}$ & 0.0010381 & 0.146 \\
\hline PY18-F50 & & 67 & 558.4 & & 0 & 0 & & 0.002 & 0.017 & & 0.065 \\
\hline BKGND & & $\begin{array}{r}45.23 \\
0.2045\end{array}$ & $\begin{array}{r}45.23 \\
0.2045\end{array}$ & & 0 & 10 & & 0 & 0 & & 0.073 \\
\hline
\end{tabular}

\begin{tabular}{|c|c|c|c|c|c|c|c|c|}
\hline TEST NO. & U, ppm & $N L(U), g / m 2$ & $\mathrm{Zn}, \mathrm{ACL}$ & $2 n, p p m$ & $N L(Z n), g / m$ & $2 r, A C L$ & $2 r, p p m$ & NL(Zr), $g / m$ \\
\hline PY1 & 5.6708 & 0.12336 & & & & -0.02 & -0.046292 & -0.014539 \\
\hline PY2 & 5.6835 & 0.12302 & & & & -0.02 & -0.045468 & -0.014209 \\
\hline PY3 & 9.0937 & 0.19723 & & & & -0.02 & -0.046754 & $\begin{array}{r}-0.01464 \\
-0.014838\end{array}$ \\
\hline PY4 & 8.762 & 0.19013 & & & & -0.02 & -0.047362 & $\begin{array}{l}-0.014838 \\
-0.014619\end{array}$ \\
\hline $\begin{array}{l}\text { PY5 } \\
\text { PYG }\end{array}$ & 8.3407 & 0.17973 & & & & -0.02 & -0.04699 & $\begin{array}{l}-0.014619 \\
-0.014468\end{array}$ \\
\hline PY6 & 8.4326 & 0.18289 & & & & -0.02 & -0.046206 & -0.014468 \\
\hline PY7 & 5.6543 & 0.12214 & 0.02 & 0.04629 & 0.11549 & $\begin{array}{l}-0.02 \\
-0.02\end{array}$ & $\begin{array}{l}-0.04629 \\
-0.04517\end{array}$ & -0.014437 \\
\hline $\begin{array}{l}\text { PY8 } \\
\text { pya }\end{array}$ & 5.7072 & 0.12081 & 0.02 & 0.04517 & 0.11044 & -0.02 & -0.04517 & $\begin{array}{r}-0.013805 \\
-0.03648\end{array}$ \\
\hline $\begin{array}{l}\text { PY9 } \\
\text { PY10 }\end{array}$ & $\begin{array}{l}4.576 \\
3.765\end{array}$ & 0.099048 & 0.02 & 0.046694 & 0.11674 & -0.05 & -0.11674 & $\begin{array}{r}-0.03648 \\
-0.037125\end{array}$ \\
\hline $\begin{array}{l}\text { PY10 } \\
\text { PY11 }\end{array}$ & $\begin{array}{l}3.765 \\
3.871\end{array}$ & 0.081412 & 0.06 & 0.1427 & $\begin{array}{r}0.3564 \\
-0\end{array}$ & -0.05 & -0.11892 & $\begin{array}{r}-0.037125 \\
-0.10215\end{array}$ \\
\hline PY11-F50 & $\begin{array}{r}3.871 \\
3.3761\end{array}$ & 0.083205 & $\begin{array}{l}-0.02 \\
-0.02\end{array}$ & $\begin{array}{l}-0.013167 \\
-0.07063\end{array}$ & -0.32688 & $\begin{array}{l}-0.05 \\
-0.05\end{array}$ & $\begin{array}{l}-0.32917 \\
-0.1758\end{array}$ & -0.10215 \\
\hline & 3.8293 & 0.082637 & -0.02 & -0.137 & -0.34149 & -0.05 & $\begin{array}{l}-0.17638 \\
-0.34251\end{array}$ & -0.16671 \\
\hline PY12-F50 & 3.1426 & & -0.02 & -0.068646 & & -0.05 & -0.17162 & \\
\hline PY13 & 3.9266 & 0.085419 & -0.02 & -0.047944 & -0.12046 & -0.05 & -0.11986 & -0.037644 \\
\hline $\begin{array}{l}\text { PY14 } \\
P Y 14\end{array}$ & 3.7683 & 0.08243 & -0.02 & -0.048034 & -0.12136 & -0.65 & -0.12009 & -0.037925 \\
\hline $\begin{array}{r}\text { PY15 } \\
\text { PY15-F50 }\end{array}$ & 1.6617 & 0.03586 & -0.02 & -0.20142 & -0.50204 & -0. & -0.50355 & -0.15689 \\
\hline PY15-F50 & 1.4347 & & -0.02 & -0.15595 & & -0.05 & -0.38987 & \\
\hline $\begin{array}{r}\text { PY16 } \\
\text { PY16-550 }\end{array}$ & $\begin{array}{r}1.618 \\
1.3916\end{array}$ & 0.035091 & -0.02 & -0.26096 & -0.65371 & -0.05 & -0.6524 & -0.20428 \\
\hline $\begin{array}{l}\text { PY16-F50 } \\
\text { BY17 }\end{array}$ & 1.3916 & & -0.02 & -0.20024 & & -0.05 & -0.50059 & \\
\hline $\begin{array}{r}\text { BY17 } \\
\text { BY17-F5a }\end{array}$ & 0.608 & 0.013134 & 0.011 & 0.044 & 0.10978 & $\stackrel{0}{0}$ & 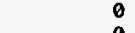 & \\
\hline $\begin{array}{l}\text { BY17-FY5 } \\
\text { BY1 }\end{array}$ & 0.515 & & $\begin{array}{l}0.0 \\
0 .\end{array}$ & 0.112 & & $:$ & 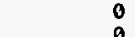 & \\
\hline $\begin{array}{r}\text { BY18 } \\
\text { BY18-F50 }\end{array}$ & $\begin{array}{l}0.601 \\
0.542\end{array}$ & 0.013167 & $\begin{array}{r}0.01 \\
0.027\end{array}$ & $\begin{array}{l}0.041 \\
0.225\end{array}$ & 0.10374 & : & : & \\
\hline $\begin{array}{r}\text { BKGND } \\
f(i)\end{array}$ & $\begin{array}{r}0 \\
0.0231^{\circ}\end{array}$ & & $\begin{array}{r}0 \\
0.0002\end{array}$ & $\begin{array}{r}0 \\
0.0002\end{array}$ & & $\begin{array}{r}0 \\
0.0016\end{array}$ & 0.0016 & \\
\hline
\end{tabular}




\begin{tabular}{|c|c|c|c|c|c|c|c|c|c|c|c|}
\hline TEST NUMBER & RXN TIME & pH & DIL FACTOR & SA/V & LEACHANT VO & ACL NUMBER & $A l, A C L$ & Al, ppm & $N L(A L), g / m$ & $B, A C L$ & B, ppm \\
\hline PZ1 & 3 & 11.94 & 7.8498 & 19988 & 5.05 & $90-0561-11$ & 0.13 & 1.0205 & 0.0013316 & 184 & 1444 \\
\hline PZ2 & 3 & 11.86 & 7.9674 & 20073 & 5.04 & $90-0561-12$ & 0.12 & 0.95609 & 0.0011406 & 195 & $\begin{array}{l}1553.6 \\
2927.5\end{array}$ \\
\hline $\begin{array}{l}\text { PZ3 } \\
\text { PZ4 }\end{array}$ & 7 & $\begin{array}{l}11.99 \\
11.98\end{array}$ & $\begin{array}{r}8.56 \\
8.9947\end{array}$ & $\begin{array}{l}20012 \\
19947\end{array}$ & 5.02 & $90-0561-13$ & 0.14 & $\begin{array}{l}1.1984 \\
.2503\end{array}$ & 0.001844 & 342 & $\begin{array}{l}2927.5 \\
2896.3\end{array}$ \\
\hline P25 & 14 & $\begin{array}{l}1.98 \\
12.05\end{array}$ & $\begin{array}{l}8.9947 \\
9.2751\end{array}$ & $\begin{array}{l}19947 \\
19954\end{array}$ & $\begin{array}{r}5.05 \\
5\end{array}$ & $\begin{array}{l}90-0561-14 \\
90-0561-15\end{array}$ & $\begin{array}{l}0.14 \\
0.14\end{array}$ & $\begin{array}{l}1.2593 \\
1.2985\end{array}$ & $\begin{array}{l}0.002026263 \\
0.0021394\end{array}$ & $\begin{array}{l}322 \\
397\end{array}$ & $\begin{array}{l}2896.3 \\
3682.2\end{array}$ \\
\hline P26 & 14 & 12.04 & 8.8848 & 19775 & 5.09 & $90-0561-16$ & 0.12 & 1.0662 & 0.0014796 & 401 & 3562.8 \\
\hline PZ7 & 28 & 12.12 & 9.7485 & 19953 & 5.01 & $91-0061-07$ & 0.14 & 1.3648 & 0.0023315 & 150 & 4386.8 \\
\hline PZ8 & 28 & 12.11 & 9.7368 & 20009 & 4.99 & $91-0061-08$ & 0.13 & 1.2658 & 0.0020389 & 465 & 4527.6 \\
\hline PZ9 & 56 & 12.12 & 15.651 & 19948 & 5.03 & $91-0134-11$ & -0.1 & -1.5651 & -0.0061578 & 378 & 5915 \\
\hline PZ10 & 56 & 12.14 & 14.446 & 19936 & 5.03 & $91-0134-08$ & -0.1 & -1.4446 & -0.0058124 & 400 & 5778 \\
\hline P211 & 98 & 12.14 & 8.3382 & 20032 & 4.99 & $91-0205-07$ & 0.14 & 1.1673 & 0.0017525 & 665 & 5544.9 \\
\hline P212 & 98 & 12.12 & 8.4729 & 20059 & 4.99 & $91-0205-08$ & 0.14 & 1.1862 & 0.0018045 & 675 & $\begin{array}{l}5719 \\
7695\end{array}$ \\
\hline P213 & 182 & 12.18 & 13.226 & 20016 & 4.98 & $91-0239-02$ & -0.1 & -1.3226 & -0.0054368 & 575 & $\begin{array}{l}7605 \\
8209\end{array}$ \\
\hline$P Z 13-F 50$ & & & 6.8415 & & & $91-0239-03$ & 0.16 & 1.0946 & & 1200 & 8209 \\
\hline PZ14 & 182 & 12.19 & 7.8436 & 19926 & 5.01 & $91-0239-05$ & 0.15 & 1.1765 & 0.0017885 & 820 & $\begin{array}{l}6431 \\
5592\end{array}$ \\
\hline PZ14-F50 & & & 7.4865 & & & $91-0239-06$ & 0.13 & 0.97324 & & 747 & $\begin{array}{r}5592 \\
129\end{array}$ \\
\hline PZ15 & 364 & 12.2 & 28.926 & 19905 & 5 & $91-6596-09$ & -0.1 & -2.8926 & -0.010026 & 448 & $\begin{array}{l}12959 \\
13534\end{array}$ \\
\hline PZ15-F50 & & & 15.592 & & & $91.0596-10$ & -0.1 & -1.5592 & & Oon & $\begin{array}{l}13534 \\
11928\end{array}$ \\
\hline PZ16 & 364 & 12.17 & 29.453 & 20029 & 5 & $91-0596-11$ & -0.1 & -2.9453 & -8.010116 & 405 & $\begin{array}{l}119 \\
111\end{array}$ \\
\hline PZ16-F50 & & & 19.341 & & & $91-0596-12$ & -0.1 & -1.9341 & & 578 & $\begin{array}{l}11179 \\
21585\end{array}$ \\
\hline PZ17 & 728 & 12.02 & 26.948 & 20000 & 5 & $92-0485-01$ & -0.1 & -2.6948 & -0.0094069 & 801 & $\begin{array}{l}21585 \\
19632\end{array}$ \\
\hline$P Z 17-F 50$ & & & 36.833 & & & $92-0485-02$ & -0.1 & -3.6833 & & 533 & $\begin{array}{l}19632 \\
19124\end{array}$ \\
\hline PZ-18 & 728 & 12.05 & 21.658 & 19900 & 5 & $92-0485-03$ & -0.1 & -2.1658 & -0.0079176 & $\begin{array}{r}883 \\
1769\end{array}$ & $\begin{array}{l}19124 \\
19987\end{array}$ \\
\hline$P Z 18-F 50$ & & & 15.863 & & & $92-0485-04$ & -0.1 & -1.5863 & & 1260 & $\begin{array}{l}19987 \\
14005\end{array}$ \\
\hline $\begin{array}{r}\text { PZ19 } \\
\end{array}$ & 552 & 12.04 & 16.594 & 19923 & 5 & 92-0485-05 & $\begin{array}{l}-0.1 \\
-0.1\end{array}$ & -1.6594 & -0.0064392 & $\begin{array}{l}844 \\
864\end{array}$ & $\begin{array}{l}14005 \\
14642\end{array}$ \\
\hline $\begin{array}{r}\text { PZ19-F50 } \\
\text { PZ20 }\end{array}$ & 1100 & 12.18 & & 20000 & 5 & $\begin{array}{r}92-0485-06 \\
\text { PACXET . }\end{array}$ & $\begin{array}{l}-0.1 \\
0.649\end{array}$ & $\begin{array}{r}-1.6947 \\
7.1163\end{array}$ & 0.018949 & $\begin{array}{l}864 \\
1925\end{array}$ & $\begin{array}{l}1464 \\
21108 \\
30535\end{array}$ \\
\hline PZZO-F25 & & & $\begin{array}{r}44.37 \\
1\end{array}$ & & & PACKET. 2 & $\begin{array}{l}0.125 \\
0.56\end{array}$ & $\begin{array}{r}5.546 \\
0.56\end{array}$ & & $\begin{array}{r}688.2 \\
0.15\end{array}$ & $\begin{array}{r}30535 \\
0.15\end{array}$ \\
\hline$f(i)$ & & & 1 & & & & 0.0173 & 0.0173 & & 0.03 & 0.03 \\
\hline
\end{tabular}


Page \#2 - "PZ.CATIONS"

\begin{tabular}{|c|c|c|c|c|}
\hline TEST NO. & $N L(B), g / m Z$ & Bo, ACL & Ba, ppm & NL(Ba), $g / m$ \\
\hline PZ1 & 2.4085 & -0.02 & -0.157 & -0.0056104 \\
\hline PZ2 & 2.5797 & -0.02 & -0.15935 & -0.0056703 \\
\hline PZ3 & 4.876 & -0.02 & -0.1712 & -0.0061106 \\
\hline PZ4 & 4.8397 & -0.02 & -0.17989 & -0.0064419 \\
\hline PZS & 6.1509 & -0.02 & -0.1855 & -0.0066403 \\
\hline P26 & 6.0053 & -0.02 & -0.1777 & -0.0064185 \\
\hline PZ7 & 7.3283 & 0.04 & 0.38994 & 0.013959 \\
\hline PZ8 & 7.5424 & 0.04 & 0.38947 & 0.013903 \\
\hline PZ9 & 9.8853 & 0.03 & 0.46951 & 0.016812 \\
\hline PZ10 & 9.6616 & 0.03 & 0.43339 & 0.015528 \\
\hline PZ11 & 9.2265 & 0.04 & 0.33353 & 0.011893 \\
\hline PZ12 & 9.5037 & 0.04 & 0.33892 & 0.012069 \\
\hline PZ13 & 12.665 & 0.04 & 0.52906 & 0.01888 \\
\hline PZ13-F50 & & 0.07 & 0.4789 & \\
\hline P214 & 10.759 & 0.06 & 0.47062 & 0.01687 \\
\hline PZ14-F50 & & 0.05 & 0.37433 & \\
\hline PZ1S & 21.701 & 0.05 & 1.4463 & 0.0519 \\
\hline PZ15-F50 & & 0.07 & 1.0914 & \\
\hline PZ16 & 19.852 & 0.04 & 1.1781 & 0.042015 \\
\hline PZ16-F50 & & 0.07 & $\begin{array}{l}1.3539 \\
3.2338\end{array}$ & \\
\hline PZ17 & 35.975 & 0.12 & 3.2338 & 0.11549 \\
\hline PZ17-F50 & 32.033 & $\begin{array}{l}0.09 \\
0.12\end{array}$ & $\begin{array}{l}3.315 \\
2599\end{array}$ & 0.093286 \\
\hline $\begin{array}{r}P Z-18 \\
P Z 18-F 50\end{array}$ & 32.035 & $\begin{array}{l}0.12 \\
0.16\end{array}$ & 2.5381 & \\
\hline PZ19 & 23.432 & 0.07 & 1.1616 & 0.041645 \\
\hline $\begin{array}{l}\text { PZ19-F50 } \\
\text { PZZO }\end{array}$ & 35.179 & 0.08 & $\begin{array}{l}1.3558 \\
8.7281\end{array}$ & \\
\hline $\begin{array}{r}P Z 20-F 25 \\
\text { BKGND }\end{array}$ & & 0.0856 & 3.7981 & \\
\hline $\mathrm{f}(\mathrm{i}$ & & 0.0014 & 0.0014 & \\
\hline
\end{tabular}

$\mathrm{Ca}, \mathrm{ACL}$

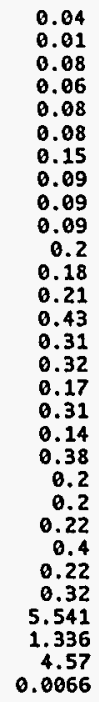

Ca, ppm NL(Ca), g/m Cr, ACl

$0.31399 \quad-0.032262$

$\begin{array}{ll}0.6848 & -0.029416\end{array}$

$53968-0.030614$

$.71078 \quad-0.029569$

$1.4623-0.023599$

.87631

1.4085

1.6676

1.5251

2.7776

2.4315

$-0.016261$

2.3957
4.9174

4.8335
4.1234

7.3496
5.3896

5.3896
7.3666
4.7648

4.7648
6.3452

3.6507

5.423
60.757
59.278

4.57
0.0066

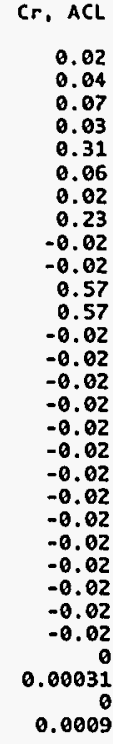

0.0009

\subsection{5}

$-0.0033783$

0.0062091

0.0014829

$-0.0069915$

0.42566

Wednesday, August $31 \quad 4.07$ PM 1994

cro

cr.

Cr. ppm

(Cr), g/a

Cu, ACL

$0.3187 \quad 0.0087272$

$-0.02$

$0.5992 \quad 0.033269$

0.015031

0.16011

0.53309

0.19497

2.2395
-0.31301

0.029953

0.12436

$-0.017435$

0.26362

0.26752
-0.014684

$-0.0087475$

$-0.032293$

$-0.032678$

$-0.029942$

$-0.024185$

$-0.018509$

0.33188

0.013755

0.0009

(2) 
Page \#3 - mpZ.CATIONS"

\begin{tabular}{|c|c|c|c|c|c|c|c|c|c|c|c|}
\hline TEST NO. & $\mathrm{Cu}, \mathrm{ppm}$ & $N L(C u), g / m$ & $\mathrm{Fe}, \mathrm{ACL}$ & Fe, ppm & $\mathrm{NL}(\mathrm{Fe}), \mathrm{g} / \mathrm{m}$ & $\mathrm{K}, \mathrm{ACL}$ & $\mathrm{K}, \mathrm{ppm}$ & $\mathrm{NL}(\mathrm{K}), g / \mathrm{m} 2$ & $\mathrm{Li}, \mathrm{ACL}$ & Li, ppm & $N L(L i), g / m$ \\
\hline PZ1 & -0.157 & -0.039273 & 0.02 & 0.157 & $8.9053 e-05$ & 88.7 & 696.28 & 1.0709 & 37.5 & 294.37 & 1.0518 \\
\hline PZ2 & -0.15935 & -0.039692 & 0.02 & 0.15935 & $9.0005 \mathrm{e}-05$ & 92.7 & 738.58 & 1.1318 & 37.9 & 301.96 & 1.0743 \\
\hline PZ3 & -0.1712 & -0.042774 & 0.02 & 0.1712 & $9.6994 \mathrm{e}-05$ & 164 & 1403.8 & 2.1676 & 36.5 & 312.44 & 1.115 \\
\hline PZ4 & -0.17989 & -0.045093 & 0.01 & 0.089947 & $5.1126 \mathrm{e}-05$ & 155 & 1394.2 & 2.1597 & 35.7 & 321.11 & 1.1497 \\
\hline P25 & -0.1855 & -0.046482 & 0.8 & 7.4201 & 0.0042161 & 192 & 1780.8 & 2.7607 & 33.1 & 307.01 & 1.0988 \\
\hline PZ6 & -0.1777 & -0.044929 & 0.02 & 0.1777 & 0.00010188 & 195 & 1732.5 & 2.7098 & 34.4 & 305.64 & 1.1038 \\
\hline PZ7 & -0.19497 & -0.048857 & 0.03 & 0.29245 & 0.00016618 & 203 & 1978.9 & 3.0692 & 32 & 311.95 & 1.1166 \\
\hline PZ8 & -0.19474 & -0.048662 & 0.03 & 0.2921 & 0.00016552 & 209 & 2035 & 3.1476 & 30.7 & 298.92 & 1.0669 \\
\hline PZ9 & 0.15651 & 0.039228 & 0.01 & 0.15651 & $8.8953 \mathrm{e}-05$ & 173 & 2707.5 & 4.2042 & 19.9 & 311.44 & 1.115 \\
\hline P210 & -0.14446 & -0.036232 & 0.01 & 0.14446 & $8.2159 \mathrm{e}-05$ & 183 & 2643.7 & 4.1073 & 20.9 & 301.93 & 1.0816 \\
\hline PZ11 & -0.16676 & -0.041624 & -0.02 & -0.16676 & $-9.4386 \mathrm{e}-05$ & 302 & 2518.1 & 3.893 & 30.7 & 255.98 & 0.91259 \\
\hline PZ12 & -0.16946 & -0.04221 & -0.02 & -0.16946 & $-9.5782 e-05$ & 303 & 2567.3 & 3.9638 & 31 & 262.66 & 0.93513 \\
\hline P213 & -0.26453 & -0.06608 & 0.08 & 1.0581 & 0.00059936 & 271 & 3584.4 & 5.5504 & 24.1 & 318.76 & 1.1373 \\
\hline PZ13-F50 & -0.13683 & & 0.09 & 0.61573 & & 556 & 3803.9 & & 49.2 & 336.6 & \\
\hline P214 & -0.15687 & -8.039364 & 0.06 & 0.47062 & 0.00026778 & 380 & 2980.6 & 4.6344 & 34.2 & 268.25 & 0.96142 \\
\hline P214-F50 & -0.14973 & & 0.09 & 0.67379 & & 350 & 2620.3 & & 31.7 & 237.32 & \\
\hline PZ15 & -0.57852 & -0.14532 & 0.02 & 0.57852 & 0.00032952 & 190 & 5495.9 & 8.5638 & 14.4 & 416.53 & 1.4945 \\
\hline PZ15-F50 & -0.31184 & & -0.02 & -6.31184 & & 360 & 5613.1 & & 13.6 & 222.05 & \\
\hline $\begin{array}{r}\text { PZ16 } \\
\text { P }\end{array}$ & -0.58906 & -0.14705 & 0.03 & 0.88359 & 0.00050018 & 168 & 4948.1 & 7.6614 & 13.6 & 400.56 & 1.4283 \\
\hline $\begin{array}{l}\text { PZ16-F50 } \\
\text { PZ17 }\end{array}$ & $\begin{array}{l}-0.38382 \\
-0.53896\end{array}$ & -0.13474 & $\begin{array}{r}0.04 \\
-0.02\end{array}$ & $\begin{array}{r}0.77364 \\
-0.53896\end{array}$ & -0.00930553 & $\begin{array}{l}241 \\
277\end{array}$ & $\begin{array}{l}4661.2 \\
7464.6\end{array}$ & & 19.4 & $\begin{array}{l}375.22 \\
638\end{array}$ & \\
\hline PZ17-F50 & -0.73666 & & $\begin{array}{l}-0.02 \\
-0.02\end{array}$ & $\begin{array}{l}-0.53896 \\
-0.73666\end{array}$ & & $\begin{array}{l}277 \\
180\end{array}$ & $\begin{array}{l}7464.6 \\
6679.9\end{array}$ & 11.58 & $\begin{array}{l}23.7 \\
15.8\end{array}$ & $\begin{array}{l}638.67 \\
581.96\end{array}$ & 2.2808 \\
\hline PZ-18 & -0.43316 & -0.10883 & -0.02 & -6.43316 & -0.00024679 & 313 & 6779 & 10.568 & 26.9 & 582.6 & 2.091 \\
\hline PZ18-F50 & -0.31726 & & 0.05 & 0.79315 & & 449 & 7122.5 & & 38.4 & 609.14 & \\
\hline $\begin{array}{r}\text { PZ19 } \\
\text { PZ19-F50 }\end{array}$ & $\begin{array}{r}0.33188 \\
-0.33894\end{array}$ & 0.083291 & $\begin{array}{r}-0.02 \\
0.03\end{array}$ & $\begin{array}{r}-0.33188 \\
0.50841\end{array}$ & -0.00018887 & $\begin{array}{l}315 \\
323\end{array}$ & $\begin{array}{l}5227.1 \\
5473.9\end{array}$ & 8.137 & $\begin{array}{l}24.2 \\
25.2\end{array}$ & $\begin{array}{l}402.57 \\
427.06\end{array}$ & 1.4396 \\
\hline PZ20 & 2.7413 & 0.68531 & 0 & $\theta$ & 0 & 711 & 7796.1 & 12.095 & 71.42 & 783.12 & 2.7967 \\
\hline $\begin{array}{r}\text { PZ20-F25 } \\
\text { BKGND }\end{array}$ & & & $\begin{array}{r}0.026 \\
0\end{array}$ & $\begin{array}{r}1.1536 \\
0\end{array}$ & & $\begin{array}{r}245.2 \\
7.04\end{array}$ & $\begin{array}{r}10880 \\
7.04\end{array}$ & & $\begin{array}{l}30.53 \\
0.05\end{array}$ & $\begin{array}{r}1354.6 \\
0.05\end{array}$ & \\
\hline$f(i)$ & 0.0002 & & 0.0882 & 9.9882 & & 9.0322 & e.e322 & & 0.024 & 0.014 & \\
\hline
\end{tabular}




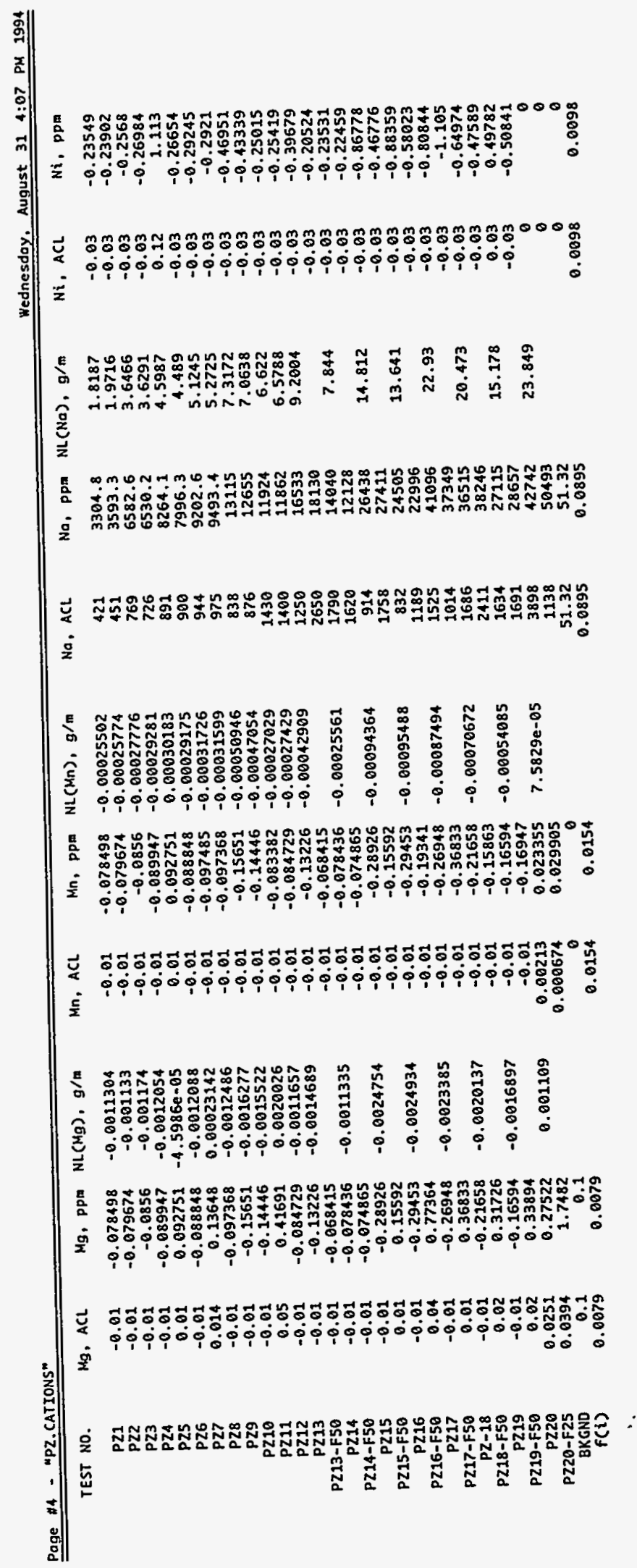




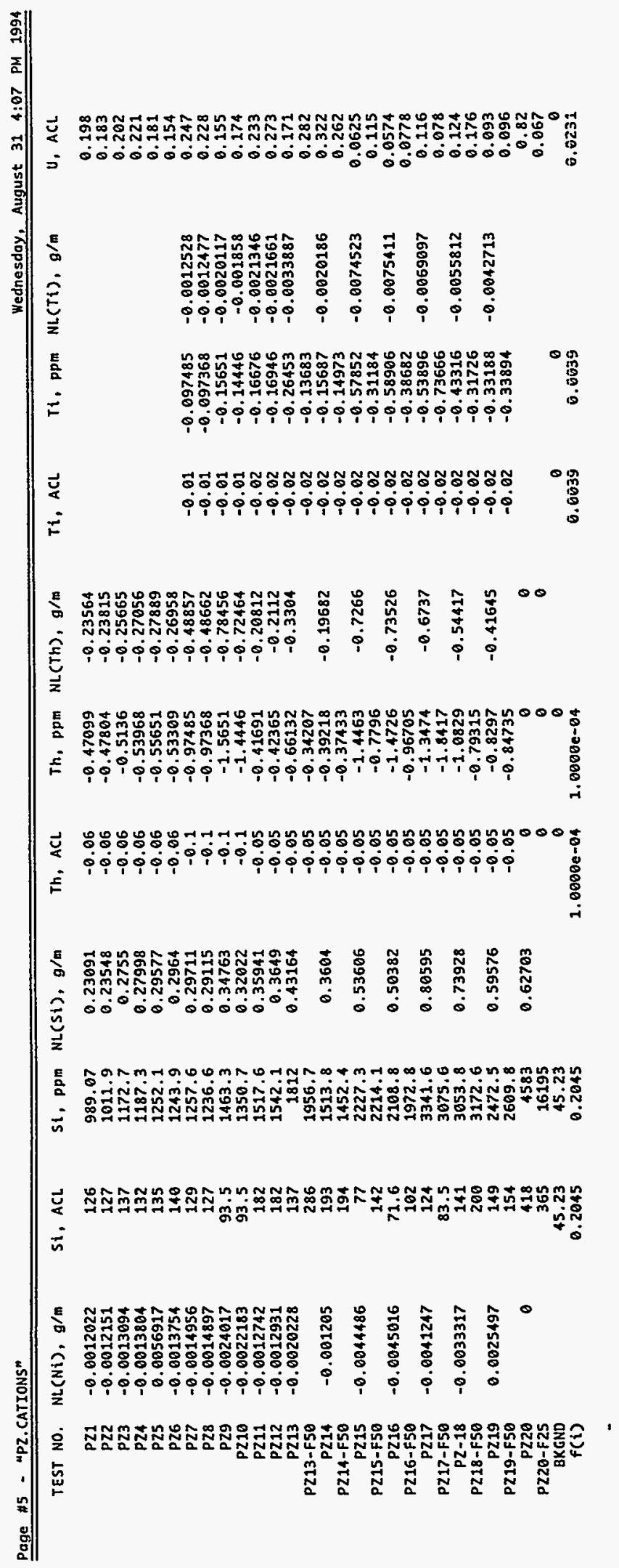


Page $n 6$ - "PZ.CATIONS"

\begin{tabular}{|c|c|c|c|c|c|c|c|c|}
\hline TEST NO. & $U, p p m$ & $N L(U), g / m 2$ & $Z n, A C L$ & $2 n, p p m$ & $N L(Z n), g / m$ & $2 r, A C L$ & $2 r$, ppm & $\mathrm{NL}(2 r), g / m$ \\
\hline & 1.5543 & 0.0033662 & & & & -0.02 & -0.157 & -0.0049091 \\
\hline PZ2 & 1.458 & 0.0032444 & & & & -0.02 & -0.15935 & -0.0049615 \\
\hline P23 & 1.7291 & 0.0037404 & & & & -0.02 & -0.1712 & -0.0053468 \\
\hline PZ4 & 1.9878 & 0.0043141 & & & & -0.02 & -0.17989 & -0.0056366 \\
\hline PZS & 1.6788 & 0.0036421 & & & & -0.02 & -0.1855 & -0.0058103 \\
\hline P26 & 1.3683 & 0.0029953 & & & & -0.02 & -0.1777 & -0.0056162 \\
\hline PZ7 & 2.4079 & 0.0052241 & 0.02 & 0.19497 & 0.048857 & -0.02 & -0.19497 & -0.0061072 \\
\hline PZ8 & 2.22 & 0.004803 & 0.01 & 0.097368 & 0.024331 & -0.02 & -0.19474 & -0.0060828 \\
\hline PZ9 & 2.4258 & 0.0052644 & 0.01 & 0.15651 & 0.039228 & -0.02 & -0.31301 & -0.0098071 \\
\hline PZ10 & 2.5137 & 0.0054583 & 0.01 & 0.14446 & 0.036232 & -0.02 & -0.28893 & -0.009058 \\
\hline PZ11 & 1.9428 & 0.0041985 & -0.02 & -0.16676 & -0.041624 & -0.05 & -0.11691 & -0.013008 \\
\hline PZ12 & 2.3131 & 0.004992 & -0.02 & -0.16946 & -0.04224 & -0.05 & -0.42365 & -0.0132 \\
\hline PZ13 & 2.2617 & 0.0048916 & 0.04 & 0.52906 & 0.13216 & -0.05 & -0.66132 & -0.02065 \\
\hline PZ13-F50 & 1.9293 & & -0.02 & -0.13683 & & -0.05 & -0.34207 & \\
\hline PZ14 & 2.5256 & 0.0054871 & 0.03 & 0.23531 & 0.059045 & -0.05 & -0.39218 & -0.012301 \\
\hline PZ14-F50 & 1.9615 & & -0.02 & -0.14973 & & -0.05 & -0.37433 & \\
\hline PZ15 & 1.8079 & 0.0039318 & -0.02 & -0.57852 & -0.14532 & -0.05 & -1.4463 & -0.045413 \\
\hline PZ15-F50 & 1.7931 & & 0.31 & 4.8335 & & -0.05 & -0.7796 & \\
\hline PZ16 & 1.6906 & 0.003654 & 0.03 & 0.88359 & 0.22058 & -0.05 & -1.4726 & -0.045954 \\
\hline PZ16-F50 & 1.5047 & & 0.45 & 8.7034 & & -0.05 & -0.96705 & \\
\hline PZ17 & 3.126 & 0.0067662 & -0.02 & -0.53896 & -0.23474 & -0.05 & -1.3474 & -0.042106 \\
\hline PZ17-F50 & 2.873 & & 0.02 & 0.73666 & & & & \\
\hline $\begin{array}{r}P Z-18 \\
P Z 18-F 50\end{array}$ & $\begin{array}{l}2.6856 \\
2.7919\end{array}$ & 0.0058422 & $\begin{array}{l}-0.02 \\
-0.02\end{array}$ & $\begin{array}{l}-0.13316 \\
-0.31726\end{array}$ & -0.10883 & $\begin{array}{l}-0.05 \\
-0.05\end{array}$ & $\begin{array}{r}-1.0829 \\
-0.79315\end{array}$ & -0.034011 \\
\hline $\begin{array}{r}\text { PZ19 } \\
\text { PZ19 }\end{array}$ & 1.5432 & 0.0033533 & 0.12 & 1.9913 & 0.49974 & -0.05 & -0.8297 & -0.026028 \\
\hline $\begin{array}{r}\text { P219-F50 } \\
\text { PZ20 }\end{array}$ & $\begin{array}{l}1.6269 \\
8.9913\end{array}$ & & -0.02 & -0.33894 & 0 & 0.00345 & $\begin{array}{r}-0.84735 \\
0.037829\end{array}$ & 0.0011822 \\
\hline PZ20-F25 & 2.9728 & & & & 0 & 0.000594 & 0.026356 & \\
\hline $\begin{array}{r}\text { BKGND } \\
f(i)\end{array}$ & $0.0231^{\circ}$ & & 0.0002 & $\begin{array}{r}0 \\
0.0002\end{array}$ & & 0.0016 & 0.0016 & \\
\hline
\end{tabular}

Wednesdoy. August $31 \quad$ 4:07 PM 2994 


\begin{tabular}{|c|c|c|c|c|c|c|c|c|c|c|c|}
\hline \multicolumn{12}{|c|}{ Tuesday, January 3 9:58 AM 1995} \\
\hline TEST NUMBER & RXN TIME & $\mathrm{pH}$ & DIL FACTOR & SA $/ V$ & LEACHANT & ACL NUMBER & Al, ACL. & Al, ppm & NL(AI), $g / m$ & B, ACL & B, ppm \\
\hline TW1 & 14 & 8.95 & 13.48 & 321 & 2.05 & $91-0532-01$ & 0.27 & 3.641 & 0.5546 & 0.69 & 9.236 \\
\hline & 14 & 9.31 & 25.03 & 327 & 2.03 & $91-0532-02$ & 0.18 & 4.506 & 0.6788 & 0.37 & 9.262 \\
\hline$T W 2-F 50$ & 14 & .... & 35.34 & rer & nos & $91-0532-03$ & -0.1 & -3.534 & & 0.24 & 8.482 \\
\hline$T W 3-F 50$ & 28 & & 24.94 & NeJ & 2.07 & $91-0533-04$ & -0.1 & -2.494 & 0.0500 & 0.41 & 10.22 \\
\hline & 28 & 9.28 & 28.89 & 319 & 2.05 & $91-0533-01$ & 0.2 & 5.778 & 0.8922 & 0.36 & 10.4 \\
\hline$T W 4-F 25$ & 28 & 30. & 19.59 & (2) & 6.00 & $91-0533-02$ & -0.1 & $\begin{aligned}-1.959 \\
-1.959\end{aligned}$ & $0.09<6$ & 0.46 & 9.013 \\
\hline & 56 & 9.5 & 11.44 & 322 & 2.05 & $91-0596-01$ & 0.4 & 4.578 & 0.7003 & 1.05 & 12.02 \\
\hline TW6 & 56 & 9.44 & 11.37 & 317 & 2.06 & $91-0596-02$ & 0.38 & 4.321 & 0.672 & 1.04 & 11.83 \\
\hline TW7 & 91 & 9.36 & 41.54 & 321 & 2.06 & $91-0639-01$ & -0.1 & $\begin{array}{r}4.321 \\
-4.154\end{array}$ & 0.082 & 0.37 & $\begin{array}{l}1.83 \\
15.37\end{array}$ \\
\hline$T W 7-F 50$ & 91 & 1.20 & 21.67 & 261 & 2.00 & $91-0639-02$ & -0.1 & -2.167 & & 0.76 & 16.47 \\
\hline TW9 & 180 & 9.4 & 29.81 & 323 & 2.05 & $92-0134-07$ & 0.17 & 5.067 & 0.7728 & 0.62 & 18.48 \\
\hline TW9-F50 & 180 & & 18.99 & & & $92-0134-08$ & -0.1 & -1.899 & & 0.99 & 18.8 \\
\hline TW10 & 180 & 9.54 & 28.94 & 317 & 2.05 & $92-0134-09$ & 0.1 & 2.894 & 0.4498 & 0.61 & 17.66 \\
\hline$T W 10-F 50$ & 180 & 5.04 & 18.23 & 218 & 6.00 & $92-0134-10$ & $\begin{array}{c}0.1 \\
-0.1\end{array}$ & $\begin{array}{r}6.874 \\
-1.823\end{array}$ & 0.4490 & 0.98 & 17.87 \\
\hline TW11 & 360 & 9.63 & 11.84 & 322 & 2.05 & PACKET. 1 & 0.411 & 4.865 & 0.7554 & $\begin{array}{l}1.93 \\
1.93\end{array}$ & 22.84 \\
\hline TW12 & 360 & 9.59 & 14.59 & 325 & 2.05 & PACKET.1 & 0.269 & 3.925 & 0.6039 & 1.66 & 24.22 \\
\hline TW13 & 540 & 10.48 & 4.6 & 320 & 2.05 & PACKET.1 & 0.200 & 3.020 & & 1.00 & 64.26 \\
\hline TW14 & 540 & 9.58 & 4.416 & 323 & 2.06 & PACKET.1 & 0.812 & 3.586 & 0.5551 & 3.9 & 17.22 \\
\hline TW15 & 720 & 9.31 & 6.795 & 319 & 2.05 & PACKET. I & 0.234 & i. 59 & 0.2492 & 2.97 & 20.18 \\
\hline TW16 & 720 & 9.75 & 6.994 & 319 & 2.05 & PACKET. I & 0.084 & 0.5875 & 0.0921 & 10.4 & 72.74 \\
\hline $\begin{array}{c}\text { BKGND } \\
f(i)\end{array}$ & & & $\begin{array}{l}1 \\
1\end{array}$ & & & & $\begin{array}{r}0 \\
0.0203\end{array}$ & $\begin{array}{r}0 \\
0.0203\end{array}$ & & $\begin{array}{r}0 \\
0.0248\end{array}$ & $\begin{array}{r}0 \\
0.0248\end{array}$ \\
\hline \multicolumn{12}{|l|}{ - } \\
\hline TEST No. & $\mathrm{NL}(B), g / \mathrm{mL}^{2}$ & $\mathrm{Ba}, \mathrm{ACL}$ & $\mathrm{Ba}, \mathrm{ppm}$ & $N L(B a), g / m$ & Co, ACL & $\mathrm{Ca}, \mathrm{ppm}$ & $N L(C a), g / m$ & $\mathrm{Cr}, \mathrm{ACL}$ & $\mathrm{Cr}, \mathrm{ppm}$ & $N L(C r), g / m$ & Su, ACL \\
\hline TW1 & 1.16 & -0.02 & -0.268 & 0 & -0.02 & -0.2677 & 0 & -0.02 & -0.2677 & 0 & -0.02 \\
\hline $\begin{array}{r}T W 2 \\
T W 2-550\end{array}$ & 1.142 & -0.02 & -0.5006 & 0 & -0.02 & -0.5006 & 0 & -0.02 & -0.5006 & 0 & -0.02 \\
\hline $\begin{array}{l}1 W 2-r 56 \\
\text { TW3 }\end{array}$ & 1.248 & $\begin{array}{l}-6.02 \\
-0.02\end{array}$ & $\begin{array}{l}-0.7068 \\
-0.6893\end{array}$ & 0 & $\begin{array}{l}-0.02 \\
-0.02\end{array}$ & $\begin{array}{l}-0.7068 \\
-0.6893\end{array}$ & 0 & $\begin{array}{l}-0.02 \\
-0.02\end{array}$ & $\begin{array}{l}-0.7068 \\
-0.6893\end{array}$ & 0 & $\begin{array}{l}-0.02 \\
-0.02\end{array}$ \\
\hline TW3-F50 & 1.248 & $\begin{array}{l}-6.02 \\
-0.02\end{array}$ & $\begin{array}{l}-0.0893 \\
-0.4987\end{array}$ & 6 & $\begin{array}{l}-0.02 \\
-0.02\end{array}$ & $\begin{array}{l}-0.6893 \\
-0.4987\end{array}$ & 0 & $\begin{array}{l}-0.02 \\
-0.02\end{array}$ & $\begin{array}{l}-0.6893 \\
-0.4987\end{array}$ & 0 & $\begin{array}{l}-6.02 \\
-0.02\end{array}$ \\
\hline & 1.315 & -0.02 & -0.5778 & 0 & -0.02 & -0.5778 & 0 & -0.02 & -0.5778 & 0 & -0.02 \\
\hline$T W 4-F 25$ & & -0.02 & -0.3919 & & -0.02 & -0.3919 & & -0.02 & -0.3919 & 6 & -0.02 \\
\hline TW5 & 1.505 & -0.02 & -0.2289 & 0 & 0.03 & 0.3433 & 0.124 & -0.02 & -0.2289 & 0 & 0.03 \\
\hline TW6 & 1.505 & -0.02 & -0.2276 & 0 & 0.04 & 0.4552 & 0.167 & -0.02 & -0.2276 & 0 & 0.03 \\
\hline TW7 & & -0.02 & -0.8308 & & -0.02 & -0.8308 & & -0.02 & -0.8308 & & -0.02 \\
\hline TW7-F50 & & -0.02 & -0.4334 & & 0.04 & 0.8668 & 0.314 & -0.02 & $\begin{array}{l}-0.4334 \\
-6.4330\end{array}$ & 0 & -0.02 \\
\hline TW8 & 2.076 & -0.02 & -0.2161 & 0 & 0.05 & 0.5402 & 0.1957 & $\begin{array}{l}-0.02 \\
-0.02\end{array}$ & -0.2161 & 0 & 0.04 \\
\hline TW9 & 2.307 & .0 .02 & -0.5962 & 0 & 0.03 & 0.8942 & 0.3219 & -0.02 & -0.5962 & 0 & -0.02 \\
\hline$T w 9-F 50$ & & -0.02 & -0.3798 & & 0.03 & 0.5696 & & -0.02 & -0.3798 & & -0.02 \\
\hline TW10 & 2.246 & $\begin{array}{l}-0.02 \\
-0.02\end{array}$ & $\begin{array}{l}-0.3798 \\
-0.5789\end{array}$ & 0 & $\begin{array}{l}0.03 \\
-0.02\end{array}$ & $\begin{array}{l}-.5696 \\
-0.5789\end{array}$ & 0 & $\begin{array}{l}-6.02 \\
-0.02\end{array}$ & $\begin{array}{l}-6.5798 \\
-6.5789\end{array}$ & 0 & $\begin{array}{l}-6.02 \\
-0.02\end{array}$ \\
\hline TW10-F50 & 2.272 & -0.02 & $\begin{array}{l}-0.3789 \\
-0.3646\end{array}$ & 0 & $\begin{array}{r}-0.06 \\
0.03\end{array}$ & $\begin{array}{r}-0.3769 \\
0.5469\end{array}$ & 0.2006 & $\begin{array}{l}-0.06 \\
-0.02\end{array}$ & $\begin{array}{l}-0.0389 \\
-0.3646\end{array}$ & $:$ & $\begin{array}{l}-0.02 \\
-0.02\end{array}$ \\
\hline TW11 & 2.838 & 0.019 & 0.2249 & 0.3492 & 0.238 & 2.817 & 0.9721 & 0.017 & 0.2012 & 1.25 & 0.062 \\
\hline TW12 & 2.981 & 0.006 & 0.0876 & 0.1347 & 0.144 & 2.101 & 0.7184 & 0.011 & 0.1605 & 0.9878 & 0.025 \\
\hline TW13 & & & 0.1016 & a 1572 & & & 07337 & & a 9578 & & 0.124 \\
\hline $\begin{array}{l}T W 14 \\
T W 15\end{array}$ & $\begin{array}{l}2.133 \\
2.531\end{array}$ & 0.023 & $\begin{array}{l}0.1016 \\
0.0747\end{array}$ & $\begin{array}{l}0.1572 \\
0.1172\end{array}$ & $\begin{array}{l}0.183 \\
0.427\end{array}$ & $\begin{array}{l}2.133 \\
2.902\end{array}$ & $\begin{array}{r}0.7337 \\
1.011\end{array}$ & $\begin{array}{l}0.038 \\
0.021\end{array}$ & $\begin{array}{l}0.1678 \\
0.1427\end{array}$ & $\begin{array}{r}1.039 \\
0.8947\end{array}$ & $\begin{array}{l}0.124 \\
0.022\end{array}$ \\
\hline TW16 & $\begin{array}{l}2.531 \\
9.121\end{array}$ & $\begin{array}{l}0.011 \\
0.003\end{array}$ & 0.021 & 0.0329 & 0.582 & 4.07 & 1.418 & 0.046 & $\begin{array}{l}0.1427 \\
0.3217\end{array}$ & $\begin{array}{r}0.8947 \\
2.017\end{array}$ & 0.005 \\
\hline $\begin{array}{r}\text { BKGND } \\
f(1)\end{array}$ & & 0.002 & 0.002 & & $\begin{array}{r}0 \\
0.0086\end{array}$ & $\begin{array}{r}0 \\
0.0086\end{array}$ & & 0.0005 & 0.0005 & & \\
\hline
\end{tabular}




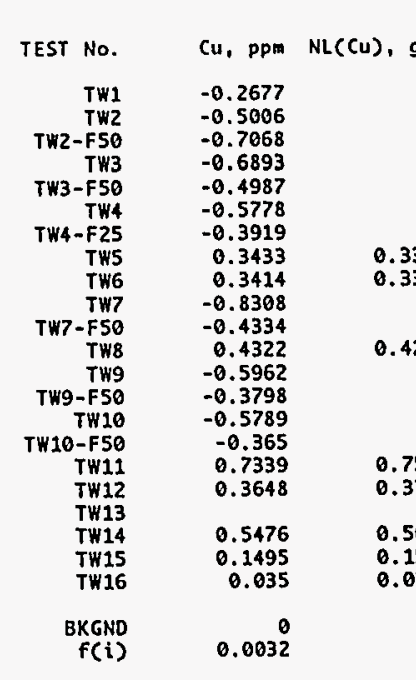

Fe, ACL

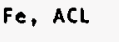

Fe, ppm NL(Fe), g/m

0.36
0.26
-0.02

-0.02
0.19

-0.02
0.31
-0.02

-0.02
0.83

0.77
0.23
-0.02

-0.02
0.94

0.32
-0.02
0.17

0.17
-0.02
0.469
0.137

1.3
0.293

0.0798
$4.819 \quad 0.1881$

-0.7068
6.549 $\quad 0.2541$

$-0.4987$

8.956
-0.3919

9.499

8.762
-0.554
-0.4334

-0.4334
10.16
9.538
-0.3798

4.921
-0.3646

5.552
1.999

5.741

0.9622

0.0798
0.3518

0.3697
0.3464

0.3965
0.3701

0.1945

0.2155
0.0769

0.2222
0.078
0.0354
K, ppm NL(K), $9 / \mathrm{m} 2$

Li, ACL

$\begin{array}{ll}11.91 & 1.201 \\ 13.52 & 1.338\end{array}$

$\begin{array}{ll}16.61 & 1.658\end{array}$

15.71
34.09

34.09

$\begin{array}{ll}12.59 & 1.265 \\ 12.52 & 1.278\end{array}$

$17.03 \quad 1.66$

$\begin{array}{ll}16.47 & 1.66 \\ 17.29 & 1.743\end{array}$

$\begin{array}{ll}16.99 & 1.702\end{array}$

$\begin{array}{ll}16.52 & 1.537\end{array}$

14.58
17.87

17.51

17.49
18.28
41.05

0

0.0309
0.64
0.35
0.22
0.26
0.35
0.31
0.35
0.95
0.96
0.34
0.54
0.37
0.5
0.5
0.
1.
1.
3.
2.
7.
Li, ppm NL(Li), g/m

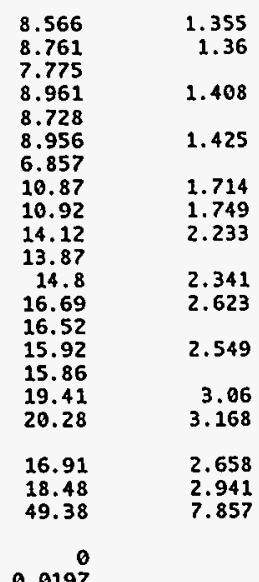

\begin{tabular}{|c|c|c|c|c|c|c|c|c|c|c|c|}
\hline TEST No. & $\mathrm{Mg}, \mathrm{ACL}$ & $\mathrm{Mg}, \mathrm{ppm}$ & $\mathrm{NL}(\mathrm{Mg}), \mathrm{g} / \mathrm{m}$ & $\mathrm{Mn}, \mathrm{ACL}$ & Mn, ppm & $N L(M n), g / m$ & $\mathrm{Na}, \mathrm{ACL}$ & $\mathrm{Na}, \mathrm{ppm}$ & $\mathrm{NL}(\mathrm{Na}), \mathrm{g} / \mathrm{m}$ & $\mathrm{Ni}, \mathrm{ACl}$ & $\mathrm{Ni}, \mathrm{ppm}$ \\
\hline$\underset{\substack{T W 1 \\
T W 2}}{T W W_{1}}$ & $\begin{array}{l}0.05 \\
0.04\end{array}$ & $\begin{array}{r}0.6693 \\
1.001 \\
-0.3534\end{array}$ & $\begin{array}{l}0.2606 \\
0.3827\end{array}$ & 0.06 & $\begin{array}{r}0.8031 \\
i .252 \\
-0.3534\end{array}$ & $\begin{array}{l}0.1787 \\
0.2734\end{array}$ & $\begin{array}{l}2.07 \\
1.12\end{array}$ & $\begin{array}{l}27.71 \\
28.04\end{array}$ & $\begin{array}{l}1.306 \\
1.297\end{array}$ & $\begin{array}{l}-0.03 \\
-0.03\end{array}$ & $\begin{array}{l}-0.4015 \\
-0.751\end{array}$ \\
\hline & $\begin{array}{r}-0.01 \\
0.02\end{array}$ & -0.3534 & & $\begin{array}{r}-0.01 \\
0.03\end{array}$ & -0.3534 & & 0.78 & 27.57 & & -0.03 & \\
\hline $\begin{array}{r}\text { TW3 } \\
-F 50\end{array}$ & $\begin{array}{r}0.02 \\
-0.01\end{array}$ & $\begin{array}{r}0.6893 \\
-0.2494\end{array}$ & 0.2668 & $\begin{array}{r}0.03 \\
-0.01\end{array}$ & $\begin{array}{r}1.034 \\
-0.2494\end{array}$ & 0.2287 & 0.89 & 30.68 & 1.437 & -0.03 & $\begin{array}{r}-1.034 \\
-0.7481\end{array}$ \\
\hline TW4 & 0.04 & 1.156 & 0.4528 & 0.05 & $\begin{array}{r}-0.2494 \\
1.444\end{array}$ & 0.3234 & $\begin{array}{l}1.23 \\
1.06\end{array}$ & $\begin{array}{l}30.67 \\
30.62\end{array}$ & 1.452 & $\begin{array}{l}-6.03 \\
-0.03\end{array}$ & $\begin{array}{l}-0.04867 \\
-0.8667\end{array}$ \\
\hline$T W 4-F 25$ & -0.01 & -0.1959 & & -0.01 & -0.1959 & & 1.48 & 29 & 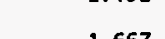 & -0.03 & -0.5878 \\
\hline TH5 & 0.11 & 1.259 & 0.4887 & 0.15 & 1.717 & 0.3808 & 3.1 & 35.48 & 1.667 & 0.03 & 0.3433 \\
\hline TW6 & 0.1 & 1.138 & 0.4487 & 0.14 & 1.593 & 0.359 & 3.07 & 34.93 & 1.667 & -0.03 & $\begin{array}{l}-0.3414 \\
-1.246\end{array}$ \\
\hline $\begin{array}{r}T W 7 \\
T W 7-F 50\end{array}$ & 0.03 & 0.246 & 0.08439 & $\begin{array}{r}0.04 \\
-0.01\end{array}$ & $\begin{array}{r}1.662 \\
-0.2167\end{array}$ & & $\begin{array}{l}1.12 \\
2.83\end{array}$ & $\begin{array}{l}46.53 \\
61.33\end{array}$ & 2.193 & $\begin{array}{l}-0.03 \\
-0.03\end{array}$ & $\begin{array}{r}-1.246 \\
-0.6501\end{array}$ \\
\hline$T W 7-F 50$ & $\begin{array}{l}0.01 \\
0.14\end{array}$ & $\begin{array}{l}0.2167 \\
1.513\end{array}$ & $\begin{array}{r}0.08439 \\
0.589\end{array}$ & $\begin{array}{r}-0.01 \\
0.17\end{array}$ & $\begin{array}{r}-0.2168 \\
1.837\end{array}$ & 0.4087 & $\begin{array}{l}2.83 \\
4.48\end{array}$ & $\begin{array}{r}48.4 \\
48.4\end{array}$ & 2.281 & -0.03 & -0.3241 \\
\hline TW9 & 0.06 & 1.788 & 0.6921 & 0.06 & 1.788 & 0.3955 & $\begin{array}{l}1.48 \\
1.88\end{array}$ & 56.04 & 2.625 & -0.03 & -0.8942 \\
\hline TW9-F50 & -0.01 & -0.1899 & & -0.01 & -0.1899 & & 3.04 & 57.72 & & -0.03 & -0.5696 \\
\hline TW10 & 0.03 & 0.8683 & 0.3424 & 0.03 & 0.8683 & 0.1957 & 1.8 & 52.1 & 2.486 & -0.03 & -0.8683 \\
\hline TW10-F50 & -0.01 & -0.1823 & & -0.01 & -0.1823 & & 3.03 & 55.24 & & -0.03 & -0.5469 \\
\hline TW11 & 0.127 & 1.503 & 0.5836 & 0.232 & 2.743 & 0.6092 & 5.24 & 62.03 & 2.914 & 0.054 & 0.6392 \\
\hline TH12 & 0.045 & 0.6567 & 0.2526 & 0.082 & 1.197 & 0.263 & 4.5 & 65.67 & 3.057 & 0.021 & 0.3065 \\
\hline $\begin{array}{l}\text { TW14 } \\
\text { TW15 }\end{array}$ & $\begin{array}{c}0.222 \\
0.04\end{array}$ & $\begin{array}{l}0.9804 \\
0.2718\end{array}$ & $\begin{array}{l}0.3794 \\
0.1065\end{array}$ & $\begin{array}{l}0.328 \\
0.072\end{array}$ & $\begin{array}{r}1.449 \\
0.4893\end{array}$ & $\begin{array}{l}0.3203 \\
0.1996 \\
0.0219\end{array}$ & $\begin{array}{r}12.6 \\
9.1 \\
26.7\end{array}$ & $\begin{array}{l}55.64 \\
61.84 \\
186.7\end{array}$ & $\begin{array}{l}2.606 \\
2.933 \\
8.856\end{array}$ & $\begin{array}{l}0.112 \\
0.041 \\
0.027\end{array}$ & $\begin{array}{l}0.4946 \\
0.2786 \\
0.1888\end{array}$ \\
\hline TW16 & 0.013 & 0.0909 & 0.0356 & 0.014 & 0.0979 & 0.0219 & 26.7 & 186.7 & 8.856 & 0.027 & \\
\hline $\begin{array}{r}\text { BKGND } \\
f(i)\end{array}$ & 0.008 & 0.008 & & $0.014^{0}$ & $\begin{array}{r}0 \\
0.014\end{array}$ & & $\begin{array}{r}0 \\
0.0661\end{array}$ & $\begin{array}{r}0 \\
0.0661\end{array}$ & & $\begin{array}{r}0 \\
0.0065\end{array}$ & $\begin{array}{r}0 \\
0.0065\end{array}$ \\
\hline
\end{tabular}




\begin{tabular}{|c|c|c|c|c|c|c|c|c|c|c|c|}
\hline TEST NO. & $N L(N i), g / m$ & Si, ACL & $\mathrm{Si}, \mathrm{ppm}$ & $N L(S i), g / m$ & $T h, A C L$ & Th, ppm & $N L(T h), g / m$ & $T i, A C L$ & $\mathrm{Ti}, \mathrm{ppm}$ & $N L(T i), g / m$ & U, ACL \\
\hline $\begin{array}{l}T_{W 1} \\
T W 2\end{array}$ & $:$ & $\begin{array}{l}4.86 \\
2.86\end{array}$ & $\begin{array}{l}65.05 \\
71.59\end{array}$ & $\begin{array}{l}0.8861 \\
0.9573\end{array}$ & $\begin{array}{l}-0.05 \\
-0.05\end{array}$ & $\begin{array}{r}-0.6693 \\
-1.252\end{array}$ & 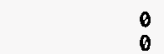 & $\begin{array}{l}-0.02 \\
-0.02\end{array}$ & $\begin{array}{l}-0.2677 \\
-0.5006\end{array}$ & $:$ & $\begin{array}{l}0.0645 \\
0.0335\end{array}$ \\
\hline$T W 2-F 50$ & & $\begin{array}{l}1.800 \\
1.74\end{array}$ & 61.49 & & $\begin{array}{l}-6.05 \\
-0.05\end{array}$ & $\begin{array}{l}-1.262 \\
-1.767\end{array}$ & & $\begin{array}{l}-6.02 \\
-0.02\end{array}$ & $\begin{array}{l}-6.03606 \\
-0.7068\end{array}$ & & $\begin{array}{l}0.0335 \\
0.0123\end{array}$ \\
\hline$T_{T W 3}^{T W 3}$ & 0 & 2.17 & $\begin{array}{r}74.79 \\
6553\end{array}$ & 1.012 & -0.05 & -1.723 & 0 & -0.02 & -0.6893 & 0 & 0.0204 \\
\hline TW4 & 0 & 2.66 & 76.84 & 1.053 & -0.05 & -1.444 & 0 & $\begin{array}{l}-0.06 \\
-0.02\end{array}$ & $\begin{array}{l}-6.49878 \\
-0.5778\end{array}$ & 0 & $\begin{array}{l}0.6097 \\
0.0268\end{array}$ \\
\hline$T W 4-F 25$ & & 2.92 & 57.21 & & -0.05 & -0.9796 & & -0.02 & -0.3919 & & 0.0124 \\
\hline IWS & 0 & 7.6 & 86.98 & 1.181 & -0.05 & -0.5722 & 0 & 0.03 & 0.3433 & 0 & 0.0742 \\
\hline TW6 & 0 & 7.48 & 85.12 & 1.174 & -0.05 & $\begin{array}{r}-0.569 \\
\end{array}$ & 0 & -0.02 & -0.2276 & 0 & 0.0767 \\
\hline TWW7-FSO & 0 & $\begin{array}{l}.466 \\
4.37\end{array}$ & 94.7 & 1.29 & $\begin{array}{l}-0.05 \\
-0.05\end{array}$ & $\begin{array}{l}-2.087 \\
-1.084\end{array}$ & 0 & $\begin{array}{l}-0.02 \\
-0.02\end{array}$ & $\begin{array}{l}-0.8308 \\
-0.433\end{array}$ & 0 & $\begin{array}{r}0.034 \\
0.0378\end{array}$ \\
\hline TW8 & 0 & 9.96 & 107.6 & 1.466 & -0.05 & -0.5402 & 0 & 0,04 & 0.4322 & 0 & 0.153 \\
\hline TH9 & 0 & 4.36 & 130 & 1.759 & -0.05 & -1.49 & 0 & -0.02 & -0.5961 & 0 & 0.0834 \\
\hline TW9-F50 & & 6.22 & 118.1 & & -0.05 & -0.9494 & & -0.02 & -0.3798 & & 0.085 \\
\hline$T^{T W 10}$ & $\stackrel{0}{\circ}$ & 3.84 & 111.1 & 1.533 & -0.05 & -1.447 & $\therefore$ & -0.02 & $\begin{array}{r}-0.5789 \\
-0.369\end{array}$ & $\therefore$ & 0.0642 \\
\hline$T W 10-F 50$ & & 5.74 & 104.6 & 1.443 & -0.05 & -0.9115 & 0 & -0.02 & -0.3646 & $\stackrel{0}{0}$ & 0.0691 \\
\hline TW11 & $\begin{array}{l}0.2836 \\
0.1347\end{array}$ & 11.94 & 141.3 & 1.917 & 0.07 & 0.8286 & 1.287 & 0.051 & 0.6037 & 0.375 & 0.179 \\
\hline $\begin{array}{l}T W 12 \\
T W 13\end{array}$ & & & & & & 0.5837 & 0.898 & 0.025 & 0.3648 & 0.2245 & \\
\hline $\begin{array}{l}\text { TW14 } \\
\text { TW15 }\end{array}$ & $\begin{array}{l}0.2188 \\
0.1248\end{array}$ & $\begin{array}{l}25.6 \\
14.4\end{array}$ & $\begin{array}{l}113.1 \\
97.85\end{array}$ & $\begin{array}{l}1.528 \\
1.339\end{array}$ & $\begin{array}{l}0.106 \\
0.097\end{array}$ & $\begin{array}{l}0.4681 \\
0.6591\end{array}$ & $\begin{array}{r}0.7246 \\
1.033\end{array}$ & $\begin{array}{l}0.085 \\
0.032\end{array}$ & $\begin{array}{l}0.3754 \\
0.2174\end{array}$ & $\begin{array}{l}0.2324 \\
0.1363\end{array}$ & $\begin{array}{l}0.301 \\
0.173\end{array}$ \\
\hline TW16 & 0.0846 & 23.4 & 163.7 & 2.24 & 0.019 & 0.1329 & 0.2083 & 0.005 & 0.035 & 0.0219 & 0.553 \\
\hline $\begin{array}{l}\text { BKGND } \\
f(i)\end{array}$ & & $\begin{array}{r}0 \\
0.2287\end{array}$ & $\begin{array}{r}0 \\
0.2287^{\circ}\end{array}$ & & $0.0023^{0}$ & $\begin{array}{r}0 \\
0.0023\end{array}$ & & $\begin{array}{r}0 \\
0.0054\end{array}$ & $\begin{array}{r}0.0054^{\circ} \\
0.0\end{array}$ & & $\begin{array}{r}0 \\
0.0164\end{array}$ \\
\hline
\end{tabular}

\begin{tabular}{|c|c|c|c|c|c|c|c|c|}
\hline TEST No. & U, ppm & $N L(U), g / m 2$ & $2 n, A C L$ & $\mathrm{Zn}, \mathrm{ppm}$ & $N L(2 n), g / m$ & $\mathrm{Zr}, \mathrm{ACL}$ & $\mathrm{Zr}, \mathrm{ppm}$ & $N L(Z r), g / m$ \\
\hline TW1 & 0.8633 & 0.164 & -0.02 & -0.2677 & 0 & -0.05 & -0.6693 & 0 \\
\hline $\begin{array}{r}T W 2 \\
T W Z-F 50\end{array}$ & 0.8386 & 0.1564 & -0.02 & -0.5006 & 0 & -0.05 & -1.252 & 0 \\
\hline $\begin{array}{l}T W 2-F 50 \\
T W 3\end{array}$ & 0.4347 & & -0.02 & -0.7068 & & -0.05 & -1.767 & \\
\hline TW3-F50 & $\begin{array}{l}0.7031 \\
0.2419\end{array}$ & 0.1327 & $\begin{array}{l}-0.02 \\
-0.02\end{array}$ & $\begin{array}{l}-0.6893 \\
-0.4987\end{array}$ & 0 & -0.05 & -1.723 & 0 \\
\hline & 0.7742 & 0.148 & $\begin{array}{l}-6.02 \\
-0.02\end{array}$ & $\begin{array}{l}-0.4988 \\
-0.5778\end{array}$ & 0 & $\begin{array}{l}-0.05 \\
-0.05\end{array}$ & $\begin{array}{l}-1.24 f \\
-1.444\end{array}$ & 0 \\
\hline TW4-F2S & 0.2429 & & 0.1 & -0.1959 & & -0.05 & -0.9796 & \\
\hline TW5 & 0.8492 & 0.1608 & -0.02 & -0.2289 & 0 & -0.05 & -0.5722 & $\theta$ \\
\hline TW6 & 0.8728 & 0.1679 & -0.02 & -0.2276 & 0 & -0.05 & -0.569 & 0 \\
\hline TW7-FSo & $\begin{array}{r}1.412 \\
0.8192\end{array}$ & 0.1556 & $\begin{array}{l}-0.02 \\
-0.02\end{array}$ & $\begin{array}{l}-0.8308 \\
-0.4334\end{array}$ & 0 & $\begin{array}{l}-0.05 \\
-0.05\end{array}$ & $\begin{array}{l}-2.077 \\
-1.084\end{array}$ & \\
\hline TW8 & 1.653 & 0.314 & $\begin{array}{l}-0.02 \\
-0.02\end{array}$ & $\begin{array}{l}-0.4334 \\
-0.2161\end{array}$ & 0 & $\begin{array}{l}-0.05 \\
-0.05\end{array}$ & $\begin{array}{r}-1.604 \\
-0.5402\end{array}$ & 0 \\
\hline TW9 & 2.486 & 0.4693 & -0.02 & -0.5962 & - & -0.05 & -1.49 & 0 \\
\hline TW9-F50 & 1.614 & & -0.02 & -0.3798 & & -0.05 & -0.9494 & \\
\hline TW10 & 1.858 & 0.3574 & -0.02 & -0.5789 & 0 & -0.05 & -1.447 & 0 \\
\hline TW10 - F50 & 1.26 & 0.2423 & -0.02 & -0.3646 & 0 & -0.05 & -0.9115 & \\
\hline TW11 & 2.119 & 0.4113 & 0.024 & 0.2841 & 4.411 & 0.011 & 0.1302 & 0.5778 \\
\hline$T W 12$ & 2.058 & 0.3957 & 0.004 & 0.0584 & 0.898 & 0.001 & 0.0146 & 0.0641 \\
\hline $\begin{array}{l}T W 13 \\
\text { TW14 }\end{array}$ & & & & & & & & \\
\hline $\begin{array}{l}\text { W } \\
\text { TW15 }\end{array}$ & 1.176 & $\begin{array}{l}0.2572 \\
0.2303\end{array}$ & $\begin{array}{l}0.108 \\
0.018\end{array}$ & 0.1223 & $\begin{array}{l}.383 \\
1.917\end{array}$ & $\begin{array}{r}0.016 \\
0.01\end{array}$ & $\begin{array}{r}0.0707 \\
0.068\end{array}$ & $\begin{array}{l}0.3125 \\
0.3043\end{array}$ \\
\hline TW16 & 3.868 & 0.7578 & 0.021 & 0.1469 & 2.302 & 0.001 & 0.007 & 0.0313 \\
\hline $\begin{array}{c}\text { BKGND } \\
f(i)\end{array}$ & $\begin{array}{r}0 \\
0.0164\end{array}$ & & $\begin{array}{r}0 \\
0.0002\end{array}$ & $\begin{array}{r}0 \\
0.0002\end{array}$ & & $\begin{array}{r}0 \\
0.0007\end{array}$ & $\begin{array}{r}0 \\
0.0007\end{array}$ & \\
\hline
\end{tabular}


Page $\# 1$ - "TW.EJ.CATIONS"

$$
\text { TEST NUMBER RXN TIME }
$$

$\begin{array}{rr}T W 31 & 14 \\ T W 32 & 14 \\ T W 32-F 50 & 14 \\ T W 33 & 28 \\ T W 33-F 50 & 28 \\ T W 34 & 28 \\ T W 34-F 25 & 28 \\ T W 35 & 56 \\ T W 36 & 56 \\ T W 37 & 91 \\ T W 37-F 50 & 91 \\ T W 38 & 91 \\ T W 39 & 180 \\ T W 39-F 50 & 180 \\ T W 40 & 180 \\ T W 40-F 50 & 180 \\ T W 41 & 360 \\ T W 42 & 360 \\ T W 43 & 540 \\ T W 44 & 540 \\ T W 45 & 720 \\ T W 46 & 720 \\ T W 40 & \end{array}$

BKGND
$f(i)$

$\begin{array}{rrr}\text { B, ppm } & \mathrm{NL}(\mathrm{B}), \mathrm{g} / \mathrm{m2} & \mathrm{Ba}, \mathrm{ACL} \\ 7.7141 & 0.93847 & -0.02 \\ 1.8794 & 0.21928 & -0.02 \\ 1.8058 & 0.41075 & -0.02 \\ 3.3995 & 0.41075 & -0.02 \\ 3.5429 & 0.34104 & -0.02 \\ 2.8819 & -0.02 \\ 2.7027 & & -0.02 \\ 7.8773 & 0.96766 & -0.02 \\ 8.7158 & 1.0693 & -0.02 \\ 10.744 & 1.3143 & -0.02 \\ 11.03 & 1.3576 & -0.02 \\ 10.856 & -0.02 \\ 20.147 & 2.4963 & -0.02 \\ 22.847 & 1.2315 & -0.02 \\ 9.984 & -0.02 \\ 9.9991 & 1.92 & -0.02 \\ 13.929 & 1.7096 & 0.008 \\ 12.607 & 1.5456 & 0.017 \\ 16.336 & 2.0524 & 0.032 \\ 18.373 & 2.2471 & 0.022 \\ 12.844 & 1.5653 & 0.003 \\ 18.762 & 2.3092 & 0.005 \\ 0.15 & & \\ 0.0248 & & 0 \\ & & 0.002\end{array}$

$\mathrm{pH}$
9.38
9.23
9.44
9.45
9.66
9.71
9.74
9.63
9.88
9.69
9.79
9.79
9.69
9.78
9.24
9.42

DIL FACTOR

12.646
17.085
36.116
26.15
25.306
36.023
19.305
12.308
10.895
28.273
18.694
11.549
125.92
26.879
38.4
23.254
10.715
11.059
4.0237
3.9942
7.0185
7.1069
1
1

$B a$, ppm NL(Ba), $g / m 2$

$$
\begin{array}{ll}
-0.25292 & -0.38911 \\
-0.3417 & -0.53727
\end{array}
$$$$
-0.72233
$$

-0.72233
-0.523
-0.50613

-0.50613
-0.72047

-0.72047
-0.3861
-0.24617

-0.24617
-0.21789

$-0.56545$

-0.37388
-0.23099

-0.23099
-2.5183
-0.53759

-0.53759
-0.768
-0.46507

$-0.46507$

0.08572
0.188
0.12876

0.087872
0.021055

0.021055
0.035535

0.002

$-1.1153$

-0.38225
-0.3373

$-0.86993$

-0.36319
-3.8983

$-1.1925$

0.13188

0.28924

0.13436
0.032195

0.054668

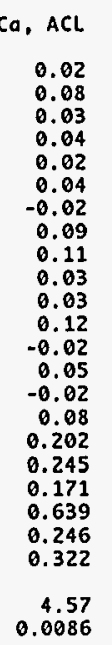

Ca, ppm NL(Ca), g/m2

-1.5088
-1.1347

0.25292
1.3668

1.0835
1.046

$-1.2481$

0.50613

1.4409
-0.3861
1.1077

1.1077
1.1984
0.84818

1.0905

0.84818
0.56082

-1.2142
-1.1778

1.3859
-2.5183

-2.344
-0.768

$-1.1277$

-0.768
1.8603
2.1644

2.1644
2.7095
0.68805

2.6895
0.68805
2.5523

1.7266

$-1.8915$

-0.86067
-0.66567

$-0.66567$

-0.71748
-1.011

\begin{tabular}{|c|c|c|}
\hline Al, ppm & $N L(A L), g / m 2$ & B, ACL \\
\hline $\begin{array}{l}1.3911 \\
1.8794\end{array}$ & $\begin{array}{l}0.05018 \\
0.12693\end{array}$ & $\begin{array}{l}0.61 \\
0.11\end{array}$ \\
\hline-3.6116 & & 0.05 \\
\hline $\begin{array}{r}2.615 \\
-2.5306\end{array}$ & 0.24013 & 0.13 \\
\hline $\begin{array}{l}-2.5306 \\
-3.6023\end{array}$ & & 0.14 \\
\hline $\begin{array}{l}-3.6023 \\
-1.9305\end{array}$ & -0.71106 & 0.08 \\
\hline 3.6925 & 0.40273 & $\begin{array}{l}0.14 \\
0.64\end{array}$ \\
\hline 4.031 & 0.45312 & 0.8 \\
\hline 3.11 & 0.31072 & 0.38 \\
\hline-1.8694 & & 0.59 \\
\hline 5.0817 & 0.623 & 0.94 \\
\hline-12.592 & -2.082 & 0.16 \\
\hline-2.6879 & & 0.85 \\
\hline-3.84 & -0.74963 & \\
\hline-2.3254 & & 0.43 \\
\hline 2.948 & 0.36175 & 1.3 \\
\hline 4.247 & 0.5588 & 1.14 \\
\hline 3.65 & 0.47921 & 4.06 \\
\hline 1.536 & 0.1473 & 4.6 \\
\hline 1.13 & 0.085866 & 1.83 \\
\hline 1.023 & 0.070238 & 2.64 \\
\hline $\begin{array}{r}0.56 \\
0.0203\end{array}$ & & $\begin{array}{r}0.15 \\
0.0248\end{array}$ \\
\hline
\end{tabular}

4.57
0.0086 


\section{$\mathrm{Cu}, \mathrm{ACL} \quad \mathrm{Cu}, \mathrm{ppm}$ NL(Cu), g/m2}

$\begin{array}{lll}-0.02 & -0.25292 & 0 \\ -0.02 & -0.3417 & 0 \\ -0.02 & -0.72233 & 0 \\ -0.02 & -0.523 & 0\end{array}$

-0.02
-0.02
-0.02

-0.02
0.03
0.04

0.03
-0.02

-0.02
0.04
-0.02

-0.02
-0.02
-0.02

-0.02
-0.02
-0.02

-0.02
0.019
0.074
0.143

0.074
0.143

0.007

0.006

0.0032

$-0.50613$

-0.72047
-0.3861

-0.3861
0.36925
0.43579

0.43579
-0.56545
-0.37388

-0.3738
0.46197
-2.5183

-2.5189
-0.53759
-0.768

-0.46507
0.20358

0.81837

0.02795

0.035092

0.0032

0
0.35836
0.42162
0

0.45398

0
0.19575
0.78689
0.56544
0.02672
0.033536

0.03672
0.03536
0.041001

NL(Li), g/m2

1.2168
0.64656

0.9075

0.72811

1.4474
1.5331
1.8027

1.9278
3.1583

1.8687

2.5862
2.3758
2.9916

2.9916
3.0676
2.1059

$\mathrm{Mg}, \mathrm{ACL}$

-0.01
0.03
-0.01

0.03
-0.01
0.03

0.008
Mg, ppm NL(Mg), g/m2

Fe, ACL.

0.08
0.14
-0.02

0.08
-0.02
0.18
-0.02

-0.02
0.09
-0.02

-0.02
0.73
0.88

0.88
0.22
0.02
0.91

0.91
-0.02
-0.02

-0.02
-0.1
-0.02

0.056

0.484
1.57
0.276
0.044

0.082

0.0798

0.51255
-0.36116

0.7845
-0.25306

0.72047
-0.19305

1.2308

1.3074
0.84818

-0.18694
1.7324
-1.2592

$-0.26879$

0.46507

0.33217

1.2795

0.056148

0.035535

0.008

0.059966

0.16634

0.1395

0.33806

0.36663
0.18776

0.53946

0.15839

0.12776
0.59974

0.59974

0.096191

0.021463
0.013667
Fe, ppm NL(Fe), g/mZ

$\begin{array}{ll}1.0117 & 0.038623 \\ 2.3919 & 0.093863\end{array}$

$\begin{array}{rr}-0.72233 & 0.18451\end{array}$

$-0.50613$

3.2421
-0.3861

-0.3861
8.9851
9.5873

9.587
6.22

0.37388

10.51
-2.5183
-0.53759

3.84
-0.46507

0.60004
5.3526

5.3526
6.3172
1.1024

0.30881

0.0798

0.12539

0.34928

0.37157
0.23944

0.41376

0.14965

0.023136
0.20638

0.26638

0.042246
0.011834

$\mathrm{Mn}, \mathrm{ACL}$

Mn, ppm NL(Mn), g/m2

K, ACL

7.04
0.0309

$0.9 / \mathrm{m} 2$
0.02779
0.11513
0.17566
0.15932
0.35494
0.38548
0.24855
0.44101
0
0.17036
0.13423
0.43621
0.34615
0.10819
0.01993
0.024991

0.091241
0.11371

0.11513

0.7845
-0.25306

1.6001
1.7432

1.1309
-0.18694

1.9634
-1.2592
-0.26879

0.23254

0.61076

1.5411

0.01993
0.01

-0.01
0.03
0.01

-0.01
0.02

0.02

0.13
0.16

0.16
0.04
-0.01

0.17
-0.01

-0.01
-0.01
0.02

-0.01
0.057

0.057
0.077
0.383

0.124
0.013

0.013
0.0169

0.014

K, ppm NL
16.44
12.814
16.975
14.644
14.425
15.49
14.479
14.77
16.342
16.681
20.75
21.251
-37.775
29.567
15.36
19.533
19.394
17.916
21.527
22.128
18.108
22.529
7.04
0.0309

$\mathrm{K}, .9 / \mathrm{mz}$
0.87825
0.52857
0.71258
0.78852
0.7186
0.87389
0.90225
1.3872
0
0.77791
1.2302
1.083
1.4743
1.4932
1.0953
1.5423

0.014
$\mathrm{Na}, \mathrm{ACL}$

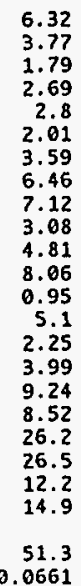

Li, ACL

Li, ppm

$\begin{array}{ll}0.62 & 7.8405 \\ 0.24 & 4.1004\end{array}$

$\begin{array}{rr}0.1 & 4.1004 \\ 0.22 & 5.716 \\ 0.22 & 5.5654\end{array}$

$\begin{array}{ll}0.21 & 4.054 \\ 0.75 & 9.2312\end{array}$ $\begin{array}{ll}0.22 & 5.5674 \\ 0.13 & 4.683 \\ 0.21 & 4.054\end{array}$

$0.41 \quad 11.592$

$1.65 \quad 12.127$

$\begin{array}{ll}0.16 & 20.147 \\ 0.79 & 21.235 \\ 0.31 & 11.904\end{array}$

$\begin{array}{ll}0.31 & 11.904 \\ 0.5 & 11.627 \\ 1.55 & 16.608\end{array}$

$\begin{array}{ll}1.38 & 15.261 \\ 4.67 & 18.791\end{array}$

$\begin{array}{ll}4.96 & 19.811 \\ 1.94 & 13.616\end{array}$

$\begin{array}{ll}0.05 & 0.05 \\ 0.0197 & 0.0173\end{array}$

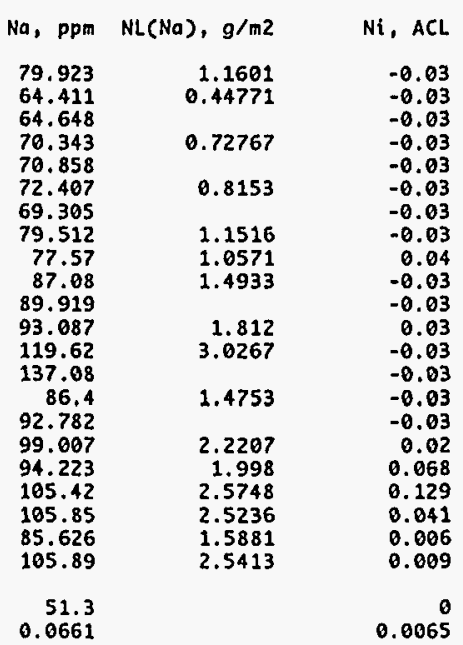

草 


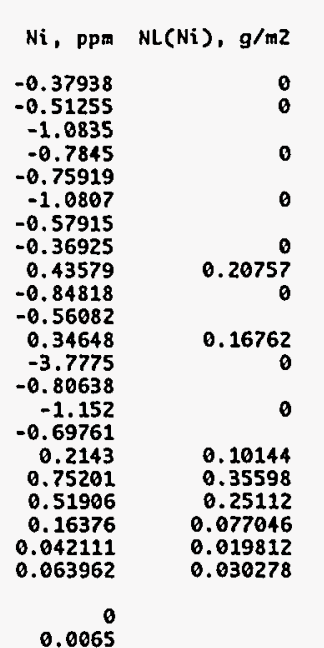

Si, ppm NL(Si), g/m2

63.356

63.356
55.697

54.536
65.375

66.989

65.562
54.44

54.44
94.528

99.033
105.17

97.957

116.65
127.18
132.78

127.18
132.78
105.22

98.131

117.86
121.65
142.44

142.44
139.8

79.369
11.229

0.2287

45.2
0.2287

$\begin{array}{rrr}\text { U. ACL } & \text { U, ppm } & \text { NLCU), g/m2 } \\ 0.142 & 1.7957 & 0.15303 \\ 0.0508 & 0.86792 & 0.09613 \\ 0.0168 & 0.60675 & 0.10197 \\ 0.0591 & 1.5455 & 0.1019 \\ 0.0439 & 1.111 & 0.1825 \\ 0.0284 & 1.0231 & 0.18225 \\ 0.0332 & 0.64092 & 0.31232 \\ 0.134 & 1.6493 & 0.40517 \\ 0.197 & 2.1463 & 0.4017 \\ 0.0792 & 2.2392 & 0.42011 \\ 0.0806 & 1.5067 & 0.55142 \\ 0.249 & 2.8758 & 0.5512 \\ 0.0125 & 1.574 & 0.29713 \\ 0.0525 & 1.4112 & 0.35122 \\ 0.0483 & 1.8547 & 0.35122 \\ 0.0489 & 1.1371 & 0.26737 \\ 0.133 & 1.4251 & 0.45439 \\ 0.219 & 2.4219 & 0.45752 \\ 0.593 & 2.3861 & 0.457 \\ 0.518 & 2.069 & 0.38581 \\ 0.117 & 0.82116 & 0.15312 \\ 0.217 & 1.5422 & 0.28934 \\ 0.0164 & 0.0164 & \\ 0.0164 & 0 & \end{array}$

Zn, ACL.

$-0.02$

-0.25292
-0.3417

-0.3417
-0.72233

-0.523
-0.50613

-0.72047
-0.3861

-0.3861
-0.24617

-0.21789
-0.56545

-0.56545
-0.37388

-0.23099
-2.5183

$-0.53759$

-0.768
-0.46507

0.04286
0.17694

0.25752
0.61511
0.1123

0.1123
0.14214

0.000
Th, ACL

0.78943
0.7015

0.83195

0.82418

1.2201
1.2773

1. 352

1.5396
1.6583

1.3652

0.97763
1.0285

1.0285
1.337

1.337
1.2649
0.4561

0.4561
-0.45705

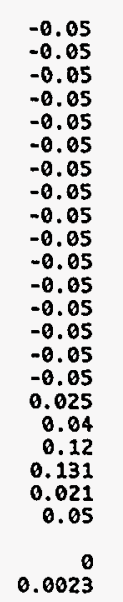

Th, ppm NL(Th), $g / m 2$ -0.6323
-0.85426

-1.8058
-1.3075

-1.2653
-1.8012

-1.8012
-0.96524
-0.65542

-0.54474
-1.4136

-1.44136
-0.9347

-0.9347
-0.57746

-6.2958
-1.344

-1.344
-1.1627

-1.2627
0.26788
0.44236

0.44236
0.48284

0.52324
0.14739

0.35535

0.0023

$Z n, p p m \quad N L(Z n), g / m 2$

$$
\begin{array}{r}
-3.8911 \\
-5.3727 \\
-11.357 \\
-8.1975 \\
-7.9331 \\
-11.153 \\
-5.9767 \\
-3.8225 \\
-3.373 \\
-8.6993 \\
-5.752 \\
-3.6319 \\
-38.983 \\
-8.3218 \\
-11.925 \\
-7.2216 \\
0.65938 \\
2.7222 \\
4.049 \\
9.4053 \\
1.7171 \\
2.1867 \\
0 \\
0.0002 \\
0
\end{array}
$$

$2 \mathrm{r}, \mathrm{ACL}$

$-0.05$

-0.05
-0.05
-0.05

$-0.05$

$-0.05$

-0.05
-0.05

$-0.05$

-0.05
-0.05

$-0.05$

-0.05
-0.05

0.003

0.029
0.022

0.019
0.002

0.002



Ti, ACL

-0.02
-0.02
-0.02

-0.02
-0.02
-0.02
-0.02

-0.02
-0.02
0.03

-0.03
0.04
-0.02

-0.02
-0.02
0.05

0.05
-0.02
-0.02

-0.02
-0.02

-0.02
0.021
0.056

0.056
0.114
0.059
0.017

0.01
0.017

0.0054

Zr, ppm NL(Zr), g/m2

-0.6323
-0.85426

$-1.8058$

-1.3075
-1.2653

-1.8012
-0.96524

-0.651542
-0.54474
-1.9 .947

$-1.4136$

-0.9347
-0.57746

-6.2958
-1.344

-1.344
-1.92
-1.1627

0.032145

0.099531

0.07589

0.014037
0.42641

0.0007

$T i, \mathrm{ppm} N L(T i), g / \mathrm{m} 2$

$\begin{array}{ll}-0.25292 & 0 \\ -0.3417 & 0\end{array}$

$-0.72233$

$-0.50613$

$0.36925 \quad 0.21236$

$-0.56545$

(1)

- 57746

0.33628

$-0.46507$

0.12821
0.35288
0.26712

0.23566
0.070185
0.039346

0.12082

0.068842

0.0054 


\begin{tabular}{|c|c|c|c|c|c|c|c|c|c|c|c|}
\hline TEST NUMBER & RXN TIME & $\mathrm{pH}$ & DIL FACTOR & $\mathrm{SA} / \mathrm{N}, / \mathrm{m}$ & LEACHANT Vo & ACL NUMBER & $\mathrm{Al}, \mathrm{ACL}$ & Al, ppm & $N L(A L), g / m 2$ & B, ACL & B, ppm \\
\hline TY1 & 3 & 9.96 & 3,004 & 1882 & 10.09 & $91-0532-07$ & 1.64 & 4.9266 & 0.12895 & 4.8 & 14.419 \\
\hline $\begin{array}{r}\text { TY1-F50 } \\
\text { TY2 }\end{array}$ & 3 & 9.93 & $\begin{array}{l}3.5765 \\
3.7059\end{array}$ & 1963 & 10.03 & $\begin{array}{l}91-0532-08 \\
91-0532-09\end{array}$ & $\begin{array}{l}1.26 \\
1.32\end{array}$ & $\begin{array}{l}4.5064 \\
4.8918\end{array}$ & 0.12276 & $\begin{array}{l}3.98 \\
3.75\end{array}$ & $\begin{array}{l}14.234 \\
13.897\end{array}$ \\
\hline TYZ -F50 & & & 3.6862 & 1903 & & $91-0532-10$ & $\begin{array}{l}1.24 \\
1.24\end{array}$ & $\begin{array}{l}4.8918 \\
4.5709\end{array}$ & $0.126 \pi 6$ & 3.89 & $\begin{array}{l}13.897 \\
14.339\end{array}$ \\
\hline TY3 & 7 & 9.99 & 2.4288 & 1889 & 10.05 & $92-0353-09$ & 2.01 & 4.8819 & 0.12731 & 7.42 & 18.022 \\
\hline TY3 $-F 50$ & & & 10.449 & & & $92-0353-10$ & 0.37 & 3,8661 & & 1.76 & 18.39 \\
\hline $\begin{array}{r}T Y 4 \\
T Y 4-F 50\end{array}$ & 7 & 10.07 & $\begin{array}{r}2.1543 \\
11.75\end{array}$ & 1908 & 10.04 & $\begin{array}{l}92-0353-11 \\
92-0353-12\end{array}$ & $\begin{array}{l}2.33 \\
0.34\end{array}$ & 5.0195 & 0.12959 & $\begin{array}{l}8.53 \\
1.59\end{array}$ & $\begin{array}{l}18.376 \\
18.683\end{array}$ \\
\hline $\begin{array}{r}\text { TY4-F50 } \\
\text { TY5 }\end{array}$ & 14 & 10.02 & 2.2371 & 1925 & 10.05 & $\begin{array}{l}92-0353-12 \\
92-0353-13\end{array}$ & & $\begin{array}{r}3.993 \\
4.4742\end{array}$ & 0.1145 & $\begin{array}{l}1.99 \\
9.93\end{array}$ & 23.214 \\
\hline TYS-FSO & & & 12.919 & & & $92-0353-14$ & 0.22 & 2,8422 & & 1.85 & $\begin{array}{r}23.9 \\
\end{array}$ \\
\hline $\begin{array}{r}\text { TY6 } \\
T Y Y-F 50\end{array}$ & 14 & 9.97 & 2.493 & 1953 & 10.01 & $92-0353-15$ & 1.75 & $\begin{array}{r}4.3628 \\
2.9237\end{array}$ & 0.11004 & $\begin{array}{l}9.05 \\
2.04\end{array}$ & $\begin{array}{r}22.562 \\
22.94\end{array}$ \\
\hline TYT-F5O & & & 11.231 & & & $92-0278-02$ & & 3.3693 & & 2.22 & 24.933 \\
\hline $\begin{array}{r}T Y 8 \\
T Y 8-550\end{array}$ & 30 & 10.12 & 2.1464 & 1955 & 10.01 & $92-0278-03$ & 1.91 & $\begin{array}{l}4.0996 \\
3.5104\end{array}$ & 0.1033 & $\begin{array}{l}11.8 \\
2.19\end{array}$ & $\begin{array}{r}25.328 \\
24.8\end{array}$ \\
\hline $\begin{array}{r}\text { TY8-F50 } \\
\text { TY9 }\end{array}$ & 70 & 10.32 & 10.403 & 1947 & 10.02 & $92-0278-05$ & 0.43 & 4,4733 & 0.11318 & 2.79 & 29.024 \\
\hline TY9-F5Q & & & 11.288 & & & $92-0278-06$ & 0.25 & 2.822 & & 2.36 & 26.64 \\
\hline $\begin{array}{r}\text { TY10 } \\
Y Y 10-F 50\end{array}$ & 70 & 10.33 & 8,7297 & 1951 & 9,99 & $92-0278-07$ & 0.51 & $\begin{array}{l}4,4521 \\
2,5619\end{array}$ & 0.11241 & 3.37 & 29.419 \\
\hline $\begin{array}{l}\text { TY10-F50 } \\
\text { TY11 }\end{array}$ & 140 & 10.17 & $\frac{11,643}{2,2804}$ & 2005 & 9.99 & $92-0087-01$ & $\begin{array}{l}0.22 \\
1.73\end{array}$ & 3.9451 & 0.096927 & $\begin{array}{l}2.34 \\
14.3\end{array}$ & $\begin{array}{r}27.249 \\
32.61\end{array}$ \\
\hline TY11-F50 & & & 24,015 & & & $92-0087-02$ & -0.1 & $-2,4015$ & & 1,26 & 30.259 \\
\hline TYiz & 140 & 10.28 & 2.3214 & 1975 & 10.04 & $92-0087-03$ & 1.79 & 4.1553 & 0.10364 & 14.4 & 33,428 \\
\hline TY12-F50 & 280 & 1939 & $\begin{array}{r}17,25 \\
\end{array}$ & 2013 & 19.94 & $\begin{array}{l}92-6087-04 \\
992-0278-89\end{array}$ & -0.1 & -1.725 & 0.1007 & 1.7 & 29.325 \\
\hline TY13-F50 & & 10.39 & 11.211 & 2613 & 10.04 & & $\begin{array}{l}0.41 \\
0.19\end{array}$ & $\begin{array}{l}4.1172 \\
2.1301\end{array}$ & 0.10075 & $\begin{array}{r}3.7 \\
3.43\end{array}$ & $\begin{array}{l}37.155 \\
38.454\end{array}$ \\
\hline TY15 & 560 & 10.11 & 1.896 & 2017 & 10.05 & PACKET.1 & 1.05 & 1.9908 & 0.048621 & 19.4 & 36.782 \\
\hline TY15-FSO & 200 & 10.11 & 2.488 & cost & 20.00 & PACKET. 1 & 0.652 & 1.5471 & & 14.5 & 36.076 \\
\hline TY16 & 560 & 10.29 & 1.78 & 2000 & 10.07 & PACKET 1 & 1.36 & 2.4208 & 0.059626 & 20.8 & 37.024 \\
\hline TY16-F50 & & & 2.063 & & & PACKET 1 & 0.6 & 1.2378 & & 13.2 & 27.232 \\
\hline TY17 & 980 & 9.95 & 2,723 & 2008 & 10.07 & PACKET. 2 & 0.687 & 1.8707 & 0.045893 & $\begin{array}{l}17.09 \\
8\end{array}$ & 46.536 \\
\hline $\begin{array}{r}T Y 17-F 25 \\
T Y 18\end{array}$ & 980 & 9.84 & $\begin{array}{l}5.341 \\
2.736\end{array}$ & 1946 & 10.07 & $\begin{array}{l}\text { PACKET. } \\
\text { PACKET . ? }\end{array}$ & $\begin{array}{l}0.132 \\
0.556\end{array}$ & $\begin{array}{r}0.70501 \\
1.5212\end{array}$ & 0.038508 & $\begin{array}{l}8.375 \\
14.57\end{array}$ & $\begin{array}{l}44.731 \\
39.864\end{array}$ \\
\hline$T Y 18-F 25$ & & & $\begin{array}{l}2.346 \\
5.342\end{array}$ & & & $\begin{array}{l}\text { PACKET.2 } \\
\text { PACKET. }\end{array}$ & 0.499 & 2.6657 & & & 48.078 \\
\hline $\begin{array}{r}f(i) \\
\text { BKGND }\end{array}$ & & & & & & & & 0.0203 & & & 0.0248 \\
\hline
\end{tabular}




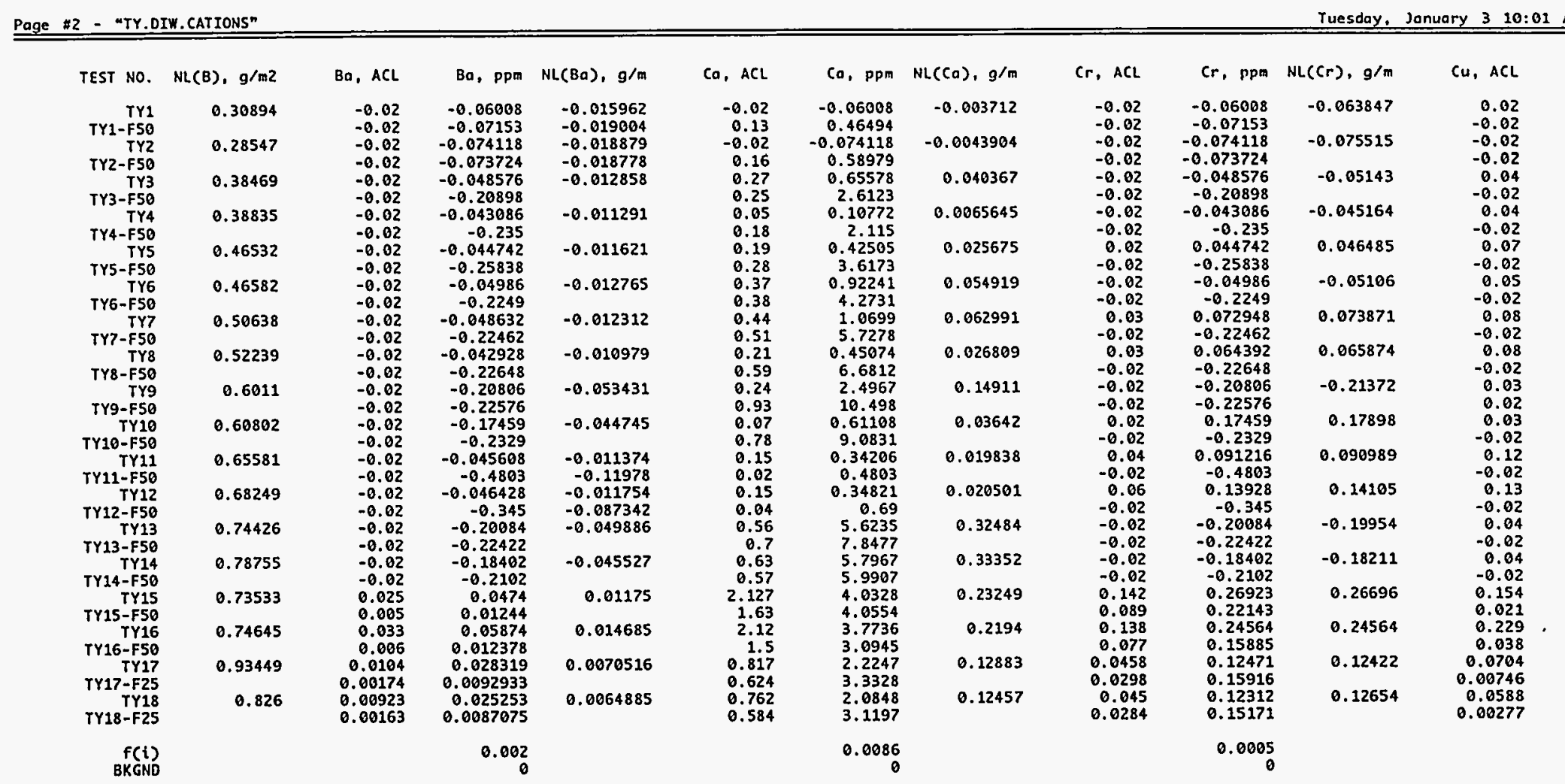




\begin{tabular}{|c|c|c|c|c|c|}
\hline TEST NO. & $\mathrm{Cu}, \mathrm{ppm}$ & $N L(C u), g / m$ & $\mathrm{Fe}, \mathrm{ACl}$ & $\mathrm{Fe}, \mathrm{ppm}$ & $\mathrm{NL}(\mathrm{Fe}), \mathrm{g} / \mathrm{m}$ \\
\hline TY1 & 0.06008 & 0.0099761 & 0.67 & 2.0127 & 0.013401 \\
\hline TY1-F50 & -0.07153 & -0.011877 & 0.08 & 0.28612 & \\
\hline & -0.074118 & -0.011799 & 0.53 & 1.9641 & 0.012539 \\
\hline $2-F 50$ & -0.073724 & -0.011737 & 0.08 & 0.2949 & \\
\hline & $\begin{array}{l}0.097152 \\
-0.20898\end{array}$ & 0.016072 & 1.18 & $\begin{array}{r}2.866 \\
0.73143\end{array}$ & 0.019012 \\
\hline $\begin{array}{c}\text { TYA } \\
\text { TS }\end{array}$ & 0.086172 & 0.014114 & $\begin{array}{l}0.07 \\
1.36\end{array}$ & $\begin{array}{r}2.9298 \\
2.929\end{array}$ & 0.019243 \\
\hline TY4-F50 & -0.235 & & & 0.235 & \\
\hline $\begin{array}{r}\text { TY5 } \\
\text { TYS-F50 }\end{array}$ & $\begin{array}{r}0.1566 \\
-0.25838\end{array}$ & 0.025422 & $\begin{array}{r}1.98 \\
-0.02\end{array}$ & $\begin{array}{r}4.4295 \\
-0.25838\end{array}$ & 0.028835 \\
\hline TY6 & 0.12465 & 0.019945 & $\begin{array}{r}-0.02 \\
1.81\end{array}$ & $\begin{array}{r}-0.25838 \\
4.5123\end{array}$ & 0.028953 \\
\hline TY6-F50 & -0.2249 & & -0.02 & -0.2249 & \\
\hline $\begin{array}{r}\text { TY7 } \\
47-F 59\end{array}$ & $\begin{array}{r}0.19453 \\
-077467\end{array}$ & 0.03078 & $\begin{array}{r}2.18 \\
-0.02\end{array}$ & $\begin{array}{r}5.3009 \\
-9.22462\end{array}$ & 0.033634 \\
\hline $\begin{array}{l}\text { TY7-F50 } \\
\text { TY8 }\end{array}$ & -0.22462 & 0.027448 & -0.02 & $\begin{array}{r}-0.22462 \\
5.1514\end{array}$ & \\
\hline $\begin{array}{l}\text { TY8 } \\
\text { Y8-F50 }\end{array}$ & $\begin{array}{r}0.1717 \\
-0.22648\end{array}$ & $0.06 r 448$ & 0.02 & $\begin{array}{l}5.1514 \\
0.22648\end{array}$ & 0.03302 \\
\hline TY9 & 0.31209 & 0.050091 & 0.66 & 6.866 & 0.044191 \\
\hline Y9-F50 & 0.22576 & & 0.02 & & \\
\hline $\begin{array}{c}T Y 1 \theta \\
\theta-F 5 \theta\end{array}$ & $\begin{array}{l}0.26189 \\
-0.2329\end{array}$ & 0.041948 & $\begin{array}{r}0.82 \\
-0.02\end{array}$ & $\begin{array}{r}7.1584 \\
-6.2329\end{array}$ & 0.045978 \\
\hline $\begin{array}{r}\text { TY10-F50 } \\
\text { TY11 }\end{array}$ & 0.27365 & 0.042651 & $\begin{array}{r}-0.02 \\
3.97\end{array}$ & $\begin{array}{r}-6.2329 \\
9.0532\end{array}$ & 0.056583 \\
\hline TY11-F50 & -0.4803 & -0.07486 & -0.02 & -0.4803 & \\
\hline TY12 & 0.30178 & 0.04775 & 3.99 & 9.2624 & 0.05877 \\
\hline $\begin{array}{l}\text { TF56 } \\
\text { TY13 }\end{array}$ & $\begin{array}{r}-0.345 \\
0.40168\end{array}$ & $\begin{array}{r}-0.054589 \\
0.062357\end{array}$ & $\begin{array}{r}-0.02 \\
1.02\end{array}$ & $\begin{array}{l}-0.345 \\
10.743\end{array}$ & 0.963764 \\
\hline TY13-F50 & $\begin{array}{r}0.042422 \\
-0.222\end{array}$ & & $\begin{array}{l}1.02 \\
0.02\end{array}$ & $\begin{array}{l}16.243 \\
0.22422\end{array}$ & \\
\hline TY14 & 0.36804 & 0.056909 & 1. & 10.857 & .067321 \\
\hline$-F 56$ & -0.2102 & & -6. & 2 & \\
\hline TY15 & 0.29198 & 0.045238 & 1. & 2.5975 & .016138 \\
\hline$-F 50$ & 0.052248 & & & & \\
\hline TY16 & 0.40762 & 0.063691 & 2.28 & 4.6 & .025 \\
\hline $\begin{array}{l}\text { 6-F50 } \\
T Y 17\end{array}$ & 0.078394 & & & & \\
\hline $\begin{array}{r}\text { TY17 } \\
\text { TY17-F25 }\end{array}$ & $\begin{array}{r}0.1917 \\
0.039844\end{array}$ & 0.029834 & $\begin{array}{r}1.973 \\
0.00697\end{array}$ & $\begin{array}{r}5.3725 \\
0.037227\end{array}$ & 0.033528 \\
\hline $\begin{array}{r}\text { TY18 } \\
\text { TY18-F25 }\end{array}$ & $\begin{array}{r}0.16088 \\
0.014797\end{array}$ & 0.025835 & $\begin{array}{r}1.345 \\
0\end{array}$ & $\begin{array}{r}3.6799 \\
0\end{array}$ & 0.023697 \\
\hline $\begin{array}{r}f(i) \\
B K G N D\end{array}$ & 0.0032 & & & .0798 & \\
\hline
\end{tabular}

\begin{tabular}{|c|c|c|}
\hline$K, A C L$ & $\mathrm{k}, \mathrm{ppm}$ & $N L(K), g / m 2$ \\
\hline $\begin{array}{l}5.11 \\
4.22\end{array}$ & $\begin{array}{r}15.35 \\
15.093\end{array}$ & 0.26396 \\
\hline 3.98 & 14.749 & 0.24316 \\
\hline 4.07 & 15.003 & \\
\hline 6.63 & 16.103 & 0.27588 \\
\hline 1.41 & 14.733 & \\
\hline 7.55 & 16.265 & 0.27588 \\
\hline 1.23 & 14.453 & \\
\hline $\begin{array}{l}8.66 \\
1.53\end{array}$ & $\begin{array}{l}19.373 \\
19.766\end{array}$ & 0.3257 \\
\hline 7.72 & 19.246 & 0.31892 \\
\hline 1.8 & 20.241 & \\
\hline $\begin{array}{l}9.26 \\
1.87\end{array}$ & $\begin{array}{l}22.517 \\
21.002\end{array}$ & 0.36896 \\
\hline 10.5 & 22.537 & 0.37307 \\
\hline 1.81 & 20.496 & \\
\hline 2.32 & 24.135 & 0.40116 \\
\hline $\begin{array}{l}1.96 \\
2.78\end{array}$ & $\begin{array}{l}22.124 \\
24.269\end{array}$ & 0.40256 \\
\hline 1.91 & 22.242 & \\
\hline $\begin{array}{l}13.5 \\
1.25\end{array}$ & $\begin{array}{l}30.785 \\
30.019\end{array}$ & 0.4969 \\
\hline 13.1 & 30.41 & 0.49831 \\
\hline $\begin{array}{l}1.65 \\
3.16\end{array}$ & $\begin{array}{l}28.462 \\
31.733\end{array}$ & 0.51016 \\
\hline 2.67 & 29.933 & \\
\hline 3.58 & $\begin{array}{r}32.94 \\
\end{array}$ & 0.52747 \\
\hline $\begin{array}{l}2.86 \\
16.2\end{array}$ & $\begin{array}{l}30.059 \\
30.715\end{array}$ & 0.49282 \\
\hline $\begin{array}{l}10.6 \\
11.7\end{array}$ & 29.21 & \\
\hline $\begin{array}{l}17.8 \\
10.8\end{array}$ & $\begin{array}{r}31.684 \\
22.28\end{array}$ & 0.51269 \\
\hline 13.47 & 36.679 & 0.59114 \\
\hline $\begin{array}{l}6.898 \\
10.87\end{array}$ & $\begin{array}{r}36.842 \\
29.74\end{array}$ & 0.49459 \\
\hline 7.182 & 38.366 & \\
\hline & 0.0309 & \\
\hline
\end{tabular}

\begin{tabular}{|c|c|c|}
\hline$L i, A C L$ & Li, ppm & $N L(L i), g / m$ \\
\hline & 13.518 & 0.36461 \\
\hline 3.74 & 13.376 & \\
\hline $\begin{array}{r}3.61 \\
3.6\end{array}$ & $\begin{array}{r}13.378 \\
13.27\end{array}$ & 0.34595 \\
\hline 6.01 & 14.597 & 0.39225 \\
\hline $\begin{array}{l}1.37 \\
7.36\end{array}$ & $\begin{array}{l}14.315 \\
15.856\end{array}$ & 0.42183 \\
\hline 1.23 & 14.453 & \\
\hline $\begin{array}{r}8.5 \\
1.44\end{array}$ & 19.015 & 0.50143 \\
\hline $\begin{array}{l}1.44 \\
7.67\end{array}$ & 19.121 & 0.49699 \\
\hline $\begin{array}{l}1.56 \\
9.33\end{array}$ & $\begin{array}{l}17.542 \\
22.687\end{array}$ & 0.5831 \\
\hline 1.88 & 21.114 & \\
\hline 10.4 & 22.323 & 0.5796 \\
\hline $\begin{array}{l}1.92 \\
2.41\end{array}$ & 25.071 & 0.65365 \\
\hline 2.02 & 22.802 & 0.67231 \\
\hline 1.98 & $\begin{array}{r}23.04 \\
23.057\end{array}$ & \\
\hline 14.1 & $\begin{array}{l}32.154 \\
28.578\end{array}$ & 0.81405 \\
\hline 13.8 & 32.035 & 0.82337 \\
\hline $\begin{array}{l}3.04 \\
3.48\end{array}$ & 34.946 & 0.88123 \\
\hline 3.09 & 34.642 & \\
\hline $\begin{array}{l}4.19 \\
3.28\end{array}$ & $\begin{array}{l}38.553 \\
34.473\end{array}$ & 0.96833 \\
\hline 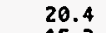 & 38.678 & 0.97341 \\
\hline & 38.066 & \\
\hline $\begin{array}{l}22.1 \\
13.6\end{array}$ & $\begin{array}{l}39.338 \\
28.057\end{array}$ & 0.99843 \\
\hline 18.4 & 50.103 & 1.2666 \\
\hline 8.432 & 45.035 & 1) 0304 \\
\hline $\begin{array}{l}14.49 \\
8.924\end{array}$ & $\begin{array}{l}39.645 \\
47.672\end{array}$ & 1.0341 \\
\hline & 0.01 & \\
\hline
\end{tabular}




\begin{tabular}{|c|c|c|c|}
\hline TEST NO. & $\mathrm{Mg}, \mathrm{ACL}$ & Mg, ppm & $N L(M g)$, \\
\hline TY1 & 0.1 & 0.3004 & 0. \\
\hline TY1-F50 & 0.03 & 0.10729 & \\
\hline TY2 & 0.08 & 0.29647 & 0.018 \\
\hline 2-F50 & 0.04 & 0.14745 & \\
\hline TY3 & 0.15 & 0.36432 & 0.024 \\
\hline 3-F50 & 0.01 & 0.10449 & \\
\hline TY4 & 0.17 & 0.36623 & 0.02 \\
\hline 4-F50 & -0.01 & -0.1175 & \\
\hline & 0.22 & 0.49216 & 0.03 \\
\hline 15-F50 & -0.01 & -0.12919 & \\
\hline & 0.2 & $\begin{array}{r}0.4986 \\
033735\end{array}$ & 0.031 \\
\hline $\begin{array}{r}-F 50 \\
\text { TY7 }\end{array}$ & $\begin{array}{r}.03 \\
0.2\end{array}$ & 0.48632 & 0.0 \\
\hline $7-F 50$ & -0.01 & -0.11231 & \\
\hline TY8 & 0.22 & 0.47221 & 0. \\
\hline 8-F50 & -0.01 & -0.11324 & \\
\hline TY9 & 0.06 & 0.62418 & 0.04 \\
\hline $\begin{array}{l}\text { Y9-F50 } \\
\text { YYY10 }\end{array}$ & 0.02 & 0.22576 & \\
\hline$T Y 10-F 50$ & 0.01 & 0.11645 & \\
\hline TY11 & 0.35 & 0.79814 & 0 \\
\hline TY11-F50 & -0.01 & -0.24015 & \\
\hline $\begin{array}{r}\text { TY12 } \\
T Y 12-F 50\end{array}$ & $\begin{array}{r}0.35 \\
-0.01\end{array}$ & 0.81249 & 9 \\
\hline TY13 & 0.09 & 0.90378 & \\
\hline $3-F 50$ & 0.02 & 0.22422 & \\
\hline TY14 & 0.1 & 0.92011 & \\
\hline $\begin{array}{r}4-F 50 \\
T Y 15\end{array}$ & -0.01 & -0.1051 & \\
\hline $\begin{array}{r}\text { TY15 } \\
\text { TY15-F50 }\end{array}$ & 0.221 & 0.41902 & 0.025 \\
\hline $\begin{array}{r}T_{13}-5160 \\
\text { TY16 }\end{array}$ & $\begin{array}{l}0.081 \\
0.309\end{array}$ & $\begin{array}{l}0.205002 \\
0.55\end{array}$ & \\
\hline TY16-F50 & 0.13 & 0.26819 & \\
\hline TY17 & 0.154 & 0.41934 & 0 \\
\hline TY17-F25 & 0.0735 & 0.39256 & \\
\hline $\begin{array}{r}T Y 18 \\
T Y 18-F 25\end{array}$ & $\begin{array}{r}0.162 \\
0.0222\end{array}$ & $\begin{array}{l}0.44323 \\
0.11859\end{array}$ & 0.02 \\
\hline $\begin{array}{r}f(i) \\
B K G N D\end{array}$ & & 0.008 & \\
\hline
\end{tabular}

\begin{tabular}{|c|c|c|}
\hline $\mathrm{Mn}, \mathrm{ACL}$ & $\mathrm{Mn}$, ppm & $N L(M n), g^{\prime m}$ \\
\hline$=0$ & & 0.0079809 \\
\hline $\begin{array}{r}-0.01 \\
0.06\end{array}$ & $\begin{array}{r}-0.035765 \\
0.22235\end{array}$ & 0.0080909 \\
\hline $\begin{array}{l}-0.01 \\
-0.01\end{array}$ & -0.036862 & \\
\hline 0.14 & 0.34003 & 0.012858 \\
\hline-0.01 & -0.16 & \\
\hline $\begin{array}{r}0.16 \\
-0.01\end{array}$ & $\begin{array}{r}0.34469 \\
-0.1175\end{array}$ & 0.012904 \\
\hline 0.26 & 0.58165 & 0.021582 \\
\hline 0.01 & $\begin{array}{r}-0.12919 \\
0.57339\end{array}$ & 0.020971 \\
\hline $\begin{array}{r}-0.02 \\
0.29\end{array}$ & $\begin{array}{r}-0.11245 \\
0.70516\end{array}$ & 0.025503 \\
\hline-0.01 & -0.11231 & \\
\hline $\begin{array}{r}0.32 \\
-0.01\end{array}$ & $\begin{array}{r}0.68685 \\
-0.11324\end{array}$ & 0.025095 \\
\hline 0.09 & 0.93627 & 0.034348 \\
\hline $\begin{array}{l}-0.61 \\
0.11\end{array}$ & $\begin{array}{r}-0.11288 \\
0.96027\end{array}$ & 0.035157 \\
\hline-0.01 & -0.11645 & \\
\hline 0.59 & 1.3454 & 0.047931 \\
\hline $\begin{array}{r}-0.01 \\
0.59\end{array}$ & $\begin{array}{r}-0.24015 \\
1.3696\end{array}$ & 0.049534 \\
\hline-0.01 & -0.1725 & \\
\hline 0.15 & 1.5 & 0.053449 \\
\hline 0.01 & -0.11211 & \\
\hline$\because \hat{a}+>$ & 1.5642 & 0.055283 \\
\hline & -0.1 & \\
\hline & & 0.034109 \\
\hline & 0.0 & \\
\hline & & 849776 \\
\hline & & 0.033321 \\
\hline $\begin{array}{l}0.344 \\
0.0026\end{array}$ & $\begin{array}{l}0.93687 \\
0.01387\end{array}$ & 0.035321 \\
\hline 0.242 & 0.66211 & 0.024303 \\
\hline 0.00085 & 0.0045407 & \\
\hline & & \\
\hline
\end{tabular}

Na, ACL
14.9
$13 . .2$
11.9
13.1
21.2
4.92
25.4
4.42
29.6
5.4
26.9
6.16
32.8
6.98
36.8
6.82
8.3
7.46
10.3
7.36
50
4.39
48.8
6.95
11.9
11.1
13.7
11.8
70.6
53.8
77
49
60.15
30.21
48.53
13.38

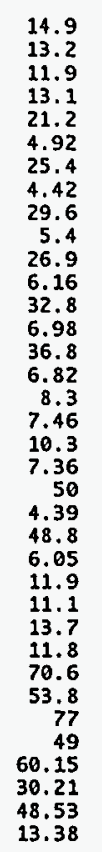

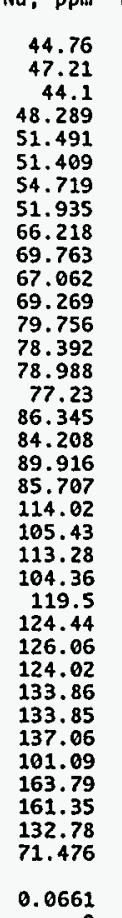

$\mathrm{Ni}, \mathrm{ACL}$

0.3598

0.33987

0.11238

0.43387

0.52041

0.51948

0.61094

0.61124

0.67092

0.69723

0.86033

0.86776

0.89809

0.94361

1.00

1.0368

1.234

1.0322

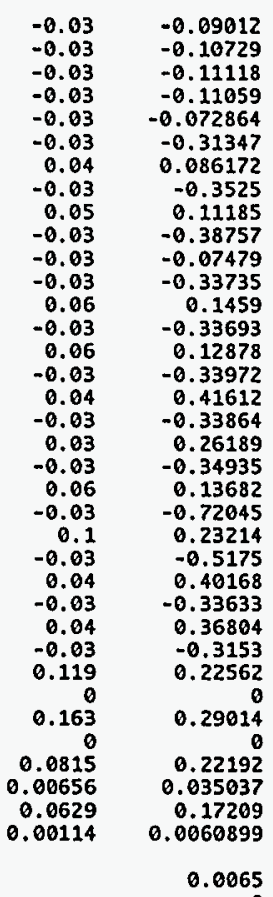

0.0065 


\begin{tabular}{|c|c|c|c|c|}
\hline TEST NO. & $N L(N i), g / m$ & $\mathrm{Si}, \mathrm{ACL}$ & Si, ppm & $N L(S i), g$ \\
\hline TY1 & -0.007367 & 31.2 & 93.725 & 0 \\
\hline TY1-F50 & & 25.7 & 91.916 & \\
\hline $\begin{array}{r}\text { TY2 } \\
\text { TYZ-F5Q }\end{array}$ & -0.0087133 & 24.6 & 91.165 & \\
\hline $\begin{array}{l}\text { Tru-rse } \\
\text { IY3 }\end{array}$ & -0.0059343 & $\begin{array}{l}24.9 \\
46.5\end{array}$ & $\begin{array}{l}91.786 \\
112.94\end{array}$ & 0.2 \\
\hline & & 9.68 & 101.15 & \\
\hline TY4 & 0.0069482 & 51.5 & 110.95 & 0.2 \\
\hline $4-F 50$ & & & 105.75 & \\
\hline TY5 & 0.0089395 & 57.4 & 128.41 & .2 \\
\hline 5-F50 & & 10.2 & 131.77 & \\
\hline TY6 & -0.0058915 & 53.4 & 133.13 & .2 \\
\hline $\begin{array}{l}5-650 \\
-7 y 7\end{array}$ & & 11.1 & 124.82 & \\
\hline TY7 & 0.011365 & 54.2 & 131.79 & .2 \\
\hline $7-F 50$ & & 11.7 & 131.4 & \\
\hline TY8 & 0.010134 & 64.7 & 138.87 & \\
\hline 8-F50 & & 12 & 135.89 & \\
\hline $\begin{array}{r}\text { TY9 } \\
Y 9-550\end{array}$ & 0.032881 & 14.9 & 155 & 0.3 \\
\hline 19-F50 & & 12.1 & 136.58 & \\
\hline $\begin{array}{r}\text { TY10 } \\
\text { TY10-F50 }\end{array}$ & 0.020651 & 18.3 & 159.75 & \\
\hline TY11 & 0.010499 & 77.4 & 176.5 & \\
\hline TY11-F50 & & 6.31 & 151.53 & \\
\hline $\begin{array}{r}\text { TY12 } \\
T Y 12-F 50\end{array}$ & 0.018083 & $\begin{array}{r}76 \\
8.52\end{array}$ & $\begin{array}{l}176.43 \\
146.97\end{array}$ & \\
\hline $\begin{array}{r}T Y 12-F 50 \\
T Y 13\end{array}$ & 0.030699 & $\begin{array}{l}8.52 \\
18.5\end{array}$ & $\begin{array}{l}146.97 \\
185.78\end{array}$ & \\
\hline TY13-F50 & & $\begin{array}{l}16.3 \\
16.3\end{array}$ & $\begin{array}{l}105.78 \\
162.74\end{array}$ & \\
\hline TY14 & 0.028017 & 22 & 202.42 & \\
\hline $\begin{array}{r}T Y 14-F 50 \\
T Y 15\end{array}$ & & 17.5 & 183.93 & \\
\hline $\begin{array}{l}T 15 \\
T Y 15-F 50\end{array}$ & 0.017269 & $\begin{array}{l}95.7 \\
70.4\end{array}$ & $\begin{array}{l}181.45 \\
175.16\end{array}$ & 0.3 \\
\hline TY16 & 0.022318 & 103 & 183.34 & \\
\hline $6-F 50$ & & 62.3 & 128.52 & \\
\hline TY17 & 0.017003 & 74.9 & 203.95 & \\
\hline TY17-F25 & & 36.9 & 197.08 & \\
\hline $\begin{array}{r}T Y 18 \\
T Y 18-F 25\end{array}$ & 0.013605 & $\begin{array}{l}72.7 \\
36.5\end{array}$ & $\begin{array}{l}198.91 \\
194.98\end{array}$ & \\
\hline $\begin{array}{r}f(i) \\
\text { BKGND }\end{array}$ & & & 0.2287 & \\
\hline
\end{tabular}

\begin{tabular}{|c|c|c|}
\hline Th, ACL & Th, ppm & $N L(T h), g / m$ \\
\hline-0.05 & -0.1502 & -0.03469 \\
\hline $\begin{array}{l}-0.05 \\
-8.05\end{array}$ & $\begin{array}{r}-0.17883 \\
-0.1853\end{array}$ & 0411 \\
\hline $\begin{array}{l}-6.03 \\
-0.05\end{array}$ & -0.18431 & \\
\hline-0.05 & -0.12144 & -0.02795 \\
\hline-0.05 & -0.5 & \\
\hline $\begin{array}{l}-0.05 \\
-0.05\end{array}$ & -0.10772 & -0.0245 \\
\hline $\begin{array}{l}-6.03 \\
-0.05\end{array}$ & $\begin{array}{l}-6.11185 \\
-0.1885\end{array}$ & -0.0252 \\
\hline-0.05 & -0.64595 & \\
\hline $\begin{array}{l}-0.05 \\
-0.05\end{array}$ & $\begin{array}{l}-0.12465 \\
-0.5625\end{array}$ & -0.027 \\
\hline $\begin{array}{l}-0.03 \\
-0.05\end{array}$ & -0.12158 & -0.026765 \\
\hline $\begin{array}{l}-0.05 \\
-0.05\end{array}$ & $\begin{array}{l}-0.56155 \\
-0.10732\end{array}$ & 0.0238 \\
\hline $\begin{array}{l}-6.03 \\
-0.05\end{array}$ & $\begin{array}{l}-0.1062 \\
-0.5662\end{array}$ & \\
\hline $\begin{array}{l}-0.05 \\
-0.05\end{array}$ & $\begin{array}{r}-0.52015 \\
-0.5644\end{array}$ & -0.116 \\
\hline $\begin{array}{l}-0.05 \\
-0.05\end{array}$ & -0.43649 & -0.0972 \\
\hline $\begin{array}{r}-0.05 \\
0.05\end{array}$ & $\begin{array}{r}-0.58225 \\
0.1402\end{array}$ & 0.02472 \\
\hline-0.05 & -1.2007 & \\
\hline 0.05 & 0.11607 & 0.02 \\
\hline-0.0 & & \\
\hline-0.0 & -0.2 & -0.16 \\
\hline-0.0 & -0.56 & \\
\hline-0.0 & -0.4 & -0.09897 \\
\hline & -0 & \\
\hline & & 0.0192 \\
\hline & 4 & \\
\hline 0.05 & $\therefore$ & 0.0193 \\
\hline 0.012 & 0.0 & \\
\hline 0.0279 & 0.0 & 0.01645 \\
\hline 0.000648 & 0.003 & \\
\hline 0. & 0.068947 & 0.015404 \\
\hline & 0.00 & \\
\hline & - & \\
\hline
\end{tabular}

\begin{tabular}{|c|c|c|}
\hline $\mathrm{Ti}, \mathrm{ACL}$ & $T i$, ppra & $N L(T i), g / m$ \\
\hline & . & 0.0059118 \\
\hline $\begin{array}{l}-0.02 \\
-0.02\end{array}$ & $\begin{array}{r}-0.07153 \\
-0.074118\end{array}$ & -0.0069921 \\
\hline $\begin{array}{l}-0.02 \\
-0.02\end{array}$ & -0.073724 & \\
\hline $\begin{array}{r}0.04 \\
-0.02\end{array}$ & $\begin{array}{l}0.097152 \\
-0.20898\end{array}$ & 0.0095241 \\
\hline $\begin{array}{r}-0.02 \\
0.04\end{array}$ & 0.086172 & 0.0083636 \\
\hline $\begin{array}{r}-0.02 \\
0.06\end{array}$ & $\begin{array}{r}-6.235 \\
0.13423\end{array}$ & 0.012913 \\
\hline-0.02 & -0.25838 & \\
\hline $\begin{array}{r}0.05 \\
-0.02\end{array}$ & $\begin{array}{r}0.12465 \\
-0.2249\end{array}$ & 0.011819 \\
\hline 0.07 & 0.17021 & 0.01596 \\
\hline $\begin{array}{r}-0.02 \\
0.08\end{array}$ & $\begin{array}{r}-0.22642 \\
0.17171\end{array}$ & 0.016265 \\
\hline-0.02 & -0.22648 & 01 \\
\hline 0.02 & $\begin{array}{r}0.20806 \\
-0.22576\end{array}$ & 0.0197 \\
\hline $\begin{array}{r}-0.02 \\
0.03\end{array}$ & 0.26189 & 0.024858 \\
\hline-0.0 & -0.2 & \\
\hline $\begin{array}{r}0.13 \\
-0.02\end{array}$ & $\begin{array}{l}0.29645 \\
-0.4803\end{array}$ & 0.0273 \\
\hline $\begin{array}{r}-6.02 \\
0.14\end{array}$ & $\begin{array}{r}-6.4863 \\
0.325\end{array}$ & 0.0304 \\
\hline-0.6 & -0.345 & \\
\hline 0.04 & 0.40 & 0.03695 \\
\hline-0.02 & -0.22 & \\
\hline 0.04 & 0.36 & 0.03372 \\
\hline-0.02 & -0.2102 & \\
\hline 0.139 & 0.26 & 0.02419 \\
\hline 0.007 & 0.017416 & \\
\hline 0.1 & 0.34 & 0.0316 \\
\hline 0.0 & & \\
\hline 0.147 & 0.40 & 0.03691 \\
\hline & & \\
\hline $\begin{array}{r}0.109 \\
0.00188\end{array}$ & $\begin{array}{r}0.29822 \\
0.010043\end{array}$ & 0.028 \\
\hline & & \\
\hline
\end{tabular}

U, ACL

0.758
0.616
0.623 0.616
0.623
0.601 0.648 0.126 0.0752
0.13
0.477 0.477
0.0667
0.435 0.435 0.0744 0.421
0.0706 0.48
0.0691 0.0691
0.119
0.0722 0.119
0.0722
0.147 0.147
0.0729 0.759 0.0531 0.768
0.0751 0.0751
0.226 0.157
0.26 0.159 1.59
1.09 1.72
1.19 1.493
0.656 0.656
1.348
0.642 


\begin{tabular}{|c|c|c|c|c|c|c|c|c|}
\hline TEST NO. & U, ppra & $N L(U), g / m 2$ & $2 n, A C L$ & $2 n$, ppm & $N L(2 n), g / m$ & $\mathrm{Zr}, \mathrm{ACL}$ & $\mathrm{Zr}, \mathrm{ppm}$ & $N L(Z r), g / m$ \\
\hline $\begin{array}{r}T Y 1 \\
T Y 1-F 50\end{array}$ & 2.277 & 0.073774 & -0.02 & -0.06008 & -0.15962 & -0.05 & -0.1502 & -0.11401 \\
\hline TYZ & 2.3088 & 0.071716 & -0.02 & -0.074118 & -0.18879 & $\begin{array}{l}-0.05 \\
-0.05\end{array}$ & $\begin{array}{r}-0.17883 \\
-0.1853\end{array}$ & -0.13485 \\
\hline$-F 50$ & 2.2154 & & -0.02 & -0.073724 & & -0.05 & -0.18431 & \\
\hline TY3 & 1.5739 & 0.050803 & 0.05 & 0.12144 & 0.32144 & -0.05 & -0.12144 & -0.09184 \\
\hline TY3-F50 & 1.3166 & & 0.05 & 0.52245 & & -0.05 & -0.52245 & \\
\hline $\begin{array}{r}\text { TY4 } \\
\text { TY4-F50 }\end{array}$ & $\begin{array}{r}0.162 \\
1.5275\end{array}$ & 0.0051773 & $\begin{array}{r}-0.02 \\
0.03\end{array}$ & -0.043086 & -0.11291 & -0.05 & $\begin{array}{r}-0.10772 \\
-0.5875\end{array}$ & -0.080649 \\
\hline TYS & 1.0671 & 0.033801 & 0.04 & 0.089484 & 0.23243 & $\begin{array}{l}-0.05 \\
-0.05\end{array}$ & $\begin{array}{l}-0.11185 \\
-0.1185\end{array}$ & -0.083009 \\
\hline $\begin{array}{l}-F 50 \\
T Y 6\end{array}$ & 0.8617 & 0.033858 & 0.05 & 0.64595 & 0.38295 & $\begin{array}{l}-0.05 \\
-0.05\end{array}$ & -0.64595 & -0.091178 \\
\hline TYE-F50 & 0.83663 & & 0.07 & 0.78715 & & $\begin{array}{l}-0.05 \\
-0.05\end{array}$ & $\begin{array}{l}-0.12465 \\
-0.56225\end{array}$ & \\
\hline TY7 & 1.0237 & 0.031606 & 0.08 & 0.19453 & 0.49248 & -0.05 & -0.12158 & -0.087942 \\
\hline-550 & 0.79291 & & 0.0 & 1.0108 & & -0.05 & -0.56155 & \\
\hline & 1.0303 & 0.032134 & 0.0 & 0.064392 & 0.16469 & -0.05 & -0.10732 & -0.078422 \\
\hline$F 50$ & 0.78249 & & 0. & 1.1324 & & -0.05 & -0.5662 & \\
\hline & 1.238 & 0.03877 & 0.05 & 0.52015 & 1.3358 & -0.05 & -0.52015 & -0.38165 \\
\hline & 0.81499 & & 0.1 & 1.8061 & & $-\theta$. & -0.5644 & \\
\hline TY10 & 1.2833 & 0.040107 & -0.0 & -0.17459 & -0.44745 & -0.05 & -0.43649 & -0.31962 \\
\hline$-F 50$ & 0.84892 & & 0.1 & 1.6303 & & -0.05 & -0.58225 & \\
\hline TY11 & 1.7308 & 0.052637 & 0.0 & 0.068412 & 0.1706 & -0. & -0.11402 & -0.08124 \\
\hline $1-F 50$ & 1.2752 & & -0.1 & -0.7 & & -0.0 & -1.2007 & \\
\hline TY12 & 1.7828 & 0.055043 & 0.0 & 0.069642 & 0.17631 & -0.05 & -0.11607 & -0.083957 \\
\hline $\begin{array}{l}2-750 \\
-50\end{array}$ & 1.2955 & & -0.0 & -0.345 & & -0.05 & -0.8625 & \\
\hline $\begin{array}{r}T Y 13 \\
T Y 13-T_{50}\end{array}$ & 2.2695 & 0.068745 & 0.1 & 1.0042 & 2.4943 & -0.05 & -0.5021 & -0.35633 \\
\hline TY13-F50 & $\begin{array}{l}1.7601 \\
2.3923\end{array}$ & 0.072178 & 0.12 & 1.3453 & 7504 & -0.05 & -0 . & - \\
\hline TY14-F50 & 1.6711 & & 0.09 & $\begin{array}{l}1.0121 \\
0.9459\end{array}$ & & $\begin{array}{l}-0.05 \\
-0.05\end{array}$ & $\begin{array}{l}-0.46006 \\
-0.5255\end{array}$ & -0.3252 \\
\hline TY15 & 3.0146 & 0.091135 & 0.034 & 0.064464 & 0.1598 & 0.011 & 0.020856 & 0.014772 \\
\hline TY15-F50 & 2.7119 & & 0.042 & 0.1045 & & 0.001 & 0.002488 & \\
\hline $\begin{array}{r}\text { TY16 } \\
\text { TY16-F50 }\end{array}$ & $\begin{array}{r}3.0616 \\
2,455\end{array}$ & 0.093341 & 0.044 & $\begin{array}{r}0.07832 \\
0.2063\end{array}$ & 0.1958 & 0.013 & 0.02314 & 0.016529 \\
\hline TY17 & 4.0654 & 0.12345 & 0.0169 & 0.046019 & 0.11459 & 00677 & 0.018435 & 0.013115 \\
\hline & 3.5037 & & 0.0136 & 0.072638 & & 2 & 0.0 & \\
\hline $\begin{array}{r}\text { TY18 } \\
\text { TY18-F25 }\end{array}$ & $\begin{array}{l}3.6881 \\
3.4296\end{array}$ & 0.11556 & $\begin{array}{l}0.0284 \\
0.0207\end{array}$ & $\begin{array}{r}0.077702 \\
0.11058\end{array}$ & 0.19965 & $\begin{array}{r}0.00471 \\
0.000146\end{array}$ & $\begin{array}{r}0.012887 \\
0.00077993\end{array}$ & 0.0094601 \\
\hline 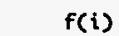 & 0.0164 & & & 0.0002 & & & 0.0007 & \\
\hline BKGENO & & & & & & & & \\
\hline
\end{tabular}


Page \#1 - "T2.DIH. CATIONS" TEST NUMBER RXN TIME

PH DIL FACTOR

SA/V LEACHANT VO

ACL. NUMBER

Al, ACL

.$A C L$
1.16
0.2
1.25
0.21
2.64
1.85
0.22
0.33
3.39
3.02
-0.1
0.46
0.14
0.13
0.24
0.28
0.24
-0.1
0.1
-0.1
-0.1
0.13
-0.1
0.13
-0.1
-0.1
0.1
-0.1
-0.1
0.0203

A1, ppm
7.3061
2.3978
7.7169
2.4172
11.362
12.02
2.2611
12.54
15.787
11.777
-2.4535
5.1955
2.1691
3.1911
1.9765
1.6254
1.5556
-1.4514
1.1274
-0.13712
-1.1431
1.1658
-1.7086
1.1424
-1.6737
-3.6605
0.96971
-2.079
-0.9111
0.0203
0
0

Tuesday, January 3 9:07 AM 1995

$\begin{array}{rr}T Z 1 & 3 \\ T Z 1-F 50 & 3 \\ T Z 2 & 3 \\ T Z 2-F 50 & 3 \\ T Z 3 & 7 \\ T Z 4 & 7 \\ T Z 4-F 50 & 7 \\ T Z 5-U F & 14 \\ T Z 5 & 14 \\ T Z 6 & 14 \\ T Z 6-F 50 & 14 \\ T 27 & 28 \\ T Z 7-F 50 & 28 \\ T Z 8 & 28 \\ T Z 8-F 50 & 28 \\ T Z 9 & 56 \\ T Z 10 & 56 \\ T Z 11 & 91 \\ T Z 11-F 50 & 91 \\ T Z 12 & 91 \\ T Z 12-F 50 & 91 \\ T Z 13 & 182 \\ T Z 13-F 50 & 182 \\ T Z 14 & 182 \\ T Z 14-F 50 & 182 \\ T Z 15 & 364 \\ T Z 15-F 50 & 364 \\ T Z 16 & 364 \\ T Z 16-F 50 & 364\end{array}$

$f(i)$
$B \times G N O$

\begin{tabular}{|c|c|c|c|c|}
\hline 11.09 & $\begin{array}{r}6.2984 \\
11.989\end{array}$ & 19846 & $\begin{array}{l}5.03 \\
5.03\end{array}$ & $\begin{array}{l}91-0532-11 \\
91-0532-12\end{array}$ \\
\hline 11.14 & $\begin{array}{l}11.989 \\
6.1735\end{array}$ & 19948 & $\begin{array}{l}5.03 \\
5.01\end{array}$ & $\begin{array}{l}91-0532-12 \\
91-0532-13\end{array}$ \\
\hline & 11.51 & & 5.01 & $91-0532-14$ \\
\hline 10.93 & 4.3036 & 20015 & 5.03 & $91-0532-15$ \\
\hline 11.21 & $\begin{array}{l}6.4973 \\
10.278\end{array}$ & 20015 & $\begin{array}{l}5.03 \\
4.98\end{array}$ & $\begin{array}{l}91-0532-16 \\
91-0532-17\end{array}$ \\
\hline & & & 4.98 & $91-0532-18$ \\
\hline .34 & 4.657 & 20076 & & $91-0532-19$ \\
\hline 11.33 & 3.8997 & 20029 & 4.99 & $91-0532-20$ \\
\hline 11.49 & $\begin{array}{l}24.535 \\
11.295\end{array}$ & 20121 & $\begin{array}{l}4.99 \\
4.99\end{array}$ & $\begin{array}{l}911-0532-21 \\
91-0533-09\end{array}$ \\
\hline & 15.065 & & 4.99 & $91-0533-10$ \\
\hline 11.46 & 4.547 & 20046 & 4.98 & $91-0533-11$ \\
\hline & & & & $91-0533-12$ \\
\hline 11.4 & 5.8051 & 20092 & 4.99 & $91-0569-05$ \\
\hline 11. & 6.4818 & 20061 & 4.99 & $91-0569-06$ \\
\hline 1.4 & 14.514 & 20154 & 4.97 & $91-0639-07$ \\
\hline 11.5 & $\begin{array}{l}11.274 \\
13.712\end{array}$ & 20032 & $\begin{array}{l}4.97 \\
4.99\end{array}$ & $\begin{array}{r}91-0639-08 \\
99-0639-09\end{array}$ \\
\hline & 11.431 & & .99 & $91-0639-10$ \\
\hline 11.62 & 8.9679 & 20143 & 4.98 & 92-0134-15 \\
\hline & 0.08 & & & $92-0134-16$ \\
\hline 11.6 & 6 & 20103 & & $92-0134-17$ \\
\hline $11.8-5$ & $\begin{array}{l}16.737 \\
36.605\end{array}$ & 20939 & $\begin{array}{l}4.98 \\
4.99\end{array}$ & $92-0134-18$ \\
\hline & $\begin{array}{l}36.6971 \\
9.6971\end{array}$ & & & $92-0388-02$ \\
\hline 11.76 & 20.792 & 19985 & 5 & $\begin{array}{r}92-0388-03 \\
92-0388-04\end{array}$ \\
\hline
\end{tabular}

0.0203

0.018135

B, ACL

8. $\mathrm{ppm}$

0.019057

12.3
6.23
12.4
6.45

77.47
74.692

0.027963

12.4
6.06

74.692
76.551

0.027963
0.029584

0.038738

0.01272

0.0078418

0.0039852

0.00382

0

0.0028511

0.0027993

0

$22.4 \quad 96.401$
14.96 .81

$\begin{array}{ll}9.41 & 96.81 \\ 2.79 & 96.714\end{array}$

$\begin{array}{ll}2.79 & 106.02 \\ 24.4 & 113.63\end{array}$

$\begin{array}{ll}24.4 & 88.133 \\ 2.47 & 109.67\end{array}$

$\begin{array}{ll}4.47 & 109.67 \\ 11.8 & 133.28 \\ 9.19 & 138.45\end{array}$

$\begin{array}{rr}9.19 & 138.45 \\ 5.1 & 125.19 \\ 16.7 & 137.53\end{array}$

$\begin{array}{ll}16.7 & 137.53 \\ 26.8 & 155.58\end{array}$

$\begin{array}{ll}25.7 & 166.58 \\ 13.6 & 197.39\end{array}$

$\begin{array}{rr}17.9 & 197.39 \\ 13.9 & 201.8 \\ 13.9 & 190.6\end{array}$

$\begin{array}{ll}17.2 & 196.61 \\ 31.9 & 286.08 \\ 15.1 & 258\end{array}$

$\begin{array}{rr}15.1 & 258 \\ 33 & 289.99\end{array}$

$\begin{array}{rr}15.9 & 26.12 \\ 113 & 4136.4\end{array}$

$\begin{array}{ll}361 & 4136.4 \\ 177 & 3500.7 \\ 364 & 3679.8\end{array}$ 


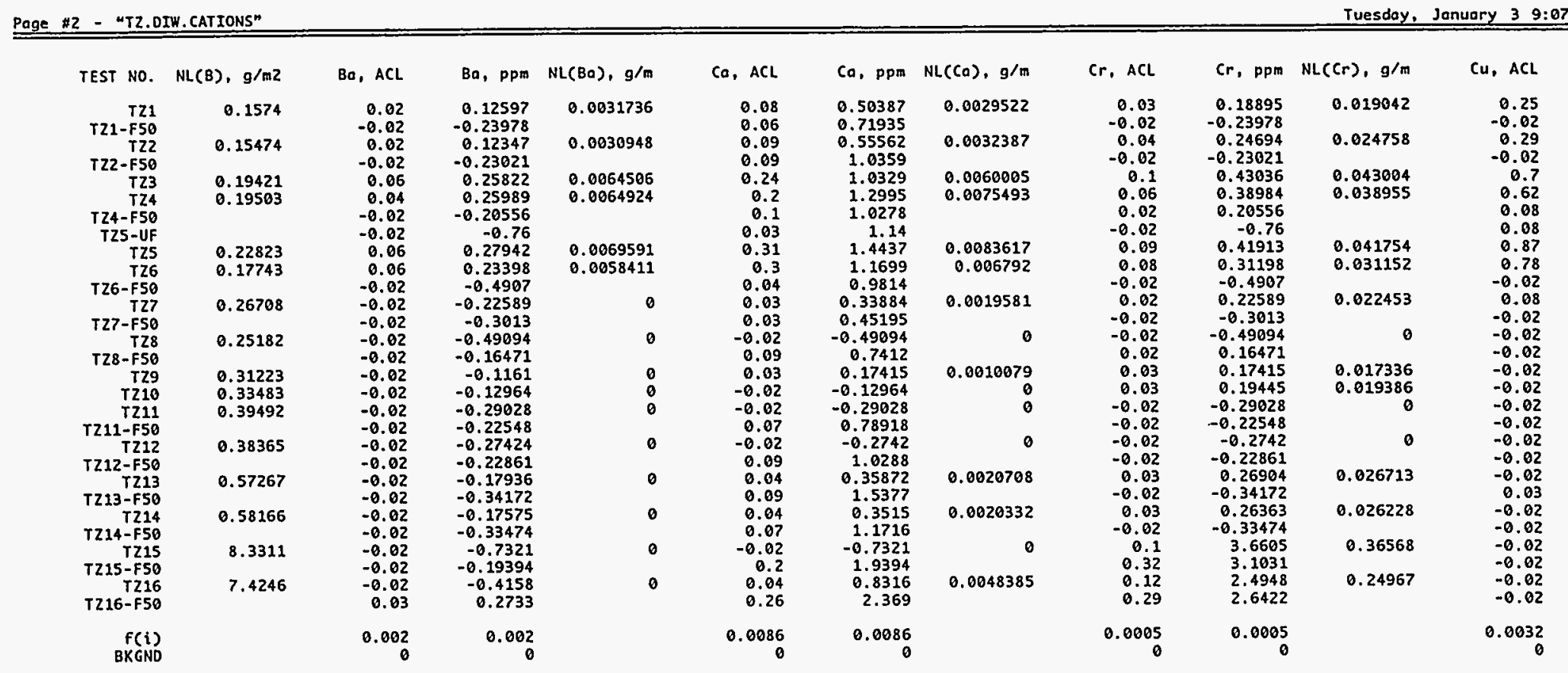




\begin{tabular}{|c|c|c|c|c|c|c|c|c|c|c|c|}
\hline TEST NO. & C11, ppm & $\mathrm{NL}(C u), g / m$ & $\mathrm{Fe}, \mathrm{ACL}$ & re, ppm & $N L(F e), g / m$ & $\mathrm{X}, \mathrm{ACl}$ & K, ppm & $N L(K), g / m 2$ & $L, A C I$ & Li, ppm & $N L(L i), g / m$ \\
\hline $\begin{array}{r}171 \\
121-F 50\end{array}$ & $\begin{array}{r}1.5746 \\
-0.23978\end{array}$ & A 024794 & $\begin{array}{l}4.66 \\
0.04\end{array}$ & $\begin{array}{r}29.351 \\
0.47956\end{array}$ & 0.018533 & $\begin{array}{l}7.84 \\
3.95\end{array}$ & $\begin{array}{l}49.379 \\
47.357\end{array}$ & 0.080522 & $\begin{array}{l}10.6 \\
5.43\end{array}$ & $\begin{array}{l}66.763 \\
65.101\end{array}$ & 0.17076 \\
\hline & 1.7903 & 0.028047 & 5.19 & 32.04 & 0.020128 & 8.05 & 49.697 & 0.080625 & 10.9 & 67.291 & 0.17123 \\
\hline$T 22-F 50$ & -0.23021 & & 0.04 & 0.46042 & & 3.87 & 44.545 & & 5.38 & 61.926 & \\
\hline & 3.0125 & 0.047035 & 12 & 51.643 & 0.032334 & 14.7 & 63.263 & 0.10229 & 19 & 81.768 & $\begin{array}{l}0.20738 \\
0.20598\end{array}$ \\
\hline $\begin{array}{r}T 24 \\
T 24-F 50\end{array}$ & $\begin{array}{r}4.8283 \\
0.82222\end{array}$ & 0.062895 & $\begin{array}{l}8.19 \\
0.17\end{array}$ & $\begin{array}{l}53.213 \\
1.7472\end{array}$ & 0.033316 & $\begin{array}{l}9.96 \\
5.99\end{array}$ & $\begin{array}{l}64.713 \\
61.564\end{array}$ & 0.10464 & $\begin{array}{l}12.5 \\
7.58\end{array}$ & $\begin{array}{l}81.216 \\
77.906\end{array}$ & \\
\hline rZS-UF & 3.04 & & 1.49 & 56.62 & & 2.16 & 82.08 & & 2.28 & 86.64 & \\
\hline I25 & 4.0516 & 0.063066 & 15.3 & 71.252 & 0.044475 & 16.8 & 78.238 & 0.12612 & & 94.537 & 0.23903 \\
\hline $\begin{array}{r}126 \\
126-F 50\end{array}$ & $\begin{array}{r}3.0118 \\
-0.4987\end{array}$ & 0.047459 & $\begin{array}{l}13.8 \\
0.03\end{array}$ & $\begin{array}{r}53.816 \\
0.73605\end{array}$ & 0.03367 & 15.8 & $\begin{array}{r}61.615 \\
73,85\end{array}$ & 0.099557 & 18.6 & 72.534 & 0.18383 \\
\hline & 0.90356 & 0.014033 & $\begin{array}{l}0.03 \\
1.33\end{array}$ & & 0.0093555 & $\begin{array}{l}3.01 \\
8.05\end{array}$ & $\begin{array}{r}73.85 \\
90.921\end{array}$ & 0.14624 & $\begin{array}{l}3.29 \\
8.71\end{array}$ & $\begin{array}{r}80.72 \\
98.375\end{array}$ & 0.24818 \\
\hline$T 27-\{50$ & -0.3013 & & -0.02 & -0.3013 & & 6.24 & 94.006 & & 6.76 & 101.84 & \\
\hline 128 & -0.49094 & 0 & 0.19 & 4.6639 & 0.0029156 & 3.55 & 87.142 & 0.14068 & 3.63 & 89.106 & 0.2256 \\
\hline$T Z 8-F 50$ & -0.16471 & & -0.02 & -0.16471 & & 11.1 & 91.415 & & & 98.827 & \\
\hline 129 & -0.1161 & 0 & 0.1 & 6.58051 & 0.00036206 & 18.7 & 108.56 & 0.17485 & 17 & 98.687 & 0.24933 \\
\hline$T 210$ & -0.12964 & 0 & 0.05 & 0.32409 & 0.00020245 & 17.9 & 116.02 & 0.18717 & 15.9 & 103.06 & $\begin{array}{l}0.26078 \\
0.29647\end{array}$ \\
\hline T211 & -0.29028 & 0 & 0.05 & 0.7257 & 0.00045122 & 10 & 145.14 & 0.23306 & 8.11 & 117.71 & 0.29647 \\
\hline $1211 . F 50$ & -0.22548 & & -0.02 & -0.22548 & & 13.1 & 147.69 & & $\begin{array}{l}10.4 \\
8.27\end{array}$ & 117.25 & \\
\hline $\begin{array}{r}T Z 12 \\
1 Z 12-F 50\end{array}$ & $\begin{array}{r}-0.2742 \\
-0.2861\end{array}$ & 0 & $\begin{array}{r}0.02 \\
-0.02\end{array}$ & $\begin{array}{r}0.2742 \\
-0.2361\end{array}$ & 0.00017 & 10.3 & $\begin{array}{l}141.23 \\
144.03\end{array}$ & 0.22817 & $\begin{array}{l}8.22 \\
9.97\end{array}$ & $\begin{array}{l}112.71 \\
113.96\end{array}$ & 0.28561 \\
\hline $\begin{array}{r}1213 \\
\end{array}$ & -0.17936 & 0 & 0.03 & 0.26904 & 0.00016737 & 22.6 & 202.67 & 0.32562 & 16 & 144.38 & 0.36385 \\
\hline $1213-F 50$ & 0.51258 & & -0.02 & -0.34172 & & 10.9 & 186.24 & & 7.7 & 131.56 & \\
\hline$T 214$ & -0.17575 & 0 & 0.04 & 0.3515 & 0.00021911 & 23.2 & 203 & 0.3282 & 16.4 & 144.12 & 0.363 \\
\hline $1214-F 50$ & -0.33174 & & -0.02 & -0.33474 & & 21.3 & 189.13 & & 8.13 & 136.07 & \\
\hline T215 & -0.7321 & - & 0.05 & 1.8303 & 0.0011456 & 16.5 & 1702.1 & 2.7515 & 10.8 & 395.33 & 1.002 \\
\hline $\begin{array}{r}1215-F 50 \\
7216\end{array}$ & $\begin{array}{r}-0.19394 \\
-0.4158\end{array}$ & 0 & $\begin{array}{r}0.09 \\
-0.02\end{array}$ & $\begin{array}{l}0.87274 \\
-0.4158\end{array}$ & 0 & $\begin{array}{r}168 \\
71.9\end{array}$ & $\begin{array}{l}1551.5 \\
1494.8\end{array}$ & 2.4286 & $\begin{array}{l}37.6 \\
16.7\end{array}$ & $\begin{array}{l}364.61 \\
347.19\end{array}$ & 0.8818 \\
\hline $1716-F$ & -0.1822 & & -0.0 & -0.1822 & & 16 & 24 & & & 346.22 & \\
\hline $\begin{array}{c}f(i) \\
B K G N D\end{array}$ & 0.0032 & & 0.0798 & 0.0798 & & $\begin{array}{r}0.0309 \\
0\end{array}$ & 0.0309 & & $\begin{array}{r}0.0173 \\
0\end{array}$ & 0.0197 & \\
\hline
\end{tabular}




\begin{tabular}{|c|c|c|c|}
\hline TEST NO. & $\mathrm{Mg}, \mathrm{ACL}$ & $\mathrm{Mg}, \mathrm{ppm}$ & $\mathrm{NL}(\mathrm{Mg}), \mathrm{g} / \mathrm{m}$ \\
\hline $\begin{array}{r}T Z 1 \\
T Z 1-F 50\end{array}$ & $\begin{array}{l}0.69 \\
0.01\end{array}$ & $\begin{array}{r}4.3459 \\
0.11989\end{array}$ & 0.027373 \\
\hline $\begin{array}{l}T 21-+560 \\
T Z 2\end{array}$ & $\begin{array}{l}0.61 \\
0.77\end{array}$ & $\begin{array}{r}.11989 \\
4.7536\end{array}$ & 0.029787 \\
\hline TZ2-F50 & 0.02 & 0.23021 & \\
\hline T23 & 1.54 & 6.6275 & 0.041391 \\
\hline$T Z 4$ & 1.06 & 6.8871 & 0.043012 \\
\hline$T Z 4-F 50$ & 0.02 & 0.20556 & \\
\hline $\begin{array}{r}T 25-U F \\
\text { T25 }\end{array}$ & $\begin{array}{l}0.18 \\
1.88\end{array}$ & $\begin{array}{r}6.84 \\
8.7552\end{array}$ & 0.054513 \\
\hline 126 & 1.68 & 6.5515 & 0.040888 \\
\hline$T 26-F 50$ & -0.01 & -0.24535 & \\
\hline T27 & 0.16 & 1.8071 & 0.011227 \\
\hline $727-F 50$ & -0.01 & -0.15065 & \\
\hline T28 & 0.02 & 0.49094 & 0.0030613 \\
\hline TZ8-F50 & -0.01 & -0.082356 & \\
\hline T29 & 0.01 & 0.058051 & 0.00036116 \\
\hline TZ10 & -0.01 & -0.064818 & $\because$ \\
\hline$T 211$ & -0.01 & -0.14514 & 0 \\
\hline TZ11-F50 & 0.01 & 0.11274 & \\
\hline TZ12 & -0.01 & -0.13712 & 0 \\
\hline TZ12-F50 & 0.01 & 0.11431 & \\
\hline $\begin{array}{l}7213 \\
7359\end{array}$ & -0.01 & -0.089679 & $\theta$ \\
\hline $\begin{array}{r}3-F 50 \\
\text { TZ14 }\end{array}$ & $\begin{array}{r}0.01 \\
-0.01\end{array}$ & -0.087876 & 0 \\
\hline TZ14-F50 & -0.01 & -0.16737 & \\
\hline $\begin{array}{r}\text { TZ15 } \\
T Z 15-F 50\end{array}$ & -0.01 & $\begin{array}{r}-0.36605 \\
0.19394\end{array}$ & 0 \\
\hline $\begin{array}{r}1216 \\
T 216 \\
T Z 16-55\end{array}$ & $\begin{array}{r}-0.01 \\
0.02\end{array}$ & $\begin{array}{r}-0.2079 \\
0.1822\end{array}$ & 0 \\
\hline & & & \\
\hline $\begin{array}{l}\text { F(t) } \\
\text { BKGND }\end{array}$ & 6 & & \\
\hline
\end{tabular}

\begin{tabular}{|c|c|c|}
\hline $\mathrm{Mn}, \mathrm{ACL}$ & Mn, ppm & $N L(M n), g / m$ \\
\hline 1.03 & 6.4874 & 0.02334 \\
\hline $\begin{array}{r}-0.01 \\
1.16\end{array}$ & $\begin{array}{r}-0.11989 \\
7.1613\end{array}$ & 0.02564 \\
\hline-0.01 & -0.1151 & \\
\hline 2.59 & 11.146 & 0.0397 \\
\hline 1.74 & 11.305 & 0.0403 \\
\hline 0.01 & 0.10278 & \\
\hline $\begin{array}{l}0.31 \\
3.22\end{array}$ & $\begin{array}{r}11.78 \\
14.996\end{array}$ & 0.0533 \\
\hline 2.86 & 11.153 & 0.0397 \\
\hline-0.01 & -0.24535 & \\
\hline 0.27 & 3.0495 & 0.010 \\
\hline $\begin{array}{r}-0.01 \\
0.04\end{array}$ & $\begin{array}{r}-0.15065 \\
0.98188\end{array}$ & 0.003 \\
\hline-0.01 & -0.082356 & \\
\hline 0.02 & 0.1161 & 0.0004 \\
\hline-0.01 & -0.064818 & \\
\hline-0.01 & -0.14514 & \\
\hline-0.01 & -0.11274 & \\
\hline-0.01 & -0.13712 & \\
\hline-0.01 & -0.11431 & \\
\hline-0.01 & -0.089679 & \\
\hline-0.01 & -0.17086 & \\
\hline-0.01 & -0.087876 & \\
\hline-0.01 & -0.16737 & \\
\hline-0.01 & -0.36605 & \\
\hline-0.01 & -0.096971 & \\
\hline $\begin{array}{l}-0.01 \\
-0.01\end{array}$ & $\begin{array}{r}-0.2079 \\
-0.09111\end{array}$ & \\
\hline 0.014 & & \\
\hline & 14 & \\
\hline
\end{tabular}

No, $\mathrm{ACl}$

37.2
18.1
37.6
18.3
68.6
45.7
27.3
8.19
74.6
69.3
12.7
37.4
27.2
15
51.9
70.3
67.3
38.2
49.7
39.3
48.2
86.7
42.1
88.6
43.5
197
721
309
706
0.0661
0
Na, ppn NL(Na), g/m

234.3
217
232.12
210.64
295.23
296.93
280.58
311.22
347.41
270.25
311.59
422.41
409.77
368.21
427.43
408.1
436.23
554.43
560.32
538.88
550.95
777.52
719.32
778.58
728.05
7211.2
6991.6
6424.1
6432.4
0.0661
0

$\mathrm{Ni}, \mathrm{ACl}$

0.17861

0.17604

0.22315
0.22444

0.20413

0.31761

0.27788

0.30728
0.32897

0.41619

0.407

0.58396

0.58592

5.4493

4.863 


\begin{tabular}{|c|c|c|c|c|}
\hline TEST NO. & $N L(\mathrm{Ni}), g / m$ & Si, ACL & si, ppm & NL(Si), $g / m$ \\
\hline $\begin{array}{r}T 21 \\
1-F 50\end{array}$ & 0.015624 & 45.2 & 284.69 & 0.062723 \\
\hline $\begin{aligned} 1-530 \\
T 22\end{aligned}$ & 0.01714 & $\begin{array}{l}20.3 \\
46.3\end{array}$ & $\begin{array}{l}243.38 \\
285.83\end{array}$ & 0.062654 \\
\hline $22-F 50$ & & 19.9 & 229.06 & \\
\hline I23 & 0.025141 & 86 & 370.11 & 0.080855 \\
\hline $\begin{array}{r}T 24 \\
T 24-F 50\end{array}$ & 0.026969 & 55.9 & 363.2 & 0.079346 \\
\hline T25-UF & & 10.8 & $\begin{array}{r}366.28 \\
410.4\end{array}$ & \\
\hline$T 25$ & 0.031405 & 96.8 & 450.8 & 0.098183 \\
\hline T26 & 0.023364 & 83.1 & 324.07 & 0.070747 \\
\hline TZ6-F50 & & 13.5 & 331.22 & \\
\hline & 0.0077722 & 35.5 & 400.95 & 0.087132 \\
\hline T27-F50 & & 26.5 & 399.22 & \\
\hline & 0 & 14.7 & 360.84 & 0.078709 \\
\hline$T Z 8-F 50$ & & 47.6 & 392.01 & \\
\hline T29 & $\circ$ & 69.5 & 403.45 & 0.087802 \\
\hline TZ10 & 0 & 66 & 427.8 & 0.093244 \\
\hline TZ11 & 0 & 32.1 & 465.9 & 0.10108 \\
\hline TZ11-F50 & & 42.1 & 474.64 & \\
\hline $\begin{array}{r}T Z 12 \\
7712-559\end{array}$ & 0 & $\begin{array}{l}32.7 \\
40.5\end{array}$ & 448.38 & 0.09787 \\
\hline $\begin{array}{r}T Z 12-F 50 \\
T Z 13\end{array}$ & 0 & $\begin{array}{l}40.5 \\
65.4\end{array}$ & $\begin{array}{r}462.94 \\
586.5\end{array}$ & 0.12731 \\
\hline rZ13-F5O & & 31.7 & 541.63 & \\
\hline $\begin{array}{r}T 214 \\
T Z 24-550\end{array}$ & 0 & $\begin{array}{l}69.1 \\
33.9\end{array}$ & 607.22 & 0.13208 \\
\hline TZ15 & 0 & 29.6 & $\begin{array}{l}368.38 \\
1083.5\end{array}$ & 0.23665 \\
\hline TZ15-F5O & & 100 & 969.71 & \\
\hline $\begin{array}{r}T Z 16 \\
T 716-550\end{array}$ & - & 46 & 956.34 & 0.20924 \\
\hline$f(i$ & & 0.2287 & 0.2 & \\
\hline & & & & \\
\hline
\end{tabular}

\begin{tabular}{|c|c|c|}
\hline$T h, A C L$ & Th, ppm & $N L(T h), g / m$ \\
\hline & 0.56686 & 0.012419 \\
\hline-0.05 & $\begin{array}{r}-0.59946 \\
0.61735\end{array}$ & 0.013456 \\
\hline $\begin{array}{l}-0.05 \\
-0.12\end{array}$ & -0.57552 & \\
\hline 0.19 & 0.81768 & 0.017762 \\
\hline 0.12 & 0.77968 & \\
\hline $\begin{array}{l}-0.05 \\
-0.05\end{array}$ & $\begin{array}{r}-0.51389 \\
-1.9\end{array}$ & \\
\hline 0.22 & 1.0245 & 0.022188 \\
\hline 0.19 & 0.74094 & 0.016084 \\
\hline-0.05 & $\begin{array}{r}-1.2267 \\
-0.56473\end{array}$ & 9 \\
\hline-0.05 & -0.75325 & \\
\hline $\begin{array}{l}-0.05 \\
-0.05\end{array}$ & $\begin{array}{r}-1.2274 \\
-0.41178\end{array}$ & $\theta$ \\
\hline-0.05 & -0.29026 & 0 \\
\hline-0.05 & -0.32409 & \\
\hline-0.05 & -0.7257 & 0 \\
\hline $\begin{array}{l}-0.05 \\
-0.05\end{array}$ & $\begin{array}{l}-0.5637 \\
-0.6856\end{array}$ & 0 \\
\hline-0.05 & -0.57153 & \\
\hline-0.05 & -0.4484 & 0 \\
\hline-0.05 & -0.8543 & \\
\hline-0.05 & -0.43938 & 0 \\
\hline$-\hat{0} .65$ & - ช. 03684 & \\
\hline-0.05 & -1.8303 & 0 \\
\hline $\begin{array}{l}-0.05 \\
-0.05\end{array}$ & $\begin{array}{r}-0.48485 \\
-1.0395\end{array}$ & 0 \\
\hline-0.05 & -0.4556 & \\
\hline 0.0023 & 0.0023 & \\
\hline & & \\
\hline
\end{tabular}

$T i, A C L$
0.25
-0.02
0.28
-0.02
0.69
0.47
-0.02
0.09
0.89
0.8
-0.02
0.08
-0.02
-0.02
-0.02
-0.02
-0.02
-0.02
-0.02
-0.02
-0.02
-0.02
-0.02
-0.02
-0.02
-0.02
-0.02
-0.02
-0.02
0.0054

$T i$, ppm NL(Ti), g/n

U, ACL

$\begin{array}{rll}1.5746 & 0.014693 & 1.261 \\ -0.23978 & 0.335 \\ 1.7286 & 0.016047 & 1.329\end{array}$

1.329
0.334

$\begin{array}{lll}2.9695 & 0.027475 & 2.58 \\ 3.0537 & 0.028254 & 1.815\end{array}$

$\begin{array}{rl}0.20556 & 0.368 \\ 3.42 & 3.053\end{array}$

$4.1447 \quad 0.038232$

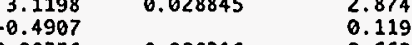

$\begin{array}{lll}0.90356 & 0.008316 & 0.662\end{array}$

$\begin{array}{rrr}-0.3013 & 0.292 \\ -0.49094 & 0 & 0.23\end{array}$

$\begin{array}{ll}-0.16471 & 0.23 \\ -0.1161 & 0.6\end{array}$

$\begin{array}{lll}-0.1161 & 0 & 0.757 \\ -0.12964 & 0 & 0.723\end{array}$

$\begin{array}{rll}-0.29028 & 0 & 0.267 \\ -0.22548 & 0.307\end{array}$

$\begin{array}{lll}-0.2742 & 0 & 0.278 \\ -0.22861 & 0.305\end{array}$

$\begin{array}{lll}-0.17936 & 0 & 0.337\end{array}$

$\begin{array}{lll}-0.17575 & 0 & 0.32 \\ 0.3347 & 0.138\end{array}$ 


\begin{tabular}{|c|c|c|c|c|c|c|c|c|}
\hline TEST NO. & $u, \mathrm{ppm}$ & $\mathrm{NL}(U), g / m 2$ & $\mathrm{Zn}, \mathrm{ACl}$ & $\mathrm{Zn}, \mathrm{ppm}$ & $N L(2 n), g / m$ & $\mathrm{Zr}, \mathrm{ACL}$ & $\mathrm{Zr}, \mathrm{ppm}$ & $\mathrm{NL}(Z r), g / \mathrm{m}$ \\
\hline $\begin{array}{r}T Z 1 \\
T Z 1-F 50\end{array}$ & $\begin{array}{l}7.9423 \\
4.0163\end{array}$ & 0.024402 & $\begin{array}{r}0.02 \\
-0.02\end{array}$ & $\begin{array}{r}0.12597 \\
-0.23978\end{array}$ & 0.031736 & $\begin{array}{l}-0.05 \\
-0.05\end{array}$ & $\begin{array}{l}-0.31492 \\
-0.59946\end{array}$ & 0 \\
\hline $\begin{array}{r}T Z 1-+560 \\
T Z 2\end{array}$ & 8.2046 & 0.025079 & 0.02 & 0.12347 & 0.030948 & -0.05 & -0.30868 & 0 \\
\hline$T 22-F 50$ & 3.8445 & & -0.02 & -0.23021 & & -0.05 & -0.57552 & \\
\hline IZ3 & 11.116 & 0.033865 & 0.08 & 0.34429 & 0.086007 & 0.05 & 0.21518 & 0.0015358 \\
\hline T24 & 11.793 & 0.035926 & 0.16 & 1.0396 & 0.2597 & -0.05 & -0.32487 & 0 \\
\hline $\begin{array}{r}\text { TZ4-F50 } \\
\text { TZ5-UF }\end{array}$ & $\begin{array}{l}3.7822 \\
116.01\end{array}$ & & $\begin{array}{r}0.08 \\
-0.02\end{array}$ & $\begin{array}{l}0.82222 \\
-0.76\end{array}$ & & -0.05 & -0.51389 & \\
\hline $\begin{array}{r}\text { TZ5-UF } \\
\text { TZ5 }\end{array}$ & 116.01 & & $\begin{array}{r}-0.02 \\
0.07\end{array}$ & $\begin{array}{r}-0.76 \\
0.32599\end{array}$ & & -0.05 & -1.9 & \\
\hline $\begin{array}{l}T 25 \\
T 26\end{array}$ & 11.208 & 0.03412 & 0.06 & $\begin{array}{l}0.32599 \\
0.23398\end{array}$ & $\begin{array}{l}0.081189 \\
0.058411\end{array}$ & $\begin{array}{r}0.07 \\
-0.05\end{array}$ & $\begin{array}{r}0.32599 \\
-0.19499\end{array}$ & $\begin{array}{r}0.0023197 \\
0\end{array}$ \\
\hline T26-F50 & 2.9197 & $0.057 \alpha$ & -0.02 & -0.4907 & & -0.05 & -1.2267 & \\
\hline & 7.477 & 0.022659 & -0.02 & -0.22589 & 0 & -0.05 & -0.56473 & 0 \\
\hline T27-F50 & $\begin{array}{r}4.399 \\
5.6458\end{array}$ & & -0.02 & -0.3013 & & -0.05 & -0.75325 & \\
\hline $\begin{array}{r}\text { T28 } \\
T 28-\mathrm{F} 50\end{array}$ & 5.6458 & 0.017173 & -0.02 & -0.49094 & 0 & -0.05 & -1.2274 & 0 \\
\hline $\begin{array}{r}\text { T28-F50 } \\
\text { TZ9 }\end{array}$ & $\begin{array}{l}4.9414 \\
4.3945\end{array}$ & 0.013336 & $\begin{array}{l}-0.02 \\
-0.02\end{array}$ & -0.16471 & & -0.05 & -0.41178 & \\
\hline $\begin{array}{r}T 29 \\
T 210\end{array}$ & $\begin{array}{l}4.3945 \\
4.6863\end{array}$ & $\begin{array}{l}0.013336 \\
0.014244\end{array}$ & $\begin{array}{l}-0.02 \\
-0.02\end{array}$ & $\begin{array}{r}-0.1161 \\
-0.12964\end{array}$ & $: 0$ & -0.05 & -0.29026 & 0 \\
\hline TZ11 & 3.8752 & 0.011724 & -0.02 & $\begin{array}{l}-0.12964 \\
-0.29028\end{array}$ & 0 & $\begin{array}{l}-0.05 \\
-0.05\end{array}$ & $\begin{array}{r}-0.32409 \\
-0.7257\end{array}$ & 0 \\
\hline TZ11-F50 & 3.4611 & & -0.02 & -0.22548 & & -0.05 & -0.5637 & \\
\hline$T 212$ & 3.812 & 0.0116 & -0.02 & -0.2742 & 0 & -0.05 & -0.6856 & 0 \\
\hline$T Z 12-F 50$ & 3.4863 & & -0.02 & -0.22861 & & -0.05 & -0.57153 & \\
\hline TZ13 & 3.0222 & 0.0091486 & -0.02 & -0.17936 & 0 & -0.05 & -0.4484 & 0 \\
\hline$T Z 13-F 50$ & 2.4091 & & 0.04 & 0.68344 & & -0.05 & -0.8543 & \\
\hline TZ14 & 2.812 & 0.0085293 & -0.02 & -0.17575 & 0 & -0.05 & -0.43938 & o \\
\hline TZ14-F50 & 2.3097 & & 0.02 & 0.33474 & & $\begin{array}{l}-0.05 \\
-0.05\end{array}$ & -0.83684 & \\
\hline TZ15 & 0.98467 & 0.0029991 & -0.02 & -0.7321 & 0 & -0.05 & $\begin{array}{r}-1.8303 \\
-0.48485\end{array}$ & 0 \\
\hline TZ15-F50 & 0.71662 & & 0.02 & 0.19394 & & $\begin{array}{l}-0.05 \\
-0.05\end{array}$ & $\begin{array}{l}-0.48485 \\
-1.0395\end{array}$ & \\
\hline $\begin{array}{r}\text { TZ16 } \\
\text { TZ16-F50 }\end{array}$ & $\begin{array}{r}0.51559 \\
0.4619\end{array}$ & 0.0015731 & $\begin{array}{l}-0.02 \\
-0.02\end{array}$ & $\begin{array}{l}-0.4158 \\
-0.1822\end{array}$ & 0 & $\begin{array}{l}-0.05 \\
-0.05\end{array}$ & $\begin{array}{l}-1.0395 \\
-0.4556\end{array}$ & 0 \\
\hline$f(i)$ & 0.0164 & & 0.0002 & 0.0002 & & 0.007 & 0.007 & \\
\hline BKGND & & & & & & & & \\
\hline
\end{tabular}


Page \#1 - "TZ.EJ.CATIONS"

test No. TARGet time

PH DIL FACTOR

SA/V

LEACHANT ACL NUMBER

Al, ACL

Al, ppa NL(AI),

Frido

TZ31
TZ31-F50

11.02

\begin{tabular}{|c|c|}
\hline $\begin{array}{l}6.276 \\
12.59\end{array}$ & $1.980 e+04$ \\
\hline $\begin{array}{l}6.528 \\
12.55\end{array}$ & $1.998 e+04$ \\
\hline $\begin{array}{l}6.603 \\
10.11\end{array}$ & $1.990 e+04$ \\
\hline $\begin{array}{l}4.317 \\
4.972\end{array}$ & $\begin{array}{l}1.963 e+04 \\
2.001 e+04\end{array}$ \\
\hline $\begin{array}{l}37.02 \\
5.139\end{array}$ & $2.005 c+04$ \\
\hline $\begin{array}{l}25.07 \\
15.91\end{array}$ & $1.996 e+04$ \\
\hline $\begin{array}{l}9.455 \\
16.74\end{array}$ & $2.000 \mathrm{e}+04$ \\
\hline $\begin{array}{l}9.672 \\
6.179\end{array}$ & $1.984 e+04$ \\
\hline $\begin{array}{l}6.098 \\
13.27\end{array}$ & $\begin{array}{l}1.980 e+04 \\
1.986 e+04\end{array}$ \\
\hline $\begin{array}{l}11.49 \\
12.25\end{array}$ & $1.986 e+04$ \\
\hline $\begin{array}{l}11.32 \\
8.653\end{array}$ & $1.975 e+04$ \\
\hline $\begin{array}{l}16.41 \\
8.643\end{array}$ & $1.978 \mathrm{e}+04$ \\
\hline & $1.974 e+04$ \\
\hline $\begin{array}{r}11.01 \\
28.6 \\
10.03\end{array}$ & $1.979 e+04$ \\
\hline & \\
\hline
\end{tabular}

$5.06 \quad 91-0532-22$

0.84

TZ32-F50

11.04

11.03

11.01
11.23

11.28

11.37

11.38

11.37

11.38

11.63

11.66

11.09

11.75

1

$5.02 \quad 91-0532-24$

$5.04 \quad \begin{array}{r}91-0532-25 \\ 91-0532-26\end{array}$

$5.11 \quad 91-0532-27$
$91-0532-28$

$5.01 \quad 91-0532-29$

$4.99 \quad \begin{array}{r}91-0532-30 \\ 91-0532-31\end{array}$

$5.01 \quad 91-0532-32$

$5 \quad 91-0533-14$

91-0533-15

$91-0533-16$
$5.04 \quad 91-0596-07$

$5.05 \quad 91-0596-08$

91-0639-12

$5.04 \quad \begin{array}{r}91-0639-13 \\ 91-0639-14\end{array}$

$5.06 \quad \begin{array}{r}91-0639-14 \\ 92-0134-19\end{array}$

$5.06 \quad 92-0134-21$

$5.07 \quad 92-0134-22$

$\begin{array}{ll}5.07 & 92-0485-07 \\ 5.06 \quad 92-0485-08 \\ 92-0485-09\end{array}$

0.84
0.1
0.83
0.1
1.39
0.13
1.93
2.31
0.28
2.35
-0.1
0.38
0.19
0.23
0.2
0.25
0.25
-0.1
-0.1
0.19
0.15
0.13
-0.1
0.14
-0.1
0.1
-0.1
0.13
0.0203
0.56

5.272

$5.272-0.01172$
1.259

B, ACL

$2: 43$ PM 1994

0.01197

$\begin{array}{lr}12.1 & 75.94 \\ 5.84 & 73.5\end{array}$

$\begin{array}{ll}1.255 & 0.02133\end{array}$

$\begin{array}{rr}1.314 & 0.02195 \\ 8.331 & 0.0195\end{array}$

$\begin{array}{ll}10.37 & 0.02689 \\ 12.08 & 0.0283\end{array}$

-2.507
6.046 $\quad 0.01354$

$\begin{array}{ll}1.796 & 0.01354 \\ 3.85 & 0.008105\end{array}$

1.85
1.934
1.555

1.545
1.525
-1.327

-1.327
-1.149

1.699

1.125
-1.641

-1.641
1.21
-1.724

1.101

1.101
-2.86
1.304
0.0203
0.56

0.002445

0.0024
-0.00468

0.004382

0.001409

0.001619

$-0.008513$

$\begin{array}{ll}11.8 & 77.02 \\ 5.98 & 75.02\end{array}$

$\begin{array}{rr}14.2 & 93.77 \\ 8.5 & 85.93 \\ 22.5 & 9.81\end{array}$

$\begin{array}{ll}21.5 & 92.81 \\ 22.3 & 110.9\end{array}$

$\begin{array}{ll}2.83 & 104.8 \\ 21.7 & 111.5 \\ 1.28 & \end{array}$

$\begin{array}{ll}4.28 & 107.3 \\ 7.46 & 118.7 \\ 13.4 & 126.7 \\ 7.22 & 128.9\end{array}$

$\begin{array}{ll}7.22 & 120.9 \\ 13.2 & 127.7\end{array}$

$\begin{array}{ll}23.9 & 147.7 \\ 24.5 & 149.5\end{array}$

$\begin{array}{ll}13.3 & 176.5 \\ 15.8 & 181.5\end{array}$

$14.7 \quad 180$

$\begin{array}{ll}28.4 & 185.2 \\ 28.7 & \end{array}$

$\begin{array}{ll}28.2 & 243.7 \\ 13.3 & 229.3\end{array}$

310
122
339
0.0248
0.15 


\begin{tabular}{|c|c|c|c|c|}
\hline TEST NO. & $\mathrm{NL}(\mathrm{B}), \mathrm{g} / \mathrm{mZ}$ & $\mathrm{Ba}, \mathrm{ACL}$ & Bo, ppm & NL(Ba). \\
\hline T231 & 0.1543 & -0.02 & -0.1255 & -0.003 \\
\hline & 0.1551 & $\begin{array}{l}-0.02 \\
-0.02\end{array}$ & $\begin{array}{l}-0.2517 \\
-0.1305\end{array}$ & -0.0032 \\
\hline$T 232-F 50$ & & -0.02 & -0.2509 & \\
\hline T233 & 0.1897 & 0.03 & 0.1981 & 0.0049 \\
\hline $\begin{array}{r}\text { TZ33-F50 } \\
\text { TZ34 }\end{array}$ & 0.1903 & $\begin{array}{r}-0.02 \\
0.03\end{array}$ & $\begin{array}{r}-0.2022 \\
0.1295\end{array}$ & \\
\hline $\begin{array}{r}\text { TZ35 } \\
\text { TZ35-UF }\end{array}$ & 0.2231 & 0.04 & 0.1989 & 0.0049 \\
\hline $\begin{array}{l}\text { TZ35-UF } \\
\text { TZ36 }\end{array}$ & 0.224 & $\begin{array}{r}-0.02 \\
0.04\end{array}$ & $\begin{array}{r}-0.7405 \\
0.2056\end{array}$ & 0.005 \\
\hline TZ36-F50 & & -0.02 & -0.5014 & \\
\hline $\begin{array}{r}\text { T237 } \\
\end{array}$ & 0.2394 & -0.02 & -0.3182 & \\
\hline $\begin{array}{r}\text { TZ37-F50 } \\
\text { TZ38 }\end{array}$ & 0.2434 & $\begin{array}{l}-0.02 \\
-0.02\end{array}$ & $\begin{array}{l}-0.1891 \\
-0.3348\end{array}$ & -0 \\
\hline$T 238-F 50$ & & -0.02 & -0.1934 & \\
\hline$T 239$ & 0.2998 & -0.02 & -0.1236 & -0.0 \\
\hline $\begin{array}{l}T Z 46 \\
T Z 41\end{array}$ & 0.3039 & -0.02 & -0.122 & -0 . \\
\hline TZ41-F50 & 0.358 & $\begin{array}{l}-6.02 \\
-0.02\end{array}$ & $\begin{array}{l}-0.2654 \\
-0.2298\end{array}$ & -0.0 \\
\hline $\begin{array}{r}T Z 42 \\
T 242\end{array}$ & 0.3652 & -0.02 & -0.245 & -0.0 \\
\hline TZ43 & 0.5013 & $\begin{array}{l}-6.02 \\
-0.02\end{array}$ & $\begin{array}{l}-0.2265 \\
-0.1731\end{array}$ & -0.00 \\
\hline$T Z 43-F 50$ & 0.4966 & -0.02 & -0.3282 & \\
\hline $\mathrm{TZ} 44-\mathrm{Fse}$ & 0.4900 & $\begin{array}{l}-6.02 \\
-0.02\end{array}$ & $\begin{array}{l}-0.1729 \\
-0.3448\end{array}$ & \\
\hline $\begin{array}{r}T 245-F 50 \\
T Z 46 \\
T Z 46-F 50 \\
F(i) \\
\text { BKGND }\end{array}$ & 7.109 & $\begin{array}{r}-0.02 \\
-0.02 \\
-0.02 \\
0.002 \\
0\end{array}$ & $\begin{array}{r}-0.2203 \\
-0.572 \\
-0.2006 \\
0.002 \\
0\end{array}$ & -0. \\
\hline
\end{tabular}

\begin{tabular}{|c|c|c|}
\hline $\mathrm{Ca}, \mathrm{ACL}$ & Co, ppm & $\mathrm{NL}(C a), g / \mathrm{m}$ \\
\hline 0.09 & 0.5648 & -0.02352 \\
\hline 0.08 & 1.007 & \\
\hline 0.08 & 0.5222 & -0.02355 \\
\hline 0.07 & 0.8782 & \\
\hline 0.16 & 1.057 & -0.02053 \\
\hline 0.18 & $\begin{array}{r}1.82 \\
0.9929\end{array}$ & -0.02119 \\
\hline 0.22 & 1.094 & -0.0202 \\
\hline $\begin{array}{r}-0.02 \\
0.21\end{array}$ & $\begin{array}{r}-0.7405 \\
1.079\end{array}$ & -0.02025 \\
\hline 0.06 & 1.504 & \\
\hline-0.02 & -0.3182 & -0.02847 \\
\hline 0.09 & 0.851 & \\
\hline-0.02 & $\begin{array}{r}-0.3348 \\
0.7738\end{array}$ & -0.02852 \\
\hline $\begin{array}{l}0.08 \\
0.02\end{array}$ & $\begin{array}{l}0.7738 \\
0.1236\end{array}$ & -0.02605 \\
\hline 0.02 & 0.122 & -0.02612 \\
\hline-0.02 & -0.2654 & -0.02831 \\
\hline 0.08 & 0.9192 & \\
\hline-0.02 & -0.245 & -0.02819 \\
\hline 0.08 & 0.9059 & \\
\hline 0.04 & 0.3461 & -0.02486 \\
\hline 0.1 & 1.641 & -0.07534 \\
\hline $\begin{array}{l}0.03 \\
0.07\end{array}$ & $\begin{array}{r}0.2593 \\
1.207\end{array}$ & -0.02534 \\
\hline & $\begin{array}{l}1.872 \\
0.572\end{array}$ & -0.02349 \\
\hline 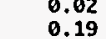 & $\begin{array}{l}0.572 \\
1.906\end{array}$ & \\
\hline 0.008 & 0.0086 & \\
\hline & 4.8 & \\
\hline
\end{tabular}

Fridoy, December 30 2:43 PM 1994

$C r, A C L$
0.03
-0.02
0.03
-0.02
0.05
-0.02
0.06
0.07
-0.02
0.07
-0.02
-0.02
0.02
-0.02
0.02
0.02
0.02
-0.02
-0.02
0.02
0.02
0.03
-0.02
0.03
-0.02
0.39
0.11
0.28
0.0005

Cr. ppm
0.1883
-0.2517
0.1958
-0.2509
0.3302
-0.2022
0.259
0.3481
-0.7405
0.3597
-0.5014
-0.3182
0.1891
-0.3348
0.1934
0.1236
0.122
-0.2654
-0.2298
0.245
0.2265
0.2596
-0.3282
0.2593
-0.344
4.295
3.14
2.80
0.0005

$\mathrm{Cu}, \mathrm{ACL}$

(Cr), g/m

0.23

$\begin{array}{rr}0.1883 & 0.01901 \\ .2517 & 0.0196\end{array}$

0.03318

0.02639

0.03589

$-0.03188$

$-0.03348$

0.01246

0.01232
-0.02673

0.02466

0.02628

0.02622

-0.02
0.24
-0.02

0.45
-0.02

-0.02
0.6
0.66

0.66
0.07
0.67

-0.02
0.08
-0.02

-0.02
0.03

-0.02
-0.02

-0.02
-0.02

-0.02
-0.02

-0.02
-0.02
-0.02

-0.02
-0.02
-0.02

-0.02
-0.02

0.3179

$-0.02$

-0.02
0.0032
0 


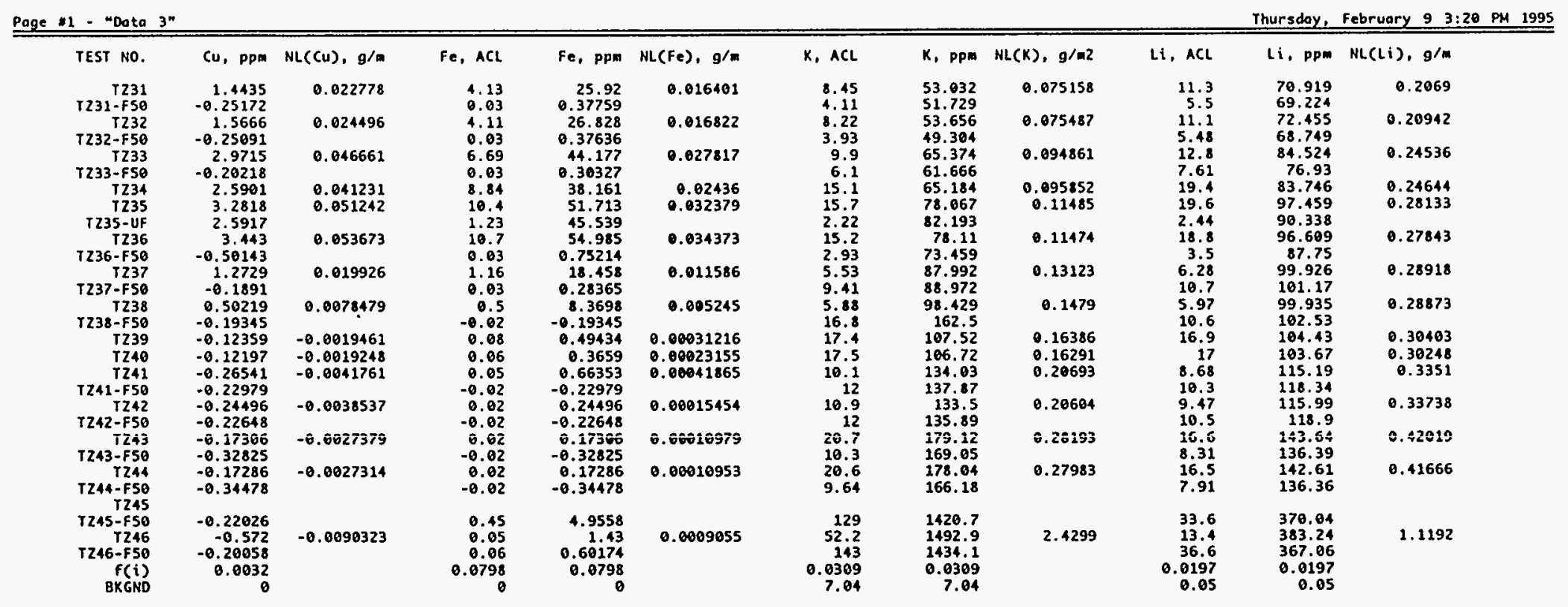




\begin{tabular}{|c|c|c|c|}
\hline TEST NO. & $\mathrm{Mg}, \mathrm{ACL}$ & Mg, ppm & $N L(M g)$, \\
\hline & 0.63 & $\begin{array}{r}3.954 \\
-0.1259\end{array}$ & 0.0 \\
\hline $\begin{array}{r}T 231-550 \\
T Z 32\end{array}$ & $\begin{array}{l}-0.01 \\
0.62\end{array}$ & $\begin{array}{l}-0.1259 \\
4.047\end{array}$ & \\
\hline$\tau 232-F 50$ & 0.01 & 0.1255 & \\
\hline$T 233$ & 0.91 & 6.009 & \\
\hline TZ33-F50 & 0.02 & 0.2022 & \\
\hline T234 & 1.19 & 5.137 & \\
\hline $\begin{array}{r}\text { T235 } \\
35-U F\end{array}$ & $\begin{array}{l}1.34 \\
0.16\end{array}$ & $\begin{array}{l}6.663 \\
5.924\end{array}$ & 0.04 \\
\hline $\begin{array}{l}336 \\
\text { T236 }\end{array}$ & $\begin{array}{l}0.16 \\
1.37\end{array}$ & 7.04 & \\
\hline$T 236-550$ & -0.01 & -0.2507 & \\
\hline $\begin{array}{r}T 237 \\
7737-58\end{array}$ & 0.14 & 2.228 & \\
\hline $\begin{array}{r}T Z 37-F 50 \\
T Z 38\end{array}$ & 0.01 & 0.09455 & \\
\hline $\begin{array}{r}T 238 \\
T 238-F 50\end{array}$ & $\begin{array}{r}0.06 \\
-0.01\end{array}$ & $\begin{array}{r}1.004 \\
-0.09672\end{array}$ & \\
\hline TZ39 & -0.01 & -0.06179 & -0.0 \\
\hline TZ40 & -0.01 & -0.06098 & -0 \\
\hline$T 241$ & -0.01 & -0.1327 & -0.0 \\
\hline TZ41-F50 & 0.01 & 0.1149 & \\
\hline${ }^{T Z 42}$ & -0.01 & -0.1225 & -0.0 \\
\hline TZ42-F50 & 0.01 & 0.1132 & \\
\hline 7243 & -0.01 & -0.08653 & -0.000 \\
\hline $\begin{array}{r}r-F 50 \\
T Z 44\end{array}$ & $\begin{array}{r}0.01 \\
-0.01\end{array}$ & $\begin{array}{r}0.1641 \\
-0.08643\end{array}$ & -0.000 \\
\hline $\begin{array}{r}T 244-F 50 \\
\text { TZ45 }\end{array}$ & 0.01 & 0.1724 & \\
\hline$T Z 45-F 50$ & & & \\
\hline $\begin{array}{r}T Z 46 \\
T Z 46-f 50\end{array}$ & $\begin{array}{r}-0.01 \\
0.02\end{array}$ & $\begin{array}{l}-0.286 \\
0.2006\end{array}$ & -0.00 \\
\hline $\begin{array}{r}f(i) \\
B K G N D\end{array}$ & 0.008 & $\begin{array}{r}0.008 \\
0\end{array}$ & \\
\hline
\end{tabular}

$\begin{array}{rrr}M n, \text { ACL } & \text { Mn, ppm } & \text { NL }(M n), g / m \\ 0.96 & 6.025 & 0.02173 \\ -0.01 & -0.1259 & 0.024 \\ 0.96 & 6.266 & 0.0224 \\ -0.01 & -0.1255 & 0.0355 \\ 1.5 & 9.905 & 0.0355 \\ -0.01 & -0.1011 & 0.0311 \\ 1.98 & 8.547 & 0.0317 \\ 2.32 & 11.54 & 0.04117 \\ 0.27 & 9.996 & 0.04358 \\ 2.38 & 12.23 & 0.0435 \\ -0.01 & -0.2507 & 0.01423 \\ 0.25 & 3.978 & -0.01423 \\ -0.01 & -0.09455 & 0.006577 \\ 0.11 & -0.841 & 0.006577 \\ -0.01 & -0.09672 & 0.000224 \\ 0.01 & 0.06179 & 0.00224 \\ 0.01 & 0.06098 & 0.0022 \\ -0.01 & -0.1327 & -0.0004773 \\ -0.01 & -0.1149 & -0.000404 \\ -0.01 & -0.1225 & -0.0004404 \\ -0.01 & -0.1132 & -0.0003129 \\ -0.01 & -0.08653 & -0.0003129 \\ -0.01 & -0.1641 & -0.0003122 \\ -0.01 & -0.08643 & -0.0003122 \\ -0.01 & -0.1724 & \\ 0.02 & 0.2203 & \\ -0.01 & -0.286 & -0.001032 \\ -0.01 & -0.1003 & \\ 0.014 & 0.014 & \\ 0 & 0 & \end{array}$

Na, ACL
45.1
20.9
44.3
20.6
51.1
29.6
76.4
76.8
9.53
74.4
14.2
25.3
47.3
25
46.4
772
71.5
41.7
48.8
44.4
49.3
84.9
42.8
84.1
40.6
590
228
644
0.0661
51.3

\begin{tabular}{|c|c|c|c|}
\hline $\mathrm{Na}, \mathrm{ppm}$ & $\mathrm{NL}(\mathrm{Na}), g^{\prime / m}$ & $\mathrm{Ni}, \mathrm{ACl}$ & $\mathrm{Ni}, \mathrm{ppm}$ \\
\hline $\begin{array}{r}283 \\
263\end{array}$ & 0.177 & 0.29 & $\frac{1.82}{3376}$ \\
\hline 289.2 & 0.1801 & 0.28 & $\begin{array}{r}-0.3776 \\
3.828\end{array}$ \\
\hline 258.4 & & -0.03 & -0.3764 \\
\hline 337.4 & 0.2175 & 0.43 & 2.839 \\
\hline 299.2 & & -0.03 & -0.3033 \\
\hline 329.8 & 0.2146 & 0.56 & 2.417 \\
\hline 381.9 & 0.2499 & 0.64 & 3.182 \\
\hline 352.8 & & 0.07 & 2.592 \\
\hline 382.3 & 0.2498 & 0.66 & 3.392 \\
\hline 356 & & -0.03 & -0.7521 \\
\hline 402.6 & 0.2662 & 0.07 & 1.114 \\
\hline 447.2 & & -0.03 & -0.2837 \\
\hline 418.5 & 0.2778 & 0.04 & 0.6696 \\
\hline 448.8 & & -0.03 & -0.2902 \\
\hline 444.9 & 0.3001 & -0.03 & -0.1854 \\
\hline 436 & 0.2939 & -0.03 & -0.1829 \\
\hline 553.4 & 0.3824 & -0.03 & -0.3981 \\
\hline $\begin{array}{l}560.7 \\
543.8\end{array}$ & 0.3751 & $\begin{array}{l}-0.03 \\
-0.03\end{array}$ & $\begin{array}{l}-0.3447 \\
-0.3674\end{array}$ \\
\hline $\begin{array}{l}558.3 \\
558.3\end{array}$ & & -0.03 & -0.3397 \\
\hline 734.6 & 0.5234 & -0.03 & -0.2596 \\
\hline $\begin{array}{l}702.5 \\
726.9\end{array}$ & 0.5168 & $\begin{array}{l}-0.03 \\
-0.03\end{array}$ & $\begin{array}{l}-0.4924 \\
-0.2593\end{array}$ \\
\hline $\begin{array}{l}26.9 \\
699.9\end{array}$ & & -0.03 & -0.5172 \\
\hline $\begin{array}{r}6498 \\
6521 \\
6459 \\
0.0661 \\
51.3\end{array}$ & 4.946 & $\begin{array}{r}0.19 \\
-0.03 \\
-0.03 \\
0.0065\end{array}$ & $\begin{array}{r}2.092 \\
-0.858 \\
-0.3009 \\
0.0065\end{array}$ \\
\hline
\end{tabular}




\begin{tabular}{|c|c|c|c|c|c|c|c|c|c|c|c|}
\hline TEST NO. & $\mathrm{NL}(\mathrm{Ni}), \mathrm{g} / \mathrm{m}$ & $\mathrm{Si}, \mathrm{ACL}$ & $\mathrm{Si}, \mathrm{ppm}$ & NL(Si), $g / m$ & Th, ACL & Th, ppm & $N L(T h), g / m$ & $T i, A C L$ & $T i$, ppm & NLC(Ti), $g / m$ & U, $A C L$ \\
\hline $\begin{array}{r}\text { T231 } \\
\text { T231-F50 }\end{array}$ & 0.01414 & $\begin{array}{l}44.4 \\
19.3\end{array}$ & $\begin{array}{l}278.7 \\
242.9\end{array}$ & 0.05154 & $\begin{array}{r}0.09 \\
-0.05\end{array}$ & $\begin{array}{r}0.5648 \\
-0.6293\end{array}$ & 0.0124 & $\begin{array}{r}0.23 \\
-0.02\end{array}$ & $\begin{array}{r}1.443 \\
-0.2517\end{array}$ & 0.0135 & $\begin{array}{l}1.309 \\
0.407\end{array}$ \\
\hline T232 & 0.01407 & 43.4 & 283.3 & 0.05209 & 0.09 & 0.5875 & 0.01278 & 0.23 & 1.501 & 0.01391 & $\begin{array}{l}1.408 \\
1.289\end{array}$ \\
\hline $\mathrm{r} 232-\mathrm{F} 50$ & & 19.4 & 243.4 & & -0.05 & -0.6273 & D.01aro & -0.02 & -0.2509 & & 0.38 \\
\hline$T 233$ & 0.02195 & 53.2 & 351.3 & 0.06725 & 0.12 & 0.7924 & 0.01731 & 0.4 & 2.641 & 0.02458 & 1.806 \\
\hline$T 233-F 50$ & $0.0<193$ & 26.4 & 266.9 & $0.00 / 6 \mathrm{~s}$ & -0.05 & -0.5055 & 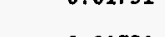 & -0.02 & -0.2022 & & 0.511 \\
\hline TZ34 & 0.01894 & 78.2 & 337.6 & 0.06512 & 0.18 & 0.777 & 0.01721 & 0.55 & 2.374 & 0.0224 & 2.628 \\
\hline$T 235$ & 0.02446 & 82.7 & 411.2 & 0.07997 & 0.21 & 1.044 & 0.02268 & 0.66 & 3.282 & 0.03037 & 2.834 \\
\hline TZ35-UF & & 10.5 & 388.7 & & -0.05 & -1.851 & & 0.07 & 2.592 & & 0.382 \\
\hline T236 & 0.02603 & 81.2 & 417.3 & 0.08116 & 0.2 & 1.028 & 0.02229 & 0.67 & 3.443 & 0.03181 & 2.841 \\
\hline T236-F50 & $0.0<063$ & 12.9 & 323.4 & 0.00110 & -0.05 & -1.254 & & -0.02 & -0.5014 & & 0.248 \\
\hline $\begin{array}{l}\text { T237 } \\
\text { T237 }\end{array}$ & 0.008583 & 22.7 & 361.2 & 0.06921 & -0.05 & -0.7956 & -0.01733 & 0.08 & 1.273 & 0.01181 & 0.588 \\
\hline TZ37-F50 & 0.000000 & 38.1 & 360.2 & 0.0056 .2 & -0.05 & -0.4728 & & -0.02 & -0.1891 & & 0.663 \\
\hline$T 238$ & 0.005151 & 20.9 & 349.9 & 0.06662 & -0.05 & -0.837 & -0.0182 & 0.03 & 0.5022 & 0.004651 & 0.463 \\
\hline T238-F50 & 8.000101 & 37.4 & 361.7 & 0.0000 & -0.05 & -0.4836 & & -0.02 & -0.1934 & & 0.657 \\
\hline TZ39 & -0.001437 & 61.4 & 379.4 & 0.07364 & -0.05 & -0.309 & -0.006769 & -0.02 & -0.1236 & -0.001153 & 0.96 \\
\hline$T 240$ & -0.001421 & 63.2 & 385.4 & 0.07512 & -0.05 & -0.3049 & -0.006695 & -0.02 & -0.122 & -0.001141 & 1.012 \\
\hline TZ41 & -0.003084 & 32.3 & 428.6 & 0.08442 & -0.05 & -0.6635 & -0.01453 & -0.02 & -0.2654 & -0.002475 & 0.395 \\
\hline TZ41-F50 & & 38.3 & 440 & & -0.05 & -0.5745 & & -0.02 & -0.2298 & & 0.391 \\
\hline TZ42 & -0.002846 & 35.6 & 436 & 0.08603 & -0.05 & -0.6124 & -0.0134 & -0.02 & -0.245 & -0.002284 & 0.431 \\
\hline$T Z 42-F 50$ & & 39.4 & 446.2 & & -0.05 & -0.5662 & & -0.02 & -0.2265 & & 0.444 \\
\hline T243 & -0.002022 & 65 & 562.5 & 0.1145 & -0.05 & -0.4327 & -6.009523 & -0.02 & -0.1731 & -0.001622 & 0.412 \\
\hline$T Z 43-F 50$ & & 32.4 & 531.8 & & -0.05 & -0.8206 & & $\begin{array}{l}-0.02 \\
-0.02\end{array}$ & $\begin{array}{l}-0.3282 \\
-0.1729\end{array}$ & -0.001619 & $\begin{array}{l}0.196 \\
0.399\end{array}$ \\
\hline $\begin{array}{r}T Z 44 \\
T Z 44-F 50\end{array}$ & -0.002017 & $\begin{array}{l}65.6 \\
31.3\end{array}$ & $\begin{array}{r}567 \\
539.6\end{array}$ & 0.1154 & $\begin{array}{l}-0.05 \\
-0.05\end{array}$ & $\begin{array}{r}-0.4321 \\
-0.862\end{array}$ & -0.0095 & $\begin{array}{l}-0.02 \\
-0.02\end{array}$ & $\begin{array}{l}-0.1729 \\
-0.3448\end{array}$ & -0.001619 & $\begin{array}{l}0.399 \\
0.181\end{array}$ \\
\hline $\begin{array}{r}T Z 45 \\
T Z 45-F 50\end{array}$ & & 83.6 & 920.7 & & & & & & & & \\
\hline TZ46 & -0.00667 & 37.2 & 1064 & 0.2251 & -0.05 & -1.43 & -0.03142 & -0.02 & -0.572 & -0.005352 & 0.0183 \\
\hline TZ46-F50 & & 96.8 & 970.8 & & 0.05 & -0.5015 & & $\begin{array}{l}-0.02 \\
0.054\end{array}$ & $\begin{array}{r}-0.2006 \\
0.0054\end{array}$ & & 0.0383 \\
\hline $\begin{array}{r}f(i) \\
B K G N D\end{array}$ & & 0.2287 & $\begin{array}{l}0.2287 \\
45.2\end{array}$ & & & 0.0 & & & o & & 0.0164 \\
\hline
\end{tabular}




\begin{tabular}{|c|c|c|c|c|c|c|c|c|}
\hline TEST NO. & U, ppm & $N L(U), g / m 2$ & $\mathrm{Zn}, \mathrm{ACl}$ & $2 n, p p m$ & $N L(Z n), g / m$ & $\mathrm{Zr}, \mathrm{ACL}$ & $2 \mathrm{r}, \mathrm{ppm}$ & $N L(Z r), g / m$ \\
\hline $\begin{array}{r}r 231 \\
r 731-850\end{array}$ & $\begin{array}{l}8.215 \\
5.123\end{array}$ & $\begin{array}{l}0.02529 \\
0.01577\end{array}$ & -0.02 & $\begin{array}{l}-0.1255 \\
-0.2517\end{array}$ & -0.03169 & -0.05 & -0.3138 & -0.02264 \\
\hline $\begin{array}{r}T Z 31-F 50 \\
T Z 32\end{array}$ & $\begin{array}{l}5.123 \\
8.414\end{array}$ & 0.02567 & $\begin{array}{l}-0.02 \\
-0.02\end{array}$ & $\begin{array}{l}-0.2517 \\
-0.1305\end{array}$ & & -0.05 & -0.6293 & \\
\hline $5232-F 50$ & 4.767 & 0.01455 & -0.02 & -0.2509 & -0.03266 & $\begin{array}{l}-0.05 \\
-0.05\end{array}$ & $\begin{array}{l}-0.3264 \\
-0.6273\end{array}$ & -0.02333 \\
\hline$T 233$ & 11.93 & 0.03654 & 0.07 & 0.4622 & 0.1161 & -0.05 & -0.3302 & -0.0237 \\
\hline TZ33-F50 & 5.166 & 0.01583 & -0.02 & -0.2022 & & -0.05 & -0.5655 & 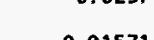 \\
\hline $\begin{array}{l}\text { T234 } \\
\text { TZ35 }\end{array}$ & $\begin{array}{l}11.34 \\
14.09\end{array}$ & $\begin{array}{l}0.03524 \\
0.04293\end{array}$ & $\begin{array}{l}0.08 \\
0.05\end{array}$ & $\begin{array}{l}0.3453 \\
0.2486\end{array}$ & $\begin{array}{l}0.08796 \\
0.06211\end{array}$ & $\begin{array}{r}-0.05 \\
0.05\end{array}$ & $\begin{array}{r}-0.2158 \\
0.2486\end{array}$ & -0.01571 \\
\hline TZ35-UF & 14.14 & 0.04309 & -0.02 & -0.7405 & & -0.05 & $\begin{array}{l}0.2480 \\
-1.851\end{array}$ & \\
\hline$T 236$ & 14.6 & 0.04441 & 0.05 & 0.2569 & 0.06409 & 0.05 & 0.2569 & 0.01831 \\
\hline $36-F 50$ & 6.218 & 0.01891 & -0.02 & -0.5014 & & -0.05 & -1.254 & \\
\hline 7237 & 9.35 & 0.02858 & -0.02 & -0.3182 & -0.0797 & -0.05 & -0.7956 & -0.05693 \\
\hline $7-F 50$ & 6.269 & 0.01915 & -0.02 & -0.1891 & & -0.05 & -0.4728 & \\
\hline$T 238$ & 7.75 & 0.02363 & -0.0 & -0.3348 & -0.08371 & -0.05 & -0.837 & -0.05979 \\
\hline $8-F 50$ & 6.355 & 0.01938 & -0.0 & -0.1934 & & -0.05 & -0.4836 & \\
\hline TZ39 & 5.932 & 0.01823 & -0.02 & -0.1236 & -0.03114 & -0.05 & -0.309 & -0.02224 \\
\hline & 6.17 & 0.019 & -0. & -0.122 & -0. & -8 & -0.3049 & -0.022 \\
\hline$T 749-F 50$ & 5.24 & 0.01609 & -0. & -0.2 & -0.06682 & & -0.6635 & -0.04773 \\
\hline $\begin{array}{r}\text { TZ41-F50 } \\
\text { TZ42 }\end{array}$ & $\begin{array}{l}4.492 \\
5.279\end{array}$ & 0.01379 & $\begin{array}{l}-0.02 \\
-0.02\end{array}$ & -0.2298 & -0.06166 & -0.05 & -0.5745 & \\
\hline $\begin{array}{r}T Z 42 \\
T Z 42-F 50\end{array}$ & $\begin{array}{l}5.279 \\
5.028\end{array}$ & $\begin{array}{l}0.0162 \\
0.01543\end{array}$ & $\begin{array}{l}-0.02 \\
-0.02\end{array}$ & $\begin{array}{r}-0.245 \\
-0.2265\end{array}$ & -0.06166 & $\begin{array}{l}-0.05 \\
-0.05\end{array}$ & $\begin{array}{l}-0.6124 \\
-0.5662\end{array}$ & -0.04404 \\
\hline TZ43 & 3.565 & 0.01 & -0. & -0.1731 & -0.04381 & -0.05 & -0.4327 & -0.03129 \\
\hline$T Z 43-F 50$ & 3.217 & 0.00993 & 0. & 0.3282 & & -0.05 & -0.8206 & \\
\hline & 3,449 & 0.01063 & -0.5 & -0.1729 & -0.0437 & -0.05 & -0.4321 & -0.03122 \\
\hline $\begin{array}{r}\text { TZ44-F50 } \\
\text { TZ45 }\end{array}$ & 3.12 & 0.00962 & $-0.0<>>$ & -0.3448 & & -0 & -0.862 & \\
\hline $\begin{array}{r}T Z 45-F 50 \\
T Z 46 \\
T Z 46-F 50\end{array}$ & $\begin{array}{r}0.141 \\
0.5234 \\
0.3841\end{array}$ & 0.001613 & $\begin{array}{r}0.08 \\
-0.02 \\
0.05\end{array}$ & $\begin{array}{r}0.881 \\
-0.572 \\
0.5015\end{array}$ & -0.1445 & $\begin{array}{l}-0.05 \\
-0.05 \\
-0.05\end{array}$ & $\begin{array}{r}-0.5507 \\
-1.43 \\
-0.5015\end{array}$ & -0.1032 \\
\hline $\begin{array}{r}f(i) \\
\text { BXGND }\end{array}$ & $\begin{array}{r}0.0164 \\
0\end{array}$ & & 0.0002 & 0.0002 & & 0.0007 & $\begin{array}{r}-0.0007 \\
0.007\end{array}$ & \\
\hline
\end{tabular}


Table D.4. Anion Results

Column Heading

TEST No.

REACTION TIME

$\mathrm{pH}$

$\mathrm{C}$ dil. factor

TC analyzed

TOC analyzed

$\mathrm{TC}, \mathrm{ppm}$

TOC, ppm

TIC, ppm

ACL Number

F-, ppm

CHO2-, ppm

$\mathrm{Cl}-, \mathrm{ppm}$

NO2-, ppm

NO3-, ppm

HPO4=, ppm

$\mathrm{SO} 4=, \mathrm{ppm}$

$\mathrm{C} 2 \mathrm{O} 4=, \mathrm{ppm}$

\section{Comment}

Test number in test matrix (Table D.1).

Actual reaction time, in days.

Leachate $\mathrm{pH}$ measured at room temperature.

Dilution factor of sample used in carbon analyses.

Total carbon in sample analyzed, in parts per million of carbon.

Total organic carbon in sample analyzed, in parts per million of carbon.

Total carbon corrected for dilution, in parts per million of carbon.

Total organic carbon corrected for dilution, in parts per million of carbon.

Total inorganic carbon calculated as TC-TOC, in parts per million of carbon.

Solution identification number.

Fluoride content, in parts per million.

Formate content, in parts per million.

Chloride content, in parts per million.

Nitrite content, in parts per million.

Nitrate content, in parts per million.

Hydrogen phosphate content, in parts per million.

Sulfate content, in parts per million.

Oxalate content, in parts per million. 
Page "1 - "BX. ANIONS"

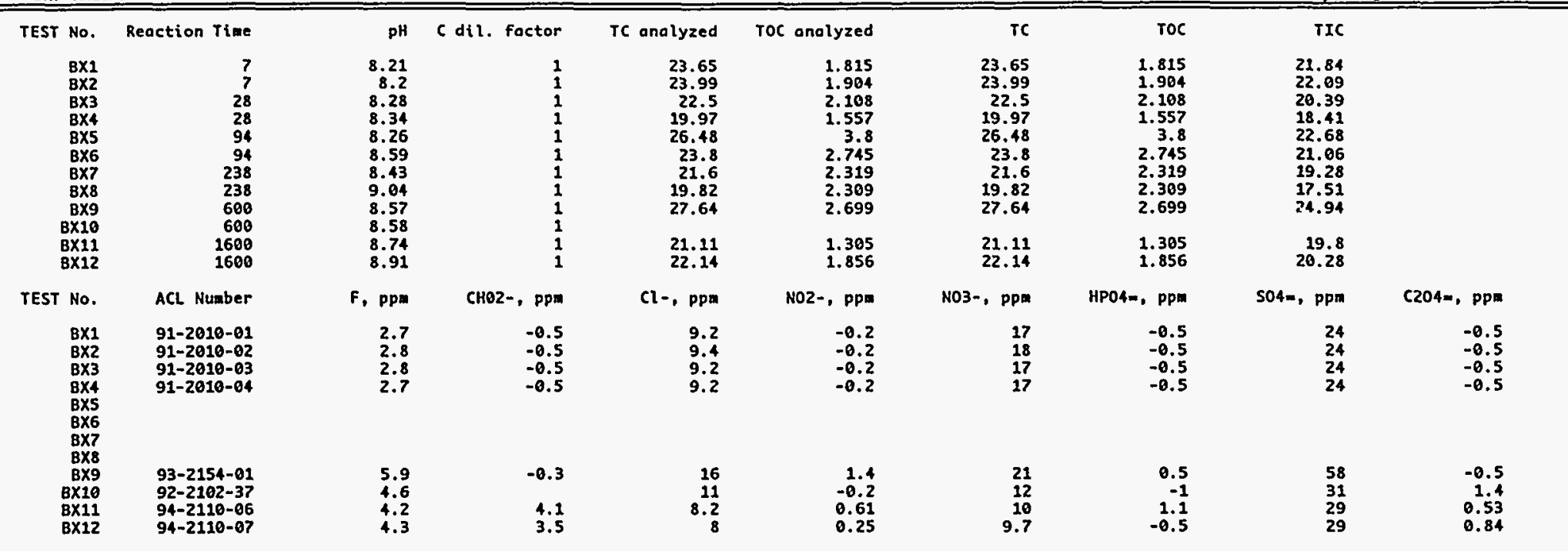




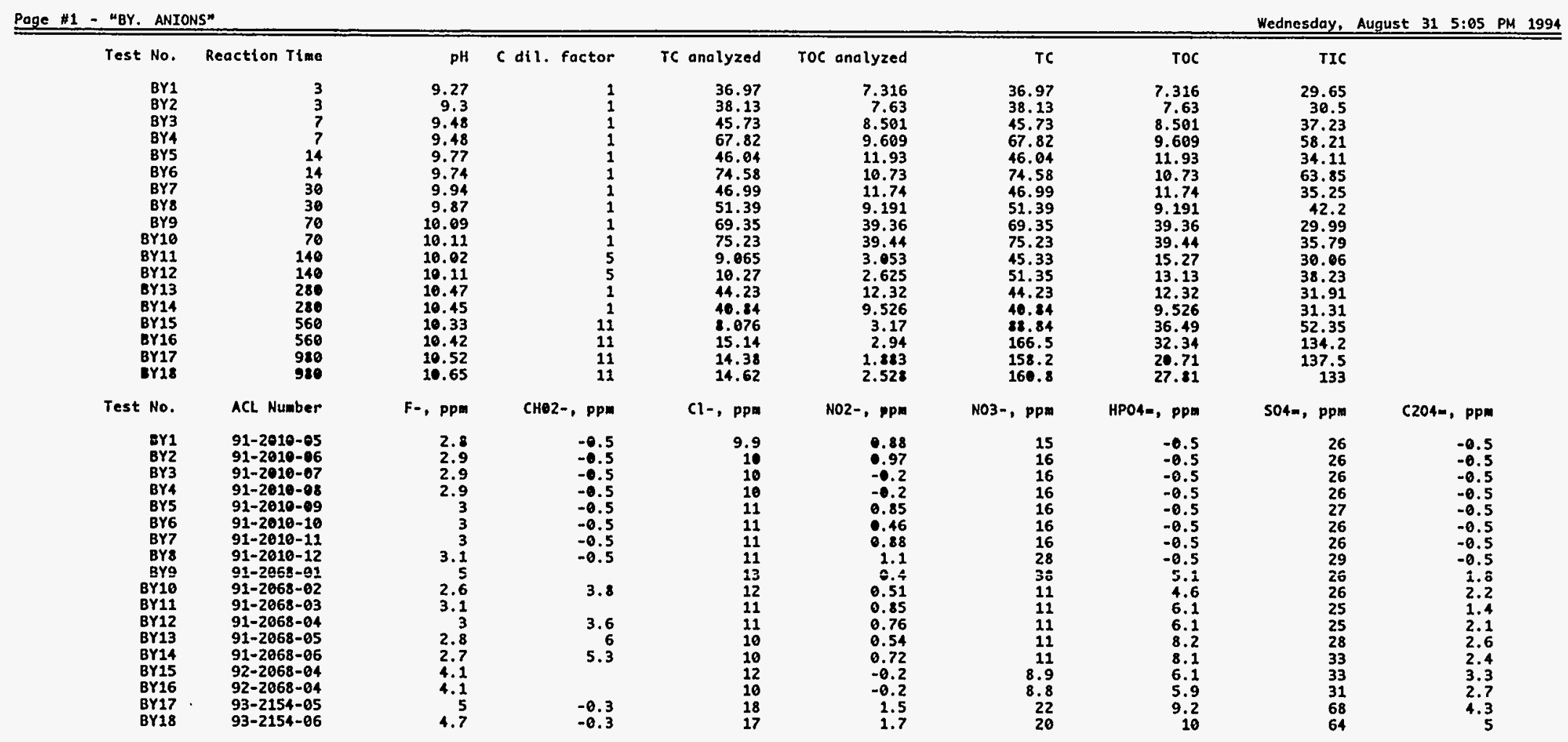


Page "1 - "BZ. ANIONS"

\begin{tabular}{|c|c|c|c|c|c|c|c|c|c|}
\hline Test No. & Reaction Time & pH & C dil. foctor & TC analyzed & TOC analyzed & TC & TOC & TIS & \\
\hline 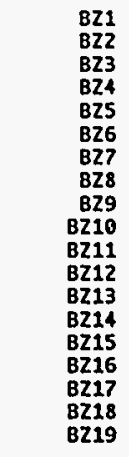 & $\begin{array}{r}3 \\
3 \\
7 \\
7 \\
14 \\
14 \\
28 \\
28 \\
56 \\
56 \\
98 \\
98 \\
182 \\
182 \\
364 \\
364 \\
736 \\
736 \\
484\end{array}$ & $\begin{array}{l}10.5 \\
10.62 \\
10.73 \\
10.48 \\
10.98 \\
11.01 \\
11.02 \\
11.1 \\
11.24 \\
11.33 \\
11.26 \\
11.43 \\
10.92 \\
11.29 \\
12.03 \\
11.02 \\
11.96 \\
11.97 \\
11.86\end{array}$ & $\begin{array}{r}2.5 \\
2.5 \\
2.5 \\
2.5 \\
2.5 \\
2.5 \\
2 \\
2 \\
1.67 \\
1.67 \\
2.5 \\
2.5 \\
11 \\
11 \\
11 \\
11 \\
11 \\
11 \\
11\end{array}$ & $\begin{array}{l}56.07 \\
47.12 \\
54.15 \\
58.28 \\
84.24 \\
57.22 \\
77.63 \\
81.08 \\
138.4 \\
144.4 \\
102.4 \\
107.8 \\
30.63 \\
25.08 \\
13.43 \\
14.86 \\
12.58 \\
15.63 \\
9.269\end{array}$ & $\begin{array}{l}23.1 \\
20.63 \\
22.12 \\
27.69 \\
27.9 \\
28.35 \\
39.88 \\
40.39 \\
993.36 \\
96.48 \\
65.59 \\
63.96 \\
20.11 \\
17.29 \\
7.117 \\
7.331 \\
8.903 \\
15.21 \\
8.465\end{array}$ & $\begin{array}{l}140.2 \\
117.8 \\
135.4 \\
145.7 \\
210.6 \\
113.1 \\
155.3 \\
162.2 \\
231.1 \\
241.2 \\
256 \\
269.5 \\
336.9 \\
275.9 \\
147.7 \\
163.5 \\
138.4 \\
171.9 \\
102\end{array}$ & $\begin{array}{l}57.75 \\
51.58 \\
55.3 \\
69.23 \\
69.75 \\
70.88 \\
79.76 \\
80.78 \\
155.9 \\
161.1 \\
164 \\
159.9 \\
221.2 \\
190.2 \\
78.29 \\
80.64 \\
97.93 \\
1967.3 \\
93.12\end{array}$ & $\begin{array}{l}82.43 \\
66.23 \\
80.08 \\
76.48 \\
140.9 \\
72.18 \\
75.5 \\
81.38 \\
75.22 \\
80.03 \\
92.03 \\
109.6 \\
115.7 \\
85.69 \\
69.44 \\
82.82 \\
40.45 \\
4.62 \\
8.844\end{array}$ & \\
\hline Test No. & ACL Number & ACL F-, ppm & $\mathrm{ACL}, \mathrm{CHOZ-,} \mathrm{PP}$ & $\mathrm{Cl}-, \mathrm{ppm}$ & N02-, ppm & NO3-, ppm & $\mathrm{HPOA}=\mathrm{ppm}$ & SO4-, ppm & $C 204=, P p m$ \\
\hline 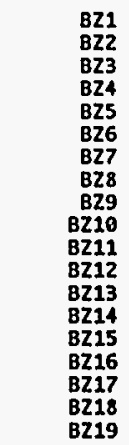 & $\begin{array}{l}91-2010-13 \\
90-4085-11 \\
91-2010-14 \\
91-2010-15 \\
91-2010-16 \\
90-4085-12 \\
91-2010-17 \\
90-1085-13 \\
91-2045-11 \\
91-2045-12 \\
91-2045-13 \\
91-2045-14 \\
91-2068-07 \\
91-2068-88 \\
92-2005-01 \\
92-2005-02 \\
93-2154-07 \\
92-2103-39 \\
92-2103-40\end{array}$ & $\begin{array}{r}3 \\
3.6 \\
3 \\
3 \\
3 \\
3 \\
3 \\
3 \\
3\end{array}$ & $\begin{array}{l}15 \\
12 \\
14 \\
18 \\
19 \\
17 \\
22 \\
23 \\
27 \\
28 \\
10 \\
31 \\
35 \\
26 \\
\\
-20\end{array}$ & $\begin{array}{r}12 \\
12 \\
15 \\
13 \\
13 \\
12 \\
14 \\
13 \\
14 \\
14 \\
15 \\
14 \\
19 \\
18 \\
-10 \\
-10 \\
147 \\
215 \\
119\end{array}$ & $\begin{array}{r}1.7 \\
0.84 \\
1.5 \\
0.95 \\
1.3 \\
1 \\
1.5 \\
1.2 \\
-0.02 \\
-0.02 \\
-0.02 \\
-0.02 \\
1.9 \\
1.9 \\
216 \\
171 \\
22 \\
-15 \\
-15\end{array}$ & $\begin{array}{r}17 \\
16 \\
19 \\
18 \\
17 \\
16 \\
18 \\
15 \\
18 \\
17 \\
22 \\
16 \\
251 \\
151 \\
12 \\
17 \\
48 \\
26 \\
28\end{array}$ & $\begin{array}{r}7.7 \\
7.4 \\
10 \\
11 \\
13 \\
12 \\
18 \\
15 \\
22 \\
23 \\
28 \\
28 \\
51 \\
71 \\
121 \\
257 \\
115 \\
103 \\
119\end{array}$ & $\begin{array}{r}34 \\
35 \\
39 \\
38 \\
37 \\
35 \\
40 \\
39 \\
12 \\
40 \\
12 \\
39 \\
44 \\
39 \\
95 \\
85 \\
612 \\
293 \\
183\end{array}$ & $\begin{array}{r}5.1 \\
3.6 \\
6.4 \\
6.4 \\
7.7 \\
7.2 \\
11 \\
11 \\
14 \\
14 \\
14 \\
14 \\
18 \\
10 \\
15 \\
17 \\
104 \\
56 \\
-50\end{array}$ \\
\hline
\end{tabular}


Page \#1 - "PX. ANIONS"

Test No. Reaction $T$

pH C dil. fact TC analyzed TOC analyze

TC

TOC

TIC

Nednesday, August 31 5:05 PM 1994

\begin{tabular}{|c|c|}
\hline PX1 & 7 \\
\hline PX2 & 7 \\
\hline PX3 & 14 \\
\hline $\begin{array}{l}\text { PX4 } \\
\text { PX5 }\end{array}$ & $\begin{array}{l}14 \\
94\end{array}$ \\
\hline PX6 & 94 \\
\hline PX7 & 238 \\
\hline $\begin{array}{l}\text { PX8 } \\
\text { PX8 }\end{array}$ & $\begin{array}{l}238 \\
669\end{array}$ \\
\hline $\begin{array}{r}\text { PX9 } \\
\text { PX10 }\end{array}$ & $\begin{array}{l}601 \\
601\end{array}$ \\
\hline PX11 & 1400 \\
\hline PX12 & 1400 \\
\hline
\end{tabular}
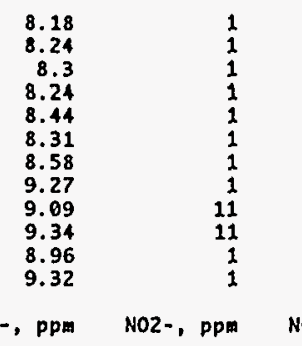

23.84
23.49
24.36
24.15
25.68
24.56
28.85
25.76
1.399
4.438
29.47
30.89

1.873
1.662
2.108
2.25
2.592
2.57
3.049
2.926
0.57
0.582
2.402
2.613

$\begin{array}{ll}23.84 & 1.873 \\ 23.49 & 1.652\end{array}$

23.49
24.36

24.36
24.15
25.68

24.56
28.85

28.85
25.76
48.39
48.82
29.47

30.89

1.873
1.662
2.108

2.108
2.25
2.592

2.57
3.049
2.926

3.049
2.926
6.27

6.27
6.402
2.402

N02-, ppm N03-, ppm HPO4=, ppm 504=, ppm C204=, ppm

$\begin{array}{ll}3.2 & -0.5 \\ 2.9 & -0.5 \\ 2.8 & -0.5 \\ 2.8 & -0.5\end{array}$

9.

-0.2
-0.2
0.41
0.93

$\begin{array}{llll}17 & -0.5 & 25 & -0.5 \\ 19 & -0.5 & 25 & -0.5 \\ 16 & -0.5 & 26 & -0.5 \\ 16 & -0.5 & 26 & -0.5\end{array}$

$\begin{array}{rrrrrrrr}6.5 & -0.3 & 20 & 0.98 & 24 & 1.4 & 67 & 0.57 \\ 4.3 & .3 & 12 & -0.2 & 12 & -1 & 29 & 1.3 \\ 4.8 & 5.1 & 12 & 0.73 & 13 & 0.63 & 37 & 0.68 \\ 4.7 & 4.1 & 9.6 & 0.61 & 11 & 0.66 & 32 & 0.82\end{array}$ 
Page \#1 - "PY. ANIONS"

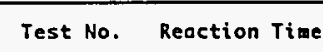

pH C dil. factor

rC anolyzed

TOS analyzed

Wednesday. August 31 5:06 PM 1994

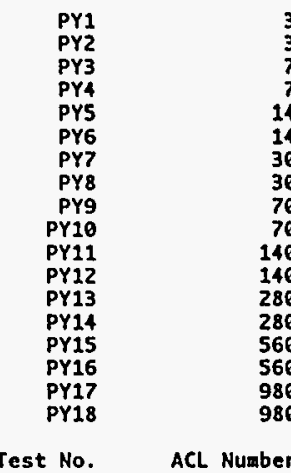

9.93
10.07

10.07
10.26

10.26
10.25
10.45

10.25
10.45
10.45

10.47

10.52
10.61

10.66
10.72

10.72
10.72

11.44
11.48

11.48
11.44

11.44
11.49
11.74

11.72 11

Test No. ACL Number

F-, ppm

CHO2-, ppm

$\begin{array}{ll}\text { PY1 } & 91-2010-22 \\ \text { PY2 } & 91-2010-23\end{array}$

PY2 $91-2010-23$

$91-2010-25$
$91-2010-26$

91-2010-27

PY7
PY8
P1-2010-28

$91-2010-29$
PY9
PY9

$91-2068-19$
PY11

PY12
PY13 91-2068-21
91-2081-09

PY14
PY15
PY16 $92-2063-01$

$\begin{array}{ll}\text { PY15 } & 92-2063-01 \\ \text { PY16 } & 92-2063-02 \\ \text { P17 } & 93-2154-12 \\ \text { PY18 } & 93-2154-13\end{array}$

$\begin{array}{lll}2.6 & -0.5 & 10 \\ 2.7 & -0.5 & 9.7 \\ 2.7 & -0.5 & 11 \\ 2.7 & -0.5 & 11 \\ 2.7 & -0.5 & 11 \\ 2.7 & -0.5 & 10 \\ 2.5 & -0.5 & 10 \\ 2.4 & -0.5 & 10 \\ 1.9 & 5.6 & 11 \\ 1.8 & 2.8 & 13 \\ 1.6 & 2.6 & 11 \\ 1.7 & 2.5 & 12 \\ 1.7 & 3.1 & 12 \\ 3.3 & & 11 \\ 2.8 & & 12 \\ 14 & -5 & 50 \\ 14 & -5 & 59\end{array}$

TC

TOC

32.56
47.99
39.6

47.99
39.6
37.45
36.53

36.53
39.41

39.18
41.46

41.46
71.21
69.5

69.5
48.91

42.4

84.19
71.19
72.28

71.19
72.28
69.2
66.55

66.55
95.91

N03-, ppm

$2-, p p m$

-0.2
0.93
-0.93
-0.2
-0.2
-0.2
-0.2
1.3
-0.1
-0.2
0.96
0.39
0.63
0.73
0.73
0.81
0.81
-8.
18
TOC

TIC

28.03
43.27

$\begin{array}{rl}5.459 & 34.14 \\ 5.834 & 31.62 \\ 10.2 & 26.33\end{array}$

12.18

9.839

10.79
30.47

30.47
33.91
14.74

33.74
7.85

7.85
27.93

20.43
12.05

12.05
13.77
17.66

HP04-, ppa

-0.5
-0.5
-0.5
-0.5
-0.5
-0.5
-0.5
-0.5
-2
-2
-2
-2
5.6
9.1
8.5
-40
-40


Page \#1 - "PZ. ANIONS"

$$
\text { Test No. Reaction Timo }
$$

PZ1
PZ2
PZ3
PZ4
PZ5
PZ6
PZ7
P28
PZ9
PZ10
PZ11
PZ12
PZ13
PZ14
PZ15
PZ16
PZ17
PZ18
PZ19
PZ20

PZ1 91-2010-30

P22
P23 $91-2010-31$
P24

P24

$91-2010-35$

$\begin{array}{ll}\text { P27 } & 91-2010-36 \\ \text { PZ8 } & 91-2010-37\end{array}$

P28
P29 91-2015-15

PZ10 91-2045-16

P11
PZ12 $91-2045-17$
P12

P112 91-2045-18
PZ13 93-2154-14

P214 93-2154-15

PZ16 92-2005-04

PZ17 93-2154-16

$\begin{array}{ll}\text { PZ18 } & 93-2154-17 \\ \text { PZ19 } & 93-2154-18 \\ \text { PZ20 } & 94-2110-03\end{array}$

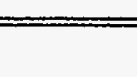

$\mathrm{pH}$ C dil. factor

TC analyzed

11.86
11.99

11.99
11.98
12.05

12.05
12.04
12.12

12.12

12.11
12.12
12.14

12.14

12.12
12.18

12.19
12.2
12.17

12.17
12.02

12.05
12.04
12.18

F-, ppp

7.3
7.3
12
12
13
13
14
14
16
16
16
16
-3
-3

-3
-3
-3
-20

CHO2-, PPD

-0.5
-0.5
-0.5
-0.5
-0.5
-0.5
-0.5
-0.5
-0.5
-0.5
-0.5
-0.5
-5
-5

-5
-5
-5
-20

$$
\begin{aligned}
& 56.95 \\
& 33.59 \\
& 34.49 \\
& 42.91 \\
& 41.68 \\
& 42.25 \\
& 44.45 \\
& 37.38 \\
& 55.21 \\
& 104.2 \\
& 72.74 \\
& 72.64 \\
& 8.807 \\
& 9.743 \\
& 9.708 \\
& 9.977 \\
& 11.81 \\
& 11.02 \\
& 12.43 \\
& 12.15
\end{aligned}
$$

$\mathrm{Cl}-, \mathrm{Ppr}$

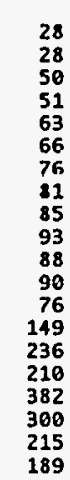

TS

242.4

142.4
83.98
86.23

86.23
107.3
104.2

104.2
105.6
111.1

111.1
93.45

26.17

49.46
86.23

64.94

64.84
2.766

2.929
9.633

11.5
10.66
11.17

11.59

HO2-, ppm

-0.2
-0.2
-0.2
-0.2
-0.2
-0.2
-0.2
-0.2
-0.2
-0.2
-0.2
-0.2
-8
-8
-15
-15
-8
-8
-8
-20

174
181.9
181.6

88.07
97.43

97.43
106.8
109.8

129.9
121.2

136.7
133.7

N03-, ppo

19
17
20
19
21
21
21
21
19
21
20
20
23
27
14
12
-15
18
20
18

Wednesday, August 31 5:06 PM 1994

TOC

TIC

41.68
28.65
70.53

70.53
88.1

96.03
91.25
102.3
65.43

65.43
82.6
144

$162.4-19.5$

24
26
56
54
66
66
79
84
66
105
193
99
166
199
280
244
574
460
381
414 
Test No. Reaction Time

$\underset{\substack{T W 1 \\ T W 2 \\ T W 3}}{T W}$

$T W 2$
$T_{W W 3}$
$T W 4$
$T W 5$
$T W 5$

$\operatorname{Tim}_{\text {Tw6 }}$
TW7
Tw8

TW8
TWY
TW10

TW11

TW12

TW14

TW15

Test No. ACL NUMBER

TW1
TW2
TW3
$T W 4$
$T W 5$

$\begin{array}{ll}T W 3 & 93-2154-19 \\ T W 4 & 93-2154-20 \\ T W 5 & 93-2154-21 \\ T W 6 & 93-2154-22 \\ T W 6 & 92-2103\end{array}$

TW7 $92-2103-49$

TW9 93-2154-23

TW10 92-2103-50

TW12 92-2103-51

Tw13

TW14

93-2154-25

$93-2154-26$
$93-2154-27$
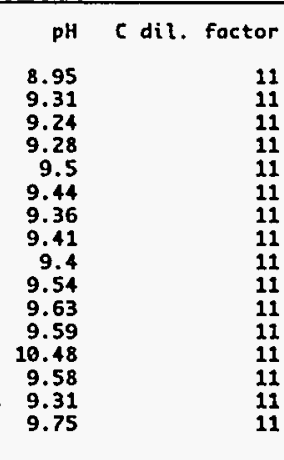

F, ppm

$\begin{array}{ll}1.6 & -0.3 \\ 1.2 & -0.3 \\ 2.5 & -0.3 \\ 2.6 & -0.3\end{array}$

-0.3
-0.3
-0.3
-0.3

$\begin{array}{ll}2.1 & -0.3 \\ 3.1 & \end{array}$

2.1
6.9
3.7

6
5
5.7

11
11
11
11
11
11
11
11
11
11
11
11
11
11
11
11

$-0.3$

-0.3
-0.3
-0.3 ic analyzed. TOC anolyzed

3.084
2.982
3.98

6.86
9.47

Cl-, ppm
2.982
3.756
8.037

4.651
3.911
4.971

3.911
4.871
4.183

4.183
6.341

6. 6419

3.317

4.828
11.91
3.223
6.86

0.95
0.63
0.95
0.98

\subsection{2}

2.181
3.153

1.946
1.435

1.435
4.681
4.064

4.064
1.358

1.358
1.381
0.622
1.108

1.108
3.883
0.976
1.085

1.085
1.235

NO2-, ppm

0.59
0.24
0.74
1.5

1.3
1.9
1.1
1.2
0.7

2.1
0.26
-0.2
1.5
-0.2

1.4
0.67
1.4
TC. ppm

33.92
32.8

52.32
88.41
51.16

43.02
53.58

46.01
69.75

67.64

36.49

53.11
131
35.45
75.46

75.46
104.2

N03 -, ppm

-0.2
-0.2
-0.2
1.2

0.66
-0.2
0.29
0.76

0.76
0.36

1.9
0.22
0.63
Wednesday, August 31 5:06 PM 1994

TOC, ppm TIC, Ppm

$$
\begin{array}{r}
23.23 \\
13.04 \\
23.99 \\
34.68 \\
21.11 \\
15.79 \\
51.49 \\
14.7 \\
14.94 \\
15.19 \\
6.84 \\
12.19 \\
42.71 \\
10.74 \\
11.94 \\
13.58
\end{array}
$$

HPO4=, ppm

2.3
2.3
3.6
4.1
3.2
5.4
2.9
8.7
3.7
5
4.4
4.5

$\begin{array}{rr}4.3 & -0.5 \\ 3.6 & -0.5 \\ 5.9 & 0.69 \\ 4.7 & -0.5 \\ 2.1 & -1 \\ 6.3 & -0.5 \\ 1.9 & -1 \\ 8.4 & -0.5 \\ 2.5 & 1.7 \\ 3.6 & -0.5 \\ 3.8 & -0.5 \\ 7.2 & 1.4\end{array}$


Page \#1 - "TW.EJ. ANIONS"

\begin{tabular}{|c|c|c|c|c|c|c|c|c|c|}
\hline TEST NO. & Reaction Time & pH & C dil. factor & TC analyzed & TOC analyzed & TC, ppm & TOC, pDm & TIC, ppm & \\
\hline $\begin{array}{l}T w 31 \\
T W 32 \\
T W 33 \\
T W 34 \\
T W 35 \\
T W 36 \\
T W 37 \\
T W 38 \\
T W 39 \\
T W 40 \\
T W 41 \\
T W 42 \\
T W 43 \\
T W 43 \\
T W 44 \\
T W 45 \\
T W 46\end{array}$ & $\begin{array}{r}14 \\
14 \\
28 \\
28 \\
56 \\
56 \\
91 \\
91 \\
180 \\
180 \\
360 \\
360 \\
540 \\
540 \\
720 \\
720\end{array}$ & $\begin{array}{l}9.38 \\
9.23 \\
9.44 \\
9.45 \\
9.66 \\
9.71 \\
9.74 \\
9.63 \\
9.88 \\
9.69 \\
9.79 \\
9.79 \\
9.69 \\
9.78 \\
9.24 \\
9.42\end{array}$ & $\begin{array}{l}11 \\
11 \\
11 \\
11 \\
11 \\
11 \\
11 \\
11 \\
11 \\
11 \\
11 \\
11 \\
11 \\
11 \\
11 \\
11\end{array}$ & $\begin{array}{l}4.419 \\
3.381 \\
5.637 \\
5.678 \\
4.405 \\
4.147 \\
5.017 \\
4.754 \\
9.483 \\
7.275 \\
3.299 \\
3.974 \\
4.461 \\
3.834 \\
6.215 \\
6.455\end{array}$ & $\begin{array}{l}1.624 \\
0.808 \\
1.52 \\
1.974 \\
3.005 \\
1.562 \\
2.166 \\
4.155 \\
2.025 \\
1.527 \\
0.564 \\
0.703 \\
0.823 \\
4.434 \\
0.733 \\
0.945\end{array}$ & $\begin{array}{r}48.61 \\
37.19 \\
62.4 \\
62.46 \\
48.46 \\
45.62 \\
55.19 \\
52.29 \\
104.3 \\
80.03 \\
36.29 \\
43.71 \\
49.07 \\
42.17 \\
68.37 \\
71.01\end{array}$ & $\begin{array}{r}17.86 \\
8.888 \\
16.72 \\
21.71 \\
33.06 \\
17.18 \\
23.83 \\
45.71 \\
22.28 \\
16.8 \\
6.204 \\
7.733 \\
9.053 \\
48.77 \\
8.06 \\
10.4\end{array}$ & $\begin{array}{r}30.75 \\
28.3 \\
45.68 \\
40.74 \\
15.4 \\
28.44 \\
31.36 \\
6.589 \\
82.04 \\
63.23 \\
30.09 \\
35.98 \\
40.02 \\
-6.6 \\
60.3 \\
60.61\end{array}$ & \\
\hline Test No. & ACL Number & F-, ppm & СHO2-, ppm & $\mathrm{Cl}-, \mathrm{ppm}$ & NO2-, ppm & NO3-, ppm & HPO4=, ppm & SO4=, ppm & C204-, ppm \\
\hline \multicolumn{10}{|l|}{$\begin{array}{l}T W 31 \\
T W 32\end{array}$} \\
\hline $\begin{array}{l}\text { Tw33 } \\
T w 34\end{array}$ & $\begin{array}{l}93-2154-28 \\
93-2154-29\end{array}$ & $\begin{array}{l}6.3 \\
8.7\end{array}$ & $\begin{array}{l}-0.3 \\
-0.3\end{array}$ & $\begin{array}{l}20 \\
22\end{array}$ & 1.4 & 21 & $\begin{array}{l}0.93 \\
-0.5\end{array}$ & $\begin{array}{l}61 \\
68\end{array}$ & $\begin{array}{l}-0.5 \\
-0.5\end{array}$ \\
\hline TW35 & $93-2154-30$ & 8 & -0.3 & 20 & $\begin{array}{l}1.0 \\
1.9\end{array}$ & 23 & $\begin{array}{r}-6.5 \\
1.8\end{array}$ & $\begin{array}{l}68 \\
62\end{array}$ & $\begin{array}{l}-6.5 \\
-0.5\end{array}$ \\
\hline $\begin{array}{l}\text { Tw36 } \\
\text { Tw37 }\end{array}$ & $\begin{array}{l}93-2154-31 \\
93-2154-32\end{array}$ & $\begin{array}{l}6.8 \\
7.5\end{array}$ & $\begin{array}{l}-0.3 \\
-0.3\end{array}$ & 18 & 1.5 & 20 & $\begin{array}{l}2.12 \\
2.5\end{array}$ & $\begin{array}{l}58 \\
57\end{array}$ & $\begin{array}{l}-0.5 \\
-0.5\end{array}$ \\
\hline TW38 & $92-2103-52$ & 4.6 & -0.3 & $\begin{array}{l}16 \\
12\end{array}$ & $\begin{array}{l}1.9 \\
-0.2\end{array}$ & $\begin{array}{l}19 \\
13\end{array}$ & $\begin{array}{l}2.5 \\
1.4\end{array}$ & $\begin{array}{l}37 \\
29\end{array}$ & $\begin{array}{r}-6.3 \\
-1\end{array}$ \\
\hline $\begin{array}{l}\text { TW39 } \\
\text { TW40 }\end{array}$ & $\begin{array}{l}93-2154-33 \\
92-2103-53\end{array}$ & $\begin{array}{r}20 \\
5.7\end{array}$ & -0.3 & 44 & $\begin{array}{r}3.3 \\
-0.2\end{array}$ & $\begin{array}{l}46 \\
12\end{array}$ & $\begin{array}{l}9.5 \\
1.3\end{array}$ & $\begin{array}{r}155 \\
27\end{array}$ & -0.5 \\
\hline TW41 & $93-2154-34$ & 12 & -0.3 & 16 & 2.2 & 16 & 3.9 & 53 & -0.5 \\
\hline $\begin{array}{l}T W 42 \\
T W 43\end{array}$ & $92-2103-54$ & 7.2 & & 10 & -0.2 & 10 & 2.2 & 26 & 1.1 \\
\hline $\begin{array}{l}\text { TW44 } \\
\text { TW45 }\end{array}$ & $\begin{array}{l}93-2154-35 \\
93-2154-36\end{array}$ & $\begin{array}{l}11 \\
13\end{array}$ & $\begin{array}{l}-0.3 \\
-0.3\end{array}$ & $\begin{array}{l}14 \\
14\end{array}$ & 1.2 & $\begin{array}{l}12 \\
14\end{array}$ & $\begin{array}{r}3.9 \\
2\end{array}$ & $\begin{array}{l}47 \\
46\end{array}$ & $\begin{array}{l}-0.5 \\
-0.5\end{array}$ \\
\hline TW46 & $93-2154-37$ & 13 & -0.3 & 15 & 1.4 & 15 & 4.2 & 48 & -0.5 \\
\hline
\end{tabular}




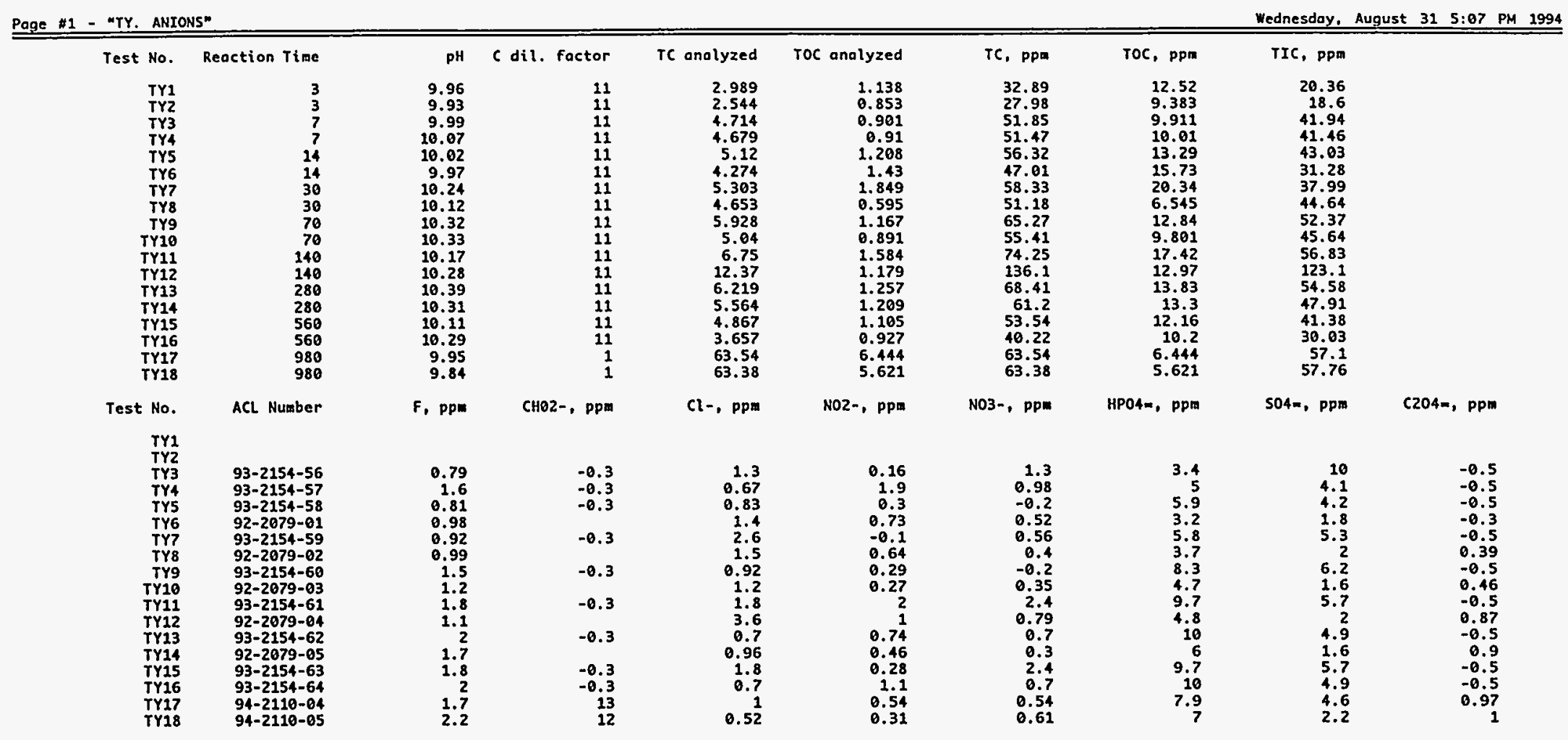




\begin{tabular}{|c|c|c|c|c|c|c|c|c|c|}
\hline Poge "11 - "T2.DIW & oNs" & & & & & & & Wednesday. & August 31 5:07 PM 1994 \\
\hline Test No. & Reaction Time & pH & C dil, factor & TC analyzed & roC analyzed & TC, ppm & TOC, ррm & $T I C, p p m$ & \\
\hline $\begin{array}{r}\text { TZ1 } \\
\text { TZ2 } \\
\text { TZ3 } \\
\text { TZ4 } \\
\text { TZ5 } \\
\text { TZ6 } \\
\text { TZ7 } \\
\text { TZ8 } \\
\text { TZ9 } \\
\text { TZ10 } \\
\text { TZ11 } \\
\text { TZ12 } \\
\text { TZ13 } \\
\text { TZ14 } \\
\text { TZ15 } \\
\text { TZ16 }\end{array}$ & $\begin{array}{r}3 \\
3 \\
7 \\
7 \\
14 \\
14 \\
28 \\
28 \\
56 \\
56 \\
91 \\
91 \\
182 \\
182 \\
364 \\
364\end{array}$ & $\begin{array}{r}11.69 \\
11.14 \\
10.93 \\
11.21 \\
11.34 \\
11.33 \\
11.49 \\
11.46 \\
11.46 \\
11.5 \\
11.43 \\
11.5 \\
11.62 \\
11.69 \\
11.72 \\
11.76\end{array}$ & $\begin{array}{l}11 \\
11 \\
11 \\
11 \\
11 \\
11 \\
11 \\
11 \\
11 \\
11 \\
11 \\
11 \\
11 \\
11 \\
11 \\
11\end{array}$ & $\begin{array}{l}3.901 \\
2.959 \\
4.933 \\
4.003 \\
4.117 \\
3.149 \\
10.87 \\
8.288 \\
4.583 \\
4.783 \\
5.871 \\
5.537 \\
10.93 \\
8.352 \\
6.233 \\
6.507\end{array}$ & $\begin{array}{l}2.027 \\
1.02 \\
2.297 \\
1.341 \\
1.88 \\
1.374 \\
4.632 \\
4.162 \\
2.382 \\
2.462 \\
2.772 \\
2.593 \\
3.668 \\
2.557 \\
1.281 \\
0.982\end{array}$ & $\begin{array}{l}42.91 \\
32.55 \\
54.26 \\
44.03 \\
45.29 \\
34.64 \\
119.6 \\
91.17 \\
50.41 \\
52.61 \\
64.58 \\
60.91 \\
120.2 \\
91.87 \\
68.56 \\
71.58\end{array}$ & $\begin{array}{r}22.297 \\
11.22 \\
25.267 \\
14.751 \\
20.68 \\
15.114 \\
50.952 \\
45.782 \\
26.202 \\
27.082 \\
30.492 \\
28.523 \\
40.348 \\
28.127 \\
14.091 \\
10.802\end{array}$ & $\begin{array}{r}20.613 \\
21.33 \\
28.993 \\
29.279 \\
24.61 \\
19.526 \\
68.648 \\
45.388 \\
24.208 \\
25.528 \\
34.088 \\
32.387 \\
79.852 \\
63.743 \\
54.469 \\
60.778\end{array}$ & \\
\hline Test No. & ACL NUMBER & $A C L F-, p p M$ & $\mathrm{ACL}, \mathrm{CHO2-,} \mathrm{PP}$ & $\mathrm{Cl}-, \mathrm{ppm}$ & $\mathrm{NO2-,} \mathrm{ppm}$ & NO3-, ppm & HPO4=, ppm & SO4=, ppw & C204=, ppm \\
\hline $\begin{array}{l}\text { TZ1 } \\
\text { TZ2 }\end{array}$ & $\begin{array}{l}93-2154-38 \\
93-2154-39\end{array}$ & $\begin{array}{l}1.4 \\
1.9\end{array}$ & $\begin{array}{l}-0.3 \\
-0.3\end{array}$ & $\begin{array}{r}2.7 \\
2\end{array}$ & 0.42 & $\begin{array}{r}2.8 \\
-0.2\end{array}$ & $\begin{array}{l}18 \\
16\end{array}$ & $\begin{array}{r}11 \\
7.3\end{array}$ & $\begin{array}{r}0.55 \\
1\end{array}$ \\
\hline 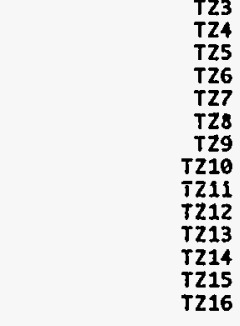 & $\begin{array}{l}93-2154-40 \\
93-2154-41 \\
93-2154-42 \\
93-2154-43 \\
92-2102-41 \\
93-2154-44 \\
92-2102-42 \\
92-2005-05 \\
92-2005-06 \\
93-2154-43 \\
92-2103-43 \\
93-2154-44 \\
92-2103-44\end{array}$ & $\begin{array}{l}2.2 \\
2.4 \\
2.4 \\
2.7 \\
2.7 \\
3.8 \\
1.7 \\
1.8 \\
2.7 \\
5.4 \\
22 \\
-5\end{array}$ & $\begin{array}{r}-0.3 \\
-0.3 \\
-0.3 \\
-0.3 \\
16 \\
-0.3 \\
5.8 \\
7.4 \\
-0.3 \\
-0.3 \\
-15\end{array}$ & $\begin{array}{l}2.1 \\
1.6 \\
2.2 \\
2.8 \\
1.6 \\
2.2 \\
1.7 \\
1.2 \\
1.5 \\
3.6 \\
2.5 \\
22 \\
-8\end{array}$ & $\begin{array}{r}1.5 \\
0.8 \\
1.1 \\
0.37 \\
-0.2 \\
0.5 \\
-0.2 \\
0.97 \\
1.1 \\
0.88 \\
-0.2 \\
2.6 \\
-15\end{array}$ & $\begin{array}{r}-0.2 \\
-0.2 \\
0.82 \\
0.42 \\
0.5 \\
0.55 \\
0.45 \\
1.9 \\
50 \\
-0.2 \\
1 \\
272 \\
7.4\end{array}$ & $\begin{array}{l}19 \\
24 \\
26 \\
34 \\
21 \\
39 \\
26 \\
36 \\
36 \\
52 \\
29 \\
58 \\
62\end{array}$ & $\begin{array}{r}7.6 \\
8.5 \\
9.9 \\
11 \\
4.3 \\
12 \\
5 \\
6.5 \\
6.2 \\
19 \\
7.9 \\
208 \\
138\end{array}$ & $\begin{array}{r}1.4 \\
2 \\
2 \\
1.5 \\
1.3 \\
1.4 \\
1.4 \\
-0.7 \\
-0.7 \\
1.5 \\
1.4 \\
-2 \\
-50\end{array}$ \\
\hline
\end{tabular}




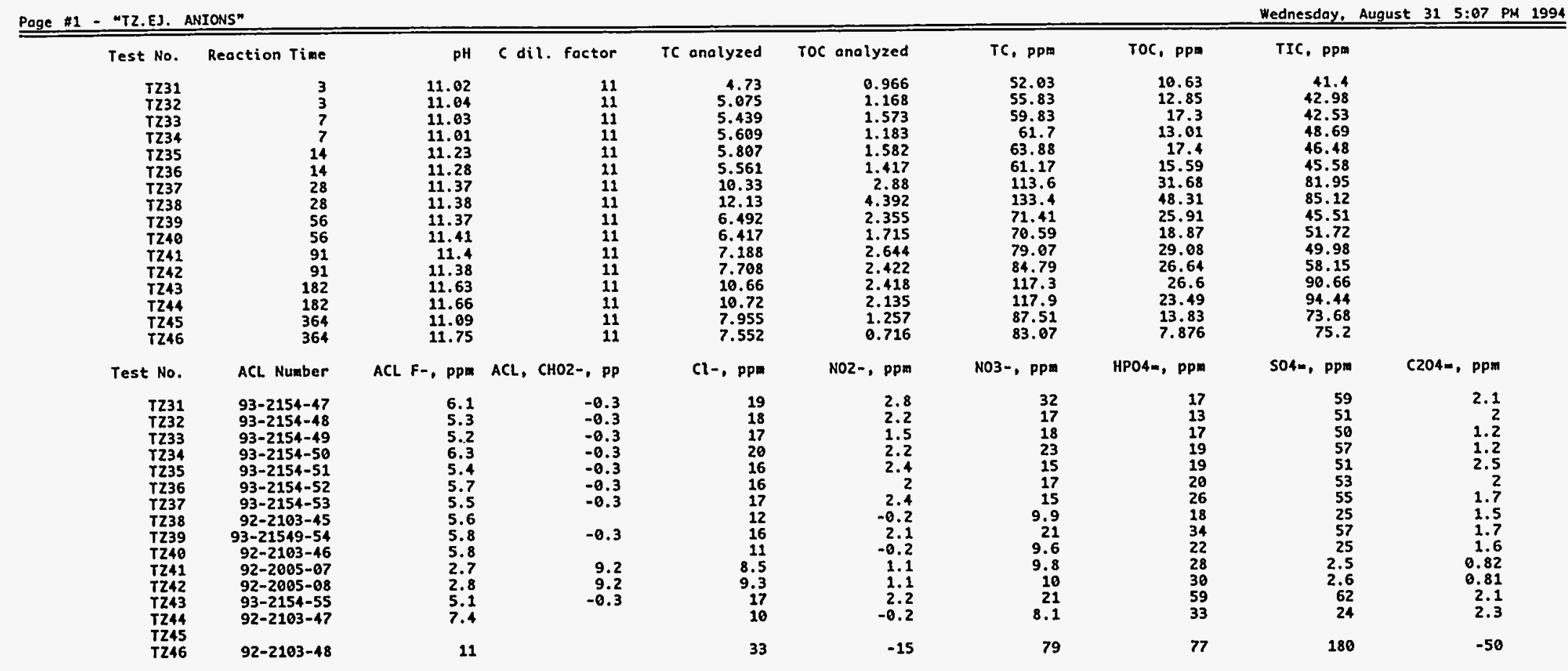


Table D.5. Actinide Results

Column Heading

SAMPLE

(FRACTION)

UF

F.45

F50

AS

ELEC

RXN TIME

SOL'N ml

ALIQUOT ml

SPEC NO. (DET)

LIVE TIME

EFFICIENCY

Np bkgnd

Pu bkgnd

Am bkgnd

Np COUNTS

$\mathrm{Np}$ ng

Pu COUNTS

Pung

Am COUNTS

Am pg

\section{Comment}

Test number in test matrix (Table D.1). Identification of sample treatment.

Unfiltered leachate.

Filtrate from $0.45 \mu \mathrm{m}$ filtration.

Filtrate from filtration through Amicon model CF50A filter.

Solution from acid soak of reaction vessel.

Sample prepared by electroplating

Reaction time, in days.

Volume of solution into which actinides were dissolved, in milliliters.

Volume of solution evaporated or electroplated onto planchet.

Spectrum number of alpha counting (detector number).

Time detector was active during analysis, in seconds.

Measured efficiency of detector, in counts/disintegration.

Background counts per second in $\mathrm{Np}$ region.

Background counts per second in Pu region.

Background counts per second in Am region.

Number of counts assigned to neptunium-237 peak.

Mass of neptunium-237 calculated, in nanograms.

Number of counts assigned to plutonium-239 peak.

Mass of plutonium-239 calculated, in nanograms.

Number of counts assigned to americium-241 peak.

Mass of americium-241 calculated, in picograms. 
Page $\# 1$ - "BX.ACTINIDES $x x^{*}$

Monday, February 6 9:38 AM 1995

\begin{tabular}{|c|c|c|c|c|c|c|c|c|}
\hline SAKPLE & RXN TIME & SOL' $N m L$ & ALIQUOT $\mathrm{ml}$ & SPEC NO. & LIVE TIME & EFFICIENCY & Np bkgnd & Pu bkgnd \\
\hline $\begin{array}{r}\text { BX1(UF) } \\
\text { BX1(F50) }\end{array}$ & 7 & $\begin{array}{l}16.62 \\
16.62\end{array}$ & $\begin{array}{l}0.05 \\
0.03\end{array}$ & $\begin{array}{l}A 129(2) \\
A 149(1)\end{array}$ & $\begin{array}{r}80646 \\
185048\end{array}$ & $\begin{array}{l}0.27 \\
0.22\end{array}$ & $\begin{array}{l}0.00004 \\
0.00003\end{array}$ & 0.00002 \\
\hline BXI(AS) & & 19 & 0.05 & $\begin{array}{r}2038 \\
2038\end{array}$ & 11977 & 0.24 & 0 & 0 \\
\hline$B \times 2$ (UF) & 7 & 16.66 & 0.05 & $A 230(4)$ & 181587 & 0.15 & 0 & 0.00001 \\
\hline $\mathrm{B} \times 2(\mathrm{~F} 50)$ & & 16.66 & 0.45 & A231(1) & 167072 & 0.21 & 0.00002 & 0.00001 \\
\hline$B \times 2(A S)$ & & 19 & 0.05 & 2039 & 12144 & 0.24 & 0 & 0 \\
\hline BX3(UF) & 28 & 17.42 & 0.05 & A233(3) & 151368 & 0.17 & 0 & 0.00002 \\
\hline$B \times 3(F 50)$ & & 17.42 & 0.5 & A175(1) & 101937 & 0.27 & 0.00038 & 0.00031 \\
\hline$B \times 3(A S)$ & & $\begin{array}{r}19 \\
169\end{array}$ & 0.05 & A330(4) & $\begin{array}{l}81720 \\
80546\end{array}$ & 0.24 & 0 & 0.00002 \\
\hline $\begin{array}{r}8 \times 4 \text { (UF) } \\
B \times 4(F 50)\end{array}$ & 28 & $\begin{array}{l}16.9 \\
16.9\end{array}$ & $\begin{array}{l}0.05 \\
0.45\end{array}$ & $\begin{array}{l}A 130(3) \\
A 150(2)\end{array}$ & $\begin{array}{r}80046 \\
185047\end{array}$ & $\begin{array}{l}0.21 \\
0.27\end{array}$ & 0.09 & $0.00031^{\circ}$ \\
\hline$B \times 4$ (AS) & & 19 & 0.05 & A232(2) & 151368 & 0.18 & 0.00005 & 0.00001 \\
\hline BX5(UF) & 91 & 17.41 & 0.05 & A117(3) & 175263 & 0.21 & 0 & 0 \\
\hline $8 \times 5(750)$ & & 17.41 & 0.4 & Al01(1) & 137887 & 0.2 & 0 & $\theta$ \\
\hline$B \times 5$ (AS) & & 19 & 0.05 & $A 102(2)$ & 137887 & 0.27 & 0.00004 & 0.00001 \\
\hline BX6(UF) & 91 & 16.48 & 0.05 & A116(2) & 175250 & 0.27 & 0.00004 & 0.00002 \\
\hline $8 \times 6(f 50)$ & & 16.48 & 0.35 & $A 104(4)$ & 137887 & 0.26 & 0.00006 & 0.00002 \\
\hline BX6(AS) & & 19 & 0.05 & A103(3) & 137887 & 0.23 & 0.00004 & 0.00004 \\
\hline BX7(UF) & 238 & 16.4 & 0.05 & A580 (1) & 248819 & 0.26 & 0.00007 & 0.00005 \\
\hline $8 \times 7(f 50)$ & & 16.4 & 1 & A581(2) & 248819 & 0.27 & 0.00005 & 0.00003 \\
\hline BX7(AS) & & $\begin{array}{r}19 \\
16.23\end{array}$ & $\begin{array}{l}0.05 \\
0.05\end{array}$ & A567(4) & $\begin{array}{r}93387 \\
\end{array}$ & 0.24 & 0.60003 & $\begin{array}{l}0.00002 \\
0.00015\end{array}$ \\
\hline $\begin{array}{r}B \times 8 \text { (UF) } \\
B \times 8(F 50)\end{array}$ & 238 & $\begin{array}{l}16.23 \\
16.23\end{array}$ & $\begin{array}{l}0.05 \\
0.05\end{array}$ & $\begin{array}{l}A 582(3) \\
A 583(4)\end{array}$ & $\begin{array}{l}248818 \\
248818\end{array}$ & $\begin{array}{l}0.24 \\
0.24\end{array}$ & $\begin{array}{l}0.00013 \\
0.00003\end{array}$ & 0.00002 \\
\hline BX8(AS) & & $\begin{array}{r}20.23 \\
19\end{array}$ & 0.05 & A566(3) & 93388 & 0.24 & 0.00013 & 0.00015 \\
\hline BX9(UF) & 600 & 16.67 & 0.05 & A996(2) & 343939 & 0.25 & 0.00031 & 0.00072 \\
\hline BX9(F50) & & 16.67 & 0.1 & A999(3) & 162908 & 0.29 & 0.00005 & 0.00004 \\
\hline BX9(AS) & & 19 & 0.05 & A997(3) & 180985 & 0.29 & 0.00005 & 0.00004 \\
\hline BXI0(UF) & 600 & 16.93 & 0.05 & $c 001(4)$ & 162908 & 0.28 & 0.00008 & 0.00007 \\
\hline $8 \times 10(F 50)$ & & 16.93 & 0.1 & A995(1) & 343939 & 0.25 & 0.00004 & 0 \\
\hline BX10(AS) & & 19 & 0.05 & A998(4) & 180984 & 0.28 & 0.00008 & 0.00007 \\
\hline BXI1(UF) & 1400 & 17.06 & 0.1 & C1006(1) & 172800 & 0.28 & 0.00003 & 0.00017 \\
\hline $8 \times 11(F 50)$ & & 17.06 & 0.1 & C2006(2) & 372800 & 0.26 & 0.00006 & \\
\hline BXII(AS) & & & 0.1 & (3006(3) & 172860 & 0.26 & 0.00005 & 0.00016 \\
\hline BX12(UF) & 1400 & 16.44 & 0.1 & C4006(4) & 172800 & 0.27 & 0.00007 & 0.0001 \\
\hline$B \times 12(F 50)$ & & 16.44 & 0.1 & C5006(5) & 172800 & 0.23 & 0.0021 & 0.00023 \\
\hline BX12(AS) & & 19 & 0.1 & $66006(6)$ & 172800 & 0.24 & & 0.00004 \\
\hline
\end{tabular}




$\begin{array}{rr}\text { SAMPLE } & \text { Am bkgnd } \\ \text { BX1CUF) } & 0.00002 \\ \text { BX1(FS0) } & 0.00001 \\ \text { BX1(AS) } & 0 \\ \text { BX2(UF) } & 0 \\ \text { BX2(F50) } & 0 \\ \text { BX2(AS) } & 0 \\ \text { BX3(UF) } & 0.00006 \\ \text { BX3(F50) } & 0.00035 \\ \text { BX3(AS) } & 0.00003 \\ \text { BX4(UF) } & 0 \\ \text { BX4(F50) } & 0.00035 \\ \text { BX1(AS) } & 0.00002 \\ \text { BXSCUF) } & 0 \\ \text { BXS(FS0) } & 0 \\ \text { BXS(AS) } & 0.00002 \\ \text { BX6(UF) } & 0.00002 \\ \text { BX6(FS0) } & 0.00006 \\ \text { BX6(AS) } & 0.00002 \\ \text { BX7(UF) } & 0.00003 \\ \text { BX7(F50) } & 0.00002 \\ \text { BX7(AS) } & 0.00002 \\ \text { BX8(UF) } & 0.00018 \\ \text { BX8(FS0) } & 0.00002 \\ \text { BX8(AS) } & 0.00018 \\ \text { BX9(UF) } & 0.00186 \\ \text { BX9(FS0) } & 0.00007 \\ \text { BX9(AS) } & 0.06007 \\ \text { BX10(UF) } & 0.00006 \\ \text { BX10(F50) } & 0.00008 \\ \text { BX10(AS) } & 0.00006 \\ \text { BX11(UF) } & 0.0011 \\ \text { BX11(FS0) } & 0 \\ \text { BX11(AS) } & 0.00035 \\ \text { BX12(UF) } & 0.0003 \\ \text { BX12(F50) } & 0.00084 \\ \text { BX12(AS) } & 0.00059 \\ & \end{array}$

Np counts

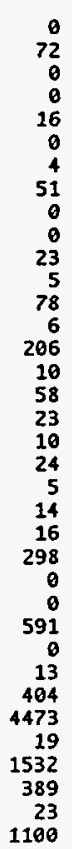

Np ng

$-1.88606$

34.63276
0

0.50439

2.1242

0.60126

$-1.36224$

$-1.40313$

27.73436

0.36269
78.37474

0.79769

2. 46349

8.12993

0.10888

1.45174

$-3.80363$

1.80709

184.8532

$-15.83383$

$-1.09326$

160.26665

$-3.66597$

-0.05667
110.68453

607.69342

67.69342
1.27999

1.27999
249.61923

50.49155

0.92224

195.45778
Pu COUNTS

$\begin{array}{rr}0 & -0.01074 \\ 56 & 0.33226 \\ 0 & 0 \\ 2 & 0.00098 \\ 2 & 0.00015 \\ 5 & 0.28305 \\ 3 & -0.00017 \\ 38 & 0.00357 \\ 0 & -0.01398 \\ 1 & 0.00854 \\ 46 & -0.00372 \\ 11 & 0.059 \\ 345 & 1.39647 \\ 1 & 0.00659 \\ 552 & 2.45039 \\ 0 & -0.01064 \\ 41 & 0.02157 \\ 337 & 1.75462 \\ 8 & -0.00978 \\ 12 & 0.00049 \\ 78 & 0.57232 \\ 84 & 0.11016 \\ 14 & 0.02175 \\ 1071 & 7.80305 \\ 3505 & 5.50677 \\ 0 & -0.00996 \\ 2557 & 7.99363 \\ 1278 & 4.05586 \\ 26 & 0.02214 \\ 2568 & 8.26591 \\ 12317 & 19.02588 \\ 11 & 0.01857 \\ 8158 & 15.16605 \\ 6268 & 9.53248 \\ 53 & 0.02433 \\ 9817 & 19.84362\end{array}$

Am COUNTS

Ax pg
$-0.19378$

4.51409
45.58751

45.58751
0.09564

11.24015

$-0.88193$

$-0.06727$

$-0.06727$

$-0.37856$

$-0.34173$

2.1298
3.02462

33.02462
0

98.66321
1.72656

$-0.0435$

29.26

$-0.01847$

0.02134

20.23632

2.09653

2.09653
0.87111

144.46269

144.46269
93.56852

$-0.3145$

280.75732

73.65108

73.

$-0.02329$

404.87817
174.59474

174.59474

7.09947

624.43964

132.74112

0.3594
40.75824 
Page $\# 1$ - "BY.ACTINIDES $x \times$ "

Monday, February $6 \quad 9: 38$ AM 1995

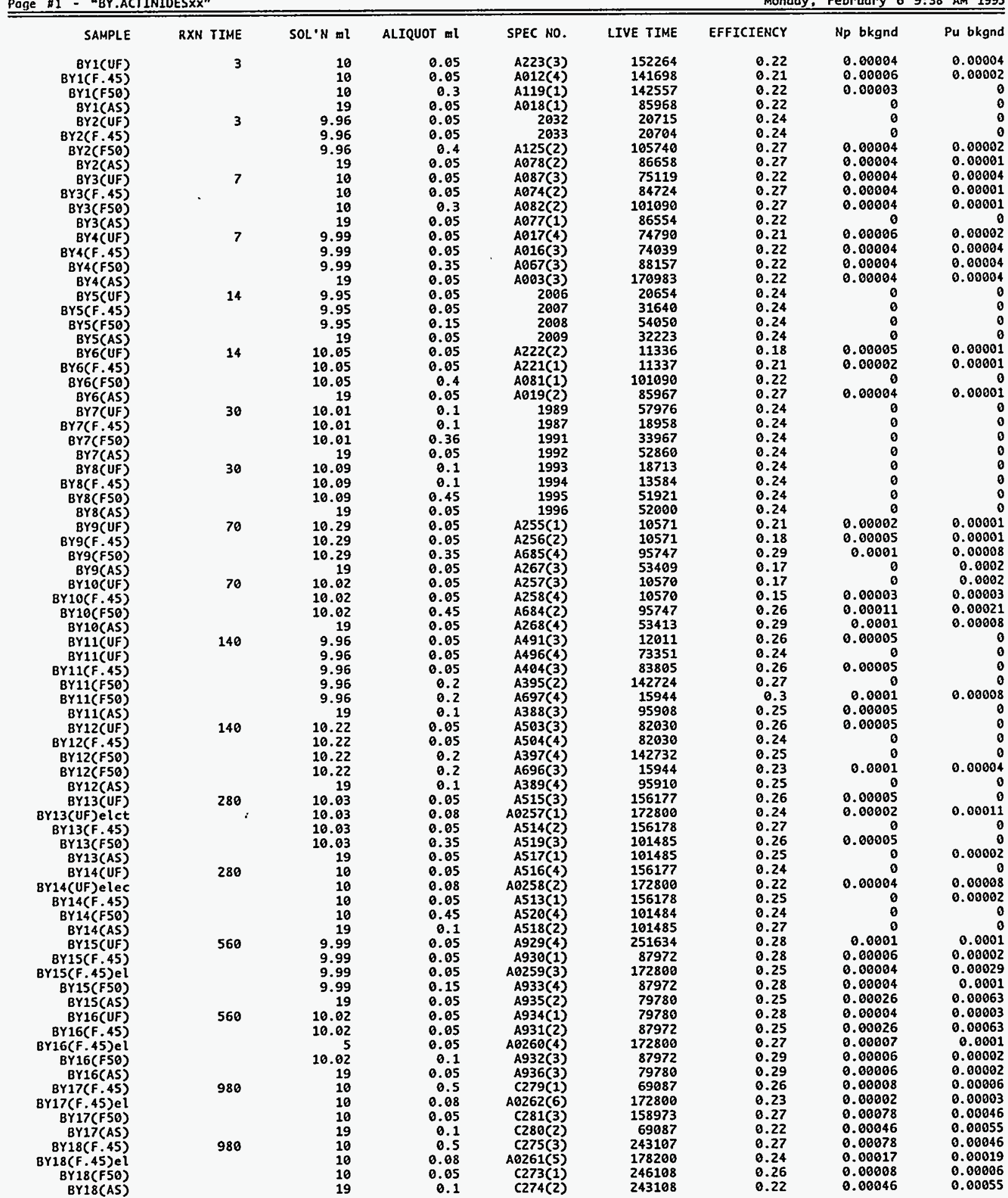


Page $\# 2$ - "BY.ACTINTDES $x x^{\prime \prime}$

Monday, February 6 9:38 AM 1995

\begin{tabular}{|c|c|c|c|c|c|c|c|}
\hline SAMPLE & Am bkgnd & Np COUNTS & Np $n g$ & Pu COUNTS & Pu ng & Am COUNTS & Am $p g$ \\
\hline BYI(UF) & 0.00002 & 509 & 116.59008 & 3033 & 7.98838 & 4054 & 192.97682 \\
\hline BY1(F.4S) & 0.00006 & 533 & 136.31563 & 2711 & 8.01244 & 3831 & 204.1385 \\
\hline BYi(F5日) & 0.00001 & 960 & 38.90437 & 2228 & 1.03245 & 2898 & 24.22852 \\
\hline BYI(AS) & 0 & 138 & 106.19448 & 841 & 7.36727 & 1349 & 213.30978 \\
\hline BY2(UF) & 0 & 224 & 342.32147 & 1000 & 17.39698 & 1285 & 403.52014 \\
\hline BY2(F.45) & 0 & 233 & 356.26465 & 1170 & 20.36528 & 1384 & 434.83936 \\
\hline $8 Y 2$ (F5O) & 0.00002 & 5078 & 169.48288 & 3787 & 1.43925 & 886 & 6.0669 \\
\hline BYZ(AS) & 0.00002 & 51 & 29.56728 & 624 & 4.41244 & 989 & 126.18869 \\
\hline BY3(UF) & 0.00002 & 276 & 128.28453 & 2457 & 13.12746 & 3901 & 376.53369 \\
\hline$B Y 3(F .45)$ & 0.00002 & 158 & 51.77249 & 4014 & 15.2979 & 5828 & 400.89233 \\
\hline BY3 (FSO) & 0.00002 & 1725 & 80.4962 & 2639 & 1.40464 & 3631 & 34.87915 \\
\hline BY3 (AS) & & 79 & 60.38091 & 924 & 8.03956 & 1561 & 245.16101 \\
\hline BY4(UF) & 0.00006 & 117 & 55.34629 & 4126 & 23.09653 & 6744 & 681.22693 \\
\hline BY4 (F.45) & 0.00002 & 887 & 421.0611 & 5124 & 27.76641 & 6968 & 681.81378 \\
\hline BY4(FSO) & 0.00002 & 1670 & 95.23096 & 2628 & 1.7073 & 2825 & 33.15148 \\
\hline BY4(AS) & 0.00002 & 106 & 38.89638 & 1012 & 4.48841 & 1632 & 131.26678 \\
\hline BYS(UF) & 0 & 325 & 497.63852 & 8805 & 153.47855 & 11633 & 3660.14502 \\
\hline $\begin{array}{r}B Y 5(F .45) \\
\text { BYS(F50) }\end{array}$ & 0 & 500 & 499.76785 & 3500 & 39.82484 & 5000 & 1026.93811 \\
\hline $\begin{array}{l}\text { BYS(F50) } \\
\text { BY5(AS) }\end{array}$ & 0 & 419 & 81.72066 & 1204 & 2.6732 & 1131 & 45.32694 \\
\hline BYS (AS) & 0 & 0 & 0 & 1100 & 23.4682 & 2700 & 654.67267 \\
\hline BY6(UF) & 0.00002 & 235 & 904.56848 & 1021 & 44.84227 & 1245 & 986.935 \\
\hline$B Y 6(F .45)$ & 0 & 200 & 636.87537 & 1200 & 43.54567 & 1676 & 1097.91077 \\
\hline BY6 (F50) & 0 & 2612 & 113.01788 & 1790 & 0.88169 & 2204 . & 19.59573 \\
\hline BYG(AS) & 0.00002 & 83 & 49.88716 & 1238 & 8.83067 & 1881 & 242.13324 \\
\hline BY7(UF) & 0 & 0 & 0 & 16500 & 51.53938 & 25000 & 1409.55994 \\
\hline BY7(F.45) & 0 & 646 & 542.07056 & 39000 & 372.54233 & 28776 & 4961.69043 \\
\hline BY7 (F50) & 0 & 769 & 100.04202 & 963 & 1.42617 & 1087 & 29.05773 \\
\hline BY7 (AS) & 0 & 49 & 55.98018 & 1763 & 22.92863 & 2161 & 507.30469 \\
\hline BY8(UF) & 0 & 0 & 0 & 9703 & 94.65057 & 7211 & 1269.69946 \\
\hline BY $8(F .45)$ & 0 & 0 & 0 & 7526 & 101.134 & 5800 & 1406.85449 \\
\hline BY8(F50) & 0 & 1347 & 92.44532 & 8690 & 6.7893 & 17795 & 250.95238 \\
\hline BY8(AS) & 0 & 47 & 54.58331 & 1765 & 23.33428 & 2476 & 590.86542 \\
\hline BYg(UF) & 0 & 303 & 1059.9563 & 1930 & 76.90768 & 2406 & 1730.69299 \\
\hline BY $9(F .45)$ & 0.00002 & 243 & 1027.24963 & 1842 & 88.83167 & 1413 & 1229.89636 \\
\hline BY9 (FSO) & 0.00004 & 5447 & 221.26686 & 12667 & 5.86438 & 9239 & 77.22251 \\
\hline BY9(AS) & 0.00006 & 34 & 55.81343 & 1339 & 24.82274 & 3271 & 1102.27673 \\
\hline BY10(UF) & 0.00006 & 132 & 577.41406 & 2477 & 123.24133 & 2261 & 2031.73999 \\
\hline$B Y 10(F .45)$ & 0.00007 & 367 & 1763.33667 & 2312 & 126.54954 & 2639 & 2606.98267 \\
\hline$B Y 10(F 50)$ & 0.0005 & 9612 & 331.47195 & 36328 & 14.26914 & 37272 & 264.06427 \\
\hline BY10 (AS) & 0.00004 & 15 & 9.1066 & 2575 & 27.59188 & 5741 & 1111.83252 \\
\hline BY1I(UF) & 0.00002 & 0 & -1.45598 & 3352 & 92.51228 & 3829 & 1907.39978 \\
\hline BY11(UF) & & 879 & 380.94275 & 17267 & 85.18742 & 25493 & 2270.21899 \\
\hline$B Y 11(F .45)$ & 0.00002 & 854 & 295.28311 & 24996 & 98.87241 & 28506 & 2035.18359 \\
\hline BY11(F50) & 0.00003 & 13696 & 682.95178 & 68486 & 38.87638 & 60819 & 623.1333 \\
\hline BY11(F50) & 0.00004 & 0 & -0.64656 & 11471 & 52.94799 & 8206 & 683.72668 \\
\hline BY11(AS) & 0.00002 & 0 & -1.47906 & 9181 & 32.23592 & 20671 & 1309.9646 \\
\hline BY12(UF) & 0.00002 & 1243 & 451.27512 & 19244 & 79.7974 & 25629 & 1918.16296 \\
\hline BY12(F.45) & 0 & 1531 & 608.79474 & 24074 & 108.9762 & 27728 & 2265.63647 \\
\hline BY12(F50) & $\theta$ & 13397 & 726.08148 & 72836 & 44.93778 & 67367 & 750.24133 \\
\hline BY12(F50) & 0.00003 & 0 & -0.65897 & 12203 & 57.41133 & 9683 & 822.30017 \\
\hline BY1Z(AS) & 0 & 0 & 0 & 6618 & 22.59344 & 17351 & 1069.22339 \\
\hline BY13(UF) & 0.00002 & 1620 & 302.71075 & 43301 & 92.55437 & 54050 & 2085.24878 \\
\hline 13 (UF)elct & 0.00009 & 5955 & 697.82617 & 107455 & 143.40198 & 162378 & 3911.82275 \\
\hline BY13(F. 45) & 0.00003 & 27475 & 5062.16455 & 51199 & 107.38602 & 59111 & 2237.73389 \\
\hline BY13(F50) & 0.00002 & 3809 & 157.02202 & 6360 & 2.98864 & 8529 & 72.3268 \\
\hline BY13(AS) & 0.00003 & 0 & 0 & 28008 & 182.88531 & 32791 & 3864.8335 \\
\hline BY14(UF) & $\theta$ & 0 & 0 & 43172 & 100.43621 & 56661 & 2379.36255 \\
\hline 14 (UF)elec & 0.00017 & 5666 & 715.92474 & 135002 & 194.40364 & 177220 & 4606.14551 \\
\hline $8 Y 14(F .45)$ & 0.00003 & 0 & 0 & 52560 & 117.37785 & 62709 & 2527.79736 \\
\hline BY 14 (F50) & 0 & 4497 & 157.14491 & 2858 & 1.13691 & 3311 & 23.77457 \\
\hline BY14(AS) & 0.00003 & 0 & 0 & 29257 & 89.44514 & 35612 & 1965.05383 \\
\hline BY15(UF) & 0.0001 & 0 & -2.7136 & 97365 & 119.49621 & 133950 & 2967.65137 \\
\hline $8 Y 15(F .45)$ & 0.00009 & 0 & -1.65755 & 38595 & 137.96501 & 41150 & 2654.80249 \\
\hline $15(F .45) e l$ & 0.00027 & 2388 & 415.13773 & 80903 & 160,47227 & 114151 & 4087.84961 \\
\hline $8 Y 15(F 50)$ & 0.00004 & $\$ 264$ & 540.88489 & 29182 & 34.14689 & 36970 & 781.02234 \\
\hline BY15(AS) & 0.00185 & 0 & -15.25823 & 13215 & 110.23934 & 17520 & 2625.86621 \\
\hline BY16(UF) & 0.00005 & 0 & -1.10835 & 18480 & 73.05604 & 34703 & 2476.37109 \\
\hline$B Y 16(F .45)$ & 0.00185 & 0 & -8.04671 & 34828 & 139.25954 & 45454 & 3274.09033 \\
\hline $16(F .45)$ el & 0.00006 & 2109 & 169.62233 & 74730 & 68.79973 & 103213 & 1715.42297 \\
\hline $8 Y 16(F 50)$ & 0.00007 & 3465 & 516.86884 & 18420 & 31.32384 & 28036 & 860.47015 \\
\hline BY16(AS) & 0.00007 & 0 & -2.99055 & 13244 & 94.18029 & 17903 & 2297.58594 \\
\hline BY $17(F .45)$ & 0.00011 & & & & & & \\
\hline $17(F .45) e l$ & 0.00006 & 2172 & 259.02945 & 68389 & 92.96471 & 87398 & 2144.38623 \\
\hline$B Y 17$ (FSO) & 0.00058 & 960 & 149.74762 & 9419 & 19.05727 & 10703 & 390.55035 \\
\hline BY17(AS) & 0.00167 & 0 & -15.4259 & 26499 & 146.21445 & 30231 & 3003.75244 \\
\hline$B Y 18(F-45)$ & 0.00058 & & & & & & \\
\hline $18(F .45) \mathrm{el}$ & 0.00068 & 2193 & 244.13025 & 66590 & 85.52636 & 86809 & 2010.75049 \\
\hline BY18 (F50) & 0.00011 & 2047 & 245.52197 & 11130 & 15.32414 & 11444 & 284.11563 \\
\hline 8Y18(AS) & 0.00167 & 0 & -15.4259 & 84172 & 131.96486 & 106275 & 3000.80786 \\
\hline
\end{tabular}


Page $\# 1$ - "B2.ACTINIDES $x \times$ "

Monday, February 6 9:39 АM 1995

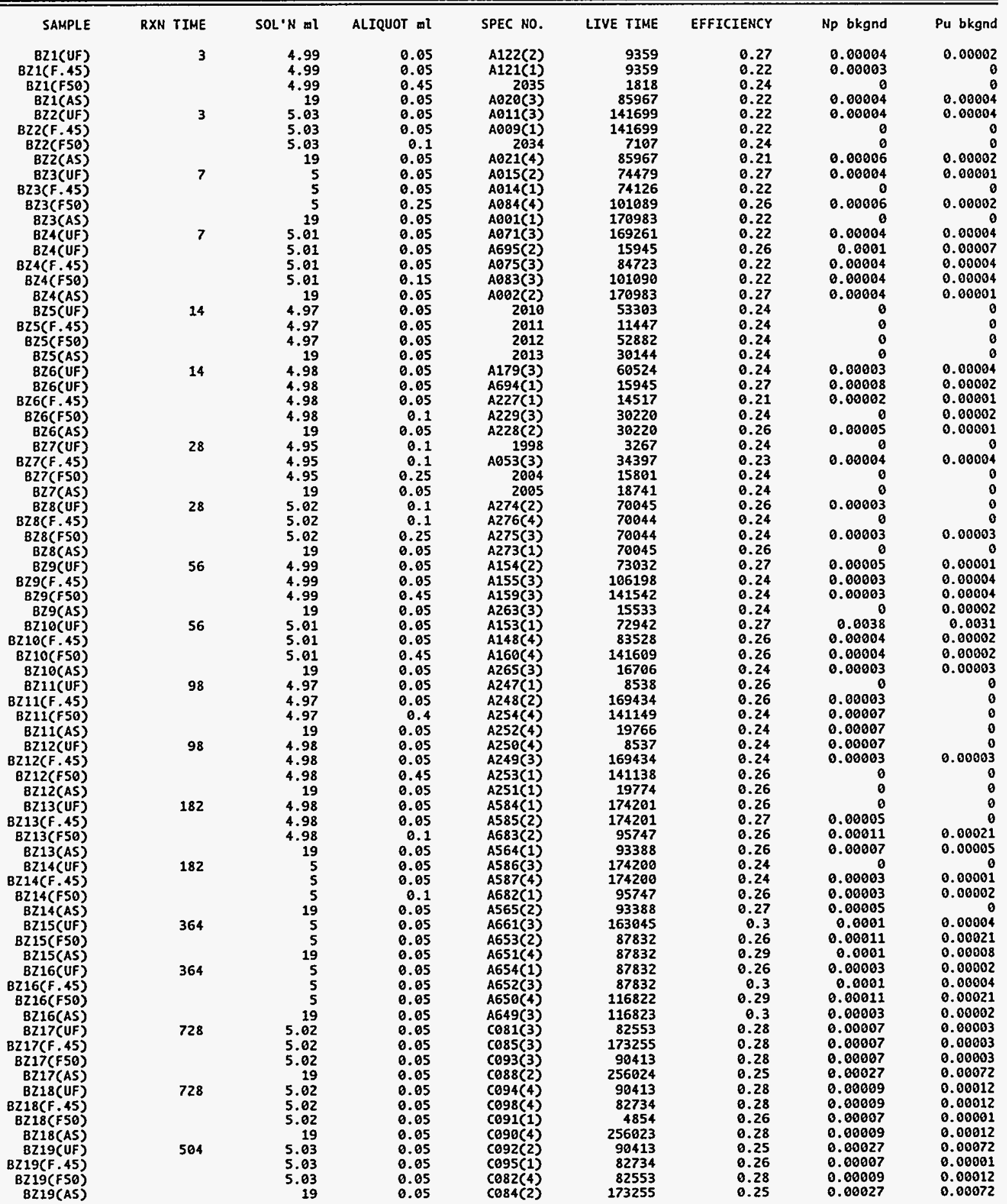




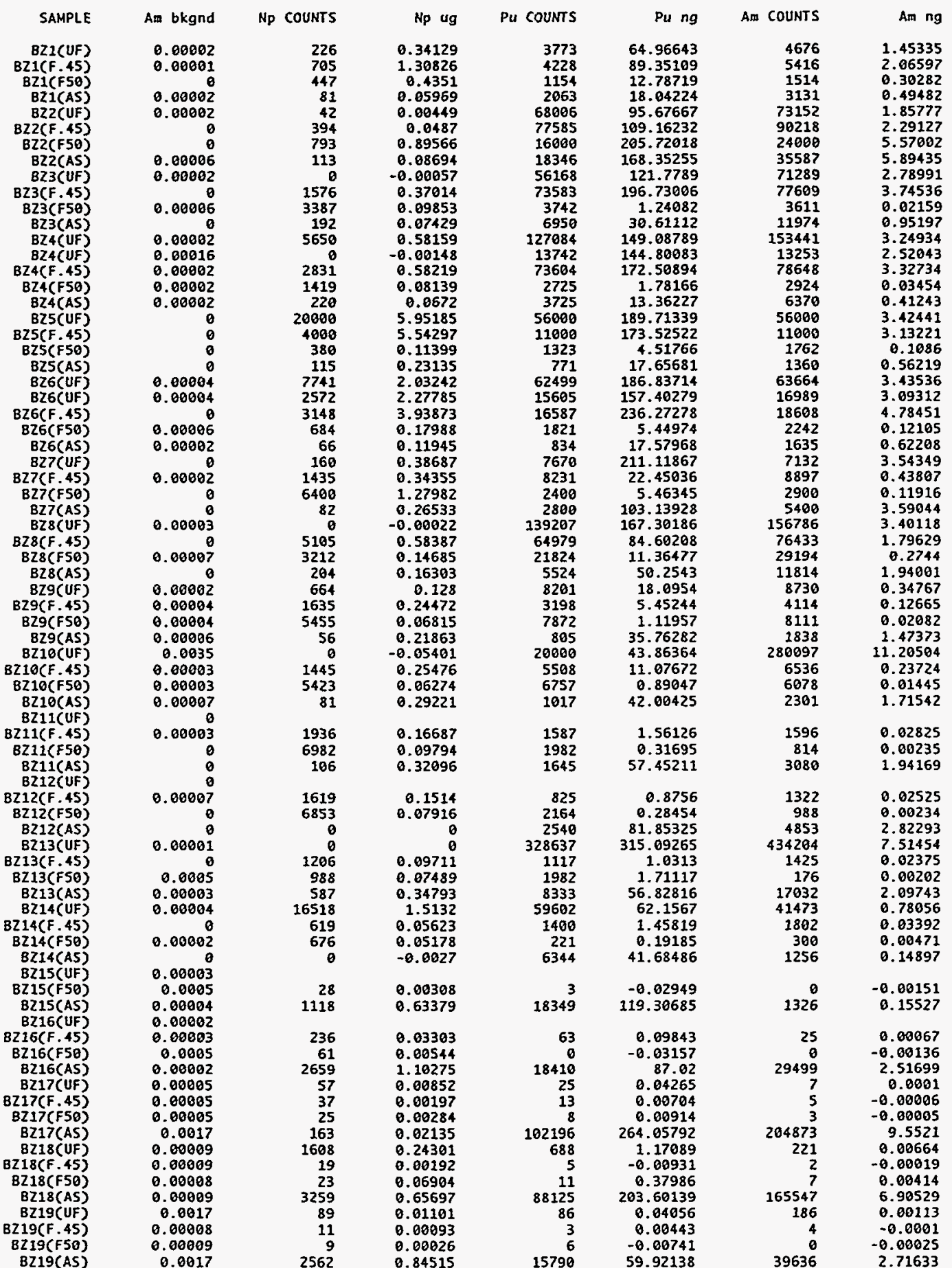


Page $\# 1$ - "PX.ACTINIDES $x \mathrm{X}^{\text {" }}$

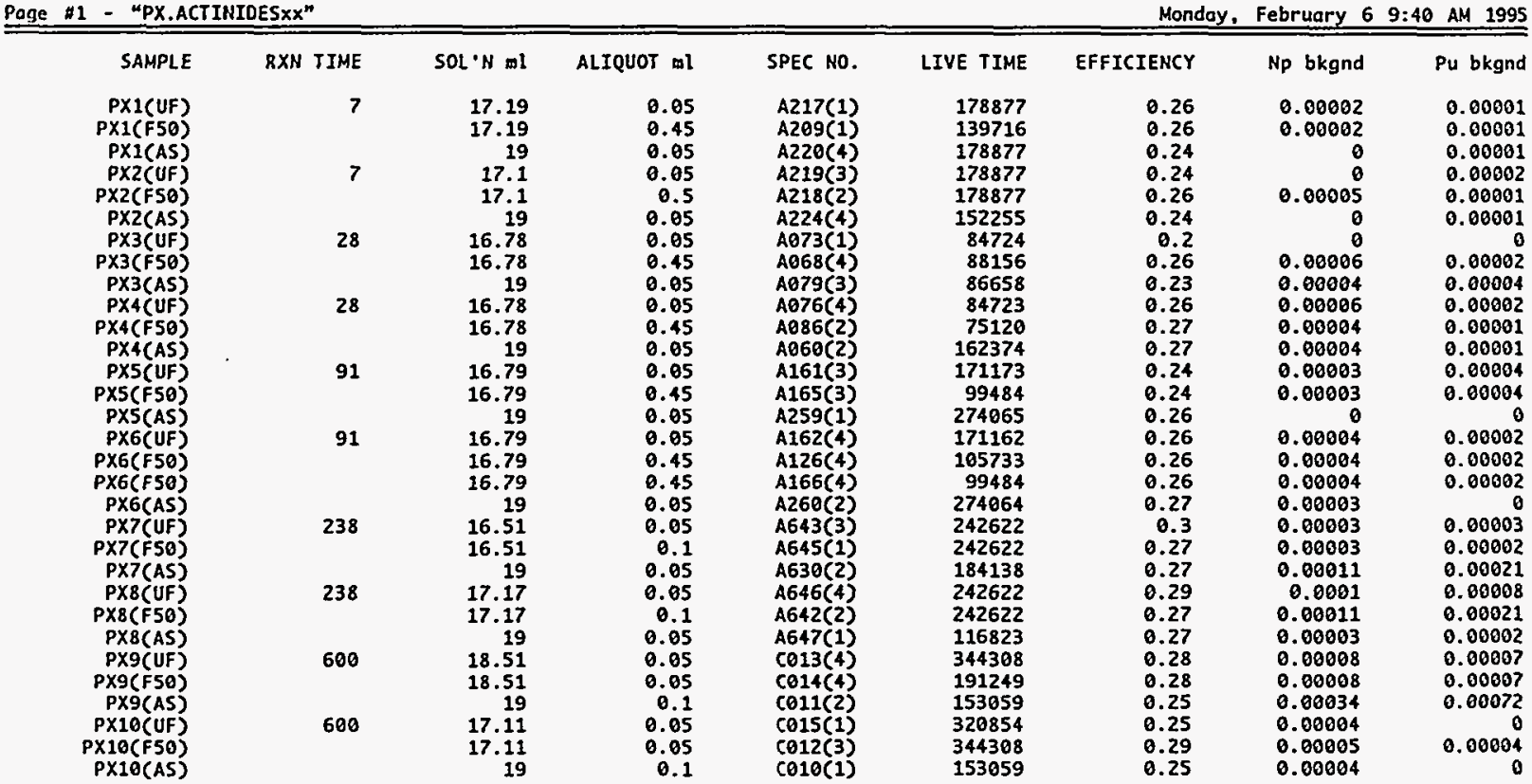

\begin{tabular}{|c|c|c|c|c|c|c|c|}
\hline SAMPLE & Am bkgnd & Np coUNTS & $N_{p} n g$ & Pu COUNTS & Pu pg & An COUNTS & Am pg \\
\hline $\begin{array}{l}\text { PX1(UF) } \\
\text { PX1(F50) }\end{array}$ & 0 & $\begin{array}{r}34 \\
149\end{array}$ & $\begin{array}{l}8.61333 \\
5.88853\end{array}$ & $\begin{array}{r}0 \\
128\end{array}$ & $\begin{array}{l}-5.76526 \\
58.04621\end{array}$ & $\begin{array}{r}0 \\
92\end{array}$ & 0.76139 \\
\hline PZ1(AS) & 0 & $\begin{array}{r}149 \\
4\end{array}$ & 1.35605 & $\begin{array}{r}120 \\
38\end{array}$ & 139.74866 & 52 & 3.6224 \\
\hline PX2(UF) & 0.00006 & 8 & 2.4409 & 0 & -12.426 & 1 & -0.61019 \\
\hline PX2(F50) & 0.00002 & 81 & 2.0294 & 61 & 18.98405 & 63 & 0.34389 \\
\hline PX2(AS) & & 0 & 0 & 15 & 61.10757 & 29 & 2.37341 \\
\hline PX3(UF) & 0 & 20 & 15.171 & 2 & 17.27038 & 0 & 0 \\
\hline PX3(F50) & 0.00006 & 175 & 10.57456 & 6 & 3.00529 & 0 & -0.06772 \\
\hline$P \times 3(A S)$ & 0.00002 & 3 & -0.34651 & 16 & 104.18668 & 14 & 1.84058 \\
\hline PX4(UF) & 0.00006 & 10 & 2.86888 & 6 & 28.5997 & 5 & -0.01 \\
\hline PX4(F50) & 0.00002 & 93 & 6.33692 & 7 & 5.00891 & 0 & -0.02174 \\
\hline PX4(AS) & 0.00002 & 8 & 0.49963 & 31 & 111.01619 & 35 & 2.16599 \\
\hline PX5(UF) & 0.00004 & 107 & 31.89019 & 2 & -17.27375 & 2 & -0.3118 \\
\hline PX5(F50) & 0.00004 & 450 & 26.75439 & 6 & 1.37673 & 3 & -0.01204 \\
\hline PXS(AS) & & 15 & 3.0637 & 54 & 125.55585 & 91 & 3.8192 \\
\hline PX6(UF) & 0.00003 & 59 & 15.0724 & 4 & 1.8975 & 0 & -0.30493 \\
\hline PX6(F50) & 0.00003 & 1 & -0.16787 & 8 & 3.48267 & 9 & $\begin{array}{r}0.06225 \\
-02753\end{array}$ \\
\hline PX6(F50) & 0.00003 & 174 & 9.39317 & 44 & 26.4213 & 1 & -0.02253 \\
\hline PX6(AS) & 0.00003 & 25 & 3.29996 & 70 & 156.73007 & 82 & 2.98174 \\
\hline PX7(UF) & 0.00007 & 222 & 37.3078 & 11 & 7.36057 & 5 & -0.42784 \\
\hline PX7(F50) & 0.00002 & 416 & 39.45292 & 20 & 16.64494 & 0 & -0.09625 \\
\hline PX7(AS) & 0.00005 & 19 & -0.36744 & 93 & 181.05508 & 101 & 5.52155 \\
\hline PX8(UF) & 0.00004 & 39 & 2.75489 & 2 & -37.04686 & 0 & -0.37277 \\
\hline PX8(F50) & 0.0005 & 55 & 2.8421 & 2 & -55.93981 & 0 & -2.50237 \\
\hline PX8(AS) & 0.00002 & 756 & 347.21143 & 648 & 3391.44043 & 308 & 28.98076 \\
\hline PX9(UF) & 0.00006 & 133 & 15.50953 & 19 & -8.54124 & 343 & 9.74142 \\
\hline PX9(F50) & 0.00006 & 42 & 7.06953 & 3 & -31.30933 & 19 & 0.40942 \\
\hline PX9(AS) & 0.00186 & 2208 & 410.00974 & 773 & 1434.90149 & 16858 & 647.64752 \\
\hline PX10(UF) & 0.00008 & 1232 & 199.20219 & 343 & 637.98895 & 290 & 8.87476 \\
\hline PX10(F50) & 0.00007 & 1440 & 186.75526 & 25 & 16.77689 & 25 & 0.02423 \\
\hline PX10(AS & 0.00008 & 768 & 144.89011 & 14768 & 31971.49023 & 12693 & 495.53525 \\
\hline
\end{tabular}


Page \#1 - "PY.ACTINIDES $x x^{n}$

Monday, Februory 6 9:40 AM 1995

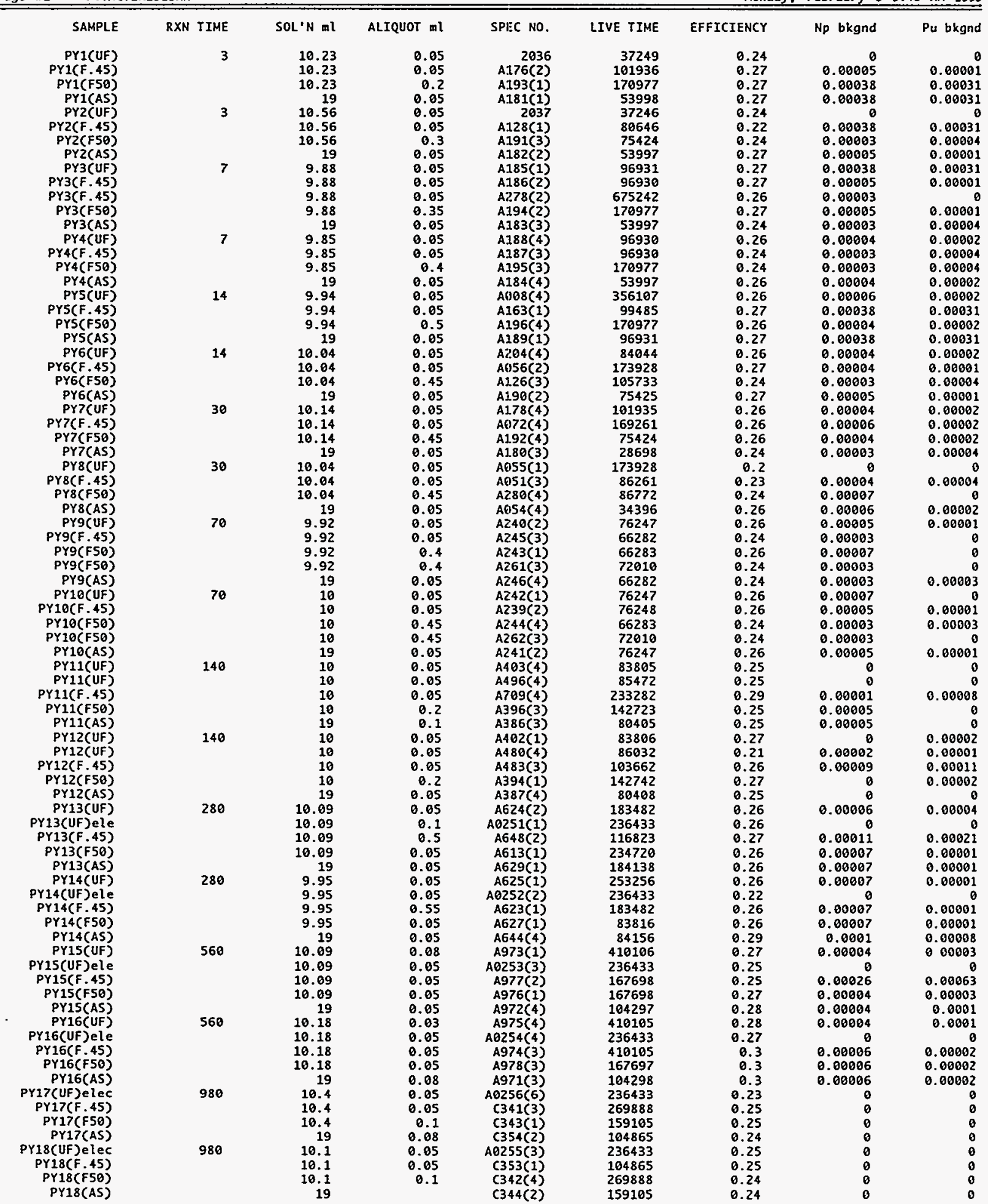


Page \#2 - "PY.ACTINIDES $x x^{n}$

Monday, February 6 9:40 AM 1995

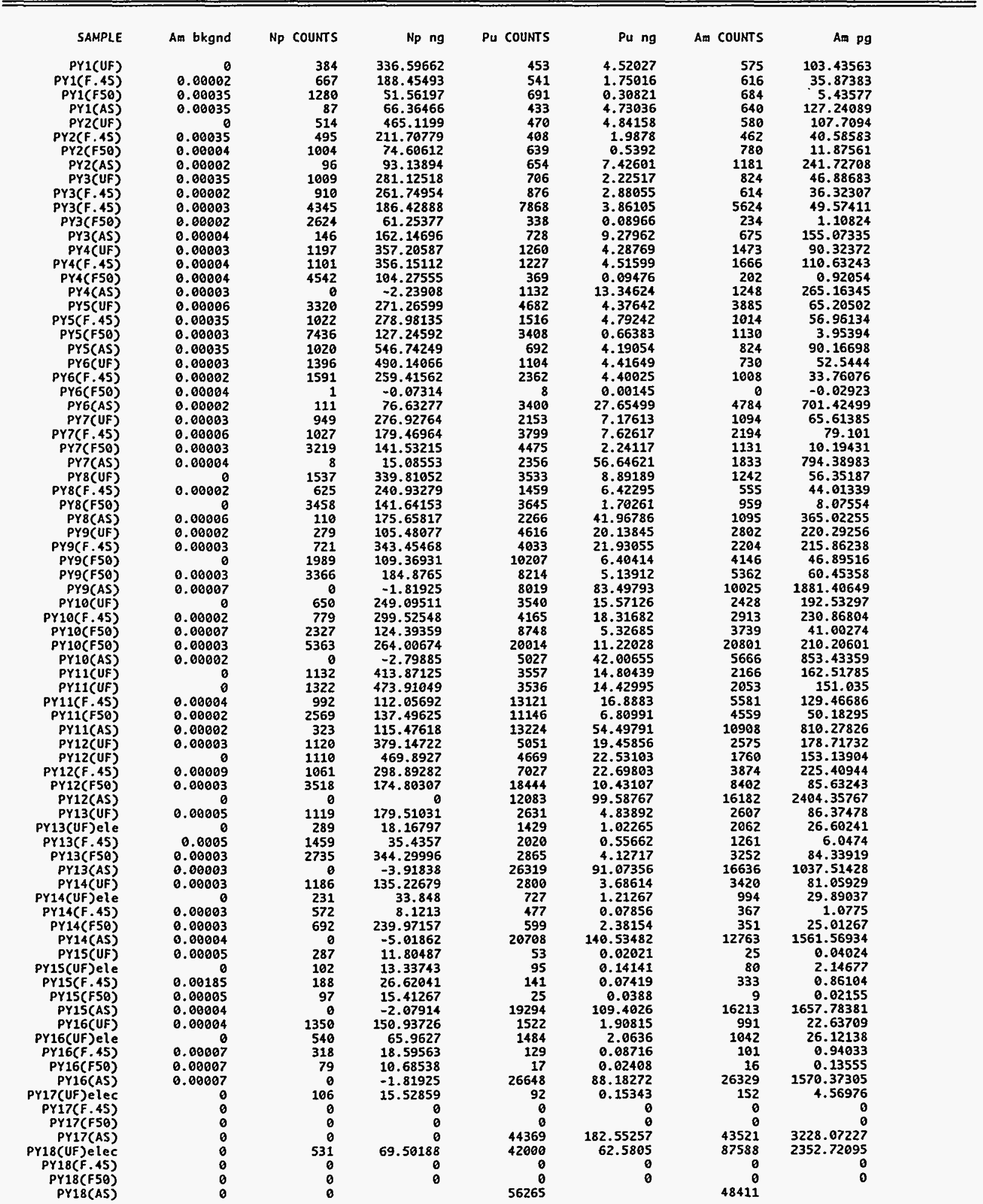


Page 11 - "PZ.ACTINIDES $x x^{\prime}$

Mondoy, February 6 9:42 AM 1995

\begin{tabular}{|c|c|c|c|c|c|c|c|c|}
\hline SAMPLE & RXN TIME & SOL'N ml & ALIQUOT ml & SPEC NO. & LIVE TIME & EFFICIENCY & Np bkgnd & Pu bkgnd \\
\hline $\begin{array}{r}\text { PZ1(UF) } \\
P Z 1(F, 45)\end{array}$ & 3 & $\begin{array}{l}5.05 \\
5.05\end{array}$ & $\begin{array}{l}0.05 \\
0.05\end{array}$ & $\begin{array}{l}A 069(1) \\
A 024(1)\end{array}$ & $\begin{array}{l}169300 \\
117091\end{array}$ & $\begin{array}{l}0.21 \\
0.21\end{array}$ & $\begin{array}{l}0 \\
0\end{array}$ & $\begin{array}{l}0 \\
0\end{array}$ \\
\hline PZi(f5O) & & 5.05 & 0.2 & A211(3) & 139716 & 0.24 & 0.00003 & 0.00004 \\
\hline PZ1(AS) & & 29 & 0.05 & $A 200(4)$ & 87992 & 0.26 & 0.00004 & 0.00002 \\
\hline PZ2(UF) & 3 & 5.04 & 0.05 & A070(2) & 169261 & 0.27 & 0.00004 & 0.00001 \\
\hline PZZ(F.45) & & 5.04 & 0.05 & $A 026(2)$ & 117091 & 0.27 & 0.00004 & 0.00001 \\
\hline P22(F50) & & 5.04 & 0.25 & A212(4) & 139716 & 0.26 & 0.00004 & 0.00002 \\
\hline PZ2(AS) & & 19 & 0.05 & A199(3) & 87992 & 0.24 & 0.00003 & 0.00004 \\
\hline PZ3 (UF) & 7 & 5.02 & 0.05 & $A 20 S(1)$ & 31919 & 0.27 & 0.00038 & 0.00031 \\
\hline$P 23(F-45)$ & & 5.02 & 0.05 & $A 206(2)$ & 31918 & 0.27 & 0.00005 & 0.00001 \\
\hline PZ3(F.45)ele & & 5.02 & & $A 0304(4)$ & 172800 & 0.27 & 0 & 0 \\
\hline P23(AS) & & 19 & 0.05 & $A 198(2)$ & 87992 & 0.27 & 0.00005 & 0.00001 \\
\hline PZ4(UF) & 7 & 5.05 & 0.05 & $A 208(4)$ & 31918 & 0.26 & 0.00004 & 0.00002 \\
\hline$P Z 4(F .45)$ & & 5.05 & 0.05 & $A 207(3)$ & 31918 & 0.24 & 0.00903 & 0.00004 \\
\hline PZ4(AS) & & 19 & 0.05 & $A 197(1)$ & 87993 & 0.27 & 0.00038 & 0.00031 \\
\hline PZ5 (UF) & 14 & 5 & 0.05 & $A 235(2)$ & 279392 & 0.26 & 0.00005 & 0.00001 \\
\hline$P 25(F .45)$ & & 5 & 0.05 & $A 234(1)$ & 204537 & 0.26 & 0.00007 & 0 \\
\hline PZ5 (F50) & & 5 & 0.15 & $A 005(1)$ & 356108 & 0.2 & 0 & 0 \\
\hline PZS(AS) & & 19 & 0.05 & A201(1) & 84045 & 0.27 & 0.00038 & 0.00031 \\
\hline PZ6(UF) & 14 & 5.09 & 0.05 & A131(4) & 80645 & 0.26 & 0.00004 & 0.00002 \\
\hline$P Z 6(F, 45)$ & & 5.09 & 0.05 & $A 124(1)$ & 105747 & 0.27 & 0.00038 & 0.00031 \\
\hline P26(F50) & & 5.09 & 0.25 & $A 006(2)$ & 356108 & 0.27 & 0.00004 & 0.00001 \\
\hline PZ6(AS) & & 19 & 0.05 & $A 202(2)$ & 84044 & 0.27 & 0.00005 & 0.00001 \\
\hline PZT(UF) & 28 & 5.01 & 0.05 & $A 088(4)$ & 75119 & 0.26 & 0.00006 & 0.00002 \\
\hline $\operatorname{PZT}(F-45)$ & & 5.01 & 0.05 & A118(4) & 32685 & 0.26 & 0.00006 & 0.00002 \\
\hline P27(F.45)ele & & 5.01 & 0.008 & $A 0385(6)$ & 172800 & 0.24 & 0 & 0 \\
\hline PZ7(F50) & & 5.01 & 0.4 & A0B5(1) & 75120 & 0.2 & 0 & 0 \\
\hline PZ7(AS) & & 19 & 0.05 & $A 080(4)$ & 86657 & 0.26 & 0.00006 & 0.00002 \\
\hline PZT(AS) & & 19 & 0.05 & $A 279(3)$ & 86772 & 0.24 & 0.00003 & 0 \\
\hline PZ8(UF) & 28 & 4.99 & 0.05 & A237(4) & 279391 & 0.24 & 0.00003 & 0.00003 \\
\hline PZ8(F.45) & & 4.99 & 0.05 & $A 236(3)$ & 279392 & 0.24 & 0.00003 & 0 \\
\hline PZ8(F50) & & 4.99 & 0.15 & $A 210(2)$ & 139716 & 0.27 & 0.00005 & 0.00001 \\
\hline PZ8 (AS) & & 19 & 0.05 & $A 203(3)$ & 34944 & 0.24 & 0.00003 & 0.00004 \\
\hline PZ9CUF) & 56 & 5.03 & 0.05 & A156(4) & 106198 & 0.26 & 0.00004 & 0.00002 \\
\hline$P 29(F .45)$ & & 5.03 & 0.05 & A157(1) & 204502 & 0.27 & 0.00038 & 0.00031 \\
\hline$P 29(F .45)$ & & 5.03 & 0.05 & $A 164(2)$ & 99484 & 0.27 & 0.00005 & 0.00001 \\
\hline PZ9(F.45)ele & & 5.03 & 0.033 & $A 0268(6)$ & 172800 & 0.23 & 0 & 0 \\
\hline PZ9(FSO) & & 5.03 & 0.4 & A151(3) & 185047 & 0.24 & 0.00003 & 0.00004 \\
\hline P29(AS) & & 19 & 0.05 & A264(4) & 15539 & 0.24 & 0.00003 & 0.00003 \\
\hline PZ9(AS) & & 19 & 0.05 & A686(1) & 241271 & 0.26 & 0.00003 & 0.00002 \\
\hline PZ10(UF) & 56 & 5.03 & 0.05 & A158(2) & 204484 & 0.27 & 0.00005 & 0.00001 \\
\hline PZ10(F.45) & & 5.03 & 0.05 & $A 277(1)$ & 675243 & 0.26 & 0.00007 & 0 \\
\hline$P Z 10$ (F50) & & 5.03 & 0.45 & A152(4) & 185047 & 0.26 & 0.00004 & 0.00002 \\
\hline PZ1O(AS) & & 19 & 0.05 & A266(4) & 16713 & 0.24 & 0.00003 & 0.00003 \\
\hline $\operatorname{PZ11}(F-45)$ & 98 & 4.99 & 0.05 & $A 272(4)$ & 83809 & 0.24 & 0.00003 & 0.00003 \\
\hline PZ11(F.45)el & & 4.99 & 0.031 & $A 0263(1)$ & 172800 & 0.24 & 0 & 0 \\
\hline PZ11(F50) & & 4.99 & 0.4 & A271(3) & 83806 & 0.24 & 0.00003 & 0 \\
\hline P211(AS) & & 19 & 0.05 & $A 270(4)$ & 32632 & 0.24 & 0.00003 & 0.00003 \\
\hline$P Z 12(F .45)$ & 98 & 4.99 & 0.05 & $A 281(3)$ & 588475 & 0.24 & 0.00003 & 0 \\
\hline PZ12(F50) & & 4.99 & 0.4 & $A 282(4)$ & 588480 & 0.24 & 0.00003 & 0.00003 \\
\hline P212(AS) & & 19 & 0.05 & $A 269(3)$ & 32628 & 0.24 & 0.60003 & 0.00003 \\
\hline PZ13(Uf) & 182 & 4.98 & 0.05 & A343(4) & 144140 & 0.24 & 0 & 0 \\
\hline $\operatorname{PZ13}(F .45)$ & & 4.98 & 0.05 & A334(3) & 143189 & 0.25 & 0.00005 & 0 \\
\hline $8213(F .45)$ el & & 4.98 & 0.032 & $A 0263(2)$ & 172800 & 0.22 & 0 & 0 \\
\hline PZ13 (F50) & & 4.98 & 0.05 & A342(3) & 144139 & 0.25 & 0.00005 & 0 \\
\hline PZ13(AS) & & 19 & 0.1 & A332(1) & 143190 & 0.27 & 0 & 0.00002 \\
\hline PZ14(UF) & 182 & 5.01 & 0.05 & A335(4) & 143188 & 0.24 & 0 & 0 \\
\hline$P 214(F .45)$ & & 5.01 & 0.05 & $A 340$ (1) & 144141 & 0.27 & 0 & 0.00002 \\
\hline PZ14(F50) & & 5.01 & 0.05 & A341(2) & 144140 & 0.27 & 0 & 0 \\
\hline P214(AS) & & 19 & 0.1 & A333(2) & 143189 & 0.27 & 0 & 0 \\
\hline PZ15(UF) & 364 & 5.02 & 0.05 & A702(1) & 173930 & 0.26 & 0.00008 & 0.00002 \\
\hline$P 215(F .45)$ & & 5.02 & 0.05 & A703(2) & 173929 & 0.26 & 0.0001 & 0.00007 \\
\hline$P 215(F .45)$ el & & 5.02 & 0.033 & $A 0264(3)$ & 172800 & 0.25 & 0 & 0 \\
\hline PZ1S(F50) & & 5.02 & 0.1 & A704(3) & 173929 & 0.3 & 0.00004 & 0.00001 \\
\hline PZ16(UF) & 364 & 5.02 & 0.05 & A708(3) & 233283 & 0.3 & 0.00004 & 0.00001 \\
\hline$P Z 16(F, 45)$ & & 5.02 & 0.05 & A706(4) & 233284 & 0.29 & 0.00006 & 0.00009 \\
\hline$P 216$ (F50) & & 5.02 & 0.05 & A707(1) & 233283 & 0.26 & 0.00008 & 0.00002 \\
\hline PZ16(AS) & & 19 & 0.1 & A710(2) & 23035 & 0.26 & 0.0001 & 0.00007 \\
\hline PZ17(UF) & 728 & 5.05 & 0.05 & C122(4) & 250872 & 0.28 & 0.00009 & 0.00012 \\
\hline$P 217$ (F.4S) & 728 & 5.05 & 0.05 & C118(4) & 111926 & 0.28 & 0.00009 & 0.00012 \\
\hline P217(F.45)el & & 5.05 & 0.033 & $A 0265(3)$ & 172800 & 0.25 & 0 & 0 \\
\hline PZ17(F50) & & 5.05 & 0.05 & $(117(3)$ & 111926 & 0.3 & 0.00007 & 0.00003 \\
\hline P217(AS) & & 19 & 0.1 & C120(2) & 250873 & 0.24 & 0.00027 & 0.00072 \\
\hline PZ18(UF) & 728 & & 0.05 & (1.19(1) & 250873 & 0.26 & 0.00007 & 0.00001 \\
\hline$P 218(F, 45)$ & 728 & 5.09 & 0.05 & $(126(4)$ & 93358 & 0.28 & 0.00009 & 0.00012 \\
\hline PZ18(F5O) & & 5.09 & 0.05 & (1.21(3) & 250872 & 0.3 & 0.00007 & 0.00003 \\
\hline PZ18(AS) & & 19 & 0.1 & C124(2) & 93358 & 0.24 & 0.00027 & 0.00072 \\
\hline P219(UF) & & & 0.05 & (1.25(3) & 93358 & 0.3 & 0.00007 & 0.00003 \\
\hline P219(F.45) & 552 & 4.99 & 0.05 & C123(1) & 93358 & 0.26 & 0.00007 & 0.00001 \\
\hline PZ19(F.45)el & & 4.99 & 0.033 & $A 0267(5)$ & 172800 & 0.24 & 0 & \\
\hline PZ19(F50) & & 4.99 & 0.65 & (1.15(1) & 111927 & 0.26 & 0.00007 & 0.00001 \\
\hline P219(AS) & & 19 & 0.1 & (1.16(2) & 111926 & 0.24 & 0.00027 & 0.00072 \\
\hline PZZOCF.45)el & 1000 & 5 & 0.032 & $A 0274(6)$ & 259200 & 0.23 & 0 & 0 \\
\hline
\end{tabular}


Page $\# 2$ - "PZ.ACTINIDES $x x^{n}$

Monday, February 6 9:42 AM 1995

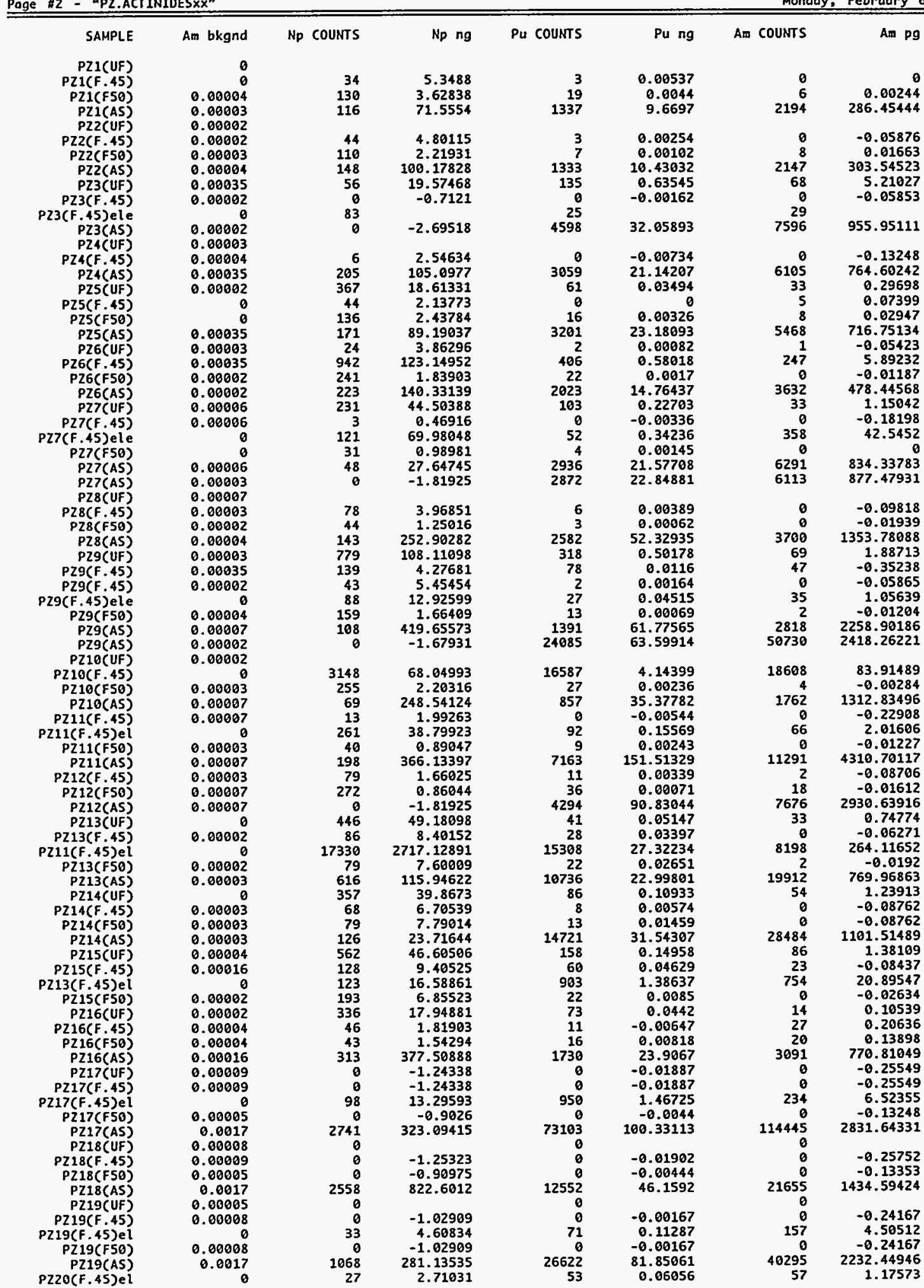


Table D.6. Spreadsheet Calculations Performed in Tables D.2 through D.5

\section{Table D.2. Test Matrices:}

Dilution factors (DF) are calculated for the unfiltered leachates (UF), the filtrates from the $0.45 \mu \mathrm{m}$ filtered solutions (F.45), and the filtrates from the CF50A filtered solutions (F50). The dilution factors are calculated from the mass of the empty solution bottle (LPE), the mass of the solution bottle plus the leachate solution (+SOLN), and the mass of deionized water and concentrated nitric acid added (+DIW+HNO3) to dilute the leachate as:

$$
\mathrm{DF}=\frac{((+\mathrm{SOLN})-(\mathrm{LPE})+(+\mathrm{DIW}+\mathrm{HNO} 3))}{((+\mathrm{SOLN})-(\mathrm{LPE}))}
$$

For example, the dilution factor for the unfiltered leachate of test BX1 is:

$$
\begin{gathered}
(\mathrm{UF} \mathrm{LPE})=24.27 \mathrm{~g} ;(+\mathrm{UF} \text { SOLN })=38.78 \mathrm{~g} ;(+\mathrm{DIW}+\mathrm{HNO})=10.97 \mathrm{~g} \\
\mathrm{DF}=(38.78-24.27+10.07) /(38.78-24.27)=1.6940
\end{gathered}
$$

\section{Table D.3. Cation Results:}

The analytical results of the diluted solutions (given in the "I, ACL" columns) are corrected for the dilution by multiplying them by the dilution factor. The dilutioncorrected concentrations are given in the "I, ppm" columns for each element.

For example, the dilution-corrected aluminum concentration for test BX1 is:

$$
\begin{gathered}
\mathrm{DF}=1.694 ; \mathrm{Al}, \mathrm{ACL}=0.54 \mathrm{ppm} \\
\mathrm{Al}, \mathrm{ppm}=(1.694) *(0.54)=0.9148 \mathrm{ppm}
\end{gathered}
$$

The normalized mass loss is calculated from the dilution-corrected concentration of the element of interest, the background concentration of the element of interest, the SA/V of the test, and the weight fraction of the element of interest. The background concentration and the weight fraction of each element, $f(i)$, are given in the tables.

For example, the normalized mass loss based on aluminum, $\mathrm{NL}(\mathrm{Al})$, in test $\mathrm{BX} 1$ is:

$$
\begin{gathered}
\mathrm{Al}, \mathrm{ppm}=0.9148 \text { ppm, Al background }=0.56 \mathrm{ppm}, \mathrm{SA} / \mathrm{V}=10.01 \mathrm{~m}^{-1}, \mathrm{f}(\mathrm{Al})=0.0203 \\
\mathrm{NL}(\mathrm{Al})=(0.9148-0.56) /(10.01 * 0.0203)=1.75 \mathrm{~g} / \mathrm{m}^{2} .
\end{gathered}
$$

Table D.4. Anion Results:

The dilution factors are calculated using the leachate volume and the volume of deionized water used to dilute the leachates prior to analysis for carbon analysis (data not included). Solutions analyzed for other anions were not diluted. 
The total carbon (TC) and total organic carbon (TOC) results were corrected for dilution by multiplying by the dilution factor ( $\mathrm{C}$ dil factor). The dilution-corrected results are given in the TC and TOC columns. The total inorganic carbon (TIC) concentration was calculated as:

$$
\mathrm{TIC}=\mathrm{TC}-\mathrm{TOC}
$$

Table D.5. Actinide Results:

The masses of neptunium, plutonium, and americium measured to be in the various fractions were determined from the number of counts under each peak in the alpha spectra. The efficiencies of the detectors and the number of background counts were determined using standards and blanks. The mass of each actinide is calculated as follows:

An, $\mu g=($ An counts/LT - An bkgnd $) *(1 / E F F) *($ SOLN ml/ALQ ml $) * A N$

where $(\mathrm{LT}=\mathrm{LIVE}$ TIME, $\mathrm{ALQ}=\mathrm{ALIQUOT})$

and $\mathrm{AN}$ is $\quad 3.83 \times 10^{-2} \mathrm{mg} \cdot \mathrm{s} /$ dissociation for $\mathrm{Np}-237$

$4.36 \times 10^{-4} \mathrm{mg} \cdot \mathrm{s} /$ dissociation for $\mathrm{Pu}-239$

$7.87 \times 10^{-6} \mathrm{mg} \cdot \mathrm{s} /$ dissociation for Am-241

For example, the mas s of plutonium in the F50 fraction of test $\mathrm{BX} 1$ is:

$\mathrm{Pu}=56$ counts

LIVE TIME $=185048$ seconds

$\mathrm{Pu}$ bkgnd $=0$ counts for detector 1

EFFICIENCY $=0.22$ counts/disintegrations for detector 1

SOL' $\mathrm{N} \mathrm{ml}=16.62 \mathrm{ml}$

ALIQUOT $\mathrm{ml}=0.03 \mathrm{ml}$

$$
(56 / 185048-0) *(1 / 0.22) *(16.62 / 0.03) * 4.36 \times 10^{-4}=3.32 \times 10^{-4} \mu \mathrm{g}
$$


Distribution for ANL-94/34

Internal:

J. K. Bates (30)

J. E. Harmon

J. J. Laidler

J. C. Cunnane

J. E. Helt

W. E. Ebert (5)

T. R. Johnson

C. C. McPheeters

TIS Files

External:

DOE-OSTI (2)

ANL-E Library (2)

ANL-W Library

Manager, Chicago Operations Office, DOE

A. Bindokas, DOE-CH

J. Haugen, DOE-CH

Chemical Technology Division Review Committee Members:

E. R. Beaver, Monsanto Company, St. Louis, MO

D. L. Douglas, Consultant, Bloomington, MN

R. K. Genung, Oak Ridge National Laboratory, Oak Ridge, TN

J. G. Kay, Drexel University, Philadelphia, PA

G. R. St. Pierre, Ohio State University, Columbus, $\mathrm{OH}$

J. Stringer, Electric Power Research Institute, Palo Alto, CA

J. B. Wagner, Arizona State University, Tempe, AZ

T. Ahn, U. S. Nuclear Regulatory Commission,Washington, DC

D. H. Alexander, USDOE, Civilian Radioactive Waste Management, Washington, DC

J. Allison, USDOE, Office of Waste Operations, Germantown, MD

S. Bates, Idaho Falls, ID

H. Benton, B\&W Fuel Company, Las Vegas, NV

A. Berusch, USDOE, Office of Civilian Radioactive Waste Management, Washington, DC

N. E. Bibler, Westinghouse Savannah River Company, Aiken, SC

J. M. Boak, USDOE, Yucca Mountain Site, Las Vegas, NV

K. Boomer, Westinghouse Hanford Company, Richland, WA

W. Bourcier, Lawrence Livermore National Laboratory, Livermore, CA

E. Bramlitt, Defense Nuclear Agency, Kirtland Air Force Base, Kirtland, NM

A. Brandstetter, Science Applications, International Corporation, Las Vegas, NV

N. Brown, USDOE, Richland Operations Office, Richland, WA

J. Bucher, Lawrence Berkeley Laboratory, Berkeley, CA

J. Canepa, Los Alamos National Laboratory, Los Alamos, NM

K. A. Chacey, USDOE, Office of Waste Management, Germantown, MD

D. Chestnut, Lawrence Livermore National Laboratory, Livermore, CA

G. R. Choppin, Florida State University, Tallahassee, FL

S. Clark, University of Georgia, Savannah River Ecology Laboratory, Aiken, SC

P. Cloke, Science Applications International Corp., Las Vegas, NV

M. O. Cloninger, Mac Technical Services, Inc., Richland, WA 
D. Codell, U. S. Nuclear Regulatory Commission, Washington, DC

G. Colten-Bradley, U. S. Nuclear Regulatory Commission, Rockville, MD

C. Colter, Los Alamos National Laboratory, Los Alamos, NM

S. Coplan, U. S. Nuclear Regulatory Commission, Washington, DC

S. P. Cowan, USDOE, Office of Waste Management, Germantown, MD

J. Davidson, U. S. Environmental Protection Agency, Washington, DC

J. Docka, Roy F. Weston, Inc., Washington, DC

R. Dresser, Roy F. Weston, Inc., Washington, DC

R. S. Dyer, Yucca Mountain Project Office, Las Vegas, NV

R. E. Erickson, USDOE, Office of Waste Operations, Germantown, MD

E. Essington, Los Alamos National Laboratory, Los Alamos, NM

F. M. Estes, Idaho State University, Pocatello, ID

R. C. Ewing, Department of Geology, University of New Mexico, Albuquerque, NM

D. Farr, Los Alamos National Laboratory, Los Alamos, NM

R. Fish, B\&W Fuel Company, Las Vegas, NV

J. Gauthier, Sandia National Laboratories, Albuquerque, NM

F. Gelbard, Sandia National Laboratories, Albuquerque, NM

C. P. Gertz, USDOE, Yucca Mountain Project Office, Las Vegas, NV

S. E. Gomberg, USDOE, Office of Civilian Radioactive Waste Management, Washington, DC

W. Gray, Battelle Pacific Northwest Laboratory, Richland, WA

P. W. Hart, USDOE, Office of Technology Development, Germantown, MD

J. Hennessey, USDOE, Office of Waste Operations, Germantown, MD

D. Hobart, LANL-TTSO/USDOE Headquarters, Germantown, MD

J. Hunt, University of California, Berkeley, Berkeley, CA

D. Hutchins, Martin Marietta Systems, Inc., Oak Ridge, TN

C. Interrante, U. S. Nuclear Regulatory Commission, Washington, DC

C. Jantzen, Westinghouse Savannah River Company, Aiken, SC

L. J. Jardine, Lawrence Livermore National Laboratory, Livermore, CA

P. Kearl, Oak Ridge National Laboratory, Grand Junction, CO

J. Keith, Daniel B. Stephens \& Associates, Albuquerque, NM

J. Kerrisk, Los Alamos National Laboratory, Los Alamos, NM

W. S. Ketola, USDOE, West Valley Project Office, West Valley, NY

D. A. Knecht, Lockheed Idaho Technology Company, Idaho Falls, ID

W. L. Kuhn, Battelle Pacific Northwest Laboratory, Richland, WA

K. S. Kung, Los Alamos National Laboratory, Los Alamos, NM

J. H. Lee, Intera, Las Vegas, NV

W. Lee, Environmental Evaluation Group, Albuquerque, NM

W. W. Lee, University of California, Berkeley, CA

J. C. Lehr, USDOE, Office of Environmental Restoration, Germantown, MD

H. Leider, Lawrence Livermore National Laboratory, Livermore, CA

R. A. Lemons, Los Alamos National Laboratory, Los Alamos, NM

S. Levy, Los Alamos National Laboratory, Los Alamos, NM

D. Livingston, USDOE, Yucca Mountain Site, Las Vegas, NV

R. Lopez, Los Alamos National Laboratory, Los Alamos, NM

J. J. Lorenz, USDOE, Yucca Mountain Site, Las Vegas, NV

R. Luce, Nuclear Waste Technical Review Board, Arlington, VA 
W. Lutze, University of New Mexico, Albuqueque, NM

H. Manaktala, Southwest Research Institute, San Antonio, TX

S. Martin, Lawrence Livermore National Laboratory, Livermore, CA

J. M. Matuszek, JMM Consulting, Del Mar, NY

J. McCarthy, Oak Ridge National Laboratory, Oak Ridge, TN

L. McDowell-Boyer, Oak Ridge National Laboratory, Grand Junction, CO

T. W. McIntosh, USDOE, Office of Waste Management, Germantown, MD

J. Meldrum, University of Nevada - Las Vegas, Las Vegas, NV

A. Mitchell, Los Alamos National Laboratory, Los Alamos, NM

R. Morissette, Science Applications International Corp., Las Vegas, NV

D. Morris, Los Alamos National Laboratory, Los Alamos, NM

P. K. Nair, Southwest Research Institute, San Antonio, TX

S. Nelson, Las Vegas, NV

B. Newman, Los Alamos National Laboratory, Los Alamos, NM

C. Novak, Sandia National Laboratories, Albuquerque, NM

E. Nuttall, University of New Mexico, Albuquerque, NM

W. O'Connell, Lawrence Livermore National Laboratory, Livermore, CA

G. C. S. Ordaz, USDOE, Office of Technology Development, Germantown, MD

C. Palmer, Lawrence Livermore National Laboratory, Livermore, CA

R. Palmer, West Valley Nuclear Services, West Valley, NY

H. Papenguth, Sandia National Laboratories, Albuquerque, NM

U-Sun Park, Science Applications International Corp., Las Vegas, NV

W. D. Pearson, Westinghouse Savannah River Company, Aiken, SC

C. Peterson, U. S. Nuclear Regulatory Commission, Washington, DC

T. H. Pigford, University of California, Berkeley, CA

M. J. Plodinec, Westinghouse Savannah River Company, Aiken, SC

W. Polzer, Los Alamos National Laboratory, Los Alamos, NM

W. G. Ramsey, Westinghouse Savannah River Company, Aiken, SC

P. Reimus, Los Alamos National Laboratory, Los Alamos, NM

B. Robinson, Los Alamos National Laboratory, Los Alamos, NM

P. Rogers, Los Alamos National Laboratory, Los Alamos, NM

R. Rundberg, Los Alamos National Laboratory, Los Alamos, NM

C. G. Russomanno, USDOE, Civilian Radioactive Waste Management, Washington, DC

J. Ryan, University of Colorado, Boulder, CO

R. Schulze, Los Alamos National Laboratory, Los Alamos, NM

W. C. Schutte, USDOE, Office of Technology Development, Germantown, MD

R. Sherman, Los Alamos National Laboratory, Los Alamos, NM

M. Siegel, Sandia National Laboratories, Albuquerque, NM

M. Silva, Environmental Evaluation Group, Albuquerque, NM

R. Silva, Lawrence Livermore National Laboratory, Livermore, CA

A. Simmons, USDOE, Las Vegas, NV

E. Springer, Los Alamos National Laboratory, Los Alamos, NM

J. Sproull, Westinghouse Savannah River Company, Aiken, SC

D. Stahl, M\&O/B\&W Fuel Company, Las Vegas, NV

W. Steinkampf, U.S. Geological Survey, Lakewood, CO

L. Stetzenbach, University of Nevada - Las Vegas, Las Vegas, NV 
R. B. Stout, Lawrence Livermore National Laboratory, Livermore, CA

D. Strachan, Battelle Pacific Northwest Laboratories, Richland, WA

M. Tomozawa, Rensselaer Polytechnic Institute, Troy, NY

I. Triay, Los Alamos National Laboratory, Los Alamos, NM

V. Trice, USDOE, Office of Waste Management, Germantown, MD

D. Turner, Southwest Research Institute, San Antonio, TN

B. Viani, Lawrence Livermore National Laboratory, Livermore, CA

J. Wan, New Mexico Tech, Socorro, NM

L. Wang, University of New Mexico, Albuquerque, NM

R. Weiner, Environmental Evaluation Group, Albuquerque, NM

M. Whitbeck, Desert Research Institute, University of Nevada, Reno, NV

C. N. Wilson, Westinghouse Hanford Company, Richland, WA

J. Wilson, New Mexico Tech, Socorro, NM

M. Wilson, Sandia National Laboratories, Albuquerque, NM

A. Wistrom, Los Alamos National Laboratory, Los Alamos, NM

J. H. Wolfram, Biotechnology-INTEL, Montana State University, Bozeman, MT

K. Wolfsberg, Los Alamos National Laboratory, Los Alamos, NM

A. Wollerman, Science Applications International Corp., Germantown, MD

A. Yang, U.S. Geological Survey, Denver, CO

M. P. Gardiner, Harwell Laboratory, Didcot, Oxon, UNITED KINGDOM

B. Grambow, Kernforschungszentrum Karlsruhe, GmbH, GERMANY

J. Kim, Kernforschungszentrum Karlsruhe, GERMANY

H. Nitsche, Forschungszentrum Rossendorf e.V., Institut fur Radiochemie, GERMANY

T. Payne, Australian Nuclear Science and Technology Organization, AUSTRALIA

P. Van Iseghem, Boeretang, BELGIUM

E. Vernaz, Centre d'Etudes Nucleares de la Valle du Rhone, Marcoule, FRANCE

P. Vilks, Atomic Energy of Canada, Whiteshell Laboratories, Pinawa, Manitoba, CANADA

L. Werme, Svensk Karnbranslehantering AB, Stockholm, SWEDEN 